\title{
AN EXPERIMENTAL AND NUMERICAL STUDY OF THE STRAIN RATE EFFECTS ON MILD STEEL FOR CYCLIC LOADING
}

\author{
A thesis submitted to \\ the Faculty of Graduate Studies and Postdoctoral Affairs \\ in partial fulfillment of the requirements for the degree \\ Master of Applied Science
}

by

Adam Walker
Department of Civil and Environmental Engineering Carleton University Ottawa, Ontario, Canada

Ottawa-Carleton Institute of Civil and Environmental Engineering September, 2012

(C) 2012, Adam Walker 
Library and Archives

Canada

Published Heritage

Branch

395 Wellington Street

Ottawa ON K1A ON4

Canada
Bibliothèque et

Archives Canada

Direction du

Patrimoine de l'édition

395 , rue Wellington

Ottawa ON K1A ON4

Canada
Your file Votre référence

ISBN: 978-0-494-93639-9

Our file Notre référence

ISBN: $978-0-494-93639-9$
NOTICE:

The author has granted a nonexclusive license allowing Library and Archives Canada to reproduce, publish, archive, preserve, conserve, communicate to the public by telecommunication or on the Internet, loan, distrbute and sell theses worldwide, for commercial or noncommercial purposes, in microform, paper, electronic and/or any other formats.

The author retains copyright ownership and moral rights in this thesis. Neither the thesis nor substantial extracts from it may be printed or otherwise reproduced without the author's permission.
AVIS:

L'auteur a accordé une licence non exclusive permettant à la Bibliothèque et Archives Canada de reproduire, publier, archiver, sauvegarder, conserver, transmettre au public par télécommunication ou par l'Internet, prêter, distribuer et vendre des thèses partout dans le monde, à des fins commerciales ou autres, sur support microforme, papier, électronique et/ou autres formats.

L'auteur conserve la propriété du droit d'auteur et des droits moraux qui protege cette thèse. $\mathrm{Ni}$ la thèse ni des extraits substantiels de celle-ci ne doivent être imprimés ou autrement reproduits sans son autorisation.
In compliance with the Canadian Privacy Act some supporting forms may have been removed from this thesis.

While these forms may be included in the document page count, their removal does not represent any loss of content from the thesis.
Conformément à la loi canadienne sur la protection de la vie privée, quelques formulaires secondaires ont été enlevés de cette thèse.

Bien que ces formulaires aient inclus dans la pagination, il n'y aura aucun contenu manquant. 


\begin{abstract}
A combined experimental and numerical study has been carried out to determine the effects of strain rate on mild steel under mainly cyclic loading. In addition, effects of loading history and cyclic mean strain on the flow stress were also investigated.

Tapered and notched round specimens of CAN/CSA G40.20/21 Grade 300W and ASTM A572 Grade 50 steels were tested under a variety of cyclic loading conditions at a strain rate ranging from $10^{-4} \mathrm{~s}^{-1}$ and $10^{-1} \mathrm{~s}^{-1}$. A positive rate sensitivity on flow stress has been found for both these materials during initial cyclic loading. However, with continual loading, tests performed at the strain rates of $10^{-2}$ and $10^{-1} \mathrm{~s}^{-1}$ can be seen to show negative flow stress rate sensitivity due to adiabatic heating. The rupture strain has been found to decrease with strain rate for the strain rate range considered in this study. On the other hand, findings on the effect of strain rate on the fatigue life are inconclusive. The loading history and mean strain effects on the final cyclic stress-strain curve at a strain range have also been found to be small if a sufficient number of loading cycles have been performed at that particular strain range.

A procedure has been developed to generate strain rate dependent input data for a numerical simulation under a general loading condition for moderate strain rate using the mixed-mode hardening model by Lemaitre and Chaboche. Finite element analyses using the input data generated from this procedure has been found to be able to simulate the strain rate effect on the load-deformation behaviour of the tests. However, the numerical simulations have been less accurate on tests performed at a strain range other than the one used in the calibration to generate the input data.
\end{abstract}




\section{ACKNOWLEDGEMENTS}

I would like to thank my supervisor Dr. Heng Aik Khoo for his unwavering guidance and support from the conception to the final submittal of this thesis. His contributions to this work have been invaluable.

This study would not be possible without the help from the staff in the Civil and Environmental Engineering Laboratory at Carleton University, special thanks to Stan Conley for his help with the experimental portion of this thesis.

I would also like to thank Dr. Yasser Hassan for lending the infrared temperature measuring equipment used in this thesis.

Finally, I would like thank Mary Compton, my family, friends and fellow graduate students for their support and advice throughout my time of study at Carleton University.

Funding for this research was provided by National Sciences and Engineering Research Council (NSERC) of Canada. 


\section{TABLE OF CONTENTS}

Chapter 1 Introduction ............................................................................................. 1

$1.1 \quad$ Objective of the Thesis .............................................................................. 2

1.2 Methodology Used in the Research .......................................................... 2

$1.3 \quad$ Organization of the Thesis .......................................................................

Chapter 2 Literature Review............................................................................. 5

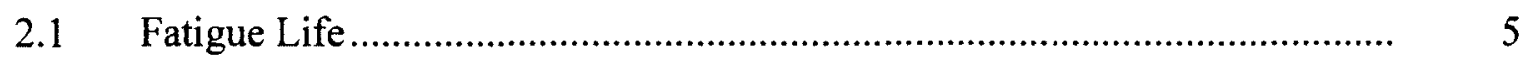

$2.2 \quad$ Specimen Profile (Stress Triaxiality) ............................................................

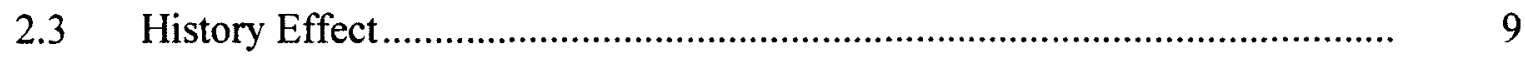

$2.4 \quad$ Mean Strain Effect .............................................................................. 12

$2.5 \quad$ Strain Rate

2.5.1 Strain Rate Effect on Stress ........................................................... 15

2.5.2 Strain Rate Effect on Fatigue Life ....................................................... 18

2.5.3 Strain Rate Effect on Fracture Strain ....................................................... 19

$2.6 \quad$ Dynamic Strain Aging ………….......................................................... 20

2.7 Constitutive Models ............................................................................. 24

Charter $3 \quad$ Testing Program .....................................................................................

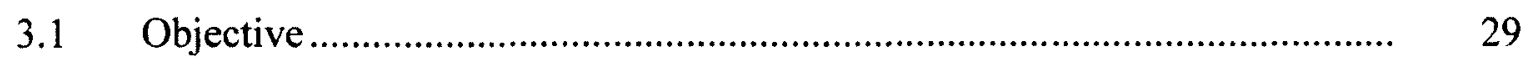

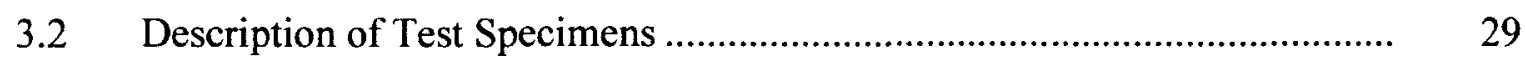

3.2.1 Specimen Designation ................................................................... 30

3.2.2 Tapered Specimens .........................................................................

3.2.3 Notch Specimens .........................................................................

$3.3 \quad$ Specimen Preparation .......................................................................... 35 
3.4 Test Setup and Instrumentation ............................................................. 36

3.5 Temperature Measurement Methods and Calibration..................................... 37

3.6 Strain Rate Control ........................................................................... 39

Charter $4 \quad$ Test Results and Discussions.......................................................................... 53

4.1 Analyses of Tapered Specimen Test Results ................................................ 54

4.1.1 Loading Control on Tapered Test Specimen ........................................... 55

4.1.2 Strain Rate Effects on Stress ............................................................. 57

4.1.3 Strain Rate Effects on Rupture Strain ..................................................

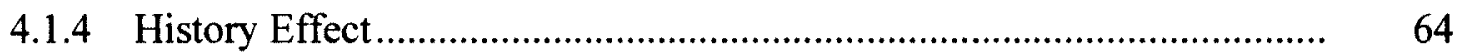

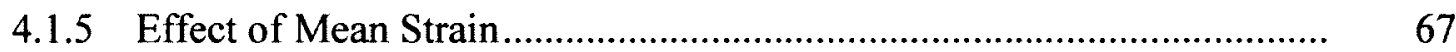

4.2 Analyses of Notched Specimen Test Results................................................. 71

4.2.1 Loading Control on Notched Specimen Tests ......................................... 71

4.2.2 Strain Rate Effects on Stress ...................................................................

4.2.3 Fatigue Life Definition for Notched Specimens ...................................... 78

4.2.4 Strain Rate and Strain Range Effects on Fatigue Life ............................. 82

Charter 5 Numerical Modelling of the Strain Rate Effects on Flow Stress .. 138

5.1 Monotonic Test Data Used for Calibration.................................................... 139

5.1.1 Full and Half Gauge Length Tapered Model Comparison ....................... 139

5.1.2 Correction for Non-Uniaxial Stress State Due to Specimen Necking ..... 141

5.1.3 Correction for Tapered Profile .............................................................. 141

5.1.4 Procedure for Generating Monotonic Flow Stress Versus True Plastic Strain Curves. 
5.2 Modelling the Effect of Strain Rate for a Mixed-Mode Hardening Plasticity Model

5.3 Validation of Optimized Yield Stress-Kinematic Hardening Parameters (Plasticity Model).

5.3.1 Numerical Simulations of the Cyclic Tests Used to Calibrate and Optimize the Kinematic Hardening Parameters

5.3.2 Numerical Simulations of Other Tests

5.3.2.1 Numerical Simulations of D Series Tests (Tapered Specimens) .......

5.3.2.2 Numerical Simulations of Notched Specimen Tests at $\pm 2 \%$ Strain Range

5.3.2.3 Effects of Strain Range and Mean Strain in Numerical Simulations.

5.3.2.4 Numerical Simulations of ATE2I, ATE3I, BTE3I, ANE4R4 and ANE4R8

5.3.2.5 Numerical Simulation of ATE2E

5.3.2.6 Numerical Simulation of ATE2C-20

5.4 Summary of Numerical Simulations.

Charter 6 Conclusions and Recommendations .................................................. 183

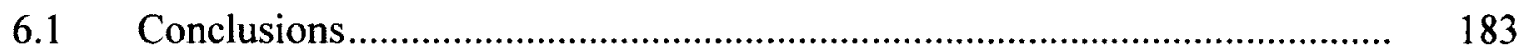

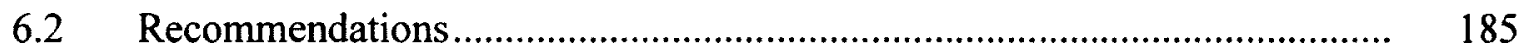

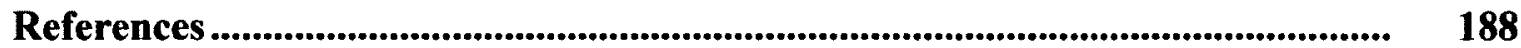

Appendix A Determination of True Strain Ranges for Notched Specimens ... 196

Appendix B Additional Test Data............................................................................... 198

Appendix C Mesh Convergence Study .......................................................................... 225 
Appendix D Larger Diameter Specimen Taper Correction Factor for Monotonic and Cyclic Loading...................................................... 233

Appendix E Representing Strain Rate Effects on Flow Stress Using A Power Law Equation ..............................................................

Appendix F Additional Results of Numerical Simulations ............................. 248

Appendix G Calibration and Optimization of the Kinematic Hardening

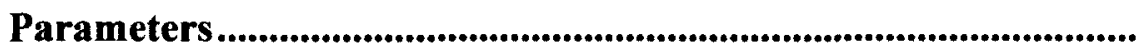




\section{LIST OF TABLES}

Table

Page

3.1 Chemical composition of the major alloying elements

44

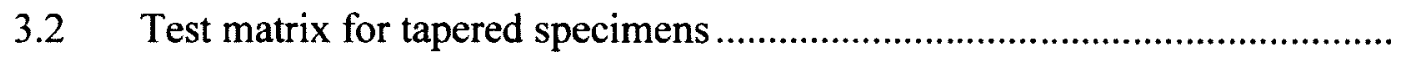

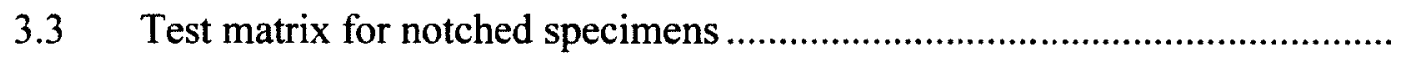

3.4 Rationale for the testing series

3.5 Values of gain used for the $25.4 \mathrm{~mm}$ (1 inch) axial extensometer

3.6 Values of gain used for the diametral extensometer...

3.7 Values of gain used for the $50.8 \mathrm{~mm}$ ( $2 \mathrm{inch}$ ) axial extensometer

4.1 Measured true strain rates and strain ranges for tapered specimens......

4.2 Temperature measurements using the IR gun

4.3 Strains at fracture

4.4 Hysteresis loop size based on average true stress for the given cycle and strain range for $\mathrm{ATE} 2 \mathrm{C}-20$

4.5 Hysteresis loop size based on average true stress for the given cycle and strain range for $\mathrm{ATE} 2 \mathrm{E}$

4.6 Measured average true strain rates and strain ranges for the notched specimens 
4.7 Peak temperature measurements during testing.

4.8 Minimum, maximum and range of measured average true strain for selected loading cycle numbers of notched specimens

4.9 Fatigue and separation lives for material A notch specimens

100

4.10 Fatigue and separation lives for material B notch specimens

4.11 Constants for Eq. (4.8) from fitted lines

5.1 Monotonic tests by Chen (2010)

159

5.2 Parameters for generating the corrected monotonic true flow stress versus true plastic strain curves

5.3 Difference in the generated (saturated) yield stress at various strain rates to that at the strain rate of $5 \times 10^{-1} \mathrm{~s}^{-1}$

5.4 Optimized $\sigma_{S}^{o}, \sigma_{L}^{o}, C_{n}$ and $\gamma_{n}$ parameters for materials A and B

A.1 Parameters used in generating the flow stress and backstress, taken from Wen (2012)

A.2 Estimated engineering strains to produce the desired true strains in the simulation

E.1 Parameters for Eq. (2.8) calibrated with monotonic test data and the assumed backstress 
E.2 Test and predicted yield stresses for monotonic tests of Chen (2010) calculated using Eq. (2.8) with calibrated parameters in Table E.1

E.3 Parameters for Eq. (2.8) and backstresses calibrated with cyclic and monotonic test data with the assumed backstress $\left(\alpha_{I}\right)$

E.4 Test and predicted yield stresses from the monotonic (Chen 2010) and cyclic tests and calculated using Eq. (2.8) with the calibrated parameters and backstresses in Table E.3 ......

G.1 Material model parameters used in finding the optimum kinematic hardening parameters and their associated error for material A.

G.2 Material model parameters used in finding the optimum kinematic hardening parameters and their associated error for material $\mathrm{B}$ 


\section{LIST OF FIGURES}

Figure

Page

2.1 Generalized flow stress versus temperature curves at a strain showing the effect of dynamic strain aging at two different strain rates (Cunningham 1999)

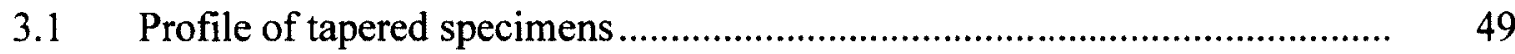

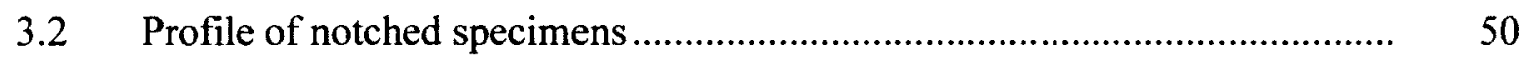

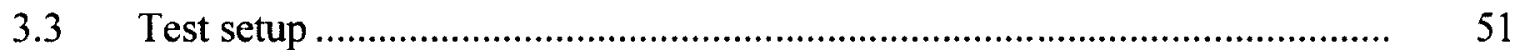

3.4 Temperature measuring device and thermocouple ................................ 52

4.1 Engineering stress versus engineering strain curves for BTE1 Is................. 103

4.2 Engineering stress versus engineering strain curves for BTE2Is................. 103

4.3 Engineering stress versus engineering strain curves for BTE3Is................. 104

4.4 Cyclic engineering stress versus engineering strain curves for BTE1 Is....... 104

4.5 Cyclic engineering stress versus engineering strain curves for BTE2Is........ 105

4.6 Cyclic engineering stress versus engineering strain curves for BTE3Is........ 105

4.7 Measurements versus time history during the drop in stress seen when the BTE2I-2 specimen was being pulled to fracture

4.8 Part of the $10^{\text {th }}$ cycle for ATE1I, ATE2I and ATE3I at the $\pm 0.5 \%$ strain range

4.9 Part of the $10^{\text {th }}$ cycle for ATE1I, ATE2I and ATE3I at the $\pm 1 \%$ strain

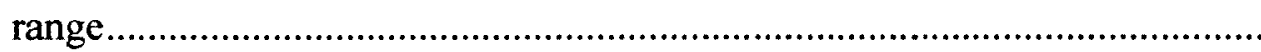

4.10 Part of the $10^{\text {th }}$ cycle for ATE1I, ATE2I and ATE3I at the $\pm 1.5 \%$ strain range 
4.11 Part of the $10^{\text {th }}$ cycle for ATE1I, ATE2I and ATE3I at the $\pm 2 \%$ strain range

4.12 Part of the $10^{\text {th }}$ cycle for ATE1D, ATE2D, ATE3D and ATE4D at the $\pm 2 \%$ strain range

4.13 Part of the $10^{\text {th }}$ cycle for ATE1D, ATE2D, ATE3D and ATE4D at the $\pm 0.5 \%$ strain range

4.14 Half engineering stress range versus cumulative plastic engineering strain at the $5^{\text {th }}$ and $10^{\text {th }}$ cycle of each strain range for ATEIs.

4.15 Half engineering stress range versus cumulative plastic engineering strain at the $5^{\text {th }}$ and $10^{\text {th }}$ cycle of each strain range for ATEDs

4.16 Magnified view of the engineering stress versus engineering strain curves for BTE1I at the $\pm 0.5 \%$ strain range

4.17 Magnified view of the engineering stress versus engineering strain curves for $\mathrm{BTE} 1 \mathrm{I}$ at the $\pm 1 \%$ strain range

4.18 Magnified view of the engineering stress versus engineering strain curves for BTE1I at the $\pm 1.5 \%$ strain range

4.19 Magnified view of the engineering stress versus engineering strain curves for BTE1I at the $\pm 2 \%$ strain range

4.20 Magnified view of the engineering stress versus engineering strain curves for $\mathrm{BTE} 1 \mathrm{D}$ at the $\pm 2 \%$ strain range

4.21 Magnified view of the engineering stress versus engineering strain curves for $\mathrm{BTE} 1 \mathrm{D}$ at the $\pm 0.5 \%$ strain range 
4.22 Magnified view of the engineering stress versus engineering strain curves close to the peak stress for I series tests of material A when being pulled to fracture

4.23 Magnified view of the engineering stress and actuator displacement versus time approximately between the strain of 0.0 and 0.1 when being pulled to fracture for ATE1I

4.24 Magnified view of the engineering stress and actuator displacement versus time approximately between the strain of 0.0 and 0.1 when being pulled to fracture for ATE2I

4.25 Magnified view of the engineering stress and actuator displacement versus time approximately between the strain of 0.0 and 0.1 when being pulled to fracture for ATE3I

4.26 Engineering strain at fracture versus strain rate.

115

4.27 True strain at fracture versus strain rate.

4.28 Fracture location of the ATE1I specimen

4.29 The 10th cycle of ATE2D and ATE2I, and the 20th cycle of ATE2D-20 at the $\pm 2 \%$ strain range

4.30 The 10th cycle of ATE3D and ATE3I at the $\pm 2 \%$ strain range...

4.31 The 10th cycle of ATE2D and ATE2I, and the 20th cycle of ATE2D-20 at the $\pm 0.5 \%$ strain range

4.32 The 10th cycle of BTE2D and BTE2I at the $\pm 0.5 \%$ strain range

4.33 The 10th cycle of ATE3D and ATE3I at the $\pm 0.5 \%$ strain range.

4.34 The 10th cycle of BTE3D and BTE3I at the $\pm 0.5 \%$ strain range 
4.35 Tensile behaviour of ATE2D-20 and ATE2I at the strain range of $\pm 0.5 \% \ldots$

4.36 Compressive behaviour of ATE2D-20 and ATE2I at the strain range of $\pm 0.5 \%$

4.37 Shifted final half-cycle at each strain range for ATE2C and ATE2I with the peak shifted stresses

4.38 Enhanced view of Fig. 4.37 for ATE2C and ATE2I

4.39 Enhanced view of the shifted final half cycle at each strain range for ATE2C-20 and ATE2I including the peak shifted stresses

4.40 Enhanced view of the shifted half cycle for $10^{\text {th }}$ and $20^{\text {th }}$ cycle at various strain ranges for $\mathrm{ATE} 2 \mathrm{C}-20$

4.41 Enhanced view of the shifted half cycle for selected cycles of ATE2C-200 and ATE2I including the peak shifted stresses.

4.42 Tensile behaviour of ATE2C-200 at the selected cycle numbers.

4.43 Enhanced view of the shifted final half cycle at each strain range for part 1 of 3 of the strain range sequence for ATE2E and $\pm 1 \%$ strain range for ATE2I

4.44 Enhanced view of the shifted final half cycle at each strain range for part 2 of 3 of the strain range sequence for ATE2E and $\pm 1 \%$ strain range for ATE2I

4.45 Enhanced view of the shifted final half cycle at each strain range for part 3 of 3 of the strain range sequence for ATE2E and $\pm 1 \%$ strain range for ATE2I 
4.46 Engineering stress versus engineering strain curve of ANE1R2 for the initial 30 cycles

4.47 Engineering stress versus engineering strain curve of ANE1R4 for the initial 30 cycles

4.48 Engineering stress versus engineering strain curve of ANE1R8

4.49 Engineering stress versus engineering strain curve of ANE4R8 for the initial 10 cycles

4.50 Peak tensile engineering stress versus cycle number for ANE1R2, ANE2R2, ANE3R2 and ANE4R2

4.51 Peak tensile engineering stress versus cycle number for ANE1R4, ANE2R4, ANE3R4 and ANE4R4

4.52 Peak tensile engineering stress versus cycle number for ANE1R8, ANE2R8, ANE3R8 and ANE4R8

4.53 Peak tensile engineering stress versus cycle number for BNE1R2, $\mathrm{BNE} 2 \mathrm{R} 2, \mathrm{BNE} 3 \mathrm{R} 2$ and BNE4R2.

4.54 Peak tensile engineering stress versus cycle number for BNE1R4, BNE2R4, BNE3R4, BNE4R4 and BNE4R4-2

4.55 Peak tensile engineering stress versus cycle number for BNE1R8, BNE2R8, BNE3R8 and BNE4R8.

4.56 Average true stress range difference between the $10^{-4}$ and $10^{-3} \mathrm{~s}^{-1}$ strain rates versus loading cycle number for material $\mathrm{A}$

4.57 Average true stress range difference between the $10^{-4}$ and $10^{-3} \mathrm{~s}^{-1}$ strain rates versus loading cycle number for material $\mathrm{B}$ 
4.58 Engineering stress versus engineering strain curve for the last few cycles of ANE3R4

4.59 Average true stress versus average true strain curve for the last few cycles of ANE1R4

4.60 Average true stress versus average true strain curve for ANE3R8.

4.61 Average true stress versus average true strain curve for the last few cycles of BNE1R4

4.62 Calculated average true plastic strain per cycle versus fatigue life for material $\mathrm{A}$ at various target strain ranges

4.63 Calculated average true plastic strain per cycle versus fatigue life for material $\mathrm{B}$ at various target strain ranges.

4.64 Constant $c$ versus strain rate

4.65 Calculated average true plastic strain per cycle versus strain rate for material $\mathrm{A}$ at various target strain ranges

4.66 Calculated average true plastic strain per cycle versus strain rate for material $\mathrm{B}$ at various target strain ranges

4.67 Fatigue life versus strain rate for material $\mathrm{A}$ at various target strain ranges .

4.68 Fatigue life versus strain rate for material B at various target strain ranges..

4.69 Cumulative average true plastic strain at failure versus strain rate for material $\mathrm{A}$ at different target strain ranges

4.70 Cumulative true plastic strain at failure versus strain rate for material $\mathrm{B}$ at different target strain ranges 
5.1 Average true stress versus true strain curves for material $G$ (material $A$ in current study), by Chen (2010)

5.2 Average true stress versus true strain curves for material $\mathrm{H}$ (material $\mathrm{B}$ in current study), by Chen (2010)

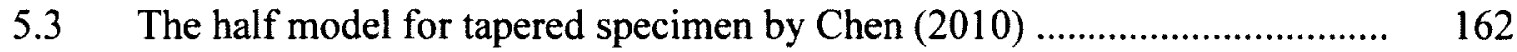

5.4 The half gauge length model of the tapered specimen by Chen (2010) ........

5.5 Engineering stress versus cross-section area ratio for half gauge length model and half model of tapered specimen GT5E5ED by Chen (2010) .......

5.6 True flow stress versus true plastic strain curves for tapered and nontapered (straight) specimens

5.7 Engineering stress versus cross section area ratio curves for tapered and non-tapered (straight) specimens

5.8 Measured and predicted engineering stress versus cross section area ratio curves for GT5E5ED

5.9 Measured and predicted engineering stress versus cross section area ratio curves for HT5E5ED

5.10 Measured and generated true flow stress versus true plastic strain curves for material $G(A)$

5.11 Measured and generated true flow stress versus true plastic strain curves for material $\mathrm{H}(\mathrm{B})$

5.12 Relationship between the flow stress, yield stress and backstress.

5.13 Yield stress versus true plastic strain curves at various strain rates for material A. 
5.14 Yield stress versus true plastic strain curves at various strain rates for material B.

5.15 Test and predicted engineering stress versus engineering strain curves for ATE4D

5.16 Test and predicted engineering stress versus engineering strain curves for BTE4D

5.17 Test and predicted engineering stress versus engineering strain curves for ANE4R2

5.18 Test and predicted engineering stress versus cross-sectional area ratio curves for ANE4R2.

5.19 Test and predicted engineering stress versus engineering strain curves of BNE4R2

5.20 Test and predicted engineering stress versus cross-sectional area ratio curves of BNE4R2

5.21 Engineering stress versus engineering strain curves between a static and a time dependent analysis for ATE1D

5.22 Average true stress versus average true strain curves between a static and a time dependent analysis for ATE1D

5.23 Test and predicted engineering stress versus engineering strain curves for the cyclic portion of ATE3D.

5.24 Test and predicted engineering stress versus engineering strain curves for the cyclic portion of ATE2D. 
5.25 Test and predicted engineering stress versus engineering strain curves for the cyclic portion of ATE1D

5.26 Test and predicted engineering stress versus engineering strain curves of ATE3D

5.27 Test and predicted engineering stress versus engineering strain curves of ATE2D

5.28 Test and predicted engineering stress versus engineering strain curves of ATE1D

5.29 Predicted and test peak tensile engineering stress versus cycle number curves for various strain rates at the strain range of $\pm 2 \%$ for material $\mathrm{A}$......

5.30 Predicted and test peak tensile engineering stress versus cycle number curves for various strain rates at the strain range of $\pm 2 \%$ for material $\mathrm{B} \ldots \ldots$.

5.31 Test and predicted engineering stress versus engineering strain curves for 50 cycles of ANE3R2

5.32 Test and predicted engineering stress versus engineering strain curves for 50 cycles of ANE2R2

5.33 Test and predicted engineering stress versus engineering strain curves for 50 cycles of ANE1R2

5.34 Test and predicted engineering stress versus engineering strain curves for the cyclic portion of ATE3I

5.35 Test and predicted engineering stress versus engineering strain curves of ATE3I 
5.36 Test and predicted engineering stress versus engineering strain curves for the cyclic portion of ATE2I

5.37 Test and predicted engineering stress versus engineering strain curves of ATE3I

5.38 Test and predicted engineering stress versus engineering strain curves for 50 cycles of ANE4R 4

5.39 Test and predicted engineering stress versus engineering strain curves for 50 cycles of ANE4R8

5.40 Test and predicted average true stress range versus cycle number for ATE2E

5.41 Test and predicted average true stress range versus cycle number for ATE2C-20

A.1 Predicted engineering strain versus average true strain

B.1 Engineering stress versus engineering strain curve for ATE3I, ATE2I and ATE1I

B.2 Engineering stress versus engineering strain curve for ATE3D, ATE2D and ATE1D

B.3 Engineering stress versus engineering strain curve for BTE3D, BTE2D and BTE1D

B.4 Part of the $10^{\text {th }}$ cycle for BTE1I, BTE2I and BTE3I at the $\pm 0.5 \%$ strain range..

B.5 Part of the $10^{\text {th }}$ cycle for BTE1I, BTE2I and BTE3I at the $\pm 1 \%$ strain range 
B.6 Part of the $10^{\text {th }}$ cycle for BTE1I, BTE2I and BTE3I at the $\pm 1.5 \%$ strain range.

B.7 Part of the $10^{\text {th }}$ cycle for BTE1I, BTE2I and BTE3I at the $\pm 2 \%$ strain range.

B.8 Part of the $10^{\text {th }}$ cycle for BTE1D, BTE2D, BTE3D and BTE4D at the $\pm 2 \%$ strain range

B.9 Part of the $10^{\text {th }}$ cycle for BTE1D, BTE2D, BTE3D and BTE4D at the $\pm 0.5 \%$ strain range

B.10 Half engineering stress range versus cumulative plastic engineering strain at the $5^{\text {th }}$ and $10^{\text {th }}$ cycle of each strain range for BTEIs

B.11 Average of the half engineering stress range at each strain rate versus cumulative plastic engineering strain at the $5^{\text {th }}$ and $10^{\text {th }}$ cycle of each strain range for BTEIs

B.12 Half engineering stress range versus cumulative plastic engineering strain at the $5^{\text {th }}$ and $10^{\text {th }}$ cycle of each strain range for BTEDs

B.13 Magnified view of the engineering stress versus engineering strain curves for ATE1I at the $\pm 0.5 \%$ strain range

B.14 Magnified view of the engineering stress versus engineering strain curves for $\mathrm{ATE} 1 \mathrm{I}$ at the $\pm 1 \%$ strain range.

B.15 Magnified view of the engineering stress versus engineering strain curves for $\mathrm{ATE} 1 \mathrm{I}$ at the $\pm 1.5 \%$ strain range

B.16 Magnified view of the engineering stress versus engineering strain curves for $\mathrm{ATE} 1 \mathrm{I}$ at the $\pm 2 \%$ strain range 
B.17 Magnified view of the engineering stress versus engineering strain curves for $\mathrm{ATE} 1 \mathrm{D}$ at the $\pm 2 \%$ strain range

B.18 Magnified view of the engineering stress versus engineering strain curves for ATE1D at the $\pm 0.5 \%$ strain range

B.19 Magnified view of the engineering stress versus engineering strain curves close to the peak stress for I series tests of material B when being pulled to fracture

B.20 Magnified view of the engineering stress and actuator displacement versus time between the approximate strain range of 0 and 0.1 during the pull to fracture for BTE1I.

B.21 Magnified view of the engineering stress and actuator displacement versus time between the approximate strain range of 0 and 0.1 during the pull to fracture for BTE2I.

B.22 Magnified view of the engineering stress and actuator displacement versus time between the approximate strain range of 0 and 0.1 during the pull to fracture for BTE3I.

B.23 The $10^{\text {th }}$ cycle of BTE2D and BTE2I at the $\pm 2 \%$ strain range

B.24 The $10^{\text {th }}$ cycle of BTE3D and BTE3I at the $\pm 2 \%$ strain range

B.25 Tensile behaviour of BTE2D and BTE2I at the strain range of $\pm 0.5 \% \ldots \ldots \ldots$

B.26 Compressive behaviour of BTE2D and BTE2I at the strain range of $\pm 0.5 \%$

B.27 Peak tensile average true stress versus cycle number for ANE1R2, ANE2R2, ANE3R2 and ANE4R2 
B.28 Peak tensile average true stress versus cycle number for ANE1R4, ANE2R4, ANE3R4 and ANE4R4

B.29 Peak tensile average true stress versus cycle number for ANE1R8, ANE2R8, ANE3R8 and ANE4R8

B.30 Peak tensile average true stress versus cycle number for BNE1R2, BNE2R2, BNE3R2 and BNE4R2

B.31 Peak tensile average true stress versus cycle number for BNE1R4, BNE2R4, BNE3R4, BNE4R4 and BNE4R4-2

B.32 Peak tensile average true stress versus cycle number for BNE1R8, BNE2R8, BNE3R8 and BNE4R8

B.33 Engineering stress versus engineering strain curve for the last few cycles of ANE1R2

B.34 Average true stress versus average true strain curve for ANE1R8.

B.35 Engineering stress versus engineering strain curve for the last few cycles of ANE2R2

B.36 Engineering stress versus engineering strain curve for the last few cycles of ANE2R4

B.37 Average true stress versus average true strain curve for ANE2R8.

B.38 Engineering stress versus engineering strain curve for the last few cycles of ANE3R2

B.39 Engineering stress versus engineering strain curve for the last few cycles of ANE4R4

B.40 Average true stress versus average true strain curve for ANE4R8. 
B.41 Engineering stress versus engineering strain curve for the last few cycles of BNE1R2.

B.42 Average true stress versus average true strain curve for BNE1R8.

B.43 Engineering stress versus engineering strain curve for the last few cycles of BNE2R2.

B.44 Engineering stress versus engineering strain curve for the last few cycles of BNE2R4.

B.45 Average true stress versus average true strain curve for BNE2R8

B.46 Engineering stress versus engineering strain curve for the last few cycles of BNE3R2.

B.47 Engineering stress versus engineering strain curve for the last few cycles of BNE3R4.

B.48 Average true stress versus average true strain curve for BNE3R8

B.49 Engineering stress versus engineering strain curve for the last few cycles of BNE4R4.

B.50 Engineering stress versus engineering strain curve for the last few cycles of BNE4R4-2

B.51 Average true stress versus average true strain curve for BNE4R8

C.1 Finite element mesh scheme-1 for the tapered specimen

C.2 Finite element mesh scheme-2 for the tapered specimen

C.3 Finite element mesh scheme-3 for the ANE4R8 specimen

C.4 Finite element mesh scheme-4 for the ANE4R8 specimen 
C.5 Average true stress versus average true strain curves from FEA simulations of the tapered specimen for Scheme-1 and Scheme-2.............

C.6 Average true stress versus average true strain curves from FEA simulations of the tapered specimen for Scheme-1 and Scheme-2 during cyclic loading.

C.7 Average true stress versus average true strain curves from FEA simulations of the notched specimen for Scheme-3 and Scheme-4..............

C.8 Peak tensile average true stress versus cycle number curves from FEA simulations of the notched specimen for Scheme-3 and Scheme-4.............

D.1 True flow stress versus true plastic strain curves for tapered and nontapered $14 \mathrm{~mm}$ diameter specimens

D.2 Engineering stress versus cross-section area ratio curves for tapered and non-tapered $14 \mathrm{~mm}$ diameter specimens

D.3 Engineering stress versus cross-section area ratio curves for half gauge length and half models of $14 \mathrm{~mm}$ diameter tapered specimens

D.4 Tensile average true stress versus average true strain curves for the nontapered and tapered $14 \mathrm{~mm}$ diameter specimens

D.5 Compressive average true stress versus average true strain curves for the non-tapered and tapered $14 \mathrm{~mm}$ diameter specimens

D.6 Shifted average true stress versus average true strain curves at the $10^{\text {th }}$ tension half-cycle of each strain range.

E.1 Test and predicted yield stress versus strain rate for the monotonic tests by Chen (2010) at a true plastic strain of 0.2 with a backstress of $200 \mathrm{MPa}$...... 
E.2 Half average true stress range versus cumulative plastic engineering strain at the $5^{\text {th }}$ and $10^{\text {th }}$ cycle of each strain range for ATEIs.

E.3 Half average true stress range versus cumulative plastic engineering strain at the $5^{\text {th }}$ and $10^{\text {th }}$ cycle of each strain range for BTEIs

E.4 Half average true stress range versus cumulative plastic engineering strain at the $5^{\text {th }}$ and $10^{\text {th }}$ cycle of each strain range for ATEDs

E.5 Half average true stress range versus cumulative plastic engineering strain at the $5^{\text {th }}$ and $10^{\text {th }}$ cycle of each strain range for BTEDs

E.6 Test and predicted yield stress versus strain rate for the combined monotonic and cyclic tests of tapered specimens with $\alpha_{I}=100 \mathrm{MPa} \ldots \ldots \ldots \ldots$

F.1 Measured and predicted engineering stress versus cross section ratio curves for GT1E4ED

F.2 Measured and predicted engineering stress versus cross section ratio curves for GT1E3ED

F.3 Measured and predicted engineering stress versus cross section ratio curves for GT1E2ED

F.4 Measured and predicted engineering stress versus cross section ratio curves for GT1E1E

F.5 Measured and predicted engineering stress versus cross section ratio curves for GT1E0E.

F.6 Measured and predicted engineering stress versus cross section ratio curves for HT1E4ED 
F.7 Measured and predicted engineering stress versus cross section ratio curves for HT1E3ED

F.8 Measured and predicted engineering stress versus cross section ratio curves for HT1E2ED

F.9 Measured and predicted engineering stress versus cross section ratio curves for HT1E1E

F.10 Measured and predicted engineering stress versus cross section ratio curves for HT1E0E

F.11 Test and predicted engineering stress versus engineering strain curves for the cyclic portion of BTE3D

F.12 Test and predicted engineering stress versus engineering strain curves for the cyclic portion of BTE2D.

F.13 Test and predicted engineering stress versus engineering strain curves for the cyclic portion of BTE1D

F.14 Test and predicted engineering stress versus engineering strain curves of BTE3D

F.15 Test and predicted engineering stress versus engineering strain curves of BTE2D

F.16 Test and predicted engineering stress versus engineering strain curves of BTE1D

F.17 Test and predicted engineering stress versus engineering strain curves for 50 cycles of BNE3R2 
F.18 Test and predicted engineering stress versus engineering strain curves for 50 cycles of BNE2R2.

F.19 Test and predicted engineering stress versus engineering strain curves for 50 cycles of BNE1R2

F.20 Test and predicted engineering stress versus engineering strain curves for the cyclic portion of BTE3I

F.21 Test and predicted engineering stress versus engineering strain curves for BTE3I.

G.1 Curve fitting of the backstress for small strain with an assumed yield stress $\left(\sigma_{S}^{o}\right)$ of $320 \mathrm{MPa}$ for ATE4D of material A

G.2 Curve fitting of the backstress for large strain with an assumed saturated yield stress $\left(\sigma_{L}^{o}\right)$ of $330 \mathrm{MPa}$ for material $\mathrm{A}$

G.3 Test and predicted engineering stress versus engineering strain for ATE4D using the kinematic hardening parameters calibrated with an assumed yield stress $\left(\sigma_{S}^{o}\right)$ of $320 \mathrm{MPa}$ and a saturated yield stress $\left(\sigma_{L}^{o}\right)$ of $330 \mathrm{MPa}$.

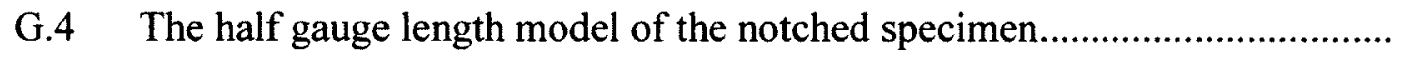
269

G.5 Locations of engineering strains where errors are calculated for the $20^{\text {th }}$ cycle of ANE4R2

G.6 Test and predicted engineering stress versus engineering strain for ANE4R2 using the kinematic hardening parameters calibrated with an assumed yield stress $\left(\sigma_{S}^{o}\right)$ of $320 \mathrm{MPa}$ and a saturated yield stress $\left(\sigma_{L}^{o}\right)$ of $330 \mathrm{MPa}$ 
G.7 Test and predicted engineering stress versus cross section change for ANE4R2 using the kinematic hardening parameters calibrated with an assumed yield stress $\left(\sigma_{S}^{o}\right)$ of $320 \mathrm{MPa}$ and a saturated yield stress $\left(\sigma_{L}^{o}\right)$ of $330 \mathrm{MPa}$. 


\section{LIST OF SYMBOLS AND ABREVIATIONS}

A $\quad-\quad$ current specimen area

$\mathrm{A}_{\mathrm{f}} \quad-\quad$ cross-sectional area at fracture

$\mathrm{A}_{\mathrm{o}} \quad-\quad$ undeformed cross-sectional area

a $\quad-\quad$ radius of minimum cross-section

ASTM - American Society for Testing and Materials

b - - Burgers vector

C - constant

c - constant

$\mathrm{c}_{\mathrm{s}} \quad-\quad$ constant

D $\quad-\quad$ current diameter

d $\quad-\quad$ constant

DSA - dynamic strain aging

$D^{1} \quad-\quad$ constant

$\mathrm{D}_{\mathrm{o}} \quad$ - original diameter

$\dot{\mathrm{D}} \quad-\quad$ specimen diameter change rate

$\mathrm{E}_{\mathrm{s}} \quad$ - elastic modulus for a tapered specimen, initial slope of average true stress-average true strain curve upon unloading and reloading in the cyclic test for a notched specimen

F $\quad-\quad$ load 


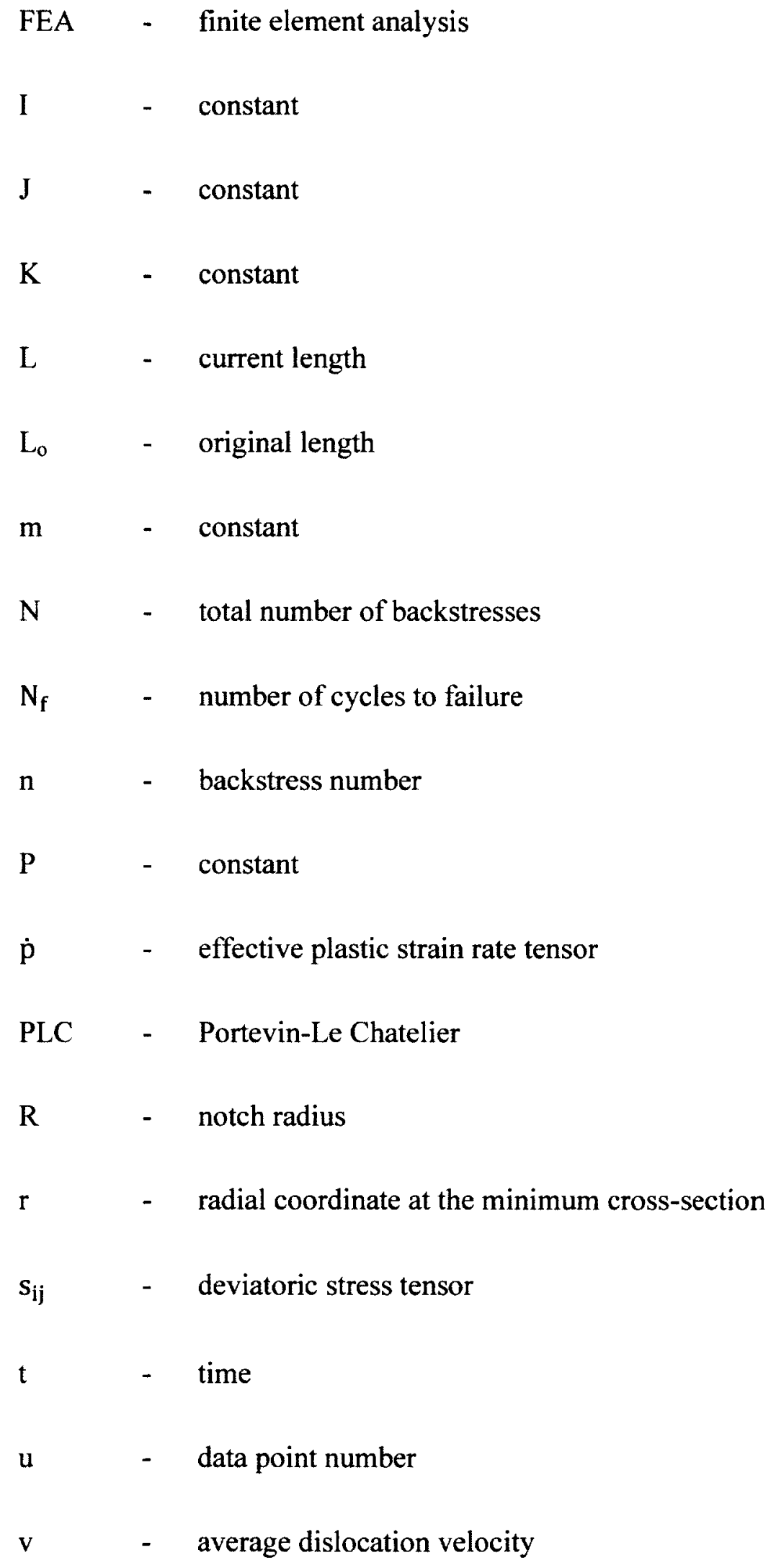




\begin{tabular}{|c|c|c|}
\hline$\alpha$ & - & total backstress \\
\hline$\alpha_{i j}$ & - & backstress tensor \\
\hline$\dot{\alpha}_{i j}$ & - & backstress rate tensor \\
\hline$\alpha_{\mathrm{ij}}^{\mathrm{dev}}$ & - & deviatoric backstress tensor \\
\hline$\Delta \mathrm{D}$ & - & specimen diameter change \\
\hline$\Delta \mathrm{L}$ & - & change in length \\
\hline$\Delta \varepsilon^{p}$ & - & plastic strain range \\
\hline$\varepsilon$ & - & strain \\
\hline$\varepsilon^{\mathrm{p}}$ & - & plastic strain \\
\hline$\varepsilon_{\mathrm{c}}$ & - & constant \\
\hline$\varepsilon_{\mathrm{e}}$ & - & engineering strain \\
\hline$\varepsilon_{\mathrm{m}}$ & - & mean strain \\
\hline$\varepsilon_{\max }$ & - & maximum strain achieved per cycle \\
\hline$\varepsilon_{\min }$ & - & minimum strain achieved per cycle \\
\hline$\varepsilon_{\mathrm{t}}$ & - & true strain \\
\hline$\varepsilon_{\mathrm{t}}^{0}$ & - & true strain at the end of the yield plateau \\
\hline$\varepsilon_{t}^{p}$ & - & true plastic strain \\
\hline$\varepsilon_{\mathrm{tc}}^{\mathrm{p}}$ & - & cumulative true plastic strain \\
\hline$\dot{\varepsilon}$ & - & strain rate \\
\hline
\end{tabular}




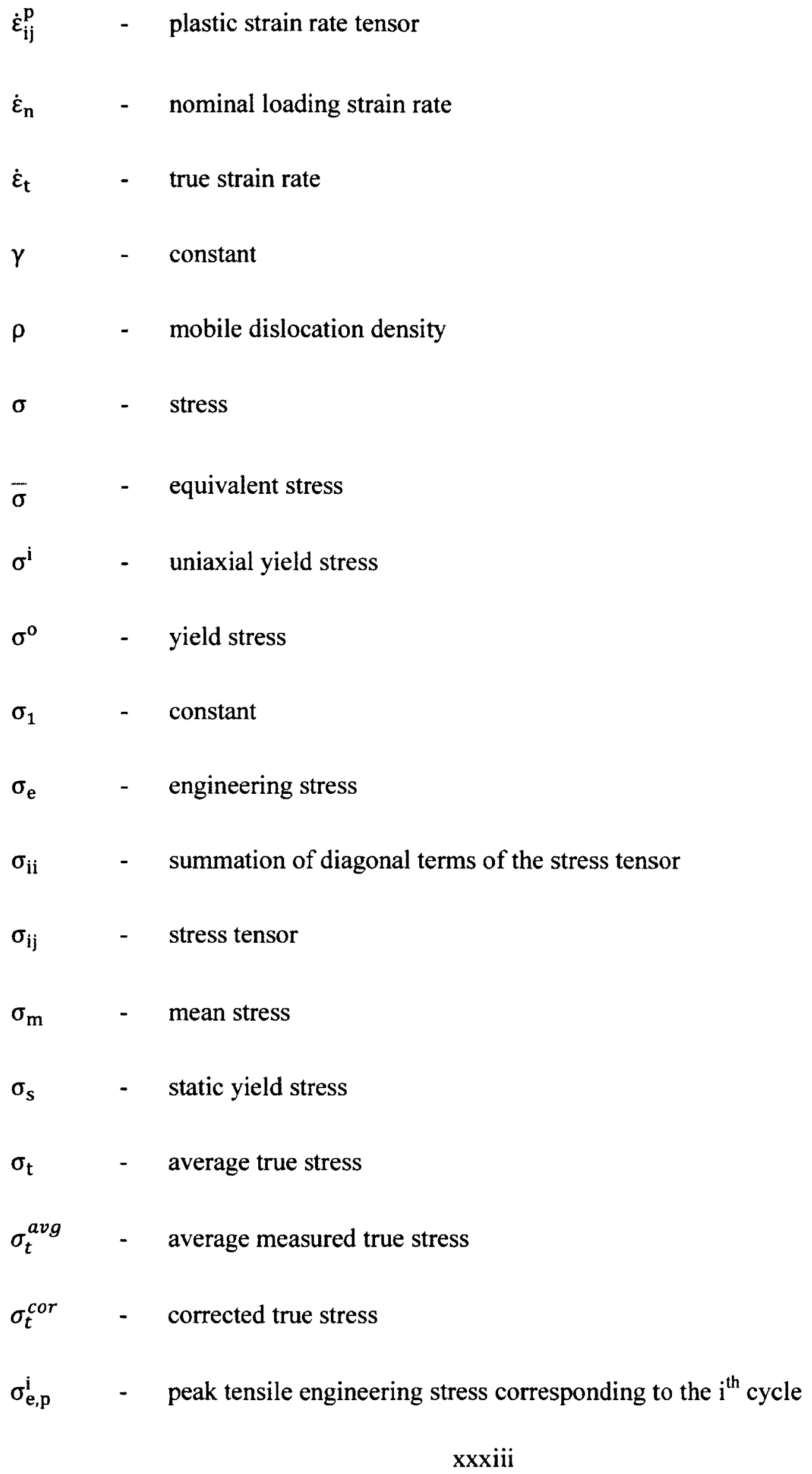




$\begin{array}{lll}\sigma_{e, p}^{i-1} & - & \text { peak tensile engineering stress corresponding to the } i-1^{\text {th }} \text { cycle } \\ \sigma_{\mathrm{t}}^{\mathrm{F}} & - & \text { true flow stress } \\ \sigma_{\mathrm{t}}^{\mathrm{y}} & - & \text { true yield stress }\end{array}$




\section{Chapter 1 Introduction}

The assessment of the performance of structural steel components requires the knowledge of some key mechanical material properties such as the elastic modulus, yield strength, ultimate tensile strength and fracture limit. Quasi-static material tests are usually performed to obtain these parameters. However, the quasi-static material properties may no longer represent the dynamic behaviour of the material during an earthquake. It is expected that the loading rate imparted on structural components to increase over that of the quasi-static condition up to a strain rate of $10^{-1} \mathrm{~s}^{-1}$ (Ngo 2007) during an earthquake. Manjoine (1944) and Cowper and Symonds (1957) have shown that the yield and flow stresses of structural steel increase with the strain rate. As well, studies have shown that the fracture limit and fatigue life may also be affected by strain rate. In addition to an increased strain rate, the shaking of the building will likely produce large inelastic deformations with a complex loading history that may lead to low cycle fatigue failure.

Since the loading experienced by structural members during an earthquake is rather complex, a thorough understanding of the material behaviour is required for an accurate simulation to predict the response of a structure to an earthquake event. Although there have been many studies carried out to study the effects of strain rate on steel, none has been found to outline an effective procedure to determine input data for material properties in a numerical simulation that can model effects of strain rate with reasonable accuracy for a general loading condition including monotonic and cyclic loading. Thus, tests have been carried out to obtian a better understanding on various 
aspects with regards to the strain rate effects that need to be considered in numerical modelling.

\subsection{Objective of the Thesis}

The objective of this thesis is to determine experimentally how the stress-strain behaviour and mechanical properties of mild steel are affected by the strain rate and strain range in consideration for numerical simulations. Specifically, the effect of strain rate and strain range on flow stress, rupture strain and low-cycle fatigue life are studied. Effects of loading history and mean cyclic loading strain on the cyclic behaviour are also investigated. Based on the test results, a procedure is developed to generate the input data of the material properties in a numerical simulation at moderate strain rate for a general loading condition in the absence of significant adiabatic heating.

\subsection{Methodology Used in the Research}

A total of 22 tapered and 25 notched specimens fabricated from CAN/CSA G40.20/21 (CSA 2004) Grade 300W and ASTM A572 (ASTM 2007) Grade 50 steel have been tested under various cyclic loading. Tapered instead of straight profiled specimens have been selected so that there was a predefined minimum cross-sectional area location to place the diametral extensometer such that cross-sectional measurement could be obtained and a loading that is close to the target true strain loading rate control can be achieved through the diametral extensometer control when the specimen was being pulled to fracture. Notched specimens were used so that large true strain ranges could be applied in a cyclic test without having the specimen buckle. 
Tapered specimens have been tested under four different series of loading sequence at strain rates of $10^{-4}$ to $10^{-1} \mathrm{~s}^{-1}$. The series differs in the number and magnitude of strain ranges considered, and as well as the number of loading cycles applied at each strain range. These allow effects of loading history, strain range, mean strain of the strain range applied and strain rate on the (cyclic) flow stress to be investigated. Most of the tapered specimens tested were pulled to fracture after being subjected to some prescribed cyclic loading. Results of tapered specimens have also been used to study the effect of strain rate on rupture strain.

The notched specimens were loaded at the nominal true strain ranges of $\pm 2 \%$, $\pm 4 \%$ and $\pm 8 \%$ at the engineering strain rates of $10^{-4}$ to $10^{-1} \mathrm{~s}^{-1}$ (nominal true strain rates of approximately $5 \times 10^{-4}$ to $5 \times 10^{-1} \mathrm{~s}^{-1}$ ). All tests were cycled until separation except for tests at the strain range of $\pm 2 \%$ and strain rate of $10^{-4} \mathrm{~s}^{-1}$, which have been loaded to only 20 cycles due to the long duration required to cycle the specimen to fracture. Tests with notched specimens enabled effects of strain rate and strain range on the (cyclic) flow stress to be studied over a large number of loading cycles (large cumulative plastic strain). Since the specimens have been cycled to separation, the strain rate effects on the fatigue life were also investigated.

Based on the test results, a procedure has been developed to generate the input data of the material properties in a numerical simulation at moderate strain rate and in the absence of significant adiabatic heating. This procedure utilizes test results from monotonic and cyclic tests. Since only cyclic tests have been conducted in the present study, data of monotonic tension tests performed on the same materials used in the current study were obtained from Chen (2010). Numerical simulations of the cyclic and 
monotonic tests were carried out with ABAQUS (Simulia 2010) using the generated input data and built-in strain rate dependent metal plasticity model. Results of the numerical simulations were validated against the test results.

\subsection{Organization of the Thesis}

Chapter 2 presents a brief literature review, which provides background information on effects of strain rate on flow stress, fracture strain and fatigue life. A short summary of studies investigating loading history and mean strain effects, as well as a brief description of dynamic strain aging and constitutive models are also provided.

Chapter 3 consists of details on the testing program that outlines the specimen type and designation, data acquisition methods and loading control. As well, the calibration procedures for the hydraulic testing machine and temperature measuring equipment are also discussed.

In Chapter 4, test results from both tapered and notched specimens on the effect of strain rate on flow stress, rupture strain, and low cycle fatigue life are analyzed. As well, discussions on the effects of loading history and mean strain of the strain range applied are also provided.

Chapter 5 describes the procedure developed to generate the input data of material properties in a numerical simulation. The proposed procedure is validated against the test results through numerical simulations. A procedure to correct for the stress measurement due to the tapered profile of specimen is also provided.

Chapter 6 contains the conclusions, and recommendations for future work with supplementary analyses and test results provided in the appendices. 


\section{Chapter 2 Literature Review}

There have been many studies conducted on fatigue life, history and strain rate effects on mechanical properties of structural steels. With many studies, it is not surprising to find differences in the experimental procedures employed and results obtained. The experimental studies differ in terms of specimen shape, material type, loading control or definition of fatigue life. At times, it may be difficult to compare results from different studies. Nevertheless, they are still important sources for understanding fatigue life and history and strain rate effects on the behaviour of structural steels. A brief literature review and discussions on these aspects of steel are provided below.

\subsection{Fatigue Life}

When structural elements are subject to cyclic loading, damage may accumulate within the matrix of the material. For steel, fatigue cracks are normally initiated on the surface of the element and propagate inward with continued loading. A sudden fracture of the element occurs in the tension half-cycle of loading (Broek, 1979) once fatigue cracks have propagated far enough to reach a critical crack size or to have minimum effective area. The mechanism of the final sudden failure of the element is normally a combination of ductile and brittle fracture.

Generally, the fatigue life is defined to correspond to a point at which enough damage has accumulated. However, since damage begins at the atomic scale, its effects are initially very subtle and difficult to observe. There have been many criteria used to 
define the fatigue life of an element. The criterion used may vary from one study to another, hence making comparisons of the results across various studies challenging. Even for ASTM E606-04el (ASTM, 2004) standard for strain-controlled fatigue testing, acceptable definitions of fatigue life include: separation, the modulus method, measurement of microcracks or a $50 \%$ stress drop from the peak tensile stress achieved during the test.

Bhanu Sankara Rao et al. (1985) investigated different definitions of fatigue life on several materials at various temperatures, strain ranges, strain rates and test specimen geometries. The objective was to determine the most reliable criteria to define the fatigue life before a specimen has fully separated. Specimens were loaded until separation occurred due to fatigue cracks propagating entirely through the specimen. Fatigue lives considered in this study were defined as load cycle number corresponding to a point at 5 , 10 and $20 \%$ fall of the maximum stress achieved during the test $\left(\mathrm{N}_{\mathrm{T} 5}, \mathrm{~N}_{\mathrm{T} 10}, \mathrm{~N}_{\mathrm{T} 20}\right), 10$ and $20 \%$ fall in the maximum stress range, cusp formation in the compressive portion of the hysteresis curve ( $\mathrm{N}_{\text {CUSP }}$ ) and occurrence of an inflection point in an elastic stiffness versus number of cycles plot. A cusp appears in the hysteretic curve due to the presence of a fatigue crack. When a large crack has formed in the specimen, there is a spike in compressive stress during the compressive half-cycle due to the closing of the crack and hence an increase in effective load-bearing area of the specimen. Results of the tests show that $\mathrm{N}_{\mathrm{T} 20}$ and $\mathrm{N}_{\mathrm{CUSP}}$ occur at nearly the same number of cycles for all tests and gave a fatigue life that was within $10 \%$ of the separation value. For uniaxial specimens, $\mathrm{N}_{\mathrm{T} 20}$ and $\mathrm{N}_{\text {CuSP }}$ are good parameters to base the fatigue life on if separation is not desired. However, for notched specimens, only $\mathrm{N}_{\text {CUSP }}$ appears to be a good choice. When testing 
notched specimens, it was observed that there was actually an increase in tensile stress at the end of the specimen life and therefore a fatigue life defined basing on stress drop would not be useful.

Number of cycles to failure can also be used to categorize the type of failure. Cyclic loading failure can be grouped as either high-cycle fatigue or low-cycle fatigue. Defining the failure of a structural element with these categories gives an idea of the loading history that the element experienced. For low-cycle fatigue, the applied stresses during the structural elements life nearly reach the tensile strength of the material. For high-cycle, the stresses are below the yield level. Manson (1966) has described the boundary between high-cycle fatigue and low-cycle fatigue as $10^{4}$ cycles. Sachs (2007) has defined the boundary somewhere between $10^{4}$ to $10^{5}$ cycles due to the fact that when high-cycle fatigue occurs in practical situations, the fatigue life of the member is generally much higher than $10^{5}$ cycles. Sachs (2007) also described a category of failure that occurs in less than 25 cycles as very-low-cycle fatigue. In practical applications, this type of failure is usually seen within 10 cycles, with large, visible plastic deformations occur a kin to an overload situation. For both high- and low-cycle fatigue, plastic deformations are not visible. The eventual failure due to fatigue loading occurs as a result of a crack initiated and grown through a member with each loading cycle. Once the crack has propagated far enough and a critical minimum effective area is reached, an instantaneous fracture occurs during the tension half-cycle. 


\subsection{Specimen Profile (Stress Triaxiality)}

When testing materials, the profile of the specimen is normally chosen such that a uniaxial state of stress exists within the specimen. Therefore, the mechanical behaviour of the material can be determined from the test data at least up until necking. Such a simple state of stress is desirable as it allows the stress in the specimen to be calculated from the applied load. For fully-reversed fatigue tests, it is a challenge to use uniaxial specimens and maintain reliable test results because the specimens tend to buckle even at a low strain range. Restrepo-Posada et al. (1994) performed cyclic tests on reinforcing bar specimens at strain rates ranging from $2.3 \times 10^{-5} \mathrm{~s}^{-1}$ to $2.3 \times 10^{-3} \mathrm{~s}^{-1}$ and strain ranges from $0.25 \%$ to $2 \%$. The specimens needed to be welded to a threaded rod in order to be tested. As a result, a large length to width ratio was used to avoid heating effects from welding and end effects. It was found that most of the specimens buckled during cyclic testing. A solution for this is to use notched specimens for tests at higher strain ranges. This allows the specimen to be loaded under fully reversed conditions at higher strain ranges while avoiding buckling. However due to the shape of the notched specimens, the stress within the notch becomes unevenly distributed. The stress triaxiality, the ratio of mean stress to equivalent stress, is elevated toward the specimens axis. This can be described by the equation proposed by Bridgman (1944),

$$
\frac{\sigma_{m}}{\bar{\sigma}}=\frac{1}{3}+\ln \left(\frac{a^{2}+2 a R-r^{2}}{2 a R}\right)
$$

where $\mathrm{a}$ is the radius of minimum cross-section, $\mathrm{R}$ is the notch radius, $\mathrm{r}$ is the radial coordinate at the minimum cross-section, $\sigma_{m}$ is the hydrostatic (mean) stress and $\bar{\sigma}$ is the equivalent stress. The mean stress and equivalent stress are given by 


$$
\begin{gathered}
\sigma_{m}=\frac{\sigma_{i i}}{3} \text { and } \\
\bar{\sigma}=\sqrt{\frac{3}{2} s_{i j} s_{i j}}
\end{gathered}
$$

where $\sigma_{i i}$ is the summation of the diagonal terms of the stress tensor and $s_{i j}$ is the deviatoric stress tensor. Equation (2.1) reaches a maximum when $r=0$ which is

$$
\frac{\sigma_{m}}{\bar{\sigma}}=\frac{1}{3}+\ln \left(1+\frac{a}{2 R}\right)
$$

Since there are very little visible plastic deformations associated with low cyclic fatigue tests, it can be assumed that the stress triaxiality in a notched specimen will remain relatively constant (Sachs 2007). This triaxiality effectively increases the stiffness of the material in the notch, which elevates the value of measured average stress required for the specimen to plastically deform above that of the uniaxial condition. For this reason, it is not possible to directly compare the test data acquired from a notched specimen to a uniaxial specimen. Nevertheless, it is still possible to determine effects of a parameter with notched specimen tests. Comparing results between specimens of similar geometry can still give the qualitative effects of that test parameter although any actual value acquired may be less useful.

\subsection{History Effect}

The history effect can be described as a change in a materials mechanical behaviour as a result of the loading conditions it has previously experienced. Many methods of testing for this effect have been developed in various studies by manipulating different loading parameters. Some of these include strain rate jump tests, cyclic strain range change tests, pre-cycling before a monotonic pull or imparting a pre-strain on a 
specimen before cycling. Manifestations of this effect are generally observed in the flow stress or fatigue life of the material. To determine if the loading history has an effect on the materials mechanical properties, a comparison is usually made between tests performed with and without a loading history. Results of these tests tend to differ depending on the material being considered. Feltner and Laird (1967) performed test which involved either a change in strain range or temperature for Copper and a CopperAluminum alloy. The strain range-temperature combinations considered were $10^{-1}$ at $-195^{\circ} \mathrm{C}, 10^{-1}$ at $25^{\circ} \mathrm{C}$ and $10^{-2}$ at $25^{\circ} \mathrm{C}$ for both materials. During the test, only one parameter (strain range or temperature) was changed at a time. When the flow stress became saturated, another change in either temperature or strain range was performed. Their results showed that there is no history effect in Copper but a notable history effect in the alloy. For the alloy, the saturated stress was always higher after a change in either strain range or temperature than that of a test performed at an unchanging strain rangetemperature combination.

Lukas and Klesnil (1973) further confirmed this result by performing tests on Copper and a Copper-Zinc alloy. Copper was cycled at the changing plastic strain ranges of $1.99 \times 10^{-4}$ to $11.05 \times 10^{-4}$ as well as $11.05 \times 10^{-4}$ to $1.99 \times 10^{-4}$. The same was considered for Copper-Zinc alloy for the plastic strain ranges of $1.19 \times 10^{-4}$ and $11.85 \times 10^{-4}$. It was found that the specimens of Copper showed no effect of loading history within the scatter of the data when a change in strain range was considered, but the Copper-Zinc alloy showed that the stress was always higher for a specimen that underwent a change in strain range compared to one than that was cycled at a single strain range. Christ et al. (1995) also performed strain range change tests on oxygen-free high conductivity copper. 
In contrast, the results showed that there is an upper limit of $2 \times 10^{-3}$ plastic strain range where history effects are not seen. In a multiple-step test, a specimen was loaded at a plastic strain range of $5 \times 10^{-4}$ to saturation, then increased to $10^{-2}$ and decreased back to $5 \times 10^{-4}$. It was found that even though some softening was seen, an increase in stress range over that of a test from the virgin state occurred when the strain range returned to $5 \times 10^{-4}$. The same qualitative results were seen for a specimen tested at $2 \times 10^{-3}$, increased to $1 \times 10^{-2}$ and decreased back down to $2 \times 10^{-3}$. However, a similar type of test was performed for the plastic strain ranges of $5 \times 10^{-4}$ and $2 \times 10^{-3}$, but no history effects were seen.

Trampczynski (1988) performed strain range change tests on $21 \mathrm{CrMoV} 57$ (similar to ASTM A193 B16 steel for bolts) and 18G2A (similar to AISI A765) steels in order to investigate the evolution of isotropic and kinematic hardening parameters. Comparisons of results for tests with increasing strain ranges to ones that decrease from the highest to the lowest strain range were conducted for both tension-compression loading as well as torsion. It was found that an increase in the saturated stress occurred at the lowest strain range for the specimen cycled at a decreasing strain range to that at an increasing strain range. It was determined that, for the $18 \mathrm{G} 2 \mathrm{~A}$ material, this change was mainly attributed to the kinematic stress calculated by the author from the flow stress. Cyclic loading tests in torsion with a pre-strain of $9.2 \%$ were also considered. An increase in the flow stress was seen in the direction of pre-straining for the first cycle. In the reversed direction, no change in flow stress was seen when the load-deformation curve is compared to that of a specimen tested without pre-straining. Consequently, the 
load-deformation curve was initially very asymmetric about zero stress. However, the flow stress in the direction of pre-straining relaxed as load cycling continued.

Studies on the strain rate history effects on 304 stainless steel have been conducted by Krempl (1979) with monotonic and cyclic uniaxial tests and by Chang and Lee (1987) on A36 structural steel tubing with cyclic tests. In both of these studies, tests were conducted at a constant strain rate as well as with varying strain rate. For tests with varying strain rates, the strain rate was increased then decreased back during the test. The portion of the stress-strain curve for a particular strain rate of a test with varying strain rates was found to be almost the same as that for another specimen tested at an equal constant strain rate.

\subsection{Mean Strain Effect}

During cyclic loading, a non-zero mean strain occurs when the maximum tension and compression strains are not equal in magnitude. Mathematically, the mean strain is defined as

$$
\varepsilon_{m}=\frac{\varepsilon_{\max }+\varepsilon_{\min }}{2}
$$

where $\varepsilon_{\max }$ is the maximum strain, and $\varepsilon_{\min }$ is the minimum strain in the loading cycle. The mean strains can be expected to vary in a structure during earthquake loading due to the ground motion. For this reason, it is important to understand how the mean strain affects material response so that it can be properly considered in design. Generally, when a non-zero constant mean strain is applied, there is an associated non-zero mean stress that may vary during the cyclic loading. 
Kliman and Bily (1980) performed multiple-step tests on cylindrical specimens of low carbon steel. Tests were performed under mean strains of $0.05 \%, 0.079 \%$ and $0.118 \%$ at frequencies of $1,5,8$ and $10 \mathrm{~Hz}$. The applied strain ranges considered in this study ranged from $2 \times 10^{-2}$ to $4 \times 10^{-5}$. For strain controlled tests, it was found that the mean stress relaxed to zero after approximately 6000 cycles for all of the mean strains considered. It was concluded that the cyclic stress-strain curve is not affected by the mean strain if sufficient number of loading cycles have been applied so that the mean stress can fully relax.

On the other hand, Chiou and Yip (2003) have noted that mean strain affected the cyclic stress-strain curve. Cyclic tests were performed on cylindrical specimens of AISI 316 stainless steel at the strain ranges of $0.5 \%$ to $1.0 \%$. Mean strains of $0.4 \%, 0.2 \%$, and $0 \%$ were considered. It was found that the specimen initially hardened then softened to a saturated stress after a few cycles. In the test, the mean stress did not fully relax if a large mean strain and a small strain range were considered. On the other hand, at smaller and zero mean strains, an almost complete relaxation of the mean stress was observed. However, the number of cycles applied at each strain range and the definition of stress saturation used were not provided. It is expected that if the same number of loading cycles is used for each mean strain and strain range combination, a larger number of cycles would be required to completely relax the mean stress for a large mean strain and small strain range combination because the initial mean stress would be larger at a high mean strain and the plastic strain per cycle would be lower at a small strain range. Thus, the findings of Chiou and Yip (2003) cannot be considered as conclusive without 
knowing the number of loading cycles applied and stress saturation definition adopted for that study.

\subsection{Strain Rate}

Dieter (1983) defined the instantaneous strain rate as the ratio of strain increment per time increment in which the straining occurs. It can be defined as

$$
\dot{\varepsilon}=\frac{d \varepsilon}{d t}=\frac{d\left[\ln \left(\frac{L}{L_{o}}\right)\right]}{d t}=\frac{1}{L} \frac{d L}{d t}
$$

where $t$ is the time, $\dot{\varepsilon}$ is the strain rate, $L$ is the current length of the specimen and $L_{o}$ is the original length of the specimen. This is an important quantity to consider when testing materials as it may affect the measured mechanical properties of a material test. Ngo (2007) has classified the strain rate in the range of $10^{-6} \mathrm{~s}^{-1}$ to $10^{-4} \mathrm{~s}^{-1}$ as quasi-static, which implies that there is insignificant strain rate effect on the material mechanical properties as well as inertia effect and adiabatic heating. A material test would be performed at this strain rate to obtain the "static" mechanical properties. Loading from an earthquake may impart strain rates of $10^{-3} \mathrm{~s}^{-1}$ to $10^{-1} \mathrm{~s}^{-1}$ on a structure (Ngo 2007). Adiabatic heating in mild steel will be noticeable at the higher bound of this range under the cyclic loading. Higher still are the strain rates that can be imposed by impact $\left(10^{0} \mathrm{~s}^{-1}\right.$ to $\left.10^{2} \mathrm{~s}^{-1}\right)$ and blast loading $\left(10^{2} \mathrm{~s}^{-1}\right.$ to $\left.10^{4} \mathrm{~s}^{-1}\right)$.

The Orowan equation given by Liu and Wang (2001) has been used to describe the strain rate effect in the context of dislocation theory and is defined as

$$
\dot{\varepsilon}=b \rho v
$$


where $b$ is the Burgers vector of dislocations in the crystal, $v$ is the average velocity of dislocations and $\rho$ is the mobile dislocation density. From this equation, it can be seen that an increase in strain rate has to be accompanied by an increase in dislocation velocity, density or both. Effects of the dislocation velocity and density on the measured flow stress are discussed in the following sections.

\subsubsection{Strain Rate Effect on Stress}

The typical strain rate effect observed in metals is an increase in flow stress with an increase in strain rate. This phenomenon is a result of the physical processes that take place within the substructure of the material. Considering Eq. (2.7) provided above, either an increase in dislocation velocity, density or both has to take place when the strain rate increases.

Johnston and Gilman (1959) performed tests on individual lithium fluoride crystals to determine the effects of dislocation velocity and density on plastic flow. A strong dependency between the dislocation velocity and the applied stress was found over the dislocation velocities of $10^{-7} \mathrm{~cm} / \mathrm{s}$ to $10^{5} \mathrm{~cm} / \mathrm{s}$. The upper limit of dislocation velocity is the speed of sound where wave effects tend to occur (Dieter 1986). Based on the relationship between dislocation velocity and applied stress, it was concluded that there are viscous forces associated with the motion of a dislocation that increase with the dislocation velocity. This shows that there is a relationship between dislocation velocity and macroscopic flow stresses.

As a metal strain hardens, there tends to be a piling up of dislocations that blocks the movement of other dislocations. Blazynski (1983) noted that the effects of strain rate on a metals substructure tend to increase strain hardening. It can be reasoned that if the 
effect of an increased strain rate is to increase the mobile dislocation density, these additional dislocations would compound the effect of dislocation blocking and hence increase strain hardening and flow stress. This could be another cause for the typical strain rate effects on stress seen in most metals.

Regardless of the exact processes that cause an increase in flow stress with increasing strain rate, the effects have been thoroughly studied and well documented. Manjoine (1944) was one of the first to conduct tensile tests to determine the effects of strain rate on the mechanical properties of mild steel. Tests were performed at strain rates ranging from $10^{-6}$ to $10^{3} \mathrm{~s}^{-1}$. The temperatures used in this study varied from room temperature up to $600^{\circ} \mathrm{C}$. It was observed that both the yield and ultimate stresses tend to increase as the strain rate increases. Cowper and Symonds (1957) have proposed an equation to relate the yield stress to the strain rate as

$$
\frac{\sigma(\dot{\varepsilon})}{\sigma_{s}}=1+\left(\frac{\dot{\varepsilon}}{I}\right)^{\frac{1}{J}}
$$

where $\sigma_{s}$ is the static value of yield stress and $I$ and $J$ are constants. There are other typical effects of strain rate on mechanical properties of metal. Soroushian and Choi (1987) gathered strain rate data for various types of steels with the yield strength ranging from 179.4 to $683.8 \mathrm{MPa}$. The results can be generalized as follows.

- Yield stress increases as strain rate increases. Lower yielding steels are more rate sensitive.

- Ultimate stress increases as strain rate increases but it is less rate sensitive than yield stress.

- Elastic modulus is not affected by strain rate. 
When considering cyclic tests, the effect of strain rate on the flow stress is the same as for monotonic tests. Benson and Hancock (1974) performed incremental step tests on five alloys consisting of two aluminums, copper, martensitic steel and a 2-phase brass. An increase in flow stress with an increase of strain rate over the range of $0.003 \mathrm{~s}^{-1}$ to $0.3 \mathrm{~s}^{-1}$ can be seen except for T651 Aluminum and martensitic steel for the strain rates from $0.03 \mathrm{~s}^{-1}$ to $0.3 \mathrm{~s}^{-1}$. These materials show a decrease in flow stress when comparing the highest and the middle strain rate. Such a decrease at a higher strain rate could be expected if there is enough adiabatic heating to soften the materials being tested. Saeki et al. (1998) performed cyclic tests on four different low yielding steels at strain rates of $10^{-3} \mathrm{~s}^{-1}$ and $5 \times 10^{-2} \mathrm{~s}^{-1}$. Results of the tests shown on a cyclic stress-strain plot indicated that there was an increase in stress with strain rate but the increase decreased with increasing strain. This effect is larger in magnitude for the steels with the lowest yield strength.

However, not all metals are rate sensitive. Zhao and Abdennadher (2004) performed compressive tests on specimens cut from heat treated square brass tubes with a Split Hopkinson pressure bar apparatus. It was found that there was no rate sensitivity of stress over the strain rates of $10^{-4} \mathrm{~s}^{-1}$ to $2500 \mathrm{~s}^{-1}$. Dusicka et al. (2007) performed tests on five different plate steels of yield strength varying from $100 \mathrm{MPa}$ to $485 \mathrm{MPa}$ at the strain rates of $10^{-3} \mathrm{~s}^{-1}$ and $10^{-1} \mathrm{~s}^{-1}$. When considering the effect of strain rate on the stress for the first two cycles, it was observed that the lower yield strength steels (100 and $225 \mathrm{MPa}$ ) have a significant increase in stress with an increased strain rate. For the other steels (345, 440 and $485 \mathrm{MPa})$, there was almost no effect. Nevertheless, further into the cyclic loading, the effects of strain rate on the flow stress diminished for all tests. 


\subsubsection{Strain Rate Effect on Fatigue Life}

When considering effects of strain rate on fatigue life, the results vary with the studies. There is no consistent trend that can be observed between strain rate and fatigue life.

Saeki et al. (1998) performed cyclic tests on $132 \mathrm{MPa}$ and $290 \mathrm{MPa}$ Nippon Steel. For these tests, the fatigue life was defined as the number of cycles in which the applied load has reduced to $75 \%$ of the maximum load achieved in the test. Strain ranges varied from $0.4 \%$ to $3 \%$ and the strain rates used were $0.001 \mathrm{~s}^{-1}$ and $0.05 \mathrm{~s}^{-1}$. For high strength steel, there was little to no strain rate effect on the fatigue life. Any evidence of the rate effect is manifested in the high cycle fatigue region as a slightly negative rate sensitivity. For the low strength steel, there is a negative rate sensitivity in the high cycle fatigue region. However, the effect is still quite small. Dusicka et al. (2007) performed tests on five different grades of plate steel. Uniaxial specimens were fabricated with a $25 \mathrm{~mm}$ effective length and a $20 \mathrm{~mm}$ diameter. Most of the tests were performed at a rate of $0.001 \mathrm{~s}^{-1}$ and only five tests were performed at the strain rate of $0.1 \mathrm{~s}^{-1}$ and strain range of $4 \%$. Failure of these specimens were defined by the number of cycles to completely fracture the specimen or the number of cycles needed to reduce the applied stress to $50 \%$ of the maximum value achieved during the test. From the results of these tests, it was concluded that the strain rate had little effect on fatigue life, hysteretic behaviour and maximum cyclic stress (peak tensile stress).

Miller and Rizk (1968) performed cyclic torsional tests until rupture on commercially pure aluminum at rates ranging from 0.0011 to $0.0212 \mathrm{~s}^{-1}$. An increase in fatigue life was observed with an increase of strain rate up to a critical value of $0.0055 \mathrm{~s}^{-1}$ 
after which the effect of strain rate on fatigue life diminished. This critical value was found to be the same for all torque ranges in this study. Begum et al. (2009) performed cyclic tests on an AZ31 magnesium alloy. The specimens were fabricated with a gauge length of $25 \mathrm{~mm}$ and a width of $6 \mathrm{~mm}$ as per the ASTM E8 standard. They were cycled with a strain range of $0.8 \%$ at three strain rates ranging from $0.001 \mathrm{~s}^{-1}$ to $0.08 \mathrm{~s}^{-1}$ until fracture. An increase in fatigue life was observed with the increase in strain rate.

\subsubsection{Strain Rate Effect on Fracture Strain}

The effect of strain rate on the fracture strain may vary depending on the definition of strain used. Engineering strain is a measure of elongation of a test specimen where as true strain is a measure of localized strain that can be related to the cross section change of the specimen. Similar to strain rate effect on fatigue life, results of strain rate effects on fracture strain tend to vary among various studies. Blazynski (1983) stated that generally materials fall into two categories with one in which there is an increase in true fracture strain up to a critical strain rate to be followed by a reduction with a further increase in the strain rate. The other category being the true fracture strain is rate insensitive up to a critical strain rate to be followed by a reduction with a higher strain rate.

Albertini and Montagnani (1980) performed high strain rate monotonic tests on three types of stainless steel. It was found that a reduction in elongation was seen with an increase in the strain rate for all types of steel over the range of strain rates of $10^{-2} \mathrm{~s}^{-1}$ to $500 \mathrm{~s}^{-1}$. Barton et al. (1991) performed tests on notched specimens of copper and iron in an attempt to model the fracture behaviour. Monotonic tests were performed with a 
"flying wedge" apparatus at separation rates of $0.1 \mathrm{~m} / \mathrm{s}$ to $10 \mathrm{~m} / \mathrm{s}$. The fracture behaviour based on final specimen diameter of the copper specimens did not show any dependency on the strain rate. Conversely, iron showed a reduction of true failure strain as the strain rate increased. Alves and Jones (1999) performed monotonic tests on notched specimens of mild steel at strain rates of $4 \times 10^{-3} \mathrm{~s}^{-1}$ to nearly $2000 \mathrm{~s}^{-1}$. Results from these tests showed that the strain rate does not have a significant effect on the strain at peak stress and the fracture strain calculated based on the cross-section change of the specimen.

\subsection{Dynamic Strain Aging}

Discontinuous plastic flow of a solid solution on a stress-strain or stress-time plot, generally referred to as serrations, is labelled as the Portevin-Le Chatelier (PLC) effect. The PLC effect is actually the manifestation of dynamic strain aging (DSA), a phenomenon in which the mobility of solute atoms (Carbon, Nitrogen, Oxygen, etc) increases under favourable testing conditions and interact with dislocations. Several theories have been presented which describe this interaction as either solute atoms causing drag on moving dislocations or solute atoms pinning a dislocation which is arrested at an obstacle (Keh et al. 1968). Considering the model where dislocations are arrested at an obstacle, solute atoms diffuse and form atmospheres around the dislocation during the waiting time. The solute atoms increase the pinning strength of the dislocation which increases the load required for a dislocation to overcome the obstacle and start moving again (Hahner 1996). The pinning and unpinning is what is believed to cause the serrated behaviour in the flow stress. However, the appearance of the PLC effect has a strong dependency on the stiffness of the testing machine used. Teresa Correia and Fortes 
(1981) have noted that load drops occur only if the rate of plastic deformation suddenly exceeds the actuator speed. As well, the rate at which data points are collected may also affect the appearance of serrations. At lower rates, there may not be enough resolution in the test data to properly view the PLC effect. Generally, DSA occurs at intermediate temperatures and strain rates. Hong et al. (2004) explained that at low temperatures and high strain rates, the velocity at which solutes move are too slow to interact with dislocations to cause aging effects. For high temperatures and low strain rates, the atmosphere of solutes around a dislocation can move with a dislocation and the DSA effect does not appear.

DSA is an important phenomenon to consider during material tests of non-pure metals as it has some interesting effects on the behaviour of the flow stress. For both monotonic and cyclic tests, a decrease of flow stress generally occurs with an increase in temperature. However, if the material is susceptible to DSA, a possible slight decrease to be followed by an increase in flow stress will occur with increasing temperature over a certain band of temperatures for specific strain rate until a peak is observed. If testing temperature is increased further, a decrease in flow stress with increasing temperature will again be observed. Keh et al. (1968) performed tension tests on low-carbon steel at two temperatures. Larger dislocation density and flow stress were observed in tests with serrated flow stress over that with no serrated flow stress. The increase in dislocation density is believed to be the cause for the increased flow stress. Rodriguez (1984) has also summarized that a peak in the work hardening rate and flow stress also occur over the band of temperatures where DSA is observed, although the temperature at which both peak may not necessarily coincide. 
Moscato et al. (1997) stated that significant increase in the strength of hightemperature alloys and stainless steels at intermediate temperatures have been noted in several studies. Both Tsuzaki et al. (1991) and Weisse et al. (1993) performed monotonic and cyclic tests on plain carbon eutectoid steel and low carbon SAE 1045 steel respectively. Tsuzaki et al. (1991) has found that the tensile stress at $10 \%$ strain under monotonic loading peaked at temperatures of $250^{\circ} \mathrm{C}$ and $300^{\circ} \mathrm{C}$ for the strain rates of $0.00033 \mathrm{~s}^{-1}$ and $0.0033 \mathrm{~s}^{-1}$, respectively. For the cyclic tests at a strain range of $\pm 0.5 \%$, the peak tensile stress was observed to occur at the temperatures of $200^{\circ} \mathrm{C}$ and $250^{\circ} \mathrm{C}$ for the strain rates of $0.00033 \mathrm{~s}^{-1}$ and $0.0033 \mathrm{~s}^{-1}$ respectively. Weisse et al. (1993) has found that the monotonic tensile strength peaked at $300^{\circ} \mathrm{C}$ for the strain rate of $7 \times 10^{-4} \mathrm{~s}^{-1}$. For cyclic tests at the strain range of $\pm 0.5 \%$ and strain rate of $5 \times 10^{-3} \mathrm{~s}^{-1}$, the peak maximum stress amplitude was observed to occur at $265^{\circ} \mathrm{C}$. For type $316 \mathrm{~L}$ stainless steel tested at a strain range of $\pm 0.25 \%$, Hong et al. (2005) has found that DSA occurs at temperature ranges of $250^{\circ} \mathrm{C}$ to $550^{\circ} \mathrm{C}, 250^{\circ} \mathrm{C}$ to $600^{\circ} \mathrm{C}$ and $250^{\circ} \mathrm{C}$ to $650^{\circ} \mathrm{C}$ for the strain rates of $10^{-4}, 10^{-3}$ and $10^{-2} \mathrm{~s}^{-1}$ respectively. Prasad et al. (2008) has shown that the peak tensile stress of IMI 834 titanium alloy increased with temperature over the temperature range of $375^{\circ} \mathrm{C}$ to $425^{\circ} \mathrm{C}$ for tests performed at a $\pm 1 \%$ strain range and a strain rate of $0.000667 \mathrm{~s}^{-1}$. A similar result has been observed by Avalos et al. (2009) for heat treated AISI 430F ferritic steel at a temperature range of $350^{\circ} \mathrm{C}$ to $450^{\circ} \mathrm{C}$ for tests performed at a $\pm 0.3 \%$ strain range and a strain rate of $10^{-3} \mathrm{~s}^{-1}$. For mild steel, Zeghib and Klepaczko (1996) have noted that the effects of DSA tend to occur in the temperature range of $75^{\circ} \mathrm{C}$ to $325^{\circ} \mathrm{C}$. Both Tsuzaki et al. (1991) and Weisse et al. (1993) have shown that the temperatures at which peak DSA 
effects on the flow stress occur are lower in cyclic than monotonic tests. However, no reason and explanation were provided.

It should be noted from the above discussions, the band of temperature in which DSA has been found to occur shifts higher when the strain rate increases. Often, this phenomenon causes a negative rate sensitivity when comparing tests of different strain rate performed at the same temperature when DSA occurs. As mentioned previously, when a dislocation is arrested at an obstacle, solute atoms will be attracted to it and thus increases the pinning strength. When DSA occurs, there is a specific temperature that produces a maximum DSA effect for a strain rate (Cunningham 1999). Corollary, there is also a specific strain rate that produces a maximum DSA effect for a temperature. Thus, a specimen tested at a constant temperature can exhibit negative strain rate strength sensitivity at strain rates higher than that to produce the maximum DSA effect, and positive strain rate sensitivity at lower strain rates. This is represented by the flow stress versus temperature curves in Fig. 2.1, which displays the effect of dynamic strain aging at two different strain rates.

It has been noted by Cheng and Nemat-Nasser (2000) that dynamic strain aging effects appear after a certain (critical) strain has been achieved in a test. It is discussed that plastic straining increases the number of vacancies within the matrix of the material and that an increase in the number of vacancies improves solute atom mobility. This effect can be seen in monotonic tension tests of mild steel by Brindley and Barnaby (1966). Serrations in the flow stress did not begin immediately during loading but only after some plastic strain has accumulated. 


\subsection{Constitutive Models}

When modelling the plastic work hardening behaviour of a metal under cyclic loading, the flow stress is often more accurately modeled if a kinematic or combined isotropic/kinematic hardening model is used over that of an isotropic hardening model. This is because kinematic hardening can better model the Baushinger effect which is commonly observed in cyclic tests. Prager (1956) was one of the first to propose a model which describes the translation of the yield surface in stress space. The yield surface can be defined by the von Mises condition as

$$
f\left(\sigma_{i j}-\alpha_{i j}\right)=\sqrt{\frac{3}{2}\left(s_{i j}-\alpha_{i j}^{d e v}\right)\left(s_{i j}-\alpha_{i j}^{d e v}\right)}-\sigma^{o}=0
$$

where $\sigma_{i j}$ is the stress tensor, $\alpha_{i j}$ is the backstress which represents the translation of the yield surface, $s_{i j}$ is the deviatoric stress tensor, $\alpha_{i j}^{d e v}$ is the deviatoric backstress tensor and $\sigma^{o}$ is the yield stress (size of the yield surface). The evolution of the backstress is assumed to follow the direction of the incremental plastic strain tensor defined simply as

$$
\dot{\alpha}_{i j}=C \dot{\varepsilon}_{i j}^{p}
$$

where $\dot{\varepsilon}_{i j}^{p}$ is the plastic strain rate tensor and $C$ is a constant. This defines a linear translation of the yield surface with an increase in plastic straining. Although less popular, Ziegler (1958) also defined a linear translation rule for the yield surface. Unlike Prager, Ziegler defined the movement of the yield surface in the radial direction of the yield surface. This is given by

$$
\dot{\alpha}_{i j}=d \mu\left(\sigma_{i j}-\alpha_{i j}\right)=C \frac{1}{\sigma^{o}} \dot{p}\left(\sigma_{i j}-\alpha_{i j}\right)
$$

where $C$ is a constant and $\dot{p}$ is the effective plastic strain rate given by 


$$
\dot{p}=\sqrt{\frac{2}{3} \dot{\varepsilon}_{i j}^{p} \dot{\varepsilon}_{i j}^{p}}
$$

The term $\left(\sigma_{i j}-\alpha_{i j}\right)$ is not deviatoric and therefore this model can account for the effect of hydrostatic stress on the backstress. However, both Prager's and Ziegler's models are the same (Lemaitre and Chaboche 1990) if incompressible plasticity is considered with yielding defined by the von Mises yield criteria given in Eq. (2.9). Since the evolution of backstress describes the movement of the yield surface, upon loading reversal, yielding will occur at a lower stress than seen in initial loading. Therefore, the Baushinger effect can be partially represented by this kinematic hardening. However, linear plastic behaviour is hardly seen during the testing of metals. Therefore, a recall term has been introduced to Eq. (2.11) by Armstrong and Frederick (1966) to obtain non-linearity with kinematic hardening. This takes the form of

$$
\dot{\alpha}_{i j}=C \frac{1}{\sigma^{0}} \dot{p}\left(\sigma_{i j}-\alpha_{i j}\right)-\gamma \alpha_{i j} \dot{p}
$$

where $\gamma$ is a constant. Further improvements of the kinematic hardening model were proposed by Chaboche et al. (1979) by introducing multiple terms of backstress to define the movement of the yield surface. The evolution of the backstress tensor for the $n^{\text {th }}$ term is defined by

$$
\dot{\alpha}_{i j_{n}}=C_{n} \frac{1}{\sigma^{o}} \dot{p}\left(\sigma_{i j}-\alpha_{i j}\right)-\gamma_{n} \alpha_{i j_{n}} \dot{p}
$$

where $C_{n}$ and $\gamma_{n}$ are constants for the $\mathrm{n}^{\text {th }}$ term, and the overall backstress tensor is given by

$$
\alpha_{i j}=\sum_{n=1}^{N} \alpha_{i j}
$$


where $N$ is the total number of backstress terms. Having multiple terms of backstress has been found to improve the accuracy in which the hardening model can model test data. However, this also increases the difficulty and time involved in calibrating the hardening parameters. Reducing Eq. (2.14) to the uniaxial tension condition gives

$$
\dot{\alpha}_{n}=C_{n} \dot{\varepsilon}_{t}^{p}-\gamma_{n} \alpha_{n} \dot{\varepsilon}_{t}^{p}
$$

where $\alpha_{n}$ is the uniaxial equivalent backstress for term $n$ given by

$$
\alpha_{n}=\sqrt{\frac{3}{2} \alpha_{i j_{n}} \alpha_{i j_{n}}}
$$

and $\varepsilon_{t}^{p}$ is the uniaxial true plastic strain. Integrating Eq. (2.16) over a half cycle and assuming there is no initial backstress gives

$$
\alpha_{n}=\frac{C_{n}}{\gamma_{n}}\left(1-e^{-\gamma_{n} \varepsilon_{t}^{p}}\right)
$$

For a combined hardening model, the kinematic as well as the isotropic component of the flow stress must be defined. The flow stress under monotonic uniaxial tension loading can be defined as

$$
\sigma_{t}^{F}\left(\varepsilon^{p}\right)=\sigma^{i}+\alpha
$$

where $\sigma_{t}^{F}$ is the true flow stress given as a function of the plastic strain, $\alpha=\sum_{n=1}^{N} \alpha_{n}$ is the total backstress and $\sigma^{i}$ is the yield stress defined for the uniaxial condition as

$$
\sigma^{i}=\sqrt{\frac{3}{2}\left(s_{i j}-\alpha_{i j}^{d e v}\right)\left(s_{i j}-\alpha_{i j}^{d e v}\right)} .
$$

Rearranging Eq. (2.19) gives

$$
\sigma^{i}=\sigma_{t}^{F}\left(\varepsilon^{p}\right)-\alpha .
$$


If the backstress is defined by Eq. (2.18), this leaves only the flow stress to be defined. Different functions have been proposed to describe the relationship of the flow stress and plastic strain. An exponential law based function has been found to be useful in describing the flow stress for modelling cyclic behaviour, as by its nature saturates at large strain similar to that of the flow stress observed in a cyclic test. Chinh et al. (2004) proposed a flow stress equation based on an exponential law as

$$
\sigma_{t}^{F}=\sigma_{t}^{y}+\sigma_{1}\left[1-\exp \left[\frac{-\left(\varepsilon_{t}^{p}\right)^{d}}{\varepsilon_{c}}\right]\right]
$$

where $\sigma_{t}^{F}$ is the true flow stress, $\sigma_{t}^{y}$ is the true stress at initial yielding, $\varepsilon_{t}^{p}$ is the true plastic strain, and $\sigma_{1}, d$, and $\varepsilon_{c}$ are constants. Equation (2.22) has been verified for both pure aluminum and pure copper at large strain up to 8.0. Another possible function to describe the flow stress is based on a power law relationship with the plastic strain. A popular form of the power law function is the Ludwik type equation of

$$
\sigma_{t}^{F}=\sigma_{t}^{y}\left[1+\left(\frac{\varepsilon_{t}^{p}}{c}\right)^{m}\right]
$$

where $c$ and $m$ are constants. A list of other functions can also be found in Sung et al. (2010).

Even though the preceding discussion on plastic work hardening behaviour under cyclic loading do not involve effects of strain rate, it is expected that strain rate in some form may affect various components of the stresses. Thus, additional discussions on strain rate effects on material behaviour modelling will be provided in the sections to follow in the analysis of test results. For modelling monotonic loading, Sung et al. (2010) have listed a number of flow stress functions that incorporate strain rate and temperature. 


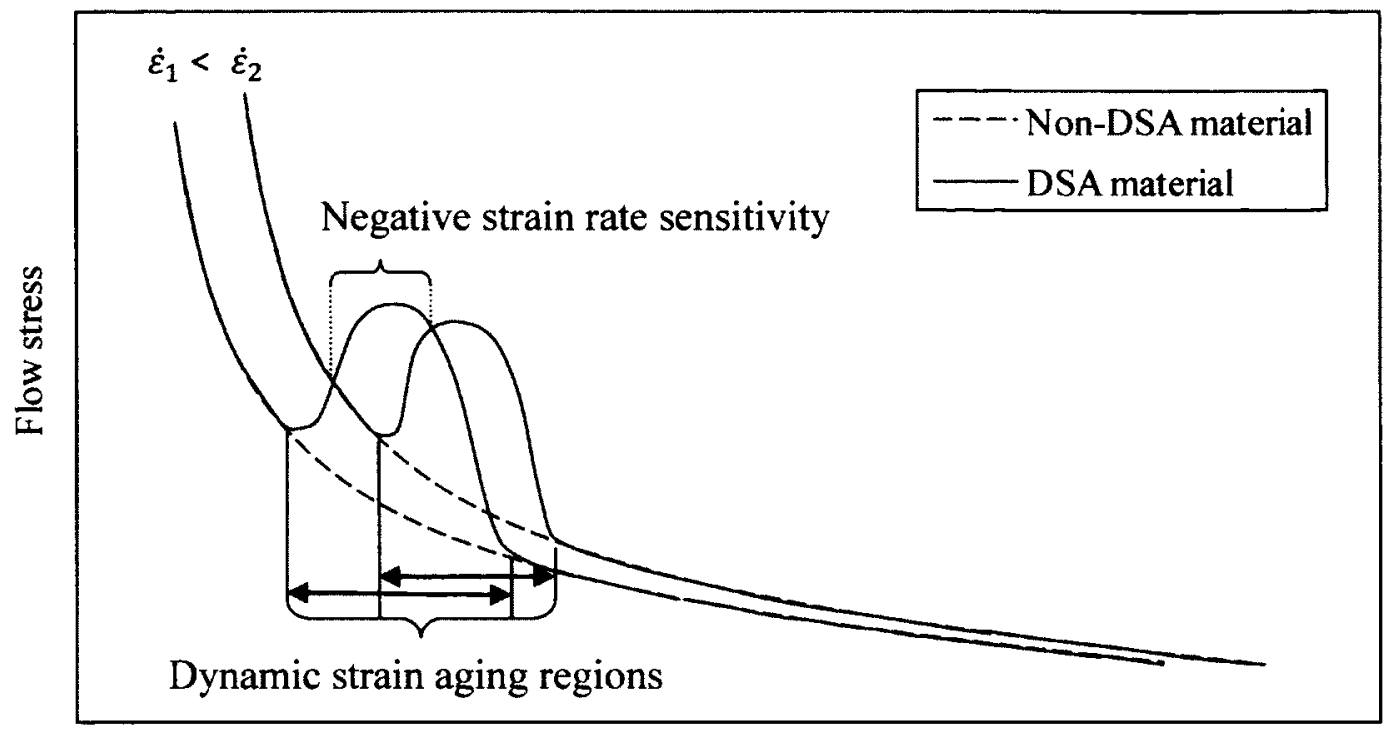

Temperature

Figure 2.1 Generalized flow stress versus temperature curves at a strain showing the effect of dynamic strain aging at two different strain rates (Cunningham 1999) 


\section{Chapter 3 Testing Program}

Effects of strain rate on the flow stress of metals have been well studied. Generally, an increase in flow stress is seen with an increase in strain rate. However, effects of strain rate and loading history on the cyclic behaviour, including the fatigue life are less well defined. Thus, tests have been carried out to further investigate the cyclic behaviour of mild steel at various strain rates.

\subsection{Objective}

The objective of the testing program is to study effects of strain rate on the cyclic behaviour of mild steel, specifically, the rate effects on flow stress, fracture limit and fatigue life. In addition, the loading history and mean strain effects on the cyclic behaviour have also been considered. The tests have been conducted at the moderate strain rate since effects of impact and blast loading are not part of the focus of the current study.

\subsection{Description of Test Specimens}

Two shapes of $14 \mathrm{~mm}$ diameter round specimens consisting of the notched and tapered profiles were considered in this study. The tapered specimens were fabricated with a $4 \%$ smaller cross-sectional area at the mid-length of the specimen while the notched specimens had a minimum diameter of $14 \mathrm{~mm}$ and a radius of curvature of 19 $\mathrm{mm}$ at the notch. The profile of the tapered specimen can be seen in Fig. 3.1 and the notched specimen can be seen in Fig. 3.2. These specimens were fabricated from 25.4 
$\mathrm{mm}$ ( 1 inch) diameter rod, from two steel grades of CAN/CSA G40.20/21 Grade 300W (44W) and ASTM A572 Grade $50(345 \mathrm{MPa})$. A total of 22 tapered and 25 notched specimens from both grades were tested. The major alloying elements of the materials used in this thesis can be found in Table 3.1.

The loading applied for tapered specimens consisted of some combination of cycling the specimen at designated strain ranges to be followed by pulling the specimen to fracture. For notched specimens the loading consisted of cycling the specimen at a designated strain range until fracture. The specific loading applied for each tapered specimen can be found in Table 3.2 and for each notched specimen in Table 3.3.

\subsubsection{Specimen Designation}

The following designation has been adopted to describe the test specimen.

\section{$\alpha \beta E \mu \delta \omega-\#$}

a The first alphabet of either A or B represents the material. A for CAN/CSA G40.20/21 Grade 300W (44W) and B for ASTM A572 Grade 50 (345 MPa).

$\beta \quad$ The second alphabet identifies the geometry of the specimen. The designation $T$ refers to specimens with the tapered profile shown in Fig. 3.1 and $\mathrm{N}$ refers to specimens with the notched profile shown in Fig. 3.2.

$\mathrm{E} \mu$ The number represents the target loading rate as in $10^{-\mu} \mathrm{s}^{-1}$

$\delta \quad$ The fifth alphabet represents the loading type and sequence. It is designated with either I, D, C or E for tapered specimens, or R for notched specimens.

I Cyclically loaded at increasing engineering strain ranges of $\pm 0.3 \%, \pm 0.5 \%$, $\pm 1 \%, \pm 1.5 \%$, and $\pm 2 \%$ for 10 cycles at each range before being pulled to 
fracture. These strain ranges were applied for each strain rate except for $10^{-1} \mathrm{~s}^{-1}$. The strain range of $\pm 0.3 \%$ was not applied at this strain rate because it was found that an accurate control could not be obtained. Therefore, the test began at the $\pm 0.5 \%$ strain range.

D Cyclically loaded at decreasing engineering strain ranges of $\pm 2 \%$ and $\pm 0.5 \%$ for 10 cycles at each range before being pulled to fracture.

C Cyclically loaded at an engineering strain range sequence of $0 / 2 \%, 0 / 4 \%$, $0 / 2 \%, \pm 2 \%, \pm 1 \%$ and $\pm 2 \%$ for 10 cycles at each range.

E Cyclically loaded for 20 cycles at each strain range twice for the engineering strain range sequence of $\pm 1 \%, 0 / 2 \%, \pm 1 \%$ and $-2 / 0 \%$, and to be followed by four times at the strain range sequence of $0 / 2 \%$ then $-2 / 0 \%$.

$\mathrm{R}$ Cyclically loaded to fracture at one nominal true strain range, except for two specimens as noted in the Table 3.3 that were loaded only for 20 cycles.

$\omega$ Represents the half nominal true strain range of the loading for notched specimens. It is either 2,4 or 8 for the nominal true strain range of $\pm 2 \%, \pm 4 \%$ and $\pm 8 \%$. This only applied to notched specimens.

\# A designation to identify tests that have been repeated. A value of 2 represents a test that is repeated while 20 represents a test with the same strain range loading sequence but loaded for 20 cycles instead of 10 at each strain range. For example, ATE1I-2 represents a repeated test for ATE1I while ATE2D-20 represents a test loaded for 20 cycles per strain range with the loading sequence as ATE2D. A value of 200 is only used for one test. The ATE2C-200 specimen was cyclically loaded at the first strain range in the $\mathrm{C}$ series of $0 / 2 \%$ for 200 cycles. 


\subsubsection{Tapered Specimens}

Tapered specimens were used in this study when the applied strain range is small and the specimen is being pulled to fracture. For a specimen with a straight profile, the exact location of necking is not known a priori. However, a tapered specimen allows for the measurement of minimum cross-sectional diameter during specimen necking. With a tapered specimen, it is possible to place the diametral extensometer at the location of the minimum cross-sectional diameter and have confidence that necking will likely occur there. This makes it possible to control the applied true strain loading rate through the diametral extensometer. Tapered specimens have been used by Chen (2010) in the study to measure the flow stress under various strain rates. The profile has been chosen such that the taper is large enough to ensure necking at the minimum cross-sectional diameter while at the same time does not significantly alter the uniaxial stress state in the specimen due to the slight taper before necking.

Two testing series were considered in this study in which the specimens were pulled to fracture after cycling. The first testing series, given the designation $\mathrm{D}$, cycled the specimens the engineering strain range of $\pm 2 \%$ and $\pm 0.5 \%$ for 10 cycles at each range, and after which they were pulled to fracture. Trampczynski (1988) noted that in both torsion and tension-compression tests kinematic and isotropic hardening parameters were found close to have stabilized after the first three half-cycles of the test. After performing fully reversed tension-compression tests on low and moderate yielding steels, Saeki et al. (1998) also noted that almost identical hysteresis loops were formed approximately 4 to 5 cycles into the loading. Therefore, it is assumed that 10 cycles is sufficient to obtain the stabilized hysteresis curve at a given strain range. The strain rates 
considered for this testing series are $10^{-1} \mathrm{~s}^{-1}, 10^{-2} \mathrm{~s}^{-1}, 10^{-3} \mathrm{~s}^{-1}$ and $10^{-4} \mathrm{~s}^{-1}$. The second testing series, designated as I, involved specimens cycled at engineering strain range sequence of $\pm 0.3 \%, \pm 0.5 \%, \pm 1 \%, \pm 1.5 \%$ and $\pm 2 \%$ for 10 cycles at each strain range before being pulled to fracture. Tests in this series were conducted at the strain rates of $10^{-1} \mathrm{~s}^{-1}, 10^{-2} \mathrm{~s}^{-1}$ and $10^{-3} \mathrm{~s}^{-1}$.

In addition to these tests, two other test series were considered that did not involve pulling the specimen to fracture after cycling. The first, given the designation $\mathrm{C}$ for the loading type and sequence involved cycling a tapered specimen at the strain rate of $10^{-2} \mathrm{~s}^{-1}$ at the strain ranges of $0 / 2 \%, 0 / 4 \%, 0 / 2 \%, \pm 2 \%, \pm 1 \%$ and $\pm 2 \%$ for 10 cycles at each strain range. The second, given the designation $E$, involved cyclically loading the specimen at the strain rate of $10^{-2} \mathrm{~s}^{-1}$ for 20 cycles and two passes of a strain range sequence of $\pm 1 \%, 0 / 2 \%, \pm 1 \%,-2 / 0 \%$, and to be followed four times at the strain range sequence of $0 / 2 \%$ and $-2 / 0 \%$. The purpose of these tests is to determine the effect of mean strain on the mechanical behaviour of the materials.

The test matrix for the tapered specimen is shown in Table 3.2. Chen (2010) has determined that it is not possible to have an adequate loading control with the diametral extensometer for tests performed at strain rates of $10^{-1} \mathrm{~s}^{-1}$ and higher. Therefore, only the axial extensometer was used to control loading when the specimen was being pulled to fracture at the strain rate of $10^{-1} \mathrm{~s}^{-1}$. The objectives for various tapered specimen tests are summarized in Table 3.4 


\subsubsection{Notch Specimens}

Unlike tapered specimens, notched specimens allow cyclic tests to be performed at large strain ranges. A tapered specimen will normally buckle in a fully reversed cyclic test at a large strain range, before reaching its fatigue life. For this reason, notched specimens were used for fully reversed fatigue tests at nominal true strain ranges of $\pm 2 \%$, $\pm 4 \%$ and $\pm 8 \%$. The engineering strain rates considered for the notched specimens are $10^{-1} \mathrm{~s}^{-1}, 10^{-2} \mathrm{~s}^{-1}, 10^{-3} \mathrm{~s}^{-1}$ and $10^{-4} \mathrm{~s}^{-1}$ at all of the true strain ranges. These strain rates and ranges were considered for both materials. All specimens were loaded until separation occurred with the exception of the test performed at the strain range of $\pm 2 \%$ and strain rate of $10^{-4} \mathrm{~s}^{-1}$. This test was only performed for 20 cycles. The test matrix for notched specimens is shown in Table 3.3. Loading for notched specimen tests was controlled through an axial extensometer because the deformation in the radial direction is too small to allow for a reliable control through a diametral extensometer. For this reason, finite element analysis (FEA) has to be carried out to estimate the axial extensions required to produce the desired true strains. Details of these analyses are discussed in Appendix A.

In a notched specimen test at a constant axial strain range, the nominal true strain (cross-sectional change) achieved in each cycle will not be constant due to the effect of material hardening and changing notched profile. Nevertheless, it was assumed that the variation in the nominal true strain range between loading cycles is small and a constant axial strain range obtained from FEA simulations can be used for each test. This assumption may not be true for tests at the $\pm 8 \%$ strain range as the overstress may significantly alter the notched profile between loading cycles. The objectives of various notched specimen tests are summarized in Table 3.4 


\subsection{Specimen Preparation}

Prior to testing, manual measurements of the minimum cross-sectional diameters of both tapered and notch specimens were taken with a Mitutoyo digital caliper. Measurements of the length of the notch and the reduced section of the tapered specimen, and the diameter outside of the notch and the reduced section were also verified. Manual measurements of the notched specimen profile were not carried out. Wen (2012) has used the notched specimen machined to the same profile. It has been verified by Wen (2012) through the shadowgraph that the radius of curvature of the notch can be considered to be accurate to $0.01 \mathrm{~mm}$ as specified.

The region around the mid length of the tapered specimens was sanded by hand with sandpaper of a progressively finer grit (400 to 1500). A smoother surface at the midlength of the tapered specimens was provided to facilitate the diametral extensometer sliding to the point of minimum cross-sectional diameter of the specimen during necking. After sanding, a Mitutoyo Type PJ-300 shadowgraph was used to measure the minimum cross-sectional diameter and mark its location. For the notched specimens, the surface outside of the notch was sanded with 400 grit sand paper to allow the clip-on axial extensometer to slide off the specimen after fracture has occurred to avoid damaging the extensometer as the specimens were erroneously machined to a rougher finish outside the notch. However, the axial extensometer was found to slip during fatigue tests of notched specimens performed at the strain rate of $10^{-2}$ and $10^{-1} \mathrm{~s}^{-1}$. Therefore, notches were filed into the specimens used for those tests so that the knife edge of the extensometer was less likely to move during the test. 


\subsection{Test Setup and Instrumentation}

An MTS 810 hydraulic testing machine was used to perform all tests. Axial strains were measured with an MTS $634.25 \mathrm{E}-2450.8 \mathrm{~mm}$ ( 2 inch) clip-on extensometer for the notch specimens and an MTS $634.12 \mathrm{E}-2425.4 \mathrm{~mm}$ (1 inch) extensometer was used for the tapered specimens. Diametral displacements were measured with an MTS 632.18E-20 extensometer. The diametral extensometer has been modified for this study to allow for a specimen of larger diameter to be tested in extension up to fracture. Extended grips were fabricated and attached to the extensometer to effectively increase the travel of the gauge. Pictures of the test setup can be seen in Fig. 3.3. All the tests were loaded by axial extensometer control except when tapered specimens were pulled to fracture. When the specimen was being pulled to fracture, the loading was switched from axial to diametral extensometer control at an axial strain of 0.1 for specimens loaded at a strain rate of $10^{-2} \mathrm{~s}^{-1}$ and lower.

To achieve the target strain rate, it was necessary to tune the testing machine for each type of specimen (extensometer) and each strain rate. In the tuning, a test specimen was loaded for 5 cycles at a given strain rate, and difference between the command signal and the recorded response by the axial extensometer, including the strain rate, were compared. Once the 5 cycles were completed, the values of $\mathrm{P}$ gain and I gain on the testing machine were varied. Several trials were conducted at various gain values and the data analyzed to determine the optimum settings for a particular specimen-rate combination. For high strain rate tests, it is necessary to use large gain values to achieve a proper strain rate control. As gain values increase, the accuracy in which the testing machine can control the strain range decreases. However, an inaccurate strain range is 
assumed to have less of an effect on the flow stress than an inaccurate strain rate. Therefore, for the $10^{-1} \mathrm{~s}^{-1}$ tests, large gain values were used. Values of gain used for the $25.4 \mathrm{~mm}(1 \mathrm{inch})$ extensometer and diametral extensometer to control the tapered specimen tests at various strain rates can be found in Table 3.5 and 3.6. Similarly, values of gain used for the $50.8 \mathrm{~mm}$ ( 2 inch) extensometer to control the notched specimen tests at various strain rates can be found in Table 3.7. For the calibration, the D gain was set to 0.001 .

\subsection{Temperature Measurement Methods and Calibration}

The response of the material may vary at high temperature compared to room temperature. It is expected that the temperature of the specimen will increase considerably during the test when the loading rate is high. Thus, temperature measurements for some high strain rate tests were recorded with a Raytek Raynger MX4 infrared measurement gun and a type $T$ thermocouple. The objective of the measurement is to provide approximate quantitative results on the temperature of the specimen so no elaborate setup was designed to measure the temperature of the specimen in detail.

To use the IR gun on a metal specimen, a calibration of the emissivity was needed. Emissivity is the ratio of energy emitted by an object to the energy emitted by a black body at the same temperature. If an object is highly reflective, such as a metal specimen, the IR gun will detect the energy emitted by the specimen as well as any energy that is reflected off the surface of the specimen. Lowering the emissivity on the IR gun will lower the value of temperature displayed by the gun based on a pre-programmed calibration curve and thus compensate for the energy reflected off of the specimen. To 
determine the emissivity within the gauge length of a specimen, an oven was used in conjunction with a thermocouple to heat a notched specimen to a known temperature given by the thermocouple. The specimen was heated for 15 minutes once the oven temperature had stabilized (taking more than one minute for the thermocouple to detect a change in temperature of $1^{\circ} \mathrm{C}$ ). After 15 minutes in the oven, the specimen was removed and placed on 2 concrete supports on a black table. The IR gun was used to take intermittent temperature measurements for approximately one minute at one emissivity setting, after which the specimen was placed back in the oven for another 15 minutes. The emissivity settings on the IR gun were adjusted and another set of measurements were taken with the new emissivity setting. Two temperatures, $146^{\circ} \mathrm{C}$ and $210^{\circ} \mathrm{C}$, were used in the calibration. Five trials were considered for the first temperature and three for the second. It was found that an emissivity of 0.15 produced a measurement closest to the temperature of the oven measured by the thermocouple.

During high strain rate tests, the gun was set up on a tripod at an optimum range of 1.15 meters from the specimen where the field of view is the smallest. Due to the fact that the gun was calibrated by taking temperature measurements of the specimen on a black table, a black garbage bag was placed behind the testing machine to replicate the condition during the calibration. This method of temperature measurement with the IR gun was only used to provide an approximate specimen temperature during high strain rate tests. The minimum field of view of the IR gun used in this study is $19 \mathrm{~mm}$ at a distance of 1.15 meters from the target. At this distance, the field of view is slightly larger than the specimen. Ideally the target should be larger than the field of view to obtain a more accurate reading. In this setup, the area of the field of view outside the 
specimen is also included in the temperature measurement given by the gun. However, this was also the case when the emissivity was calibrated. Nevertheless, this is not considered to be an accurate use of the gun. In addition, the low emissivity set for these tests is outside of the optimum operating range for the gun. This may affect measurements of temperature as well.

In addition to the IR gun, a thermocouple was used to take the surface temperature of a notched specimen after it had failed. Pictures of the temperature measuring device and thermocouple can be seen in Fig. 3.4. This method of temperature measurement has its own set of shortcomings. The thermocouple was held to the specimen by hand after failure has occurred and the machine had stopped moving. During this time, the specimen underwent rapid cooling. There was also a time delay for the thermocouple measurement to stabilize. Thus, the measured temperature was not the actual temperature at the end of the test. Similar to the IR gun, only the temperature on the surface of the specimen was measured with the thermocouple. Ideally, the temperature profile of the specimen from the centre to the surface should be measured.

\subsection{Strain Rate Control}

In this testing program, two types of loading rate control were employed; one through the axial extensometer and the other through the diametral extensometer. All tests were carried out with the axial extensometer control except for tapered specimens that were loaded at strain rates of $10^{-2} \mathrm{~s}^{-1}$ and lower that were pulled to fracture. For those tapered specimens, the loading switched from axial extensometer control to diametral extensometer control at an axial strain of 0.1 when being pulled to fracture. 
Diametral extensometer control is the more accurate form of control for testing at a constant true strain range and rate due to the fact that the change in diameter is directly related to the true strain, especially for notched specimens and tapered specimens after necking. However for the tests considered in this testing program, the largest applied true strain range is approximately $\pm 8 \%$. This translates to a diameter change of approximately $\pm 0.27 \mathrm{~mm}$ for the notched specimens. With a small deformation range, it is more difficult to maintain an accurate control with a diametral extensometer in cyclic loading. Thus, axial extensometer control was used for cyclic loading. For the notched specimens, the required applied loading deformations in the axial direction for the target true strain range were calculated indirectly through finite element analysis (FEA). However, when the tapered specimen was being pulled to fracture, its cross-sectional deformation was consistently larger and its diameter reduced monotonically. Thus, the loading was switched to diametral extensometer control at the axial strain of 0.1 so that the target true strain rate during necking can be more accurately maintained when the specimen was being pulled to fracture. Previous tests by Chen (2010) have shown that the onset of necking occurs at an axial strain of approximately 0.1 to 0.2 in mild steel. Once necking occurs, the local strain rate at the neck may be several times higher than the axial strain rate. For this reason, it is useful to control the loading to close to a constant true strain rate through the diametral extensometer measurement. For tests performed at a strain rate of $10^{-1} \mathrm{~s}^{-1}$, it was not possible to switch to diametral extensometer control and maintain an accurate loading rate after the switch due to the brief duration it took to fracture the specimen from the start of the pull. Therefore, only axial extensometer control was used for these tests. 
The diametral extensometer measures the deformation and not the strain. For this reason, it was necessary to determine the relationship between the deformation measured by the diametral extensometer (cross-section change) to the axial strain. To control the true strain rate after necking with the diametral extensometer, a relationship between the desired true strain rate and the rate of change in the diameter (extensometer) must be established. The engineering strain is defined as

$$
\varepsilon_{e}=\frac{\Delta L}{L_{o}}=\frac{L-L_{o}}{L_{o}}=\frac{L}{L_{o}}-1
$$

where $\Delta L$ is the change in length, $L$ is the current length and $L_{o}$ is the original length. The true strain can be defined as

$$
\varepsilon_{t}=\int_{L_{o}}^{L} \frac{d L}{L}=\ln \left(\frac{L}{L_{o}}\right)
$$

If it is assumed that no volume change takes place within the gauge length during straining (plastic deformation), the relationship

$$
A_{o} L_{o}=A L
$$

can be established where $A_{o}$ is the original cross-sectional area of the specimen and $A$ is the current cross-sectional area of the specimen. Equation (3.3) can be rearranged and substituted into Eq. (3.2) to approximate the true strain to the cross-sectional dimension as

$$
\varepsilon_{t}=\ln \left(\frac{A_{o}}{A}\right)=2 \ln \left(\frac{D_{o}}{D}\right)
$$

where $D_{o}$ is the original diameter and $D$ is the current diameter of the specimen. Taking the derivative of Eq. (3.4) with respect to time, the true strain rate is given by 


$$
\dot{\varepsilon}_{t}=-2 \frac{\dot{D}}{D}
$$

where the superimposed "." represents the time rate of change. The relationship between $\dot{\varepsilon}_{t}$ and $\dot{D}$ is inherently given in Eq. (3.4). However, Chen (2010) has linearized Eq. (3.4) between a true strain of 0 and 1.0 to provide a simplified relationship between the true strain rate and the diameter change rate, with a true strain of 1.0 being the approximate upper limit of the true strain at fracture for mild steel under monotonic tension loading. Equation (3.4) can be rearranged to give

$$
\frac{D}{D_{o}}=e^{-0.5 \varepsilon_{t}} .
$$

Since the diametral extensometer measures the change in the diameter, Eq. (3.6) can be rewritten as

$$
\frac{\Delta D}{D_{o}}=1-e^{-0.5 \varepsilon_{t}}
$$

where

$$
\Delta D=D_{o}-D
$$

with the reduction in diameter taken to be positive. Linearizing Eq. (3.7) according to Chen (2010) gives

$$
\frac{\Delta D}{D_{o}}=0.3935 \varepsilon_{t} .
$$

Substituting Eq. (3.8) into Eq. (3.9) and rearranging gives

$$
D=D_{o}-0.3935 D_{o} \varepsilon_{t} .
$$

Taking the derivative of Eq. (3.10) with respect time gives

$$
\dot{D}=-0.3935 D_{o} \dot{\varepsilon}_{t} .
$$


According to Chen (2010), the actual true strain rate achieved using the rate of change in the diameter calculated by Eq. (3.11) are $79 \%$ and $121 \%$ of the target rate at true strains of 0 and 1.0 respectively. Equation (3.11) was used to determine the applied rate of change of the diameter for a target true strain rate when the specimen was being pulled to fracture using diametral extensometer control. In the tests, the applied rate of diameter change for tapered specimens was calculated according to Eq. (3.11) as

$$
\dot{D}=-0.3935 D_{o} \dot{\varepsilon}_{n}
$$

where $\dot{\varepsilon}_{n}$ is the nominal loading strain rate. 
Table 3.1 Chemical composition of the major alloying elements

\begin{tabular}{|c|c|c|c|c|c|c|}
\hline Material & Designation & $\mathrm{C} \%$ & $\mathrm{P} \%$ & $\mathrm{~S} \%$ & $\mathrm{Mn} \%$ & $\mathrm{Si} \%$ \\
\hline CAN/CSA G40.20/21 300W & $\mathrm{A}$ & 0.20 & 0.013 & 0.043 & 0.72 & 0.24 \\
\hline ASTM A572 Gr. 50 & $\mathrm{B}$ & 0.17 & 0.030 & 0.029 & 1.03 & 0.22 \\
\hline
\end{tabular}


Table 3.2 Test matrix for tapered specimens

\begin{tabular}{|c|c|c|c|c|c|}
\hline Specimen & Material & $\begin{array}{l}\text { Nominal } \\
\text { loading } \\
\text { strain rate } \\
\left(\mathrm{s}^{-1}\right)\end{array}$ & $\begin{array}{l}\text { Target engineering strain } \\
\text { range }\end{array}$ & $\begin{array}{l}\text { Pull to } \\
\text { fracture }\end{array}$ & $\begin{array}{c}\text { Loading control } \\
\text { (A - axial extensometer control, } \\
\text { D - diametral extensometer control) }\end{array}$ \\
\hline ATE1I & $\mathrm{A}$ & $1.00 \mathrm{E}-01$ & $\pm 0.5 \%, \pm 1 \%, \pm 1.5 \%, \pm 2 \%$ & Yes & $\mathrm{A}=0.1 \mathrm{~mm} / \mathrm{mm} / \mathrm{s}$ \\
\hline ATE2I & A & $1.00 \mathrm{E}-02$ & $\begin{array}{c} \pm 0.3 \%, \pm 0.5 \%, \pm 1 \%, \pm 1.5 \% \\
\pm 2 \%\end{array}$ & Yes & $\mathrm{A}=0.01 \mathrm{~mm} / \mathrm{mm} / \mathrm{s}, \mathrm{D}=0.0289 \mathrm{~mm} / \mathrm{s}$ \\
\hline ATE3I & A & $1.00 \mathrm{E}-03$ & $\begin{array}{c} \pm 0.3 \%, \pm 0.5 \%, \pm 1 \%, \pm 1.5 \% \\
\pm 2 \%\end{array}$ & Yes & $\begin{array}{c}\mathrm{A}=0.001 \mathrm{~mm} / \mathrm{mm} / \mathrm{s} \\
\mathrm{D}=0.00289 \mathrm{~mm} / \mathrm{s}\end{array}$ \\
\hline ATE1D & $\mathrm{A}$ & $1.00 \mathrm{E}-01$ & $\pm 2 \%, \pm 0.5 \%$ & Yes & $\mathrm{A}=0.1 \mathrm{~mm} / \mathrm{mm} / \mathrm{s}$ \\
\hline ATE2D & $\mathrm{A}$ & $1.00 \mathrm{E}-02$ & $\pm 2 \%, \pm 0.5 \%$ & Yes & $\mathrm{A}=0.01 \mathrm{~mm} / \mathrm{mm} / \mathrm{s}, \mathrm{D}=0.0289 \mathrm{~mm} / \mathrm{s}$ \\
\hline ATE2D-20 ${ }^{1}$ & $\mathrm{~A}$ & $1.00 \mathrm{E}-02$ & $\pm 2 \%, \pm 0.5 \%$ & Yes & $\mathrm{A}=0.01 \mathrm{~mm} / \mathrm{mm} / \mathrm{s}, \mathrm{D}=0.0289 \mathrm{~mm} / \mathrm{s}$ \\
\hline ATE3D & A & $1.00 \mathrm{E}-03$ & $\pm 2 \%, \pm 0.5 \%$ & Yes & $\begin{array}{c}\mathrm{A}=0.001 \mathrm{~mm} / \mathrm{mm} / \mathrm{s} \\
\mathrm{D}=0.00289 \mathrm{~mm} / \mathrm{s}\end{array}$ \\
\hline ATE4D & $\mathrm{A}$ & $1.00 \mathrm{E}-04$ & $\pm 2 \%, \pm 0.5 \%$ & No & $\mathrm{A}=0.0001 \mathrm{~mm} / \mathrm{mm} / \mathrm{s}$ \\
\hline ATE2C & A & $1.00 \mathrm{E}-02$ & $\begin{array}{c}0 / 2 \%, 0 / 4 \%, 0 / 2 \% \\
\pm 2 \%, \pm 1 \%, \pm 2 \%\end{array}$ & No & $\mathrm{A}=0.01 \mathrm{~mm} / \mathrm{mm} / \mathrm{s}$ \\
\hline ATE2C $-20^{1}$ & A & $1.00 \mathrm{E}-02$ & $\begin{array}{c}0 / 2 \%, 0 / 4 \%, 0 / 2 \% \\
\pm 2 \%, \pm 1 \%, \pm 2 \%\end{array}$ & No & $\mathrm{A}=0.01 \mathrm{~mm} / \mathrm{mm} / \mathrm{s}$ \\
\hline ATE2C- $200^{2}$ & $\mathrm{~A}$ & $1.00 \mathrm{E}-02$ & $0 / 2 \%$ & No & $\mathrm{A}=0.01 \mathrm{~mm} / \mathrm{mm} / \mathrm{s}$ \\
\hline ATE2E & A & $1.00 \mathrm{E}-02$ & $\begin{array}{c}( \pm 1 \%, 0 / 2 \%, \pm 1 \%-2 / 0 \%) \times 2 \\
(0 / 2 \%,-2 / 0 \%) \times 4\end{array}$ & No & $\mathrm{A}=0.01 \mathrm{~mm} / \mathrm{mm} / \mathrm{s}$ \\
\hline
\end{tabular}

Notes:

1 Specimens loaded for 20 cycles at each strain range instead of 10 cycles.

2 Specimen loaded for 200 cycles at the $0 / 2 \%$ strain range. 
Table 3.2 (continued)

\begin{tabular}{|c|c|c|c|c|c|}
\hline Specimen & Material & $\begin{array}{l}\text { Nominal } \\
\text { loading } \\
\text { strain rate } \\
\left(\mathrm{s}^{-1}\right)\end{array}$ & $\begin{array}{l}\text { Target engineering strain } \\
\text { range }\end{array}$ & $\begin{array}{l}\text { Pull to } \\
\text { fracture }\end{array}$ & $\begin{array}{c}\text { Loading control } \\
\text { (A - axial extensometer control, } \\
\text { D - diametral extensometer control) }\end{array}$ \\
\hline BTE1I & $\mathrm{B}$ & $1.00 \mathrm{E}-01$ & $\pm 0.5 \%, \pm 1 \%, \pm 1.5 \%, \pm 2 \%$ & Yes & $\mathrm{A}=0.1 \mathrm{~mm} / \mathrm{mm} / \mathrm{s}$ \\
\hline BTE1I-2 & $\mathrm{B}$ & $1.00 \mathrm{E}-01$ & $\pm 0.5 \%, \pm 1 \%, \pm 1.5 \%, \pm 2 \%$ & Yes & $\mathrm{A}=0.1 \mathrm{~mm} / \mathrm{mm} / \mathrm{s}$ \\
\hline BTE2I & B & $1.00 \mathrm{E}-02$ & $\begin{array}{c} \pm 0.3 \%, \pm 0.5 \%, \pm 1 \%, \pm 1.5 \% \\
\pm 2 \%\end{array}$ & Yes & $\mathrm{A}=0.01 \mathrm{~mm} / \mathrm{mm} / \mathrm{s}, \mathrm{D}=0.0289 \mathrm{~mm} / \mathrm{s}$ \\
\hline BTE2I-2 & B & $1.00 \mathrm{E}-02$ & $\begin{array}{c} \pm 0.3 \%, \pm 0.5 \%, \pm 1 \%, \pm 1.5 \% \\
\pm 2 \%\end{array}$ & Yes & $\mathrm{A}=0.01 \mathrm{~mm} / \mathrm{mm} / \mathrm{s}, \mathrm{D}=0.0289 \mathrm{~mm} / \mathrm{s}$ \\
\hline BTE3I & B & $1.00 \mathrm{E}-03$ & $\begin{array}{c} \pm 0.3 \%, \pm 0.5 \%, \pm 1 \%, \pm 1.5 \% \\
\pm 2 \%\end{array}$ & Yes & $\begin{array}{c}\mathrm{A}=0.001 \mathrm{~mm} / \mathrm{mm} / \mathrm{s} \\
\mathrm{D}=0.00289 \mathrm{~mm} / \mathrm{s}\end{array}$ \\
\hline BTE3I-2 & B & $1.00 \mathrm{E}-03$ & $\begin{array}{c} \pm 0.3 \%, \pm 0.5 \%, \pm 1 \%, \pm 1.5 \% \\
\pm 2 \%\end{array}$ & Yes & $\begin{array}{c}\mathrm{A}=0.001 \mathrm{~mm} / \mathrm{mm} / \mathrm{s} \\
\mathrm{D}=0.00289 \mathrm{~mm} / \mathrm{s}\end{array}$ \\
\hline BTE1D & $\mathrm{B}$ & $1.00 \mathrm{E}-01$ & $\pm 2 \%, \pm 0.5 \%$ & Yes & $\mathrm{A}=0.1 \mathrm{~mm} / \mathrm{mm} / \mathrm{s}$ \\
\hline BTE2D & $\mathrm{B}$ & $1.00 \mathrm{E}-02$ & $\pm 2 \%, \pm 0.5 \%$ & Yes & $\mathrm{A}=0.01 \mathrm{~mm} / \mathrm{mm} / \mathrm{s}, \mathrm{D}=0.0289 \mathrm{~mm} / \mathrm{s}$ \\
\hline BTE3D & B & $1.00 \mathrm{E}-03$ & $\pm 2 \%, \pm 0.5 \%$ & Yes & $\begin{array}{c}\mathrm{A}=0.001 \mathrm{~mm} / \mathrm{mm} / \mathrm{s} \\
\mathrm{D}=0.00289 \mathrm{~mm} / \mathrm{s}\end{array}$ \\
\hline BTE4D & $\mathrm{B}$ & $1.00 \mathrm{E}-04$ & $\pm 2 \%, \pm 0.5 \%$ & No & $\mathrm{A}=0.0001 \mathrm{~mm} / \mathrm{mm} / \mathrm{s}$ \\
\hline
\end{tabular}


Table 3.3 Test matrix for notched specimens

\begin{tabular}{|c|c|c|c|c|}
\hline Specimen & Material & $\begin{array}{c}\text { Target nominal } \\
\text { engineering strain rate } \\
\left(\mathrm{s}^{-1}\right)\end{array}$ & $\begin{array}{c}\text { Target nominal } \\
\text { true strain range }\end{array}$ & $\begin{array}{c}\text { Engineering } \\
\text { strain range }\end{array}$ \\
\hline ANE1R2 & A & $1.00 \mathrm{E}-01$ & $\pm 2 \%$ & $-0.433 / 0.426 \%$ \\
\hline ANE2R2 & A & $1.00 \mathrm{E}-02$ & $\pm 2 \%$ & $-0.433 / 0.426 \%$ \\
\hline ANE3R2 & A & $1.00 \mathrm{E}-03$ & $\pm 2 \%$ & $-0.433 / 0.426 \%$ \\
\hline ANE4R2 & A & $1.00 \mathrm{E}-04$ & $\pm 2 \%$ & $-0.433 / 0.426 \%$ \\
\hline ANE1R4 & A & $1.00 \mathrm{E}-01$ & $\pm 4 \%$ & $-0.819 / 0.795 \%$ \\
\hline ANE2R4 & A & $1.00 \mathrm{E}-02$ & $\pm 4 \%$ & $-0.819 / 0.795 \%$ \\
\hline ANE3R4 & A & $1.00 \mathrm{E}-03$ & $\pm 4 \%$ & $-0.819 / 0.795 \%$ \\
\hline ANE4R4 & A & $1.00 \mathrm{E}-04$ & $\pm 4 \%$ & $-0.819 / 0.795 \%$ \\
\hline ANE1R8 & A & $1.00 \mathrm{E}-01$ & $\pm 8 \%$ & $-1.69 / 1.55 \%$ \\
\hline ANE1R8 & A & $1.00 \mathrm{E}-02$ & $\pm 8 \%$ & $-1.69 / 1.55 \%$ \\
\hline ANE3R8 & A & $1.00 \mathrm{E}-03$ & $\pm 8 \%$ & $-1.69 / 1.55 \%$ \\
\hline ANE4R8 & A & $1.00 \mathrm{E}-04$ & $\pm 8 \%$ & $-1.69 / 1.55 \%$ \\
\hline BNE1R2 & B & $1.00 \mathrm{E}-01$ & $\pm 2 \%$ & $-0.433 / 0.426 \%$ \\
\hline BNE2R2 & B & $1.00 \mathrm{E}-02$ & $\pm 2 \%$ & $-0.433 / 0.426 \%$ \\
\hline BNE3R2 & B & $1.00 \mathrm{E}-03$ & $\pm 2 \%$ & $-0.433 / 0.426 \%$ \\
\hline BNE4R2 & B & $1.00 \mathrm{E}-04$ & $\pm 2 \%$ & $-0.433 / 0.426 \%$ \\
\hline BNE1R4 & B & $1.00 \mathrm{E}-01$ & $\pm 4 \%$ & $-0.819 / 0.795 \%$ \\
\hline BNE2R4 & B & $1.00 \mathrm{E}-02$ & $\pm 4 \%$ & $-0.819 / 0.795 \%$ \\
\hline BNE3R4 & B & $1.00 \mathrm{E}-03$ & $\pm 4 \%$ & $-0.819 / 0.795 \%$ \\
\hline BNE4R4 & B & $1.00 \mathrm{E}-04$ & $\pm 4 \%$ & $-0.819 / 0.795 \%$ \\
\hline BNE4R4-2 & B & $1.00 \mathrm{E}-04$ & $-0.819 / 0.795 \%$ \\
\hline BNE1R8 & B & $1.00 \mathrm{E}-01$ & $-1.69 / 1.55 \%$ \\
\hline BNE2R8 & B & $1.00 \mathrm{E}-02$ & $-1.69 / 1.55 \%$ \\
\hline BNE3R8 & B & $1.00 \mathrm{E}-03$ & $1.00 \mathrm{E}-04$ & $-1.69 / 1.55 \%$ \\
\hline BNE4R8 & B & & $-1.69 / 1.55 \%$ \\
\hline B & & & \\
\hline
\end{tabular}

Notes:

1 Only 20 cycles considered for $10^{-4} \mathrm{~s}^{-1}$ tests at the $\pm 2 \%$ strain range. 
Table 3.4 Rationale for the testing series

\begin{tabular}{|c|l|}
\hline Test Series & \multicolumn{1}{c|}{ Objectives } \\
\hline Tapered - I & $\begin{array}{l}\text { Rate effects on flow stress } \\
\text { Rate effects on fracture strain } \\
\text { Loading history effects }\end{array}$ \\
\hline Tapered - D & $\begin{array}{l}\text { Rate effects on flow stress } \\
\text { Rate effects on fracture strain } \\
\text { Loading history effects }\end{array}$ \\
\hline Tapered - E & Mean strain effects \\
\hline Tapered - C & Mean strain effects \\
\hline Notch - R2, R4, R8 & $\begin{array}{l}\text { Rate effects on flow stress } \\
\text { Rate effects on fatigue life }\end{array}$ \\
\hline
\end{tabular}

Table 3.5 Values of gain used for the $25.4 \mathrm{~mm}$ ( 1 inch) axial extensometer

\begin{tabular}{|c|c|c|}
\hline Strain rate $\left(\mathrm{s}^{-1}\right)$ & P gain & I gain \\
\hline 0.1 & 7000 & 70 \\
\hline 0.01 & 7000 & 70 \\
\hline 0.001 & 1658.2 & 29.09 \\
\hline 0.0001 & 1658.2 & 29.09 \\
\hline
\end{tabular}

Table 3.6 Values of gain used for the diametral extensometer

\begin{tabular}{|c|c|c|}
\hline Strain rate $(\mathrm{mm} / \mathrm{s})$ & $\mathrm{P}$ gain & I gain \\
\hline 0.0289 & 98.18 & 5.69 \\
\hline 0.00289 & 98.18 & 5.69 \\
\hline
\end{tabular}

Table 3.7 Values of gain used for the $50.8 \mathrm{~mm}$ ( 2 inch) axial extensometer

\begin{tabular}{|c|c|c|}
\hline Strain rate $\left(\mathrm{s}^{-1}\right)$ & P gain & I gain \\
\hline 0.1 & 25000 & 250 \\
\hline 0.01 & 15000 & 150 \\
\hline 0.001 & 1600 & 160 \\
\hline 0.0001 & 3000 & 300 \\
\hline
\end{tabular}




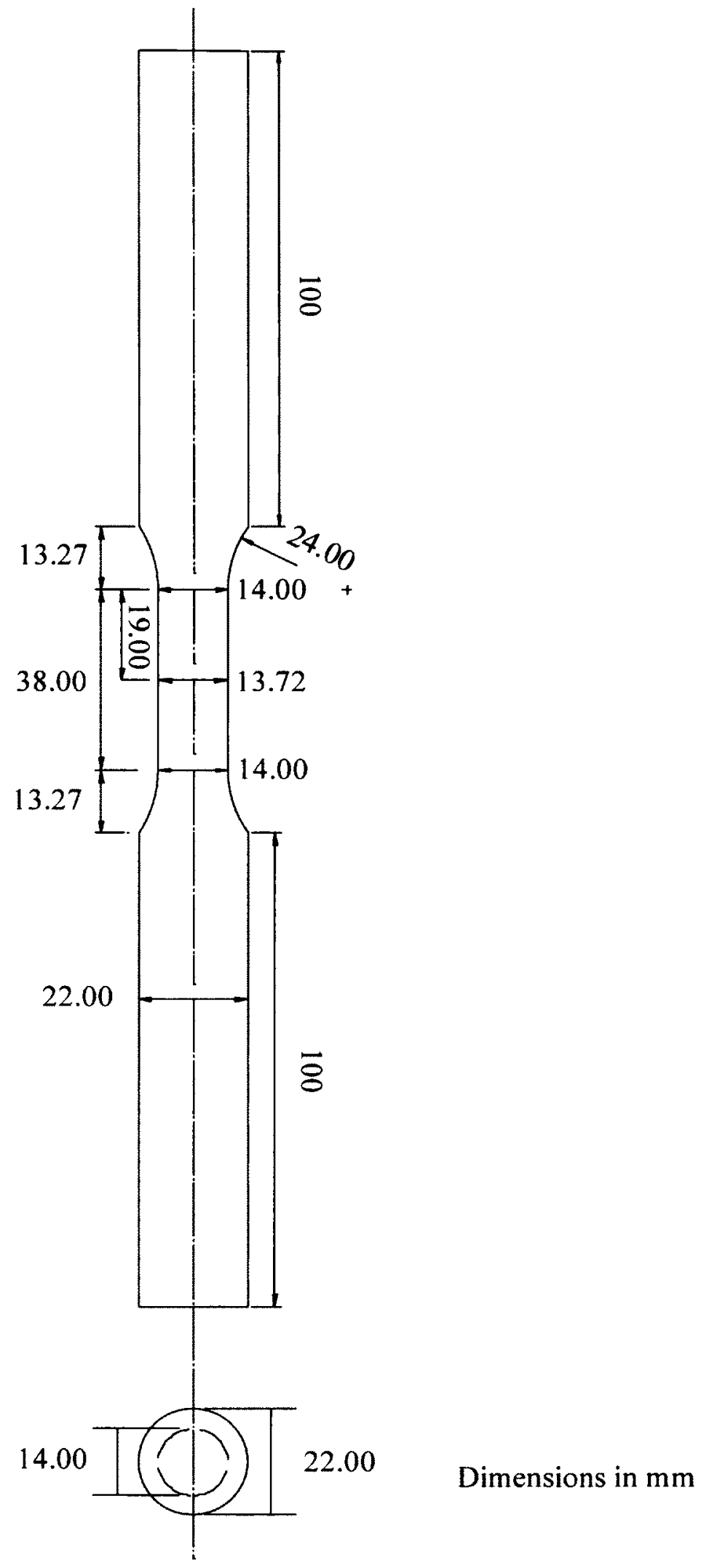

Figure 3.1 Profile of tapered specimens 


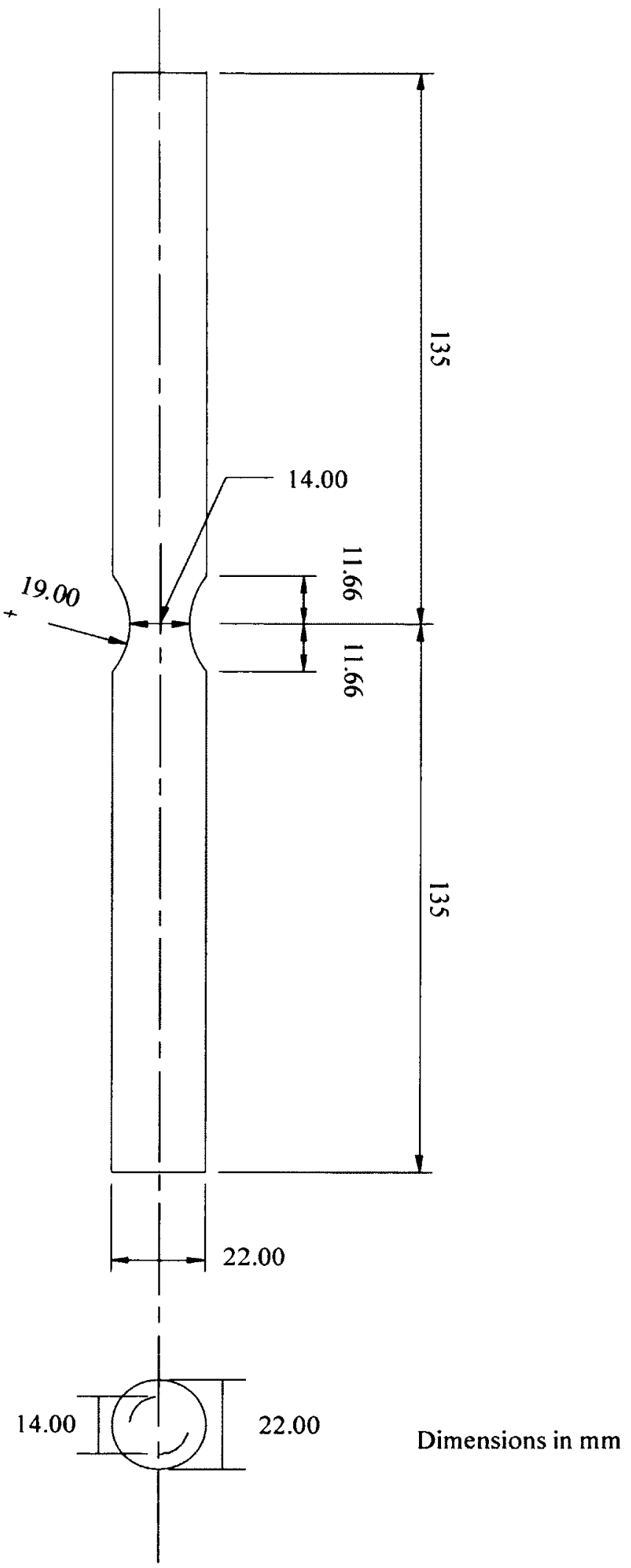

Figure 3.2 Profile of notched specimens 


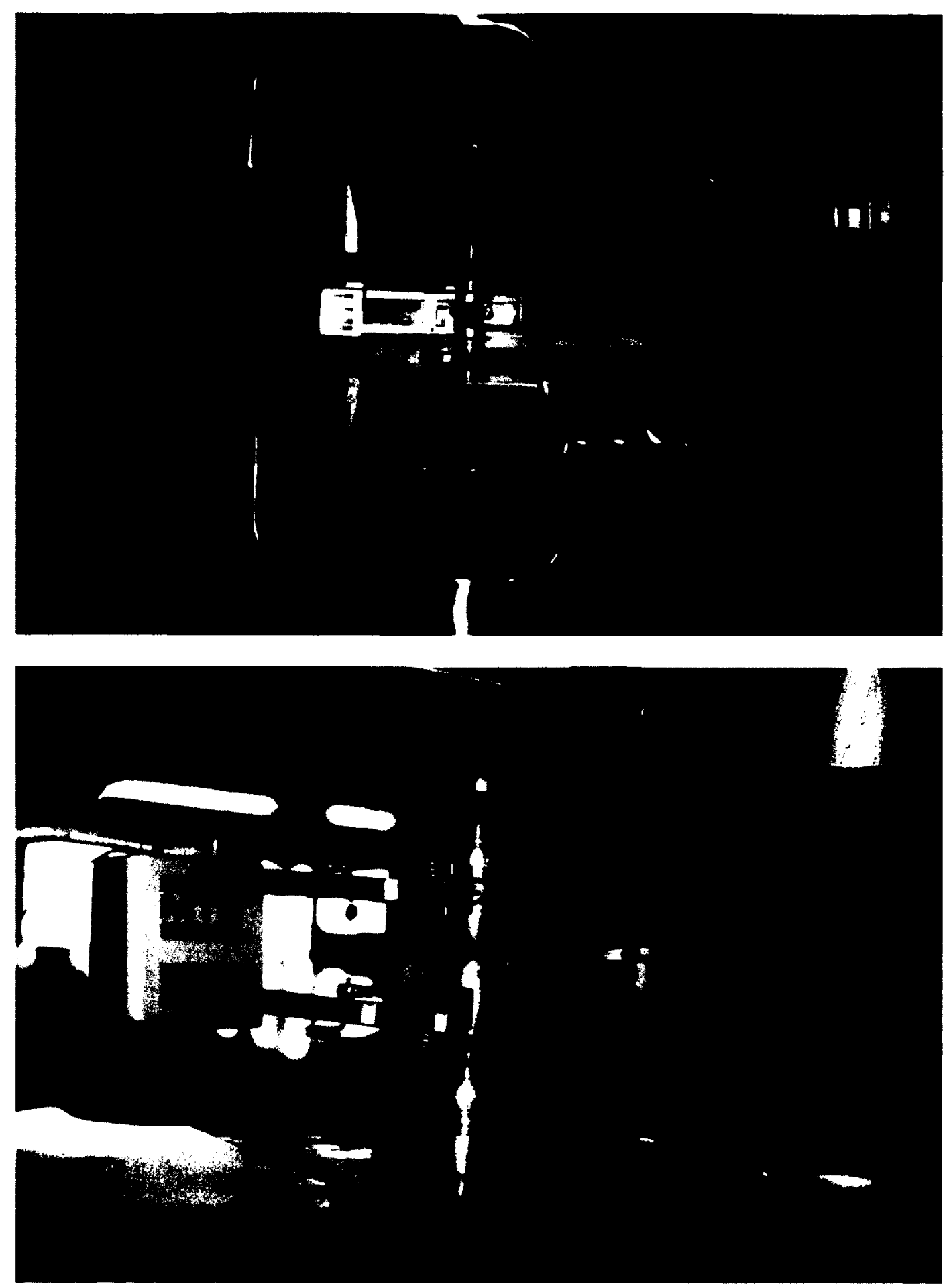

Figure 3.3 Test setup 

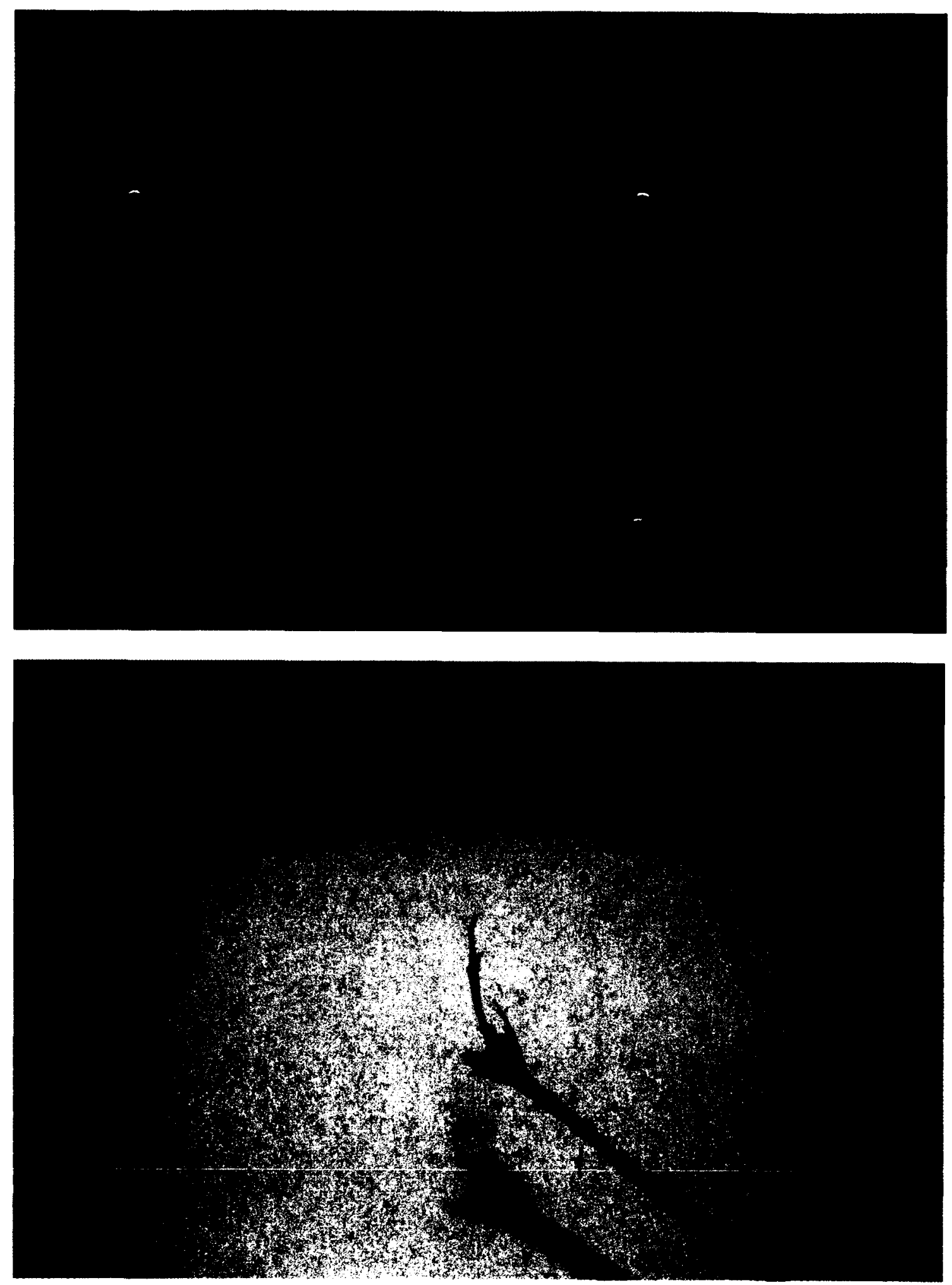

Figure 3.4 Temperature measuring device and thermocouple 


\section{Chapter 4 Test Results and Discussion}

In this chapter, test results from both tapered and notched specimens over a range of strain rates are presented and discussed. The effects of strain rate on the flow stress, rupture strain and fatigue life are evaluated. Effects of loading history and mean strain on the flow stress are also considered in the evaluation.

In order to facilitate the discussions, the following relationships between engineering stress, engineering strain, true stress, true strain and deformations are defined. In a uniaxial test, true strain as shown by Chen (2010) can be taken as

$$
\varepsilon_{t}=\ln \left(1+\varepsilon_{e}\right)
$$

before necking occurs, where $\varepsilon_{e}$ is the engineering strain defined by Eq. (3.1). The true strain can be approximated by

$$
\varepsilon_{t}=\ln \left(\frac{A_{o}}{A}\right)=2 \ln \left(\frac{D_{o}}{D}\right)
$$

where $A$ is the current cross-sectional area, $A_{o}$ is the original cross-sectional area, $D$ is the current diameter and $D_{o}$ is the original diameter. Equation (4.2) can be used to calculate the average true strain for a notched specimen or a tapered (straight) specimen after necking. For uniaxial specimens, the true stress can be calculated as

$$
\sigma_{t}=\frac{F}{A},
$$

which can be approximated for a straight (slightly tapered) specimen before necking as

$$
\begin{aligned}
& \sigma_{t}=\sigma_{e}\left(1+\varepsilon_{e}\right) \text { and } \\
& \sigma_{e}=\frac{F}{A_{o}}
\end{aligned}
$$


where $F$ is the load and $\sigma_{e}$ is the engineering stress. Equation (4.3) can be used to calculate the average true stress for a notch specimen or a tapered (straight) specimen after necking. Based on these relationships, true plastic strain can be calculated as

$$
\varepsilon_{t}^{p}=\varepsilon_{t}-\frac{\sigma_{t}}{E_{s}}
$$

where $E_{s}$ is the elastic modulus for a tapered specimen and the initial slope of the average true stress-average true strain curve upon unloading and reloading in the cyclic test for a notched specimen. For notched specimens, the true strain is calculated according to Eq. (4.2). However, once necking occurs, the stress state is no longer uniaxial and the strain is no longer uniform in a straight (slightly tapered) specimen. Therefore, only Eqs. (4.2) and (4.3) can be used to calculate the average true strain and true stress respectively for a straight (slightly tapered) specimen after necking or a notched specimen. The cumulative plastic strain of the test is taken as

$$
\varepsilon_{t c}^{p}=\sum_{i=1}^{u}\left|\varepsilon_{t}^{p, i+1}-\varepsilon_{t}^{p, i}\right|
$$

where $u$ is the data point number.

\subsection{Analyses of Tapered Specimen Test Results}

Tapered specimens were tested at strain rates of $10^{-4}, 10^{-3}, 10^{-2}$ and $10^{-1} \mathrm{~s}^{-1}$ under various loading conditions as outlined in the previous chapter. Effects of strain rate on the flow stress as well as rupture strain including effects of loading history and mean strain on the cyclic behaviour are discussed based on results of these tests. 


\subsubsection{Loading Control on Tapered Test Specimens}

Before analyzing the test data, a brief discussion is provided to explain effects of the loading control on the tests performed and test results. A loading calibration has to be performed in order to maintain the target loading strain rate in the test of a tapered specimen. Gain values have been adjusted until an optimal control has been obtained for the $25.4 \mathrm{~mm}$ ( 1 inch) extensometer. The target loading strain rate was able to be obtained for all strain ranges at the strain rates of $10^{-4}, 10^{-3}$ and $10^{-2} \mathrm{~s}^{-1}$. However, the loading control has been found to deteriorate with decreasing strain range for the loading strain rate of $10^{-1} \mathrm{~s}^{-1}$. For this reason, the lowest strain range of $\pm 0.3 \%$ in the I test series was not performed at the highest strain rate of the test program. Table 4.1 shows the measured values of true strain rate and strain range obtained in the compression half-cycle of the $10^{\text {th }}$ loading cycle at the first strain range of the loading sequence. True strain is calculated according to Eq. (4.1).

Duplicate tests for BTE1I, BTE2I and BTE3I were performed to determine the reproducibility of the test results. Comparisons of the engineering stress versus engineering strain plots of the original and duplicate tests can be seen in Figs. 4.1 to 4.3 respectively for BTE1I, BTE2I and BTE3I. For the duplicate tests in Figs. 4.2 and 4.3, a loop can be seen in the plots as the specimen has fractured at the start of the loop, but did not separate until it was reloaded at the end of the loop. Thus, the fracture strain for tests exhibiting this phenomenon is taken as the strain at the point when the stress drops abruptly at the start of the loop.

An enhanced view of the cyclic engineering stress versus engineering strain curves can be seen in Figs. 4.4 to 4.6 respectively for BTE1I, BTE2I and BTE3I and their 
duplicate tests. There is a slight difference in the stress of BTE1I and BTE2I and their duplicates. These differences could partly be attributed to a slight difference in the prestressing imparted on the specimen when it was clamped into the testing machine under zero force control and the variation in the material or geometry. For example, BTE1I and BTE1I-2 show the largest difference in engineering yield stress of $489 \mathrm{MPa}$ compared to $510 \mathrm{MPa}$. Such a difference could be the result of a slight tensile pre-stressing imparted on BTE1I or compressive pre-stressing imparted on BTE1I-2. However, the loading rate control for the high strain rate test was poorer than the lower strain rate tests. Thus, it is conceivable that the actual strain rate at yielding was much higher for BTE1I-2 than for BTE1I, and thus giving a larger yield stress due to a higher strain rate. Nevertheless, at the end of the loading for each strain range, the difference in the flow stress between these two tests was only less than $1 \%$. Thus it can be concluded that for the most part, the tests results are reproducible and not much difference in the stress versus strain curve is expected from a duplicate test.

When the specimen was being pulled to fracture, there were a few occurrences of small unloading and reloading for some of the tests. An example of this can be seen in Fig. 4.2 for BTE2I-2. These only occurred for tests performed with the diametral extensometer control. Figure 4.7 shows the loading history and the displacement history of the actuator and diametral extensometer for BTE2I-2 during an occurrence of unloading and reloading. It can be seen that there is a sharp decrease in the diameter measurement before the actuator displacement and load decreases. This was probably due to the sudden movement of the diametral extensometer sliding to the location of the minimum cross-sectional area as necking occurred. When sliding takes place, the 
extensometer will register an abrupt decrease in the diameter that translates to a very high apparent strain rate. This has resulted in an abrupt decrease in the actuator displacement to compensate for the sudden reduction in the diameter reading in an attempt to maintain the target strain diameter reduction rate. Similar results can be seen on the engineering stress versus engineering strain curves of other D and I series tests for both materials A and B shown in Figs. B.1 to B.3 of Appendix B.

\subsubsection{Strain Rate Effects on Stress}

Studies by Benson and Hancock (1974), Saeki et al. (1998) and Dusicka et al. (2007) have shown that the flow stress of steel under cyclic loading can be affected by the loading rate. While these studies provide the effects of strain rate on the flow stress under cyclic loading qualitatively, more quantitative information is desirable to assist in the numerical modelling of the cyclic behaviour. Thus, effects of strain rate on the cyclic behaviour of steel are being further investigated in the present study.

Figures 4.8 to 4.11 show part of the $10^{\text {th }}$ cycle engineering stress versus engineering strain curves at each strain range of I test series for material A loaded at strain rates of $10^{-3}, 10^{-2}$ and $10^{-1}$. Similarly, the $10^{\text {th }}$ cycle engineering stress versus engineering strain curve at each strain range for material $A$ of $D$ test series are shown in Fig. 4.12 and 4.13. The strain range for the I test series increases with the loading cycle number while the range for the $D$ test series decreases. It can be seen in Figs. 4.8 to 4.11 for I test series and Figs. 4.12 and 4.13 for D test series that the flow stress shifts from positive strain rate sensitivity to negative as the loading cycle increases. The same behaviour can be seen for material B in Figs. B.4 to B.7 for I test series and Figs. B.8 and 
B.9 for D test series in Appendix B. Figure 4.14 shows the half engineering stress range at the end of the $5^{\text {th }}$ and $10^{\text {th }}$ cycles versus the accumulated plastic engineering strain for all strain rates of the I test series of material A. A similar plot is generated for the D test series of material A in Fig 4.15. It can be seen that the rate sensitivity for both test series switches from positive to negative at about the same accumulated plastic strain. In Fig. 4.14 , it can be seen that the differences of the half engineering stress range between tests at different strain rates are close to constant between the cumulative plastic strain of 0.2 and 0.3. Similar results can be seen in tests of material B in Figs. B.10 to B.12 of Appendix B. Since duplicate tests were performed for material B under the I test series, the average values of half engineering stress range at the $5^{\text {th }}$ and $10^{\text {th }}$ cycle with the duplicate tests have been calculated and plotted in Fig. B.11.

A major factor contributing to the negative rate sensitivity of stress as the loading strain rate increases to $10^{-1} \mathrm{~s}^{-1}$ is adiabatic heating. For tests performed at the $10^{-1} \mathrm{~s}^{-1}$ strain rate, effects of heating can be seen in the cyclic behaviour. For BTE1I, the 10 cycles performed at each strain range initially show an increase in engineering stress with each loading cycle up to the approximate mid-cycle number and to be followed by a decrease in engineering stress with the subsequent cycle. Examples of this behaviour can be seen in Figs. 4.16 to 4.19 on the engineering stress versus engineering strain curves of BTE 11 at the strain range of $\pm 0.5 \%, \pm 1 \%, \pm 1.5 \%$ and $\pm 2 \%$ respectively. The magnitude of engineering stress at the end of the $10^{\text {th }}$ cycle is lower than the maximum achieved for that strain range except for the first strain range where no softening is seen. Similarly, this behaviour can be seen for BTE1D in Figs. 4.20 and 4.21 at the $\pm 2 \%$ and $\pm 0.5 \%$ strain ranges. Similar behaviour can be seen for tests of material A for ATE1I in Figs. B.13 to 
B.16 and for ATE1D in Figs. B.17 and B.18 of Appendix B for the same strain ranges. However unlike BTE1I, strain softening occurred in BTE1D after reaching the maximum at close to the mid-cycle number even at the first strain range of $\pm 2 \%$. This softening behaviour found for the high strain rate can be attributed to the interaction of two competing phenomena. The peak engineering stress in tension initially increases for the first cycle at the new larger strain range. However, the temperature rise due to the heat generated from the subsequent loading cycles at the same strain range may soften the steel. Depending on the rate of strain hardening with increasing loading cycle and softening due to temperature rise, there may either be an increase or reduction in the flow stress with the loading cycle at a strain range.

An infrared gun was used in this study to obtain an approximate temperature at the surface of the specimen for some of the tests. The measured temperatures are tabulated in Table 4.2. It was found that surface temperatures had risen to approximately $180^{\circ} \mathrm{C}$ and $190^{\circ} \mathrm{C}$ for ATE1I and BTE1I respectively at the end of cyclic loading before the specimen was being pulled to fracture. However, the temperatures at the centre (axis) of the specimen can be considerably higher. It has been found by Harmathy and Stanzak (1970) that the stress-strain behaviour of ASTM A36 mild carbon structural steel under monotonic tension loading can be significantly affected when the temperature rises above room temperature. A temperature increase to $100^{\circ} \mathrm{C}$ caused a drop in yield stress and flow stress compared to that at room temperature. As the specimen temperature rises above $100^{\circ} \mathrm{C}$, the yield stress continues to decrease, but an increase in the rate of work hardening resulted in the flow stress to eventually rise above values seen for specimens tested at room temperature with the deformation until the specimen fractures. This effect 
is at its maximum at a temperature of $260^{\circ} \mathrm{C}$. As the testing temperature rises further, the flow stress begins to decrease again until reaching the values for a specimen tested at room temperature at an approximate temperature of $370^{\circ} \mathrm{C}$. The materials in this study can be expected to be affected by temperature similar to that for ASTM A36 steel, albeit at different temperatures. It can also be seen in Table 4.2 that the approximate temperature measured for tests loaded at the strain rate of $10^{-2} \mathrm{~s}^{-1}$ are close to $65^{\circ} \mathrm{C}$ for the I series and $45^{\circ} \mathrm{C}$ for the $\mathrm{D}$ series. There may be some slight effects of temperature in reducing the flow stress for the I series tests since the temperature at the specimen centre may be higher. However, temperatures measured during the $\mathrm{D}$ series tests can be considered not high enough to significantly affect the measured flow stress when compared to that for a specimen tested at room temperature $\left(20^{\circ} \mathrm{C}\right.$ to $\left.30^{\circ} \mathrm{C}\right)$. Another possible mechanism which may cause the negative rate sensitivity seen for these specimens loaded at the strain rate higher than $10^{-2} \mathrm{~s}^{-1}$ is dynamic strain aging (DSA) instead of due to the temperature rise alone. Zeghib and Klepaczko (1996) have noted that DSA can occur at a temperature as low as $75^{\circ} \mathrm{C}$ for mild steel.

Generally, serrations on the stress-strain curve, known as the Portevin-Le Chatelier (PLC) effect, can be seen when DSA occurs. This phenomenon was first described by LeChatelier in 1909 while studying the effects of temperature on mild steel (Cunningham 1999). In more recent years, PLC has been observed in many different materials including Ductalex aluminum alloy (Teresa Correia and Fortes 1981), plain carbon eutectoid steel (Tsuzaki 1991) and 316L stainless steel (Hong 2005) amongst others. For the mild steel considered in this study, very slight serrations of about $1 \mathrm{MPa}$ are seen on the stress-strain curve for some of the tests. Figure 4.22 shows the magnified view of the 
engineering stress versus engineering strain curves of I series tests for material $A$ when being pulled to fracture. Jaggedness in the stress-strain curves can be seen for ATE1I and ATE2I. Figures $4.23,4.24$ and 4.25 show the magnified view of the engineering stress and actuator displacement versus time approximately between strains of 0.0 and 0.1 when the specimen is being pulled to fracture for ATE1I, ATE2I and ATE3I respectively. It can be seen that motion of the actuator is equally smooth while the engineering stress is more jagged for all three tests. Although DSA is not expected for ATE3I, the flow stress shown in Fig. 4.25 is not completely smooth. Schoeck (1984) has mentioned that the movement of dislocations, which are related to plastic flow, always move in a jerky motion and hence could be the cause of jaggedness of the engineering stress curve for ATE3I. Thus, jerkiness of the engineering stress curves seen in Figs. 4.23, 4.24 and 4.25 is not the manifestation of unsteady actuator movement during the test, but can possibly be attributed to DSA or unsteady dislocation movement. Similar results are seen in Figs. B.19 to B.22 for tests of material B in Appendix B. The instability of flow stress seems to improve for ATE2I after a strain of 0.1 when the loading switched to diametral extensometer control. For specimen ATE1I, only axial extensometer control was used and the serrated behaviour is seen for the entire test. Nevertheless, the absence of the PLC effect does not necessarily indicate that DSA does not occur (Weisse et al. 1993).

Nevertheless from the test results shown in Figs. 4.14, B.10 and B.11, there appears to be a constant difference in the stress range between tests at different strain rates at the accumulated plastic strain of 0.2 to 0.3 and possibly before the effect of adiabatic heating became significant. It can also be seen in Figs. 4.15 and B.12 that close 
to a constant difference in the stress range of the stabilized curve between tests at $10^{-3}$ and $10^{-4} \mathrm{~s}^{-1}$ strain rate is maintained throughout the whole duration of the tests.

\subsubsection{Strain Rate Effects on Rupture Strain}

In both the $\mathrm{D}$ and I test series, specimens were pulled to fracture after being subjected to various cyclic loading sequences. Engineering and true strain at fracture from these tests are shown in Table 4.3, and engineering and true strain at fracture versus logarithmic strain rate are plotted in Figs. 4.26 and 4.27, respectively. The true strain at fracture is taken as $\ln \left(A_{0} / A_{f}\right)$ where $A_{o}$ is the initial undeformed cross-sectional area and $A_{\mathrm{f}}$ is the cross-sectional area at fracture. Although there is some scatter in the test results for the duplicate tests, it can be seen in Fig. 4.26 that the engineering strain at fracture shows the trend of decreasing value with increasing strain rate for tests of the same material and loading sequence. The rate of decrease of the fracture engineering strain appears to increase with the strain rate. It is possible that the heat generated from the high strain rate tests has the effect of reducing the fracture strain further than what occurs at lower strain rates. Albertini and Montagnani (1980) have shown that fracture strain of monotonic tension tests performed at room temperature over the strain rate of approximately $10^{-2}$ to $500 \mathrm{~s}^{-1}$ is higher than that for tests performed at $550^{\circ} \mathrm{C}, 400^{\circ} \mathrm{C}$ and $400^{\circ} \mathrm{C}$ for stainless steel $316 \mathrm{~L}, 304 \mathrm{~L}$ and $321 \mathrm{~L}$, respectively.

The measured engineering strain at fracture decreases with the increase in the number of loading cycles applied (accumulated plastic strain due to loading cycle) before the specimen was being pulled to fracture. The ranking of measured fracture strain is in the same order according to the test series at every strain rate for each material. 
According to Manson (1953) and Coffin (1954), the relationship between the cyclic strain range and the number of cycles to fracture can be expressed as

$$
N_{f}=D^{1}\left(\Delta \varepsilon^{p}\right)^{c}
$$

where $\Delta \varepsilon^{p}$ is the cyclic plastic strain range, $N_{f}$ is the number of cycles to fracture and $D^{1}$ and $c$ are material constants. Among the tests, $\mathrm{D}$ test series has the lowest number of loading cycles and correspondingly the highest engineering fracture strain. The number of cycles to fracture is inverse to the strain range applied. Thus, I series tests have a lower fracture strain than the standard D series tests. Even though specimens in I test series were loaded for 50 cycles, the effect of cyclic loading on the fracture strain is less than that for D series at 20 cycles. This is because there were 20 cycles applied at the largest strain range of $\pm 2 \%$ for ATE2D-20 compared to only 10 cycles for I test series.

Figure 4.27 shows a similar trend of decreasing true fracture strain with increasing strain rate for most of the tests considered except for ATE1I. As it is difficult to place the diametral extensometer at the exact location of the minimum cross-section and the diametral extensometer may not have slid to the location of the minimum crosssection during necking, the measured change in the cross-sectional dimension at fracture is always smaller than the actual value. It can be assumed that the largest of the measured true strain at fracture among duplicate tests to be the more accurate representation for the material and loading. Unlike the true strain at fracture based on the calculated change in cross-sectional area, the value of engineering strain is very dependent on the specimen profile and the gauge length considered. Nevertheless, the measured engineering strain at fracture of tapered specimens may provide reliable data to study the effect of strain rate on the rupture strain quantitatively. 
For ATE1I, necking and fracture occurred far outside of the location of initial minimum cross-section of the tapered profile. It can be seen in Fig. 4.28 that the diametral extensometer was placed on the pre-defined location of initial minimum crosssection, but the specimen fractured at approximately $25 \mathrm{~mm}$ away. A similar result was obtained when the test was repeated. The shift in the location of fracture can be attributed to effects of high strain rate. It is expected that there is a higher stress concentration in the specimen close to the end of the reduced section (location of fracture) due to the change in the specimen diameter. The region of stress concentration is noted on Fig. 4.28. When loaded at a higher strain rate, the rate of stress increase at this region is correspondingly higher compared to the rest of the reduced section due to the stress concentration. There is also a shorter time for the stress (strain) to redistribute at a higher strain rate of loading. This is especially more acute under cyclic loading at a small strain range compared to a larger strain range since there is less time for the stress (strain) to redistribute due to the higher frequency of loading reversal resulting in the deformation being more localized to this region. This may be the reason ATE1I fractured close to the end of the reduced section. However, the effect may be less severe for ATE1D, which fractured at the midlength of the specimen, because there were fewer loading cycles at low strain ranges compared to ATE1I.

\subsubsection{History Effect}

Effects of loading history on the flow stress are studied by comparing results of D and $I$ test series at the strain ranges of $\pm 0.5 \%$ and $\pm 2 \%$ for $10^{-2}$ and $10^{-3} \mathrm{~s}^{-1}$ strain rates. The engineering stress versus engineering strain curves of the $10^{\text {th }}$ cycle of ATE2D and 
ATE2I, and the $20^{\text {th }}$ cycle of ATE2D-20 at the $\pm 2 \%$ strain range and the strain rate of $10^{-2} \mathrm{~s}^{-1}$ for material A can be seen in Fig. 4.29. There is only a slight difference in the stress if any between the curves of the $D$ test series in which the loading at $\pm 2 \%$ strain range was performed first and that of the I test series in which the loading at $\pm 2 \%$ strain range was performed last. Trampczynski (1988) has found comparable results with 18G2A (similar to AISI A765) steel in tension-compression as well as torsion. Similar results can be seen for loading at the strain rate of $10^{-3} \mathrm{~s}^{-1}$ for material $\mathrm{A}$ in Fig. 4.30, and for loading at strain rates of $10^{-2}$ and $10^{-3} \mathrm{~s}^{-1}$ for material B in Figs. B.23 and B.24 of Appendix B. It appears that there is minimal history effect on the stabilized cycle stressstrain curve if the loading involves an increasing strain range. Comparisons are not made for tests loaded at the strain rate of $10^{-1} \mathrm{~s}^{-1}$ because of different levels of heating may have occurred for D and I series tests that will change the material response differently.

Comparisons are also made to assess the history effect if the loading involves a decreasing strain range. Figure 4.31 shows comparisons of the engineering stress versus engineering strain curves of the $10^{\text {th }}$ cycle of ATE2D and ATE2I, and the $20^{\text {th }}$ cycle of ATE2D-20 at $\pm 0.5 \%$ strain range for material A loaded at $10^{-2} \mathrm{~s}^{-1}$ strain rate. In Fig. 4.32, the same comparisons are made for the $10^{\text {th }}$ cycle of BTE2D and BTE2I at $\pm 0.5 \%$ strain range for material $\mathrm{B}$ loaded at $10^{-2} \mathrm{~s}^{-1}$ strain rate. The peak engineering stress in tension is about $22 \mathrm{MPa}$ higher for ATE2D than ATE2I, and $40 \mathrm{MPa}$ higher for BTE2D than BTE2I. Figures 4.33 and 4.34 show a difference of 28 and $43 \mathrm{MPa}$ between tests of the D and I series at $10^{-3} \mathrm{~s}^{-1}$ strain rate for materials $\mathrm{A}$ and $\mathrm{B}$ respectively. However, it can be seen in Figs. 4.31 and 4.32 that the peak engineering stress difference in compression is smaller between ATE2D and ATE2I, but BTE2D still has a larger compression stress 
than BTE2I. Figures 4.35 and 4.36 show the tension and compression halves of the engineering stress versus engineering strain curves for ATE2D-20 and ATE2I at the strain range of $\pm 0.5 \%$. It can be seen that there is a significant difference in the peak tensile engineering stress between ATE2D-20 and ATE2I in the first few cycles, but not in the peak compressive engineering stress. The peak tensile engineering stress of ATE2D-20 decreases at a slower rate with the increase in loading cycle while the peak tensile engineering stress remains relatively constant for ATE2I. Nevertheless, the peak tensile flow stress of ATE2D-20 at the $20^{\text {th }}$ cycle is still slightly larger $(6 \mathrm{MPa})$ than that for ATE2I at the $10^{\text {th }}$ cycle, but slightly smaller $(5 \mathrm{MPa})$ for the peak compressive engineering stress. Based on the trend of the engineering stress versus engineering strain curve of ATE2D- 20 at the $20^{\text {th }}$ cycle at the $\pm 0.5 \%$ strain range, it can be expected that the stress-strain curve of ATE2D-20 to be very close but seldom equal to that for ATE2I if additional loading cycles have been applied. This is because ATE2D-20 has a different strain hardening history before being subject to the $\pm 0.5 \%$ strain range of loading. Although no test was carried out for 20 cycles in the D test series of material B, comparisons of the engineering stress versus engineering strain curves between BTE2I and BTE2D in Figs. B.25 and B.26 in Appendix B show a trend of decreasing peak tensile stress difference with increasing loading cycle for BTE2D. Thus, the reduction in peak tensile engineering stress difference to a level similar to that between ATE2D-20 and ATE2I can be expected if 20 cycles per strain range has been applied to BTE2D. Overall, it may be concluded that the history effect on the stabilized cycle stress-strain curve for materials $\mathrm{A}$ and $\mathrm{B}$ due to the loading sequence considered in the current study is small if sufficient number of loading cycles have been applied at that strain range. 
From Figs. 4.4 to 4.6 , and 4.35 and 4.36 , there appears to be very little change in the peak tensile engineering stress between the $9^{\text {th }}$ and $10^{\text {th }}$ cycle. Thus, the cyclic stressstrain curve may be considered to have stabilized at the $10^{\text {th }}$ if the specimen is loaded with an increasing strain range. However, it may be more appropriate to consider the cyclic stress-strain curve to be stabilized at the $20^{\text {th }}$ cycle rather than the $10^{\text {th }}$ cycle for the test that involves a decreasing strain range loading sequence, such as the D test series.

\subsubsection{Effect of Mean Strain}

Test series $\mathrm{C}$ and $\mathrm{E}$ were designed to study the effects of mean strain on the cyclic behaviour. In these series, tests were carried out at various mean strains and strain ranges. Figure 4.37 shows the average true stress versus true strain curves of the final tensile half cycle of each strain range for ATE2C. For tapered specimens, true strain during cycling is calculated using Eq. (4.1). These curves are shifted such that the point of maximum compressive stress and strain is at the origin. The final tensile half-cycle from the I test series at $\pm 1 \%$ and $\pm 2 \%$ strain ranges are also included in the figure. A magnified view of this figure can be seen in Fig. 4.38. Similarly, Fig. 4.39 shows the enhanced view shifted average true stress versus true strain curves of the final tensile half-cycle of each strain range for ATE2C-20. Comparing Figs. 4.38 and 4.39 for the 0/2\% strain range of ATE2C and ATE2C-20 with $\pm 1 \%$ for ATE2I, it can be seen that 10 cycles may not be sufficient to achieve a stabilized stress-strain curve when the mean strain varies. Comparisons of the shifted true stress-true strain curves and the size of the hysteresis loop between the $10^{\text {th }}$ and $20^{\text {th }}$ cycle at each strain range for ATE2C-20 are shown in Fig. 4.40 and Table

4.4. It can be seen in Fig. 4.40 that the flow stress difference between the $10^{\text {th }}$ and $20^{\text {th }}$ 
cycle is smaller when the strain range is larger. Similarly, Table 4.4 shows that the largest change in the hysteresis loop size from the $10^{\text {th }}$ to $20^{\text {th }}$ cycle at $2 \%$ strain range is $1.20 \%$ while it is only $0.21 \%$ at the $4 \%$ strain range. Based on the small change between the $10^{\text {th }}$ and $20^{\text {th }}$ cycle in the peak shifted stress in Fig. 4.40 and the hysteresis loop size in Table 4.4 , the stress-strain curve may be considered to have stabilized at the $20^{\text {th }}$ cycle when the mean strain varies. Nevertheless, another test was carried out to explore the changes of stress at the $0 / 2 \%$ strain range beyond 20 cycles up to 200 cycles.

In ATE2C-20, the peak shifted stress at the first loading strain range of $0 / 2 \%$ has been found to exhibit the largest change from the $10^{\text {th }}$ to the $20^{\text {th }}$ cycle. Thus, test ATE2C-200 was carried to extend the behaviour at the $0 / 2 \%$ strain range beyond the $20^{\text {th }}$ cycle. Figure 4.41 shows the enhanced view of the shifted half cycle for selected cycles of ATE2C-200 and the final half cycle of ATE2I at the strain range of $\pm 1 \%$. The average true stress versus true strain curves of ATE2C-200 at selected cycles are shown in Fig. 4.42. In test ATE2I which cycled at zero mean strain, the specimen exhibited continual strain hardening (increase in peak shifted stress) after the first few cycles. Strain hardening occurs when the stress increases with an increase in plastic strain. Unlike ATE2I, Figs. 4.41 and 4.42 show that ATE2C-200 with non-zero mean strain (1\%) exhibited strain softening (decrease in peak shifted stress) after the first cycle. Strain softening is considered to have occurred when the stress decreases with an increase in plastic strain. The minimum peak shifted stress for ATE2C-200 occurred at the $70^{\text {th }}$ cycle, which is slightly smaller than that for the $10^{\text {th }}$ cycle of ATE2I. After the $70^{\text {th }}$ cycle, ATE2C-200 exhibited continual strain hardening (increase in peak shifted stress) to the end of the test. The difference in the peak shifted stress of the $20^{\text {th }}$ or $70^{\text {th }}$ cycle to that at 
the $10^{\text {th }}$ cycle of ATE2I is only about $0.5 \%$. Nevertheless, the change in peak shifted stress from the $20^{\text {th }}$ to $70^{\text {th }}$ cycle is less than $1 \%$. Thus, the stress-strain curve may be considered to have stabilized at the $20^{\text {th }}$ cycle when loaded at a strain range of $2 \%$ and larger.

In order to study the effect of mean strain on the stabilized stress-strain curve further, ATE2E was tested under a series of strain range of $2 \%(-2 / 0 \%, \pm 1 \%$, and $0 / 2 \%)$ for 20 cycles at each mean strain. The enhanced view of the shifted final half cycle of the average true stress versus true strain curve at each strain range of ATE2E and $\pm 1 \%$ strain range for ATE2I are shown in Figs. 4.43 to 4.45 with the peak shifted stress. Results of the test are shown separately in three figures for clarity. Table 4.5 also shows the maximum tension, compression and shifted stresses at the $1^{\text {st }}, 2^{\text {nd }}, 10^{\text {th }}$ and $20^{\text {th }}$ cycle of loading, and the change in shifted stress from the $2^{\text {nd }}$ to the $10^{\text {th }}$ cycle and $10^{\text {th }}$ to the $20^{\text {th }}$ cycle. Results in Table 4.5 and Figs. 4.43 to 4.45 are presented in the order of strain range applied according to the test. Figure 4.43 shows results of the loading sequence of $\pm 1 \%$, $0 / 2 \%, \pm 1 \%$ and $-2 / 0 \%$ and Fig. 4.44 shows results of the subsequent same loading sequence. The last figure shows results for the loading sequence between $-2 / 0 \%$ and $0 / 2 \%$ strain ranges (-1\% and $1 \%$ mean strains). It can be seen in Table 4.5 that the change in hysteresis loop size from the $10^{\text {th }}$ to $20^{\text {th }}$ cycle is less than $1 \%$. Thus, the stress-strain curve can be considered to have stabilized at the $20^{\text {th }}$ cycle. Table 4.5 also shows that ATE2E experienced strain softening between the $10^{\text {th }}$ and $20^{\text {th }}$ cycle at each strain range. This behaviour has also been observed in other studies.

Wood (1963) has proposed a mechanism that describes cyclic strain softening after a monotonic pre-strain is applied. During the pre-straining, dislocation pile up 
occurs at grain boundaries and point defects. Stress tends to build up during this process that produces the observed strain hardening effect. During reversed cyclic loading, dislocations move back and forth, and can jump from one atomic plane to another. This allows dislocations to bypass the point defects. As piled up dislocations at point defects decrease with each cycle, the stress at the obstacle reduces accordingly, and thus produces softening.

Chai and Laird (1987) attributed the observed cyclic strain softening during cyclic tests on pre-strained low carbon steel to two microscopic processes. These processes involve dislocations within the cell structure of the material moving to areas of higher dislocation density (cell boundaries) that leads to the overall decrease in the dislocations as the mutual annihilation of dislocations increases with the increase in the dislocation density at the cell boundaries.

Results of the test in Fig. 4.43 to 4.45 and Table 4.5 show that $-2 / 0 \%$ loading range produced a slightly larger peak shifted stress compared to the other strain ranges of $\pm 1 \%$ and $0 / 2 \%$, although the difference is small. It can be seen in Fig. 4.45 that the difference in the peak shifted stress for the $0 / 2 \%$ strain range is less than $1 \%$ of the average of the peak shifted stresses at the preceding and succeeding $-2 / 0 \%$ strain ranges. The test also shows the trend of increasing strain hardening (increase in peak shifted stress) with successive blocks of the same loading sequences, as in Fig. 4.43 to 4.44 and the successive blocks of same loading strain ranges in Fig. 4.45. Although not as well defined, results of ATE2C-20 in Fig. 4.39 show the similar trend of strain hardening with successive strain range and the small effect of mean strain on the stabilized stress-strain curve. 
Thus, based on test results of ATE2C-20, ATE2C-200 and ATE2E, the effect of mean strain in the stabilized stress-strain curve can be considered to be small and may be neglected in constitutive modelling. However, additional tests should be conducted to investigate the effect at larger mean strain differences and repeatability of the test results.

\subsection{Analyses of Notched Specimen Test Results}

Fatigue test were performed on notched specimens at strain rates of $10^{-4}, 10^{-3}, 10^{-2}$ and $10^{-1} \mathrm{~s}^{-1}$ at target true strain ranges of $\pm 2 \%, \pm 4 \%$ and $\pm 8 \%$ for both materials $\mathrm{A}$ and $\mathrm{B}$. All tests were performed until the specimen had separated except for ANE4R2 and BNE4R2. These tests were performed for only 20 cycles due to the long duration required to reach separation.

Notched specimens were considered so that fatigue tests could be performed at higher strain ranges under a fully reversed condition without specimen buckling. However, due to the non-uniform state of stress within the notch, a direct comparison of test results has not been made between the tapered and notched specimens in the following discussions.

\subsubsection{Loading Control on Notched Specimen Tests}

A brief discussion on the effects of loading control on the test data is provided to facilitate the analyses of the test data. The $50.8 \mathrm{~mm}$ ( $2 \mathrm{inch}$ ) axial extensometer was used to control the notched specimen tests. Therefore, the testing machine has to be re-tuned, separately from that for the $25.4 \mathrm{~mm}$ ( $1 \mathrm{inch})$ extensometer, which was performed for the tapered specimen tests. Gain values have been adjusted until an optimal control has been 
achieved. The target loading strain rate was able to be achieved for the strain rates of $10^{-4}$ and $10^{-3} \mathrm{~s}^{-1}$. However, at the strain rate of $10^{-2} \mathrm{~s}^{-1}$, extensometer vibration and the slippage of the extensometer grip (knife edge) were observed during tuning. The vibration was alleviated by either adding a mass to the end of the extensometer (used in the tests) or holding the end of the extensometer during the test. Two grooves were filed onto the side of the specimen spaced over the root of the notch at $50.8 \mathrm{~mm}$ apart so that the extensometer knife edge would be prevented from slipping. After these modifications, the target loading strain rate of $10^{-2} \mathrm{~s}^{-1}$ was able to be achieved during tuning.

Similar to the $25.4 \mathrm{~mm}$ ( 1 inch) extensometer tuning, an accurate control was not able to be obtained for the target loading strain rate of $10^{-1} \mathrm{~s}^{-1}$. The testing machine generated an audible hum after a short duration into the test at all strain ranges. Also, when loaded at the target loading strain rate of $10^{-1} \mathrm{~s}^{-1}$ unsteady actuator movement was observed. As a result, the measured flow stress (load), exhibited drops and jaggedness with the deformation, especially during the tension half-cycle of loading. These can be seen in Figs. 4.46, 4.47, and 4.48 for tests at strain ranges of $\pm 2 \%, \pm 4 \%$ and $\pm 8 \%$ respectively for material $\mathrm{A}$. The instability worsened with increasing target strain range. Some slight instability was also noticed for tests performed at $10^{-4} \mathrm{~s}^{-1}$, as shown for ANE4R8 in Fig. 4.49 but not for the $10^{-2}$ and $10^{-3} \mathrm{~s}^{-1}$ strain rates. However, the effect of the instability on the stress (load) is much smaller than what is seen for the higher strain rate.

The loading for all tests is summarized in Table 4.6. As the deformation of notched specimen is mainly localized to the notch root, the average true (cross-section area change) strain rate is about 4 to 5 times of the applied engineering strain rate. The 
stabilized true strain ranges attained during a fatigue test were much lower than those predicted by the numerical simulation. The range of cross-section area change within a loading cycle will decrease with the increase in the cycle number as the material hardens. As most of the hardening is expected to occur within the first few loading cycles, the cross-section area change per cycle tends to decrease to a stabilized value rapidly.

\subsubsection{Strain Rate Effects on Stress}

The effect of strain rate on flow stress at large strain ranges and over a large number of cycles can be studied from the test data of notched specimens. Peak tensile engineering stress versus cycle number at different strain rates for the $\pm 2 \%, \pm 4 \%$ and $\pm 8 \%$ target strain ranges can be seen in Figs. $4.50,4.51$ and 4.52 respectively for material A and Figs. 4.53, 4.54 and 4.55 respectively for material B.

The figures show that both materials exhibit a positive rate sensitivity of stress over the first few loading cycles for all strain ranges. With continued cycling, the rate sensitivity of stress eventually turns negative for tests at a strain rate higher than $10^{-3} \mathrm{~s}^{-1}$. This negative rate sensitivity continued for the remainder of the tests at all strain ranges with the exception of the tests at $10^{-1} \mathrm{~s}^{-1}$ strain rate for material A. Tests at the $10^{-1} \mathrm{~s}^{-1}$ strain rate for material A initially show an increase in peak tensile stress with the loading cycle. This is followed by the reduction in peak tensile stress to a level below that for tests at the rates of $10^{-4} \mathrm{~s}^{-1}$ to $10^{-2} \mathrm{~s}^{-1}$ with continual cyclic loading, before eventually rising above that for tests at other strain rates until the specimen separated. Unlike material A tests of material $\mathrm{B}$ at the $10^{-1} \mathrm{~s}^{-1}$ strain rate only shows a very slight increase in the peak tensile stress towards the end of the test. This is more apparent at the $\pm 4 \%$ target 
strain range in Fig. 4.54 compared to the other strain ranges. However, this slight increase in peak tensile stress with the loading cycle is insufficient to change the negative rate sensitivity. Tests performed at the strain rates of $10^{-4}$ and $10^{-3} \mathrm{~s}^{-1}$ for both materials show a positive rate sensitivity of stress for all strain ranges. Similar results to these can be seen for the peak tensile average true stress versus cycle number plots for the $\pm 2 \%, \pm 4 \%$ and $\pm 8 \%$ target strain ranges for material A and B in Figs. B. 27 to B.32 in Appendix B.

As stated in Section 4.1.2 the discussions for tapered specimens, significant heat can be generated during high strain rate tests. Temperature measurements taken during notched specimen tests can be found in Table 4.7. It can be seen that the measured temperature of the specimen at fracture by IR gun is higher than that by the thermocouple. The reading from the IR gun was taken when the specimen fractured, while the reading with the thermocouple was taken immediately after the actuator had stopped moving. Thus, some heat was lost between the time taken for the actuator to come to a stop and for the temperature reading by the thermocouple to reach its peak value. Harmathy and Stanzak (1970) noted that strain softening occurred for ASTM A36 steel tested at close to and above $100^{\circ} \mathrm{C}$. Thus, the level of temperature measured during the tests performed at the $10^{-1} \mathrm{~s}^{-1}$ strain rate was high enough to cause significant softening. Since the interior temperature of the specimen was expected to be higher than at the surface, even temperatures measured during tests at the $10^{-2} \mathrm{~s}^{-1}$ strain rate may also be large enough to cause some softening.

As discussed previously, dynamic strain aging (DSA) may also cause negative rate sensitivity for different cumulative plastic strain/strain rates/temperature combinations. Zeghib and Klepaczko (1996) have noted that DSA may occur in mild 
steel anywhere from $75^{\circ} \mathrm{C}$ to $325^{\circ} \mathrm{C}$. One of the known phenomena of DSA is a significant increase in the work hardening rate of the material over a specific band of temperatures at a given strain rate. At a given value of strain, Keh et al. (1968) observed in tension tests on low-carbon steel specimens that larger dislocation densities and flow stress have been found in tests during serrated stress flow than those other tests with no serrated stress flow. The increase in dislocation density is believed to be the cause for the increased flow stress. It is possible that the heat generated in the $10^{-1} \mathrm{~s}^{-1}$ strain rate test is large enough to cause DSA in material A to produce a higher work hardening compared to tests at other strain rates.

However, a similar increase in work hardening has not been observed in tests at the $10^{-1} \mathrm{~s}^{-1}$ strain rate over those other tests for material $\mathrm{B}$. It can be expected that a different material will have a different band of temperatures in which DSA occurs. As can be seen in Table 3.1, materials $\mathrm{A}$ and $\mathrm{B}$ have a different composition of major alloying elements. Figure 4.54 shows that at slightly after the mid-point of the test for the $10^{-1} \mathrm{~s}^{-1}$ strain rate at $\pm 4 \%$ strain range, there appears to be some hardening. However, the amount of hardening is much less than what is observed for material $\mathrm{A}$. It is possible that the temperature generated during the $10^{-1} \mathrm{~s}^{-1}$ strain rate test is not high enough to have a large DSA effect in material B.

An increase in work hardening rate has not been observed in the I series cyclic tests of the tapered specimen performed at $10^{-1} \mathrm{~s}^{-1}$ strain rate for both materials. Figure 4.50 shows that for material A, significant work hardening only occurs after about 100 cycles at the $\pm 2 \%$ target strain range. However, the tapered specimens in I test series only have a total of 50 cycles performed over different strain ranges, but with only 10 cycles at 
the largest strain range of the test at $\pm 2 \%$. Table 4.2 shows that the temperature of $180^{\circ} \mathrm{C}$ measured with the IR gun for ATE1I at the end of the test is lower than $240^{\circ} \mathrm{C}$ as measured for ANE1R2 at fracture, shown in Table 4.7. Thus, the tapered specimens may not have been cyclically loaded long enough to undergo enough straining (cumulative plastic strain) and generate sufficient heat to have a significant hardening due to DSA.

For both materials, the shape of the peak tensile engineering stress versus cycle number curve of $10^{-1} \mathrm{~s}^{-1}$ strain rate tests is different from that for $10^{-2} \mathrm{~s}^{-1}$ strain rate. Both the curves for tests at $10^{-1} \mathrm{~s}^{-1}$ and $10^{-2} \mathrm{~s}^{-1}$ strain rates are also different from that for strain rates of $10^{-3} \mathrm{~s}^{-1}$ and $10^{-4} \mathrm{~s}^{-1}$. However, there appears to be a constant difference in the peak tensile engineering stress at each cycle between tests at the strain rates of $10^{-3} \mathrm{~s}^{-1}$ and $10^{-4} \mathrm{~s}^{-1}$ throughout the loading once the peak tensile engineering stress has come close to stabilized. This occurs approximately between the $10^{\text {th }}$ to the $20^{\text {th }}$ cycle at the $\pm 2 \%$ strain range, the $10^{\text {th }}$ to the $40^{\text {th }}$ cycle at the $\pm 4 \%$ strain range and the $4^{\text {th }}$ to the $15^{\text {th }}$ cycle at the $\pm 8 \%$ strain range. The average true stress range difference between the tests at $10^{-3}$ and $10^{-4} \mathrm{~s}^{-1}(\mathrm{E} 3-\mathrm{E} 4)$ strain rates at each cycle versus loading cycle number for $\pm 2 \%, \pm 4 \%$ and $\pm 8 \%$ target true strain ranges are shown in Figs. 4.56 and 4.57 for materials $\mathrm{A}$ and $\mathrm{B}$ respectively together with the mean of the difference over the range of loading cycle number where the difference is considered to have stabilized as noted earlier. These curves show that the effect of strain rates on flow stress does not diminish with increasing loading cycle number. The magnitude of the average true stress range difference appears to remain somewhat constant for material $\mathrm{A}$, but increases with material B. This apparent increase in the average true stress range difference for material $\mathrm{B}$ is partly the result of the differences of the actual maximum average true strain and 
average true strain range at each cycle between the tests at $10^{-3}$ and $10^{-4} \mathrm{~s}^{-1}$ strain rates. A larger maximum average true strain for $10^{-3} \mathrm{~s}^{-1}$ strain rate test increases the stress range difference because of the higher stress triaxiality. Similarly, a higher average true strain range for the $10^{-3} \mathrm{~s}^{-1}$ strain rate test also increases the stress range difference due to the longer duration of strain hardening per cycle for the $10^{-3} \mathrm{~s}^{-1}$ strain rate test. Table 4.8 lists the minimum, maximum and range of measured average true strain for selected cycle numbers for notched specimens. The last cycle in Table 4.8 represents the last cycle considered in the calculation of the mean average true stress range, and not the last cycle of the test. For material A, the difference in the maximum average true strain and average true strain range between tests at $10^{-3}$ and $10^{-4} \mathrm{~s}^{-1}$ are small. Thus, the differences in the mean average true stress range between the tests at $\pm 2 \%, \pm 4 \%$ and $\pm 8 \%$ are also small. However for material B at the last cycle, the differences in the maximum average true strain and average true strain range are small for test at $\pm 2 \%$ strain range, but slightly higher for tests at $\pm 4 \%$ and higher still for tests at $\pm 8 \%$ strain range. BNE3R 8 shows an increasing higher average true strain and average true strain range compared to BNE4R8 with the loading cycle number. This is reflected in the increasing difference in the average true stress range for tests at the $\pm 8 \%$ strain range in Fig. 4.57 , and the much larger difference compared to tests at the $\pm 2 \%$ strain range.

For material $\mathrm{A}$, the average of the average true stress range difference between the strain rates of $10^{-3}$ and $10^{-4} \mathrm{~s}^{-1}$ is approximately $19 \mathrm{MPa}, 18 \mathrm{MPa}$ and $17 \mathrm{MPa}$ at the $\pm 2 \%, \pm 4 \%$ and $\pm 8 \%$ target strain ranges respectively. Similarly for material $\mathrm{B}$, the average of the average true stress range differences are approximately $12 \mathrm{MPa}, 17 \mathrm{MPa}$ 
and $31 \mathrm{MPa}$ between the $10^{-3}$ and $10^{-4} \mathrm{~s}^{-1}$ strain rates at the $\pm 2 \%, \pm 4 \%$ and $\pm 8 \%$ target strain ranges respectively.

\subsubsection{Fatigue Life Definition for Notched Specimens}

In low cycle fatigue, damage may accumulate within the structural element before complete failure occurs. However, the instance in a test to be considered as the point of fatigue failure where sufficient damage has accumulated is often left to the discretion of the researcher. An indicator of damage accumulation during a low cycle fatigue test is an initially subtle reduction in the peak tensile stress that increases with the loading cycle. This is observed close to the separation point of the specimen in the test. If the test displays this pre-separation behaviour, fatigue life is often defined as either a drop in peak tensile stress of a designated magnitude from the maximum or as a percentage drop from the maximum stress achieved. These definitions are useful because defining fatigue life as the point at which peak tensile stress begins to decrease is challenging as the initial decrease may be very small. Another possible pre-separation behaviour may occur in ultra low cycle fatigue. In ultra low cycle fatigue, a large amount of plastic deformation is induced every cycle that may result in a sudden failure without exhibiting a gradual reduction in peak tensile stress from previous cycles. There may be no indication of accumulated damage in the stress-strain curve before failure happens. Often, the flow stress peaks at mid-cycle and separation occurs soon after. For the notched cyclic tests in this study, the two pre-separation behaviours described above have been observed. Thus, a different definition of fatigue life is used for each pre-separation behaviour. 
The first pre-separation behaviour is seen for specimens loaded at the $\pm 2 \%$ and $\pm 4 \%$ target strain ranges with the exception of ANE1R4 and BNE1R4. ANE1R4 and BNE1R4 are found to have the second pre-separation behaviour discussed below. An example of a test exhibiting the first pre-separation behaviour can be seen in Fig. 4.58 for ANE3R4. When the specimen is near separation, the peak tensile engineering stress begins to decrease with each successive loading cycle and a cusp forms during the compressive half-cycle. However, the exact cycle number at which the peak tensile stress begins to decrease and cusp formation occurs is not obvious. In addition, some materials may show cyclic softening through the majority of their fatigue life which increases the effort required to identify a failure point. For both materials, a decrease in the peak tensile engineering stress with loading cycle can be observed for the majority of the tests at a strain rate of $10^{-2} \mathrm{~s}^{-1}$. For material $\mathrm{B}$, a more pronounced reduction in the peak tensile engineering stress with loading cycle can be observed for tests performed at the strain rate of $10^{-1} \mathrm{~s}^{-1}$. The shape of the peak tensile engineering stress versus cycle number curves can be seen in Figs. 4.50 to 4.55. Therefore, the definition of fatigue life must be robust enough to estimate the fatigue life for the variety of behaviours observed in the test data while not being overly-conservative.

For material A, it may be possible to define fatigue life based on a percentage decrease of peak tensile engineering stress from the maximum achieved during the test. However if this definition is used, the softening behaviour observed for material $B$ at the $10^{-2}$ and $10^{-1} \mathrm{~s}^{-1}$ strain rates will require a percentage decrease from the maximum peak tensile engineering stress that will over-estimate the fatigue life of the tests for material A in which no significant softening occurs. Another possible definition of fatigue life may 
be based on a reduction of diametral strain sensitivity. Bui-Quoc and Biron (1977) have noted that there is a reduction in the sensitivity of the diametral strain during a fatigue test once a major fatigue crack is present in the specimen. In the tension half-cycle, strain is localized to the remaining connected material at the root of the crack. However, the diametral extensometer measures diameter change at the surface of the specimen, which is some distance away from the root of the crack. Since the extensometer is not measuring at the location where a majority of the straining is taking place, a reduction in the sensitivity of the measured diametral deformation is expected. The sensitivity is expected to reduce as the length of the crack increases. This can be seen on the average true stress versus average true strain curve as a reduction in the width of the hysteresis loop from the right hand (tensile) side of the average true stress versus average true strain curve with increasing loading cycle. However, the average true stress versus average true strain curve of some tests moves in the negative direction along the $\ln \left(\mathrm{A}_{0} / \mathrm{A}\right)$ axis from the beginning of the test. This behaviour obscures the point when the narrowing of the hysteresis loop has started. Due to this, it is difficult to establish a failure limit based on a reduction in the diametral deformation sensitivity.

As stated above, cusp formation has consistently been observed in tests exhibiting the first pre-separation behaviour. Cusp formation occurs due to the presence of a large fatigue crack in the test specimen. At the beginning of the compression half-cycle, the specimen is extended to the maximum tensile strain limit of the test. This causes any fatigue crack present within the specimen to be pulled open. As the specimen is compressed, fatigue cracks begin to close. When contact is made and the crack has closed, the effective compressive area of the specimen is increased and a sudden increase 
in the slope of the stress-strain curve is observed in the mid-cycle of loading. Since the presence of a cusp in the stress-strain curve indicates a significant fatigue crack has developed within the specimen, the fatigue life for the first pre-separation behaviour can be defined as the cycle number at which cusp formation is observed in the stress-strain curve. However, it is tedious and at times difficult to detect the first formation of cusp. For these reasons, the percentage drop in the peak tensile engineering stress between successive cycles is used as the limit to define fatigue failure. It is expected that the drop in peak tensile engineering stress between successive cycles to increase rapidly with the formation of cusp in the engineering stress versus engineering strain curve as can be seen in Fig. 4.58 for ANE3R4. Thus, a fatigue failure is assumed to have occurred when the criterion

$$
\frac{\sigma_{e, p}^{i-1}-\sigma_{e, p}^{i}}{\sigma_{e, p}^{i}}>0.01
$$

is met, where $\sigma_{e, p}^{i-1}$ and $\sigma_{e, p}^{i}$ are the peak tensile engineering stresses at the $\mathrm{i}-1^{\text {th }}$ and $\mathrm{i}^{\text {th }}$ cycles. The criterion is applied from the mid-point of the test until the specimen separates. The fatigue life is taken as the cycle number $\mathrm{i}-1$, but not larger than the cycle number when a cusp is found on the engineering stress versus engineering strain curve. It was found that a limit of $0.01(1 \%)$ is able to consistently give the cycle number for fatigue failure that is close to the initiation of cusp formation. A smaller limit was found to be unsuitable as it cannot accommodate the fluctuation in the peak tensile stress due to the poor loading control in high strain rate tests. On the other hand, a larger limit gives a cycle number at failure corresponding to one that a significant cusp formation has been observed in the stress-strain curve. 
The second pre-separation behaviour can be seen for tests performed at the $\pm 8 \%$ and some of the $\pm 4 \%$ target strain ranges. Typical average true stress versus average true strain curves for tests with this pre-separation behaviour can be seen in Fig. 4.59, 4.60 and 4.61 for ANE1R4, ANE3R8 and BNE1R4, respectively. At some point during the test, a peak in the tensile average true stress can be observed part way through the tension half-cycle. Either within the same cycle or at the beginning of the next cycle, the specimen separates. This point of failure is easy to locate. The average true stress versus average true strain curve per cycle does not exhibit any decrease in peak tensile average true stress per cycle or cusp formation. Therefore, for this type of observed behaviour, the fatigue life is defined as the cycle number of the last full cycle before a peak in the tensile average true flow stress is observed in the mid-tension half cycle of the loading. The stress versus strain curves of other notched specimen tests shown in Figs. B.33 to B.51 in Appendix B can be found to display one of the two pre-separation behaviours.

\subsubsection{Strain Rate and Strain Range Effects on Fatigue Life}

As discussed in the literature review, the strain rate effects on fatigue life have been observed to vary between studies. Positive, negative and zero rate sensitivity have all been observed in different studies for various materials. To help clarify the effects of strain rate on fatigue life for mild steel, all notched specimens were cycled until separation except for ANE4R2 and BNE4R2. Fatigue lives for material A and B, calculated as defined in the previous section, are listed in Tables 4.9 and 4.10 respectively. 
Figures 4.62 and 4.63 show the logarithmic plots of the calculated average true plastic strain per cycle versus fatigue life for materials A and B, respectively. Average true plastic strain per cycle is calculated by dividing the accumulated true plastic strain up to the end of the last full cycle before the defined fatigue life is reached by the corresponding cycle number. These values are also shown in Table 4.9 and 4.10 for material A and B, respectively. It can be seen that specimens subjected to the $\pm 8 \%$ target true strain range have a large average true plastic strain per cycle and lower fatigue life. Conversely, specimens subject to the $\pm 2 \%$ target strain range have a lower average true strain per cycle and a higher fatigue life. These results agree with the Manson (1953) and Coffin (1954) relationship shown in Eq. (4.8), which suggests that fatigue life decreases with the increase in strain range. Linear least-square error lines are fitted through the data at each strain rate. These lines are also shown in Fig. 4.62 and 4.63. The constant $c$ in Eq. (4.8) is the inverse of the exponent of the function for the least-square fit line. The parameters $D^{\prime}$ and $c$ calculated from the fitted lines in Figs. 4.62 and 4.63 are listed in Table 4.11. The inverse of the slope of these lines $(c)$ versus strain rate data for both material $\mathrm{A}$ and $\mathrm{B}$ are shown in Fig. 4.64. For the average true strain range of approximately 0.03 to 0.15 and the average true strain rate of $4 \times 10^{-3} \mathrm{~s}^{-1}$ to $0.5 \mathrm{~s}^{-1}$, there is very little change in the slope of the line for material B except for a slight decrease at the highest strain rate. However, there is a greater dependency of the slope to strain rate for material A. The slope in terms of strain rate appears to be able to be represented by the equation in the form similar to the power law function by Cowper-Symonds (1957), Eq. (2.8), that relates the yield stress to the strain rate. This equation can be expressed as 


$$
\frac{c(\dot{\varepsilon})}{c_{s}}=1+\left(\frac{\dot{\varepsilon}}{P}\right)^{\frac{1}{K}}
$$

where $c_{s}, P$ and $K$ are constants to be determined. Parameters $c_{s}, P$ and $K$ have been calculated to be $-1.19,0.96$ and 5.72 for material $\mathrm{A}$ through the method of least-square error fitting. For material B, a reasonable fit of Eq. (4.10) has not been able to be obtained due to the rate insensitivity and the limited strain rate range of the test data. However, a reasonable fit of the test data can be achieved for material $\mathrm{B}$ if the parameters $c_{s}$ and $P$ are solved with the $K$ parameter constrained to the values of 2, 3 and 4 . The curves generated by Eq. (4.10) using these parameters are shown in Fig. 4.64. Thus, Eq. (4.10) may still be used to represent the relationship of constant $c$ versus strain rate if more test data above the strain rate of $10^{-1} \mathrm{~s}^{-1}$ are available for material B. Nevertheless, the results of at least material A show that constants in Eq. (4.8) may vary with strain rate.

Average true plastic strain per cycle versus logarithmic strain rate for materials A and $\mathrm{B}$ are shown in Figs. 4.65 and 4.66, respectively. Also, logarithmic plots of the fatigue life versus strain rate for materials A and B are shown in Figs. 4.67 and 4.68. Figures 4.65 and 4.66 show that, for the most part, strain rate has very little effect on the amount of average true plastic strain per cycle. This is to be expected since the test was carried out under displacement control. At the highest strain rate tested, a slight increase in average true plastic strain per cycle can be seen for material B. For material B, BNE1R8 shows an unusually high average true plastic strain per cycle. This is the result of unstable (poor) loading control that has produced a large number of partial unloading and reloading in every loading cycle, as can be seen in Fig. B.42 in Appendix B. As a result, additional plastic strain is accumulated in these mini hysteresis loops. BNE1R8 
also has an unusually low fatigue life compared to other tests at the same strain range, which may be caused by the higher average true plastic strain per cycle. In spite of the relatively constant average true plastic strain per cycle with the strain rate, Fig. 4.67 shows that the fatigue life for material A varies with the strain rate. The fatigue life increases with increasing strain rate at the $\pm 2 \%$ target strain range. This effect appears to diminish at a large target strain range with almost no rate sensitivity seen in tests at the $\pm 8 \%$ target strain range. Nevertheless, the absence of fatigue life rate sensitivity at the $\pm 8 \%$ target strain range may be due to the low number of cycles required to reach failure and the large loading strain range, which this produced ultra low cycle fatigue failure that is different from the failure mode for tests at $\pm 2 \%$ and $\pm 4 \%$ strain range. The ratios of maximum to the minimum fatigue life between the tests are 1.84 and 1.67 for the strain range $\pm 2 \%$ and $\pm 4 \%$ respectively. At the strain range of $\pm 4 \%$, ANE4R 4 and ANE3R 4 show a slight negative rate sensitivity while positive rate sensitivity is seen at the higher strain rates. However, the slight negative rate sensitivity shown for ANE4R4 and ANE3R4 may just be due to the scatter of test results. For material B, Fig. 4.68 shows that there is little rate sensitivity in fatigue life. With the exception for BNE1R2, which has a higher fatigue life and BNE4R4, which has a somewhat lower fatigue life. BNE1R8 also displays a fatigue life which is lower than the rests of the tests at the same target strain range. However, the calculated average true plastic strain per cycle for this test is unusually high as can be seen in Fig. 4.66. Based on Eq. (4.8), a decrease in fatigue life would be expected compared to other tests at the $\pm 8 \%$ target strain range. Unlike material A, the fatigue life for material $B$ at the average true strain ranges of around 0.03 to 0.15 and the average true strain rates $4 \times 10^{-3} \mathrm{~s}^{-1}$ to $0.5 \mathrm{~s}^{-1}$ does not appear to be rate sensitive. 
Figures 4.69 and 4.70 show logarithmic plots of accumulated true plastic strain at the end of fatigue life versus strain rate for materials A and B, respectively. Accumulated plastic strain at failure seems to be affected by strain rate in the same way as fatigue life. This can be seen by comparing Figs. 4.67 and 4.69 for material A and Figs. 4.68 and 4.70 for material B. This is expected since the average true plastic strain per cycle is almost the same between tests at the same target strain ranges, except for BNE1R4 and BNE1R8. However, the cumulative average true plastic strain to failure may be a better representation of fatigue life if there is considerable difference in the calculated true plastic strain per cycle between tests at the same target strain range. This is particularly true for material $\mathrm{B}$ tested at the target engineering strain rate of $10^{-1} \mathrm{~s}^{-1}$ where a good loading control was not achieved, which has resulted in a higher calculated average true plastic strain per cycle compared to tests at lower loading rates. As can be seen in Table 4.10 for BNE1R4 and BNE1R8, the calculated average true plastic strain per cycle are $17 \%$ and $44 \%$ higher than other tests at the same target strain range respectively. These have resulted in a lower recorded number of cycles to fatigue life for BNE1R4 compared to BNE2R4 and BNE1R8 compared to BNE2R8. Unlike Fig. 4.68 that considers fatigue life as number of cycles to failure, Figure 4.70 shows that all specimens tested at the target engineering strain rate of $10^{-1} \mathrm{~s}^{-1}$ have acquired a larger cumulative average true plastic strain to fatigue failure compared to tests at lower target engineering strain rate. Nevertheless, there are insufficient data for both materials A and B, especially at the strain rate higher than that considered in the current study, to come up with the form of equation that can best represent the number of cycle or cumulative true plastic strain to failure versus the strain rate. 
There is also a significant flow stress increase at the latter half of tests conducted at the strain rate of $10^{-1} \mathrm{~s}^{-1}$, as shown in Figs. 4.50 to 4.52 , which is suspected to be the result of dynamic strain aging (DSA). These tests for material A, except for ANE1R8, also display the largest fatigue life compared to other strain rates. However, material A shows an increasing rate sensitivity of fatigue life with decreasing strain range. Therefore, it is difficult to conclude whether the higher fatigue life displayed for ANE1R2 and ANE1R4 is an effect of DSA.

Srinivasan et al. (1991) have found that the fatigue life of 316L stainless steel tested under strain control at the strain rate of $3 \times 10^{-3} \mathrm{~s}^{-1}$ to increase from 2850 cycles to 3740 cycles when the temperature increased from $25^{\circ} \mathrm{C}$ to $300^{\circ} \mathrm{C}$. As the temperature increased above $300^{\circ} \mathrm{C}$, the fatigue life was found to decrease. Weisse (1993) has also found that the fatigue life of low carbon SAE 1045 steel during incremental step tests at the strain rate of $7 \times 10^{-4} \mathrm{~s}^{-1}$ to increase as the temperature of the test increased from $25^{\circ} \mathrm{C}$ to $200^{\circ} \mathrm{C}$. An increase in stress range caused by DSA was also observed for the test performed at $200^{\circ} \mathrm{C}$ over that of the test performed at $25^{\circ} \mathrm{C}$. However, on the tests at $265^{\circ} \mathrm{C}$, where the effects of DSA were found to be the highest, the fatigue life was found to be lower than that at $25^{\circ} \mathrm{C}$. On fatigue tests performed at a constant strain range and at a strain rate of $0.0033 \mathrm{~s}^{-1}$ on plain carbon eutectoid steel at room temperature, $250^{\circ} \mathrm{C}$ (peak DSA effects) and $350^{\circ} \mathrm{C}$ (outside of DSA regime), Tsuzaki (1991) concluded that the fatigue life can decrease by a factor of more than 2 when DSA effects are at their peak compared to that at room temperature. Noted by Tsuzaki (1991) that during DSA marked with an increase in stress range per loading cycle and serrations in the flow stress, 
the fatigue life is expected to be reduced since a larger stress will accelerate the initiation and propagation of fatigue cracks.

During strain controlled fatigue tests, an increase in overall flow stress decreases the amount plastic strain per cycle. Equation (4.8) suggests that this will increase fatigue life. However, as noted by Tsuzaki (1991), an increase in flow stress due to DSA is expected to reduce the fatigue life as higher stress accelerates the initiation and propagation of fatigue cracks. In addition, at high temperature, oxidization and creep may also act to reduce the fatigue life. Oxidization has been found to embrittle metals while creep increases the amount of plastic strain per cycle for notched specimens where the axial extensometer is placed outside of the notch. Higher temperatures are expected for tests at the $10^{-1} \mathrm{~s}^{-1}$ strain rate, as recorded with the IR gun in Table 4.7. However, the effects of oxidation and creep are not expected to be significant due to the short duration of the test. These are some competing factors that may affect the fatigue life. Thus, some metals may display an increased fatigue life with increasing temperature over a limited temperature range when DSA is present. It is possible that this effect may have increased the fatigue life of ANE1R2, ANE1R4 and all the tests at the target strain rate of $10^{-1} \mathrm{~s}^{-1}$ (BNE1Rx) for material B. Nevertheless, a more detailed study is required to get a better understanding on effects of strain rate on fatigue life.

From the test results, it can be considered that material A mostly shows a positive rate sensitivity for fatigue life. This effect appears to diminish at a large target strain range. Material B remains relatively rate insensitive with the exception seen at the highest strain rate tested. A change in the strain range does not appear to have an effect on the rate sensitivity in material $\mathrm{B}$. On the other hand, the rupture strain for the tapered 
specimen has been found to decrease with strain rate for materials A and B. Nevertheless, more test data are needed as there is enough variability between results of duplicate tests to make any definitive conclusion on the fatigue life rate sensitivity of materials $A$ and $B$. 
Table 4.1 Measured true strain rates and strain ranges for tapered specimens

\begin{tabular}{|c|c|c|c|c|c|}
\hline Specimen & Material & $\begin{array}{c}\text { Nominal loading rate } \\
\left(\mathrm{s}^{-1}\right)\end{array}$ & Target true strain range & $\begin{array}{c}\text { Measured true } \\
\text { strain rate }^{\prime} \\
\left(\mathrm{s}^{-1}\right)\end{array}$ & $\begin{array}{c}\text { Measured true } \\
\text { strain range }\end{array}$ \\
\hline ATE1I & A & $1.00 \mathrm{E}-01$ & $\pm 0.5 \%, \pm 1 \%, \pm 1.5 \%, \pm 2 \%$ & $1.50 \mathrm{E}-01$ & 0.0102 \\
\hline ATE2I & A & $1.00 \mathrm{E}-02$ & $\pm 0.3 \%, \pm 0.5 \%, \pm 1 \%, \pm 1.5 \%, \pm 2 \%$ & $1.01 \mathrm{E}-02$ & 0.0059 \\
\hline ATE3I & A & $1.00 \mathrm{E}-03$ & $\pm 0.3 \%, \pm 0.5 \%, \pm 1 \%, \pm 1.5 \%, \pm 2 \%$ & $1.02 \mathrm{E}-03$ & 0.0060 \\
\hline ATE1D & A & $1.00 \mathrm{E}-01$ & $\pm 2 \%, \pm 0.5 \%$ & $9.94 \mathrm{E}-02$ & 0.0403 \\
\hline ATE2D & A & $1.00 \mathrm{E}-02$ & $\pm 2 \%, \pm 0.5 \%$ & $1.00 \mathrm{E}-02$ & 0.0401 \\
\hline ATE2D-20 & A & $1.00 \mathrm{E}-02$ & $\pm 2 \%, \pm 0.5 \%$ & $1.01 \mathrm{E}-02$ & 0.0401 \\
\hline ATE3D & A & $1.00 \mathrm{E}-03$ & $\pm 2 \%, \pm 0.5 \%$ & $9.88 \mathrm{E}-04$ & 0.0401 \\
\hline ATE4D & A & $1.00 \mathrm{E}-04$ & $\pm 2 \%, \pm 0.5 \%$ & $1.00 \mathrm{E}-04$ & 0.0400 \\
\hline ATE2C & A & $1.00 \mathrm{E}-02$ & $\begin{array}{r}0 / 2 \%, 0 / 4 \%, 0 / 2 \%, \\
\pm 2 \%, \pm 1 \%, \pm 2 \%\end{array}$ & $1.02 \mathrm{E}-02$ & 0.0199 \\
\hline ATE2C-20 & A & $1.00 \mathrm{E}-02$ & $\begin{array}{r}0 / 2 \%, 0 / 4 \%, 0 / 2 \%, \\
\pm 2 \%, \pm 1 \%, \pm 2 \%\end{array}$ & $1.02 \mathrm{E}-02$ & 0.0199 \\
\hline ATE2C-200 & A & $1.00 \mathrm{E}-02$ & $\pm 2 \%$ & $1.01 \mathrm{E}-02$ & 0.0198 \\
\hline ATE2E & A & $1.00 \mathrm{E}-02$ & $( \pm 1 \%, 0 / 2 \%, \pm 1 \%-2 / 0 \%) \times 2$, & $1.01 \mathrm{E}-01$ & 0.0200 \\
\hline
\end{tabular}


Table 4.1 (continued)

\begin{tabular}{|c|c|c|c|c|c|}
\hline Specimen & Material & $\begin{array}{c}\text { Nominal loading rate } \\
\left(\mathrm{s}^{-1}\right)\end{array}$ & Target true strain range & $\begin{array}{c}\text { Measured true } \\
\text { strain rate }^{1} \\
\left(\mathrm{~s}^{-1}\right)\end{array}$ & $\begin{array}{c}\text { Measured true } \\
\text { strain range }\end{array}$ \\
\hline BTE1I & B & $1.00 \mathrm{E}-01$ & $\pm 0.5 \%, \pm 1 \%, \pm 1.5 \%, \pm 2 \%$ & $1.43 \mathrm{E}-01$ & 0.0098 \\
\hline BTE1I-2 & B & $1.00 \mathrm{E}-01$ & $\pm 0.5 \%, \pm 1 \%, \pm 1.5 \%, \pm 2 \%$ & $1.44 \mathrm{E}-01$ & 0.0097 \\
\hline BTE2I & B & $1.00 \mathrm{E}-02$ & $\pm 0.3 \%, \pm 0.5 \%, \pm 1 \%, \pm 1.5 \%, \pm 2 \%$ & $1.03 \mathrm{E}-02$ & 0.0059 \\
\hline BTE2I-2 & B & $1.00 \mathrm{E}-02$ & $\pm 0.3 \%, \pm 0.5 \%, \pm 1 \%, \pm 1.5 \%, \pm 2 \%$ & $9.95 \mathrm{E}-03$ & 0.0059 \\
\hline BTE3I & B & $1.00 \mathrm{E}-03$ & $\pm 0.3 \%, \pm 0.5 \%, \pm 1 \%, \pm 1.5 \%, \pm 2 \%$ & $1.06 \mathrm{E}-03$ & 0.0060 \\
\hline BTE3I-2 & B & $1.00 \mathrm{E}-03$ & $\pm 0.3 \%, \pm 0.5 \%, \pm 1 \%, \pm 1.5 \%, \pm 2 \%$ & $1.02 \mathrm{E}-03$ & 0.0060 \\
\hline BTE1D & B & $1.00 \mathrm{E}-01$ & $\pm 2 \%, \pm 0.5 \%$ & $9.95 \mathrm{E}-02$ & 0.0404 \\
\hline BTE2D & B & $1.00 \mathrm{E}-02$ & $\pm 2 \%, \pm 0.5 \%$ & $9.98 \mathrm{E}-03$ & 0.0401 \\
\hline BTE3D & B & $1.00 \mathrm{E}-03$ & $\pm 2 \%, \pm 0.5 \%$ & $9.99 \mathrm{E}-04$ & 0.0401 \\
\hline BTE4D & B & $1.00 \mathrm{E}-04$ & $\pm 2 \%, \pm 0.5 \%$ & $9.88 \mathrm{E}-05$ & 0.0400 \\
\hline
\end{tabular}

Notes:

$1 \quad$ Measurements calculated from the compression half-cycle of the $10^{\text {th }}$ loading cycle at the first strain range of the loading sequence. 
Table 4.2 Temperature measurements using the IR gun

\begin{tabular}{|c|c|c|c|}
\hline Specimen & $\begin{array}{c}\text { Initial } \\
\left({ }^{\circ} \mathrm{C}\right)\end{array}$ & $\begin{array}{c}\text { End of cyclic loading } \\
\left({ }^{\circ} \mathrm{C}\right)\end{array}$ & $\begin{array}{c}\text { After fracture } \\
\left({ }^{\circ} \mathrm{C}\right)\end{array}$ \\
\hline ATE1I & 26 & 180 & 240 \\
\hline ATE2I & 26 & 60 & 51 \\
\hline ATE1D & 23 & 90 & 145 \\
\hline ATE2D & 21 & 40 & 40 \\
\hline BTE1I & 29 & 190 & 250 \\
\hline BTE2I & 28 & 67 & 57 \\
\hline BTE3I & 20 & 28 & 22 \\
\hline BTE1D & 24 & 109 & 130 \\
\hline BTE2D & 20 & 46 & 39 \\
\hline
\end{tabular}

Table 4.3 Strains at fracture

\begin{tabular}{|c|c|c|c|}
\hline Specimen & $\begin{array}{c}\text { Strain Rate } \\
\left(\mathrm{s}^{-1}\right)\end{array}$ & $\begin{array}{c}\text { Extensometer } \\
\text { Engineering } \\
\text { Strain }\end{array}$ & True Strain \\
\hline ATE1I & 0.1 & 0.348 & 0.175 \\
\hline ATE2I & 0.01 & 0.381 & 0.735 \\
\hline ATE3I & 0.001 & 0.392 & 0.797 \\
\hline BTE1I & 0.1 & 0.340 & 0.771 \\
\hline BTE1I-2 & 0.1 & 0.336 & 0.762 \\
\hline BTE2I & 0.01 & 0.371 & 0.754 \\
\hline BTE2I-2 & 0.01 & 0.359 & 0.738 \\
\hline BTE3I & 0.001 & 0.385 & 0.900 \\
\hline BTE3I-2 & 0.001 & 0.387 & 0.893 \\
\hline ATE1D & 0.1 & 0.353 & 0.622 \\
\hline ATE2D & 0.01 & 0.384 & 0.761 \\
\hline ATE2D-20 & 0.01 & 0.380 & 0.717 \\
\hline ATE3D & 0.001 & 0.397 & 0.748 \\
\hline BTE1D & 0.1 & 0.354 & 0.789 \\
\hline BTE2D & 0.01 & 0.393 & 0.895 \\
\hline BTE3D & 0.001 & 0.399 & 0.929 \\
\hline
\end{tabular}


Table 4.4 Hysteresis loop size based on average true stress for the given cycle and strain range for $\mathrm{ATE} 2 \mathrm{C}-20$

\begin{tabular}{|c|c|c|c|c|c|c|c|}
\hline $\begin{array}{l}\text { Cycle } \\
\text { number }\end{array}$ & & $0 / 2 \%$ & $0 / 4 \%$ & $0 / 2 \% \# 2$ & $\pm 2 \%$ & $\pm 1 \%$ & $\pm 2 \% \# 2$ \\
\hline \multirow{3}{*}{$1 \mathrm{st}$} & Tens. (MPa) & 389.6 & 493.5 & 437.2 & 424.8 & 472.3 & 468.5 \\
\hline & Comp. (MPa) & -391.2 & -477.4 & -480.3 & -507.7 & -457.2 & -494.3 \\
\hline & Size (MPa) & 780.8 & 970.9 & 917.5 & 932.5 & 929.5 & 962.8 \\
\hline \multirow{3}{*}{2 nd } & Tens. (MPa) & 430.1 & 503.5 & 434.6 & 479.7 & 456.0 & 488.6 \\
\hline & Comp. (MPa) & -413.5 & -487.9 & -470.2 & -514.0 & -452.5 & -502.2 \\
\hline & Size (MPa) & 843.6 & 991.4 & 904.8 & 993.7 & 908.5 & 990.8 \\
\hline \multirow{3}{*}{10 th } & Tens. (MPa) & 437.0 & 502.7 & 426.1 & 496.3 & 436.0 & 500.2 \\
\hline & Comp. (MPa) & -424.3 & -498.9 & -446.2 & -510.3 & -441.1 & -509.2 \\
\hline & Size $(\mathrm{MPa})$ & 861.3 & 1001.6 & 872.4 & 1006.5 & 877.1 & 1009.4 \\
\hline \multirow{3}{*}{ 20th } & Tens. (MPa) & 430.1 & 501.1 & 425.0 & 498.5 & 433.3 & 501.3 \\
\hline & Comp. (MPa) & -421.0 & -500.8 & -439.0 & -509.3 & -439.1 & -510.2 \\
\hline & Size $(\mathrm{MPa})$ & 851.1 & 1001.9 & 864.0 & 1007.7 & 872.3 & 1011.5 \\
\hline \multicolumn{2}{|c|}{$\begin{array}{c}\text { Size change between } \\
\text { 10th and 20th cycle } \\
(\mathrm{MPa})\end{array}$} & -10.2 & 0.4 & -8.4 & 1.2 & -4.8 & 2.1 \\
\hline \multicolumn{2}{|c|}{$\%$ Change } & $-1.20 \%$ & $0.04 \%$ & $-0.97 \%$ & $0.12 \%$ & $-0.55 \%$ & $0.21 \%$ \\
\hline
\end{tabular}


Table 4.5 Hysteresis loop size based on average true stress for the given cycle and strain range for ATE2E

\begin{tabular}{|c|c|c|c|c|c|c|c|c|c|}
\hline $\begin{array}{c}\text { Cycle } \\
\text { number }\end{array}$ & & $\pm 1 \%$ & $0 / 2 \%$ & $\pm 1 \% \# 2$ & $-2 / 0 \%$ & $\pm 1 \% \# 3$ & $0 / 2 \% \# 2$ & $\pm 1 \% \# 4$ & $-2 / 0 \% \# 2$ \\
\hline \multirow{3}{*}{ 1st } & Tens. (MPa) & 364.6 & 446.9 & 359.9 & 411.7 & 447.8 & 450.3 & 359.2 & 350.1 \\
\hline & Comp. (MPa) & -395.7 & -409.6 & -450.7 & -429.6 & -422.9 & -406.4 & -457.2 & -434.4 \\
\hline & Size $(\mathrm{MPa})$ & 760.3 & 856.4 & 810.6 & 841.4 & 870.7 & 856.7 & 816.4 & 784.5 \\
\hline \multirow{3}{*}{ 2nd } & Tens. (MPa) & 398.3 & 440.5 & 410.3 & 346.3 & 438.8 & 443.3 & 409.5 & 406.3 \\
\hline & Comp. (MPa) & -424.6 & -413.8 & -445.2 & -429.3 & -425.3 & -411.2 & -450.8 & -473.5 \\
\hline & Size (MPa) & 822.9 & 854.3 & 855.5 & 775.6 & 864.2 & 854.5 & 860.3 & 879.8 \\
\hline \multirow{3}{*}{10 th } & Tens. (MPa) & 419.4 & 423.8 & 411.4 & 413.8 & 423.7 & 428.0 & 415.6 & 417.9 \\
\hline & Comp. (MPa) & -433.9 & -416.9 & -434.1 & -450.6 & -427.2 & -416.4 & -436.9 & -454.9 \\
\hline & Size (MPa) & 853.3 & 840.6 & 845.6 & 864.4 & 850.9 & 844.3 & 852.5 & 872.8 \\
\hline \multirow{3}{*}{20 th } & Tens. (MPa) & 416.1 & 419.0 & 411.7 & 415.7 & 418.6 & 423.6 & 416.9 & 420.5 \\
\hline & Comp. (MPa) & -430.5 & -417.3 & -429.3 & -446.1 & -426.8 & -418.9 & -434.4 & -450.3 \\
\hline & Size $(\mathrm{MPa})$ & 846.6 & 836.2 & 841.0 & 861.8 & 845.4 & 842.5 & 851.3 & 870.7 \\
\hline \multicolumn{2}{|c|}{$\begin{array}{c}\text { Size decrease between } \\
\text { 2nd and 10th cycle } \\
(\mathrm{MPa})\end{array}$} & -30.4 & 13.7 & 9.9 & -88.8 & 13.3 & 10.2 & 7.8 & 6.9 \\
\hline \multicolumn{2}{|c|}{$\begin{array}{c}\text { Size decrease between } \\
\text { 10th and 20th cycle } \\
(\mathrm{MPa})\end{array}$} & 6.7 & 4.4 & 4.5 & 2.6 & 5.5 & 1.8 & 1.3 & 2.1 \\
\hline \multicolumn{2}{|c|}{$\begin{array}{l}\% \text { decrease from } \\
10 \text { th to } 20 \text { th }\end{array}$} & $0.80 \%$ & $0.53 \%$ & $0.54 \%$ & $0.31 \%$ & $0.65 \%$ & $0.22 \%$ & $0.15 \%$ & $0.24 \%$ \\
\hline
\end{tabular}


Table 4.5 (continued)

\begin{tabular}{|c|c|c|c|c|c|c|c|c|c|}
\hline $\begin{array}{c}\text { Cycle } \\
\text { number }\end{array}$ & & $0 / 2 \% \# 3$ & $-2 / 0 \% \# 3$ & $0 / 2 \% \# 4$ & $-2 / 0 \% \# 4$ & $0 / 2 \% \# 5$ & $-2 / 0 \% \# 5$ & $0 / 2 \% \# 6$ & $-2 / 0 \% \# 6$ \\
\hline \multirow{3}{*}{$1 \mathrm{st}$} & Tens. (MPa) & 476.7 & 416.4 & 478.3 & 419.4 & 480.6 & 419.7 & 482.2 & 421.4 \\
\hline & Comp. (MPa) & -416.2 & -493.4 & -418.7 & -505.4 & -419.8 & -497.5 & -420.7 & -508.4 \\
\hline & Size $(\mathrm{MPa})$ & 892.9 & 909.9 & 897.0 & 924.8 & 900.4 & 917.2 & 902.9 & 929.8 \\
\hline \multirow{3}{*}{ 2nd } & Tens. (MPa) & 465.3 & 420.5 & 466.7 & 423.8 & 468.9 & 424.1 & 469.8 & 425.9 \\
\hline & Comp. (MPa) & -420.8 & -481.5 & -423.1 & -493.1 & -424.3 & -485.2 & -425.2 & -495.7 \\
\hline & Size $(\mathrm{MPa})$ & 886.2 & 902.0 & 889.7 & 916.9 & 893.2 & 909.3 & 895.0 & 921.7 \\
\hline \multirow{3}{*}{ 10th } & Tens. (MPa) & 436.2 & 422.8 & 437.7 & 424.0 & 439.7 & 426.1 & 441.1 & 425.9 \\
\hline & Comp. (MPa) & -422.1 & -459.6 & -424.1 & -463.3 & -425.5 & -462.3 & -426.9 & -463.9 \\
\hline & Size $(\mathrm{MPa})$ & 858.4 & 882.4 & 861.8 & 887.3 & 865.2 & 888.5 & 868.1 & 889.8 \\
\hline \multirow{3}{*}{20 th } & Tens. (MPa) & 428.9 & 422.9 & 430.7 & 425.3 & 432.4 & 426.3 & 433.7 & 427.5 \\
\hline & Comp. (MPa) & -423.0 & -454.2 & -425.0 & -455.6 & -426.7 & -457.5 & -427.6 & -457.5 \\
\hline & Size (MPa) & 851.9 & 877.1 & 855.7 & 881.0 & 859.1 & 883.7 & 861.2 & 885.0 \\
\hline \multicolumn{2}{|c|}{$\begin{array}{c}\text { Size decrease between } \\
\text { 2nd and 10th cycle } \\
(\mathrm{MPa})\end{array}$} & 27.8 & 19.7 & 27.9 & 29.6 & 28.0 & 20.9 & 27.0 & 31.8 \\
\hline \multicolumn{2}{|c|}{$\begin{array}{c}\text { Size decrease between } \\
10 \text { th and 20th cycle } \\
(\mathrm{MPa})\end{array}$} & 6.4 & 5.3 & 6.1 & 6.3 & 6.1 & 4.7 & 6.8 & 4.8 \\
\hline \multicolumn{2}{|c|}{$\begin{array}{l}\% \text { decrease from } \\
10 \text { th to } 20 \text { th }\end{array}$} & $0.76 \%$ & $0.60 \%$ & $0.72 \%$ & $0.72 \%$ & $0.71 \%$ & $0.53 \%$ & $0.79 \%$ & $0.55 \%$ \\
\hline
\end{tabular}


Table 4.6 Measured average true strain rates and strain ranges for the notched specimens

\begin{tabular}{|c|c|c|c|c|c|c|}
\hline Specimen & Material & $\begin{array}{c}\text { Target engineering } \\
\text { strain rate } \\
\left(\mathrm{s}^{-1}\right)\end{array}$ & $\begin{array}{c}\text { Target nominal } \\
\text { true strain } \\
\text { range }\end{array}$ & $\begin{array}{c}\text { Measured } \\
\text { average true } \\
\text { strain rate } \\
\left(\mathrm{s}^{-1}\right)\end{array}$ & $\begin{array}{c}\text { Measured } \\
\text { average true } \\
\text { strain range }\end{array}$ & $\begin{array}{c}\text { Measured average } \\
\text { true plastic strain } \\
\text { range }\end{array}$ \\
\hline ANE1R2 & $\mathrm{A}$ & $1.00 \mathrm{E}-01$ & 0.04 & $4.50 \mathrm{E}-01$ & 0.0344 & 0.0323 \\
\hline ANE2R2 & $\mathrm{A}$ & $1.00 \mathrm{E}-02$ & 0.04 & $4.57 \mathrm{E}-02$ & 0.0288 & 0.0263 \\
\hline ANE3R2 & $\mathrm{A}$ & $1.00 \mathrm{E}-03$ & 0.04 & $4.87 \mathrm{E}-03$ & 0.0299 & 0.0276 \\
\hline ANE4R2 & $\mathrm{A}$ & $1.00 \mathrm{E}-04$ & 0.04 & $4.52 \mathrm{E}-04$ & 0.0304 & 0.0281 \\
\hline ANE1R4 & $\mathrm{A}$ & $1.00 \mathrm{E}-01$ & 0.08 & $4.35 \mathrm{E}-01$ & 0.0638 & 0.0613 \\
\hline ANE2R4 & $\mathrm{A}$ & $1.00 \mathrm{E}-02$ & 0.08 & $4.18 \mathrm{E}-02$ & 0.0541 & 0.0515 \\
\hline ANE3R4 & $\mathrm{A}$ & $1.00 \mathrm{E}-03$ & 0.08 & $4.37 \mathrm{E}-03$ & 0.0563 & 0.0533 \\
\hline ANE4R4 & $\mathrm{A}$ & $1.00 \mathrm{E}-04$ & 0.08 & $4.48 \mathrm{E}-04$ & 0.0561 & 0.0534 \\
\hline ANE1R8 & $\mathrm{A}$ & $1.00 \mathrm{E}-01$ & 0.16 & $3.96 \mathrm{E}-01$ & 0.104 & 0.101 \\
\hline ANE2R8 & $\mathrm{A}$ & $1.00 \mathrm{E}-02$ & 0.16 & $5.15 \mathrm{E}-02$ & 0.134 & 0.130 \\
\hline ANE3R8 & $\mathrm{A}$ & $1.00 \mathrm{E}-03$ & 0.16 & $4.07 \mathrm{E}-03$ & 0.131 & 0.128 \\
\hline ANE4R8 & $\mathrm{A}$ & $1.00 \mathrm{E}-04$ & 0.16 & $4.45 \mathrm{E}-04$ & 0.130 & 0.126 \\
\hline
\end{tabular}


Table 4.6 (continued)

\begin{tabular}{|c|c|c|c|c|c|c|}
\hline Specimen & Material & $\begin{array}{c}\text { Target engineering } \\
\text { strain rate } \\
\left(\mathrm{s}^{-1}\right)\end{array}$ & $\begin{array}{c}\text { Target nominal } \\
\text { true strain } \\
\text { range }\end{array}$ & $\begin{array}{c}\text { Measured } \\
\text { average true } \\
\text { strain rate }^{1} \\
\left(\mathrm{~s}^{-1}\right)\end{array}$ & $\begin{array}{c}\text { Measured } \\
\text { average true } \\
\text { strain range }\end{array}$ & $\begin{array}{c}\text { Measured average } \\
\text { true plastic strain } \\
\text { range }\end{array}$ \\
\hline BNE1R2 & B & $1.00 \mathrm{E}-01$ & 0.04 & $4.85 \mathrm{E}-01$ & 0.0325 & 0.0299 \\
\hline BNE2R2 & B & $1.00 \mathrm{E}-02$ & 0.04 & $4.67 \mathrm{E}-02$ & 0.0267 & 0.0243 \\
\hline BNE3R2 & B & $1.00 \mathrm{E}-03$ & 0.04 & $4.80 \mathrm{E}-03$ & 0.0279 & 0.0254 \\
\hline BNE4R2 & B & $1.00 \mathrm{E}-04$ & 0.04 & $5.15 \mathrm{E}-04$ & 0.0294 & 0.0267 \\
\hline BNE1R4 & B & $1.00 \mathrm{E}-01$ & 0.08 & $4.35 \mathrm{E}-01$ & 0.0578 & 0.0553 \\
\hline BNE2R4 & B & $1.00 \mathrm{E}-02$ & 0.08 & $4.66 \mathrm{E}-02$ & 0.0605 & 0.0573 \\
\hline BNE3R4 & B & $1.00 \mathrm{E}-03$ & 0.08 & $5.19 \mathrm{E}-03$ & 0.0623 & 0.0592 \\
\hline BNE4R4 & B & $1.00 \mathrm{E}-04$ & 0.08 & $4.73 \mathrm{E}-04$ & 0.0609 & 0.0580 \\
\hline BNE4R4-2 & B & $1.00 \mathrm{E}-04$ & 0.08 & $4.36 \mathrm{E}-04$ & 0.0627 & 0.0596 \\
\hline BNE1R8 & B & $1.00 \mathrm{E}-01$ & 0.16 & $5.13 \mathrm{E}-01$ & 0.159 & 0.156 \\
\hline BNE2R8 & B & $1.00 \mathrm{E}-02$ & 0.16 & $5.07 \mathrm{E}-02$ & 0.146 & 0.143 \\
\hline BNE3R8 & B & $1.00 \mathrm{E}-03$ & 0.16 & $5.38 \mathrm{E}-03$ & 0.148 & 0.145 \\
\hline BNE4R8 & B & $1.00 \mathrm{E}-04$ & 0.16 & $5.00 \mathrm{E}-04$ & 0.140 & 0.137 \\
\hline
\end{tabular}

Notes:

Measurements calculated from the compression half-cycle of the specimens $30^{\text {th }}$ loading cycle except for ANE4R2, BNE4R2, and tested at 0.16 strain range, which are calculated from the compression half-cycle of the $15^{\text {th }}$ loading cycle. 
Table 4.7 Peak temperature measurements during testing

\begin{tabular}{|c|c|c|c|c|}
\cline { 2 - 5 } \multicolumn{1}{c|}{} & \multicolumn{2}{c|}{ Initial $\left({ }^{\circ} \mathrm{C}\right)$} & \multicolumn{2}{c|}{ Fracture $\left({ }^{\circ} \mathrm{C}\right)$} \\
\hline Specimen & IR Gun & Thermocouple & IR Gun & Thermocouple \\
\hline ANE1R2 & 23 & 24 & 240 & 116 \\
\hline ANE2R2 & 25 & & 52 & \\
\hline ANE1R4 & 27 & 25 & 190 & 96 \\
\hline ANE2R4 & 26 & & 65 & \\
\hline ANE1R8 & 24 & 23 & 100 & 72 \\
\hline ANE2R8 & 23 & 23 & 89 & 40 \\
\hline BNE1R2 & 28 & 23 & 190 & 109 \\
\hline BNE2R2 & 26 & & 50 & \\
\hline BNE1R4 & 26 & 26 & 130 & 83 \\
\hline BNE2R4 & 25 & & 58 & \\
\hline BNE1R8 & 26 & 25 & 133 & 78 \\
\hline BNE2R8 & 25 & & 58 & \\
\hline
\end{tabular}


Table 4.8 Minimum, maximum and range of measured average true strain for selected loading cycle numbers of notched specimens

\begin{tabular}{|c|c|c|c|c|c|c|c|c|c|}
\hline \multirow{2}{*}{ Specimen } & \multicolumn{9}{|c|}{ Average true strain, In(Ad/A) } \\
\cline { 2 - 10 } & \multicolumn{3}{|c|}{ Cycle \#2 } & \multicolumn{3}{c|}{ Cycle \#10 } & \multicolumn{3}{c|}{ Last cycle $^{1}$} \\
\cline { 2 - 10 } & Min. & Max. & Range & Min. & Max. & Range & Min. & Max. & Range \\
\hline ANE3R2 & -0.014 & 0.017 & 0.031 & -0.014 & 0.017 & 0.031 & -0.014 & 0.017 & 0.031 \\
\hline ANE4R2 & -0.014 & 0.017 & 0.031 & -0.013 & 0.018 & 0.031 & -0.012 & 0.018 & 0.030 \\
\hline & & & & & & & & & \\
\hline ANE3R4 & -0.025 & 0.035 & 0.060 & -0.021 & 0.038 & 0.059 & -0.012 & 0.044 & 0.056 \\
\hline ANE4R4 & -0.024 & 0.036 & 0.060 & -0.020 & 0.039 & 0.060 & -0.010 & 0.046 & 0.056 \\
\hline & & & & & & & & & \\
\hline ANE3R8 & -0.037 & 0.086 & 0.123 & -0.002 & 0.126 & 0.128 & 0.017 & 0.147 & 0.131 \\
\hline ANE4R8 & -0.037 & 0.085 & 0.122 & -0.001 & 0.126 & 0.127 & 0.018 & 0.147 & 0.129 \\
\hline & & & & & & & & & \\
\hline BNE3R2 & -0.014 & 0.015 & 0.029 & -0.013 & 0.015 & 0.028 & -0.013 & 0.016 & 0.028 \\
\hline BNE4R2 & -0.014 & 0.016 & 0.030 & -0.013 & 0.016 & 0.030 & -0.012 & 0.017 & 0.029 \\
\hline & & & & & & & & & \\
\hline BNE3R4 & -0.028 & 0.035 & 0.063 & -0.024 & 0.038 & 0.062 & -0.015 & 0.048 & 0.063 \\
\hline BNE4R4-2 & -0.028 & 0.035 & 0.063 & -0.024 & 0.039 & 0.062 & -0.020 & 0.043 & 0.062 \\
\hline BNE4R4 & -0.028 & 0.033 & 0.061 & -0.023 & 0.038 & 0.061 & -0.014 & 0.047 & 0.061 \\
\hline & & & & & & & & & \\
\hline BNE3R8 & -0.053 & 0.083 & 0.136 & -0.021 & 0.123 & 0.144 & -0.003 & 0.145 & 0.148 \\
\hline BNE4R8 & -0.053 & 0.079 & 0.132 & -0.022 & 0.114 & 0.136 & -0.005 & 0.135 & 0.140 \\
\hline
\end{tabular}

Notes:

1 Last cycle represents the cycle number $20^{\text {th }}$ for $\pm 2 \%, 40^{\text {th }}$ for $\pm 4 \%$ and $15^{\text {th }}$ for $\pm 8 \%$ 
Table 4.9 Fatigue and separation lives for material A notch specimens

\begin{tabular}{|c|c|c|c|c|c|c|}
\hline Specimen & $\begin{array}{c}\text { Target } \\
\text { engineering } \\
\text { strain rate } \\
\left(\mathrm{s}^{-1}\right)\end{array}$ & $\begin{array}{c}\text { Target } \\
\text { true strain } \\
\text { range }\end{array}$ & $\begin{array}{c}\text { Separation } \\
\text { life } \\
\text { (cycle) }\end{array}$ & $\begin{array}{c}\text { Fatigue } \\
\text { Life } \\
(\text { cycle })\end{array}$ & $\begin{array}{c}\text { Cumulative average true } \\
\text { plastic strain up to the } \\
\text { end of the last full cycle } \\
\text { before fatigue life is } \\
\text { reached }\end{array}$ & $\begin{array}{c}\text { Average true } \\
\text { plastic strain } \\
\text { per cycle }\end{array}$ \\
\hline ANE3R2 & 0.001 & $\pm 2 \%$ & 309 & 273 & 13.83 & 0.0509 \\
\hline ANE2R2 & 0.01 & $\pm 2 \%$ & 329 & 304 & 14.77 & 0.0487 \\
\hline ANE1R2 & 0.1 & $\pm 2 \%$ & 512 & 503 & 25.44 & 0.0507 \\
\hline ANE4R4 & 0.0001 & $\pm 4 \%$ & 76 & 62 & 6.74 & 0.110 \\
\hline ANE3R4 & 0.001 & $\pm 4 \%$ & 72 & 60 & 6.48 & 0.110 \\
\hline ANE2R4 & 0.01 & $\pm 4 \%$ & 108 & 90 & 9.23 & 0.104 \\
\hline ANE1R4 & 0.1 & $\pm 4 \%$ & 102 & 100 & 11.40 & 0.115 \\
\hline ANE4R8 & 0.0001 & $\pm 8 \%$ & 21 & 19 & 4.76 & 0.264 \\
\hline ANE3R8 & 0.001 & $\pm 8 \%$ & 22 & 20 & 5.08 & 0.267 \\
\hline ANE2R8 & 0.01 & $\pm 8 \%$ & 22 & 19 & 4.87 & 0.271 \\
\hline ANE1R8 & 0.1 & $\pm 8 \%$ & 18 & 18 & 4.67 & 0.275 \\
\hline
\end{tabular}


Table 4.10 Fatigue and separation lives for material B notch specimens

\begin{tabular}{|c|c|c|c|c|c|c|}
\hline Specimen & $\begin{array}{c}\text { Target } \\
\text { engineering } \\
\text { strain rate } \\
\left(\mathrm{s}^{-1}\right)\end{array}$ & $\begin{array}{c}\text { Target } \\
\text { true strain } \\
\text { range }\end{array}$ & $\begin{array}{c}\text { Separation } \\
\text { life } \\
\text { (cycle) }\end{array}$ & $\begin{array}{c}\text { Fatigue } \\
\text { Life } \\
(\text { cycle })\end{array}$ & $\begin{array}{c}\text { Cumulative average true } \\
\text { plastic strain up to the } \\
\text { end of the last full cycle } \\
\text { before fatigue life is } \\
\text { reached }\end{array}$ & $\begin{array}{c}\text { Average true } \\
\text { plastic strain } \\
\text { per cycle }\end{array}$ \\
\hline BNE3R2 & 0.001 & $\pm 2 \%$ & 394 & 343 & 17.59 & 0.0514 \\
\hline BNE2R2 & 0.01 & $\pm 2 \%$ & 365 & 321 & 15.95 & 0.0499 \\
\hline BNE1R2 & 0.1 & $\pm 2 \%$ & 487 & 473 & 27.01 & 0.0572 \\
\hline BNE4R4 & 0.0001 & $\pm 4 \%$ & 71 & 66 & 7.70 & 0.119 \\
\hline BNE4R4-2 & 0.0001 & $\pm 4 \% \# 2$ & 85 & 79 & 9.44 & 0.121 \\
\hline BNE3R4 & 0.001 & $\pm 4 \%$ & 108 & 96 & 11.48 & 0.121 \\
\hline BNE2R4 & 0.01 & $\pm 4 \%$ & 117 & 103 & 11.81 & 0.116 \\
\hline BNE1R4 & 0.1 & $\pm 4 \%$ & 99 & 97 & 13.08 & 0.136 \\
\hline BNE4R8 & 0.0001 & $\pm 8 \%$ & 21 & 20 & 5.38 & 0.283 \\
\hline BNE3R8 & 0.001 & $\pm 8 \%$ & 23 & 21 & 5.97 & 0.299 \\
\hline BNE2R8 & 0.01 & $\pm 8 \%$ & 24 & 22 & 6.20 & 0.295 \\
\hline BNE1R8 & 0.1 & $\pm 8 \%$ & 18 & 17 & 6.79 & 0.425 \\
\hline
\end{tabular}


Table 4.11 Constants for Eq. (4.8) from fitted lines

\begin{tabular}{|c|c|c|c|}
\hline Material & $\begin{array}{c}\text { Strain rate } \\
\left(\mathrm{s}^{-1}\right)\end{array}$ & $D^{\prime}$ & $c$ \\
\hline \multirow{4}{*}{$\mathrm{A}$} & 0.1 & 1.411 & -1.972 \\
\cline { 2 - 4 } & 0.01 & 2.299 & -1.618 \\
\cline { 2 - 4 } & 0.001 & 2.168 & -1.595 \\
\cline { 2 - 4 } & 0.0001 & 3.133 & -1.355 \\
\hline \multirow{3}{*}{$\mathrm{B}$} & 0.1 & 3.421 & -1.706 \\
\cline { 2 - 4 } & 0.01 & 3.604 & -1.513 \\
\cline { 2 - 4 } & 0.001 & 3.147 & -1.590 \\
\cline { 2 - 4 } & 0.0001 & 2.448 & -1.587 \\
\hline
\end{tabular}




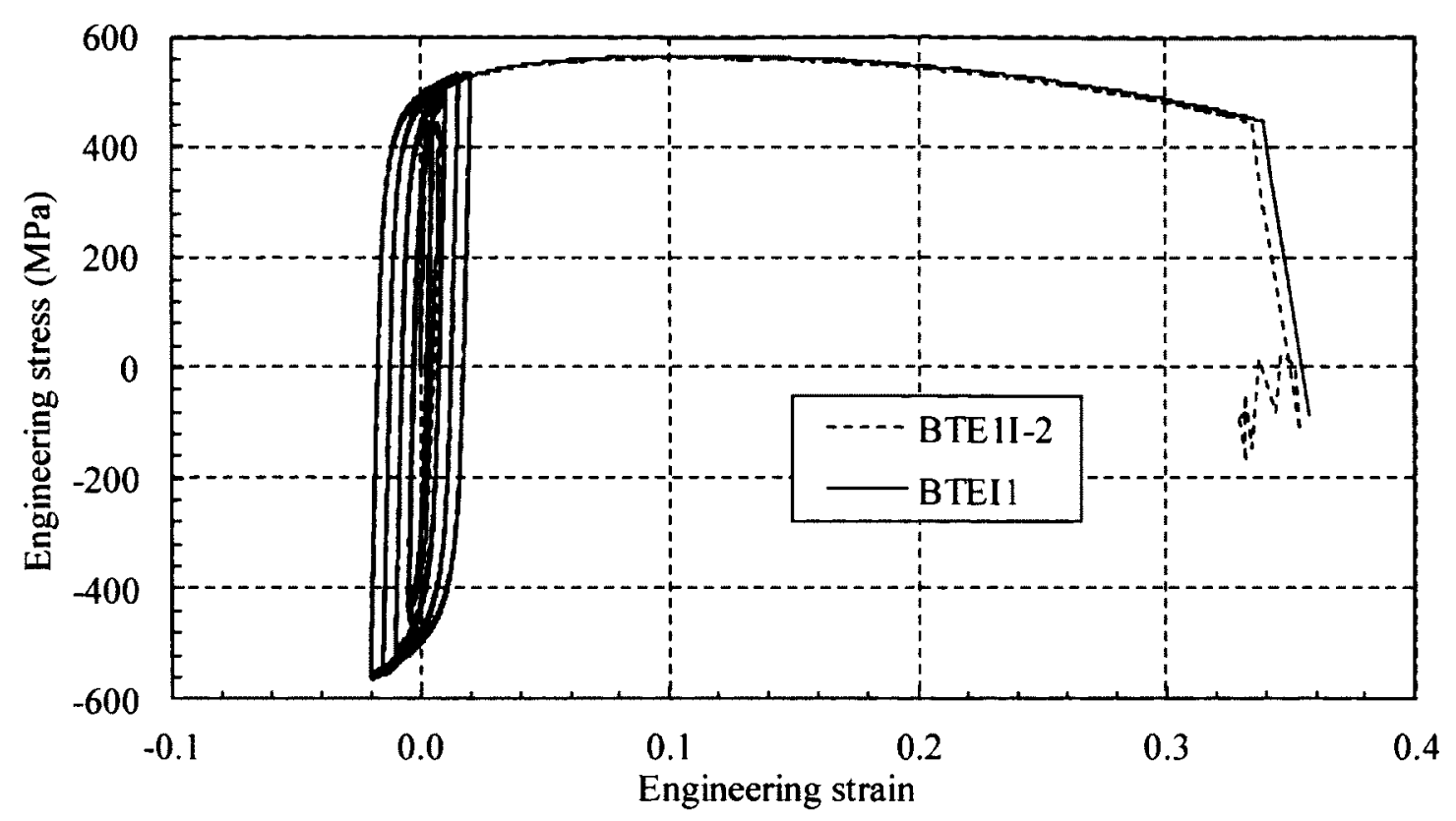

Figure 4.1 Engineering stress versus engineering strain curves for BTE1 Is

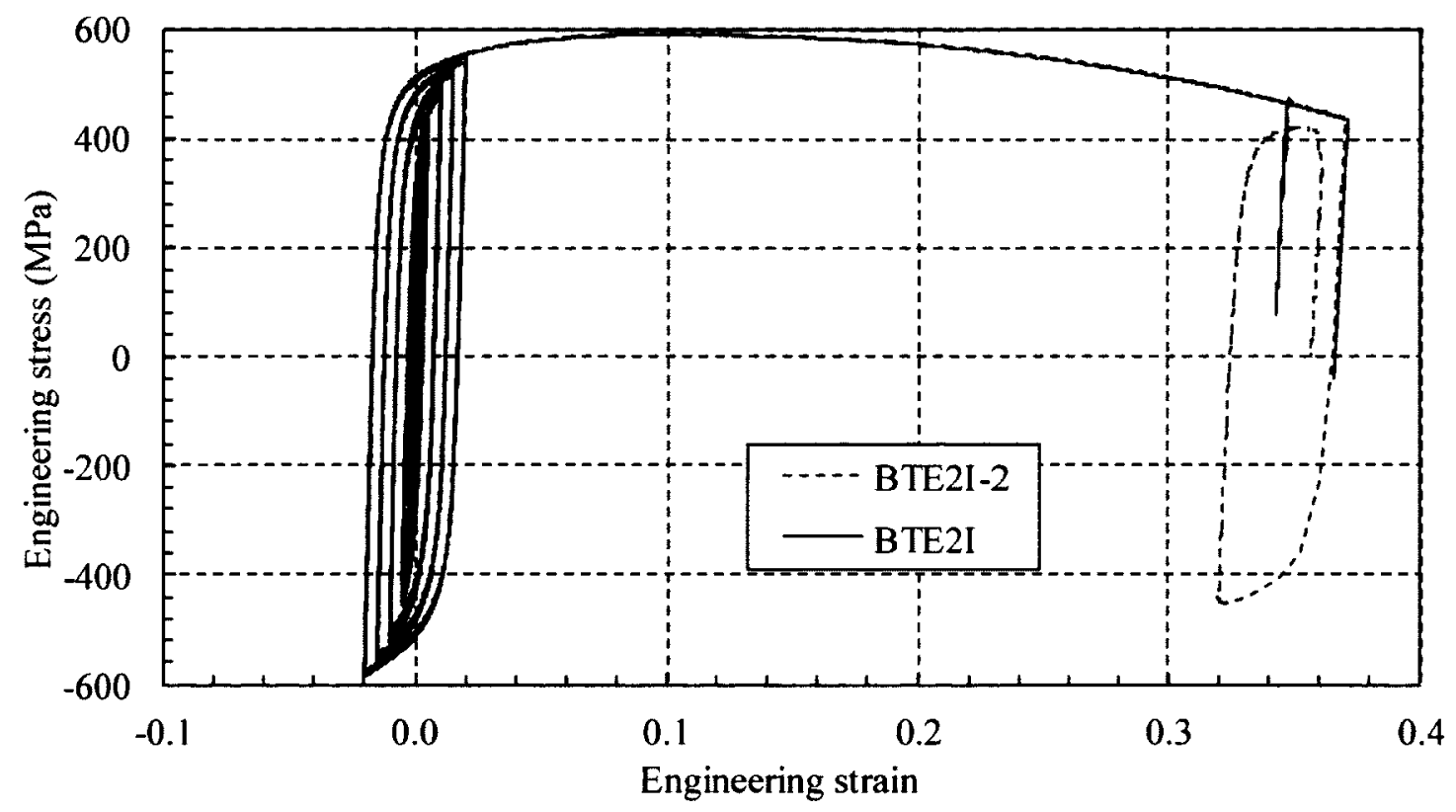

Figure 4.2 Engineering stress versus engineering strain curves for BTE2Is 


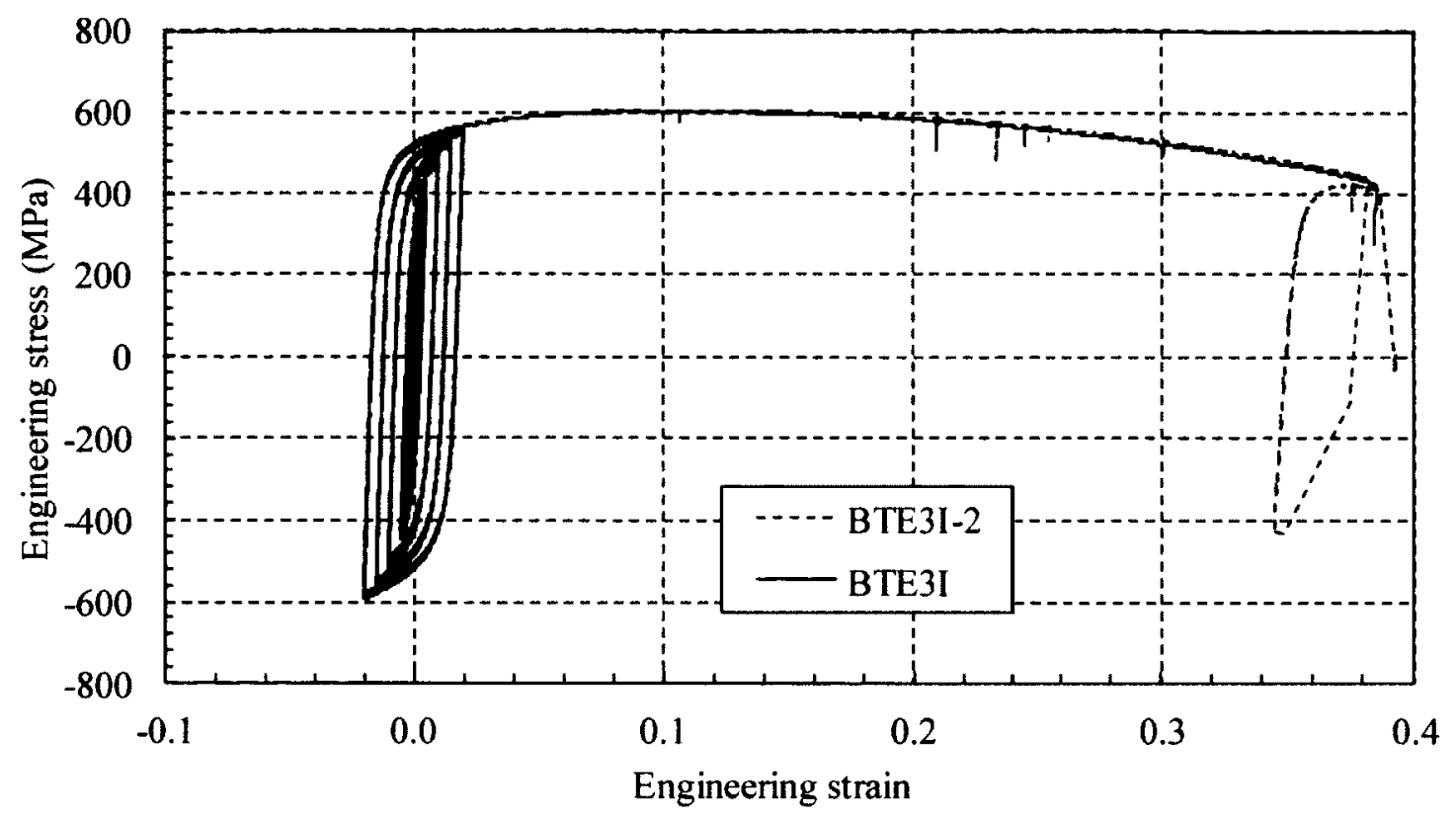

Figure 4.3 Engineering stress versus engineering strain curves for BTE3Is

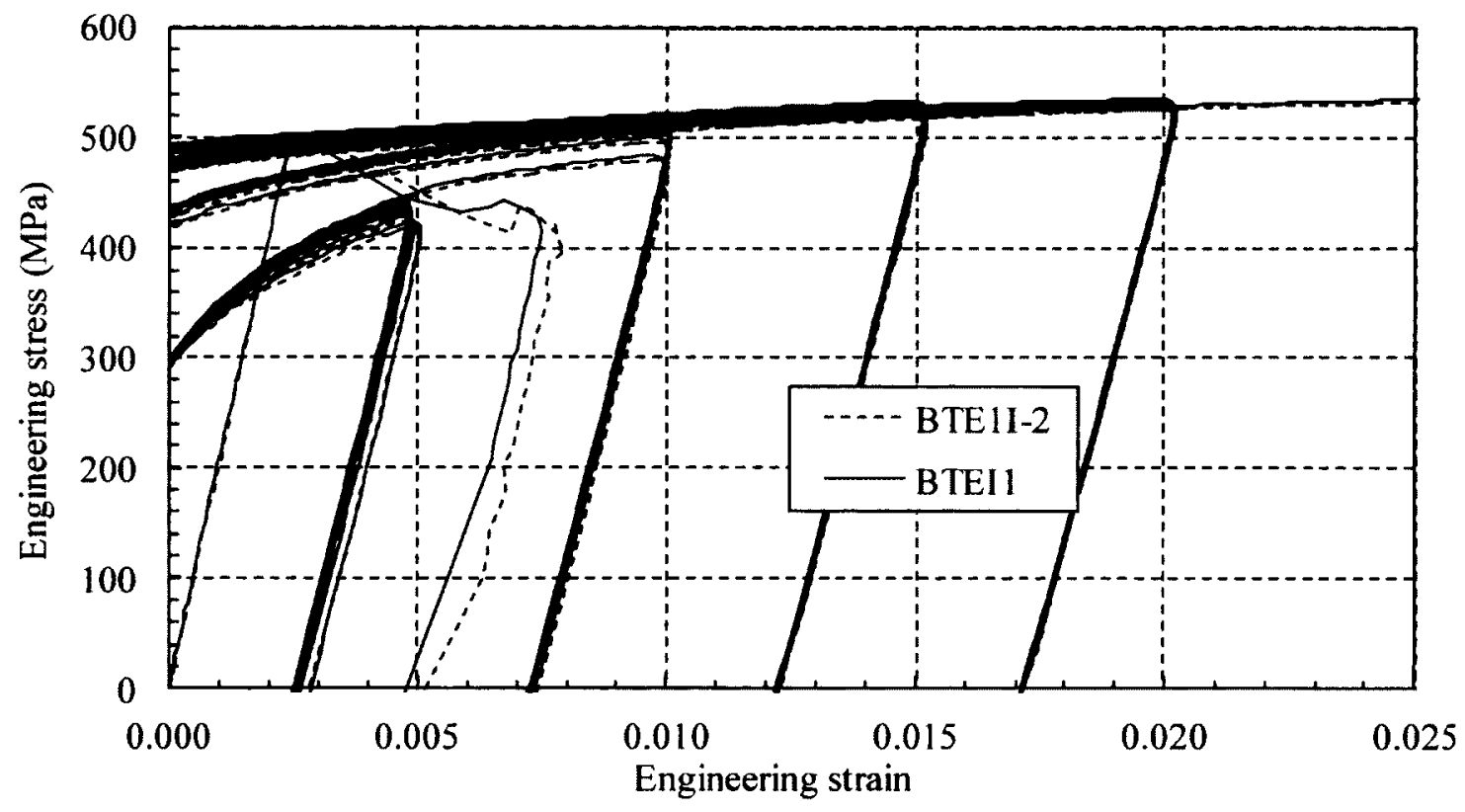

Figure 4.4 Cyclic engineering stress versus engineering strain curves for BTE1Is 


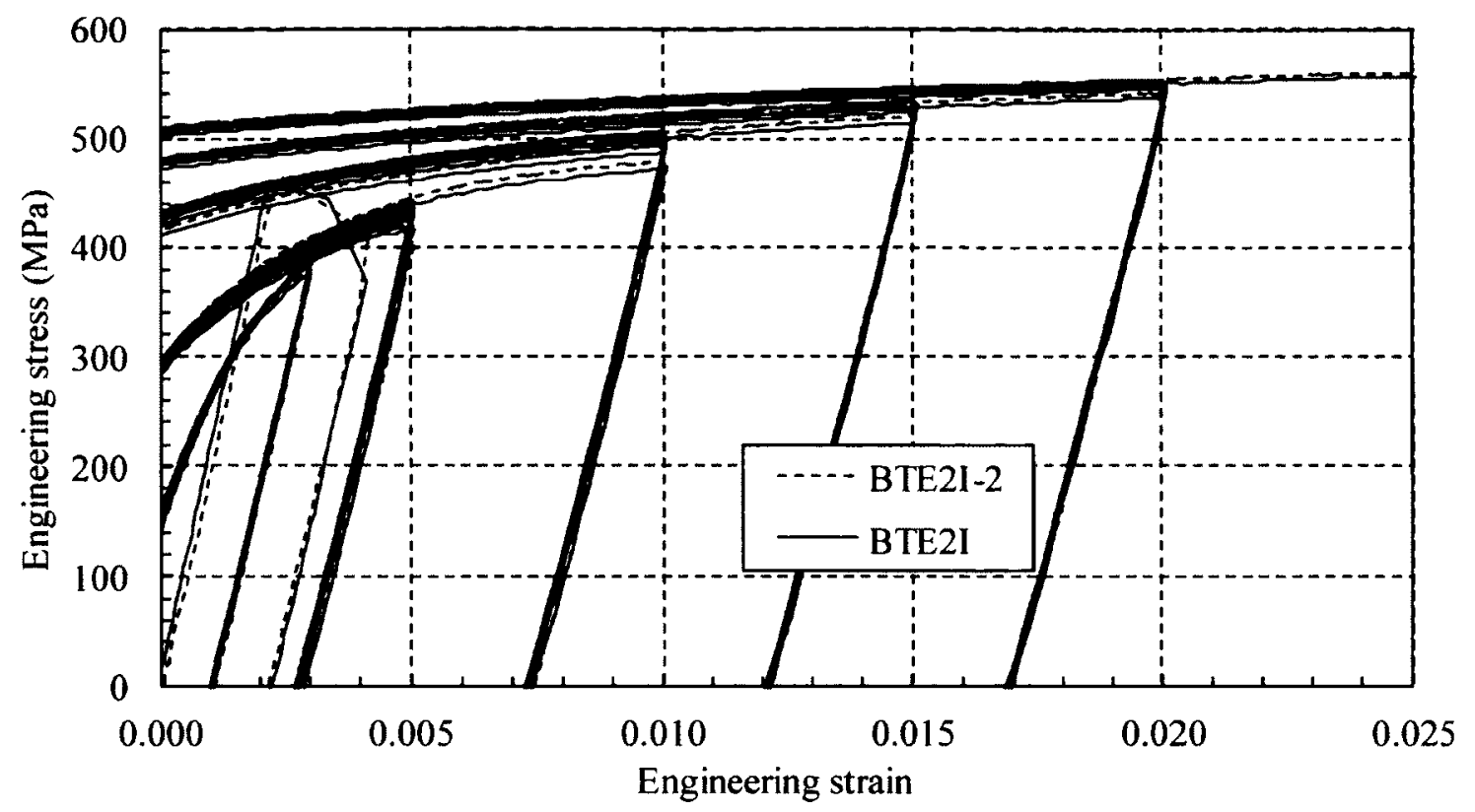

Figure 4.5 Cyclic engineering stress versus engineering strain curves for BTE2Is

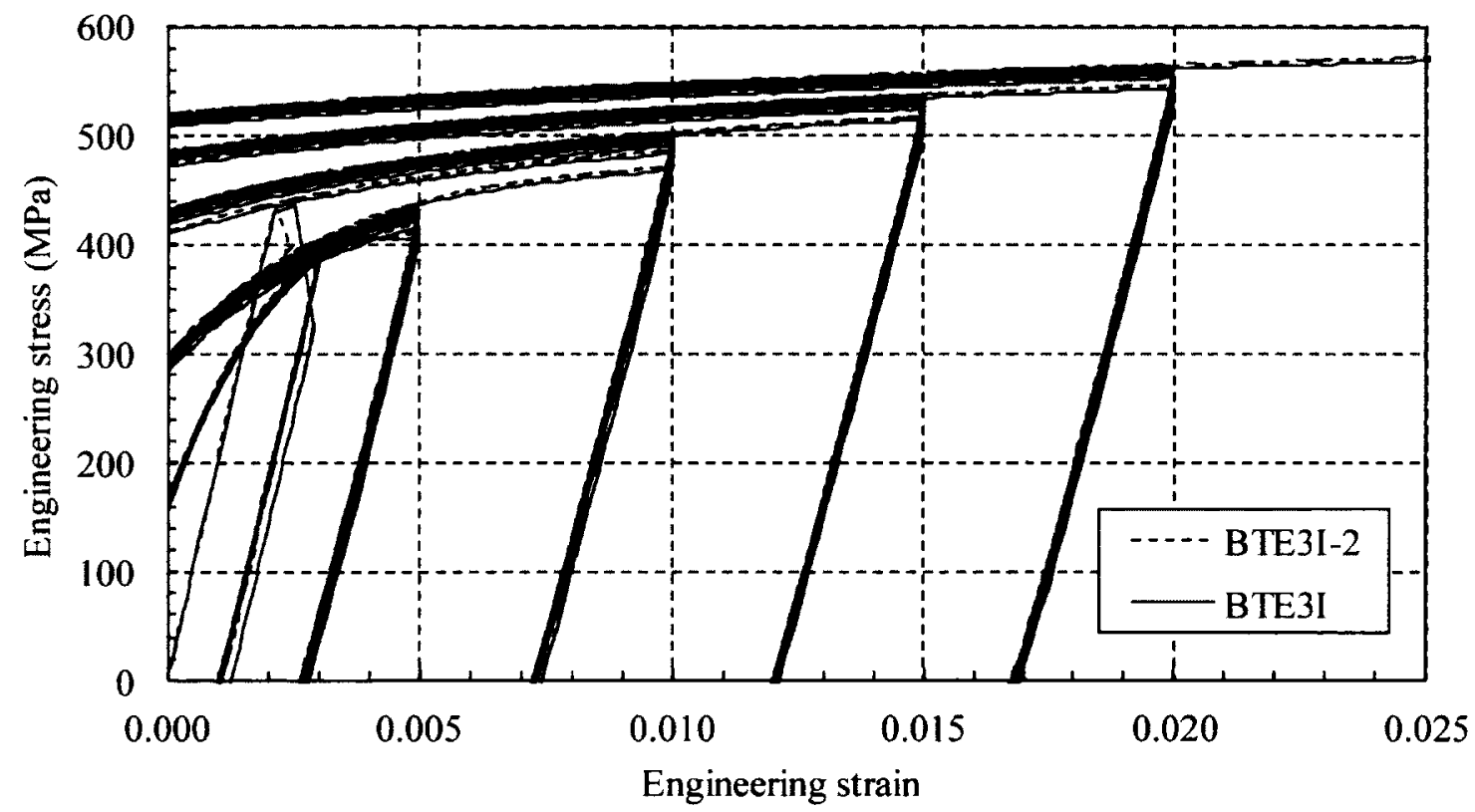

Figure 4.6 Cyclic engineering stress versus engineering strain curves for BTE3Is 


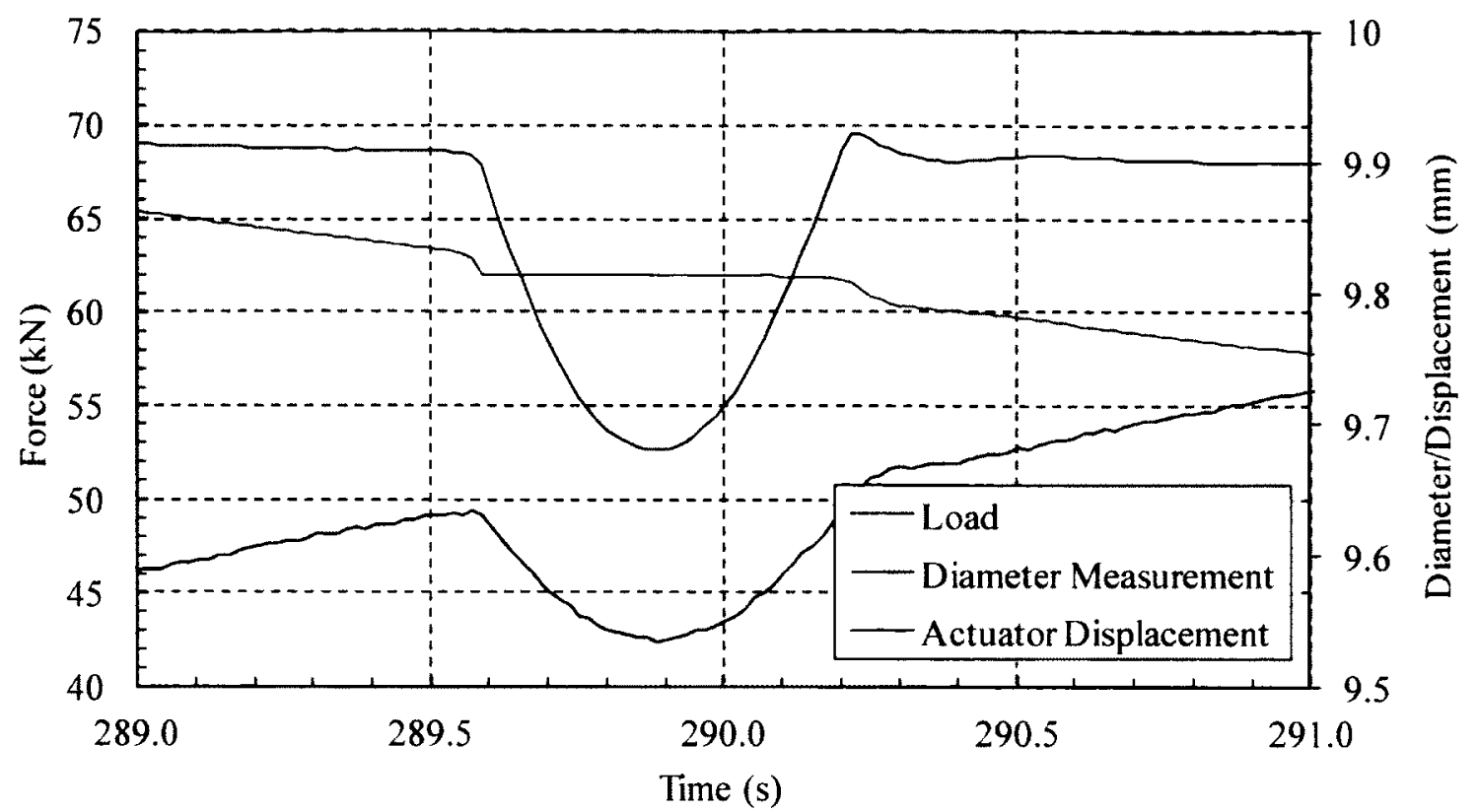

Figure 4.7 Measurements versus time history during the drop in stress seen when the BTE2I-2 specimen was being pulled to fracture

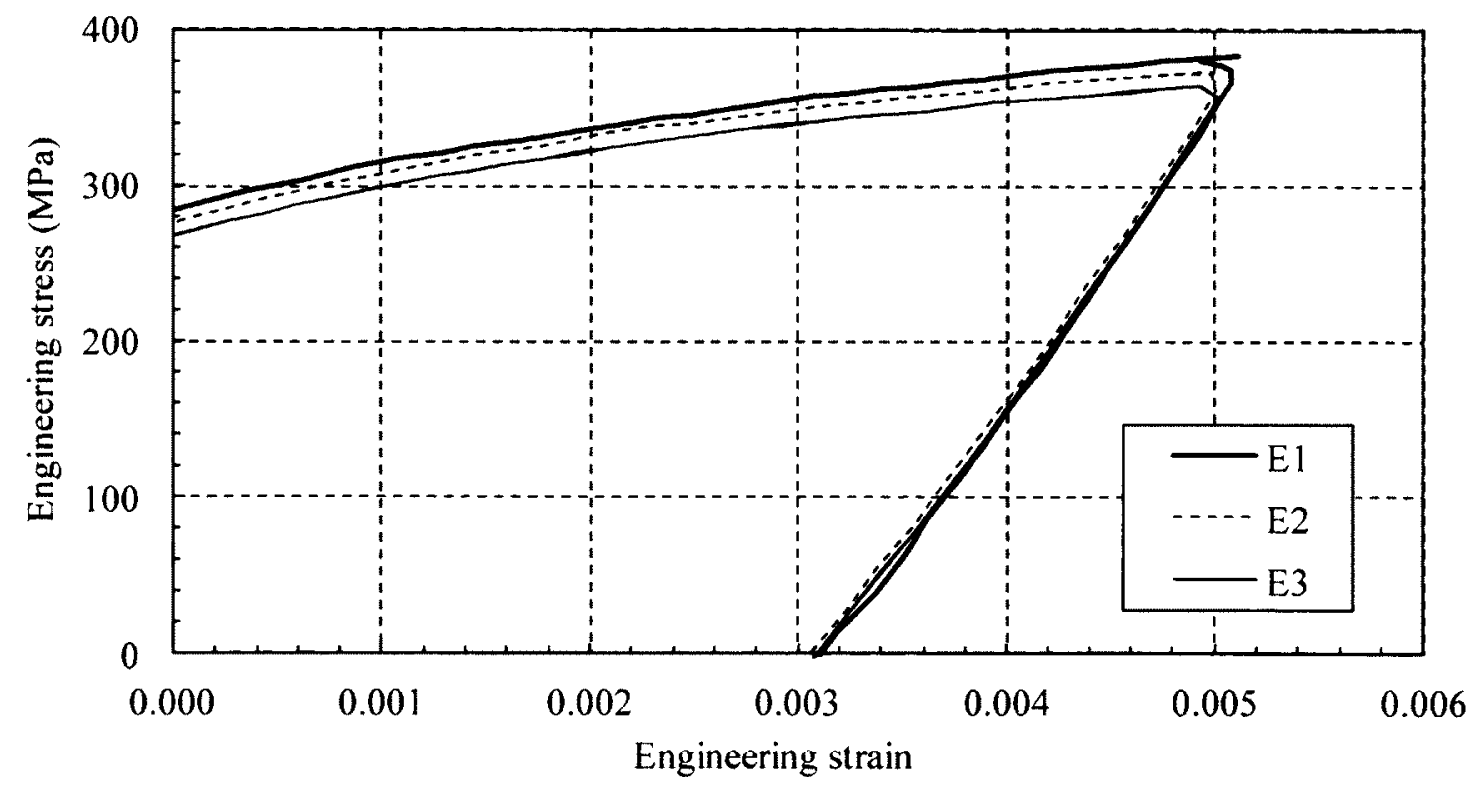

Figure 4.8 Part of the $10^{\text {th }}$ cycle for ATE1I, ATE2I and ATE3I at the $\pm 0.5 \%$ strain range 


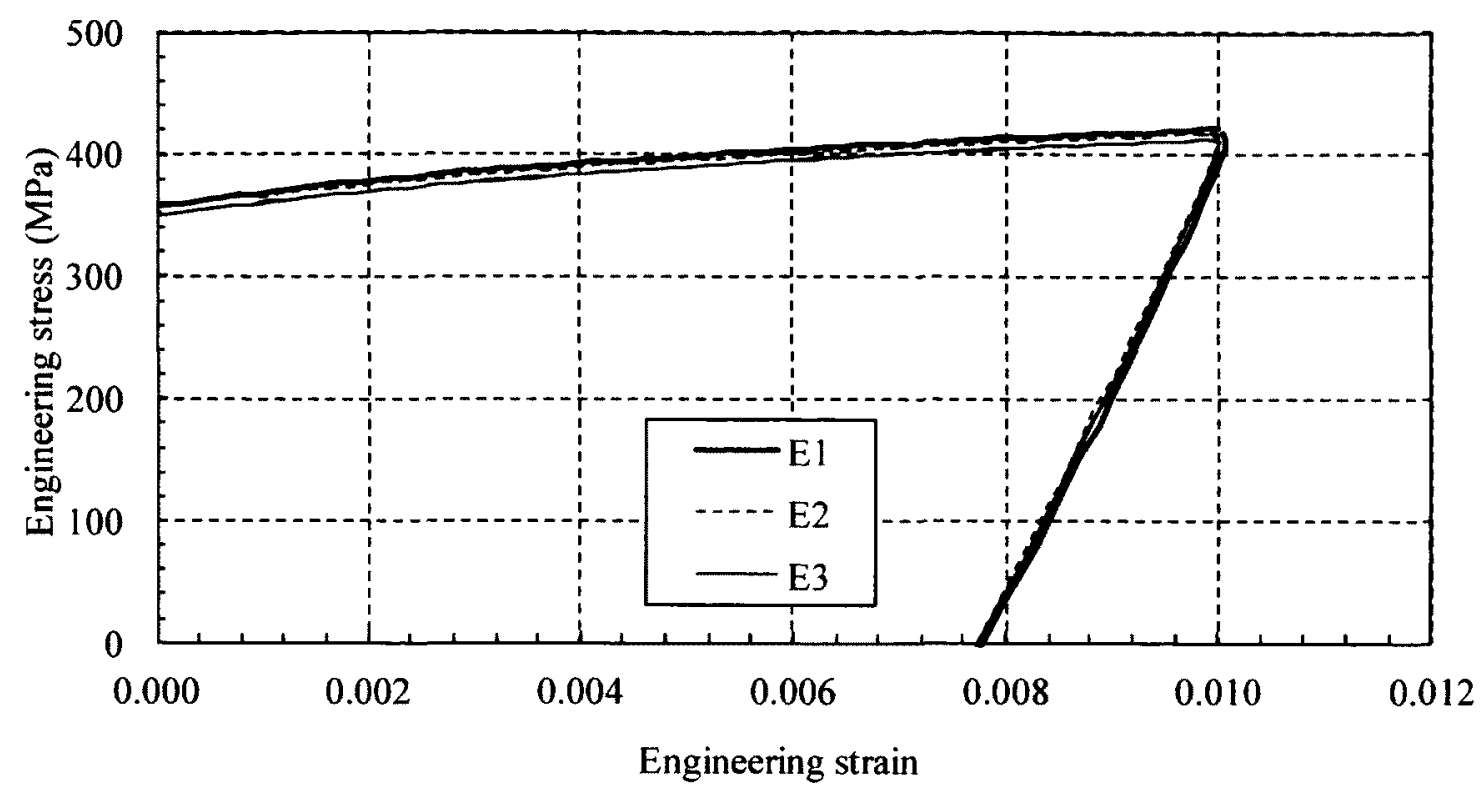

Figure 4.9 Part of the $10^{\text {th }}$ cycle for ATE1I, ATE2I and ATE3I at the $\pm 1 \%$ strain range

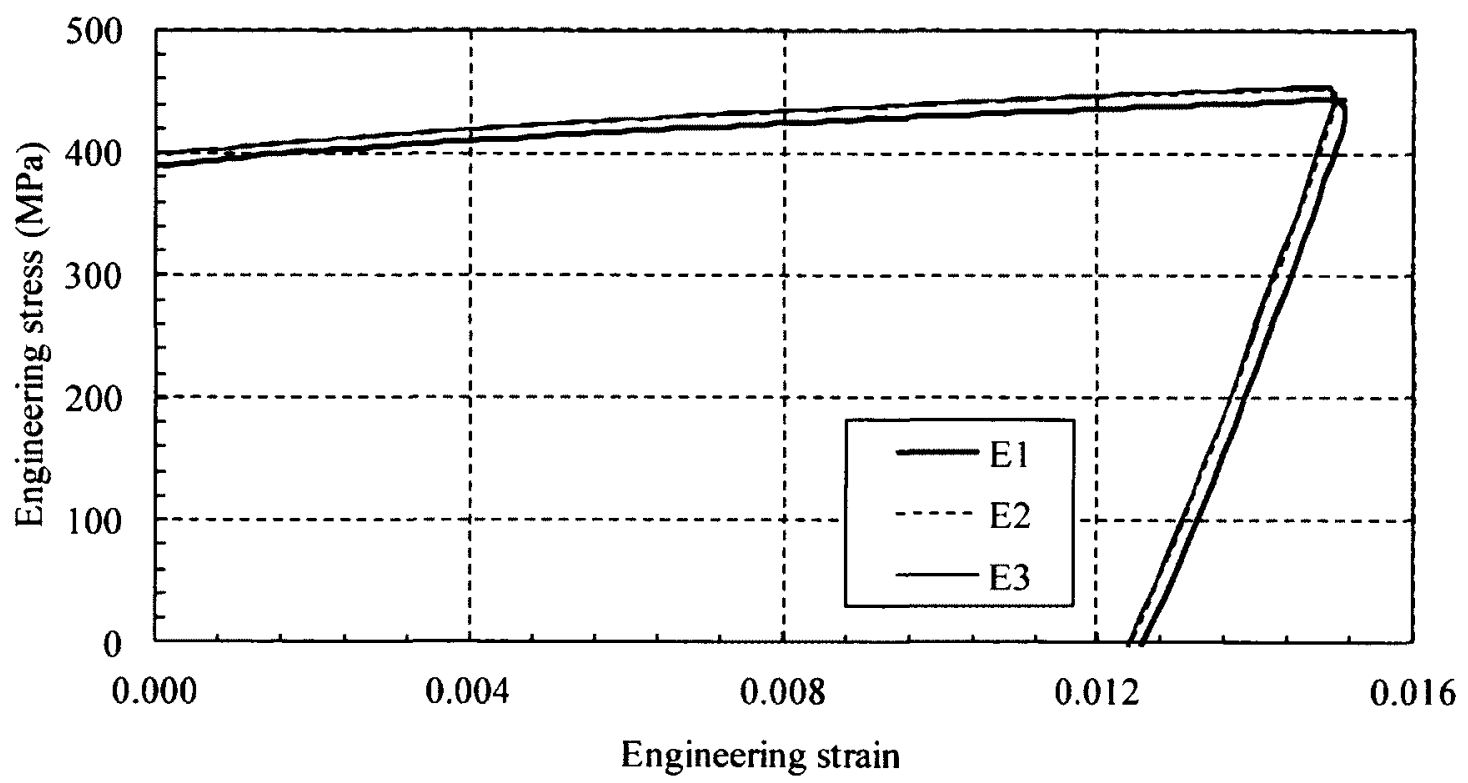

Figure 4.10 Part of the $10^{\text {th }}$ cycle for ATE1I, ATE2I and ATE3I at the $\pm 1.5 \%$ strain range 


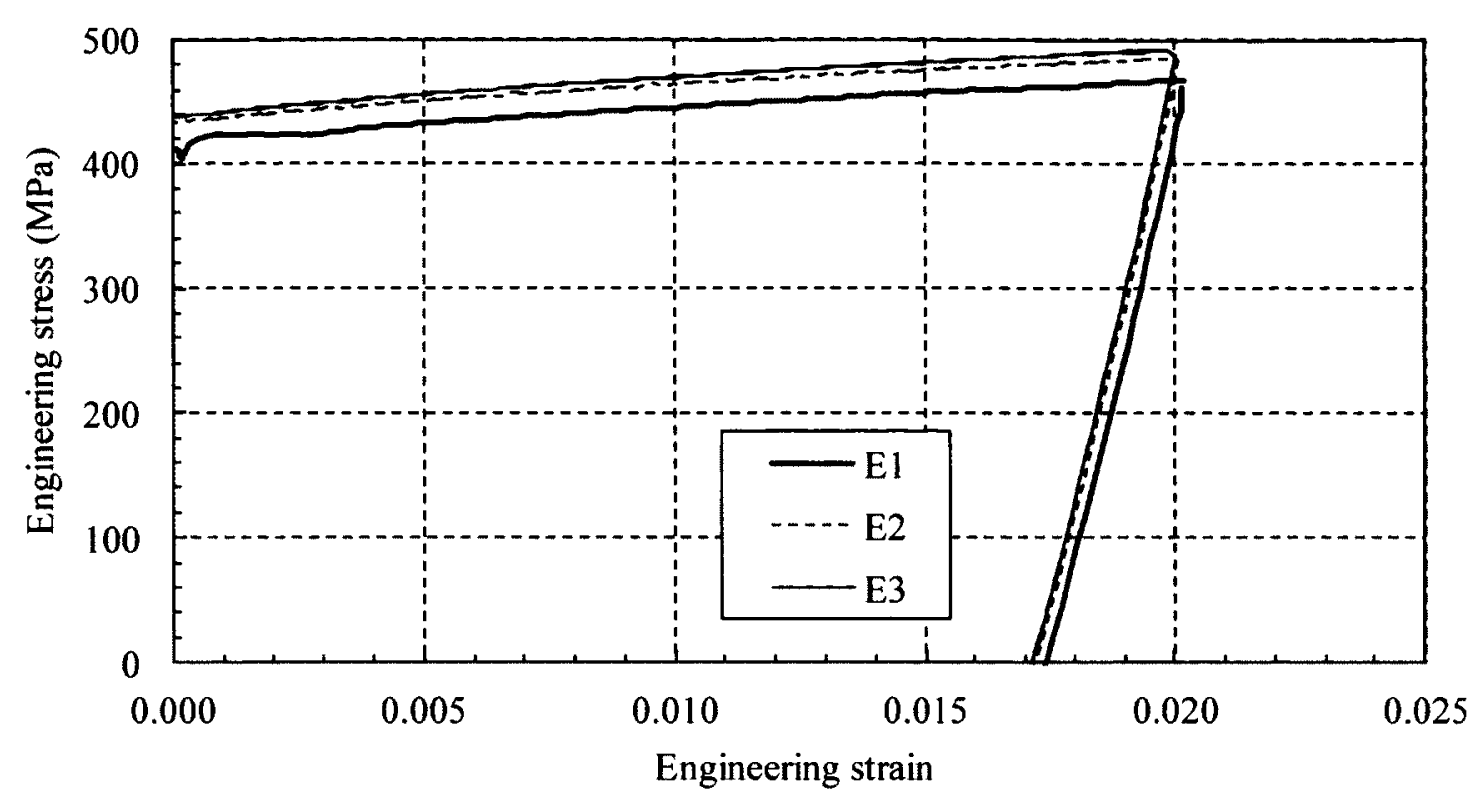

Figure 4.11 Part of the $10^{\text {th }}$ cycle for ATE1I, ATE2I and ATE3I at the $\pm 2 \%$ strain range

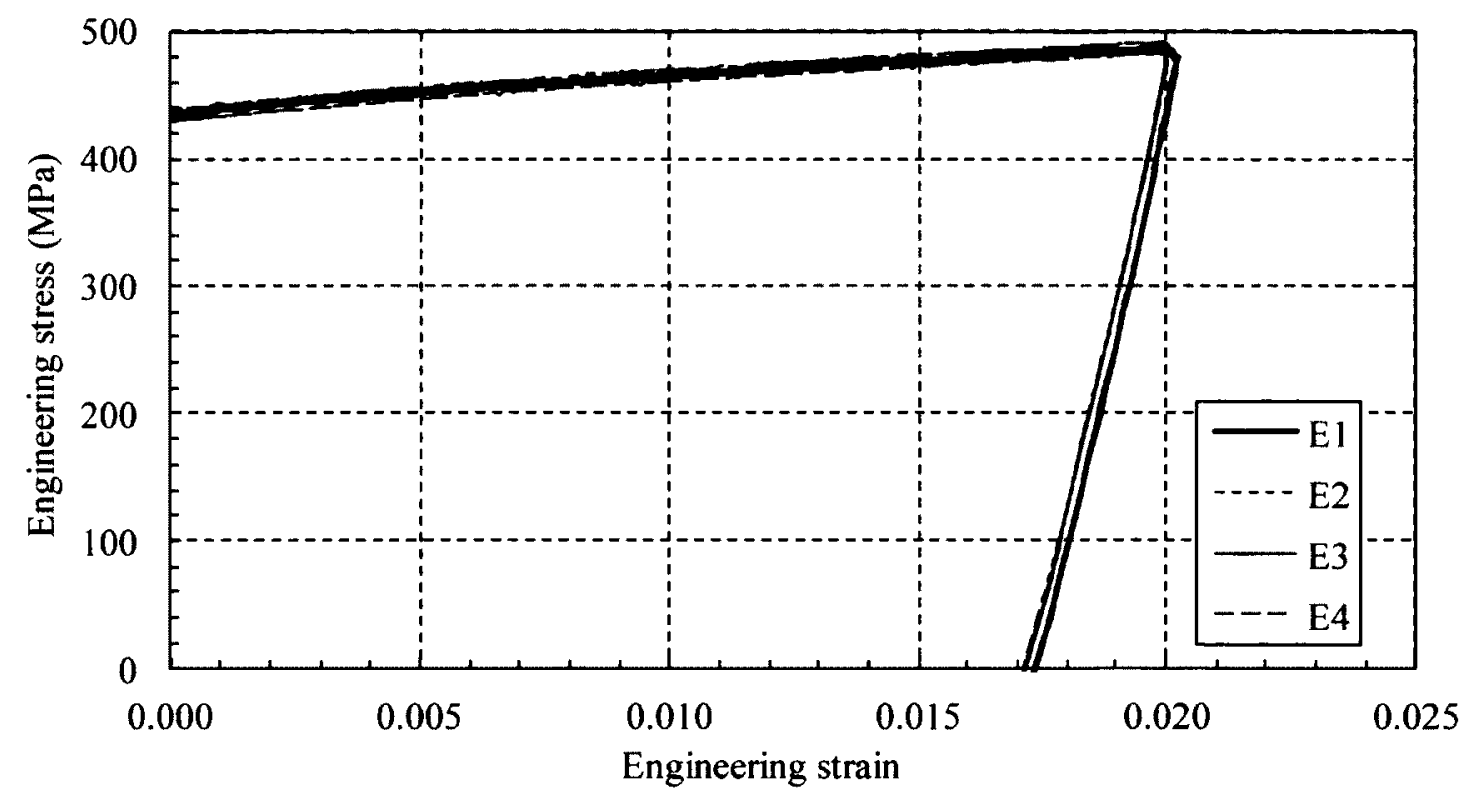

Figure 4.12 Part of the $10^{\text {th }}$ cycle for ATE1D, ATE2D, ATE3D and ATE4D at the $\pm 2 \%$ strain range 


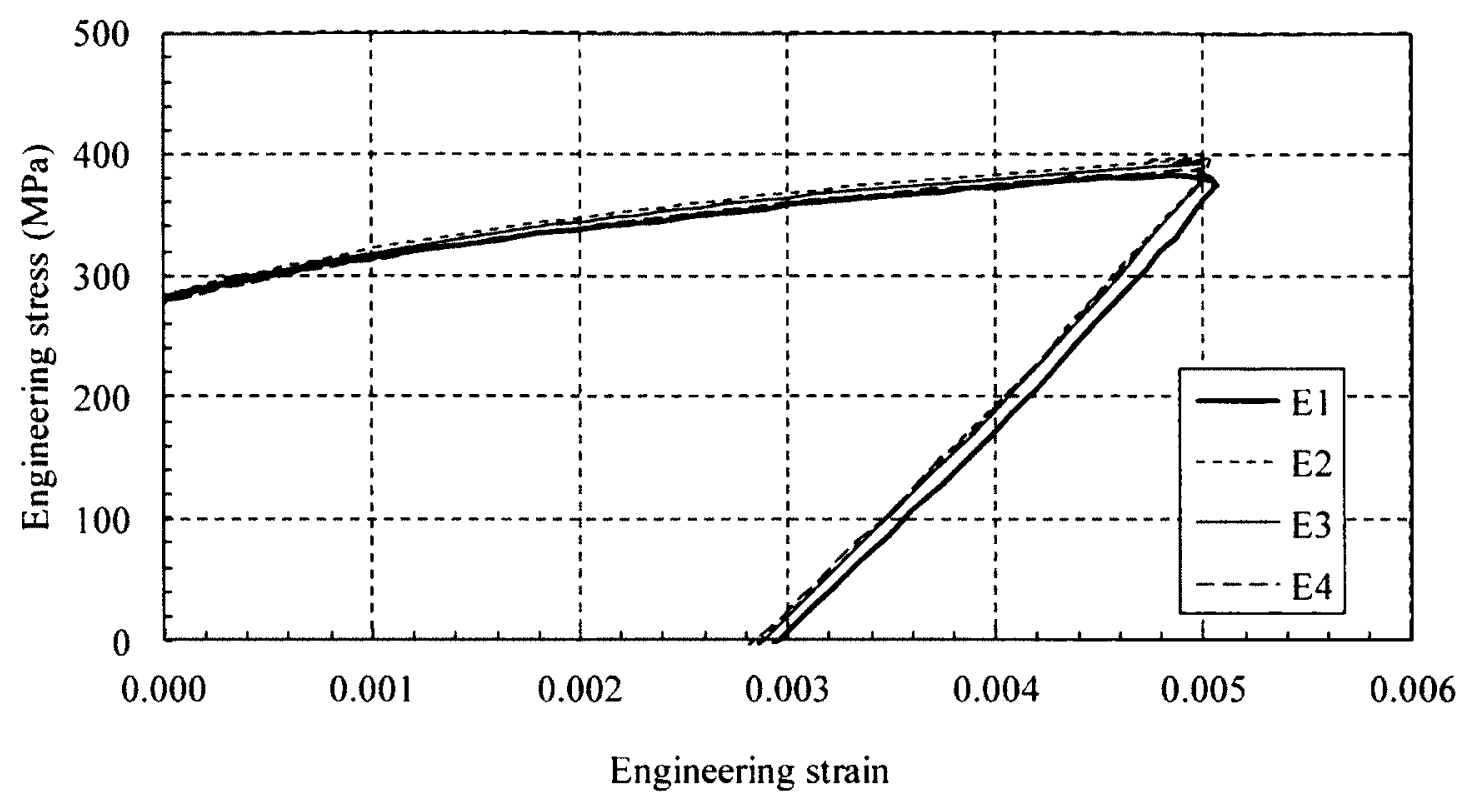

Figure 4.13 Part of the $10^{\text {th }}$ cycle for ATE1D, ATE2D, ATE3D and ATE4D at the $\pm 0.5 \%$ strain range

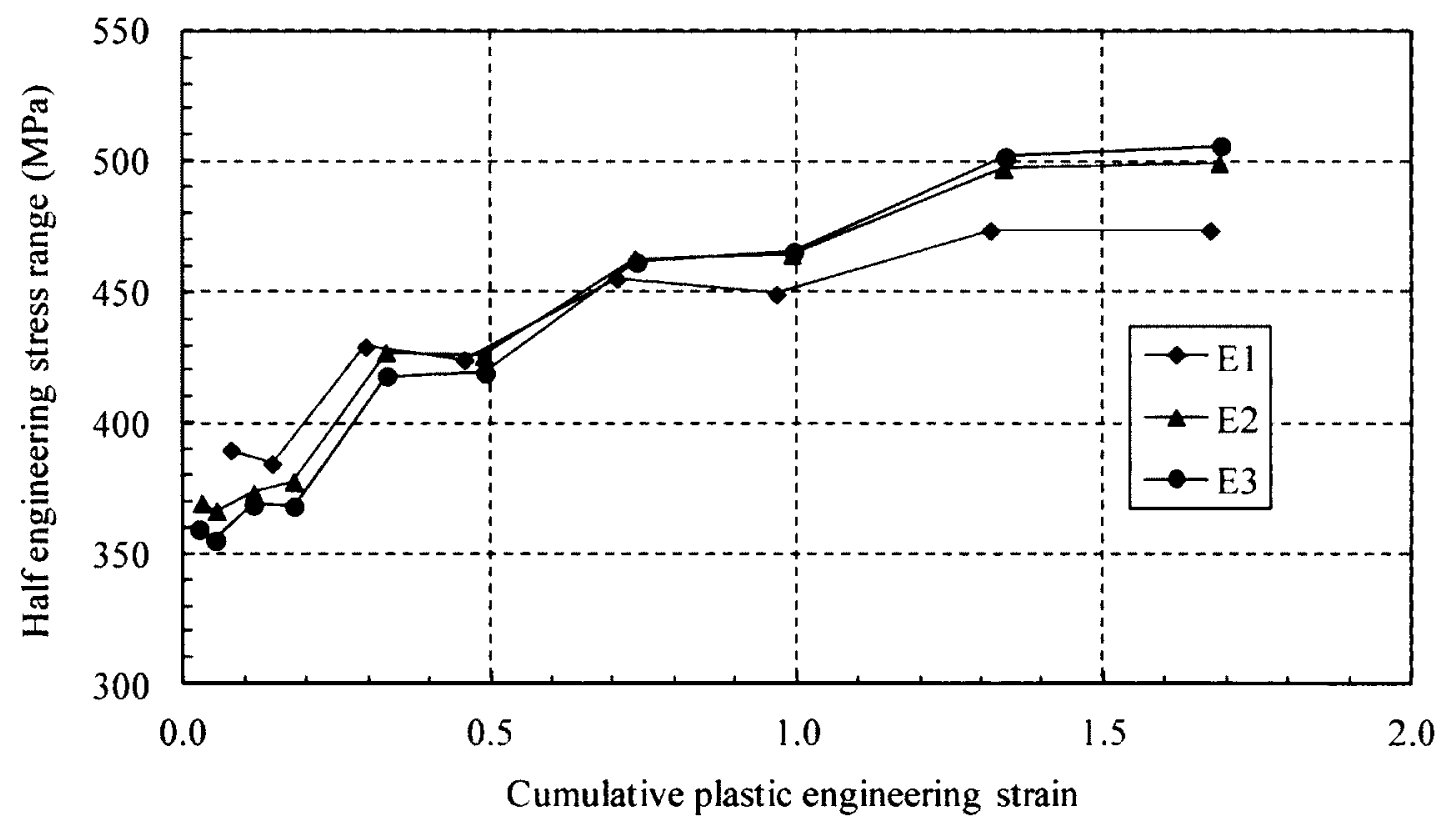

Figure 4.14 Half engineering stress range versus cumulative plastic engineering strain at the $5^{\text {th }}$ and $10^{\text {th }}$ cycle of each strain range for ATEIs 


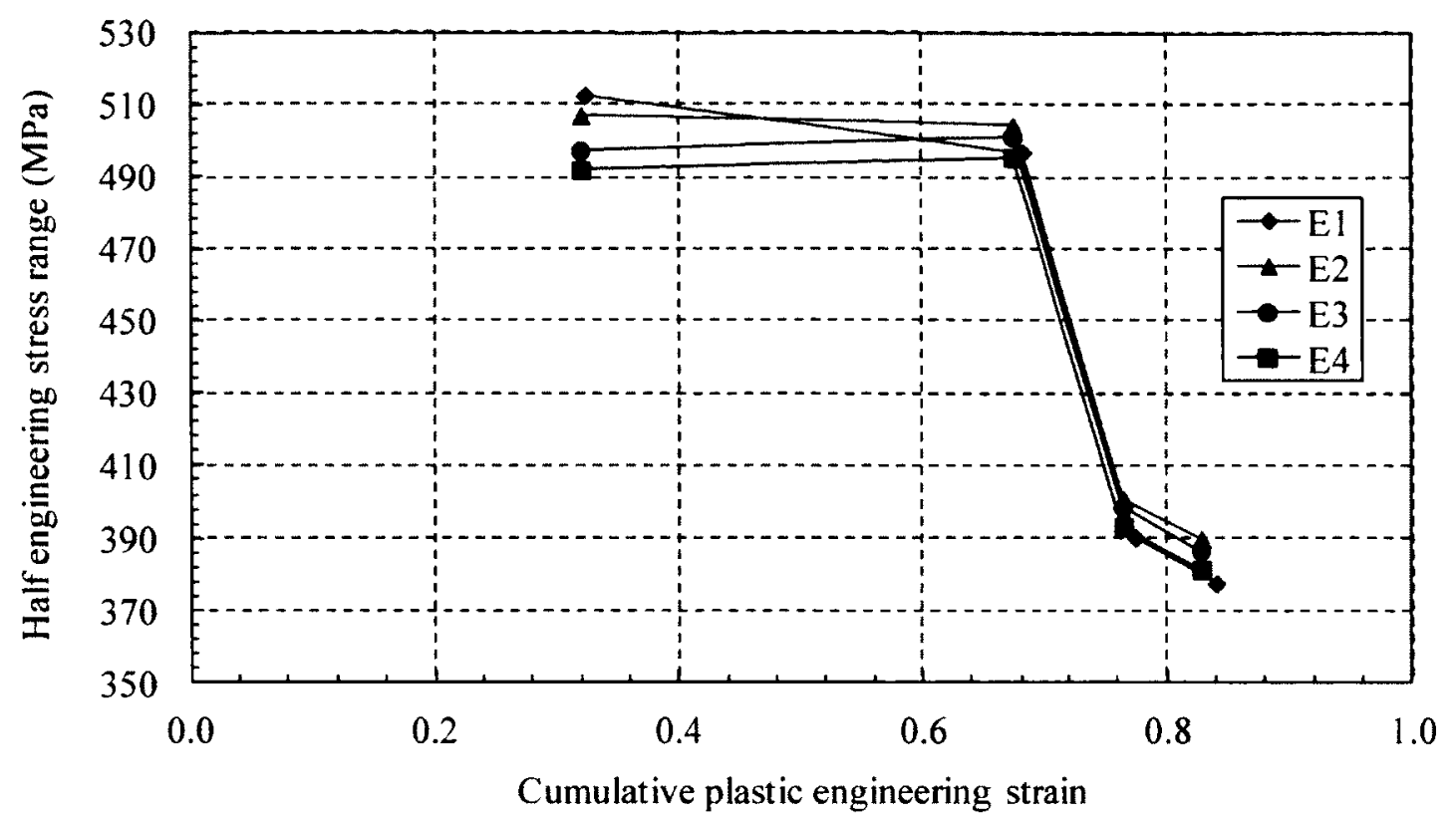

Figure 4.15 Half engineering stress range versus cumulative plastic engineering strain at the $5^{\text {th }}$ and $10^{\text {th }}$ cycle of each strain range for ATEDs

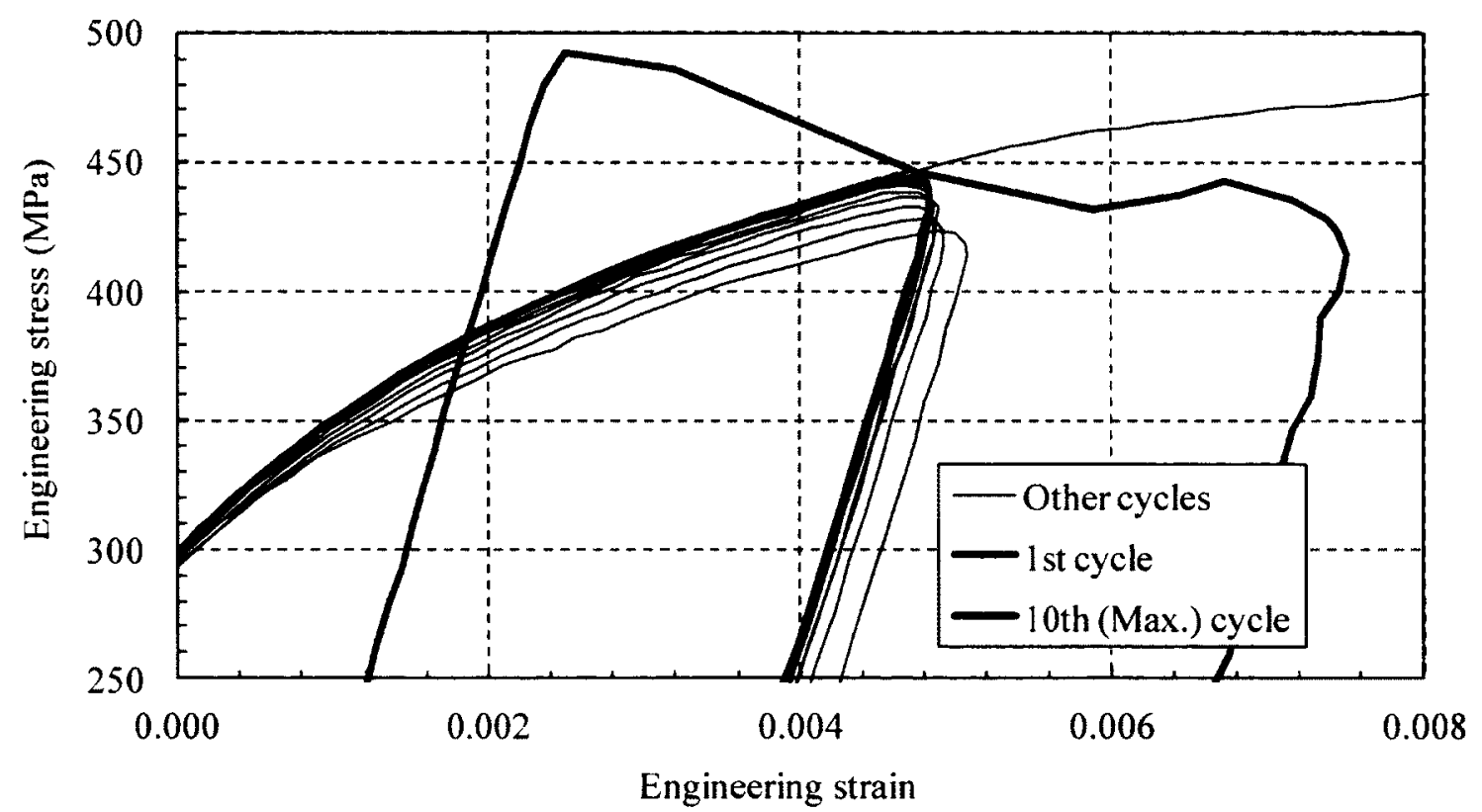

Figure 4.16 Magnified view of the engineering stress versus engineering strain curves for BTE1I at the $\pm 0.5 \%$ strain range 


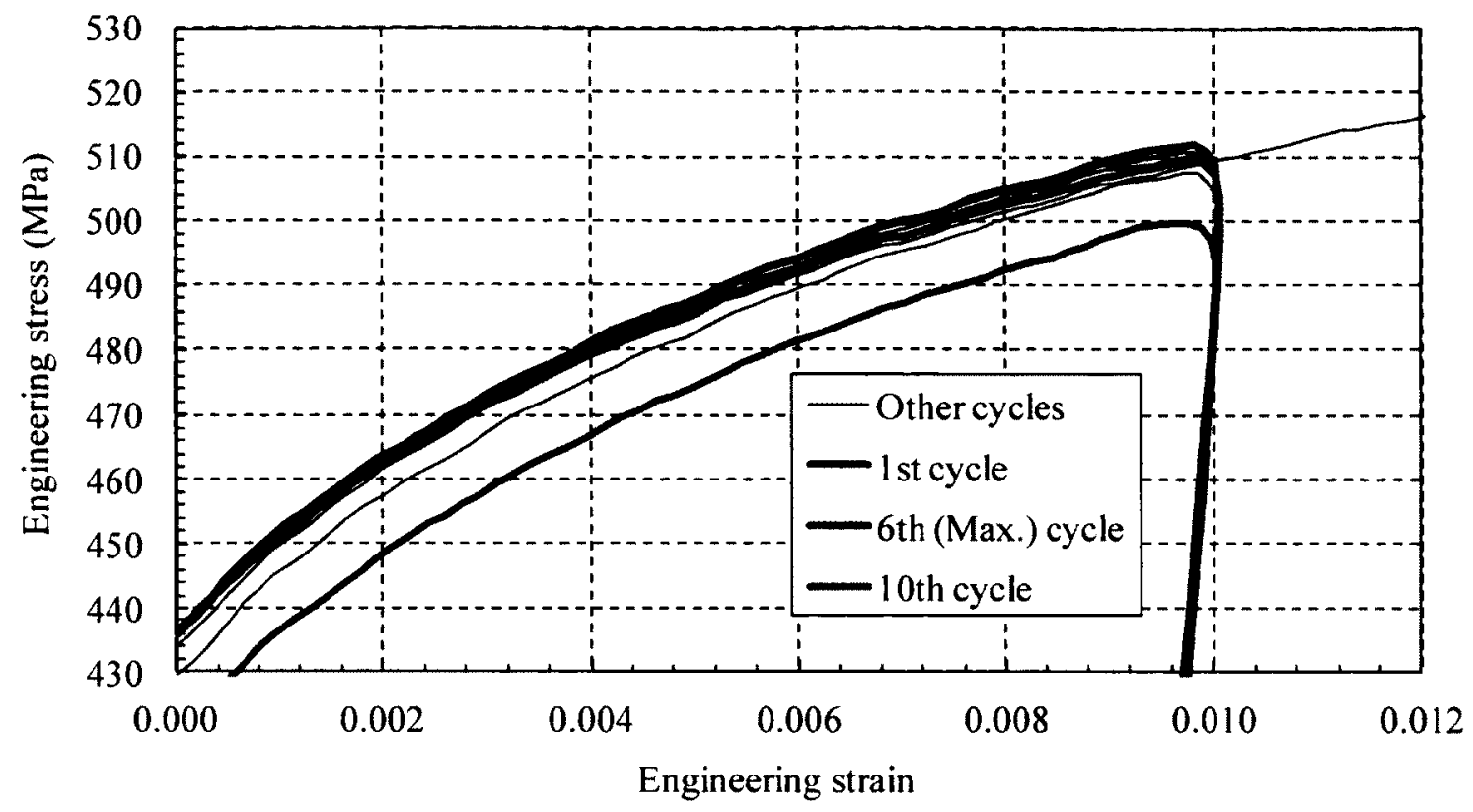

Figure 4.17 Magnified view of the engineering stress versus engineering strain curves for $\mathrm{BTE} 1 \mathrm{I}$ at the $\pm 1 \%$ strain range

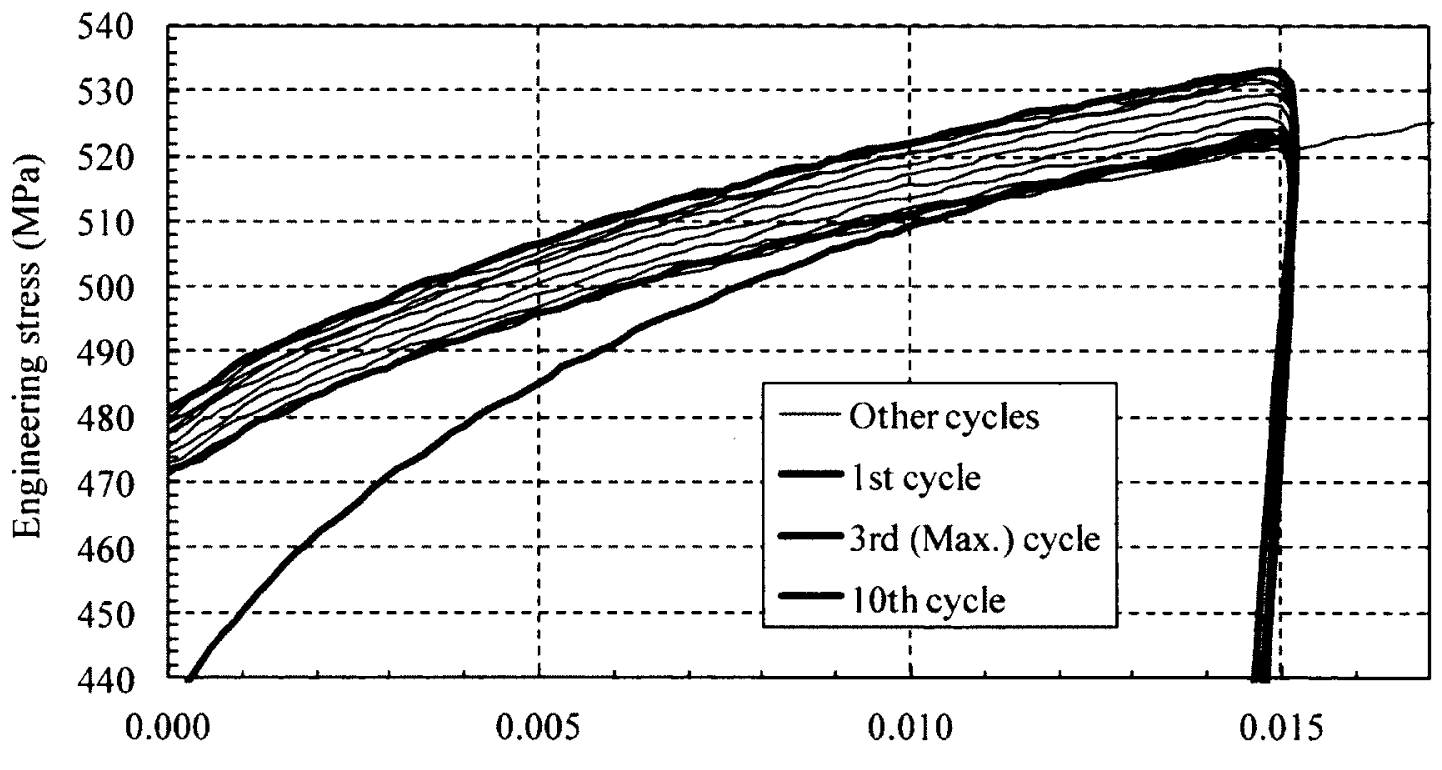

Engineering strain

Figure 4.18 Magnified view of the engineering stress versus engineering strain curves for BTE II at the $\pm 1.5 \%$ strain range 


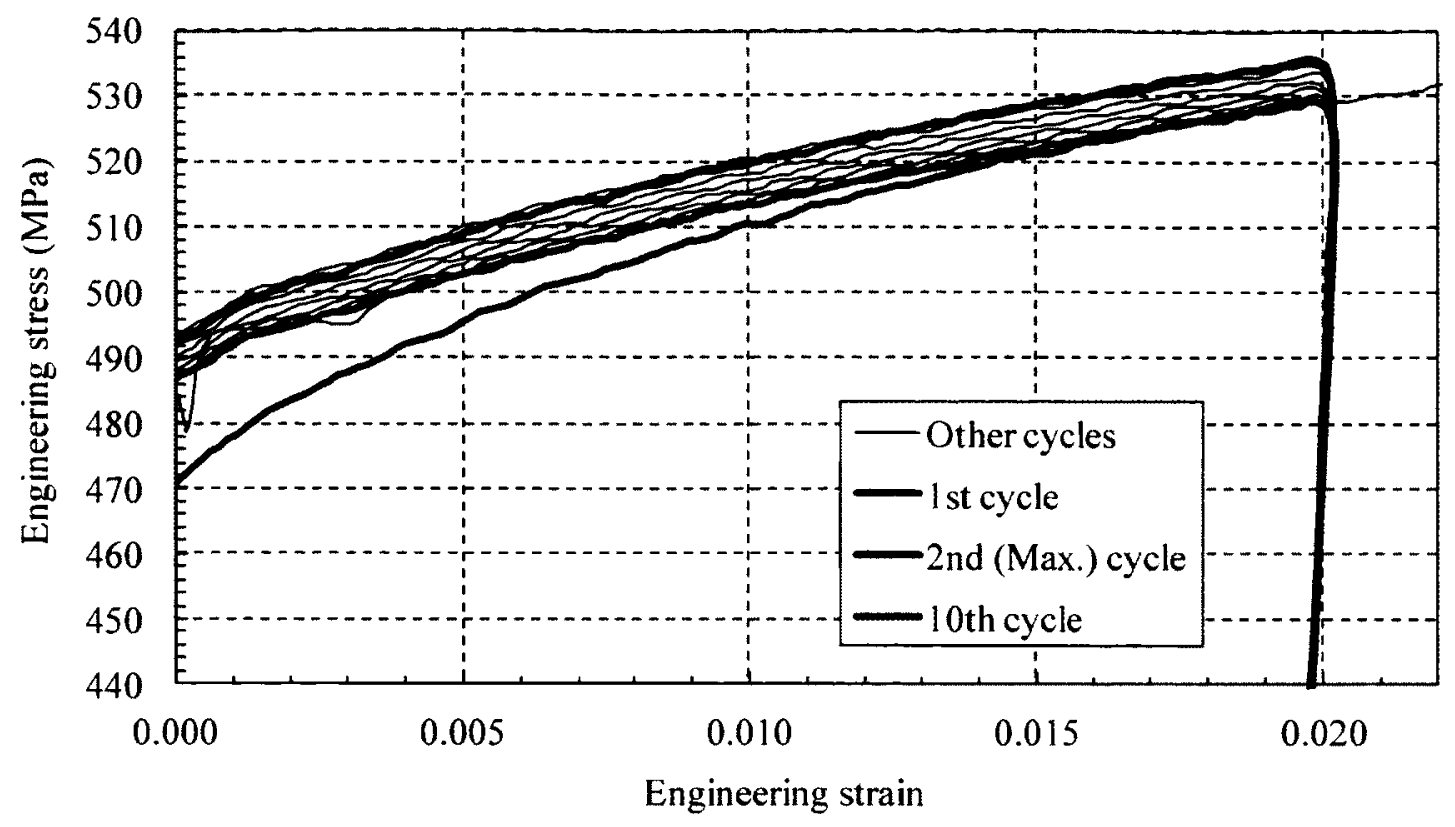

Figure 4.19 Magnified view of the engineering stress versus engineering strain curves for BTE1I at the $\pm 2 \%$ strain range

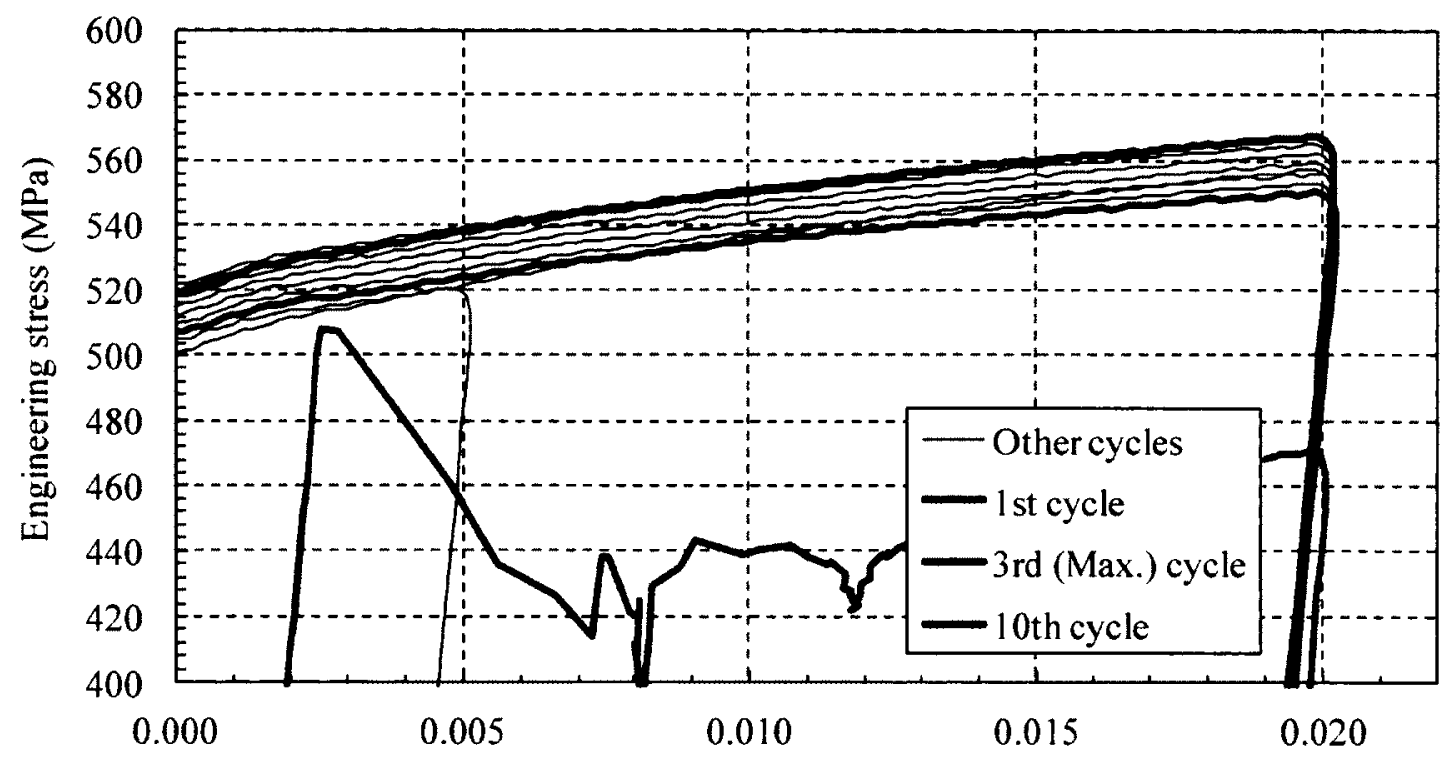

Engineering strain

Figure 4.20 Magnified view of the engineering stress versus engineering strain curves for $\mathrm{BTE} 1 \mathrm{D}$ at the $\pm 2 \%$ strain range 


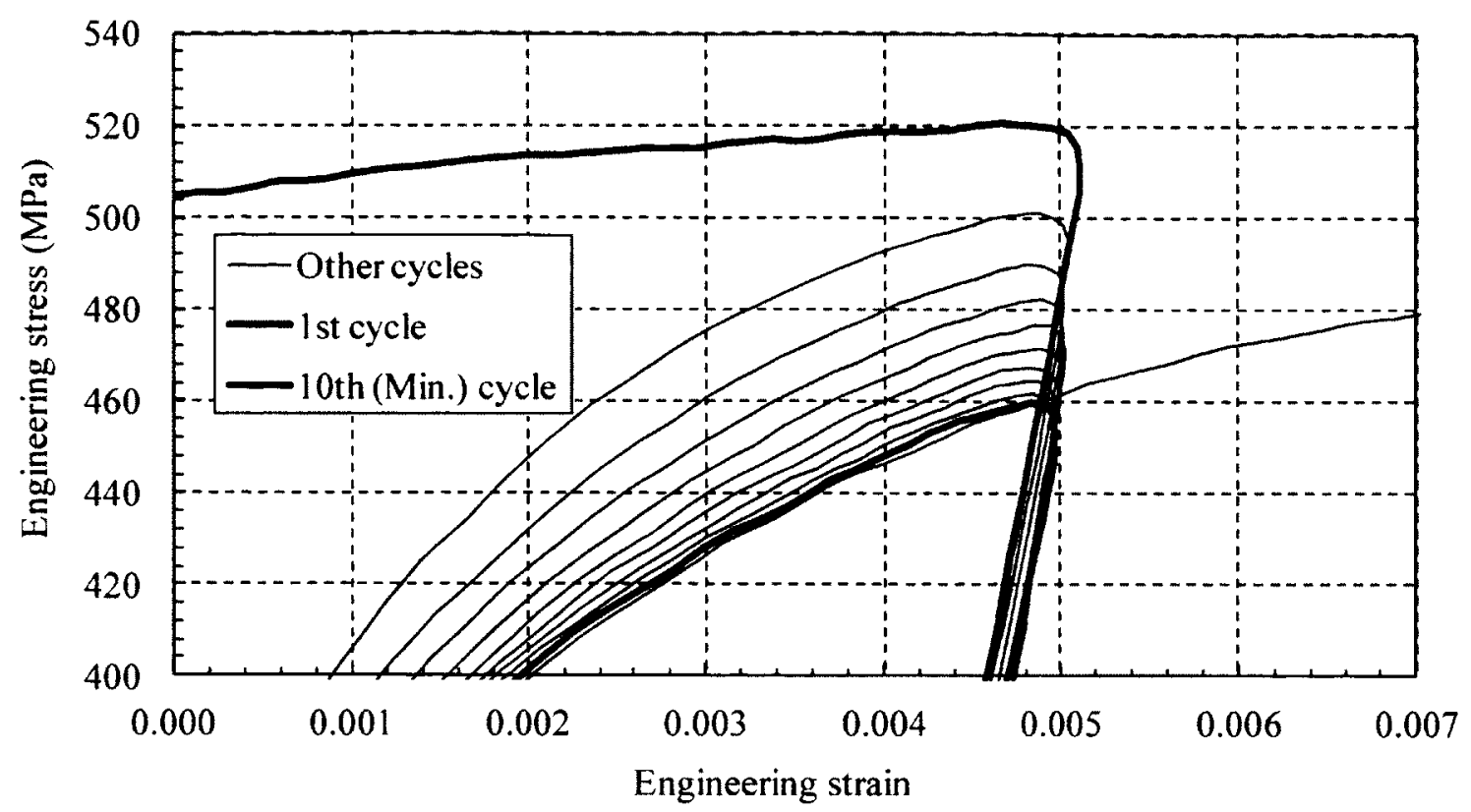

Figure 4.21 Magnified view of the engineering stress versus engineering strain curves for BTE1D at the $\pm 0.5 \%$ strain range

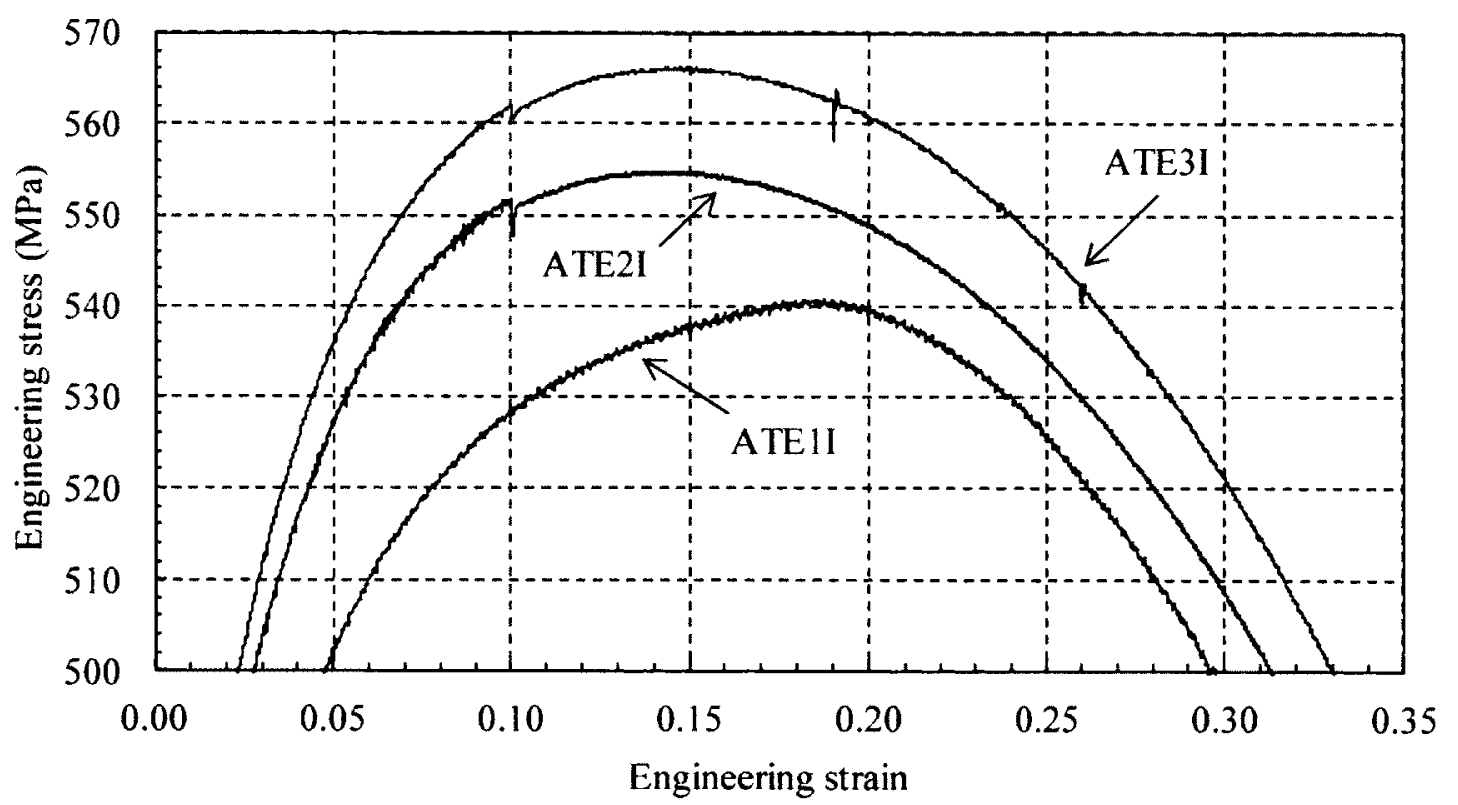

Figure 4.22 Magnified view of the engineering stress versus engineering strain curves close to the peak stress for I series tests of material A when being pulled to fracture 


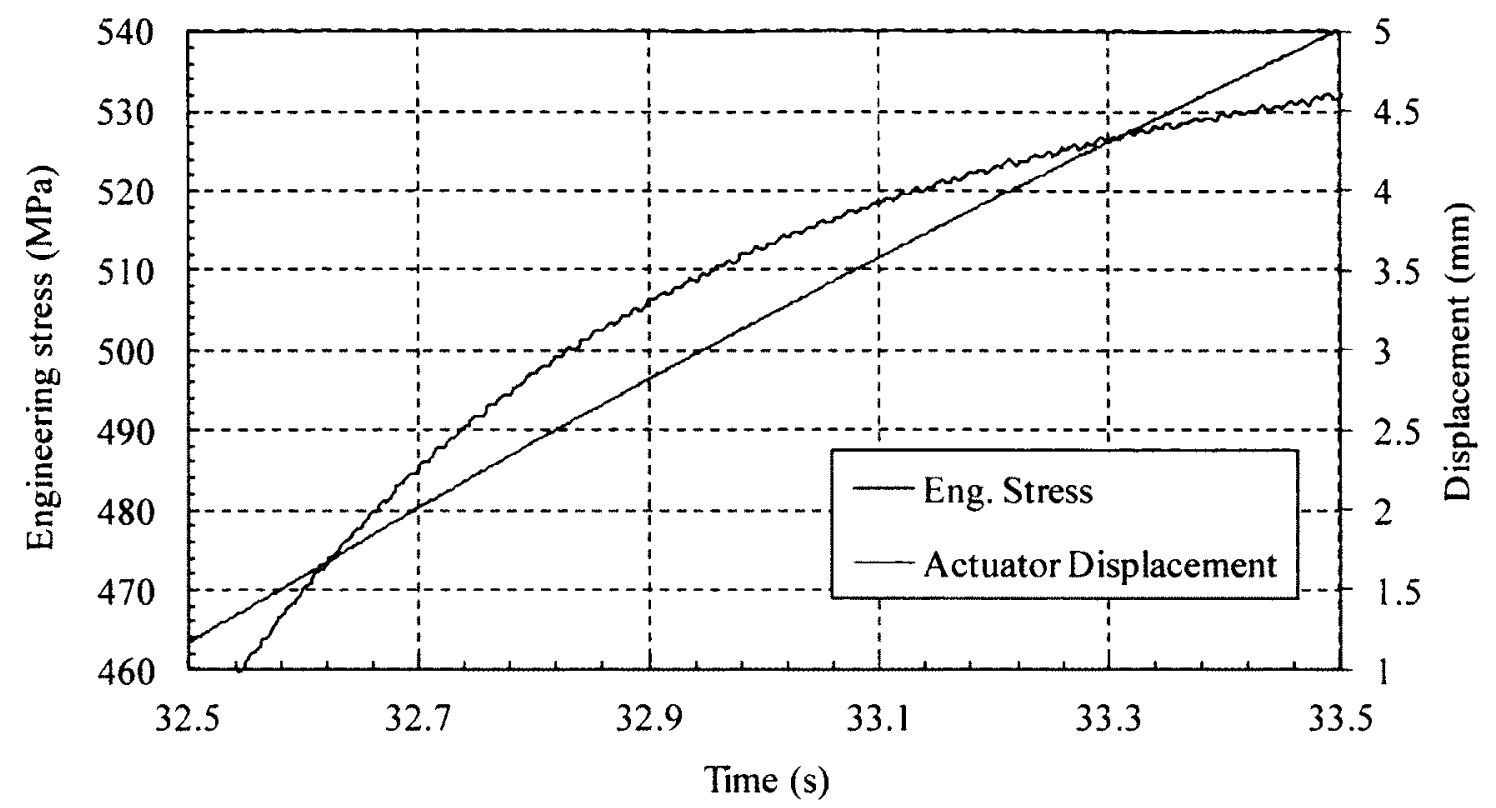

Figure 4.23 Magnified view of the engineering stress and actuator displacement versus time approximately between the strain of 0.0 and 0.1 when being pulled to fracture for ATE1I

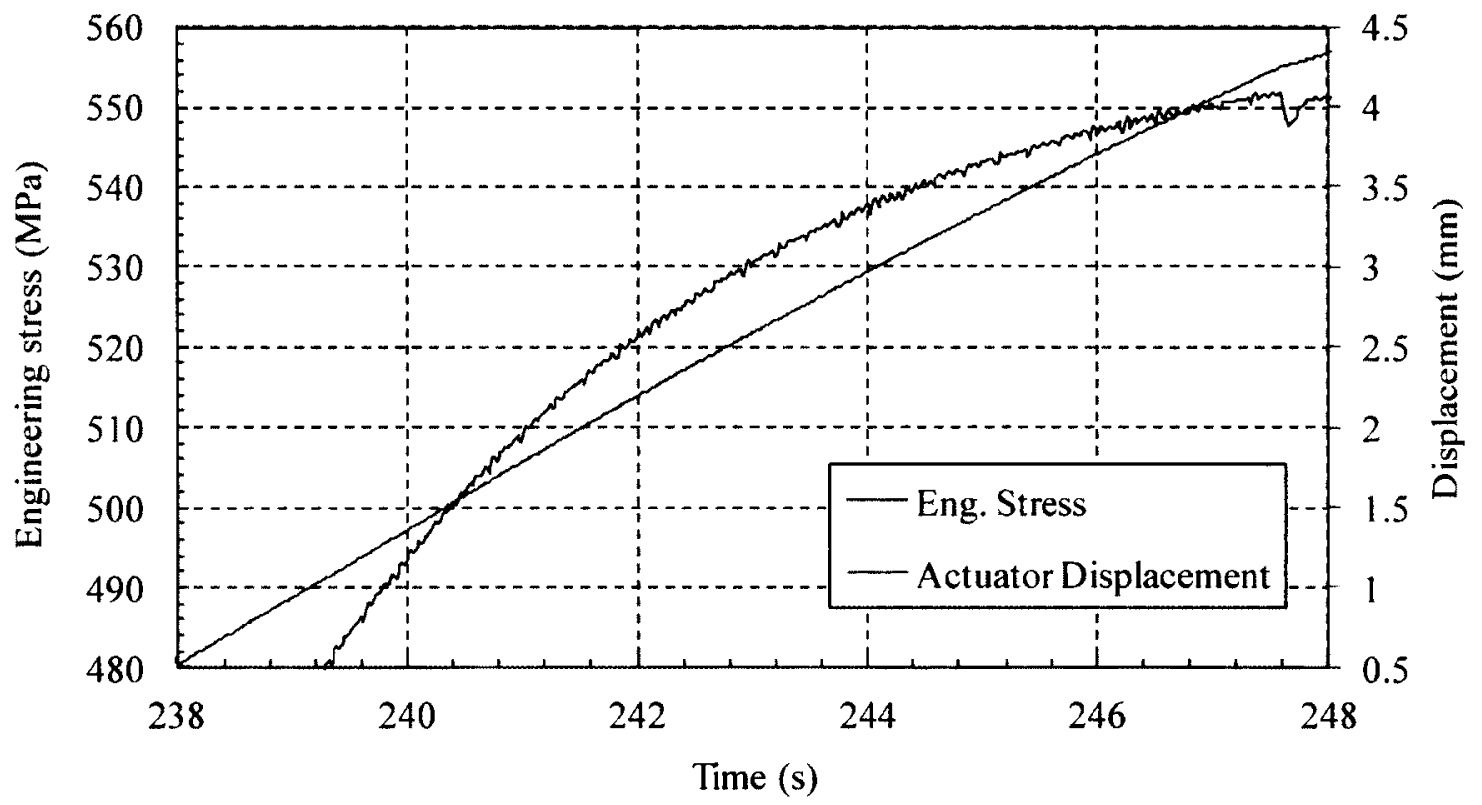

Figure 4.24 Magnified view of the engineering stress and actuator displacement versus time approximately between the strain of 0.0 and 0.1 when being pulled to fracture for ATE2I 


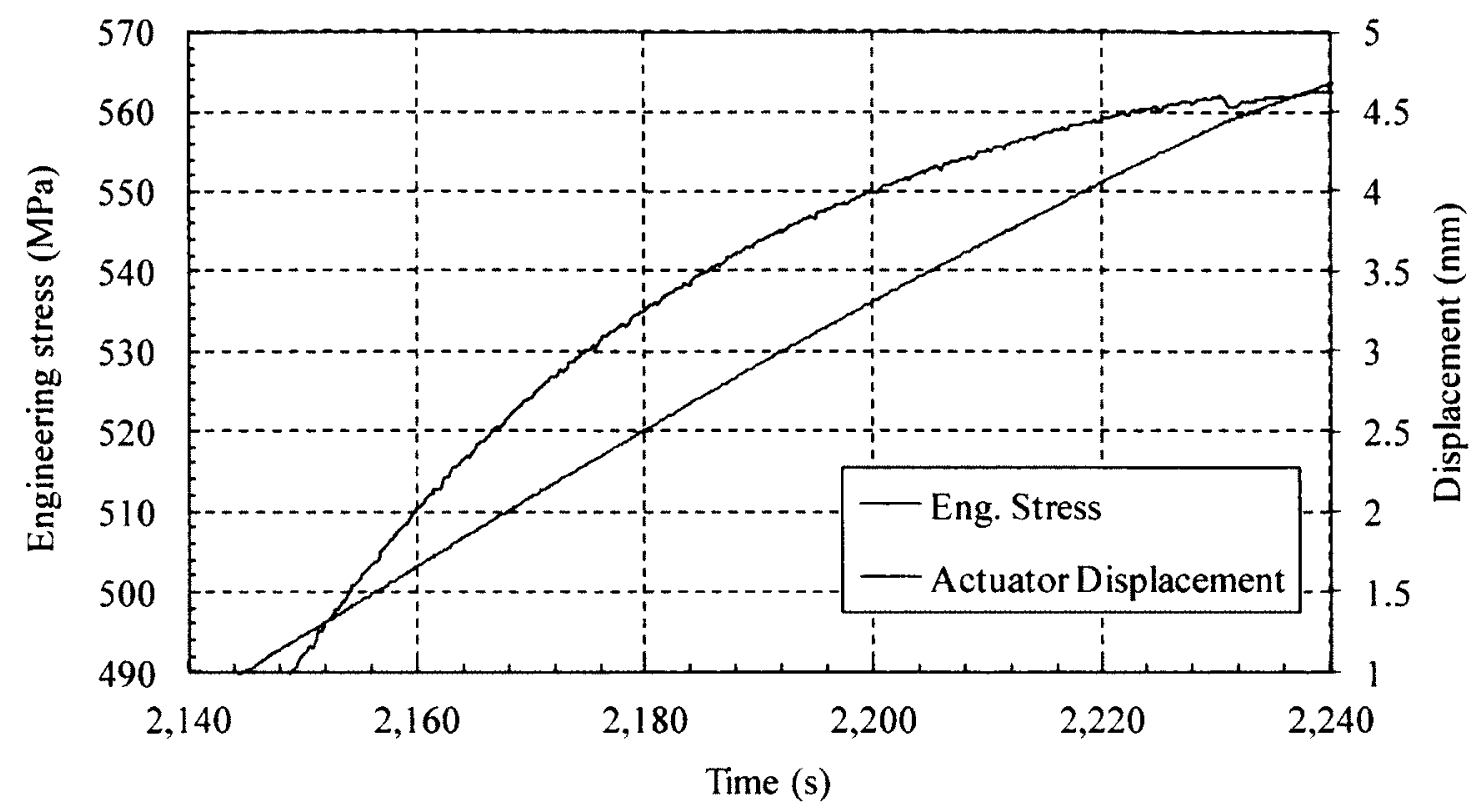

Figure 4.25 Magnified view of the engineering stress and actuator displacement versus time approximately between the strain of 0.0 and 0.1 when being pulled to fracture for ATE3I

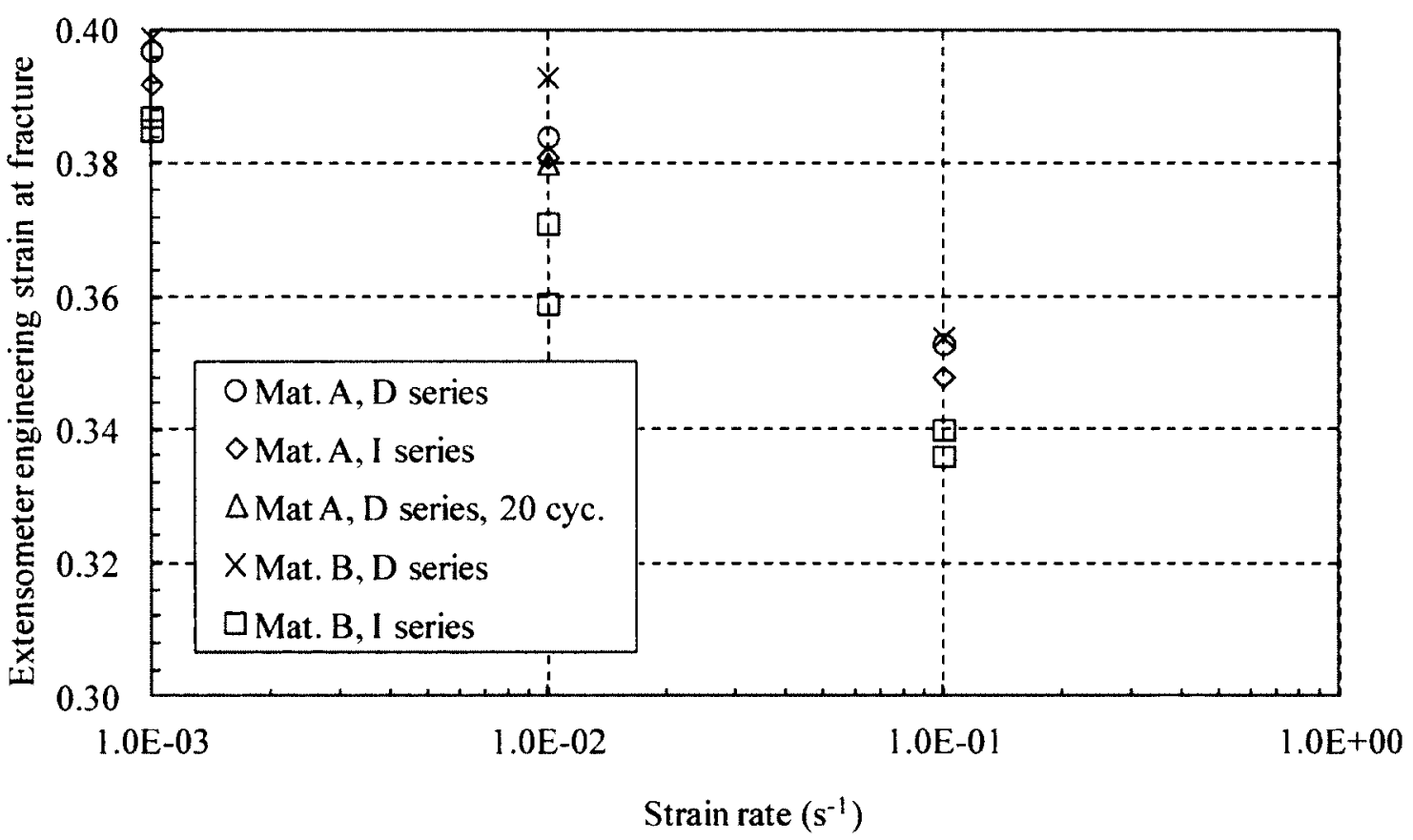

Figure 4.26 Engineering strain at fracture versus strain rate 


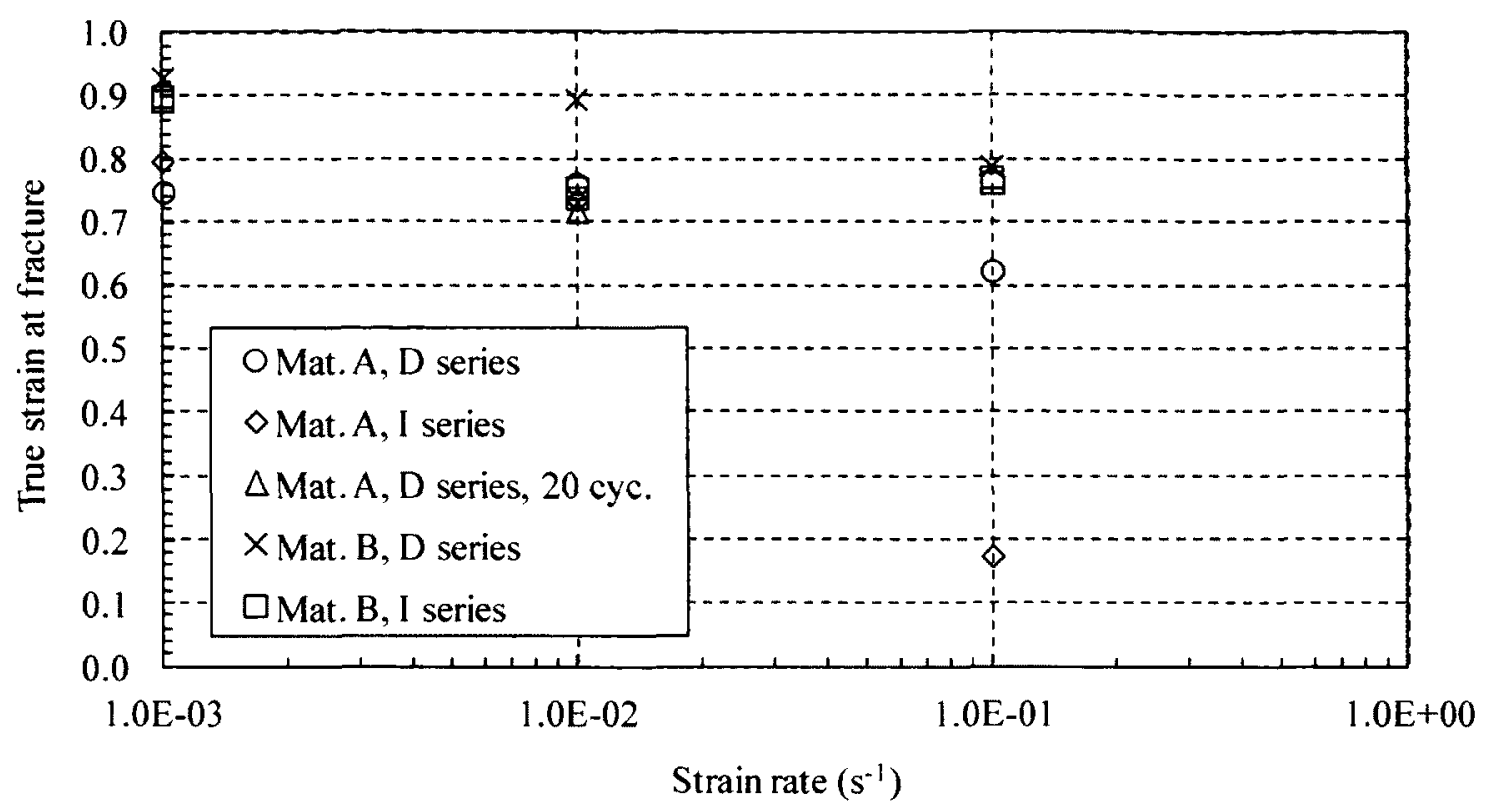

Figure 4.27 True strain at fracture versus strain rate

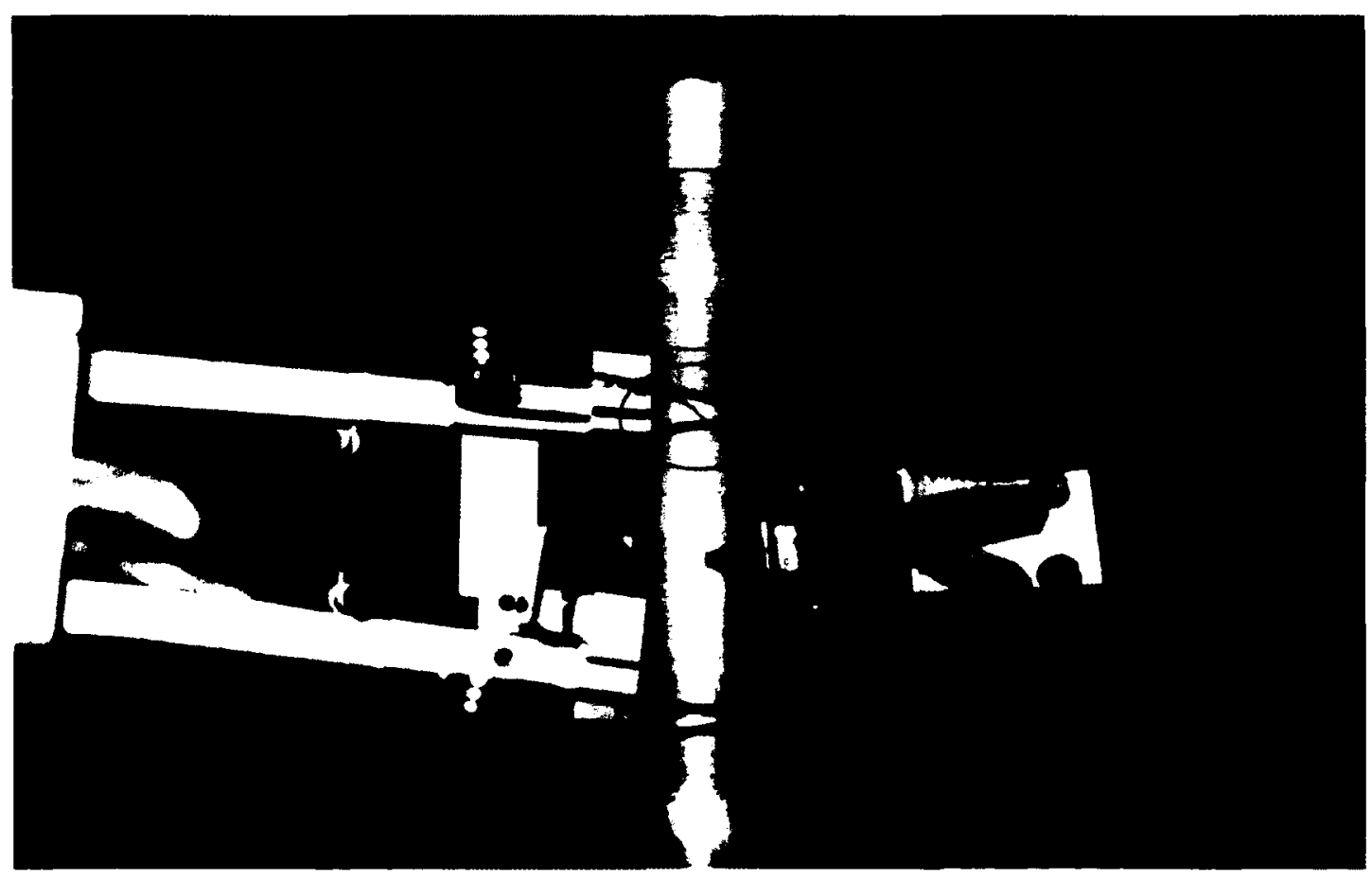

Figure 4.28 Fracture location of the ATE1I specimen 


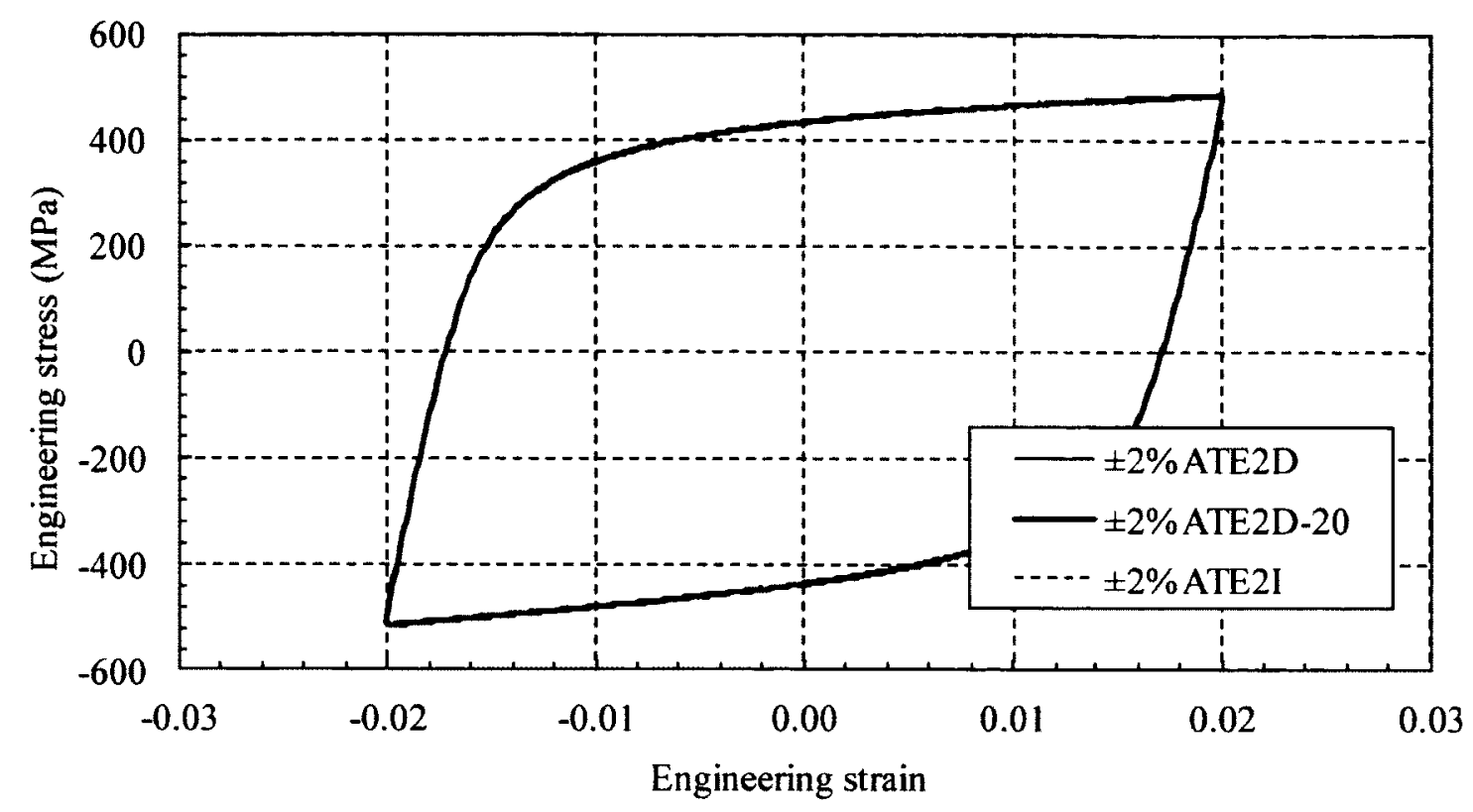

Figure 4.29 The 10th cycle of ATE2D and ATE2I, and the 20th cycle of ATE2D-20 at the $\pm 2 \%$ strain range

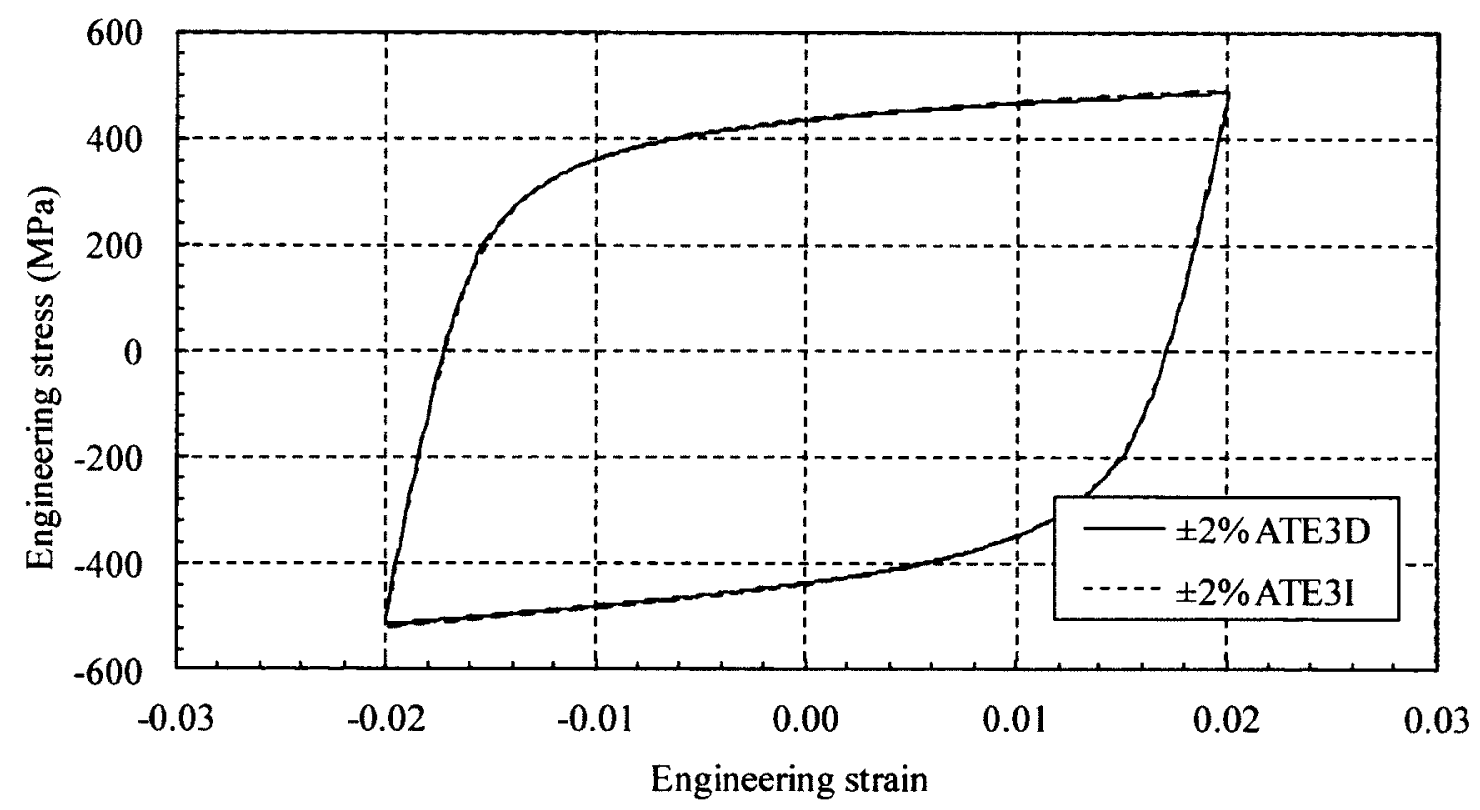

Figure 4.30 The 10th cycle of ATE3D and ATE3I at the $\pm 2 \%$ strain range 


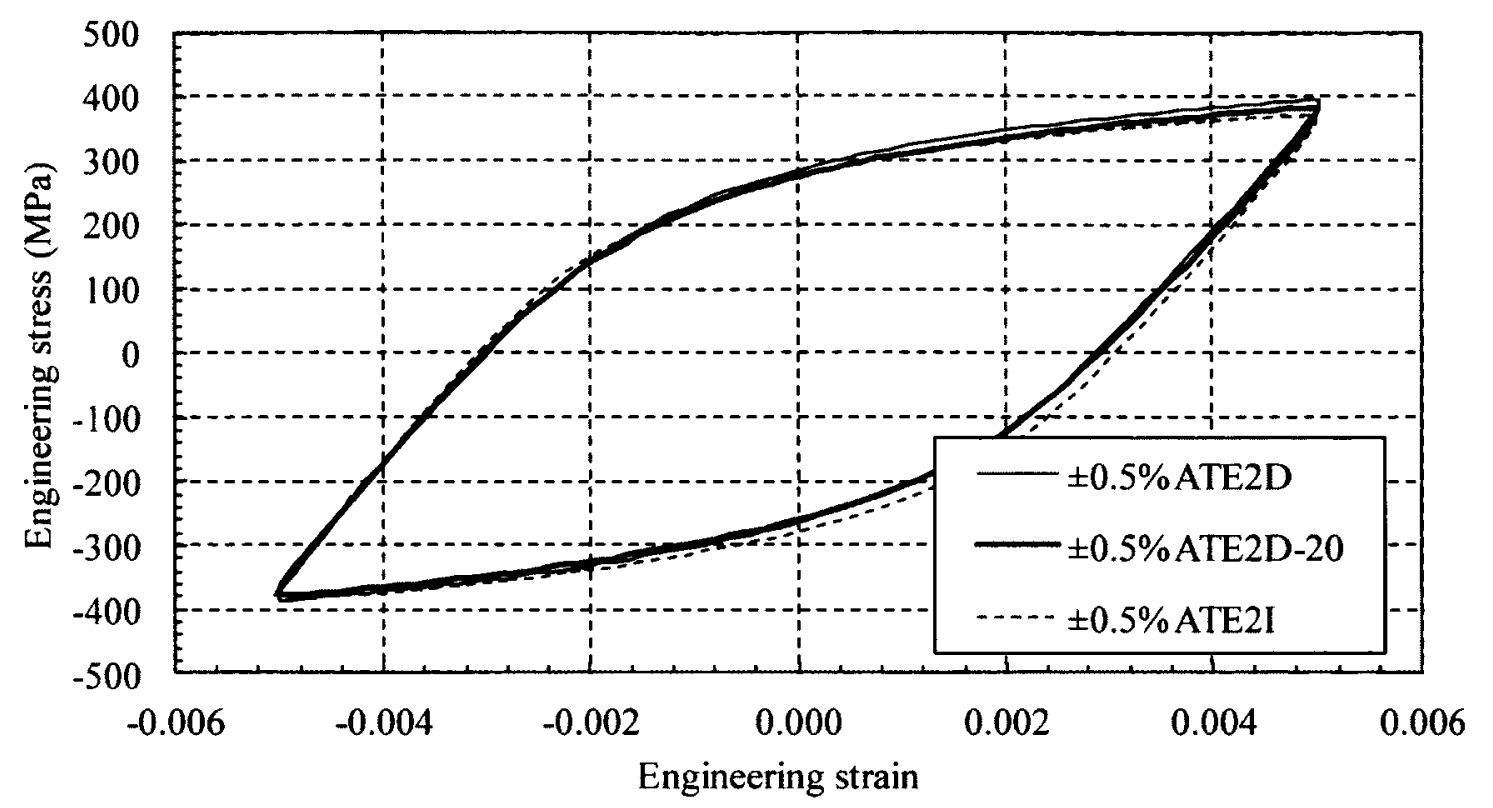

Figure 4.31 The 10th cycle of ATE2D and ATE2I, and the 20th cycle of ATE2D-20 at the $\pm 0.5 \%$ strain range

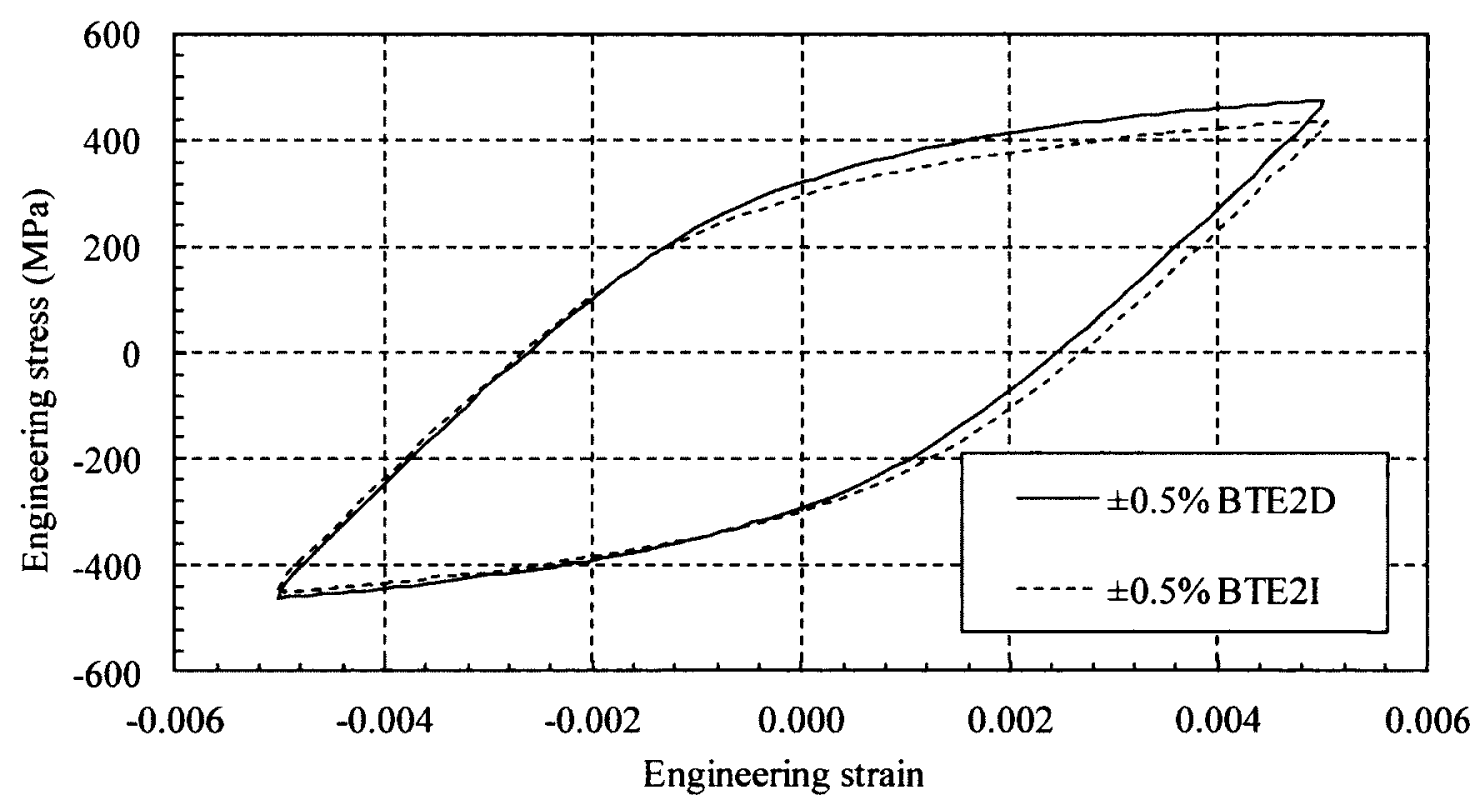

Figure 4.32 The 10th cycle of BTE2D and BTE2I at the $\pm 0.5 \%$ strain range 


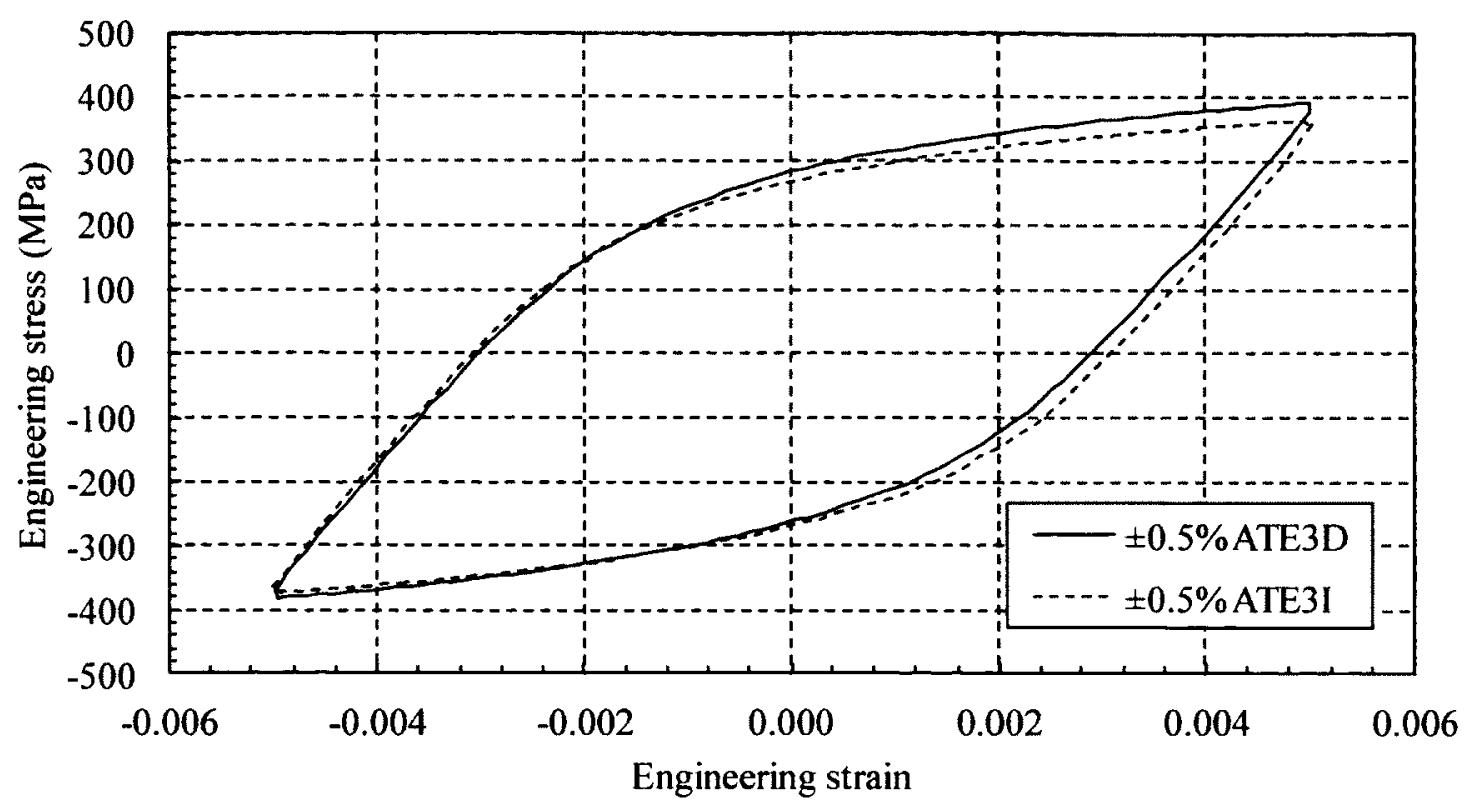

Figure 4.33 The 10 th cycle of ATE3D and ATE3I at the $\pm 0.5 \%$ strain range

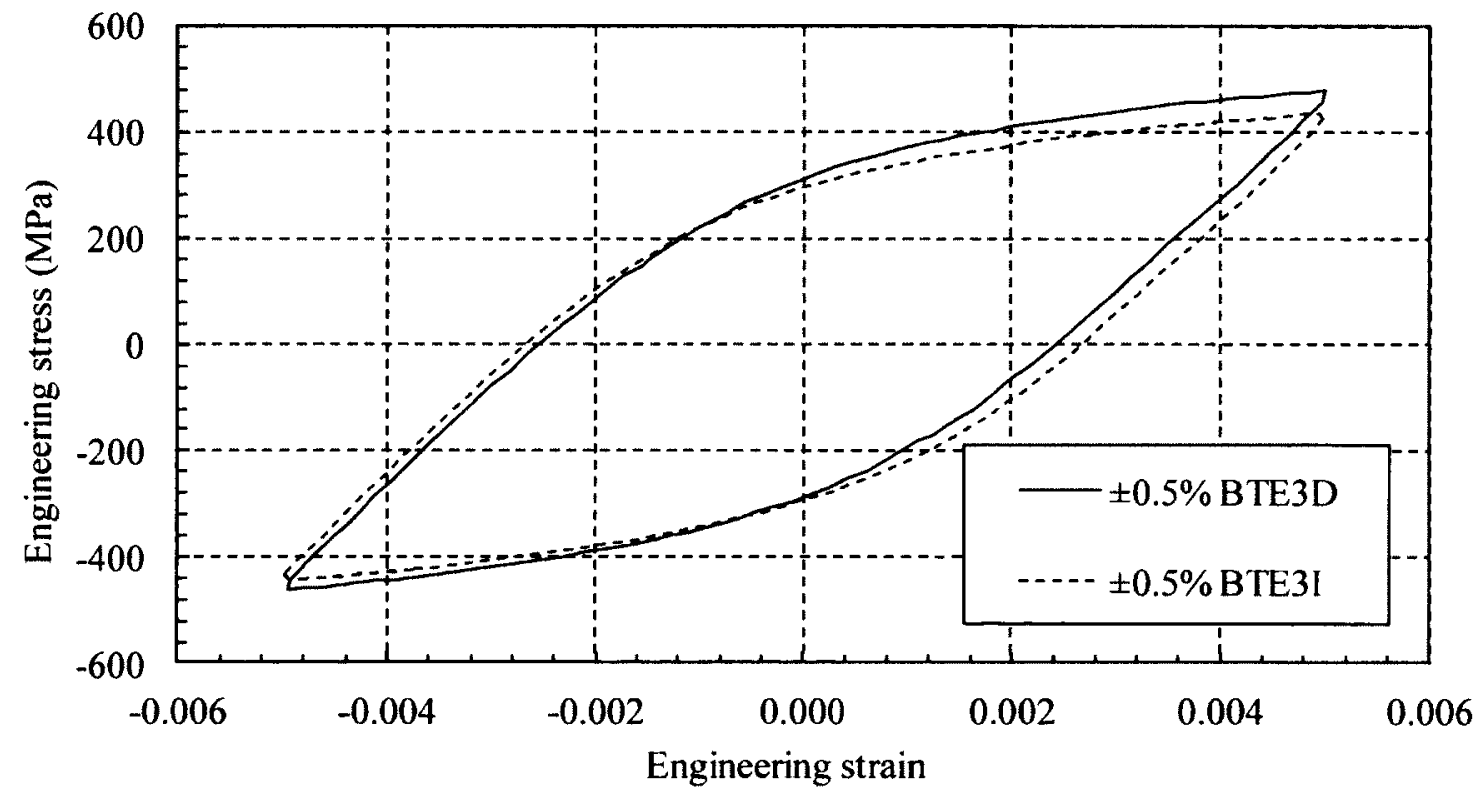

Figure 4.34 The 10th cycle of BTE3D and BTE3I at the $\pm 0.5 \%$ strain range 


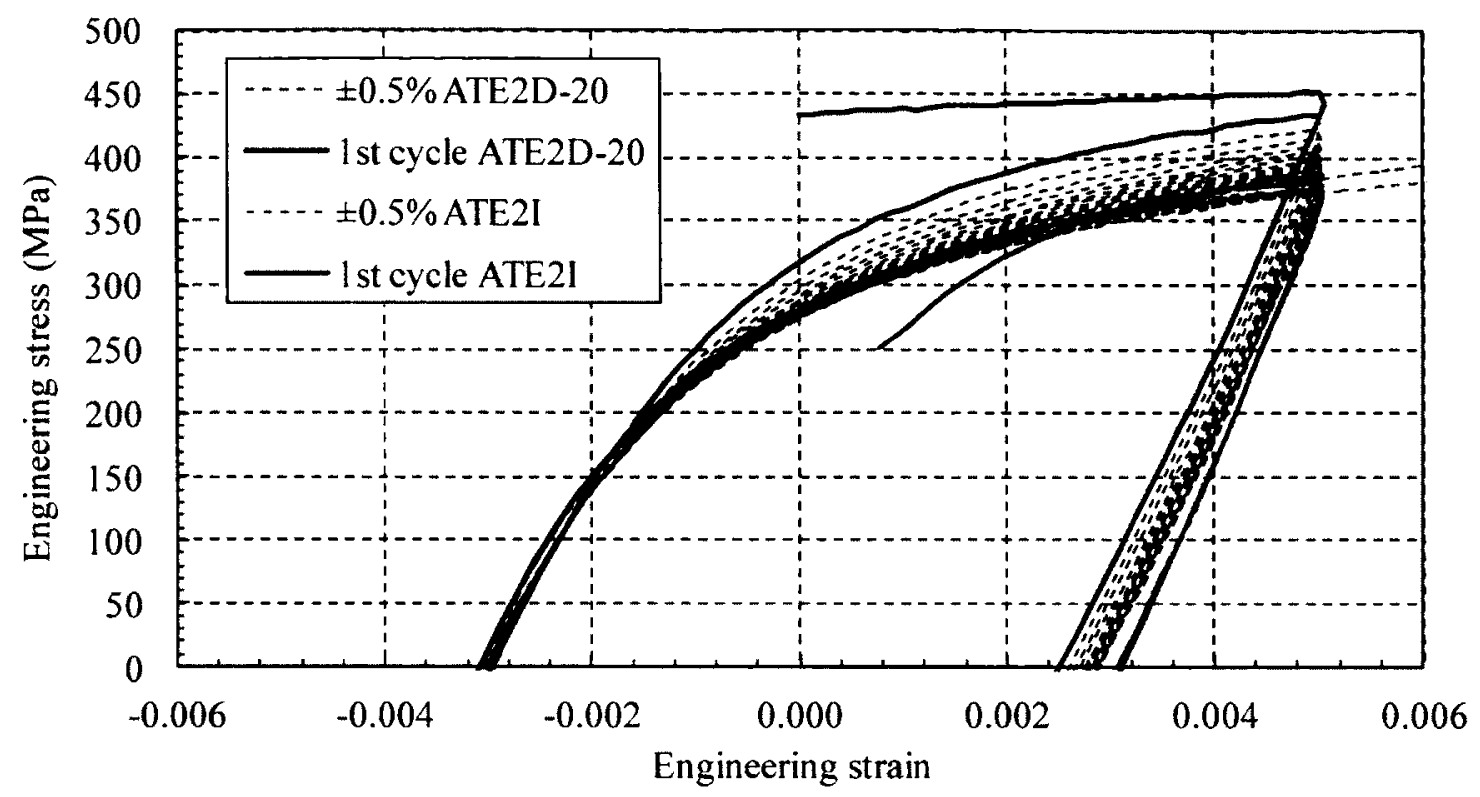

Figure 4.35 Tensile behaviour of ATE2D-20 and ATE2I at the strain range of $\pm 0.5 \%$

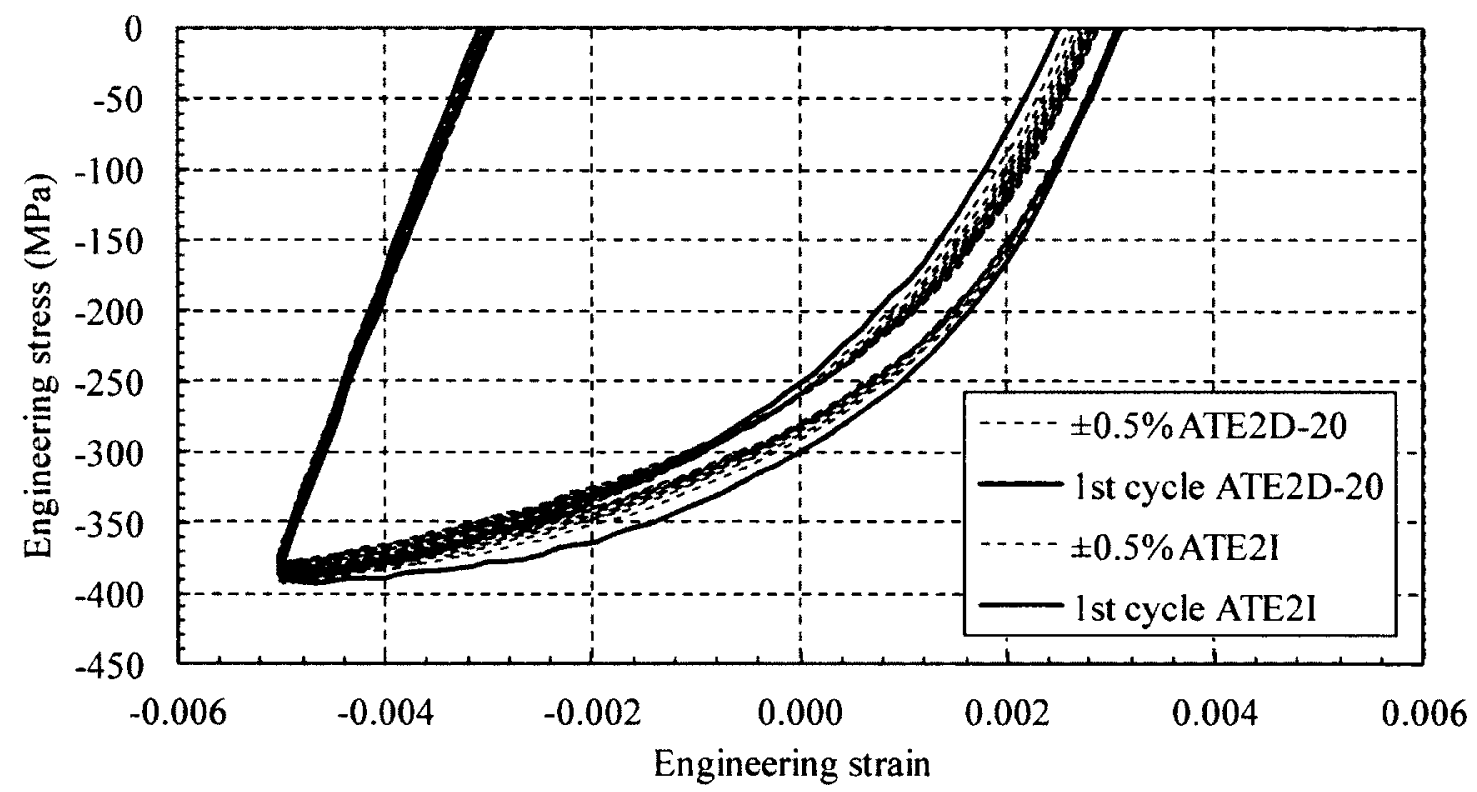

Figure 4.36 Compressive behaviour of ATE2D-20 and ATE2I at the strain range of $\pm 0.5 \%$ 


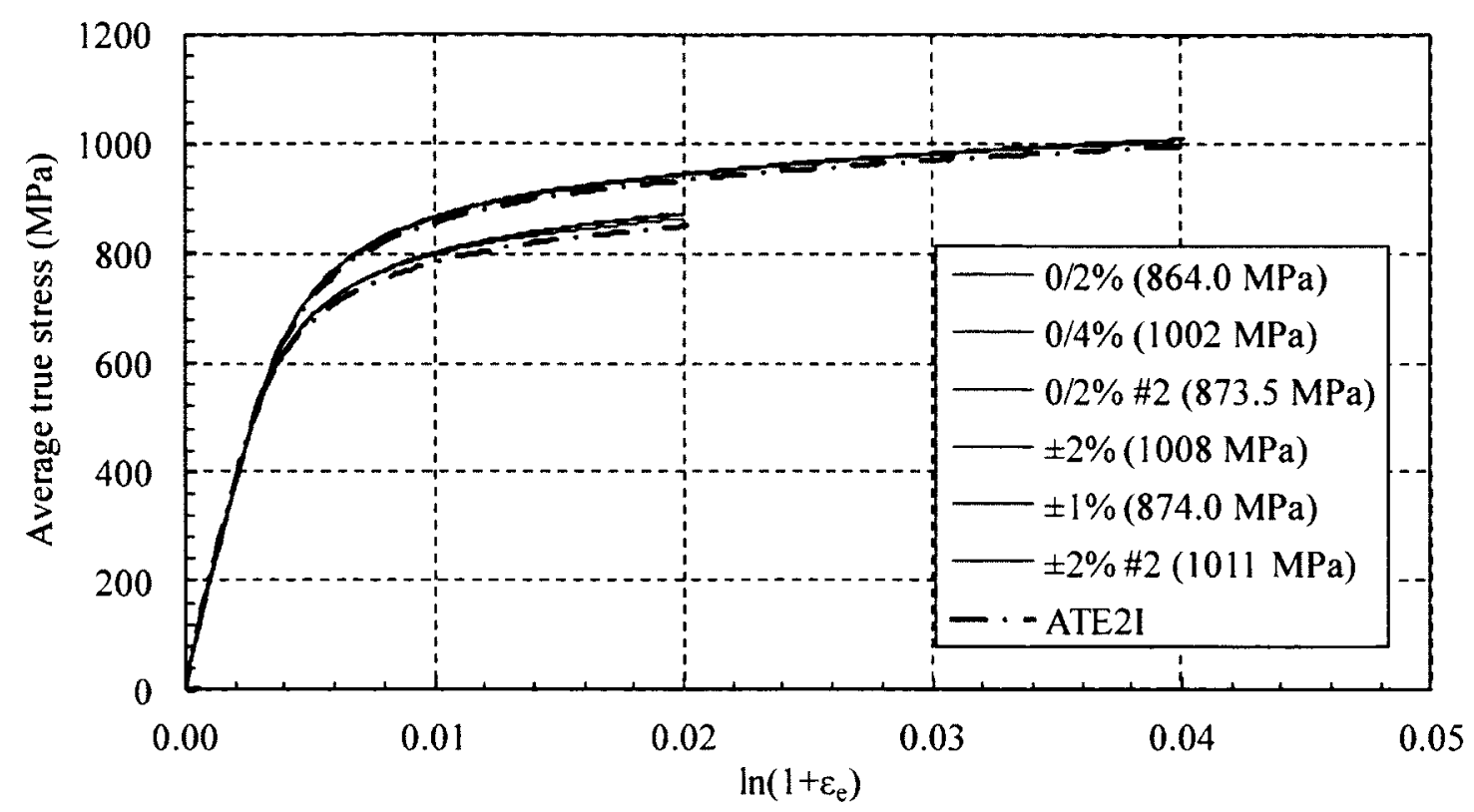

Figure 4.37 Shifted final half-cycle at each strain range for ATE2C and ATE2I with the peak shifted stresses

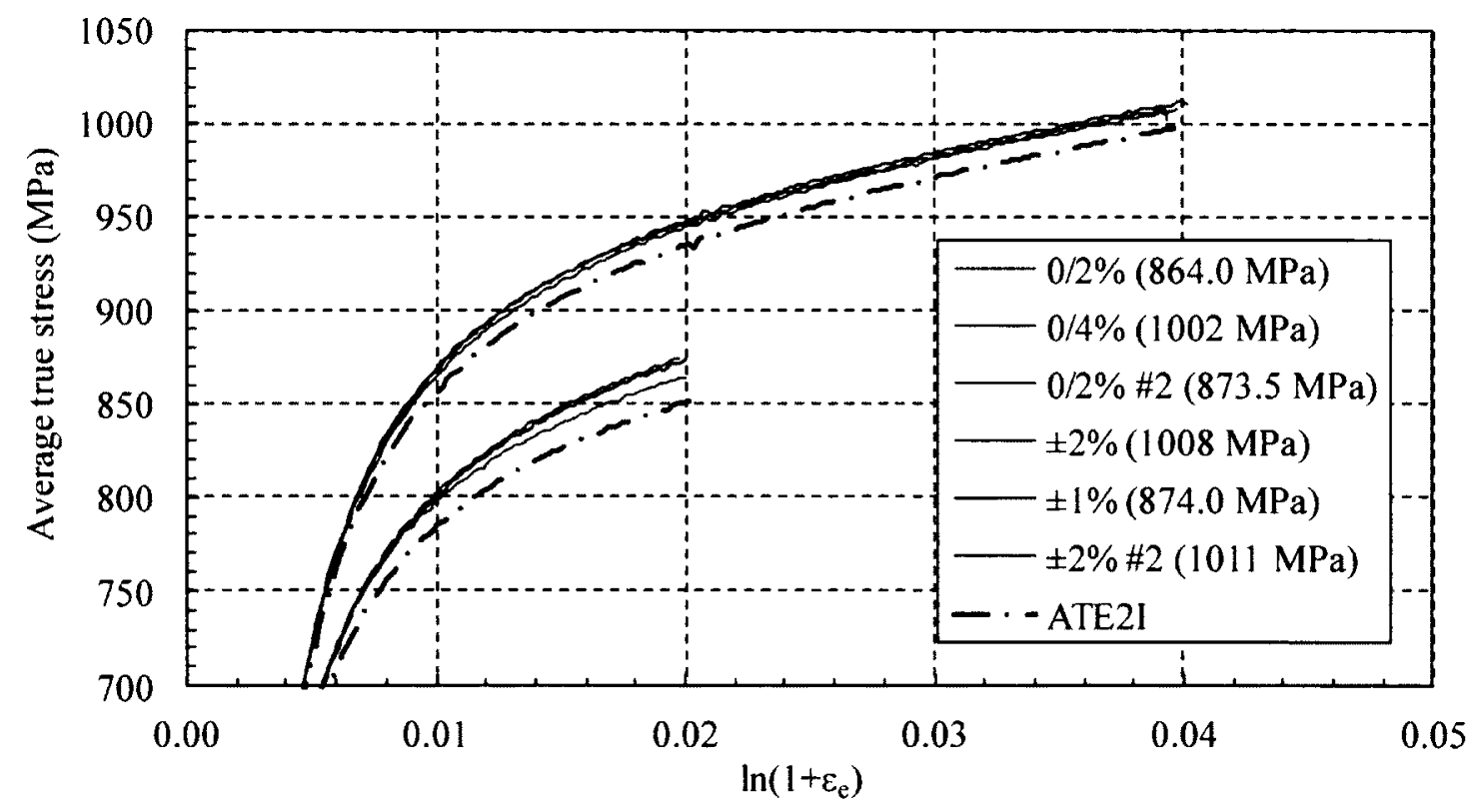

Figure 4.38 Enhanced view of Fig. 4.37 for ATE2C and ATE2I 


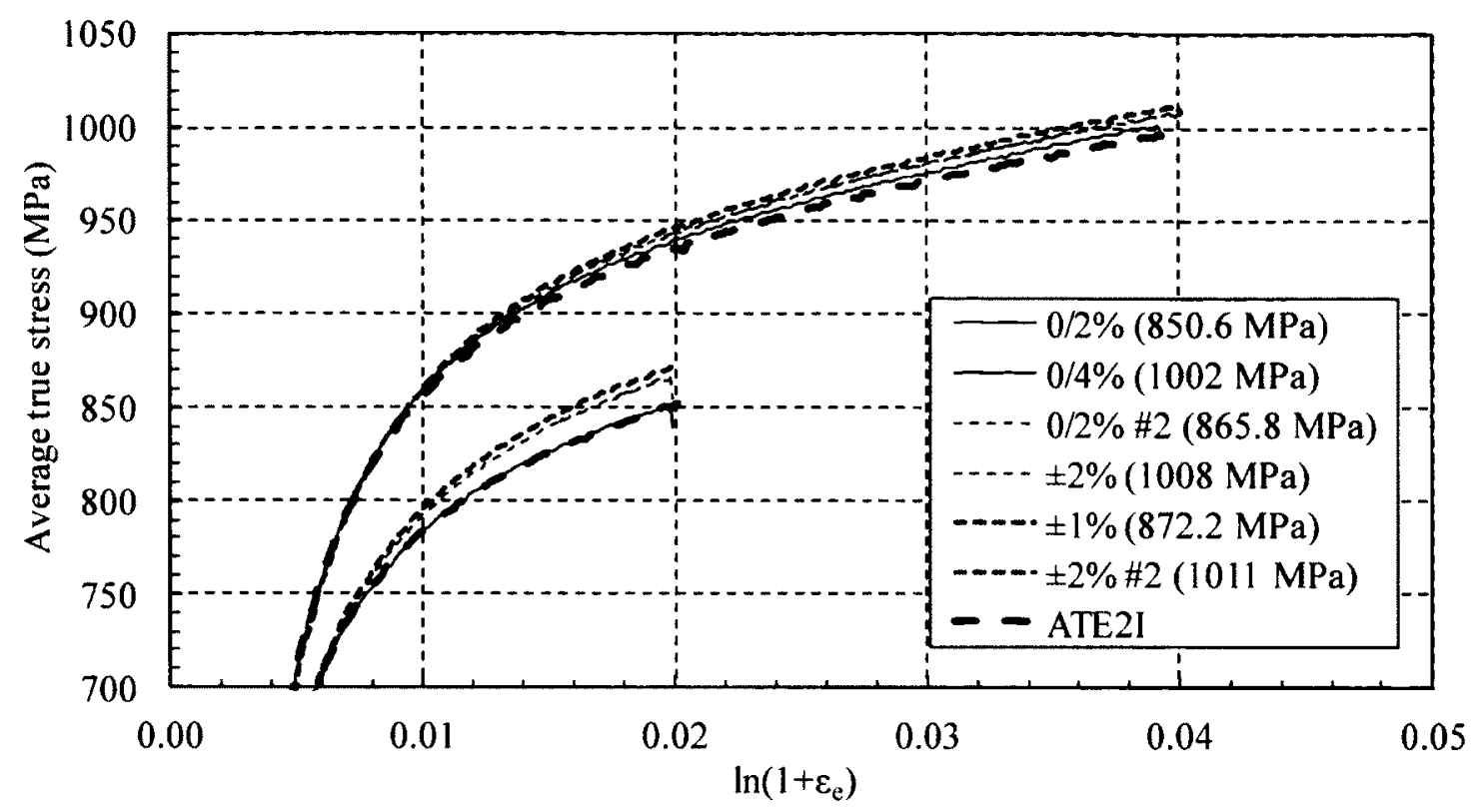

Figure 4.39 Enhanced view of the shifted final half cycle at each strain range for ATE2C-20 and ATE2I including the peak shifted stresses

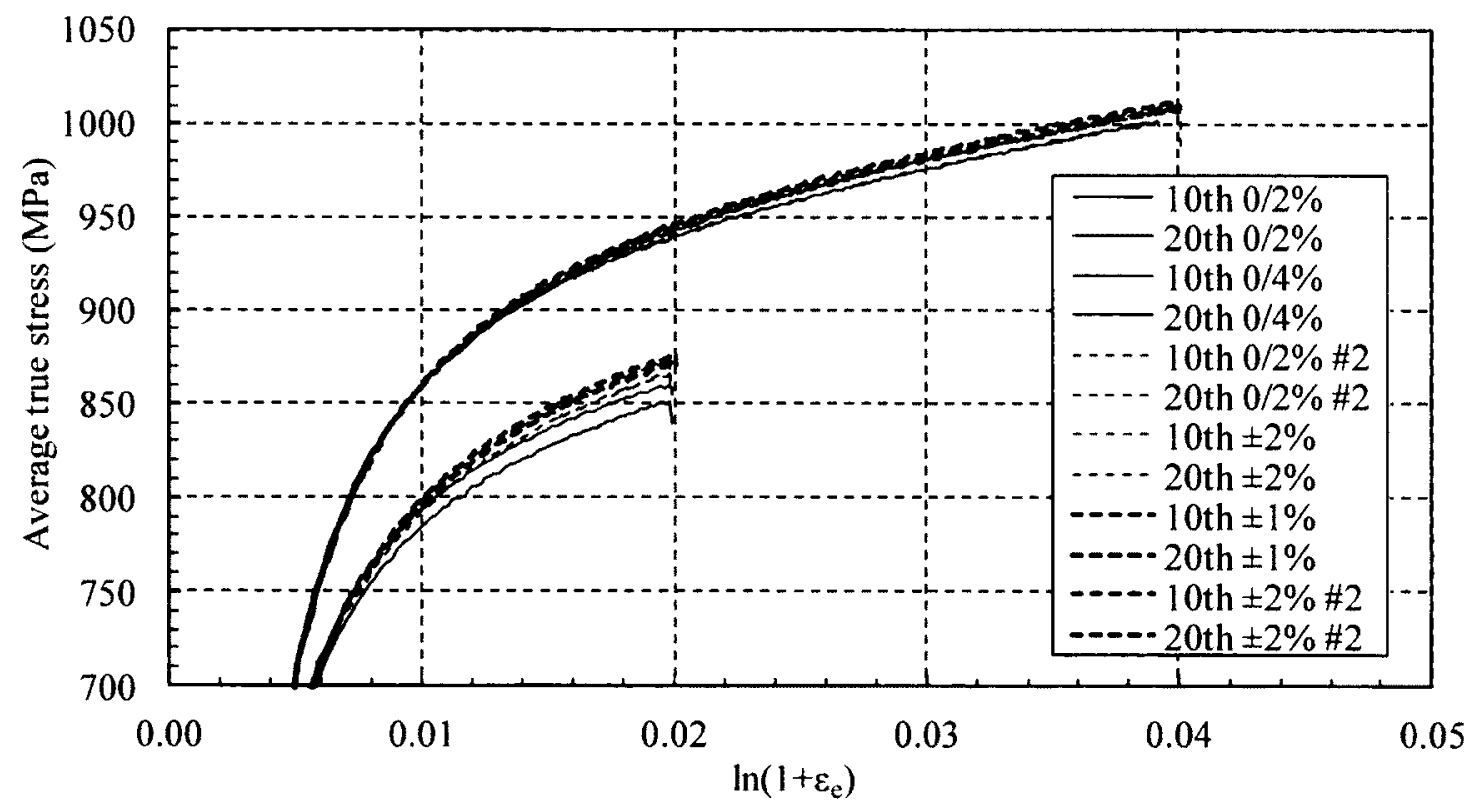

Figure 4.40 Enhanced view of the shifted half cycle for $10^{\text {th }}$ and $20^{\text {th }}$ cycle at various strain ranges for ATE2C-20 


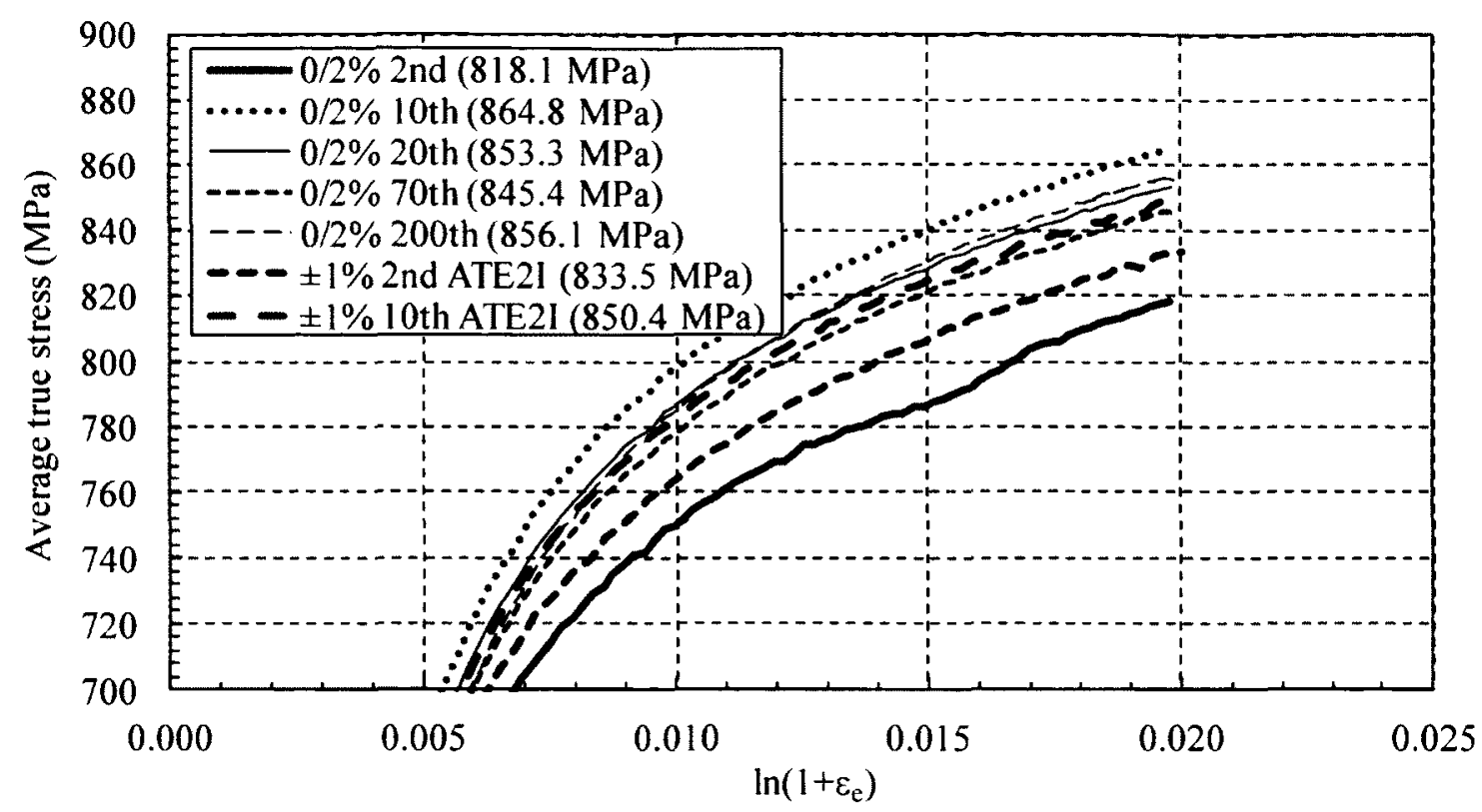

Figure 4.41 Enhanced view of the shifted half cycle for selected cycles of ATE2C-200 and ATE2I including the peak shifted stresses

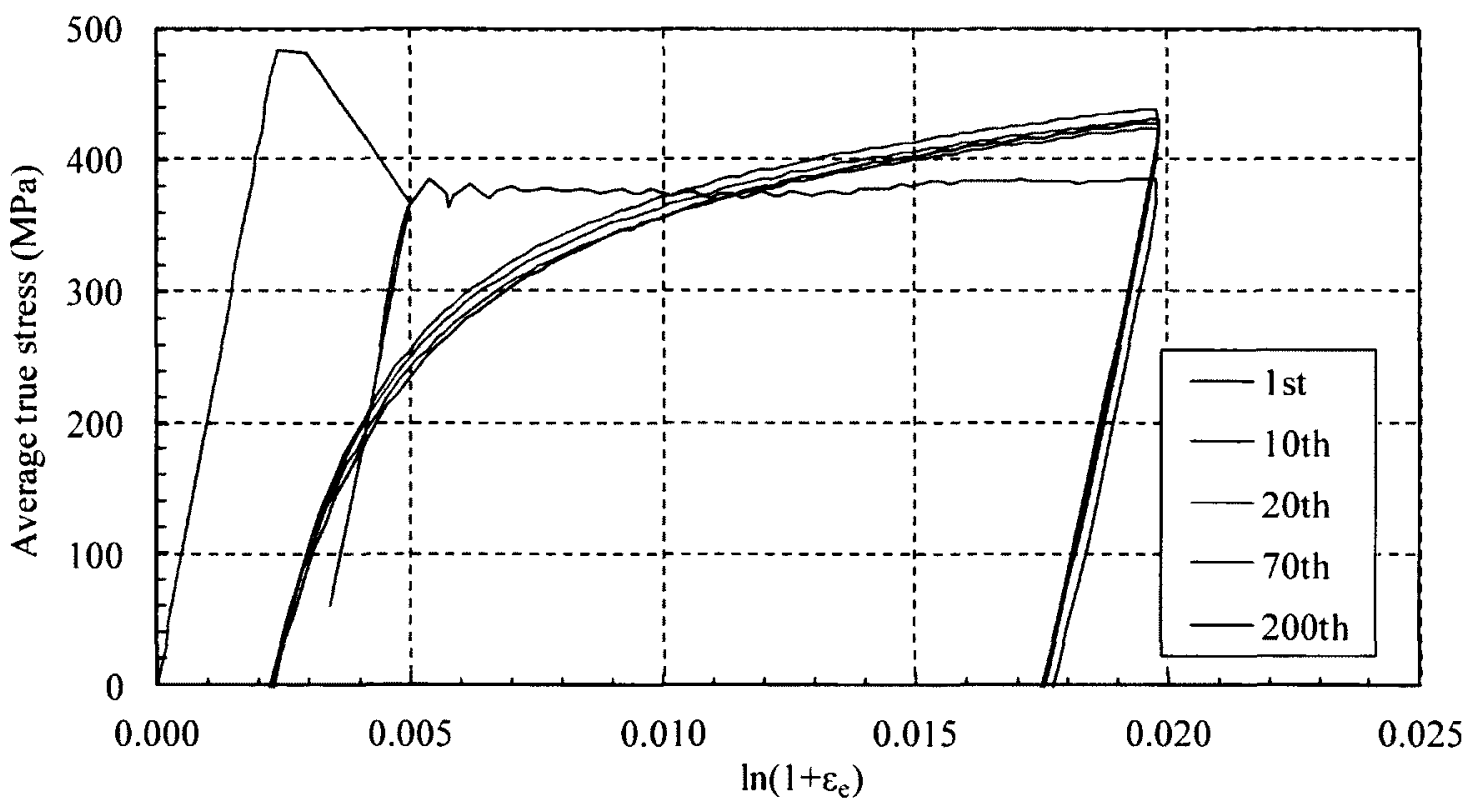

Figure 4.42 Tensile behaviour of ATE2C-200 at the selected cycle numbers 


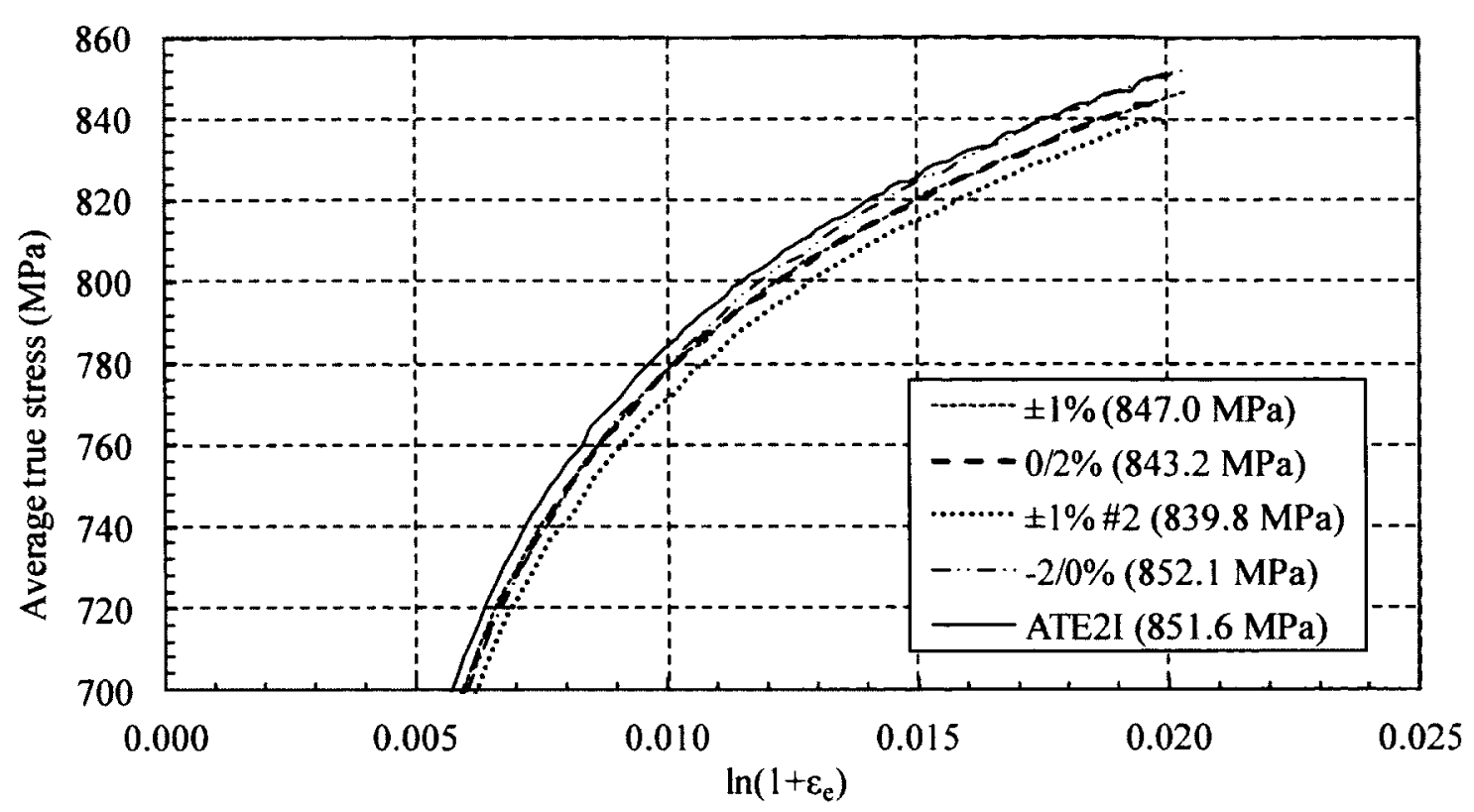

Figure 4.43 Enhanced view of the shifted final half cycle at each strain range for part 1 of 3 of the strain range sequence for ATE2E and $\pm 1 \%$ strain range for ATE2I

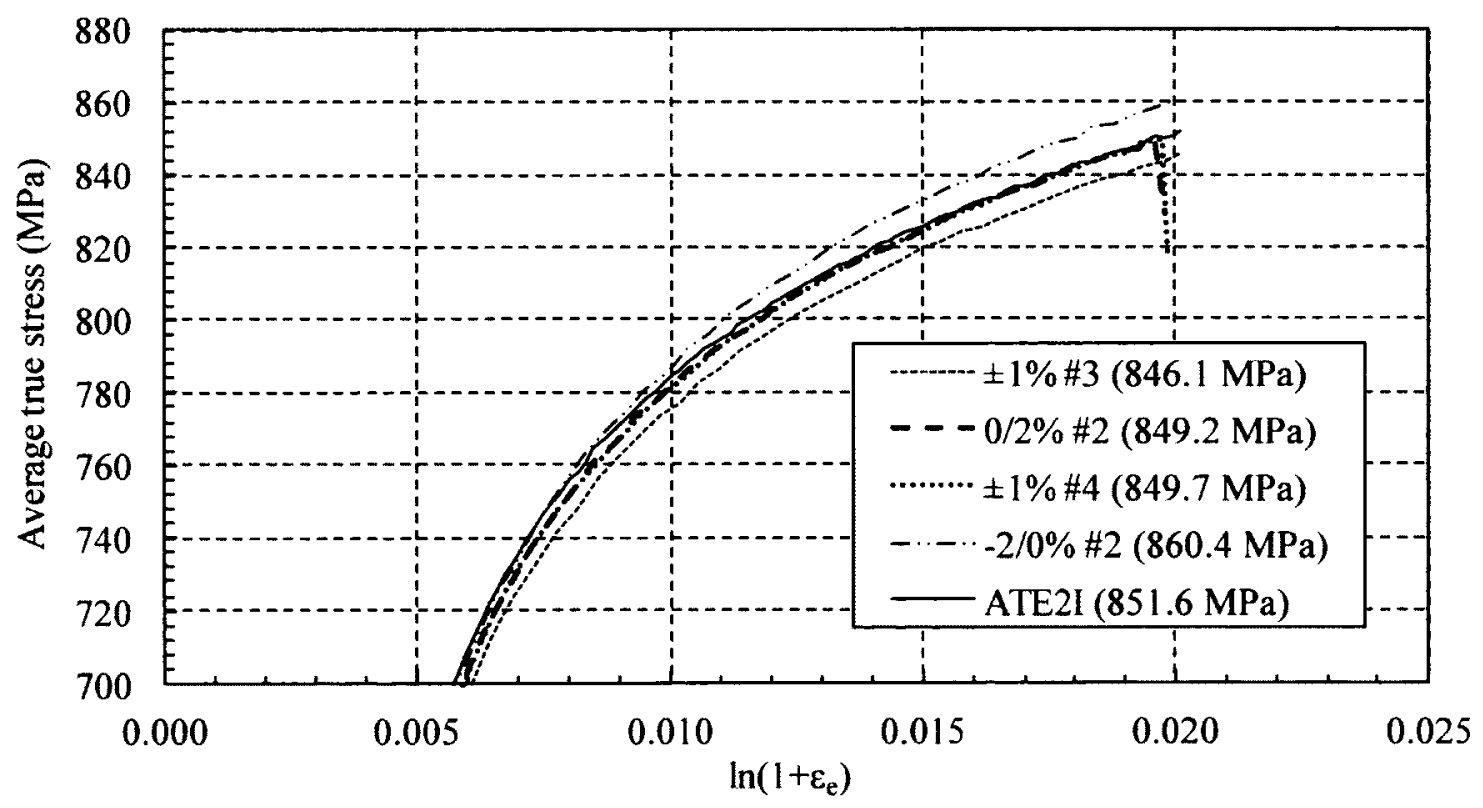

Figure 4.44 Enhanced view of the shifted final half cycle at each strain range for part 2 of 3 of the strain range sequence for ATE2E and $\pm 1 \%$ strain range for ATE2I 


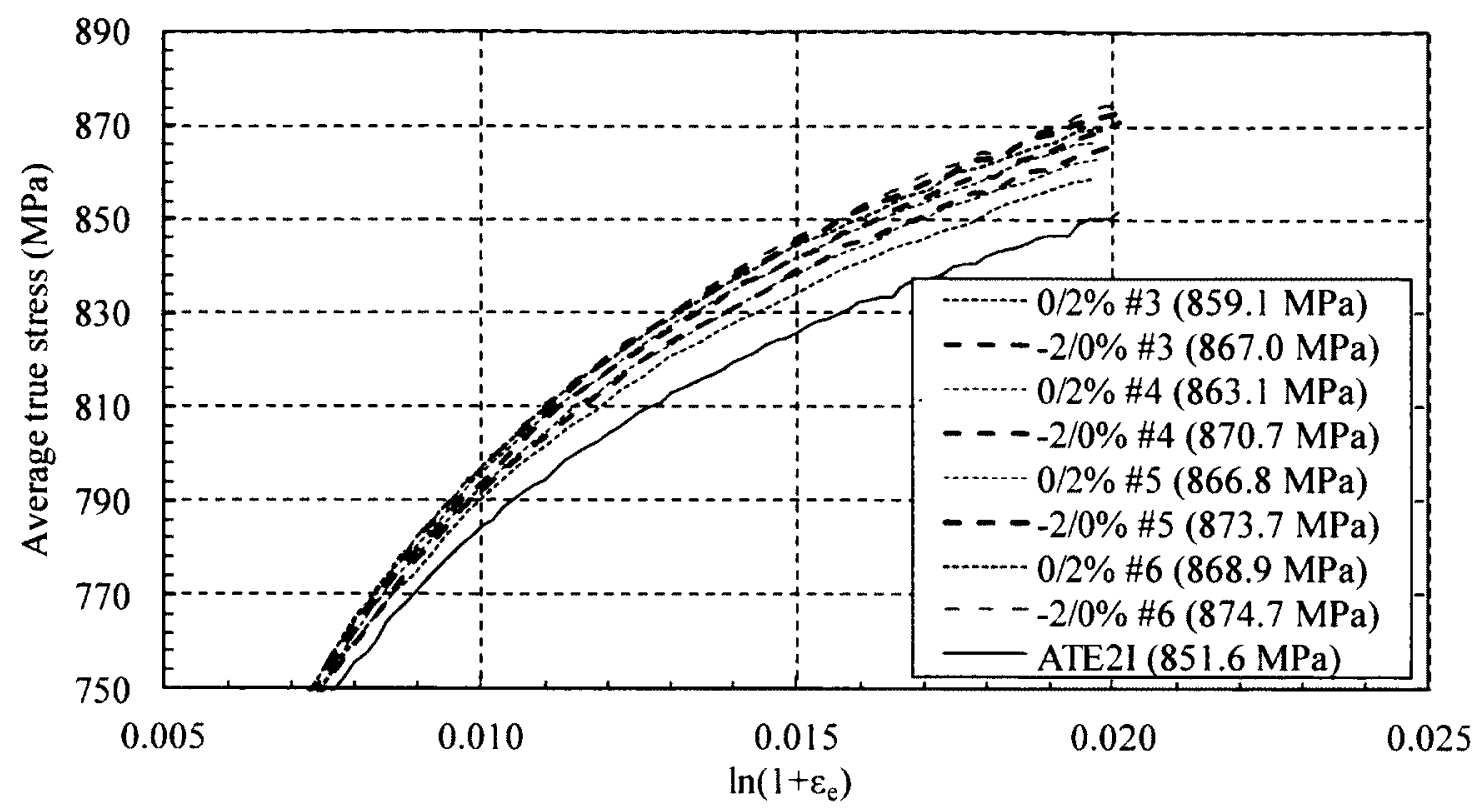

Figure 4.45 Enhanced view of the shifted final half cycle at each strain range for part 3 of 3 of the strain range sequence for $\mathrm{ATE} 2 \mathrm{E}$ and $\pm 1 \%$ strain range for ATE2I

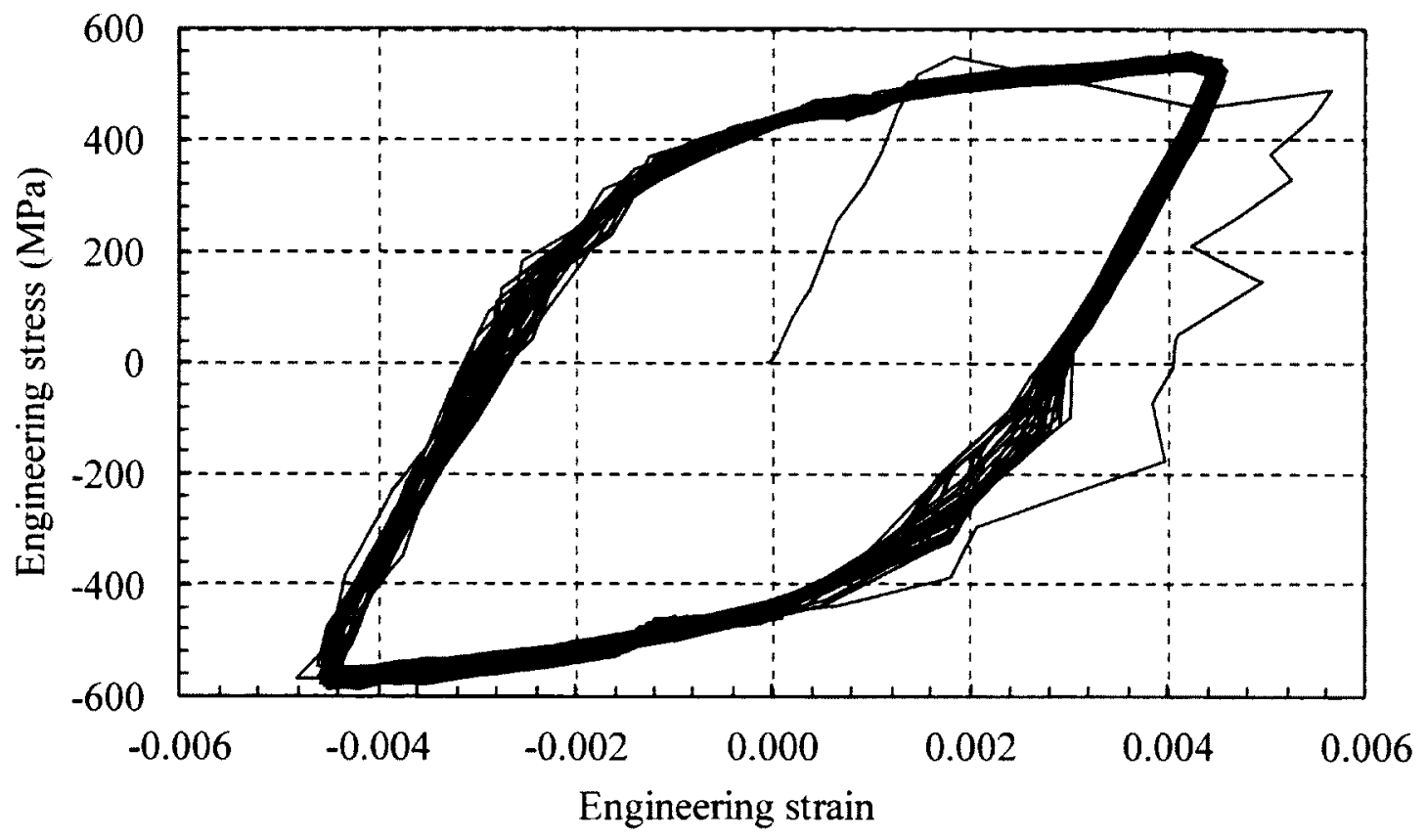

Figure 4.46 Engineering stress versus engineering strain curve of ANE1R2 for the initial 30 cycles 


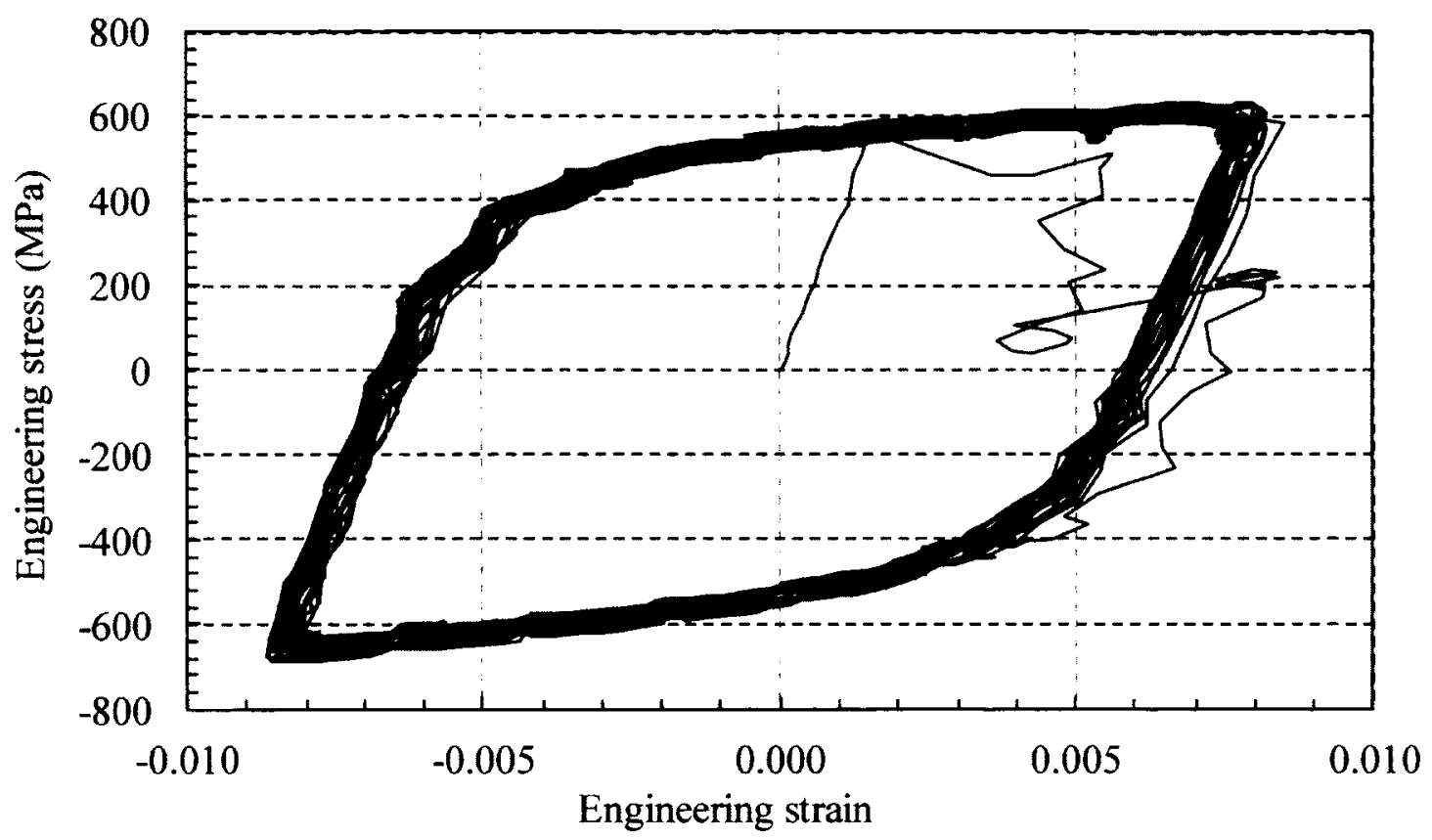

Figure 4.47 Engineering stress versus engineering strain curve of ANE1R4 for the initial 30 cycles

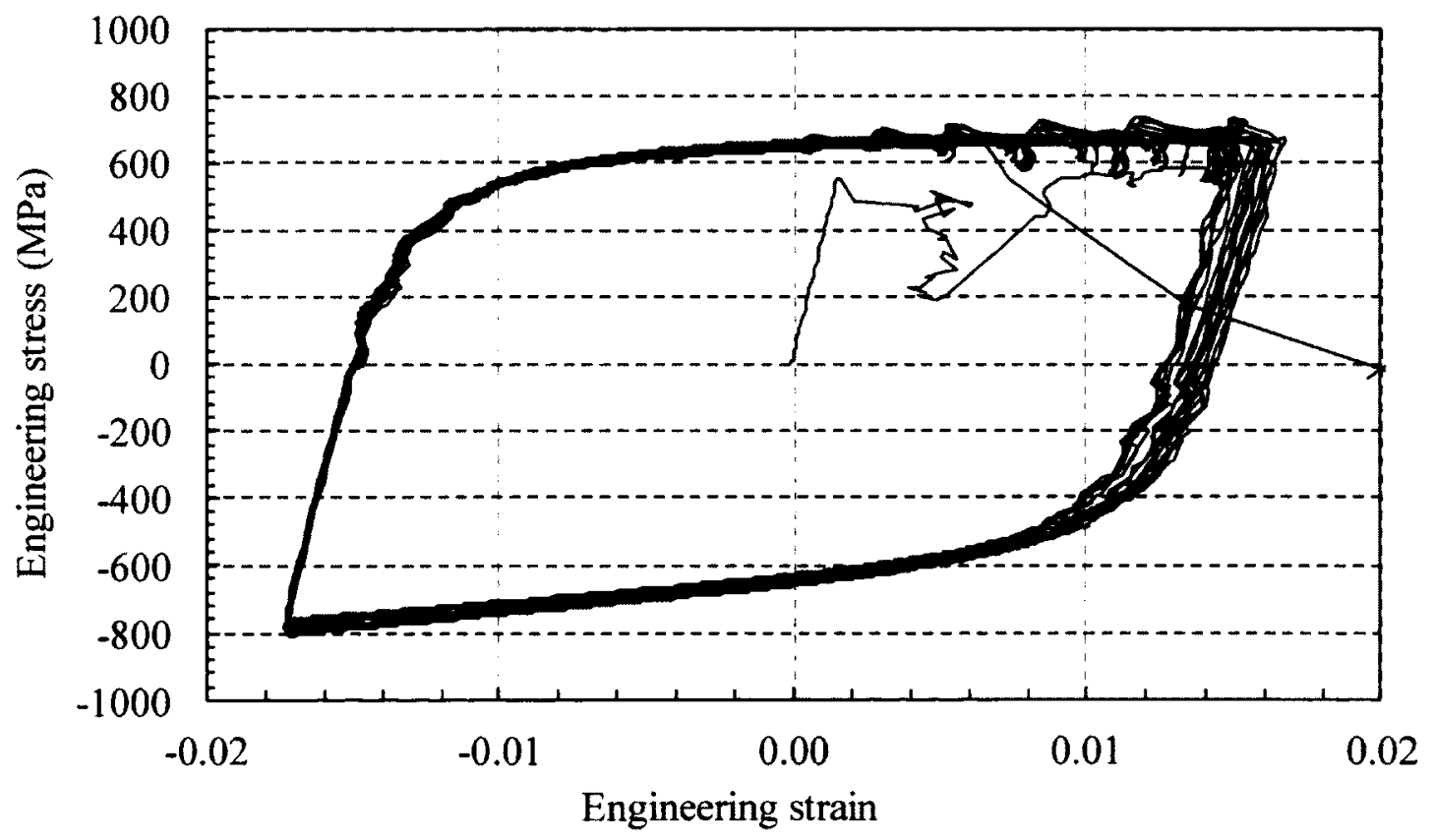

Figure 4.48 Engineering stress versus engineering strain curve of ANE1R8 


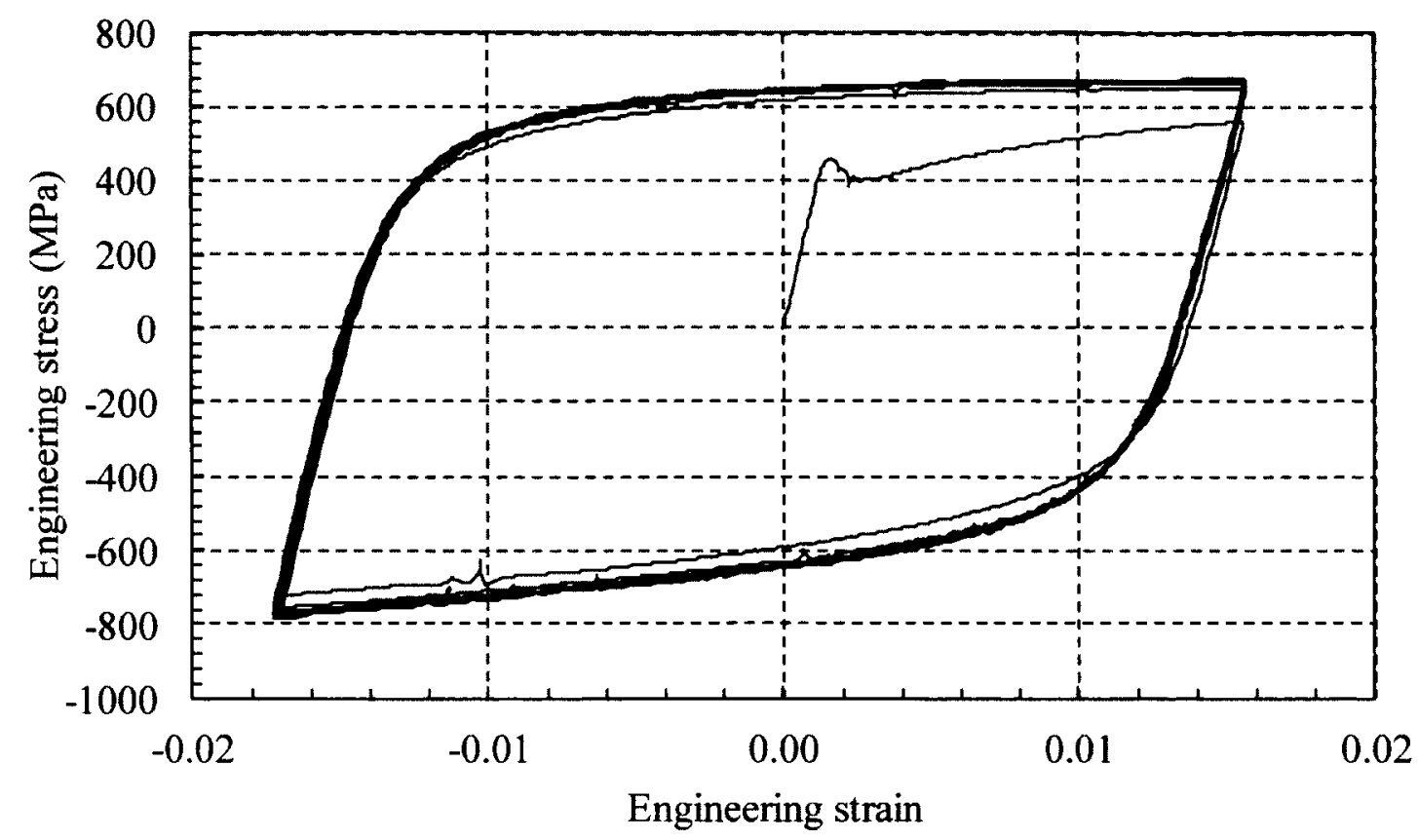

Figure 4.49 Engineering stress versus engineering strain curve of ANE4R8 for the initial 10 cycles

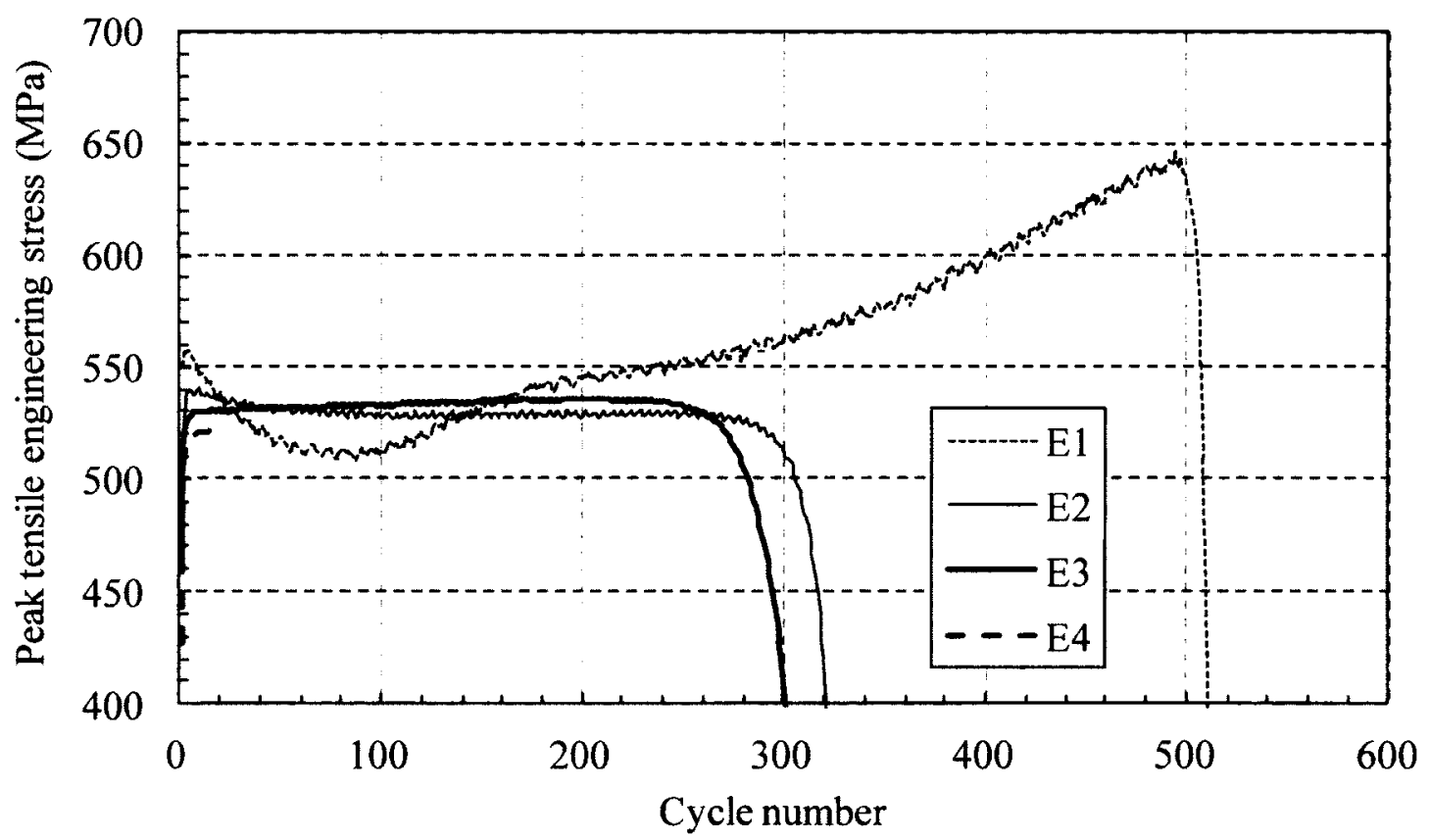

Figure 4.50 Peak tensile engineering stress versus cycle number for ANE1R2, ANE2R2, ANE3R2 and ANE4R2 


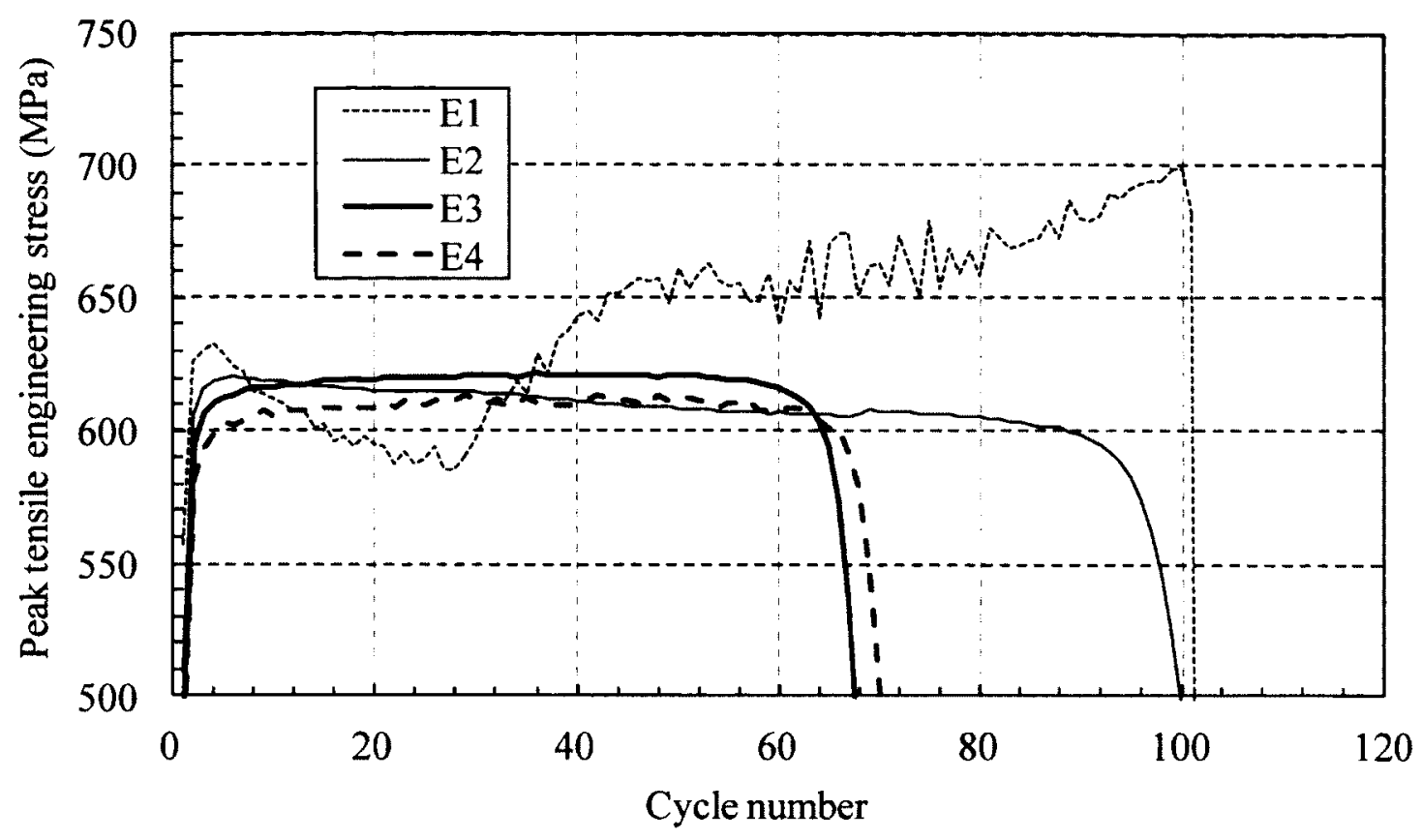

Figure 4.51 Peak tensile engineering stress versus cycle number for ANE1R4, ANE2R4, ANE3R4 and ANE4R4

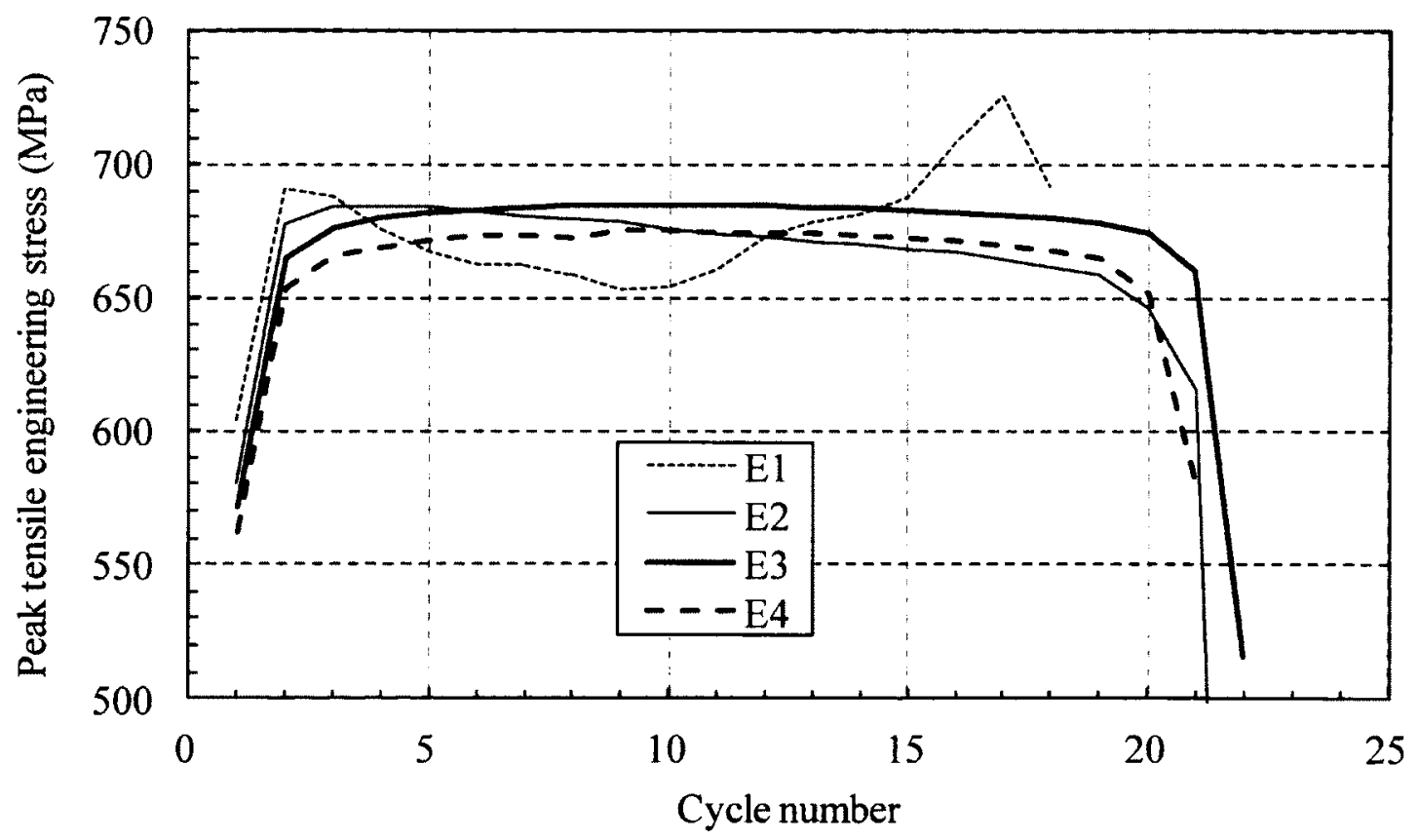

Figure 4.52 Peak tensile engineering stress versus cycle number for ANE1R8, ANE2R8, ANE3R8 and ANE4R8 


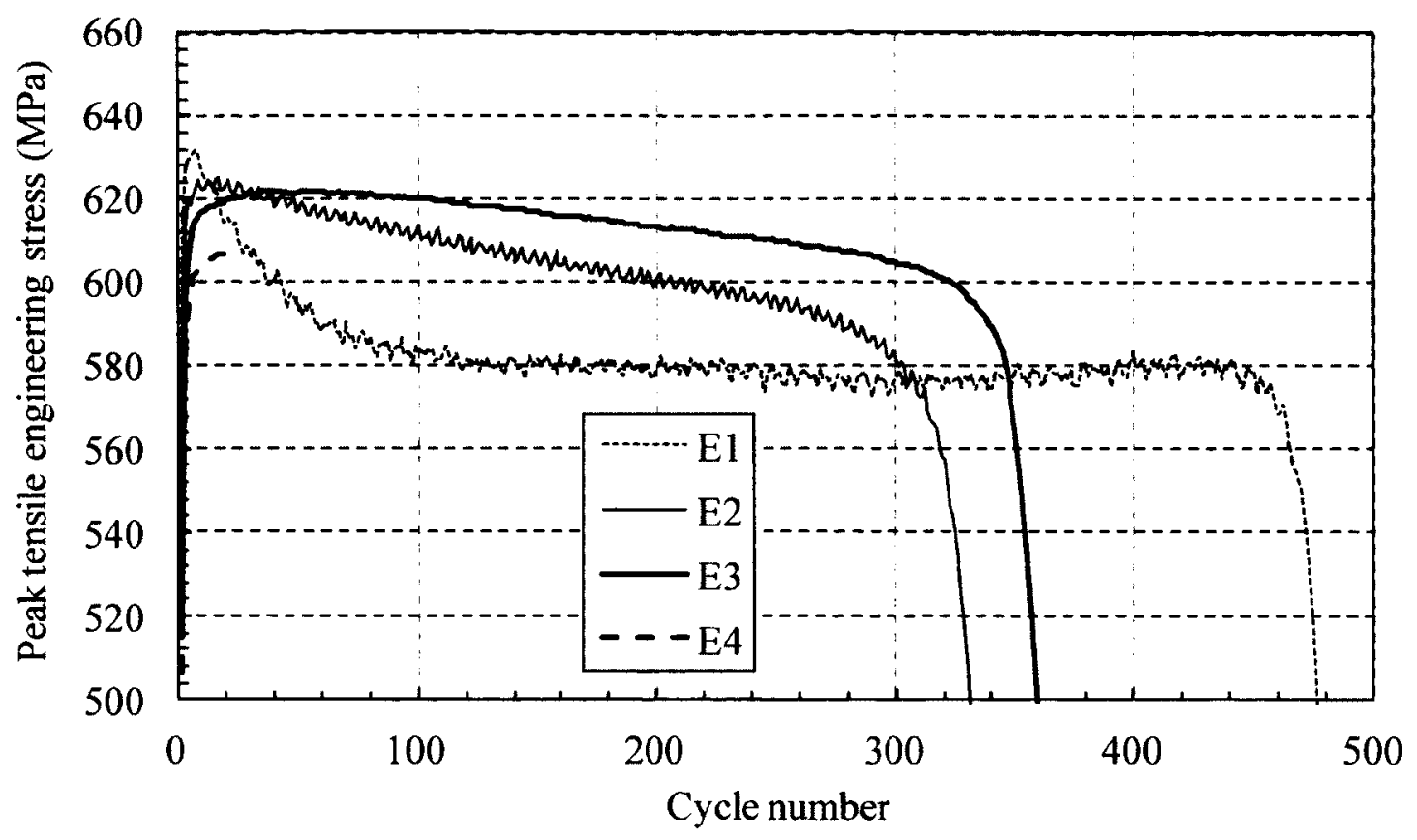

Figure 4.53 Peak tensile engineering stress versus cycle number for BNE1R2, BNE2R2, BNE3R2 and BNE4R2

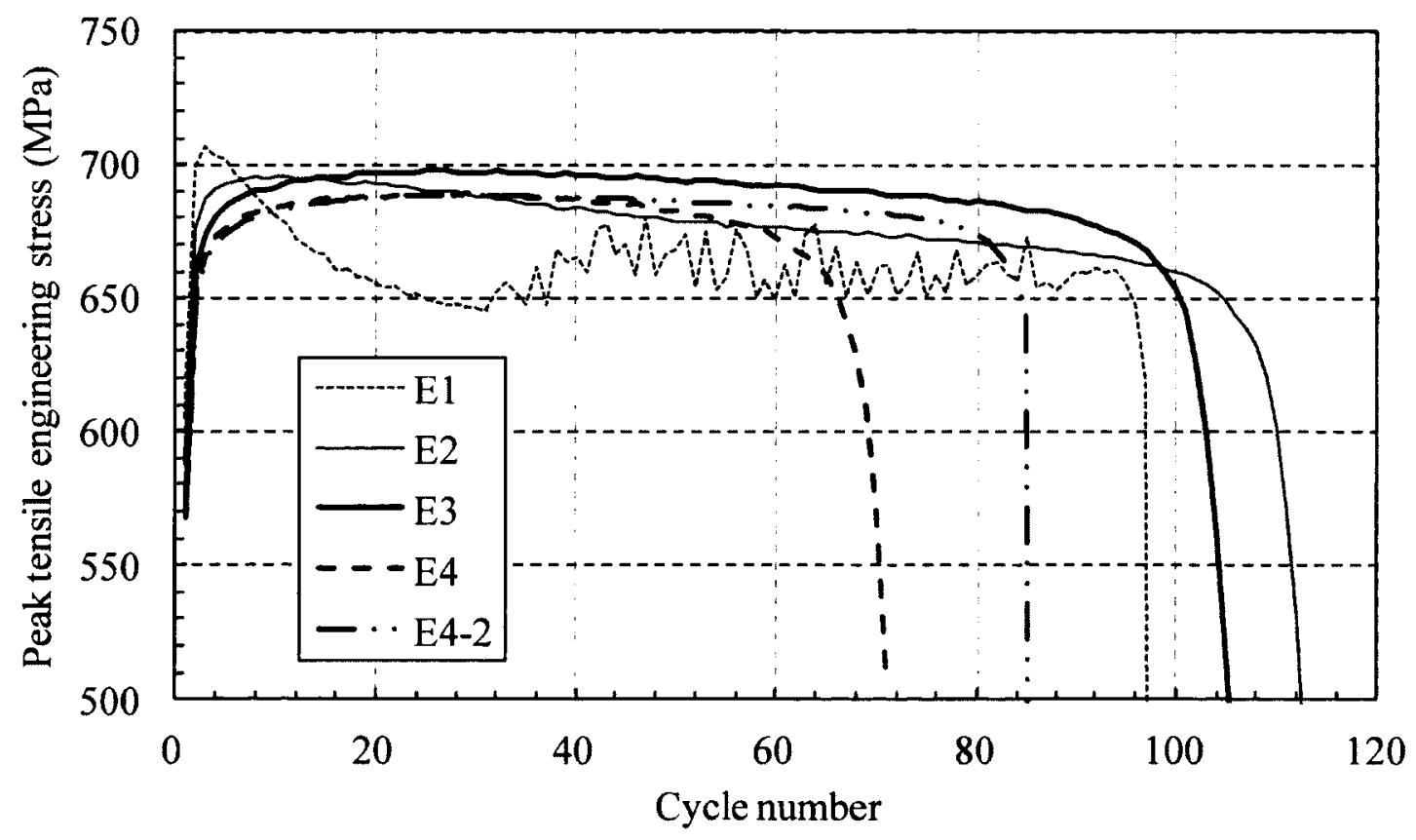

Figure 4.54 Peak tensile engineering stress versus cycle number for BNE1R4, BNE2R4, BNE3R4, BNE4R4 and BNE4R4-2 


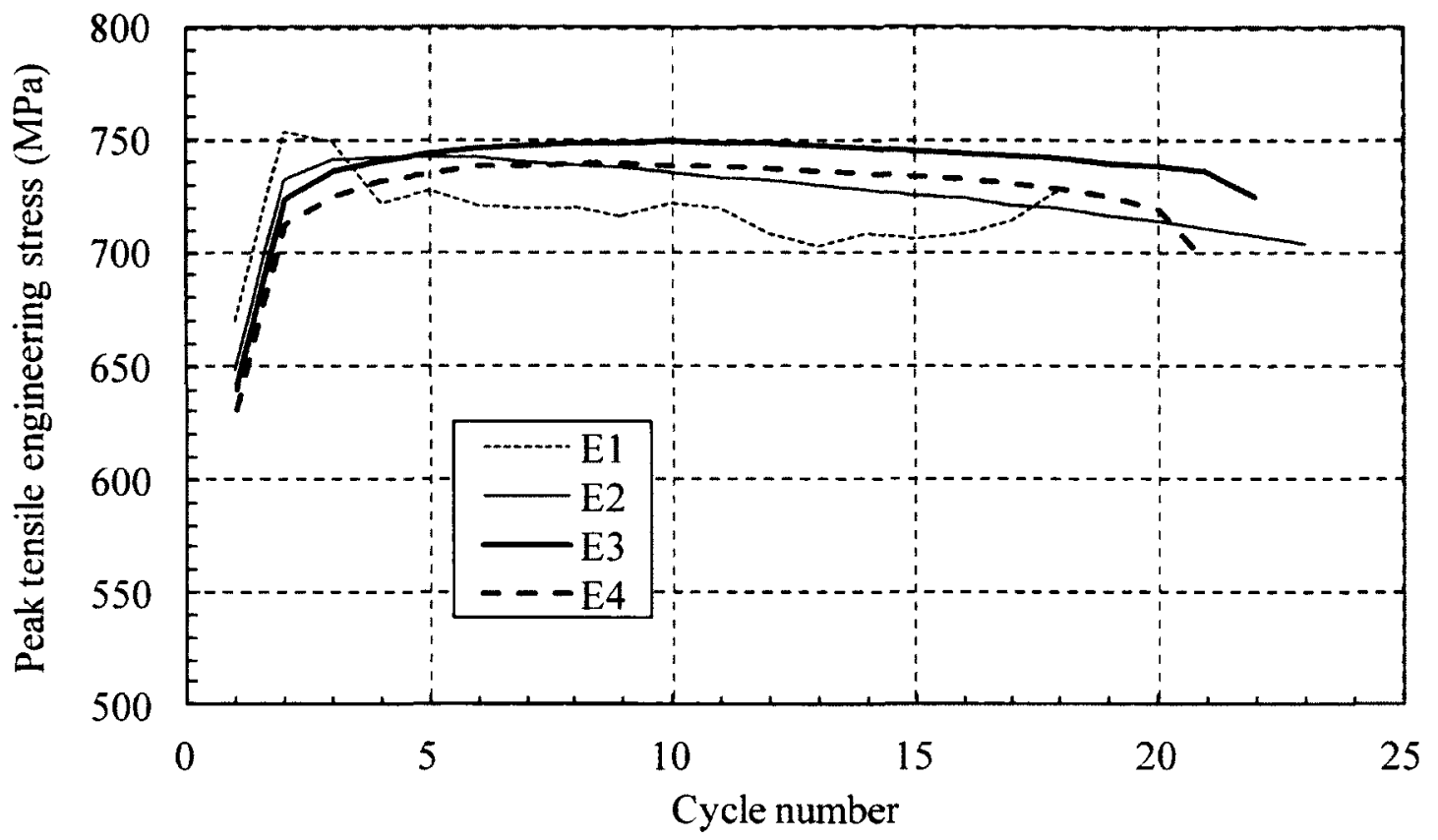

Figure 4.55 Peak tensile engineering stress versus cycle number for BNE1R8, BNE2R8, BNE3R8 and BNE4R8

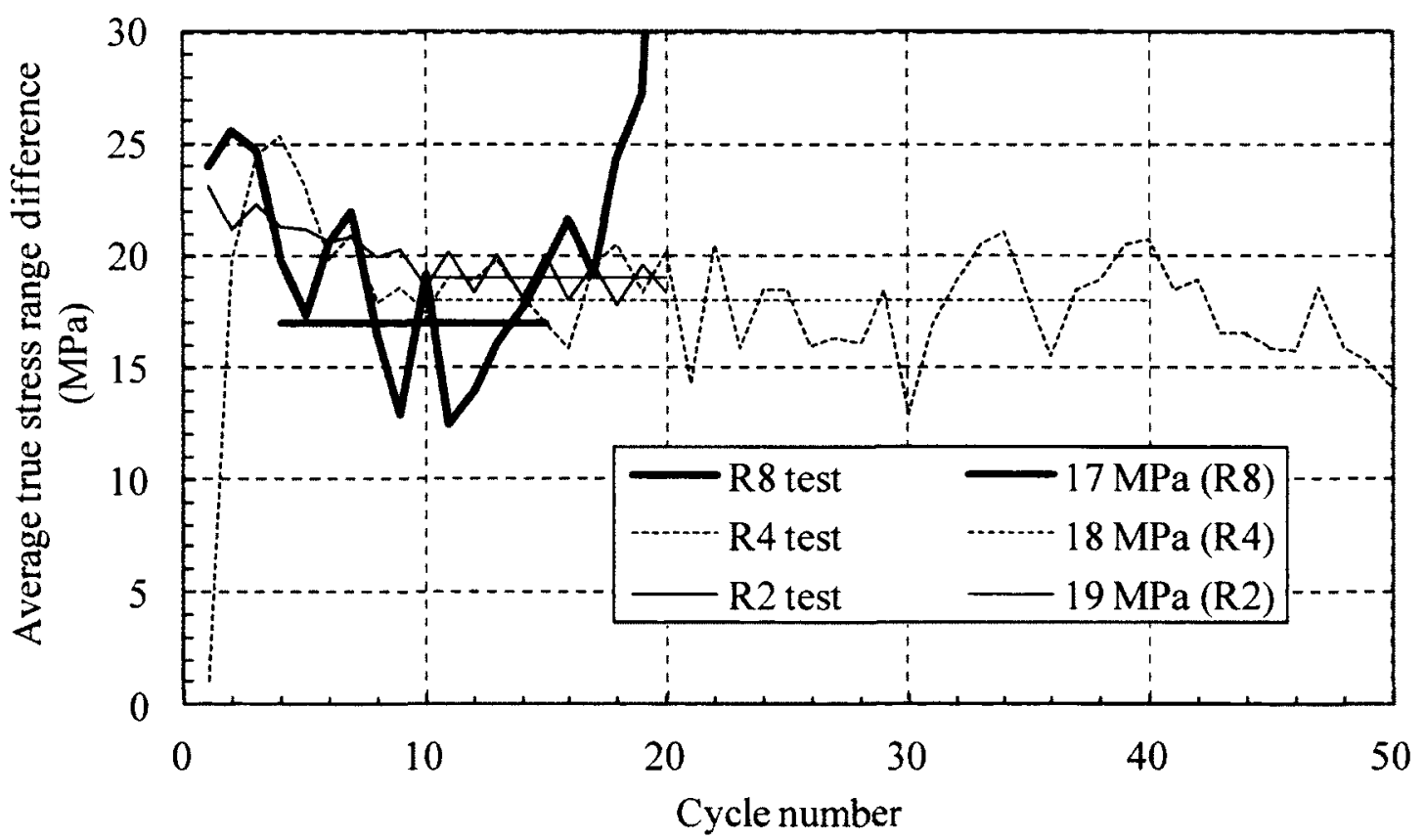

Figure 4.56 Average true stress range difference between the $10^{-4}$ and $10^{-3} \mathrm{~s}^{-1}$ strain rates versus loading cycle number for material $\mathrm{A}$ 


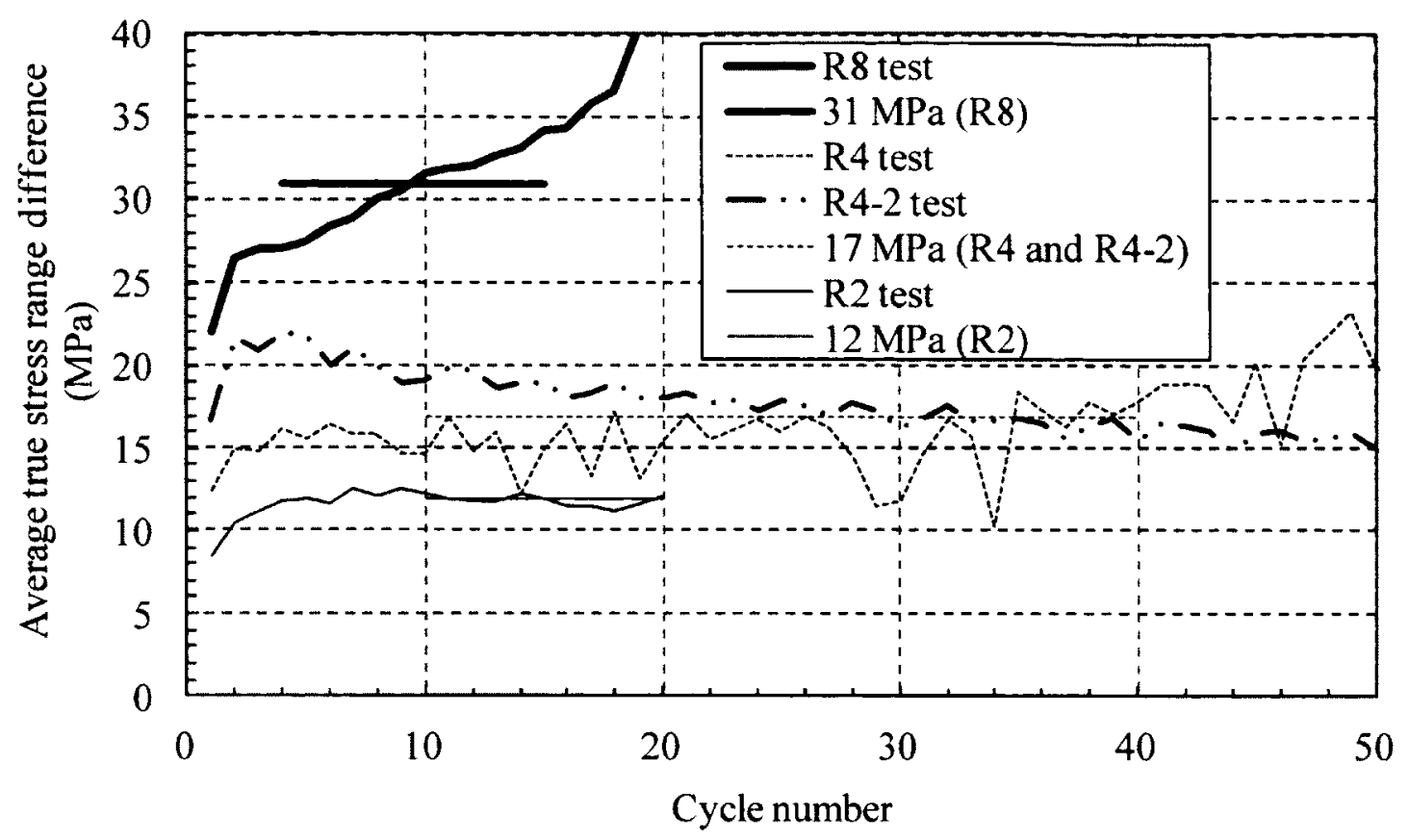

Figure 4.57 Average true stress range difference between the $10^{-4}$ and $10^{-3} \mathrm{~s}^{-1}$ strain rates versus loading cycle number for material $\mathrm{B}$

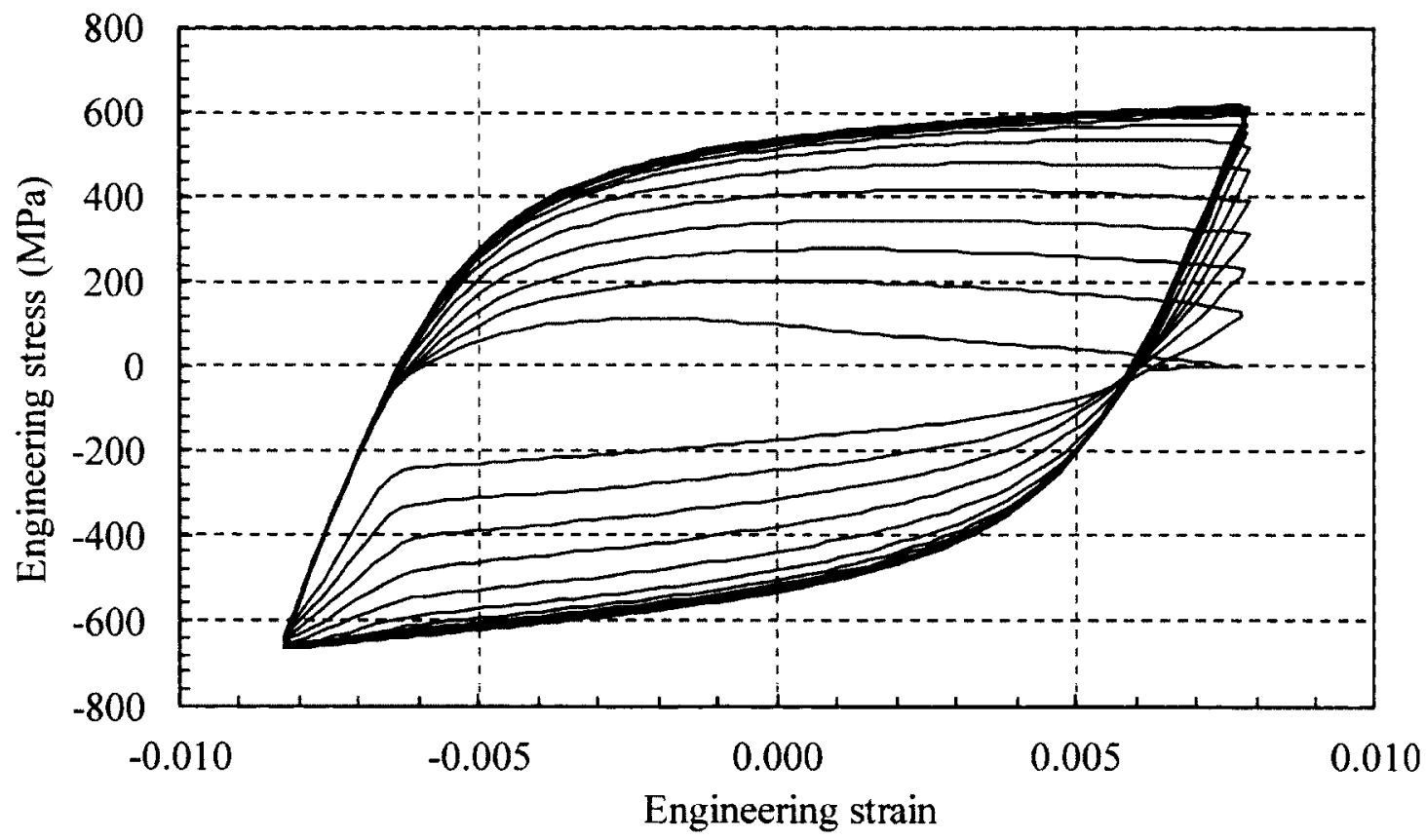

Figure 4.58 Engineering stress versus engineering strain curve for the last few cycles of ANE3R4 


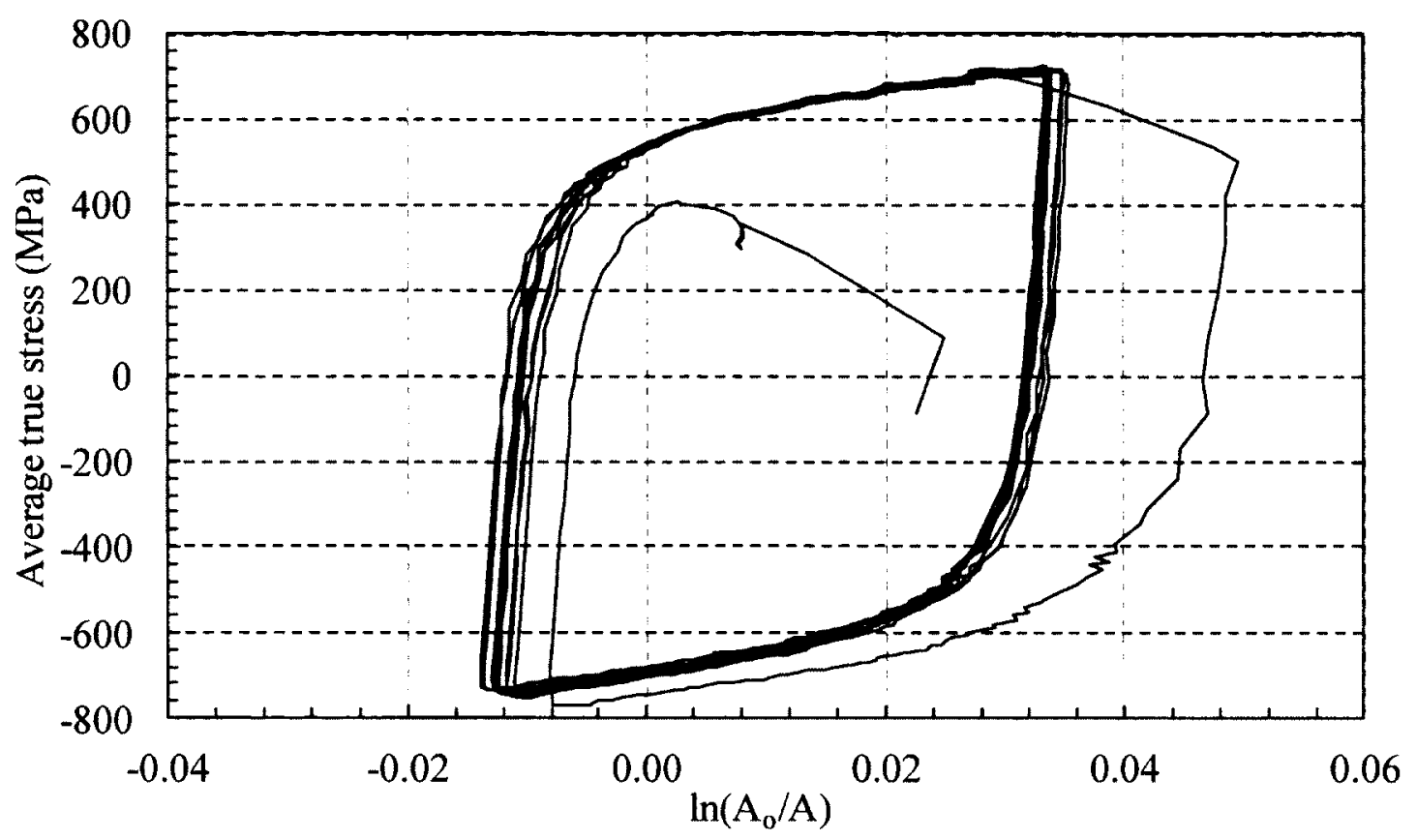

Figure 4.59 Average true stress versus average true strain curve for the last few cycles of ANE1R4

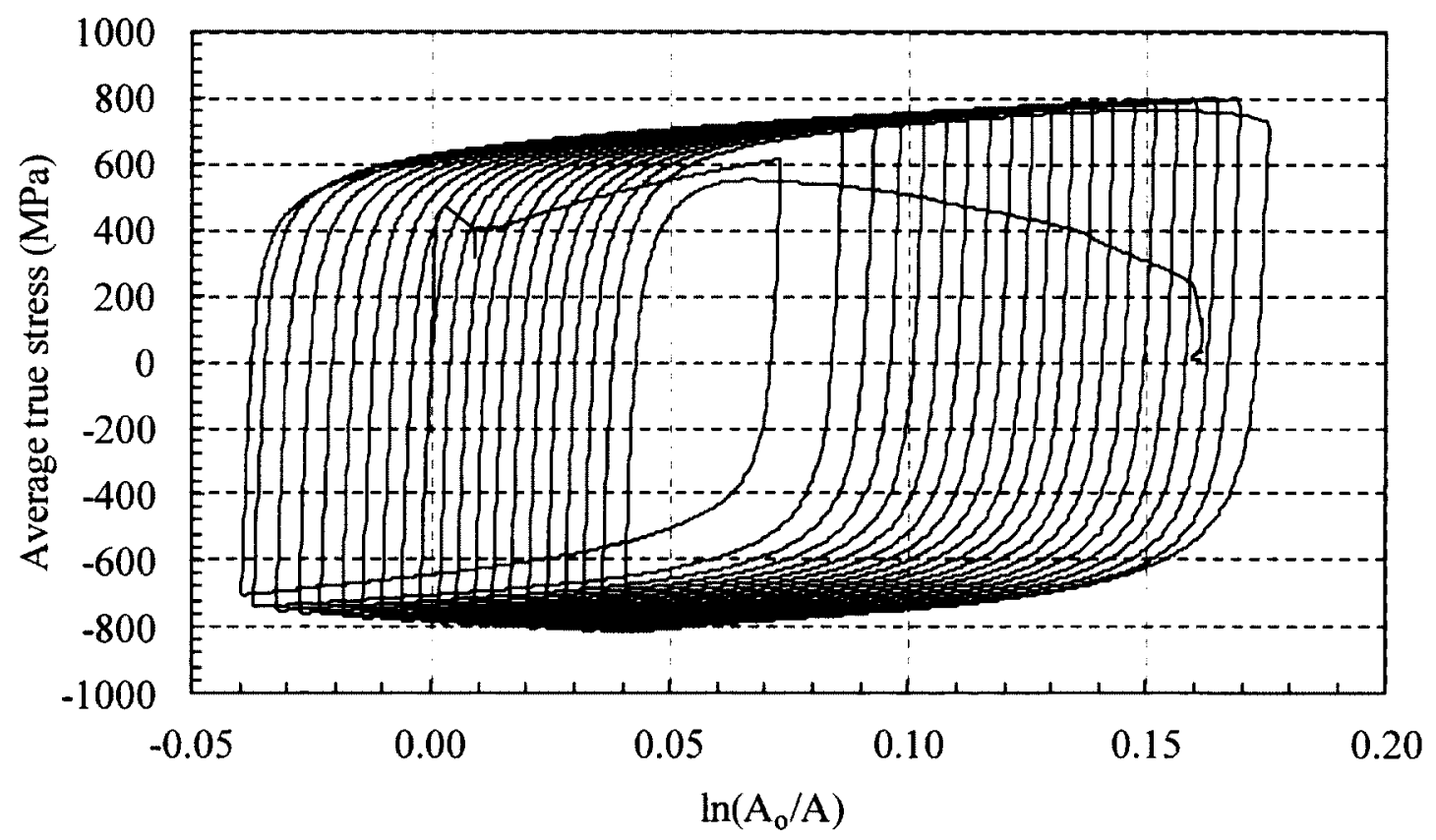

Figure 4.60 Average true stress versus average true strain curve for ANE3R8 


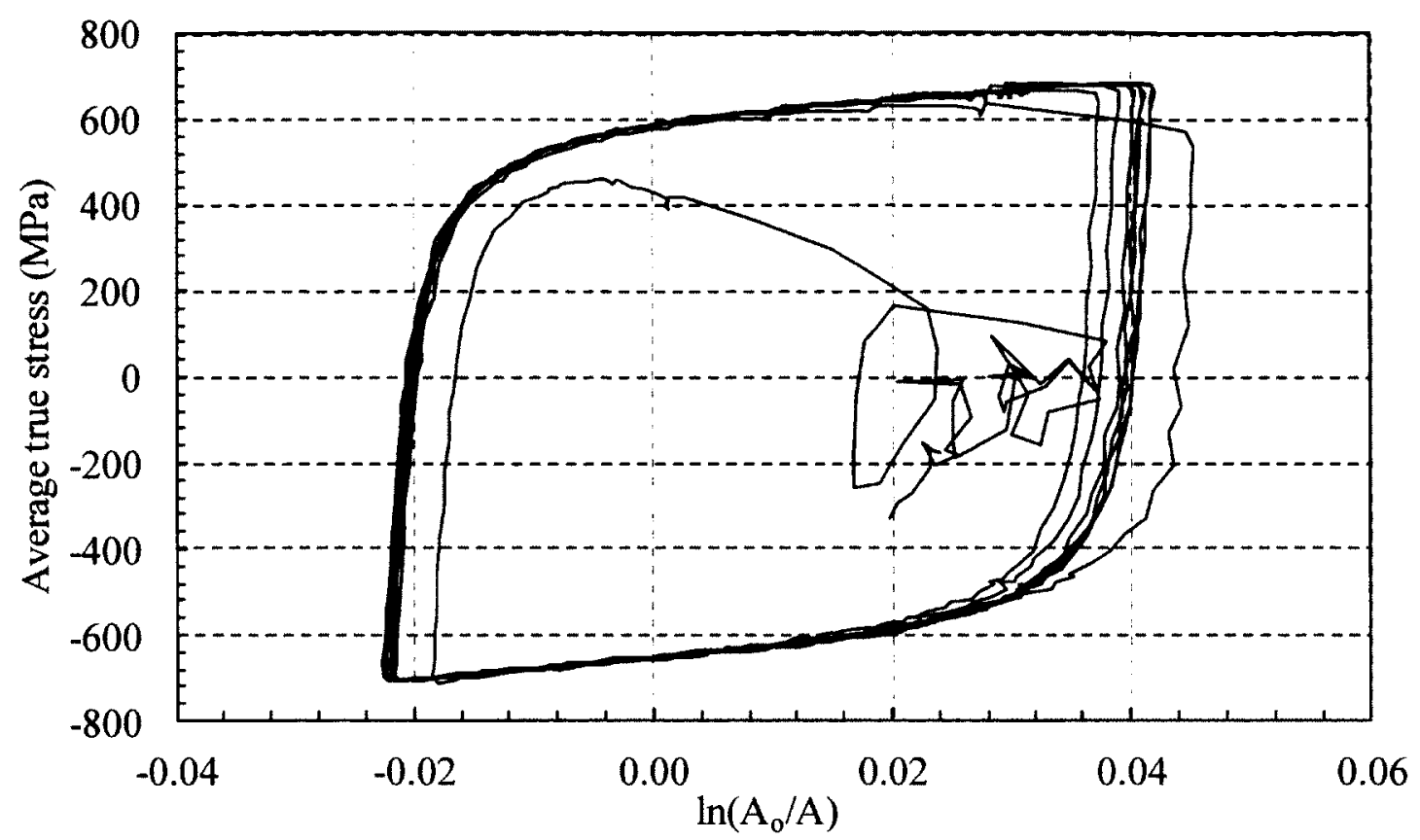

Figure 4.61 Average true stress versus average true strain curve for the last few cycles of BNE1R4

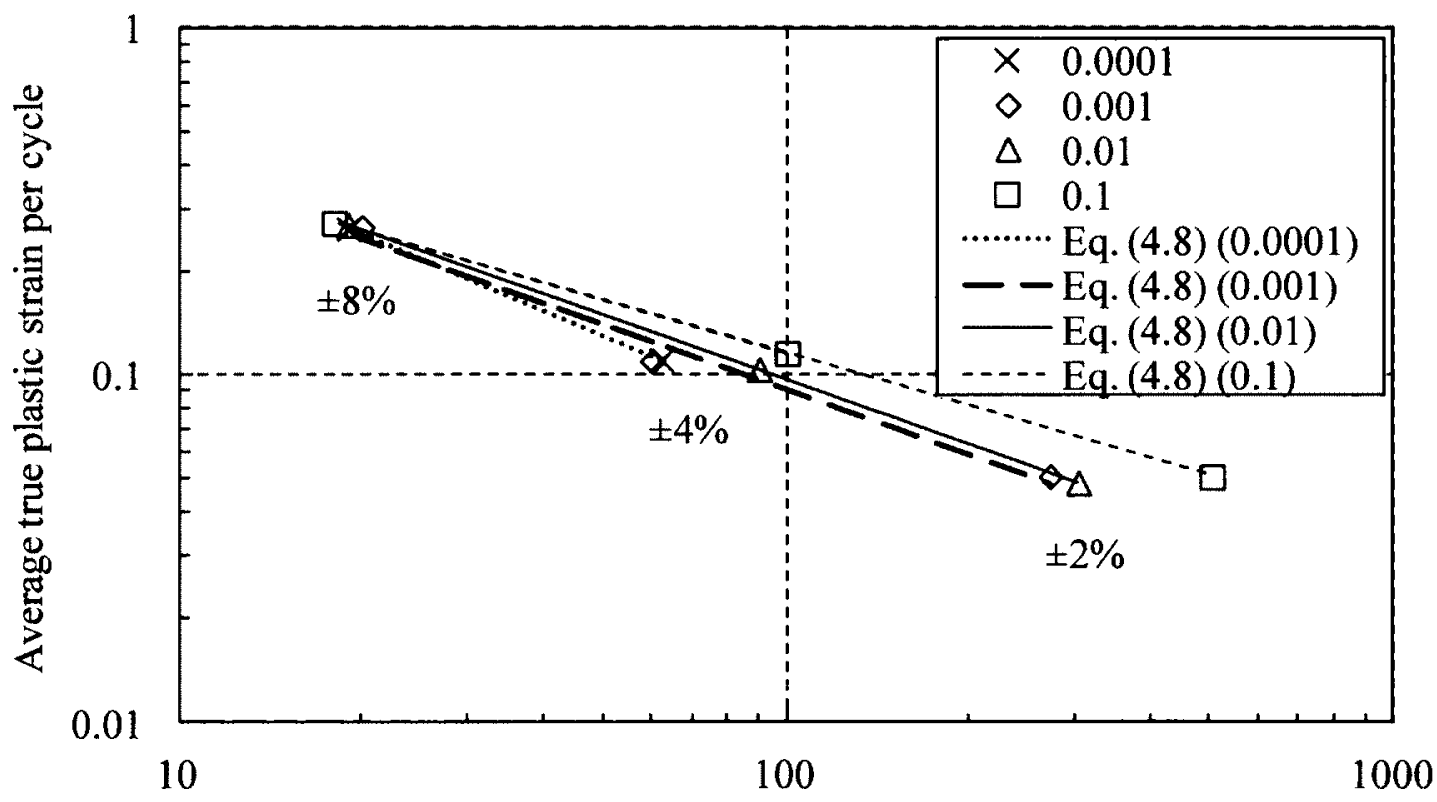

Fatigue life, cycle

Figure 4.62 Calculated average true plastic strain per cycle versus fatigue life for material $\mathrm{A}$ at various target strain ranges 


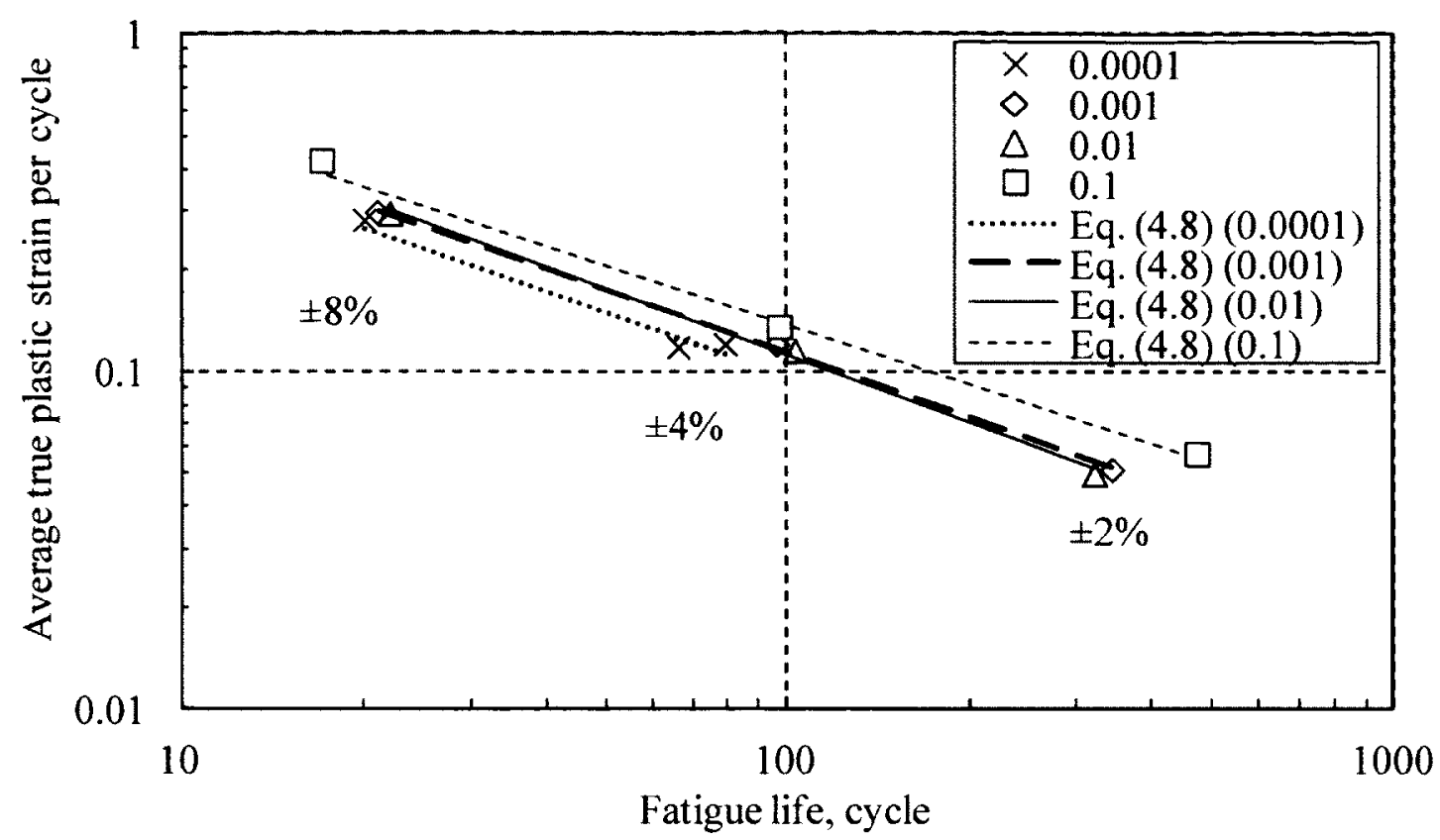

Figure 4.63 Calculated average true plastic strain per cycle versus fatigue life for material $B$ at various target strain ranges

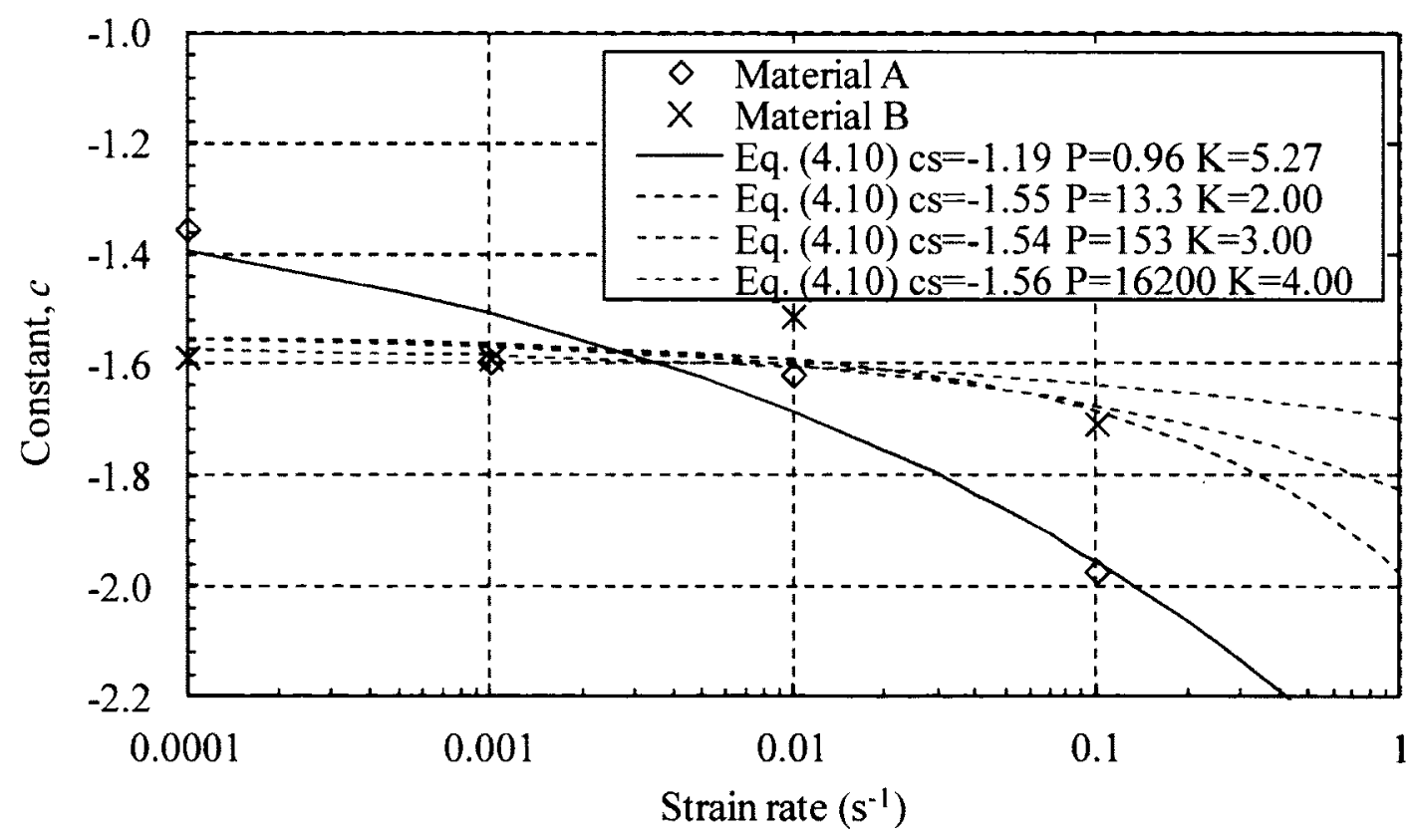

Figure 4.64 Constant $c$ versus strain rate 


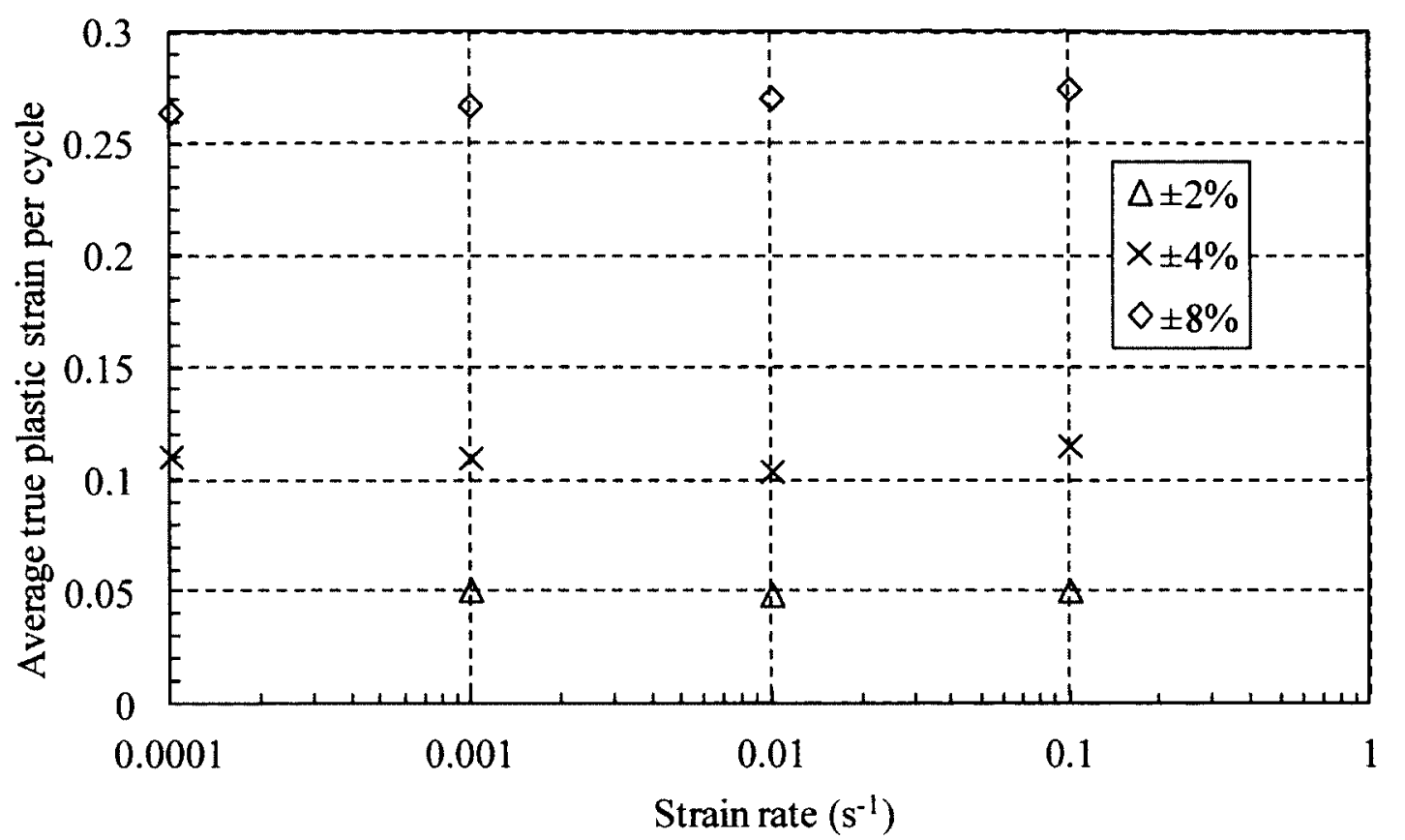

Figure 4.65 Calculated average true plastic strain per cycle versus strain rate for material $A$ at various target strain ranges

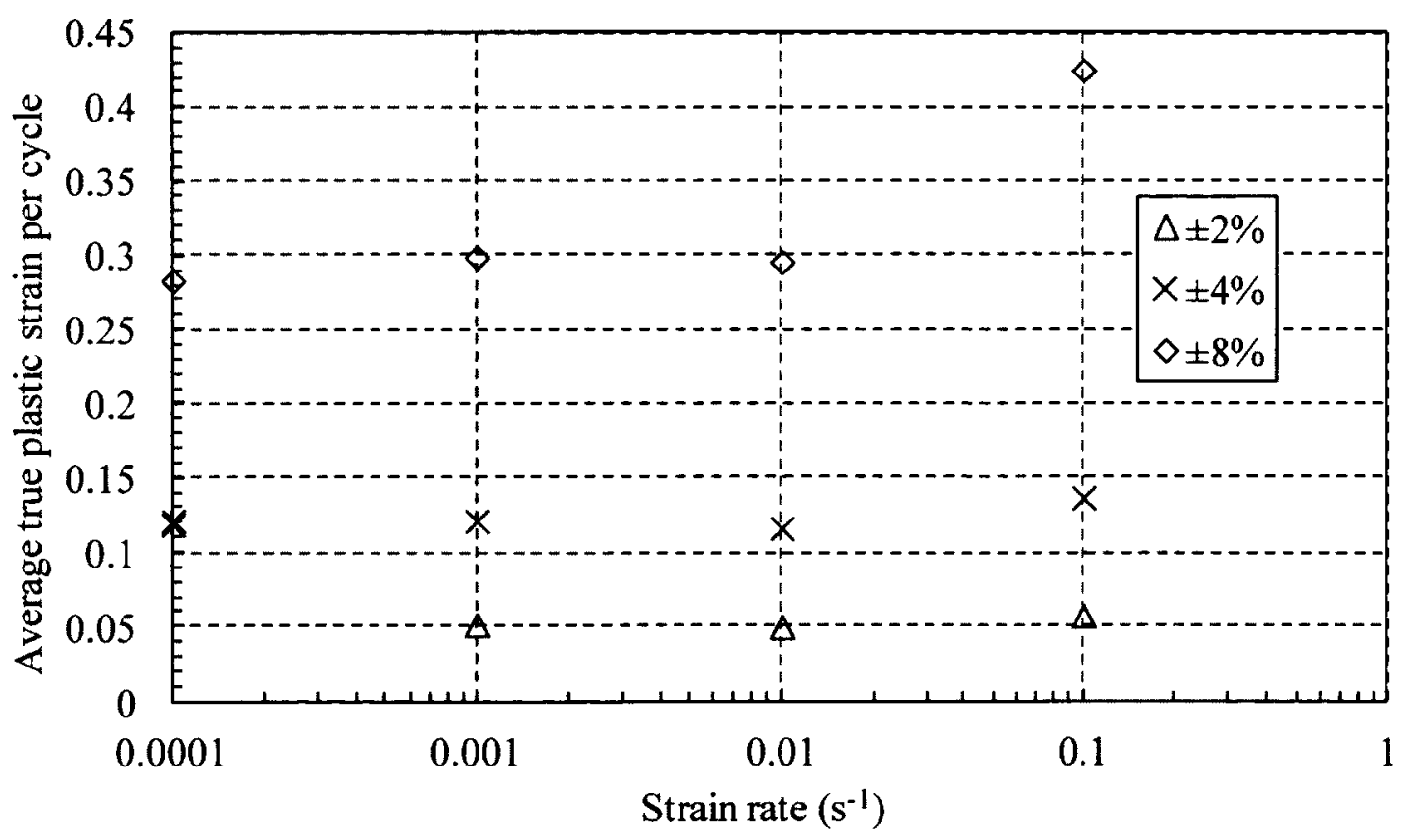

Figure 4.66 Calculated average true plastic strain per cycle versus strain rate for material $\mathrm{B}$ at various target strain ranges 


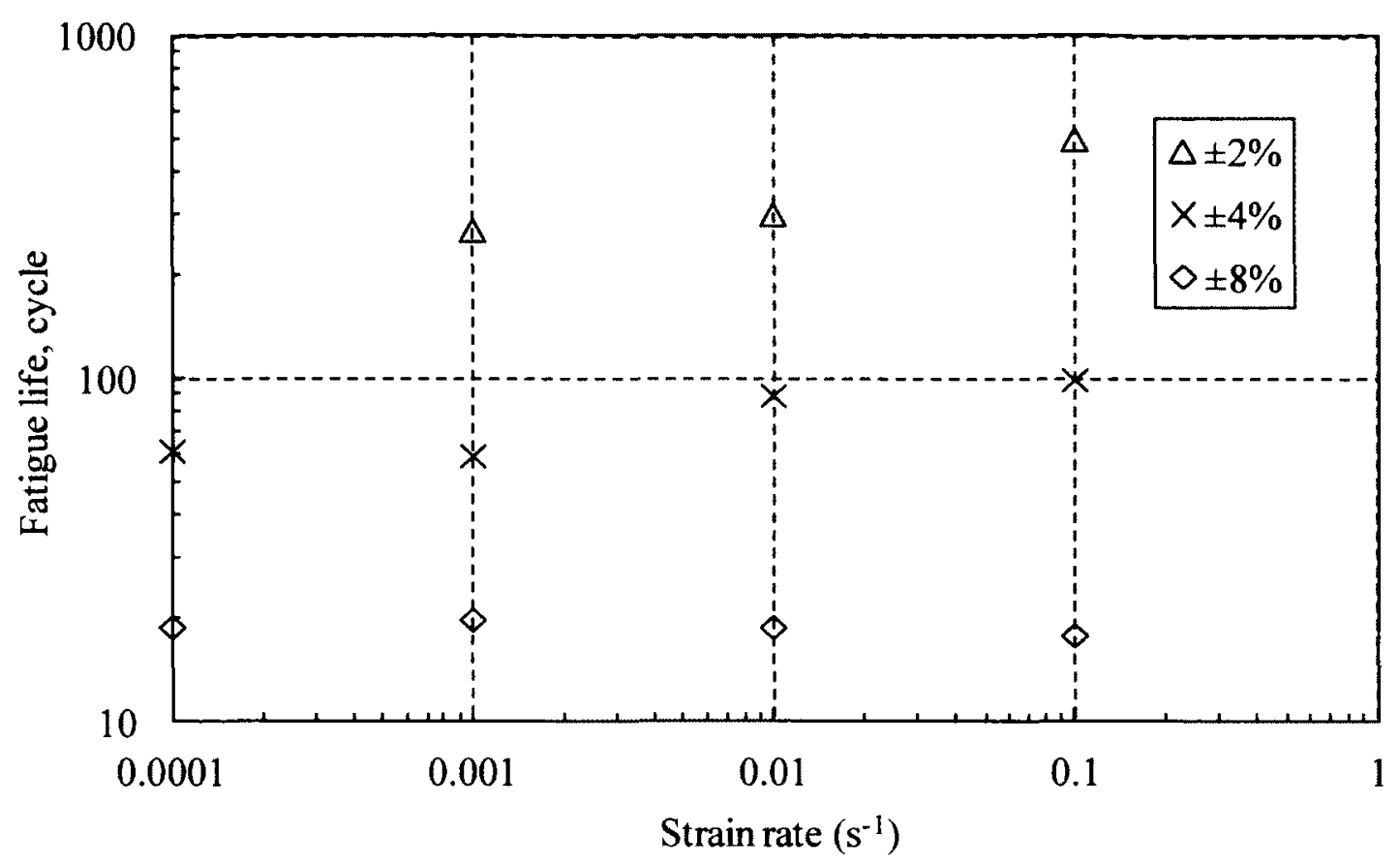

Figure 4.67 Fatigue life versus strain rate for material $\mathrm{A}$ at various target strain ranges

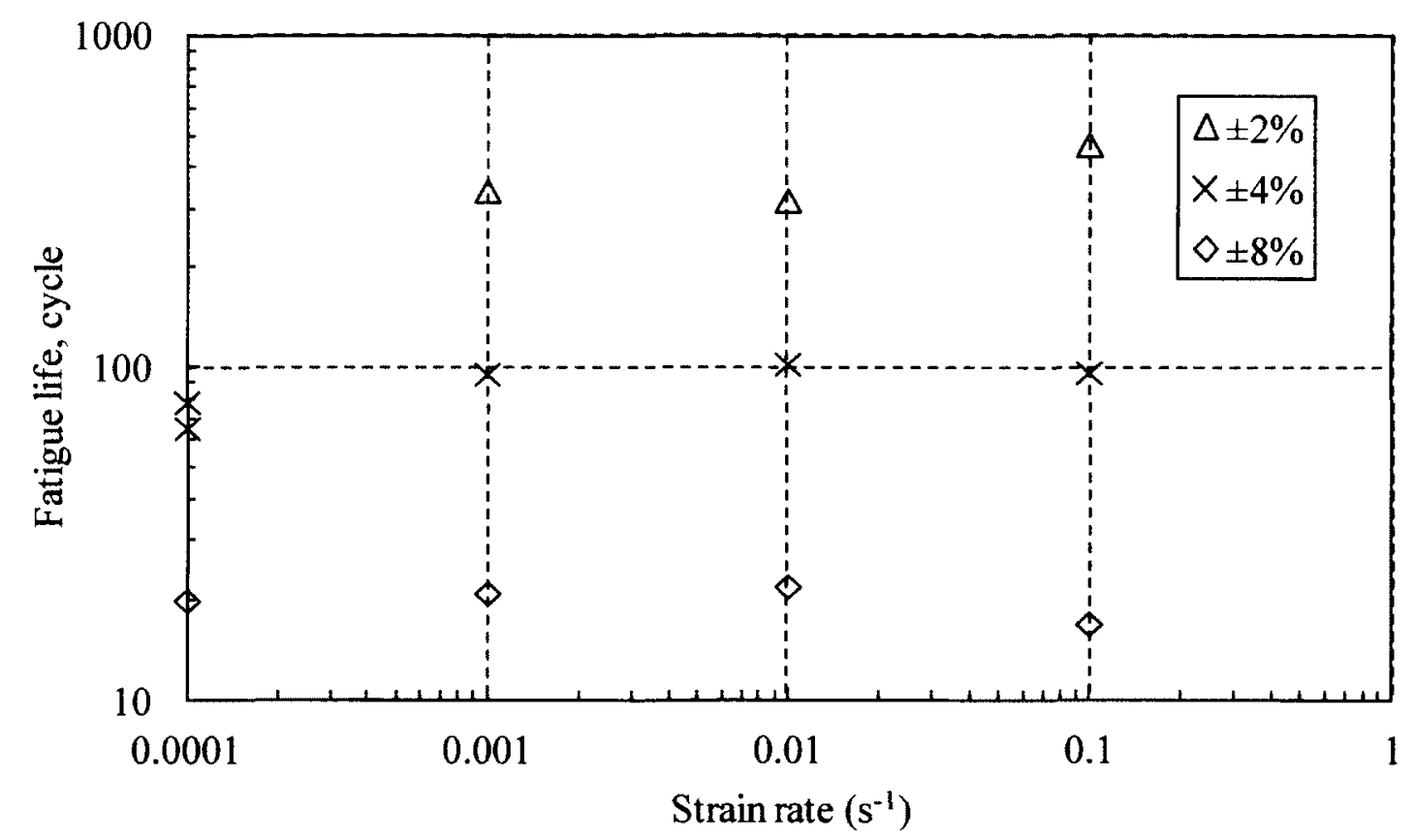

Figure 4.68 Fatigue life versus strain rate for material $\mathrm{B}$ at various target strain ranges 


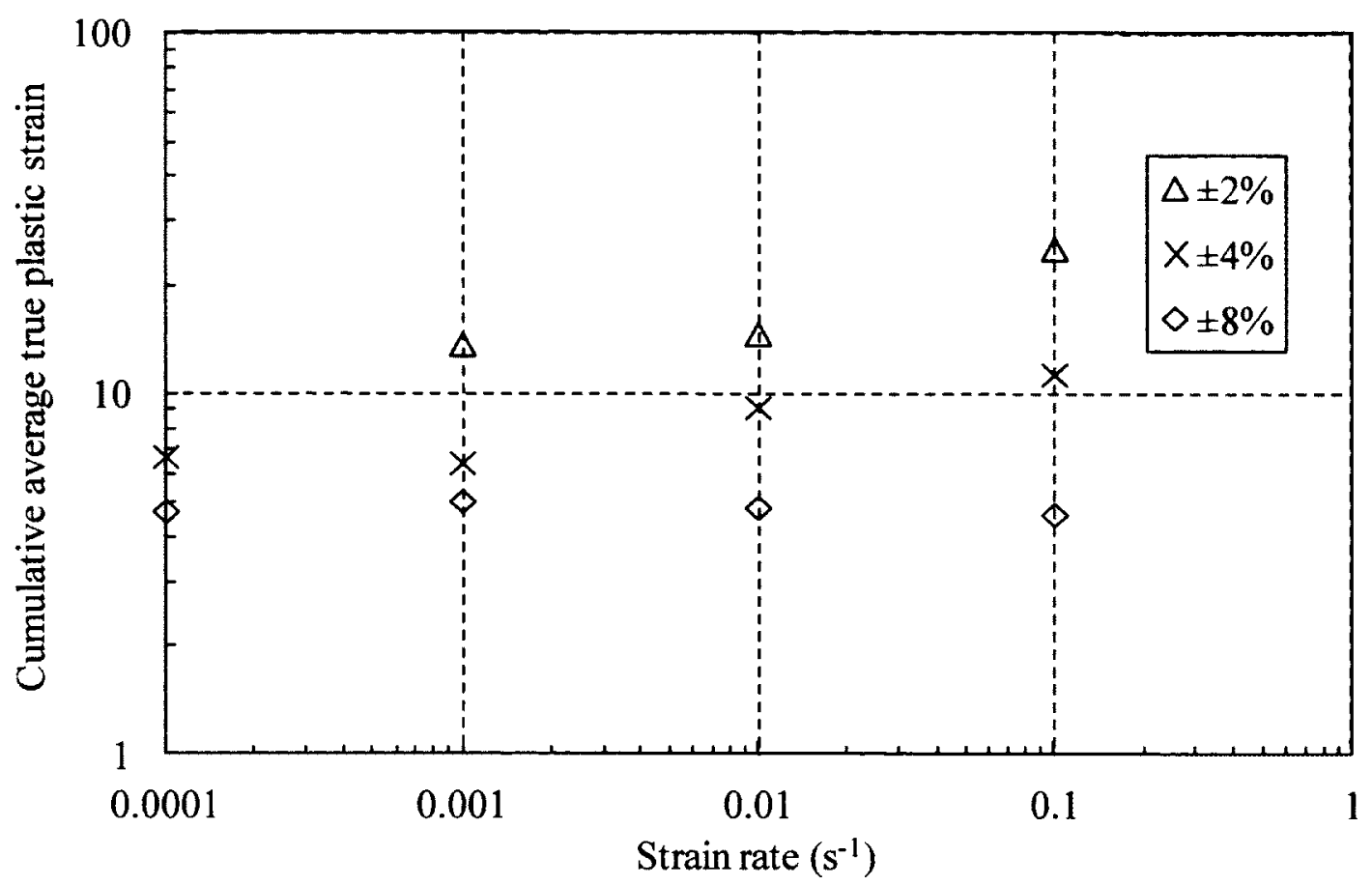

Figure 4.69 Cumulative average true plastic strain at failure versus strain rate for material $\mathrm{A}$ at different target strain ranges

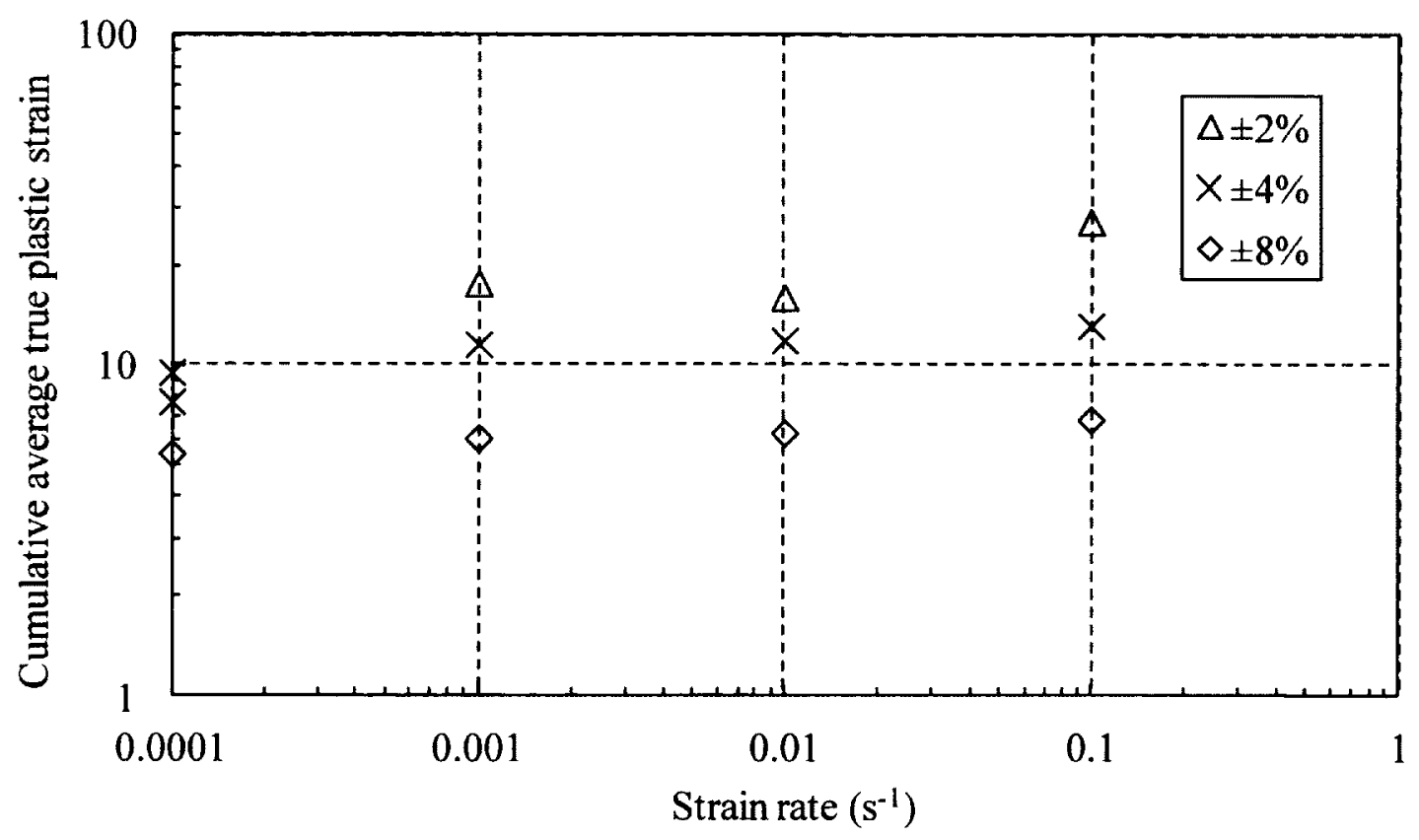

Figure 4.70 Cumulative true plastic strain at failure versus strain rate for material $\mathrm{B}$ at different target strain ranges 


\section{Chapter 5 Numerical Modelling of the Strain Rate Effects on Flow Stress}

Lemaitre and Chaboche (1990) have proposed a mixed-mode hardening plasticity model that uses multiple terms of backstress to define the non-linear movement of the yield surface centre. The model assumes that only the size of the yield surface is dependent on the strain rate while the movement of the yield surface centre is insensitive to the strain rate, but the effect of temperature has not been considered. Studies by Krempl (1979) and Chang and Lee (1987) in which a strain rate change was considered, have shown that the cyclic flow stress is affected by the current strain rate, but not the strain rate history. This implies that parameters for kinematic hardening in the plasticity model may be assumed to be independent of strain rate.

Numerical simulations of the tests have been carried out using the finite element software ABAQUS (Simulia 2010). Parameters $C_{n}$ and $\gamma_{n}$ from Eq. (2.18), which define the kinematic hardening, have been calibrated using a procedure developed by Wen (2012). This calibration procedure requires the use of both monotonic and cyclic test data. Since no monotonic tests have been included in the current experimental program, data from the monotonic tests by Chen (2010) on the same heat of steel used for the current cyclic tests have been used instead. Based on the results of the tests, a procedure has been developed to generate the strain rate dependent flow stress versus true plastic strain relationship to be used in numerical simulations. Results of numerical simulations are validated against results of the tests. 


\subsection{Monotonic Test Data Used for Calibration}

As stated above, monotonic test data used in calibrating parameters for the plasticity model have been taken from tests performed by Chen (2010). In that study, 9 $\mathrm{mm}$ round specimens that tapered to a minimum diameter of $8.82 \mathrm{~mm}$ at the centre of the reduced section were used. The specimens were fabricated from the same heat of mild steel used in the present test program. Monotonic tests were performed using axial extensometer control at the strain rate higher than $10^{-2} \mathrm{~s}^{-1}$. At the strain rates between $10^{-5}$ to $10^{-2} \mathrm{~s}^{-1}$, the test was carried out with axial extensometer control until the axial strain of 0.1 , at which point the loading was switched to diametral extensometer control. The monotonic tests by Chen (2010) can be found in Table 5.1. Materials A and B in the current study were designated as $\mathrm{G}$ and $\mathrm{H}$ respectively by Chen (2010). The final true stress versus cross-sectional area ratio curves for material $\mathrm{G}$ and $\mathrm{H}$ given by Chen (2010) can be seen in Figs. 5.1 and 5.2 respectively.

In order to use the test data given by Chen (2010) in calibrating the parameters for the plasticity model, it is necessary to correct the data to that of a uniaxial condition for the non-uniaxial stress state due specimen necking, the slight non-uniaxial stress state due to the tapered profile of the specimen and the measurement error caused by a misplaced diametral extensometer. These various corrections and finite element models used to arrive at the corrections are discussed below.

\subsubsection{Full and Half Gauge Length Tapered Model Comparison}

Finite element analysis (FEA) has been employed in an attempt to simulate the monotonic tests of Chen (2010). Since these tests are performed at a range of strain rates, 
it is desirable to use a time dependent analysis such that effects of strain rate on the flow stress of the materials are considered. This can be achieved through the use of a model that only considers the segment of the specimen that is within gauge length of the axial extensometer. The model of this type allows for the application of the exact displacement loading for a given test such that the actual displacement loading rate can be applied accurately in the simulation. However, since only the segment of the specimen within the gauge length of the axial extensometer is modelled, differences may be expected in the results between modelling the full specimen and modelling only a portion within the axial extensometer gauge length.

Using symmetry, half the specimen and half of the specimen length within the axial extensometer gauge have been modelled in ABAQUS (Simulia 2010) with CAX8R, an axisymmetric bi-quadratic element with reduced integration. Figure 5.3 shows the finite element mesh of the half model for the tapered specimen by Chen (2010). A mesh refinement has been provided around the mid-length of the specimen where necking occurs to improve the accuracy of the numerical simulation. Figure 5.4 shows the finite element mesh for the half gauge length tapered specimen model. The half model had the same mesh configuration as the half gauge length within the gauge length of the axial extensometer. A mesh convergence study in Appendix C for a $14 \mathrm{~mm}$ specimen model that has the same number of elements shows that further refinement to the mesh in Fig. 5.4 does not significantly change results of the numerical simulations. The material properties used in the numerical simulations have been taken from the true stress versus true plastic strain given by Chen (2010) for GT5E5ED, with the elastic modulus of $205600 \mathrm{MPa}$ and a Poisson's ratio of 0.3 . 
Comparisons of the predicted engineering stress versus cross-sectional area ratio curves calculated with Eqs. (4.2) and (4.5) with both models are shown in Fig. 5.5. Almost no difference can be seen between results of the numerical simulations carried out with the half model and the half gauge length model. Therefore, the half gauge length model can substitute the half model in carrying out the numerical simulations.

\subsubsection{Correction for Non-Uniaxial Stress State Due to Specimen Necking}

As the specimen necks with increasing extension, the state of stress at the region of necking always becomes increasingly non-uniaxial. Thus, the true stress is no longer given by Eq. (4.3) for defining the uniaxial true stress versus true strain relationship. However, Chen (2010) has proposed an equation to correct the calculated average true stress after the initiation of necking in calculating the uniaxial true stress versus true strain relationship as $\sigma_{t}^{\text {cor }}$ versus $\ln \left(\mathrm{A}_{\mathrm{o}} / \mathrm{A}\right)$. The corrected true stress is given by

$$
\sigma_{t}^{c o r}=0.5\left(\sigma_{t}^{a v g}\right)\left(1+\sqrt{\frac{A}{A_{p}}}\right)
$$

where $\sigma_{t}^{c o r}$ is the corrected true stress, $\sigma_{t}^{a v g}$ is the average measured true stress, $A$ is the current minimum cross-sectional area, $A_{p}$ is the minimum cross-sectional area at peak load and $A_{o}$ is the undeformed cross-sectional area. This correction is applied to the calculated average true stress after the peak load when necking has been initiated.

\subsubsection{Correction for Tapered Profile}

As test specimens were fabricated with a slight taper in the profile, the stress state of the specimen within the gauge length of the axial extensometer is not truly uniaxial. A 
slightly higher measured stress at a given axial strain is expected for a tapered specimen compared to a straight specimen as the taper increases the stress triaxiality $\left(\sigma_{m} / \sigma_{e q}\right)$, as given by Eq. (2.1). Therefore, a conversion factor is required to remove effects of the tapered profile on the measured stress when calculating the uniaxial true stress from the measured average stress with Eq. (4.3).

Numerical simulations have been carried out using the material properties in Section 5.1.1 to quantify the effect of the tapered profile on the measured stress. The half model of the tapered specimen used in the numerical simulation is shown in Fig 5.3. A similar meshing scheme without the taper has also been adopted to model the non-tapered (straight) specimen. Comparisons of the true flow stress versus true plastic strain curves, calculated with Eqs. (4.2), (4.3) and (4.6), and modified with Eq. (5.1) are shown in Fig. 5.6. Similarly, the engineering stress versus cross-sectional area ratio curves are shown in Fig. 5.7. It can be seen that the engineering stress and true flow stress of the tapered specimen are about $1 \%$ higher than that for the non-tapered specimen.

Since tapered specimens with a larger diameter are used in the current study for cyclic tests, it is desirable to determine if the same conversion factor is applicable for the larger diameter specimen. A short study to determine the conversion factor for the $14 \mathrm{~mm}$ diameter tapered specimen has been conducted and discussed in Appendix D. It has been found that the same conversion factor of $99 \%$ can be applied to the measured load to calculate the true flow stress and engineering stress of an equivalent non-tapered specimen. 


\subsubsection{Procedure for Generating Monotonic Flow Stress Versus True Plastic Strain Curves}

The calibration of the kinematic hardening parameters proposed by Wen (2012) and discussed in Appendix $G$ utilizes the quasi-static monotonic true flow stress versus true plastic strain curve (data) of a material for large strain. However, the monotonic tests performed by Chen (2010) only provide data for values of true plastic strain lower than 1.0. As well, some parts of the test data may not be representative of a material test at constant temperature due to adiabatic heating and the diametral extensometer not placed perfectly. For this reason, it might become necessary to approximate the monotonic test data with a function such that it may be used to generate the true (flow) stress-true plastic strain relationship up to a large strain.

As discussed above, some of the test data may not be representative of the actual material behaviour due to measurement errors such as the diametral extensometer not placed perfectly. This has manifested as a decreasing true stress with increasing true strain on the true stress versus true plastic strain curve near the end of the test. This effect is most obvious for GT1E4ED and HT1E4ED in Figs. 5.1 and 5.2 respectively. However, based on the test results, the placement of the diametral extensometer appears to be better for the $5 \times 10^{-5} \mathrm{~s}^{-1}$ strain rate tests compared to that for $10^{-4} \mathrm{~s}^{-1}$ strain rate tests. Furthermore, there is only expected to be a slight difference between flow stress of tests performed at strain rates of $5 \times 10^{-5} \mathrm{~s}^{-1}$ and $10^{-4} \mathrm{~s}^{-1}$. Therefore, GT5E5ED and HT5E5ED have been selected to represent the quasi-static monotonic test for both materials. Since the procedure to obtain the generated monotonic curve outlined below is the same for both materials, only the process to generate the curve for material $\mathrm{G}$ has been described in more detail in the following discussion. 
A true flow stress versus true plastic strain curve can be generated for the material using the modified Eq. (2.19) that takes into account of the yield plateau in the test data. This equation takes the form

$$
\sigma_{t}^{F}=\sigma_{t}^{y}+\sigma_{1}\left[1-\exp \left(\frac{-\left(\varepsilon_{t}^{p}-\varepsilon_{t}^{o}\right)^{d}}{\varepsilon_{c}}\right)\right] \text { for }\left(\varepsilon_{t}^{p}-\varepsilon_{t}^{o}\right)>0
$$

where $\varepsilon_{t}^{o}$ is the true plastic strain at the end of the yield plateau. In an attempt to fit the test data, the true flow stress is constrained to the value of yield stress from the test at a true plastic strain of 0 and a trial stress at a true plastic strain of 5.0. It has been found in the cyclic test that peak tensile stress per cycle does not vary much after a cumulative strain of 1.0 up to 5.0, as can be seen in Figs. 4.51 for tests at strain rates of $10^{-4}$ and $10^{-3}$ $\mathrm{s}^{-1}$, and the strain range of $\pm 4 \%$ for material $\mathrm{A}$. The measured yield stress used to constrain the curve at 0 true plastic strain for all monotonic tests can be found in Table 5.2. A least-square error fitting approach is used to find values of parameters $d, \varepsilon_{c}$ and $\sigma_{1}$ that minimize the difference between the test data and the generated true flow stress from the true plastic strain of 0 to 0.15 . The range of test data at which the equation is required to fit affects the shape of the generated curve. If a higher upper limit of true plastic strain is considered, the saturation of the generated curve usually occurs at a higher strain. If a lower limit is used, the generated curve may not capture the material behaviour before peak load occurs. For the materials $\mathrm{G}$ and $\mathrm{H}$, peak load has been found to occur at an approximate true plastic strain of 0.15 or slightly lower. Since the measured true (flow) stress versus true plastic strain curve before peak load can be assumed to be relatively accurate, an upper limit of true plastic strain of 0.15 was imposed when fitting through the test data with Eq. (5.2). 
Numerical simulations have been carried out with ABAQUS (Simulia 2010) with the generated curves and a Poisson's ratio of 0.3 using the half gauge length model of the tapered specimen shown in Fig. 5.4. Since the elastic modulus is not affected by strain rate, the average measured from the tests by Chen (2010) of $208000 \mathrm{MPa}$ has been used as the elastic modulus. Iterations are performed by changing the trial stress at a true plastic strain of 5.0 until a good agreement between the measured and predicted engineering stress versus cross-sectional area ratio curves considered to have been achieved visually. Using the method outlined above, true stresses of $765 \mathrm{MPa}$ and 781 $\mathrm{MPa}$ at the true plastic strain of 5.0 have been found to be able to generate the true flow stress versus true plastic strain curve that can closely simulate the load deformation behaviour of GT5E5ED and HT5E5ED respectively. Results of the analyses on the predicted engineering stress versus cross-sectional area ratio curves using an input curve with these stresses at a true plastic strain of 5.0 along with those when the stresses vary by $\pm 10 \mathrm{MPa}$ are shown in Figs. 5.8 and 5.9 . It can be seen that changing the stress at the true plastic strain of 5.0 by $10 \mathrm{MPa}$ only has a slight effect on the predicted curve.

The procedure to determine parameters for generating the true flow stress versus true plastic strain curves at the higher strain rate is similar to that for GT5E5ED. However for higher strain rates, a time dependent analysis has to be considered. Thus, another procedure has been used to estimate the true flow stress at a true plastic strain of 5.0 to generate the true flow stress versus true plastic strain at other strain rates. It can be assumed that there is a difference in the flow stress between various strain rates up to a true plastic strain of 5.0. This can be seen in the test data in Fig. 4.51 where there is a nearly constant difference in the peak tensile engineering stress per cycle between 
ANE3R4 and ANE4R4 from the $5^{\text {th }}$ up to approximately the $50^{\text {th }}$ cycle. The accumulated true plastic strain is approximately 5.0 at the $50^{\text {th }}$ cycle of loading. Thus, it may be possible to use the difference in the (saturated) yield stress between various strain rates to help define the true flow stress at the plastic strain of 5.0 in generating the true flow stress versus true plastic strain curves at strain rates other than $5 \times 10^{-5} \mathrm{~s}^{-1}$. It has been found that the difference in the saturated yield stress between various strain rates can be approximated with results from monotonic tension and cyclic loading tests using Eq. (2.8), the power law equation proposed by Cowper and Symonds (1957). Thus, the difference between the true flow stress at the true plastic strain of 5.0 used in generating the true flow stress versus true plastic strain curve can be taken as the difference between the generated (saturated) yield stress between various strain rates, as shown in Table 5.3. The true flow stress at the true plastic strain of 5.0 for any strain rate can be calculated by adding the difference in the generated (saturated) yield stress to that at the strain rate of $5 \times 10^{-5} \mathrm{~s}^{-1}$ to $765 \mathrm{MPa}$ for material $\mathrm{G}$ and $781 \mathrm{MPa}$ for material $\mathrm{H}$. The procedure to calculate difference in the generated (saturated) yield stress is discussed in greater detail in Appendix $\mathrm{E}$.

The generated true flow stress versus true plastic strain curves for material $G$ (A) and $H(B)$ together with the curves calculated directly from the test data are shown in Figs. 5.10 and 5.11 respectively. Parameters $\sigma_{t}^{y}, d, \varepsilon_{c}$ and $\sigma_{1}$ together with the true (flow) stress at the true plastic strain of 5.0 used in generating the monotonic true flow stress versus true plastic strain curves can be found in Table 5.2. Results of the numerical simulations are compared to the measured engineering stress versus cross-section area ratio curves in Figs. F.1 to F.10 of Appendix F. Generally, good agreement has been 
achieved between the test and predicted engineering stress versus cross-sectional change curves for monotonic tension tests of tapered specimens except after the peak load in tests at strain rates of $10^{-1} \mathrm{~s}^{-1}$ and $10^{0} \mathrm{~s}^{-1}$ where adiabatic heating can be expected and at $10^{-4} \mathrm{~s}^{-1}$ where the diametral extensometer may have been slightly more misplaced than other tests.

\subsection{Modelling the Effect of Strain Rate for a Mixed-Mode Hardening Plasticity Model}

As stated earlier, the yield stress (size of the yield surface) is dependent on the strain rate while the kinematic hardening term is insensitive. Therefore, the numerical simulation of a non-quasi-static test requires the definition of a series of yield stress versus true plastic strain curves for each strain rate as inputs while the kinematic hardening parameters can be considered to remain the same for all strain rates. Figure 5.12 shows the relationship between the true flow stress, yield stress and backstress according to Eq. (2.18) under a monotonic uniaxial constant strain rate loading condition. Based on this relationship, a unique yield stress versus true plastic strain curve can be established for each strain rate from the true flow stress versus true plastic strain curve at that strain rate and the backstress versus true plastic strain curve.

Monotonic true flow stress versus true plastic strain curves have been generated for each strain rate as outlined in Section 5.1.4. Since the kinematic hardening parameters have been considered to be strain rate insensitive, data from the quasi-static tests may be used to calibrate and optimize the kinematic hardening parameters to be used in the numerical simulations of problems involving non-static loading conditions. For this reason, the procedure proposed by Wen (2012) to calibrate and optimize the kinematic 
hardening parameters under a static loading condition has been adopted for this study. The calibration and optimization involved using test data of ATE4D and ANE4R2, and the generated monotonic true flow stress versus true plastic strain curve for GT1E4ED (at the strain rate of $10^{-4} \mathrm{~s}^{-1}$ ) for material A, and the corresponding BTE4D, BNE4R2 and HT1E4ED for material B. The detailed calibration and optimization procedure of the kinematic hardening parameters has been outlined in Appendix G. It has been noted by Wen (2012) that using only two kinematic hardening terms is sufficient to model the evolution of the backstress in the numerical simulations. Thus, two kinematic hardening terms have been used in this study.

The kinematic hardening parameters optimized for materials A and B are shown in Table 5.4. Using the optimized kinematic hardening parameters, the backstress versus true plastic strain curve under monotonic uniaxial loading can be generated using Eq. (2.18). The yield stress versus true plastic strain curves calculated by subtracting the backstress from the generated true flow stress versus true plastic strain curves for the strain rates of $5 \times 10^{-5} \mathrm{~s}^{-1}$ to $10^{0} \mathrm{~s}^{-1}$ are shown in Figs. 5.13 and 5.14 for material $\mathrm{A}$ and B, respectively.

\subsection{Validation of Optimized Yield Stress-Kinematic Hardening Parameters (Plasticity Model)}

A procedure for a static analysis has been adopted from Wen (2012) to calibrate and optimize the kinematic hardening parameters to be used in time dependent analyses. This procedure is being validated against results of selected tests through numerical simulations using the optimized yield stress-kinematic hardening parameters in Table 5.4 


\subsubsection{Numerical Simulations of the Cyclic Tests Used to Calibrate and Optimize the Kinematic Hardening Parameters}

Figures 5.15 and 5.16 show the test and predicted engineering stress versus engineering strain curves for ATE4D and BTE4D using the optimized yield stresskinematic hardening parameters listed in Table 5.4. A slight disagreement can be seen between the test and predicted curves upon yielding during loading reversals for both $\pm 2 \%$ and $\pm 0.5 \%$ strain ranges. Results of the numerical simulations show a well defined point at which the linear elastic limit has been reached and the non-linear behaviour begins, while the test data show a more gradual yielding with no clearly defined starting point. On the other hand, there is a good agreement between the test and predicted engineering stress versus engineering strain curves immediately after yielding. It is possible that a better agreement can be achieved if more terms of backstress are considered and a lower yield stress is assumed in calibrating the kinematic hardening parameters. However, Wen (2012) has shown that using more than two backstress terms did not significantly improve the overall prediction of the numerical simulation compared to the test, and using a smaller yield stress in the calibration of the kinematic hardening parameters improved the prediction at yielding (elbow of the curve), but worsened at the other region. Thus, two backstress terms have been deemed adequate to represent kinematic hardening and the optimized assumed yield stress acceptable to be used in calibrating the kinematic hardening parameters, even though there will be some disagreement at the elbow of the test and predicted stress-strain curve. Furthermore, the region of disagreement is small compared to the rest of the region if the loading strain range is sufficiently large (greater than $\pm 0.5 \%$ ). 
When the strain range decreases from $\pm 2 \%$ to $\pm 0.5 \%$, the predicted engineering stress versus engineering strain curve appears to have stabilized within only one to two loading cycles after the strain range reduction. However, there is a more gradual relaxation of the tensile stress in which more loading cycles are required for the curve to stabilize in the test. Nevertheless, there is a good agreement between the test and predicted engineering stress versus engineering strain curves at the $10^{\text {th }}$ cycle of the $\pm 0.5 \%$ loading strain range for both materials $\mathrm{A}$ and $\mathrm{B}$.

Test and predicted engineering stress versus engineering strain, and engineering stress versus cross-section area ratio curves for the notched specimens based on the final optimized parameters are shown in Figs. 5.17 and 5.18 for ANE4R2, and Figs. 5.19 and 5.20 for BNE4R2. Unlike the tapered specimens, the predicted engineering stress versus engineering strain curves for the notched specimens agree very well with the test results for both materials A and B even at the elbow of the curve. There is also a good agreement between the test and predicted engineering stress and cross-sectional area ratio (average true strain) curves at the initial first few loading cycles. However, the curves start to deviate along the $\ln \left(\mathrm{A}_{\mathrm{o}} / \mathrm{A}\right)$ axis as the number of loading cycle increases, similar to the findings by Wen (2012). This may be resolved by using a different or an improved plasticity model. However, it is not known if any proposed plasticity model has been found to be able to predict both the measured engineering stress versus engineering strain and engineering stress versus cross-sectional area ratio, particularly for notched specimens over many cycles of plastic deformation. Nevertheless, for most applications, the accuracy of the predicted stress and strain in the axial direction is much more important that the deformation in the transverse direction. In view of this, the validation 
of the procedure to calibrate and optimize the yield stress-kinematic hardening parameters will focus mainly on the engineering stress versus engineering strain curves. It is expected that the predicted engineering stress versus cross-sectional area ratio curve will not agree very well with that of the test using the mixed-mode hardening plasticity model by Lemaitre and Chaboche (1990) in the numerical simulations, and the calibration and optimization procedure adopted.

\subsubsection{Numerical Simulations of Other Tests}

Effects of strain rate on the flow stress are considered in the numerical simulations using the procedure described in Section 5.2. The main assumption made in this procedure is that the yield stress is dependent on the strain rate while the kinematic hardening term is insensitive. From this procedure, yield stress versus true plastic strain curves have been generated using the optimized kinematic hardening parameters for a strain rate of $5 \times 10^{-5} \mathrm{~s}^{-1}$ to $10^{0} \mathrm{~s}^{-1}$. The yield stress versus true plastic strain curves at these strain rates can be found in Figs. 5.13 and 5.14 for material A and B respectively. In the numerical simulation, the material properties inputs on the yield stress versus true plastic strain curve at a higher strain rate has to be higher than that at a lower strain rate in order to have a unique yield stress for a strain increment. Otherwise, computational difficulties may arise if there can be more than one yield stress. Since the effects of strain rate on the yield stress is small at a strain rate below $10^{-4} \mathrm{~s}^{-1}$, the generated yield stress versus true plastic strain curves at $10^{-4} \mathrm{~s}^{-1}$ strain rate may occasionally drop below that at $5 \times 10^{-5} \mathrm{~s}^{-1}$. For the purpose of carrying out the numerical simulations, the yield stress versus true plastic strain curve at $5 \times 10^{-5} \mathrm{~s}^{-1}$ strain is taken as no less than $1.9 \mathrm{MPa}$ and $0.9 \mathrm{MPa}$ 
smaller than the curve at $10^{-4} \mathrm{~s}^{-1}$ for materials $\mathrm{A}$ and $\mathrm{B}$ respectively. The stresses of 1.9 $\mathrm{MPa}$ and $0.9 \mathrm{MPa}$ being the differences in saturated yield stress between the strain rates of $5 \times 10^{-5} \mathrm{~s}^{-1}$ and $10^{-4} \mathrm{~s}^{-1}$ for materials $\mathrm{A}$ and $\mathrm{B}$. For the numerical simulation performed at the strain rate of $10^{-2} \mathrm{~s}^{-1}$ and higher, some difficulties have been encountered in reaching convergence in the numerical solution due to the small difference between yield stress versus true plastic strain curves at strain rates of $5 \times 10^{-5} \mathrm{~s}^{-1}$ and $10^{-4} \mathrm{~s}^{-1}$. For this reason, the yield stress versus true plastic strain data at the strain rate of $5 \times 10^{-5} \mathrm{~s}^{-1}$ have been omitted in the material properties data input for numerical simulations at the strain rate of $10^{-2} \mathrm{~s}^{-1}$ and higher. Since little difference exists between the yield stress versus true plastic strain curves at the strain rates $5 \times 10^{-5} \mathrm{~s}^{-1}$ and $10^{-4} \mathrm{~s}^{-1}$, no significant change in the simulation results are expected from the omission of the $5 \times 10^{-5} \mathrm{~s}^{-1}$ strain rate curve.

For simulations performed at the $10^{-1} \mathrm{~s}^{-1}$ strain rate, convergence could not be obtained unless the yield stress versus true plastic strain curves have been defined only for the $10^{-3}$ to $10^{0} \mathrm{~s}^{-1}$ strain rates. Even with this consideration, the simulation could only be completed for some specific time steps that are too large to show sufficient resolution in the output. However if a static analysis is considered, a smaller time step can be used and a more detailed representation of the simulation can be obtained. Figure 5.21 shows the engineering stress versus engineering strain curves and Fig. 5.22 shows the average true stress versus average true strain curve for the numerical simulations of ATE1D performed with a rate dependent analysis where the yield stress versus true plastic strain curves between the strain rates of $10^{-3}$ to $10^{0} \mathrm{~s}^{-1}$ have been defined and a static analysis where only the curve at the $10^{-1} \mathrm{~s}^{-1}$ strain rate has been taken as the static curve. Since the difference between results of the time dependent analysis and the static analysis is small, 
static analyses have been adopted for simulations of tests performed at the $10^{-1} \mathrm{~s}^{-1}$ strain rate.

As noted previously, the elastic modulus is not affected by the strain rate. Thus, the averaged measured by Chen (2010) of $208000 \mathrm{MPa}$ has been used as the elastic modulus and the Poisson's ratio taken as 0.3 .

\subsubsection{Numerical Simulations of D Series Tests (Tapered Specimens)}

The numerical simulations of the D series tests at the strain rates of $10^{-3}$ to $10^{-1} \mathrm{~s}^{-1}$ have been carried out using the half gauge length model of the tapered specimen shown in Fig. 5.4 to validate the proposed procedure for generating the input data that can account for effects of strain rate in numerical simulations. Figures 5.23 to 5.28 show the test and predicted cyclic engineering stress versus engineering strain curves for ATE3D, ATE2D and ATE1D respectively for material A. Besides ATE1D, there is good agreement between the test and predicted engineering stress versus engineering strain curves other than at the elbow of the stress-strain curve and the rate of stress relaxation at $\pm 0.5 \%$ loading strain range during cyclic loading, similar to ATE4D. There is also good agreement between the test and predicted engineering stress versus engineering strain curves when the specimens were being pulled to fracture.

For ATE1D, the temperature in the specimen increased considerably towards the end of the test due to adiabatic heating. Since the constitutive model does not take into account the temperature effect, the numerical simulation expectedly over-estimates the stress towards the end of the test, as shown in Figs. 5.25 and 5.28. However, there is a good agreement between the test and predicted engineering stress versus engineering 
strain curves at the first few cycles of $\pm 2 \%$ loading strain range before the effect of temperature rise due to adiabatic heating becomes significant. Results similar to that for ATE3D, ATE2D and ATE1D of material A can be seen for BTE3D, BTE2D and BTE1D of material B in Figs. F.11 to F.16 of Appendix F.

\subsubsection{Numerical Simulations of Notched Specimen Tests at $\pm 2 \%$ Strain Range}

Numerical simulations of the notched specimen tests for the strain rates of $10^{-3}$ to $10^{-1} \mathrm{~s}^{-1}$ at the $\pm 2 \%$ strain range have been performed for 50 cycles with the half gauge length model shown in Fig. G.4 of Appendix $G$ for the validation of the proposed procedure. Test and predicted peak tensile engineering stress versus cycle number of these tests are shown in Figs. 5.29 and 5.30 for material A and B respectively. For both materials $\mathrm{A}$ and $\mathrm{B}$, the simulations slightly overestimate the peak tensile engineering stress to a maximum of about $10 \mathrm{MPa}$ at the first few cycles of loading. This is small relative to the peak tensile engineering stress. Overall, there is a good agreement between the test and predicted peak tensile engineering stress at loading strain rates of $10^{-4} \mathrm{~s}^{-1}$ and $10^{-3} \mathrm{~s}^{-1}$. However, the difference starts to increase with the loading cycle for tests at $10^{-2}$ $\mathrm{s}^{-1}$ and $10^{-1} \mathrm{~s}^{-1}$, with the tests at $10^{-1} \mathrm{~s}^{-1}$ having a larger difference. This is expected since the temperature rise due to adiabatic heating increases with the rate of loading and duration of loading.

Test and predicted engineering stress versus engineering strain curves for ANE3R2, ANE2R2 and ANE1R2 are shown in Figs. 5.31 to 5.33. It can be seen that there is a good agreement between test and predicted curves for ANE3R2. However, the prediction deteriorates with the increase in the loading cycle for ANE2R2 and ANE1R2, 
with ANE1R2 being worse than ANE2R2. Similar results can be seen in the test and predicted engineering stress versus engineering strain curves for BNE3R2, BNE2R2 and BNE1R2 of material B in Figs. F.17 to F.19 of Appendix F.

\subsubsection{Effects of Strain Range and Mean Strain in Numerical Simulations}

Numerical simulations of a number of tapered and notched specimen tests were carried out to determine if effects of cyclic mean strain and strain range can be accounted for using input data obtained from the calibration and optimization procedures in Section 5.2. The tests considered are ATE2I, ATE3I, BTE3I, ATE2C-20, ATE2E, ANE4R4 and ANE4R8.

\subsubsection{Numerical Simulations of ATE2I, ATE3I, BTE3I, ANE4R4 and ANE4R8}

Tapered specimens in I test series were loaded for 10 cycles at each incremental strain range that varied from $\pm 0.3 \%$ to $\pm 2 \%$. Results of the test and predicted engineering stress versus engineering strain curves of ATE3I and ATE2I are shown in Figs. 5.34 to 5.37 respectively. It can be seen that the numerical simulation slightly overestimates the engineering stress for all strain ranges except for $\pm 2 \%$. However, there is a good agreement between the test and predicted curves for $\pm 2 \%$ strain range and when the specimens were subsequently pulled to fracture. A similar result to that of ATE3I can be seen for BTE3I in Figs. F.20 and F.21 of Appendix F.

Notched specimens were tested at a constant strain range until fracture. Test and predicted engineering stress versus engineering strain curves of ANE4R4 and ANE4R8 are shown in Figs. 5.38 and 5.39 respectively. It can be seen that the numerical 
simulation slightly underestimates the stress for ANE4R4 but the underestimation is larger for ANE4R8. It appears that the numerical simulation underestimates the stress when the applied strain range is larger than the value used in the calibration of kinematic hardening parameters, and overestimates when the applied strain range is smaller. Thus, additional study is required to determine if the numerical simulation can be improved to model the response for different loading strain ranges by using more backstress (kinematic hardening) terms and/or using test data from more strain ranges in calibrating the kinematic hardening parameters.

\subsubsection{Numerical Simulation of ATE2E}

For ATE2E, the test involved cyclically loading the specimen at the strain rate of $10^{-2} \mathrm{~s}^{-1}$ for 20 cycles at each pass of the strain range that includes twice at the cyclic strain range sequence of $\pm 1 \%, 0 / 2 \%, \pm 1 \%$ and $-2 / 0 \%$, and to be followed by four times at the strain range sequence of $0 / 2 \%$ and $-2 / 0 \%$. In this test, the strain range remains at $2 \%$, while the cyclic mean strain varies. The test and predicted average true stress versus loading cycle plots are shown in Figs. 5.40. Results of the numerical simulation adjusted for the overestimation of the stress range $(51 \mathrm{MPa})$ for ATE2I at the $\pm 1 \%$ strain range are also shown in Fig 5.40. The solid marker in Fig. 5.40 represents the presumed stabilized

measured average true stress range at the $20^{\text {th }}$ (last) cycle of each loading strain range. It can be seen in Fig. 5.40 that the adjusted curve is close to the average stabilized true stress range of $0 / 2 \%$ and $-2 / 0 \%$. There is also only small difference between the adjusted curve and the stabilized average true stress range. Thus, the error by neglecting the effect of cyclic mean strain in the constitutive modelling can be considered to be small. 


\subsubsection{Numerical Simulation of ATE2C-20}

ATE2C-20 considered changes in both strain range and mean strain. The test and predicted results of the average true stress versus cycle number are shown in Fig. 5.41. There is good agreement between the test and predicted average true stress range at $4 \%$ strain range $(0 / 4 \%$ and $\pm 2 \%)$ at both mean cyclic strains of $2 \%(0 / 4 \%)$ and zero mean strain $( \pm 2 \%)$. However, the numerical simulation overestimates the average true stress range at the applied strain range of $2 \%(0 / 2 \%$ and $\pm 1 \%)$. These are consistent with results of ATE2I in the overestimation of the stress at a strain range of $\pm 1 \%$ and ATE2E in showing that the effect of mean cyclic strain on a stabilized average true stress range is small.

\subsection{Summary of Numerical Simulations}

Overall, the proposed procedure is able to provide the material properties input data that can account for effects of strain rate up to $10^{-1} \mathrm{~s}^{-1}$ in the numerical simulations with the absence of significant adiabatic heating. Although still reasonable, the numerical simulation is less accurate when the loading involved conditions other than what has been used in the calibration of the kinematic hardening parameters. The peak stress is overestimated by the numerical simulation if the applied strain range is below the strain range used in the calibration and underestimated if it is above. However, the error on the stabilized true stress versus true strain curve associated with ignoring the effect of mean cyclic strain in numerical simulation (constitutive modelling) is small. As expected, the numerical simulation also overestimates the stress in tests at high strain rate where there is considerable temperature rise due to adiabatic heating. With the calibrated material 
properties for different strain rates, the mixed-mode hardening model can be used to simulate the structural response under a general loading condition, such as that in an earthquake. 
Table 5.1 Monotonic tests by Chen (2010)

\begin{tabular}{|c|c|c|}
\hline Specimen & $\begin{array}{c}\text { Nominal loading rate } \\
\left(\mathrm{s}^{-1}\right)\end{array}$ & Loading Control $^{2}$ \\
\hline GT1E5E & $1.00 \mathrm{E}-05$ & $\mathrm{E}=0.00001 \mathrm{~s}^{-1}$ \\
\hline GT5E5ED & $5.00 \mathrm{E}-05$ & $\mathrm{E}=0.00005 \mathrm{~s}^{-1}, \mathrm{D}=0.0001735 \mathrm{~mm} / \mathrm{s}$ \\
\hline GT1E4ED & $1.00 \mathrm{E}-04$ & $\mathrm{E}=0.0001 \mathrm{~s}^{-1}, \mathrm{D}=0.0003471 \mathrm{~mm} / \mathrm{s}$ \\
\hline GT1E3ED & $1.00 \mathrm{E}-03$ & $\mathrm{E}=0.001 \mathrm{~s}^{-1}, \mathrm{D}=0.003471 \mathrm{~mm} / \mathrm{s}$ \\
\hline GT1E2ED & $1.00 \mathrm{E}-02$ & $\mathrm{E}=0.01 \mathrm{~s}^{-1}, \mathrm{D}=0.03471 \mathrm{~mm} / \mathrm{s}$ \\
\hline GT1E1E & $1.00 \mathrm{E}-01$ & $\mathrm{E}=0.1 \mathrm{~s}^{-1}$ \\
\hline GT1E0E & $1.00 \mathrm{E}+00$ & $\mathrm{E}=1 \mathrm{~s}^{-1}$ \\
\hline HT1E5E & $1.00 \mathrm{E}-05$ & $\mathrm{E}=0.00001 \mathrm{~s}^{-1}$ \\
\hline HT5E5ED & $5.00 \mathrm{E}-05$ & $\mathrm{E}=0.00005 \mathrm{~s}^{-1}, \mathrm{D}=0.0001735 \mathrm{~mm} / \mathrm{s}$ \\
\hline HT1E4ED & $1.00 \mathrm{E}-04$ & $\mathrm{E}=0.0001 \mathrm{~s}^{-1}, \mathrm{D}=0.0003471 \mathrm{~mm} / \mathrm{s}$ \\
\hline HT1E3ED & $1.00 \mathrm{E}-03$ & $\mathrm{E}=0.001 \mathrm{~s}^{-1}, \mathrm{D}=0.003471 \mathrm{~mm} / \mathrm{s}$ \\
\hline HT1E2ED & $1.00 \mathrm{E}-02$ & $\mathrm{E}=0.01 \mathrm{~s}^{-1}, \mathrm{D}=0.03471 \mathrm{~mm} / \mathrm{s}$ \\
\hline HT1E1E & $1.00 \mathrm{E}-01$ & $\mathrm{E}=0.1 \mathrm{~s}^{-1}$ \\
\hline HT1E0E & $1.00 \mathrm{E}+00$ & $\mathrm{E}=1 \mathrm{~s}^{-1}$ \\
\hline
\end{tabular}

Notes:

$1 \mathrm{G}$ is material $\mathrm{A}, \mathrm{H}$ is material $\mathrm{B}, \mathrm{T}$ is tapered specimen, $(1$ or 5$) \mathrm{E}(0-5)$ is target strain rate, $\mathrm{ED}$ is test that make the transition from axial extensometer strain rate control to diametral strain rate control at the axial strain of $0.1, \mathrm{E}$ is axial extensometer strain rate control only test.

$2 \mathrm{E}$ is the target axial strain rate, $\mathrm{D}$ is the target diameter change rate. 
Table 5.2 Parameters for generating the corrected monotonic true flow stress versus true plastic strain curves

\begin{tabular}{|c|c|c|c|c|c|}
\hline Specimen & $\begin{array}{c}\sigma_{t}^{y} \\
(\mathrm{MPa})\end{array}$ & $\begin{array}{c}\sigma_{1} \\
(\mathrm{MPa})\end{array}$ & $\varepsilon_{c}$ & $d$ & $\begin{array}{c}\text { True stress at a true } \\
\text { plastic strain of 5.0 } \\
(\mathrm{MPa})\end{array}$ \\
\hline GT5E5ED & 352.9 & 412.1 & 0.229 & 0.6906 & 765.0 \\
\hline GT1E4ED & 353.8 & 413.1 & 0.215 & 0.7157 & 766.9 \\
\hline GT1E3ED & 360.1 & 414.5 & 0.223 & 0.6938 & 774.6 \\
\hline GT1E2ED & 382.3 & 402.4 & 0.254 & 0.6253 & 784.7 \\
\hline GT1E1E & 399.4 & 398.5 & 0.232 & 0.6671 & 797.8 \\
\hline GT1E0E & 420.0 & 395.0 & 0.214 & 0.7019 & 815.0 \\
\hline HT5E5ED & 383.4 & 397.6 & 0.236 & 0.6595 & 781.0 \\
\hline HT1E4ED & 383.2 & 398.7 & 0.231 & 0.6649 & 781.9 \\
\hline HT1E3ED & 388.4 & 398.0 & 0.197 & 0.7162 & 786.4 \\
\hline HT1E2ED & 396.9 & 397.4 & 0.218 & 0.6784 & 794.3 \\
\hline HT1E1E & 416.2 & 392.3 & 0.196 & 0.7216 & 808.5 \\
\hline HT1E0E & 438.7 & 395.3 & 0.211 & 0.6888 & 834 \\
\hline
\end{tabular}

Table 5.3 Difference in the generated (saturated) yield stress at various strain rates to that at the strain rate of $5 \times 10^{-1} \mathrm{~s}^{-1}$

\begin{tabular}{|c|c|c|}
\hline \multirow{2}{*}{$\begin{array}{c}\text { Strain Rate } \\
\left(\mathrm{s}^{-1}\right)\end{array}$} & \multicolumn{2}{|c|}{$\begin{array}{c}\text { Difference to } 5 \times 10^{-5} \mathrm{~s}^{-1} \\
(\mathrm{MPa})\end{array}$} \\
\cline { 2 - 3 } & $\begin{array}{c}\text { Material G } \\
(\mathrm{A})\end{array}$ & $\begin{array}{c}\text { Material H } \\
(\mathrm{B})\end{array}$ \\
\hline 0.00005 & 0.0 & 0.0 \\
\hline 0.0001 & 1.9 & 0.9 \\
\hline 0.001 & 9.6 & 5.4 \\
\hline 0.01 & 19.7 & 13.3 \\
\hline 0.1 & 32.8 & 27.5 \\
\hline 1 & 50.0 & 53.0 \\
\hline
\end{tabular}

Table 5.4 Optimized $\sigma_{S}^{o}, \sigma_{L}^{o}, C_{n}$ and $\gamma_{n}$ parameters for materials A and B

\begin{tabular}{|c|c|c|c|c|c|c|}
\hline Material & $\begin{array}{c}\sigma_{S}^{o} \\
(\mathrm{MPa})\end{array}$ & $\begin{array}{c}\sigma_{L}^{o} \\
(\mathrm{MPa})\end{array}$ & $\begin{array}{c}C_{1} \\
(\mathrm{MPa})\end{array}$ & $\gamma_{1}$ & $\begin{array}{c}C_{2} \\
(\mathrm{MPa})\end{array}$ & $\gamma_{2}$ \\
\hline $\mathrm{A}$ & 334 & 329 & 19852 & 175.20 & 1997 & 6.17 \\
\hline $\mathrm{B}$ & 390 & 385 & 27427 & 199.33 & 1383 & 5.35 \\
\hline
\end{tabular}




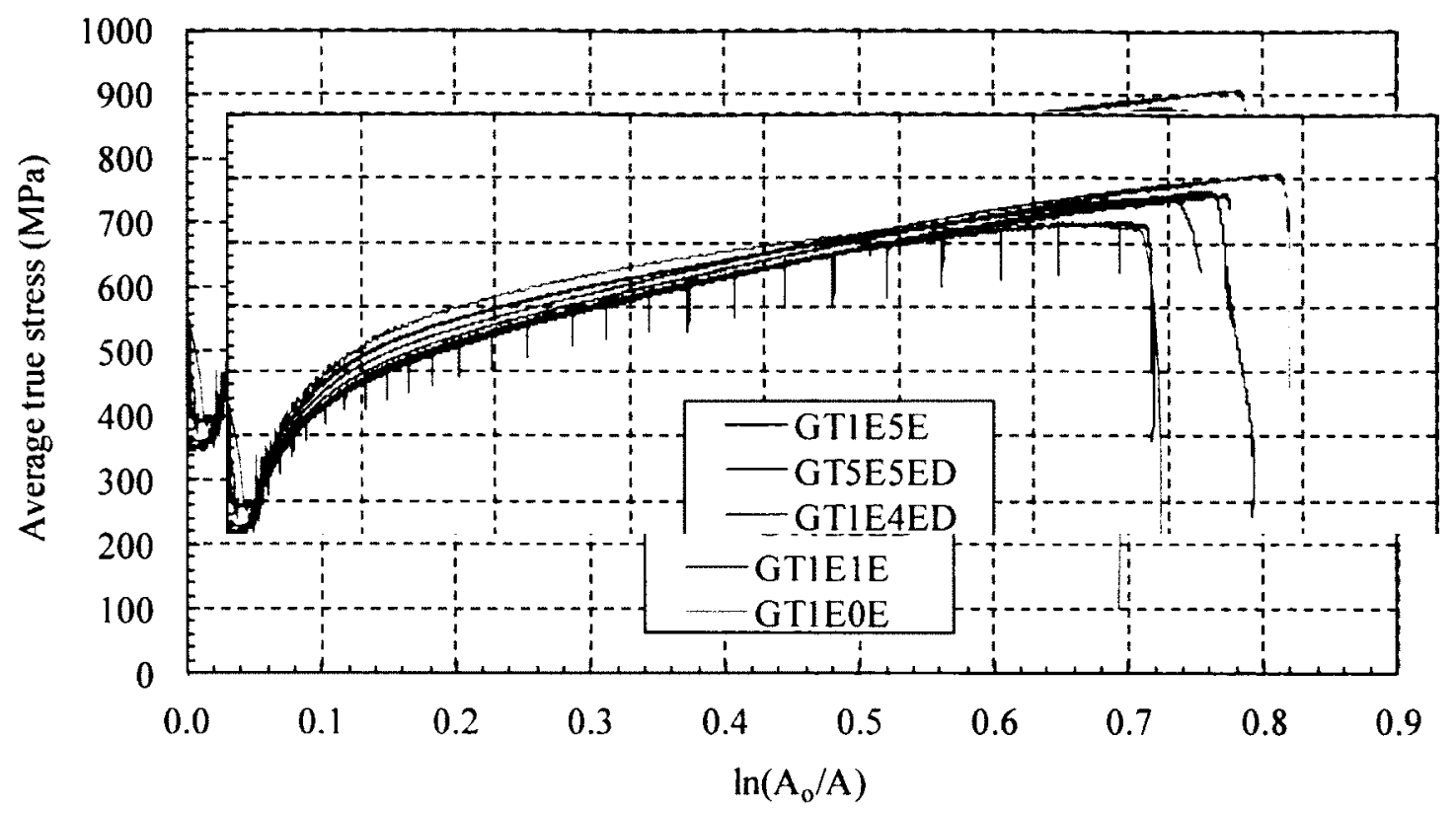

Figure 5.1 Average true stress versus cross-sectional area ratio curves for material $G$ (material A in current study), by Chen (2010)

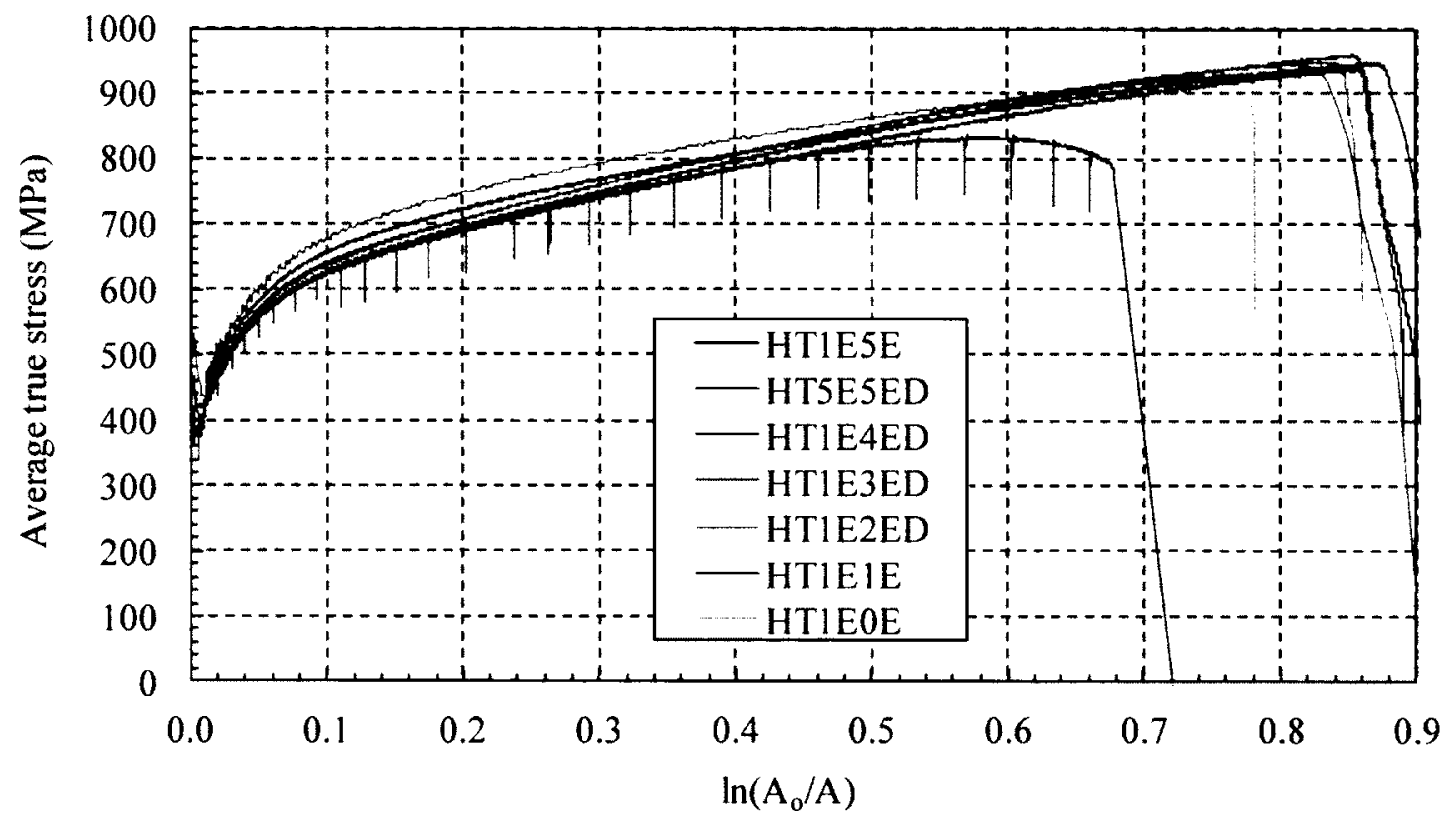

Figure 5.2 Average true stress versus cross-sectional area ratio curves for material $\mathrm{H}$ (material B in current study), by Chen (2010) 


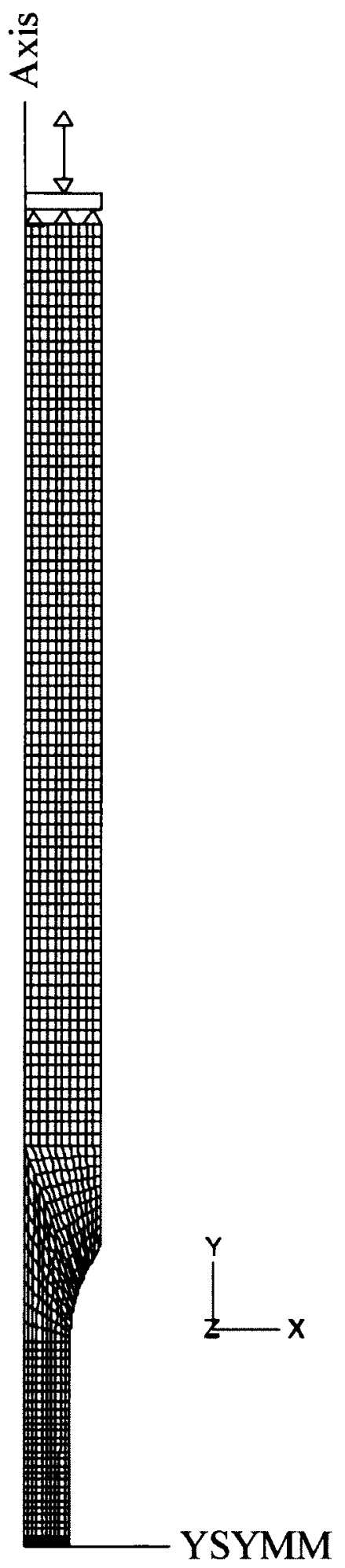

Figure 5.3 The half model for tapered specimen by Chen (2010) 


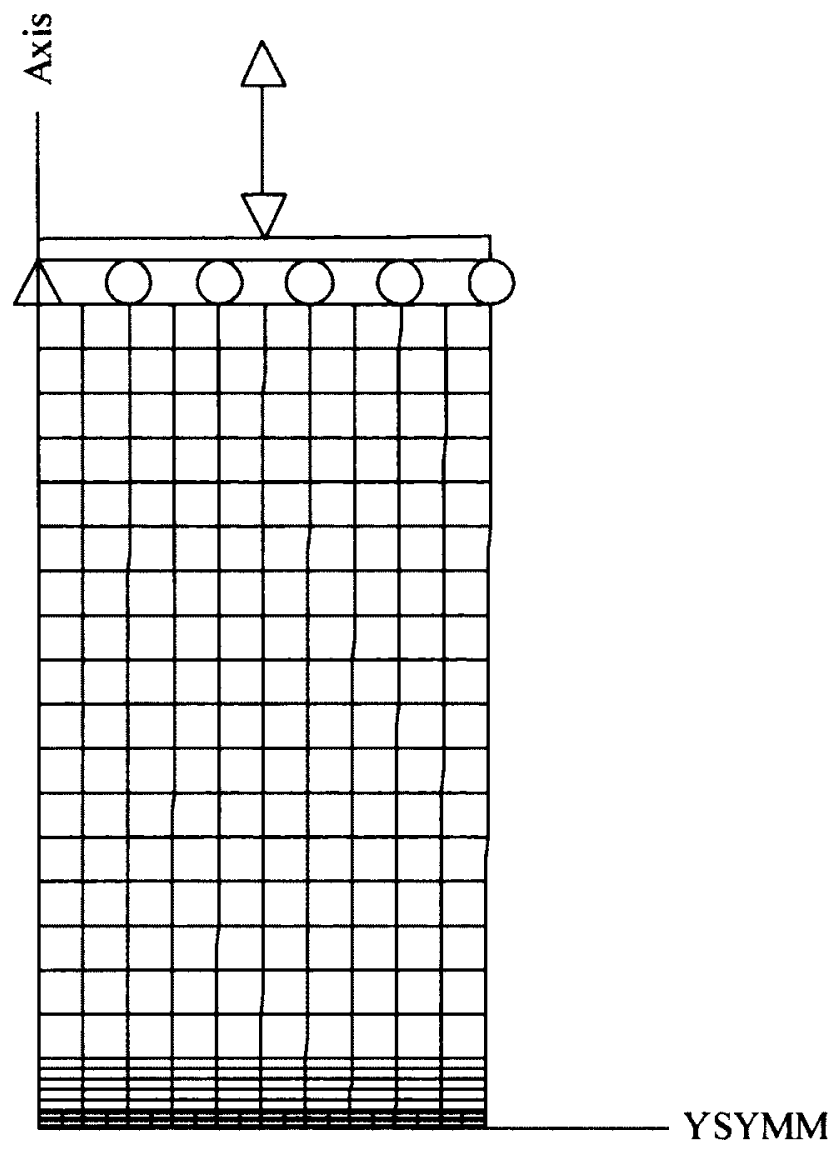

Figure 5.4 The half gauge length model of the tapered specimen by Chen (2010) 


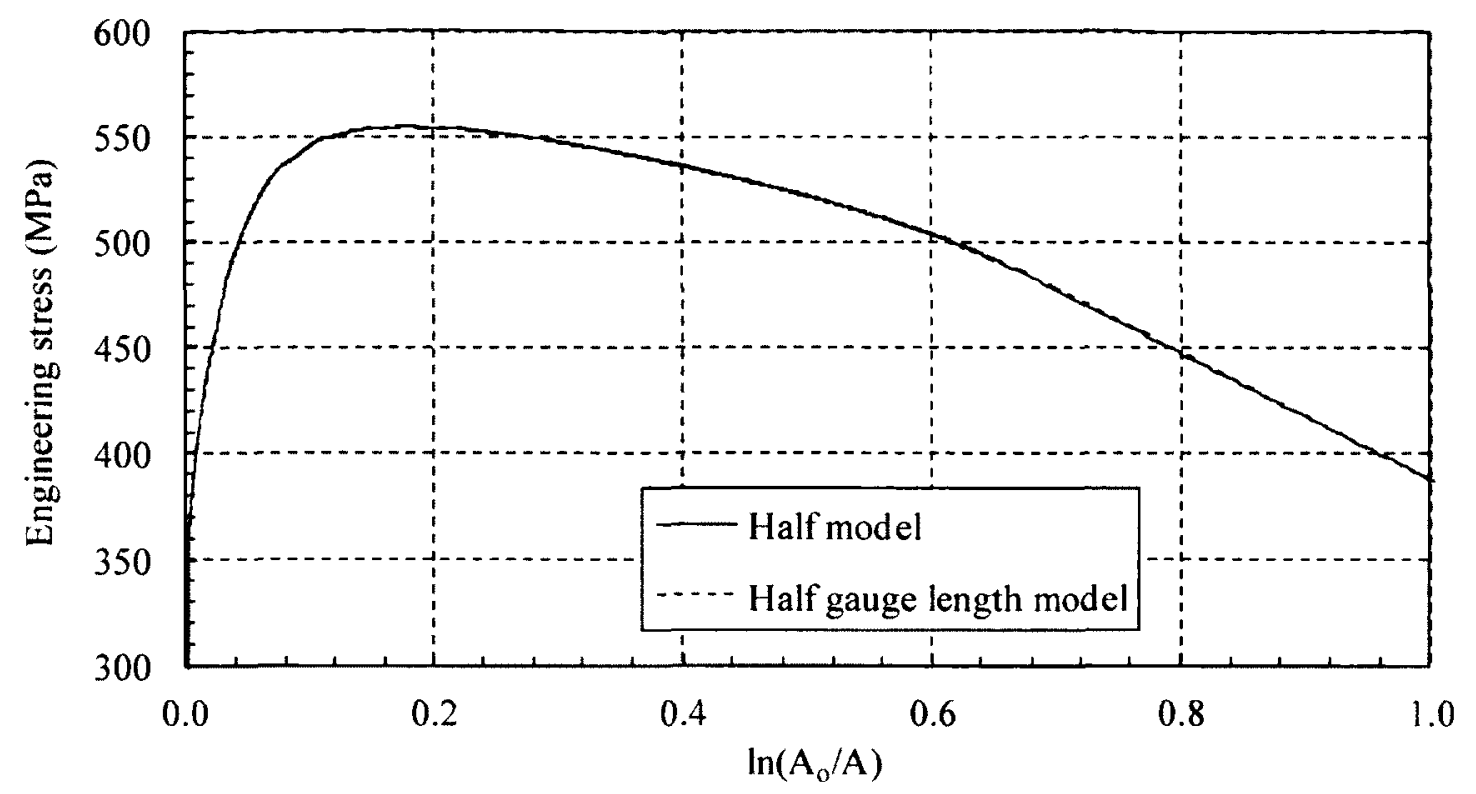

Figure 5.5 Engineering stress versus cross-section area ratio for half gauge length model and half model of tapered specimen GT5E5ED by Chen (2010)

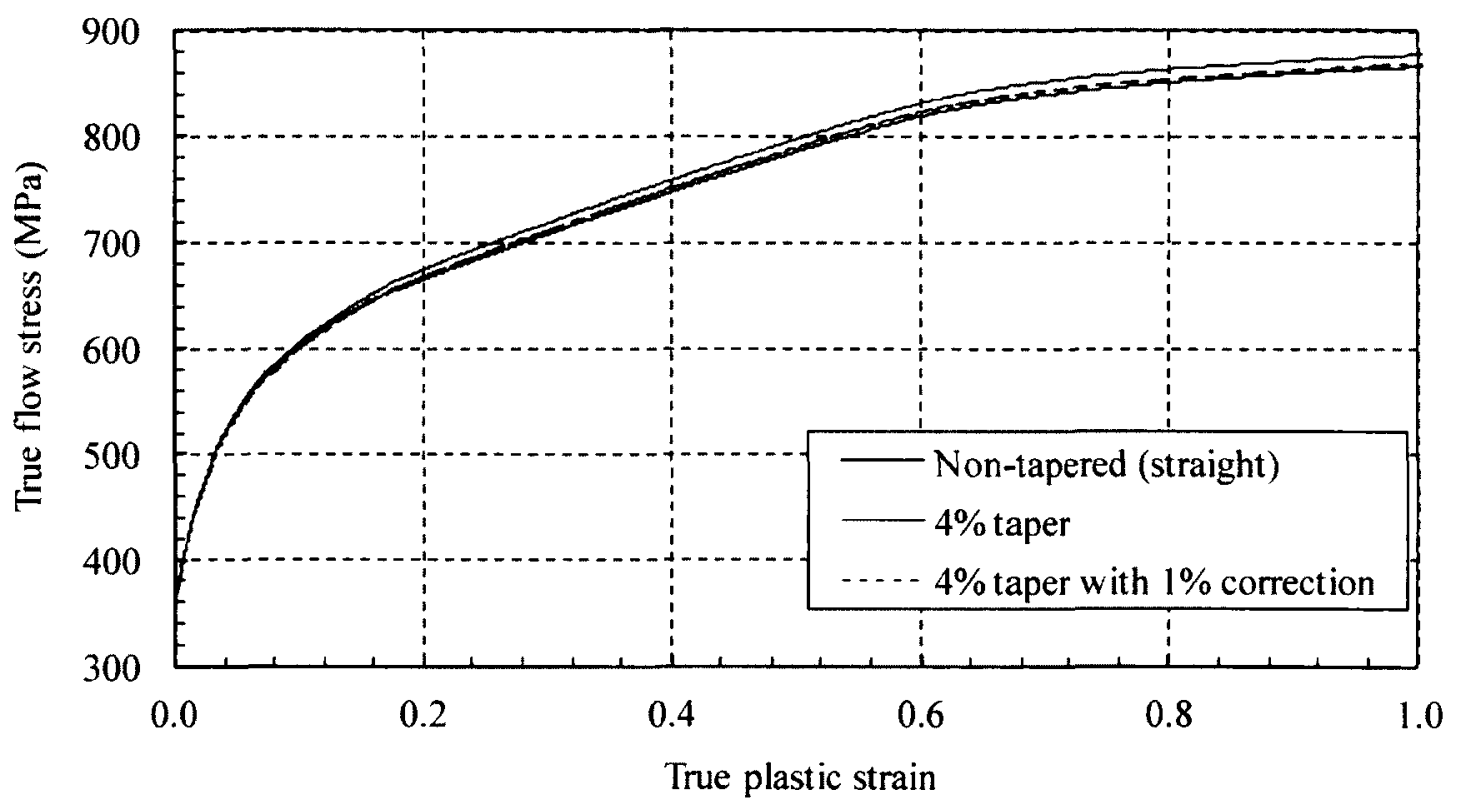

Figure 5.6 True flow stress versus true plastic strain curves for tapered and non-tapered (straight) specimens 


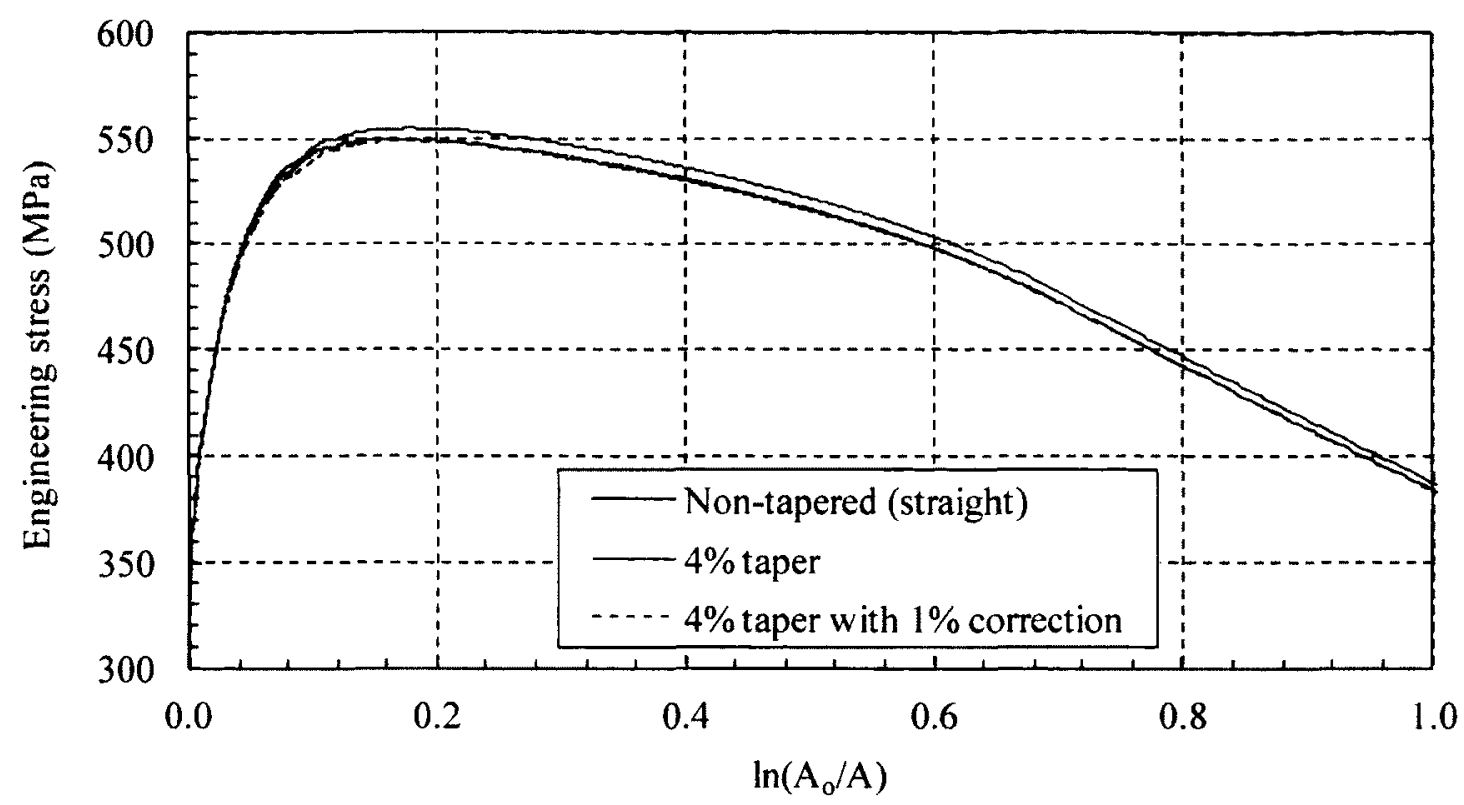

Figure 5.7 Engineering stress versus cross section area ratio curves for tapered and non-tapered (straight) specimens

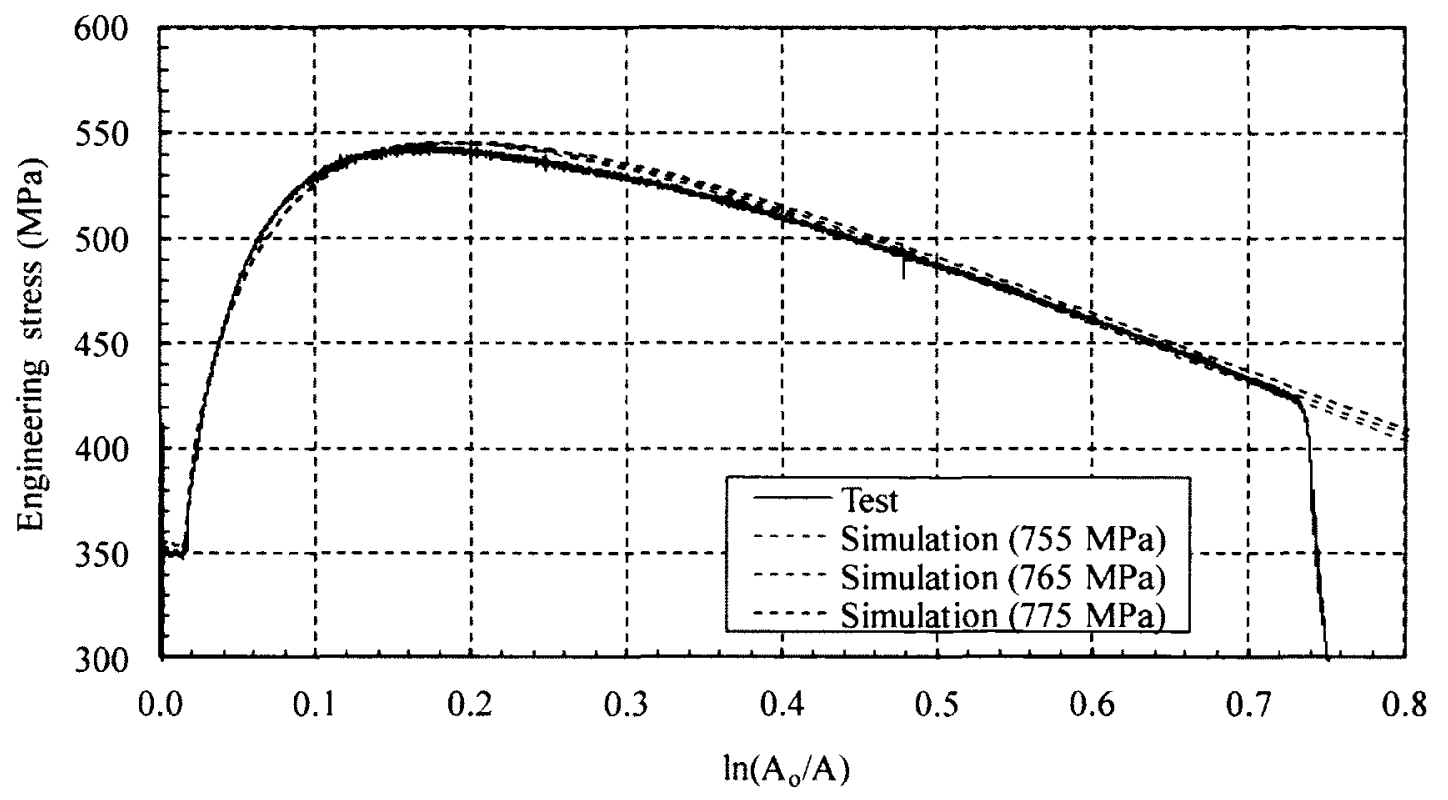

Figure 5.8 Measured and predicted engineering stress versus cross section area ratio curves for GT5E5ED 


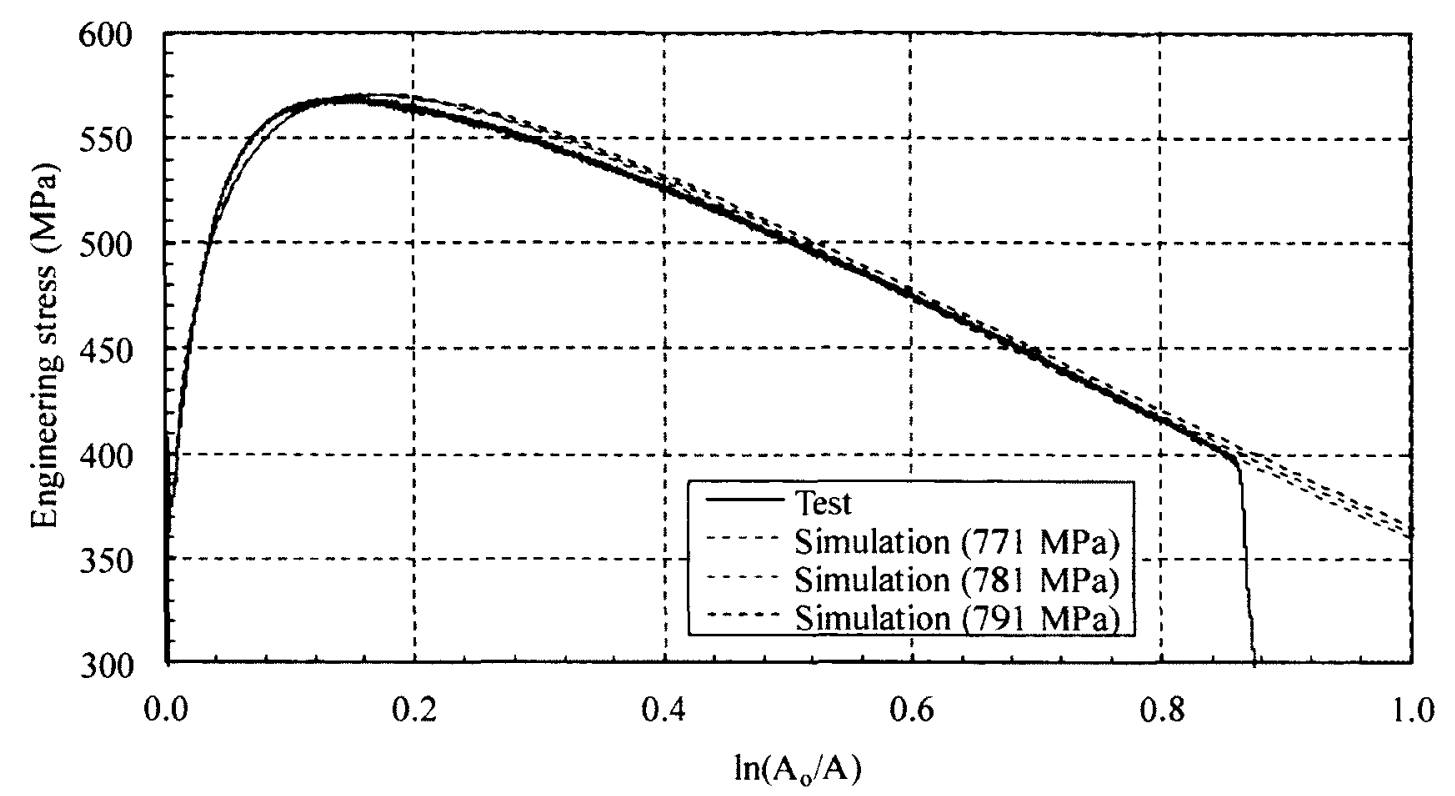

Figure 5.9 Measured and predicted engineering stress versus cross section area ratio curves for HT5E5ED

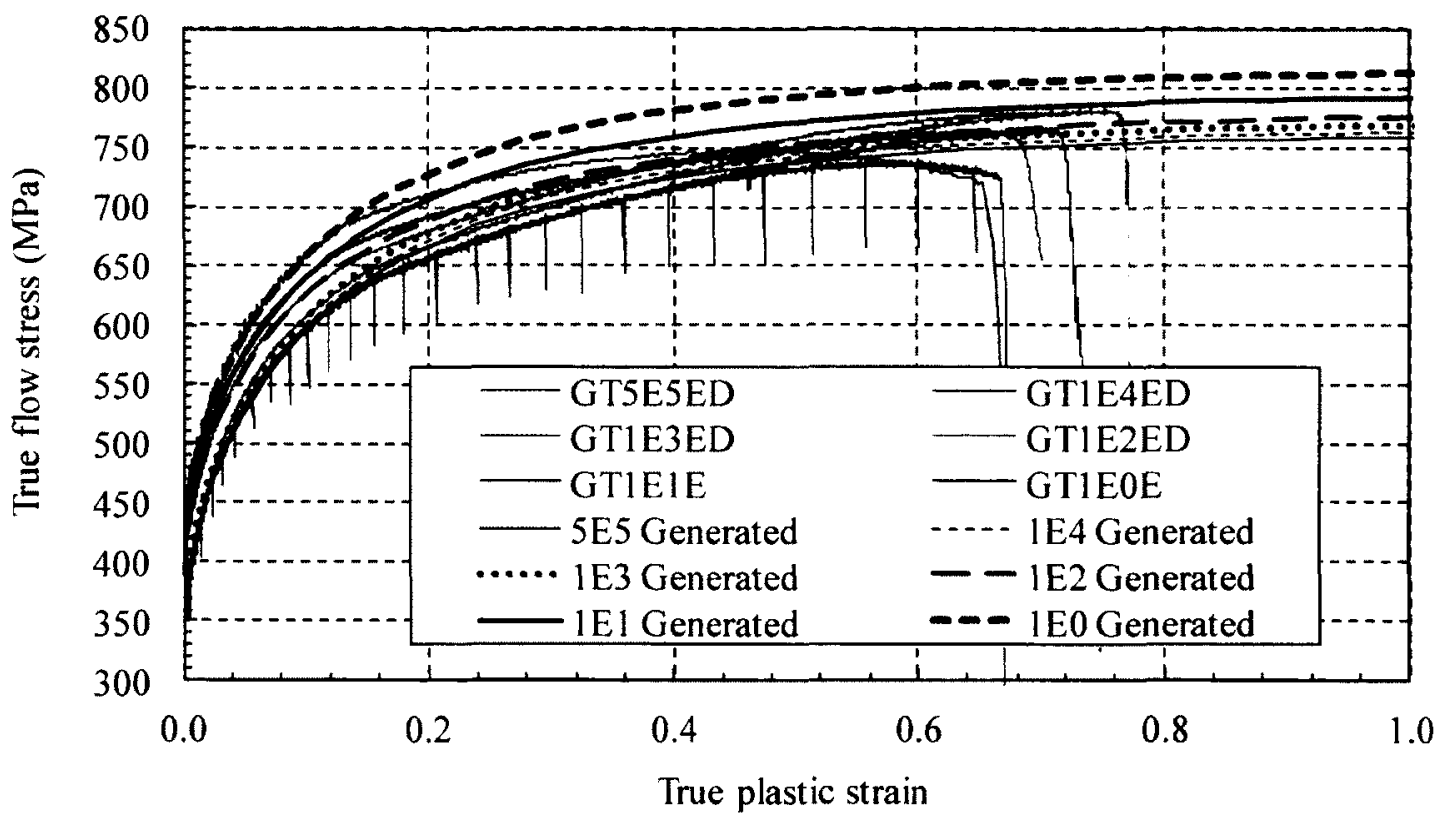

Figure 5.10 Measured and generated true flow stress versus true plastic strain curves for material G (A) 


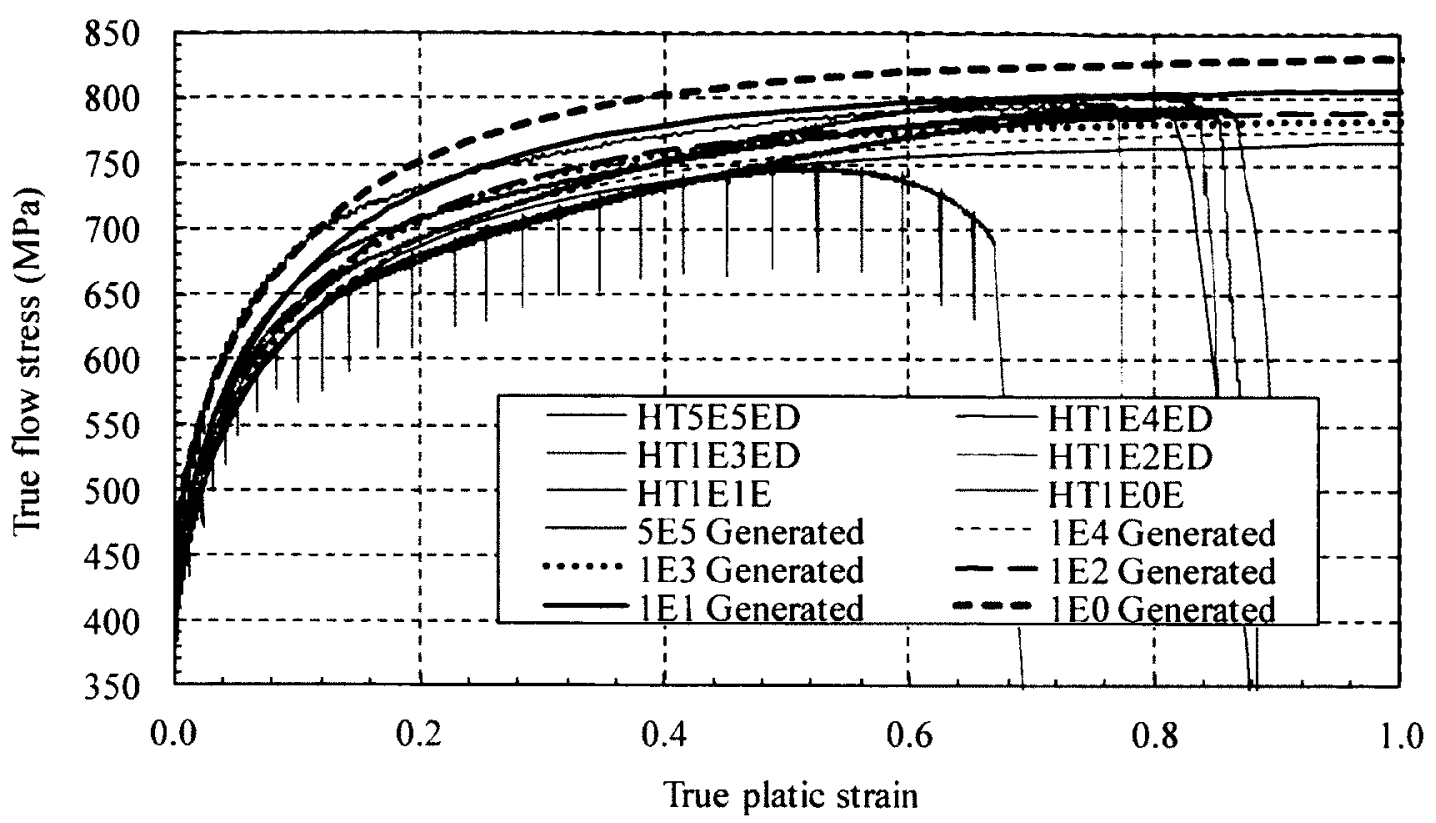

Figure 5.11 Measured and generated true flow stress versus true plastic strain curves for material $\mathrm{H}(\mathrm{B})$

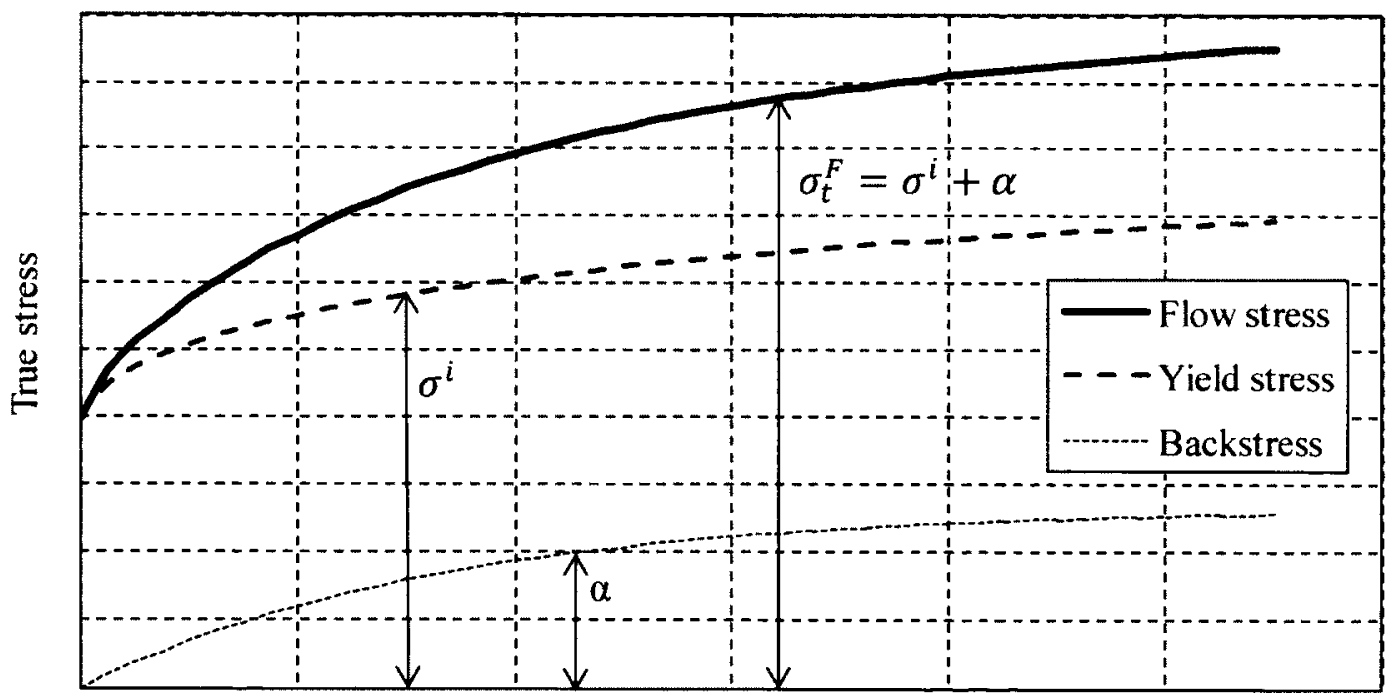

True plastic strain

Figure 5.12 Relationship between the flow stress, yield stress and backstress 


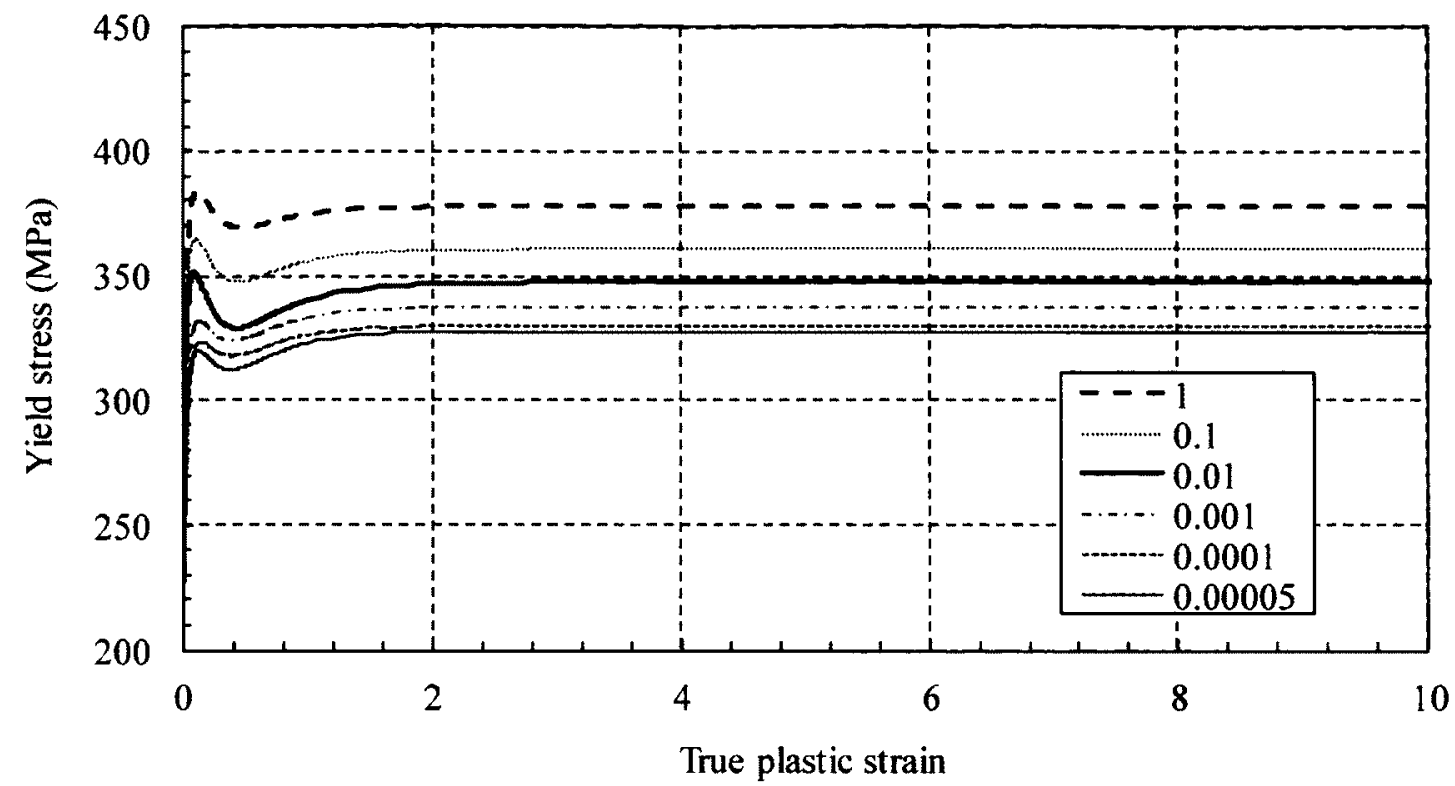

Figure 5.13 Yield stress versus true plastic strain curves at various strain rates for material A

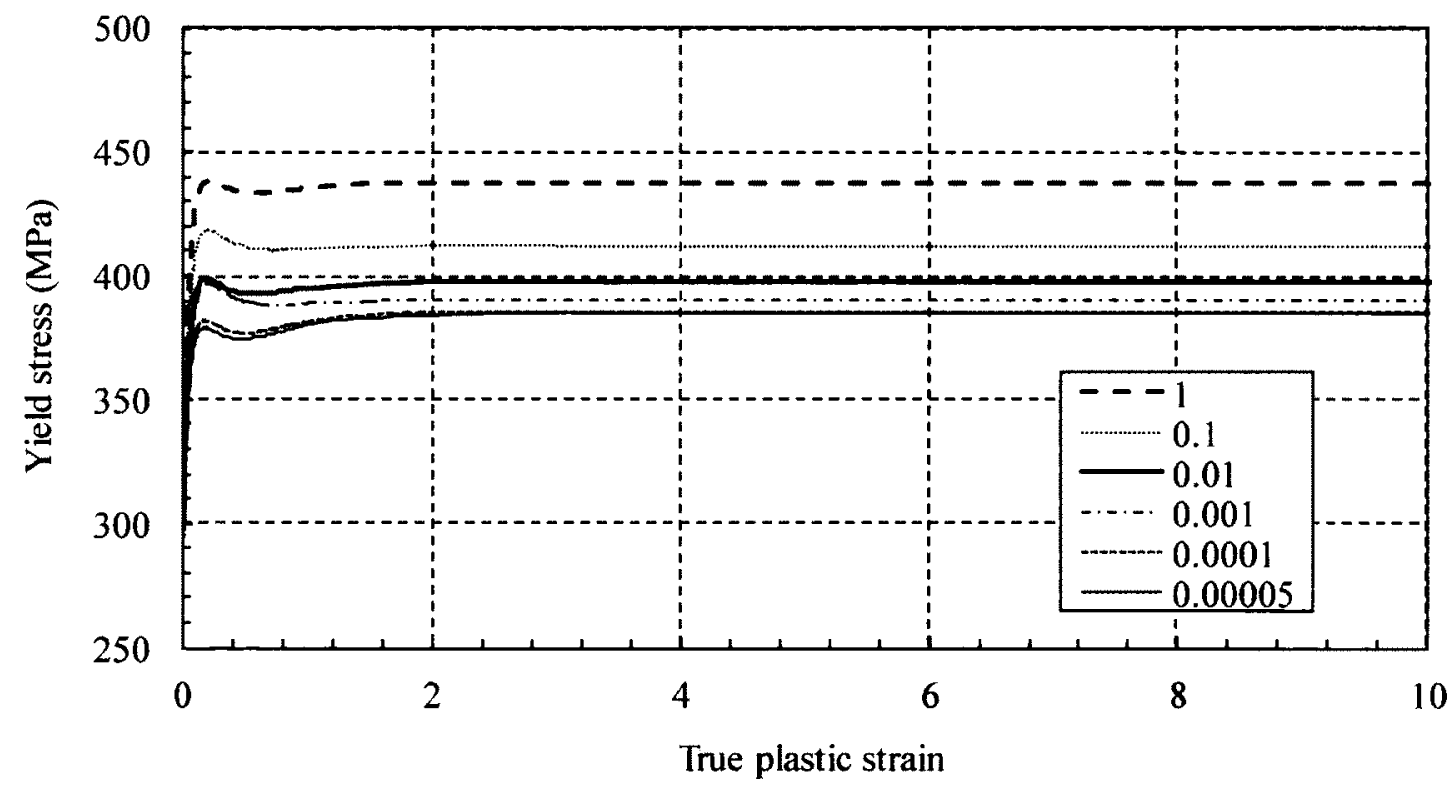

Figure 5.14 Yield stress versus true plastic strain curves at various strain rates for material B 


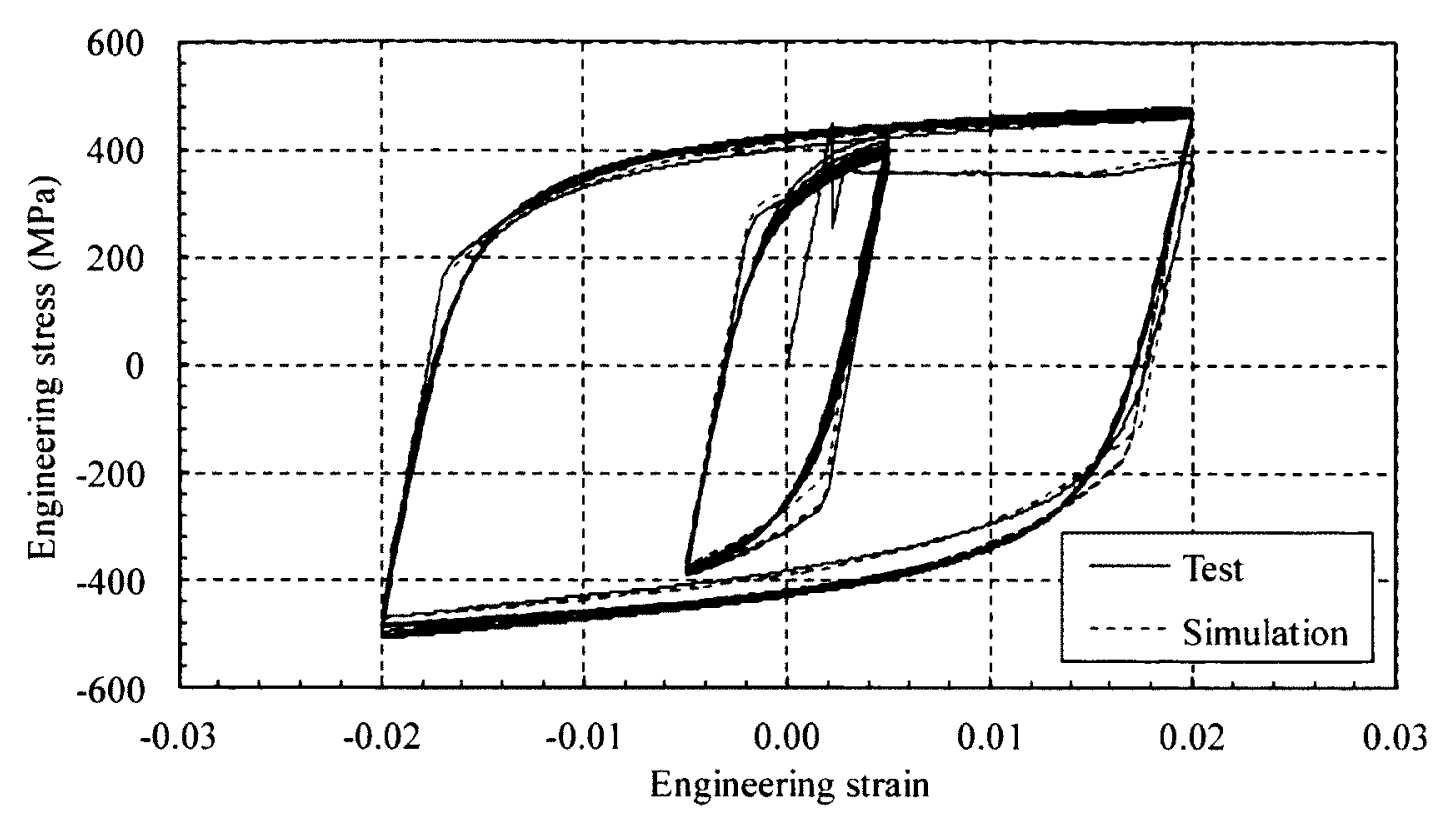

Figure 5.15 Test and predicted engineering stress versus engineering strain curves for ATE4D

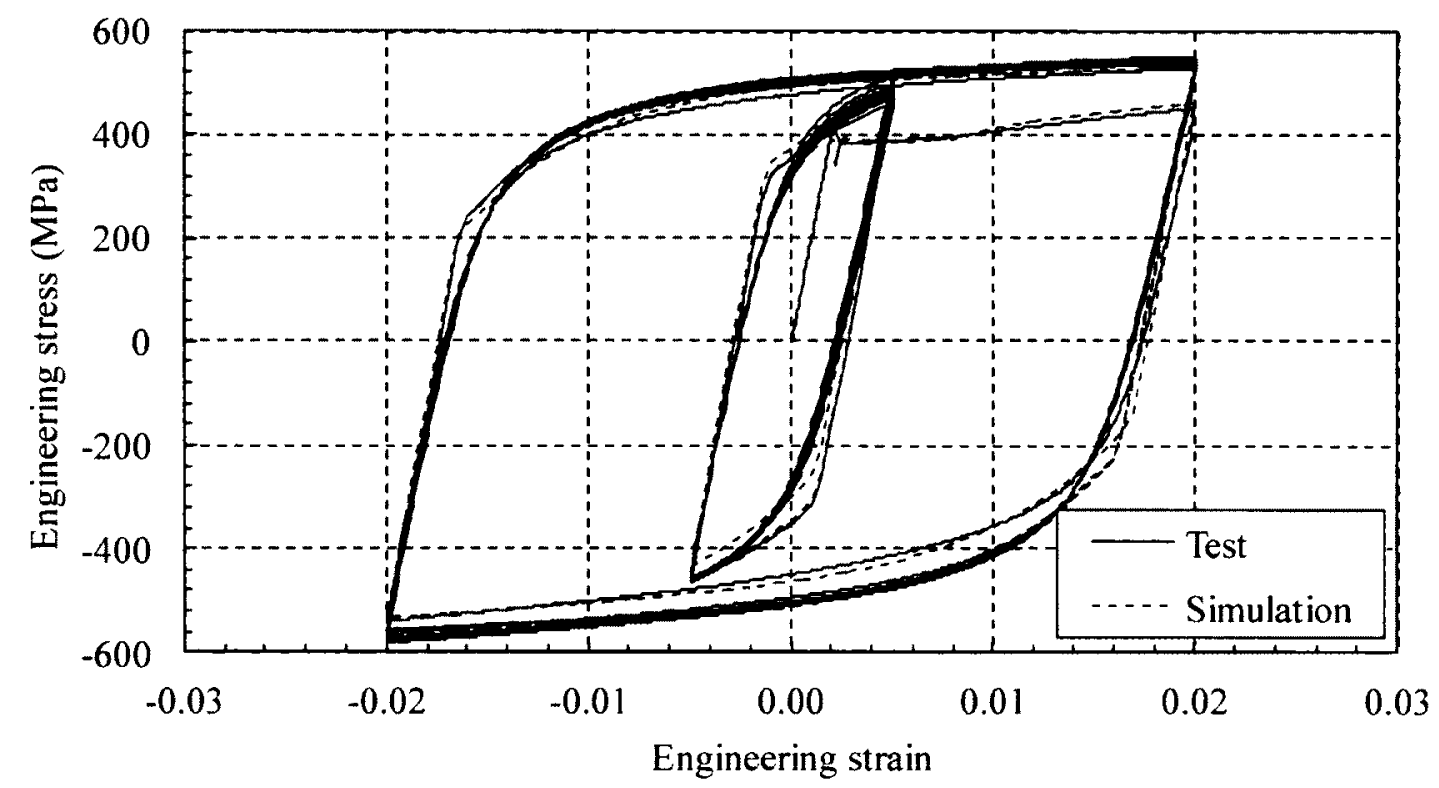

Figure 5.16 Test and predicted engineering stress versus engineering strain curves for BTE4D 


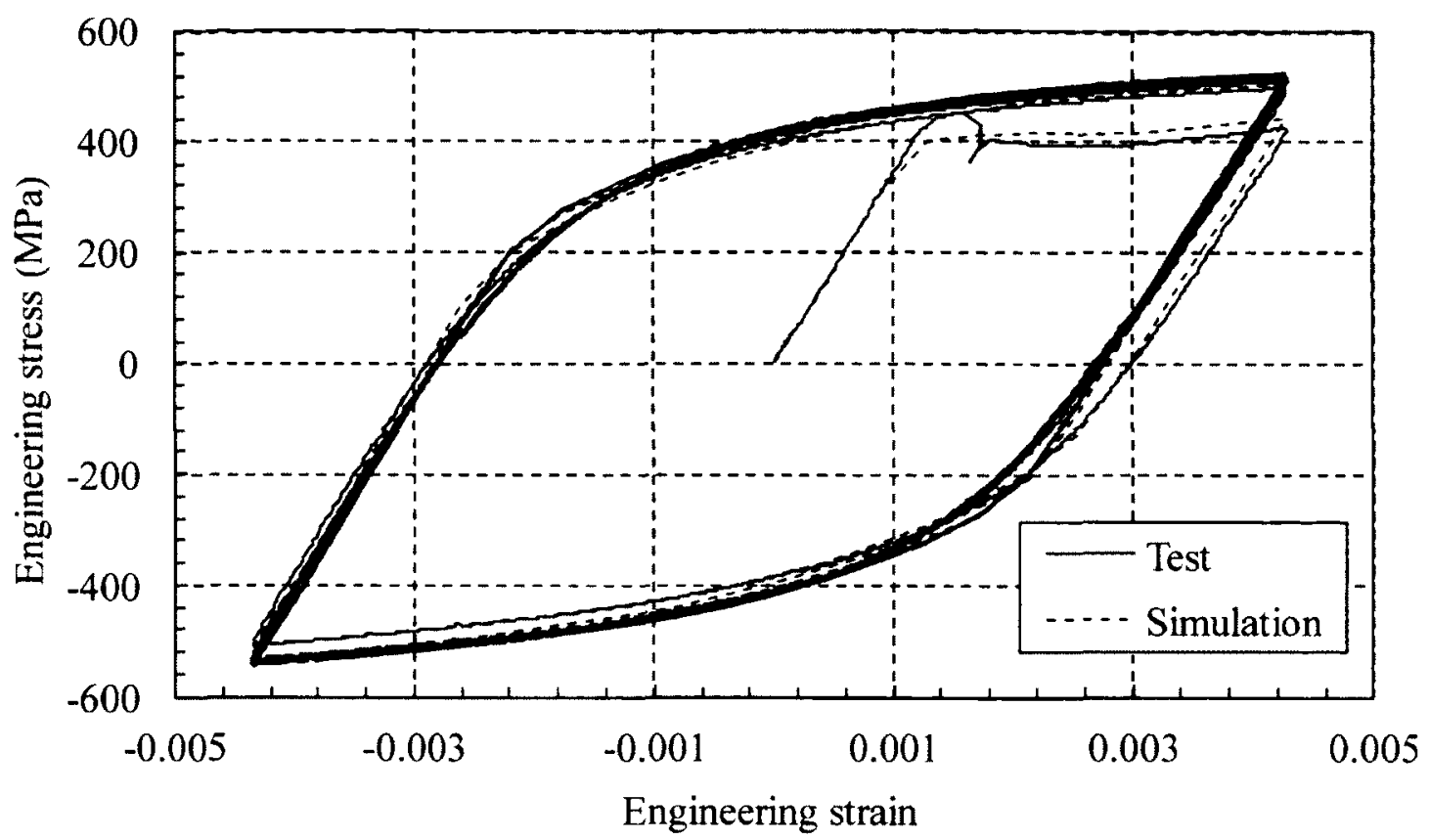

Figure 5.17 Test and predicted engineering stress versus engineering strain curves for ANE4R2

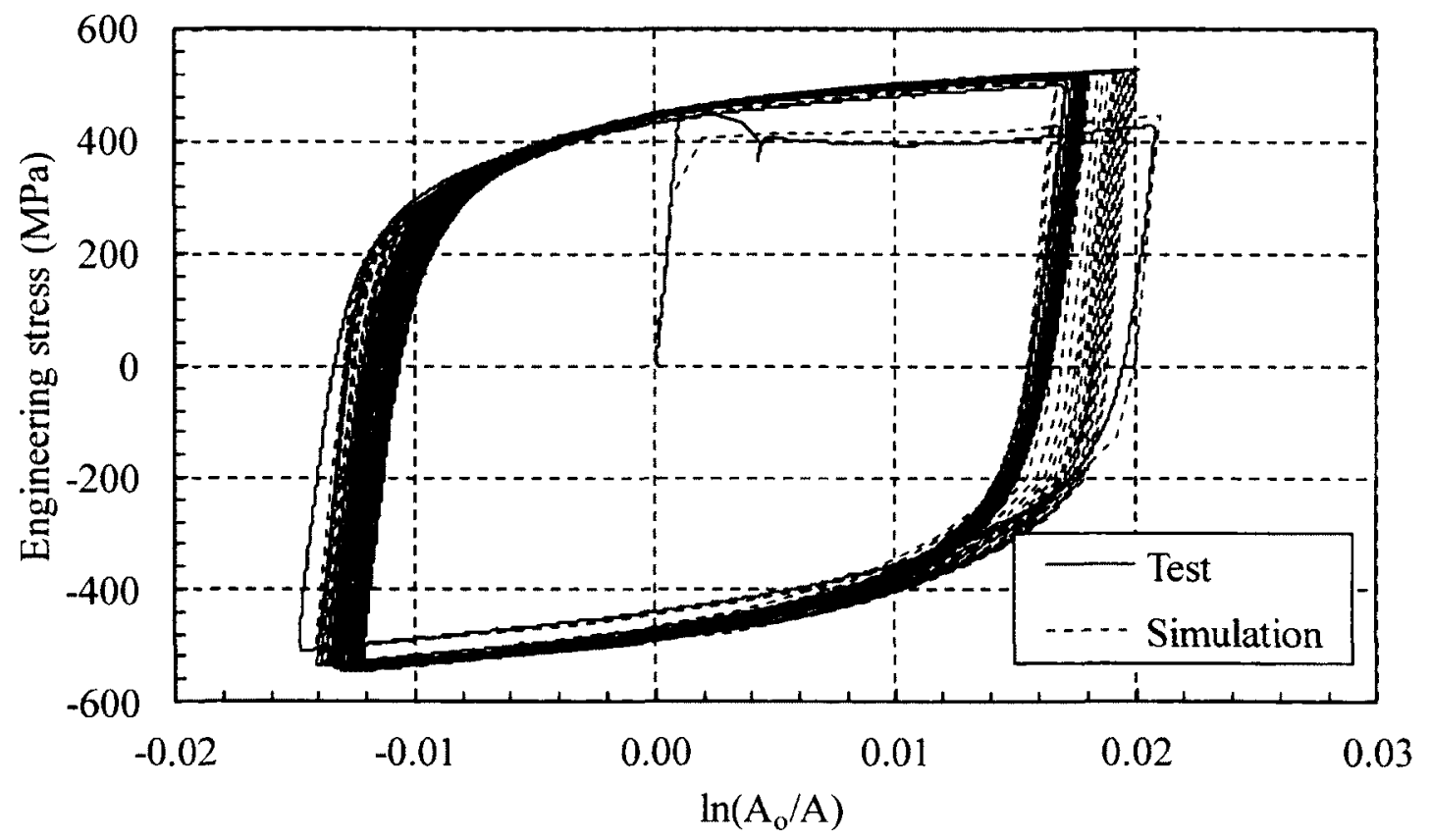

Figure 5.18 Test and predicted engineering stress versus cross-sectional area ratio curves for ANE4R2 


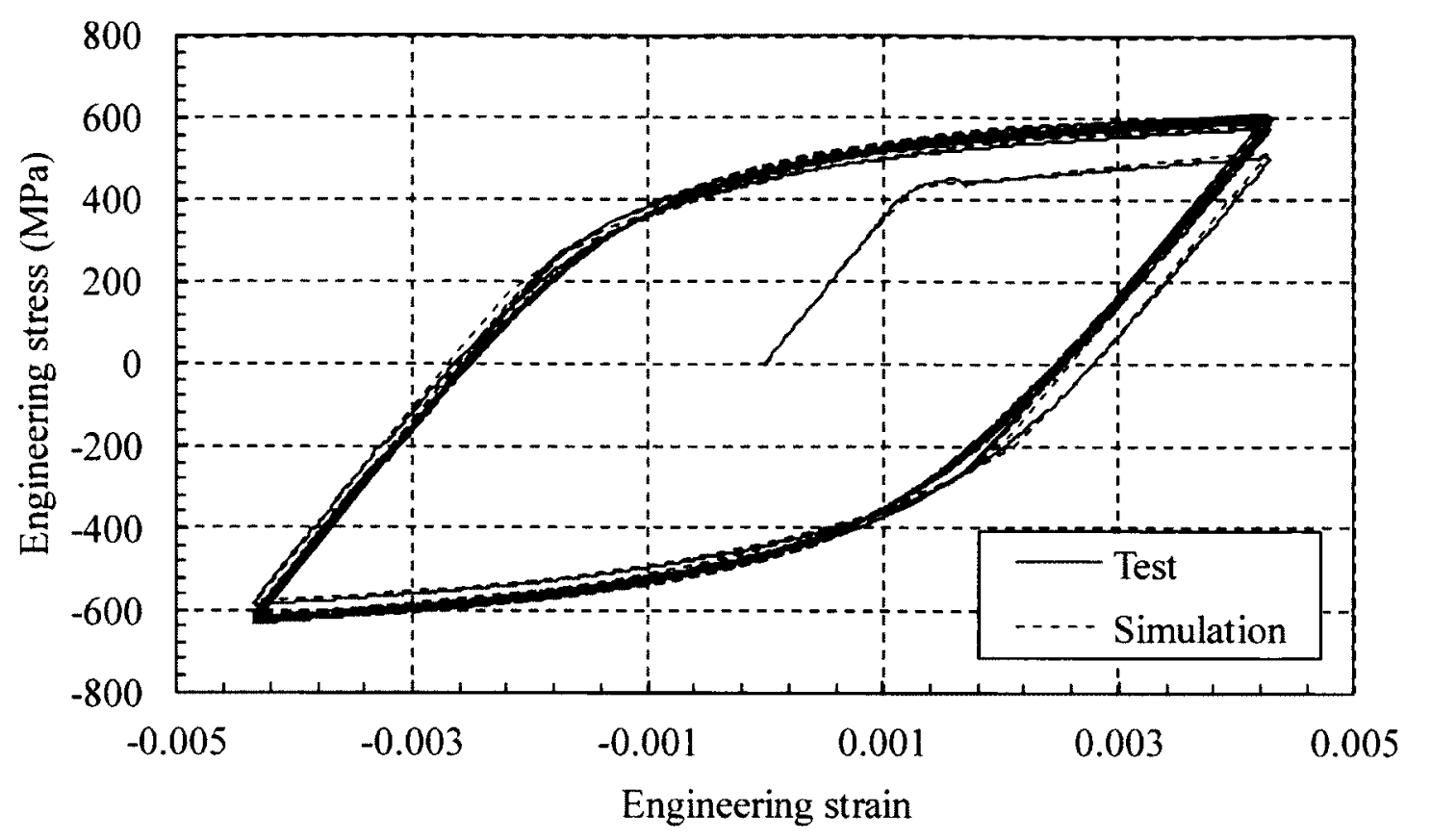

Figure 5.19 Test and predicted engineering stress versus engineering strain curves of BNE4R2

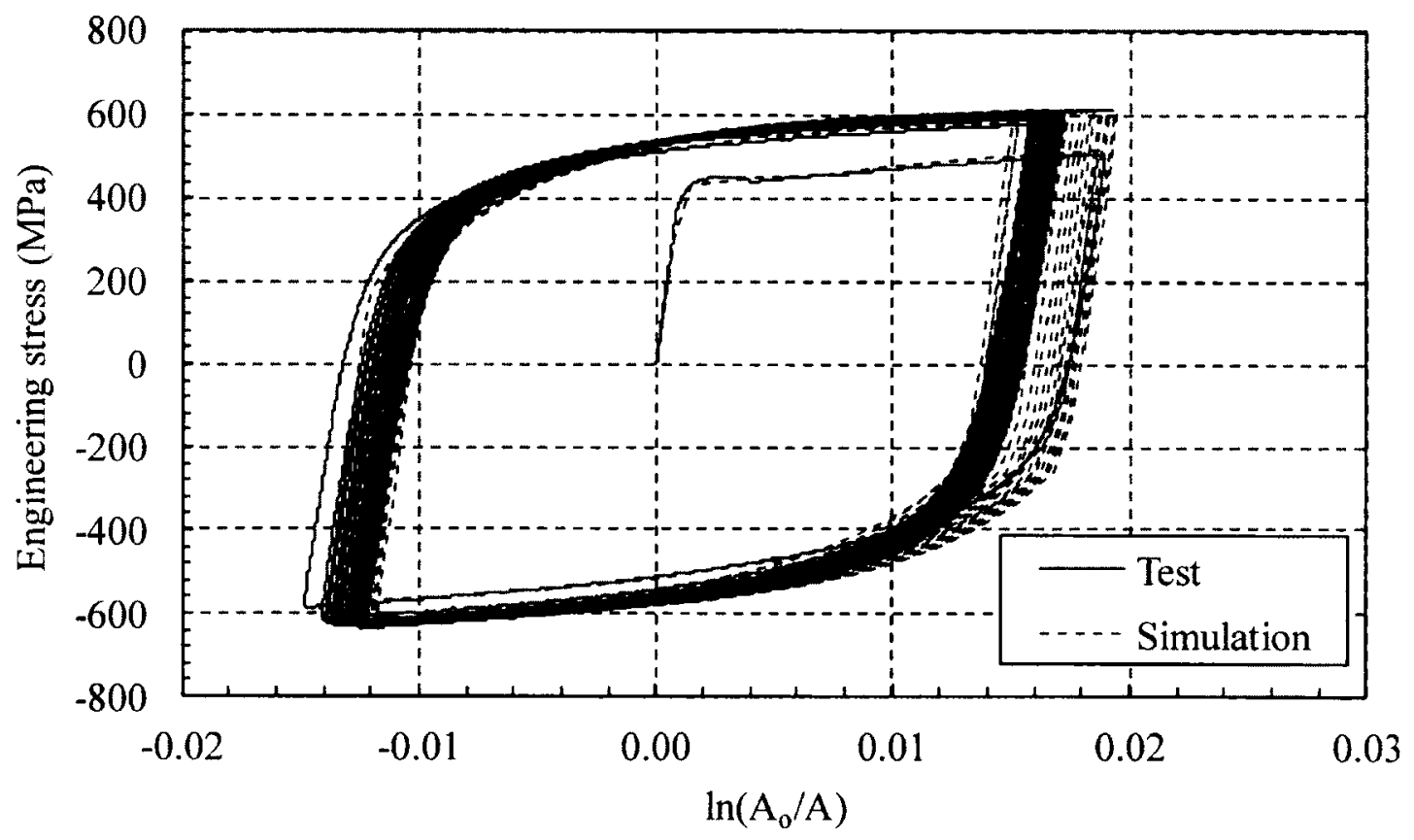

Figure 5.20 Test and predicted engineering stress versus cross-sectional area ratio curves of BNE4R2 


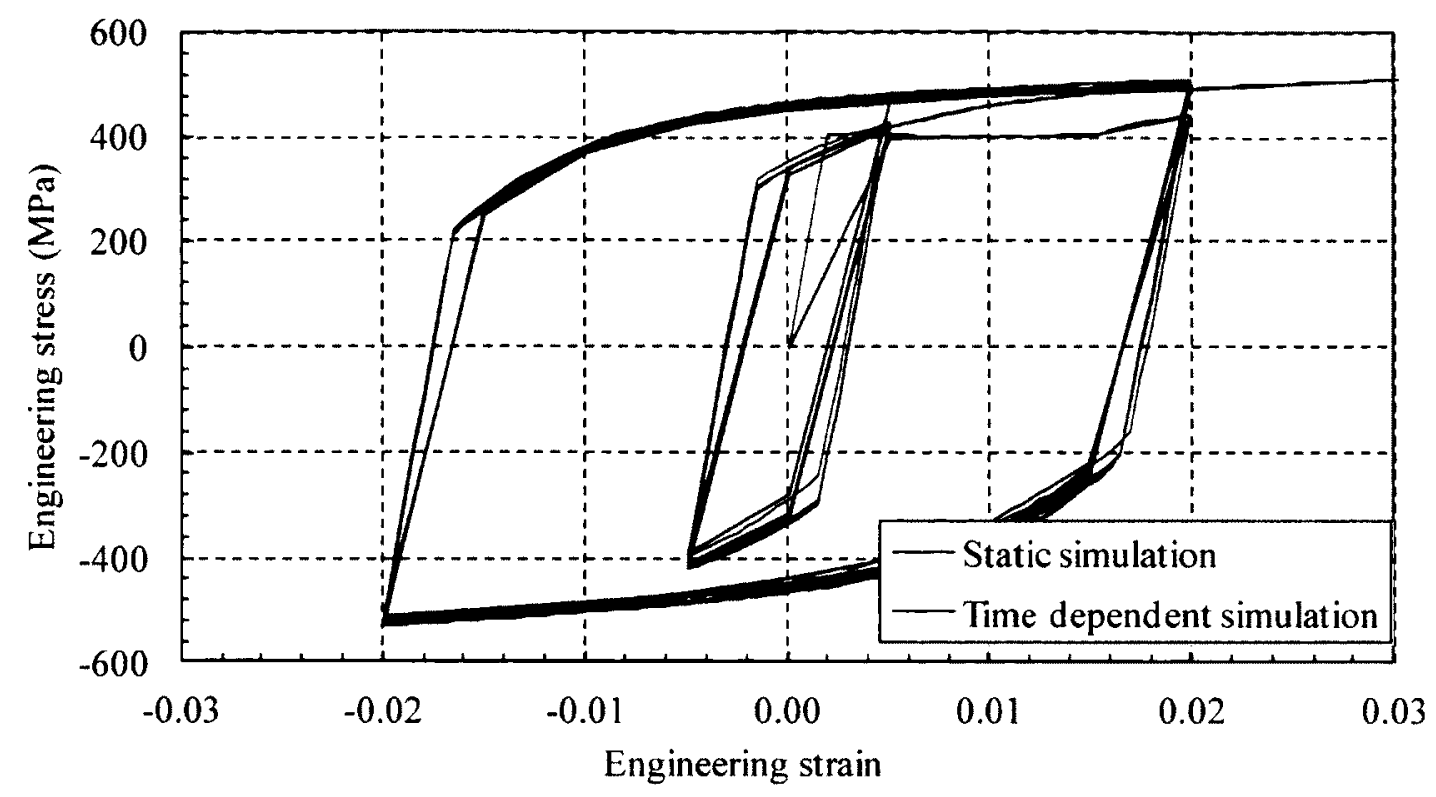

Figure 5.21 Engineering stress versus engineering strain curves between a static and a time dependent analysis for ATEID

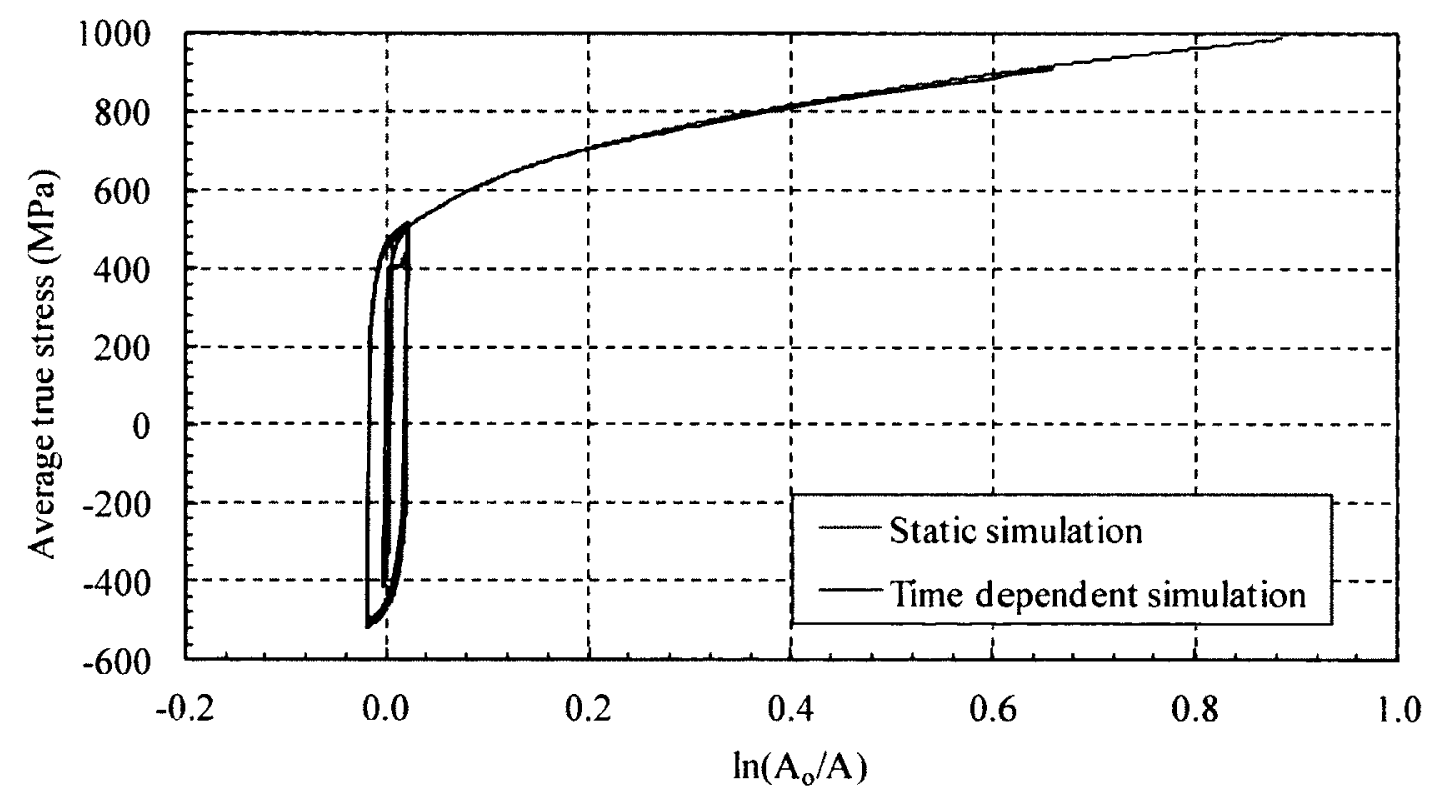

Figure 5.22 Average true stress versus average true strain curves between a static and a time dependent analysis for ATE1D 


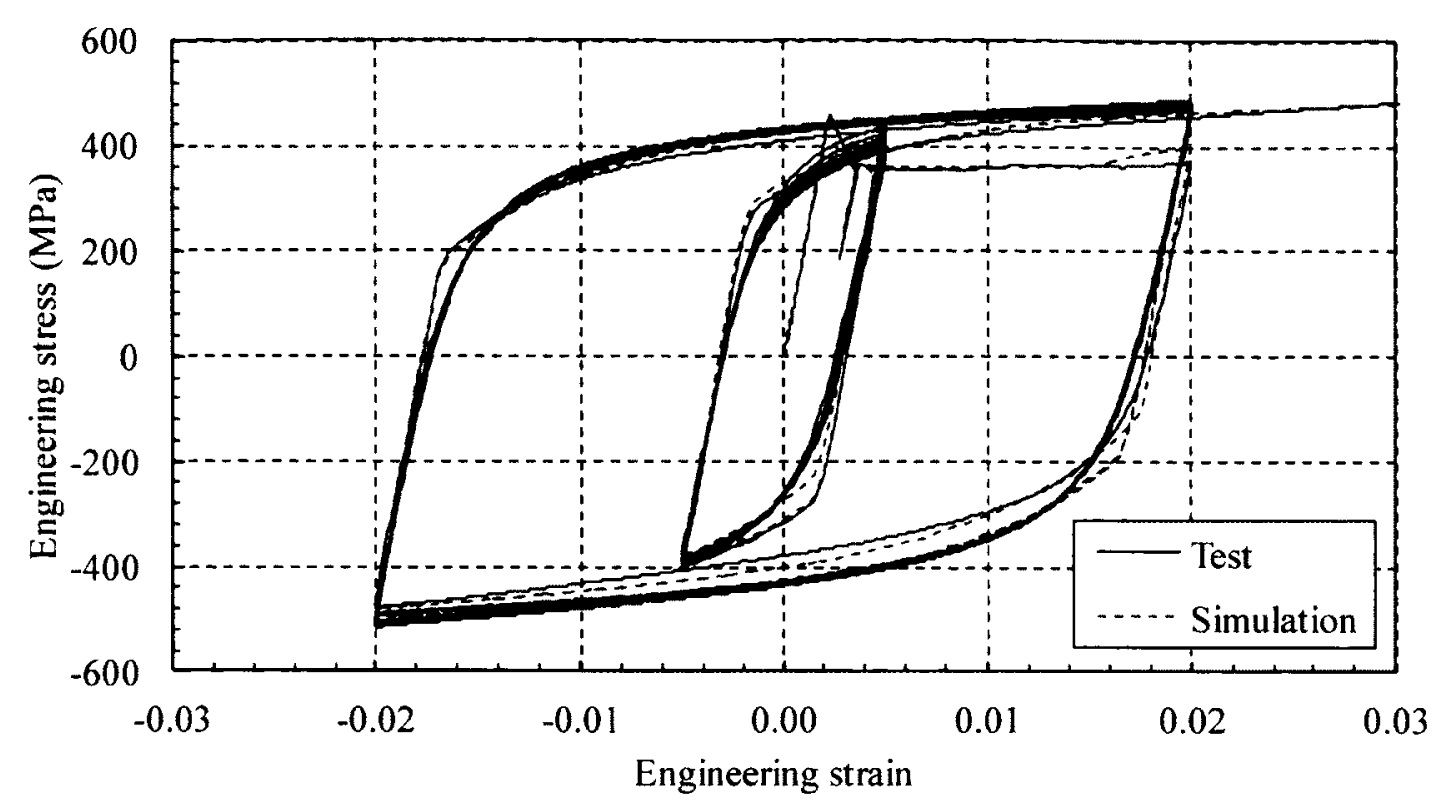

Figure 5.23 Test and predicted engineering stress versus engineering strain curves for the cyclic portion of ATE3D

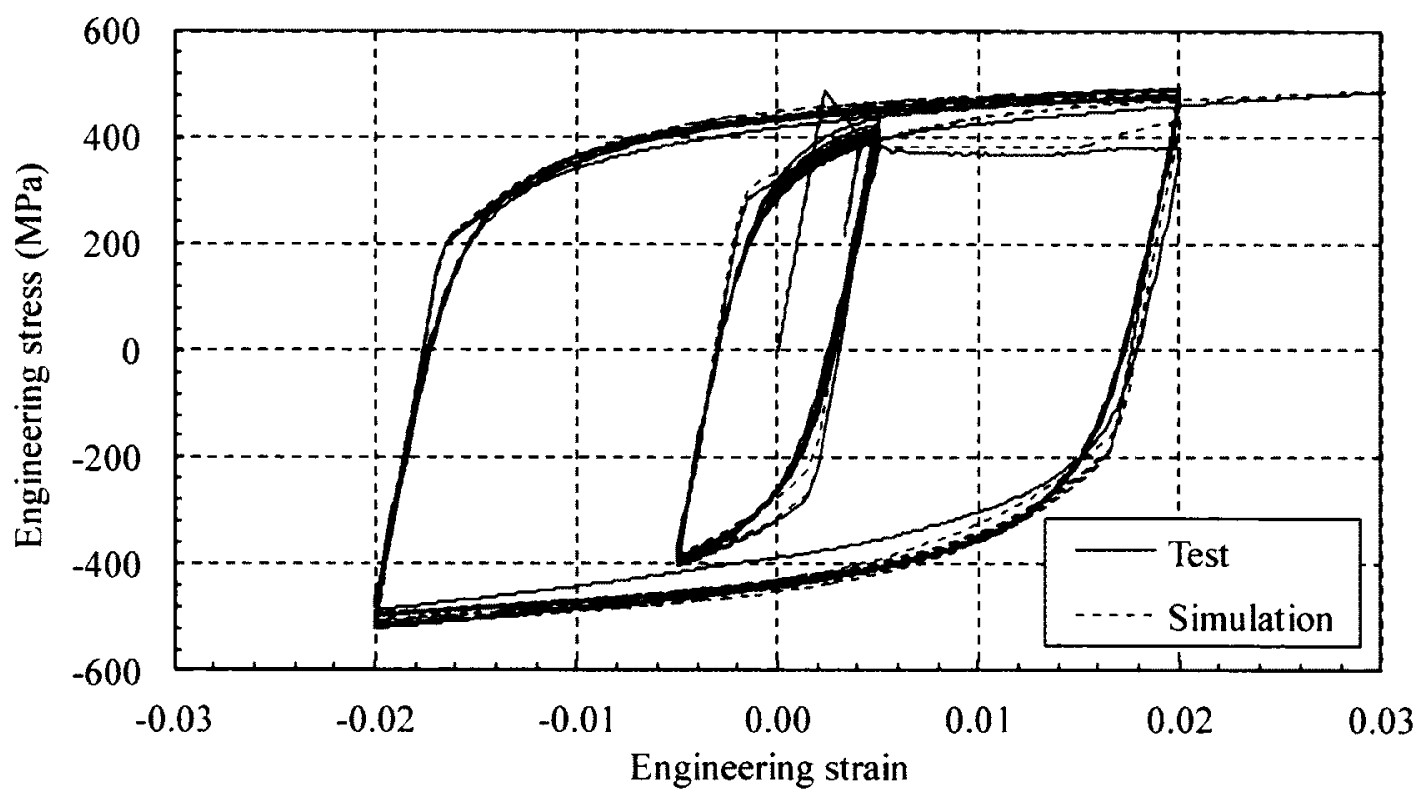

Figure 5.24 Test and predicted engineering stress versus engineering strain curves for the cyclic portion of ATE2D 


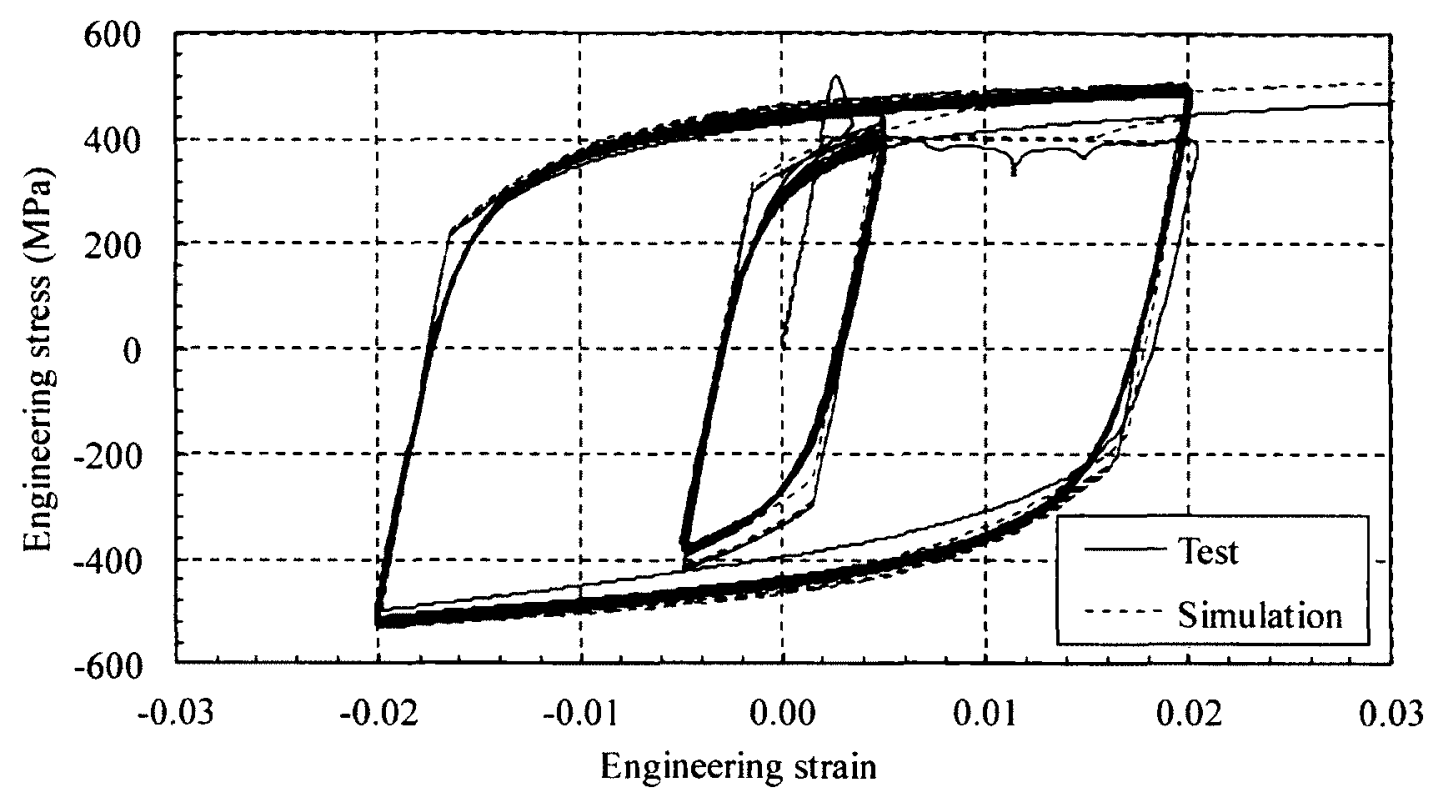

Figure 5.25 Test and predicted engineering stress versus engineering strain curves for the cyclic portion of ATE1D

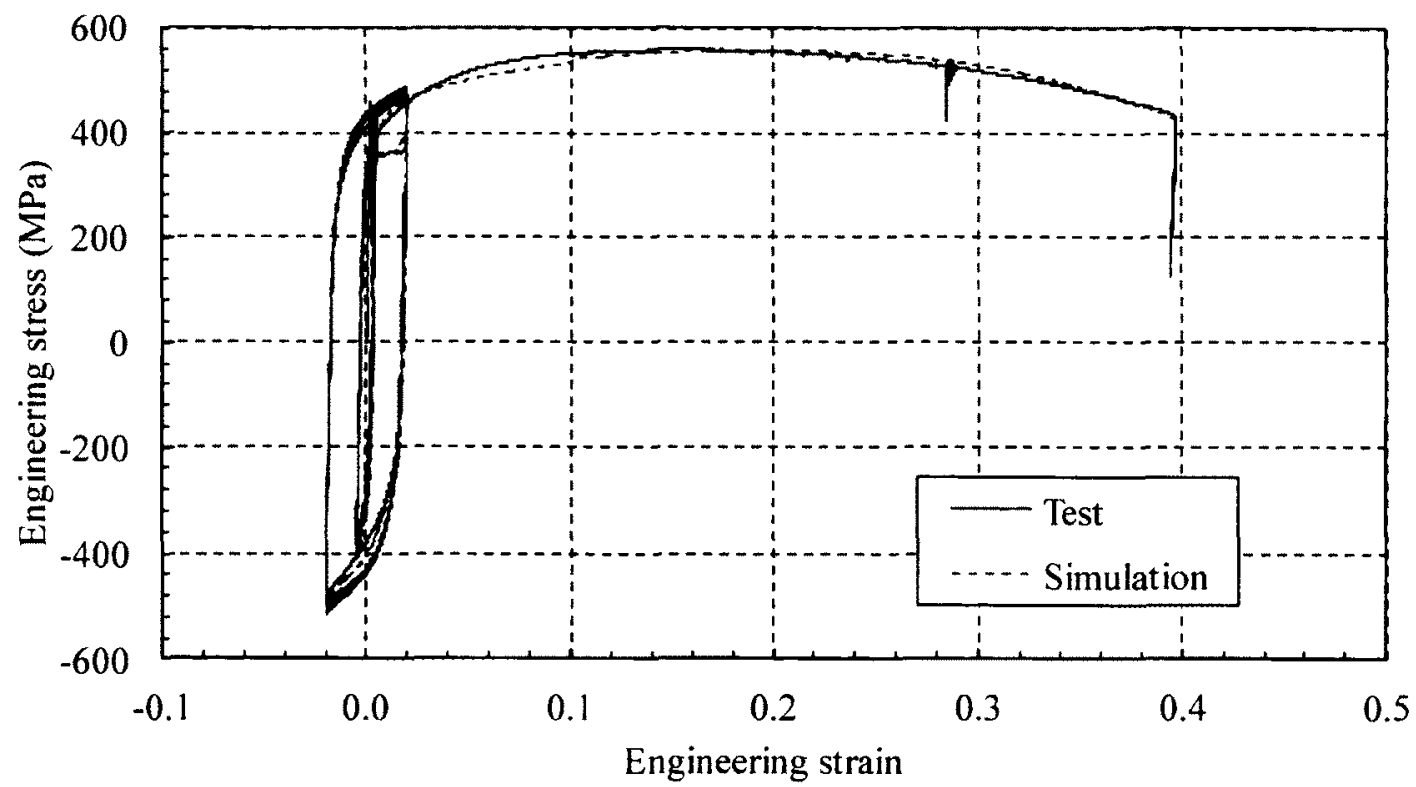

Figure 5.26 Test and predicted engineering stress versus engineering strain curves of ATE3D 


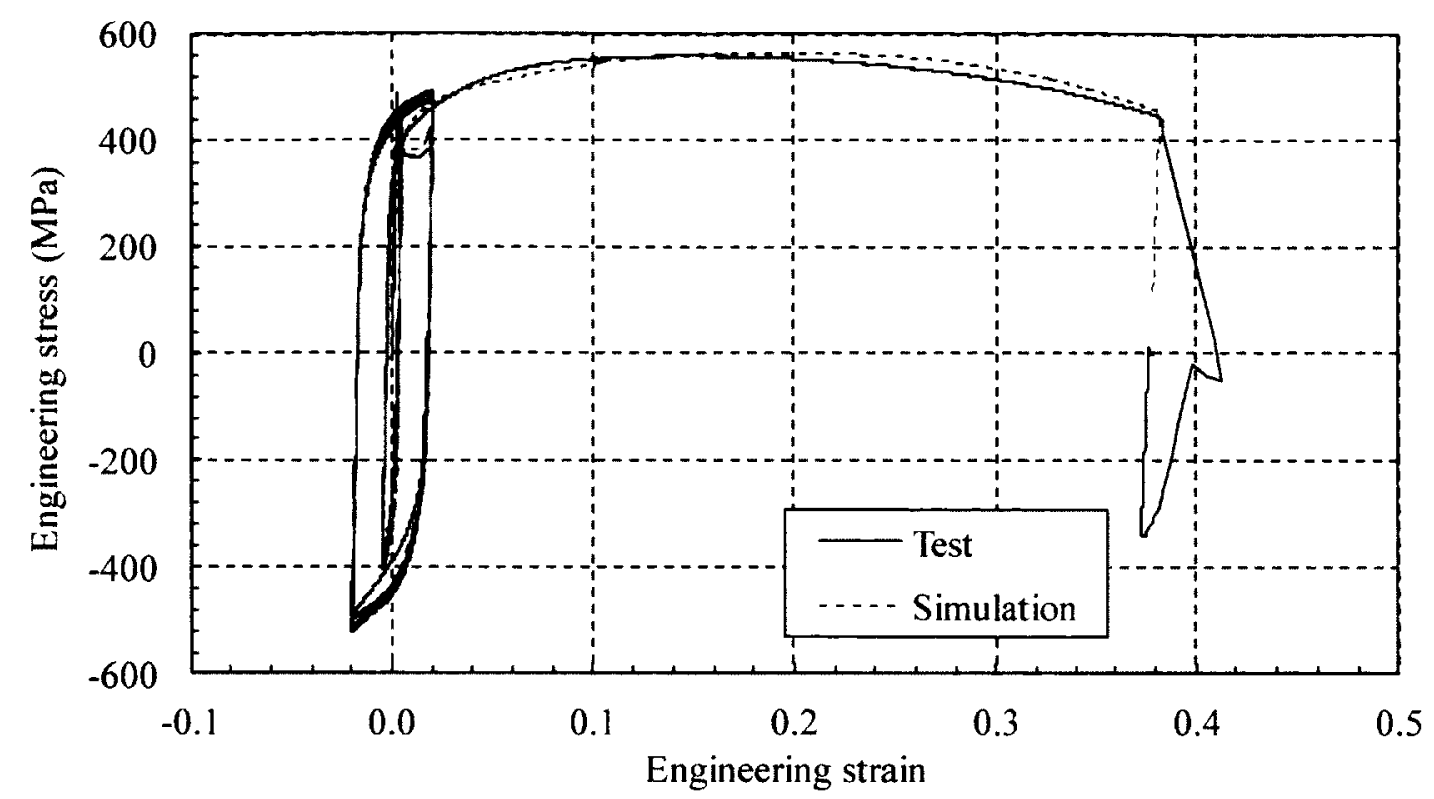

Figure 5.27 Test and predicted engineering stress versus engineering strain curves of ATE2D

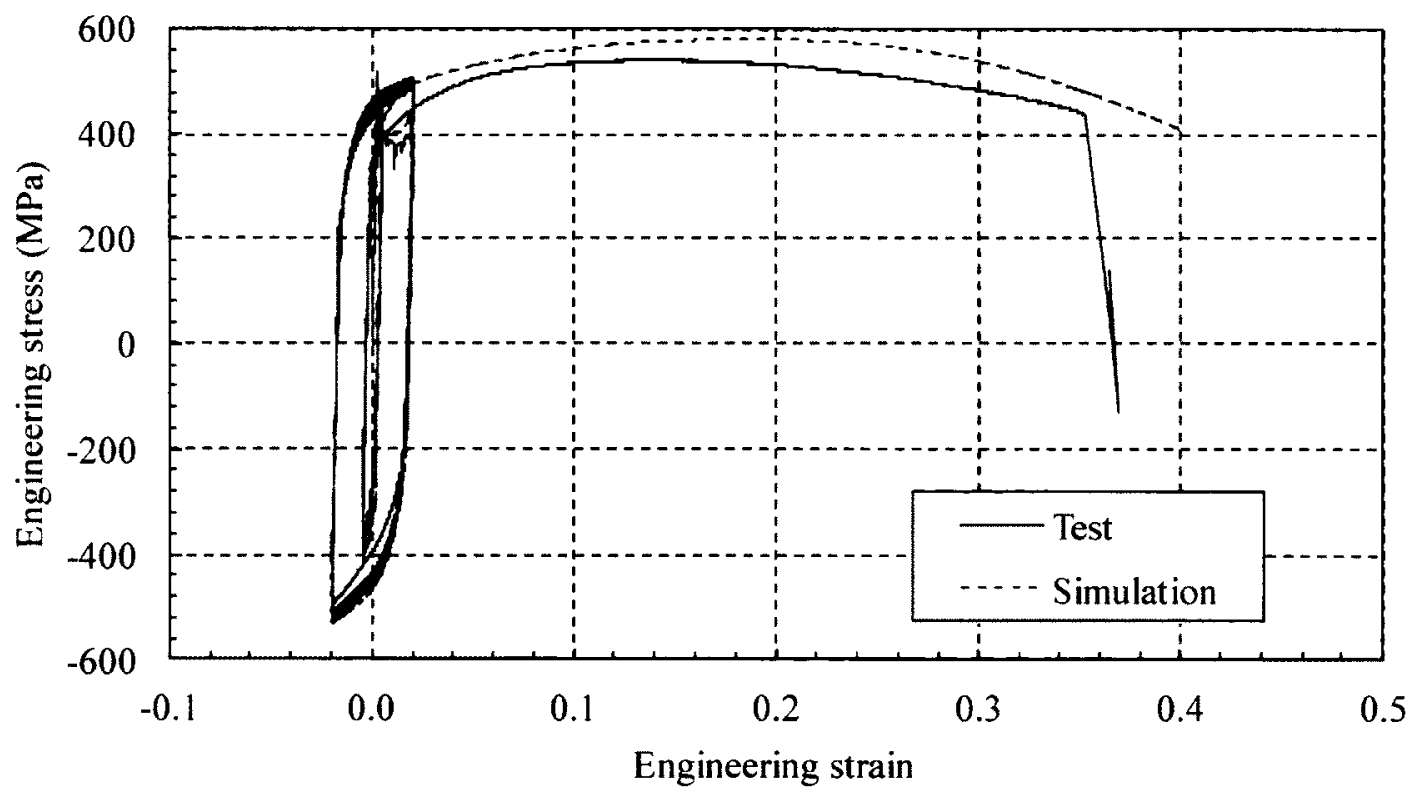

Figure 5.28 Test and predicted engineering stress versus engineering strain curves of ATE1D 


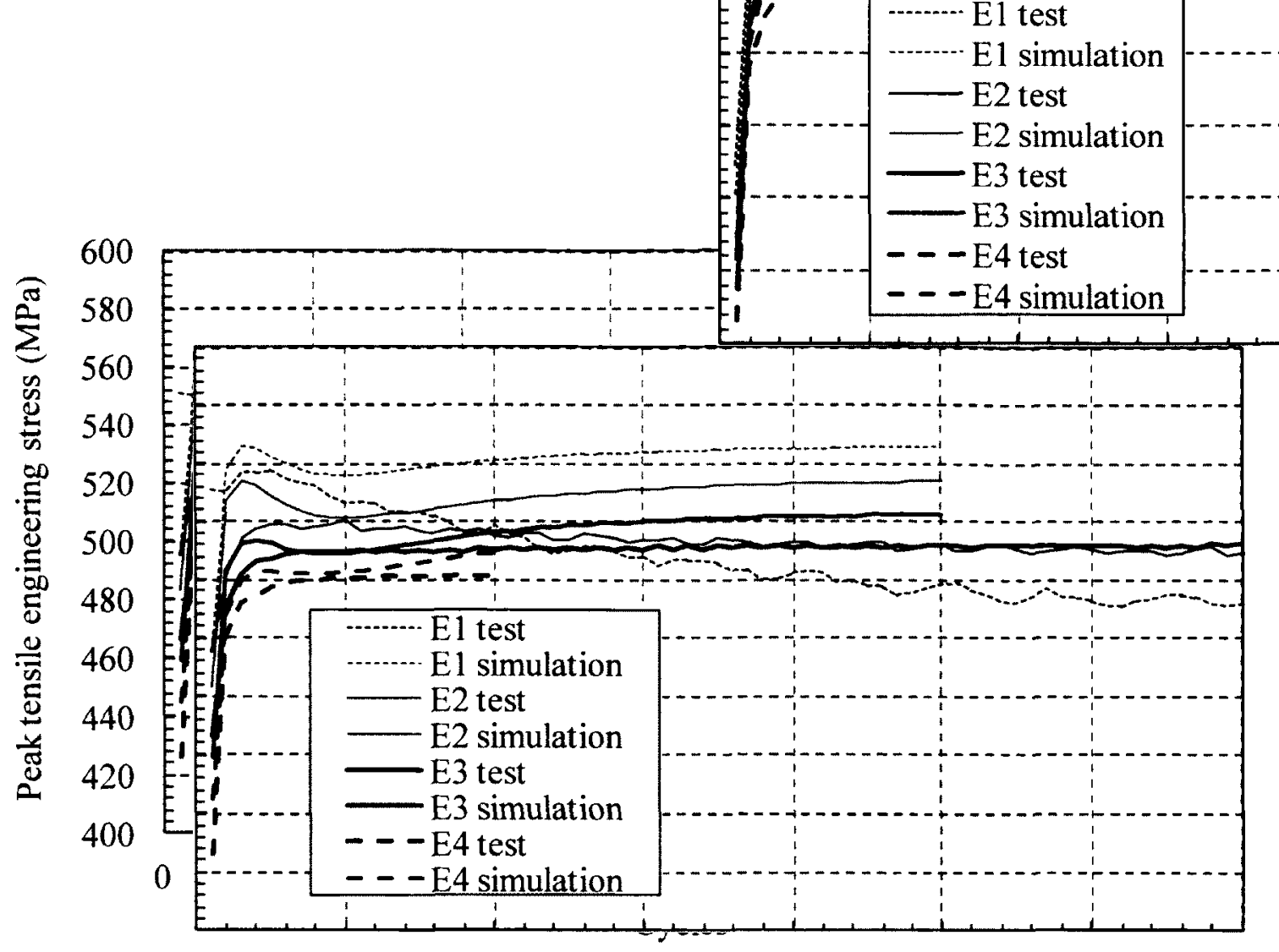

Figure 5.29 Predicted and test peak tensile engineering stress versus cycle number curves for various strain rates at the strain range of $\pm 2 \%$ for material $\mathrm{A}$

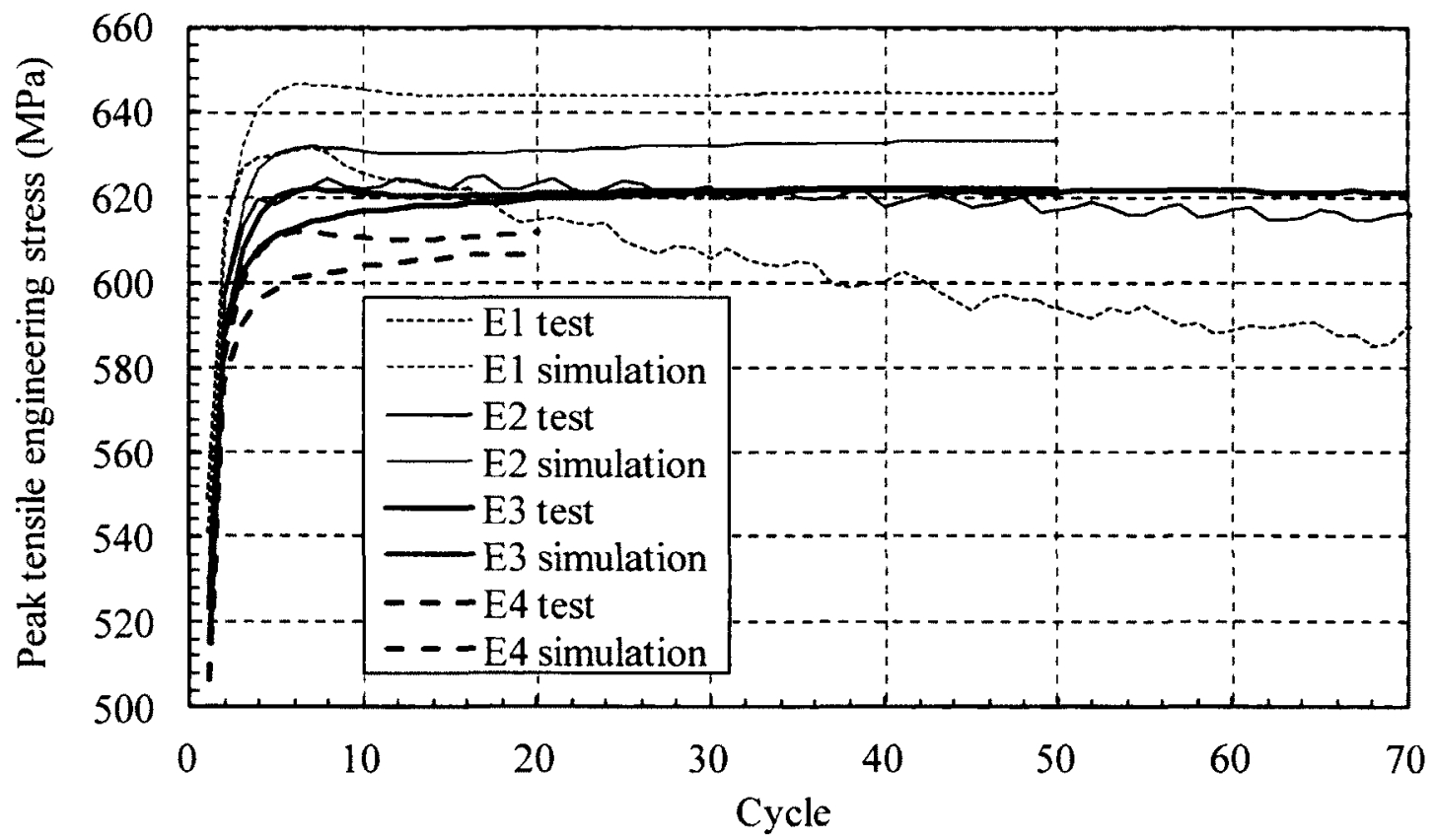

Figure 5.30 Predicted and test peak tensile engineering stress versus cycle number curves for various strain rates at the strain range of $\pm 2 \%$ for material $B$ 


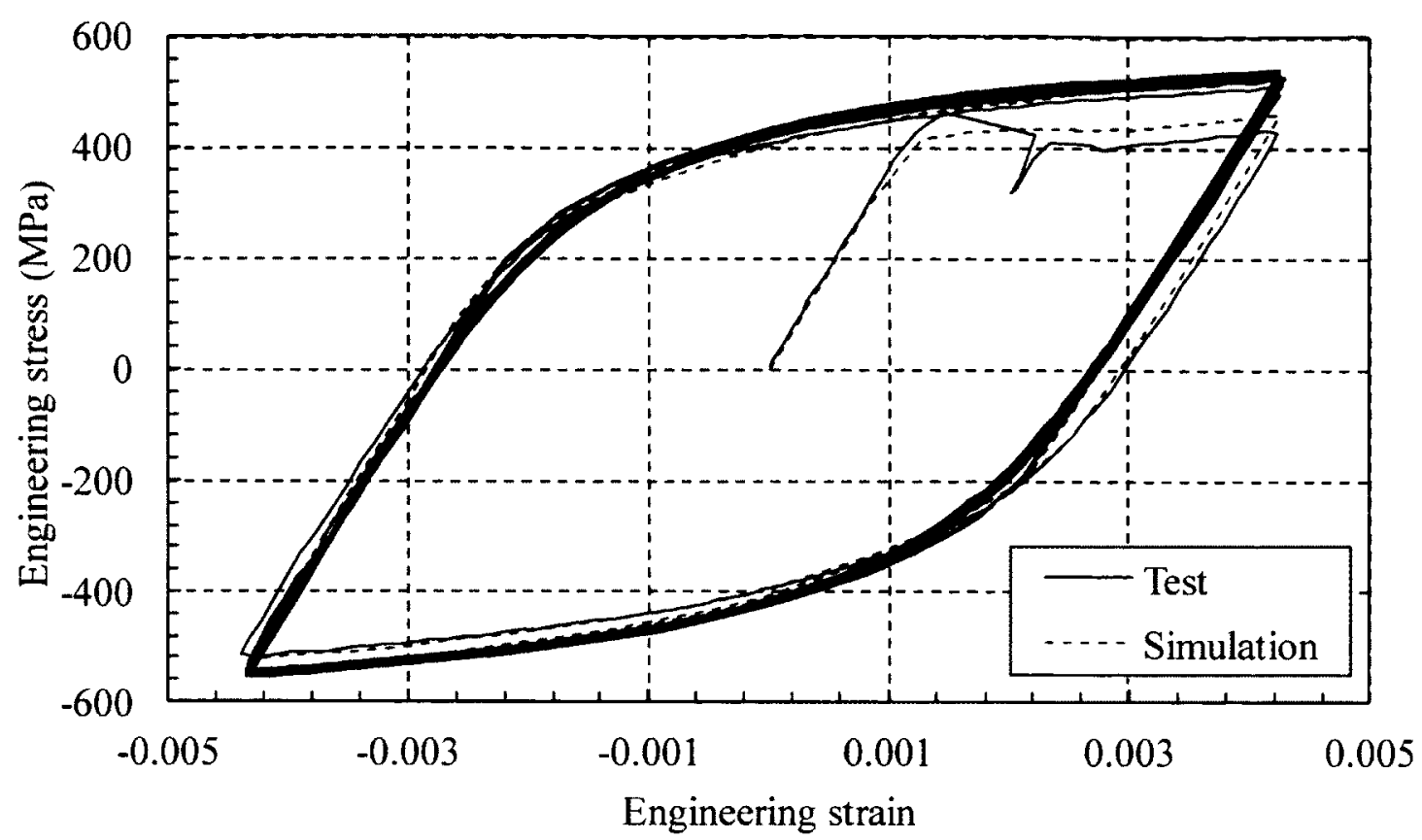

Figure 5.31 Test and predicted engineering stress versus engineering strain curves for 50 cycles of ANE3R2

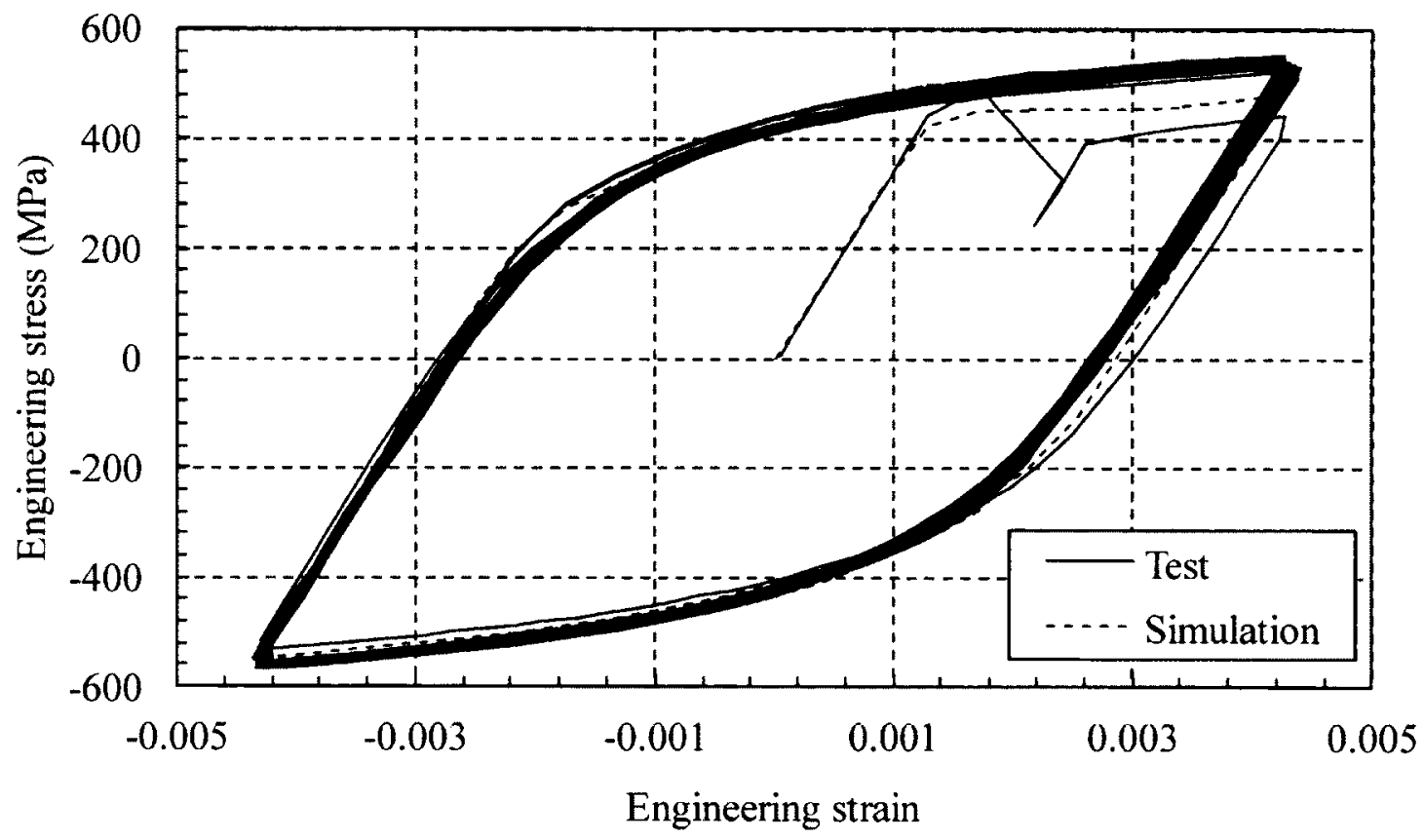

Figure 5.32 Test and predicted engineering stress versus engineering strain curves for 50 cycles of ANE2R2 


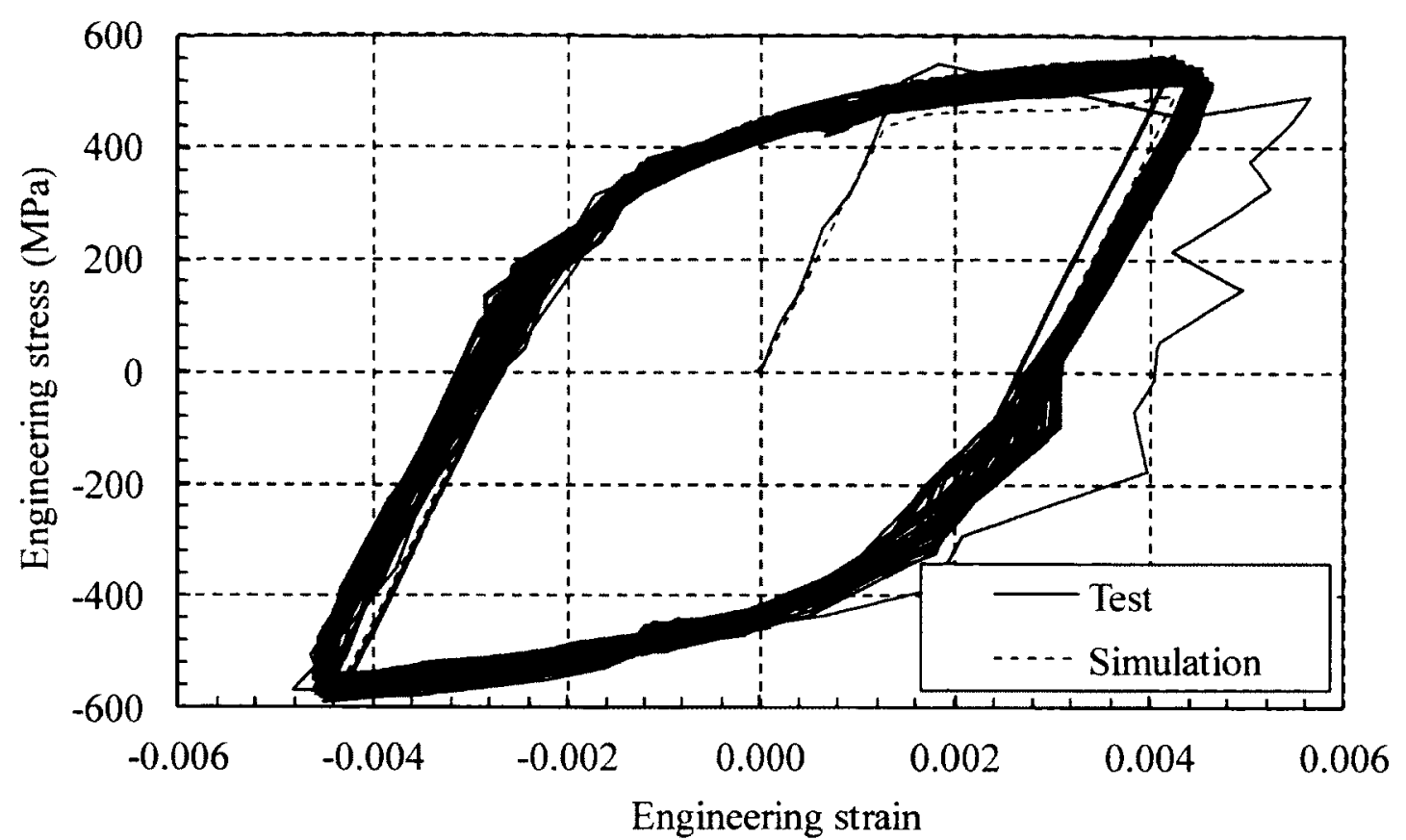

Figure 5.33 Test and predicted engineering stress versus engineering strain curves for 50 cycles of ANE1R2

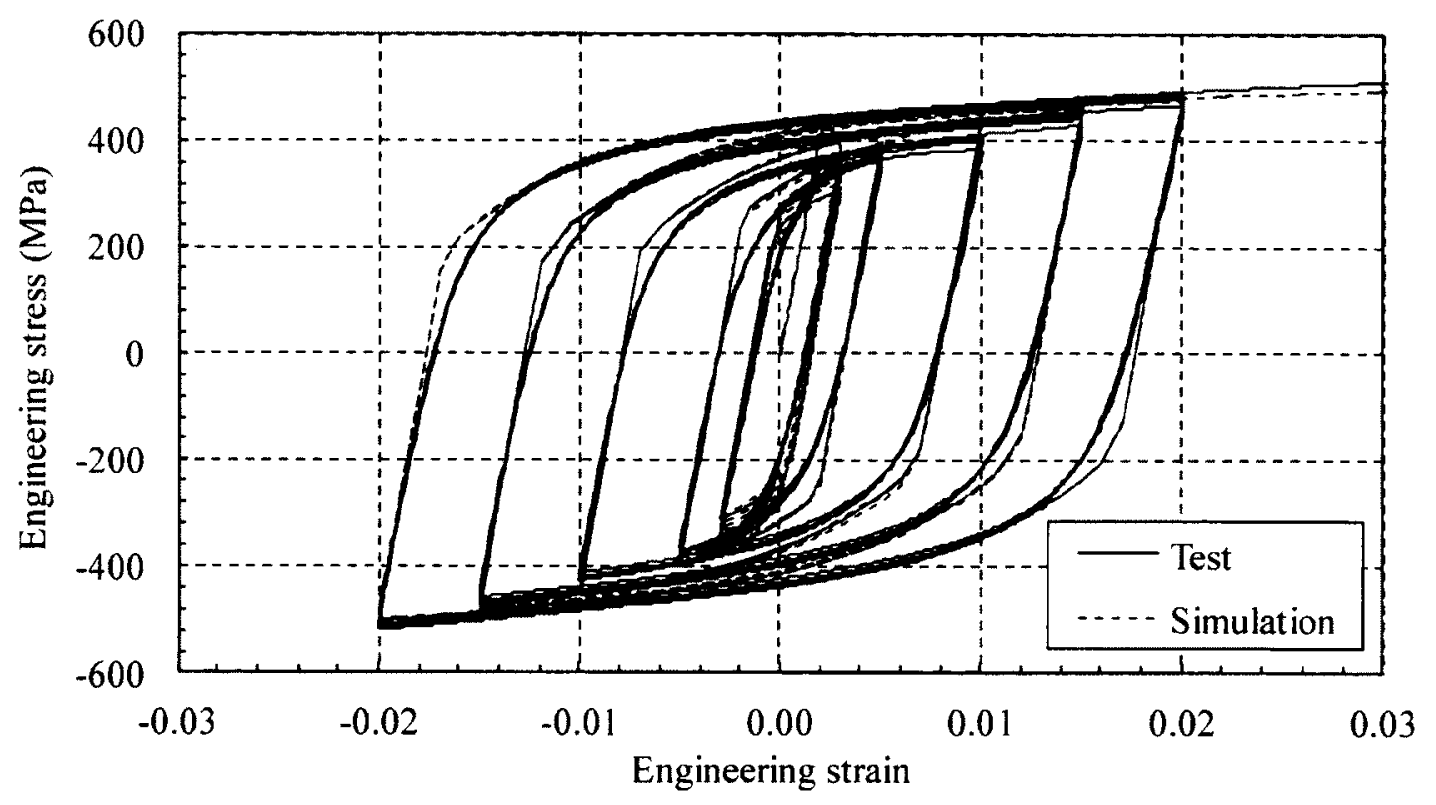

Figure 5.34 Test and predicted engineering stress versus engineering strain curves for the cyclic portion of ATE3I 


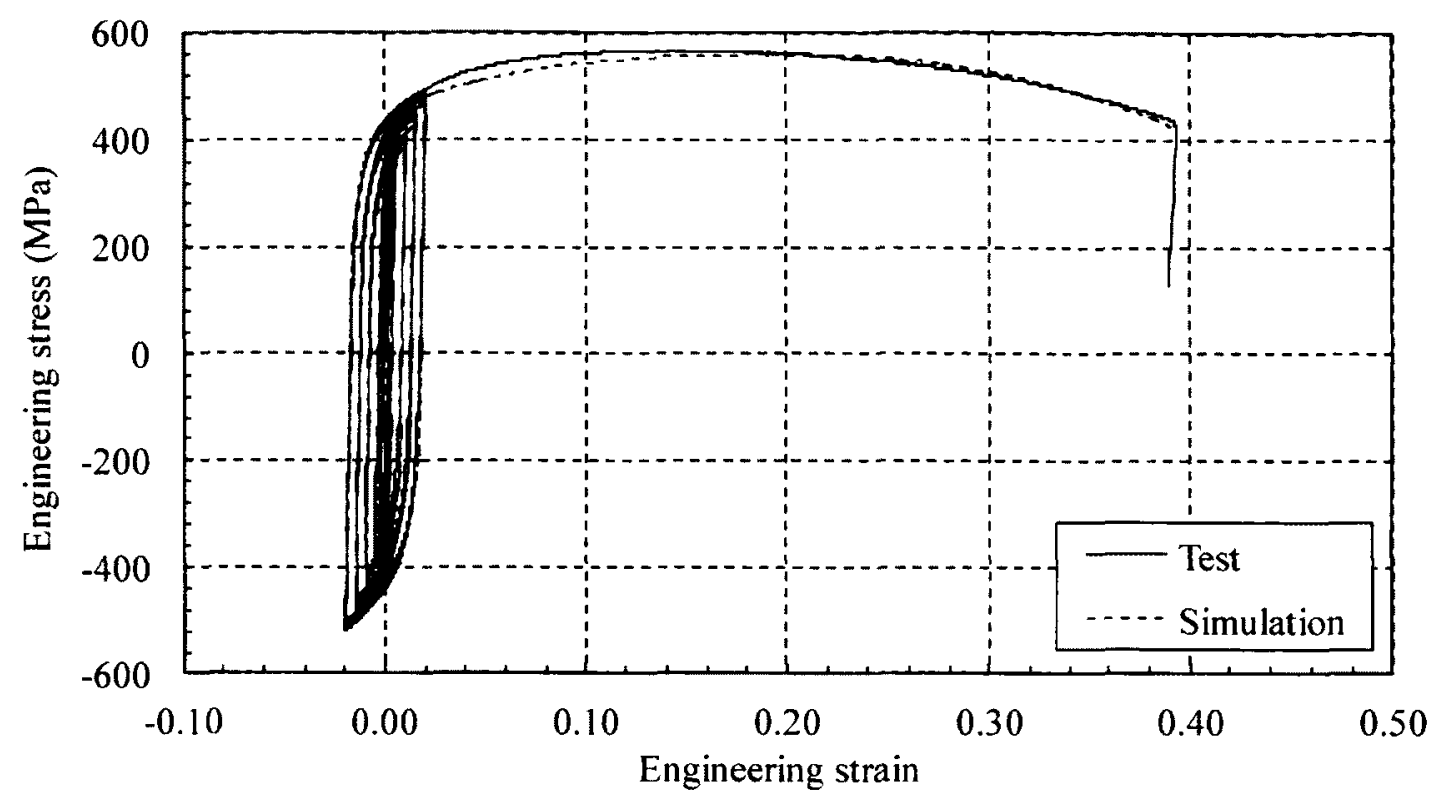

Figure 5.35 Test and predicted engineering stress versus engineering strain curves of ATE3I

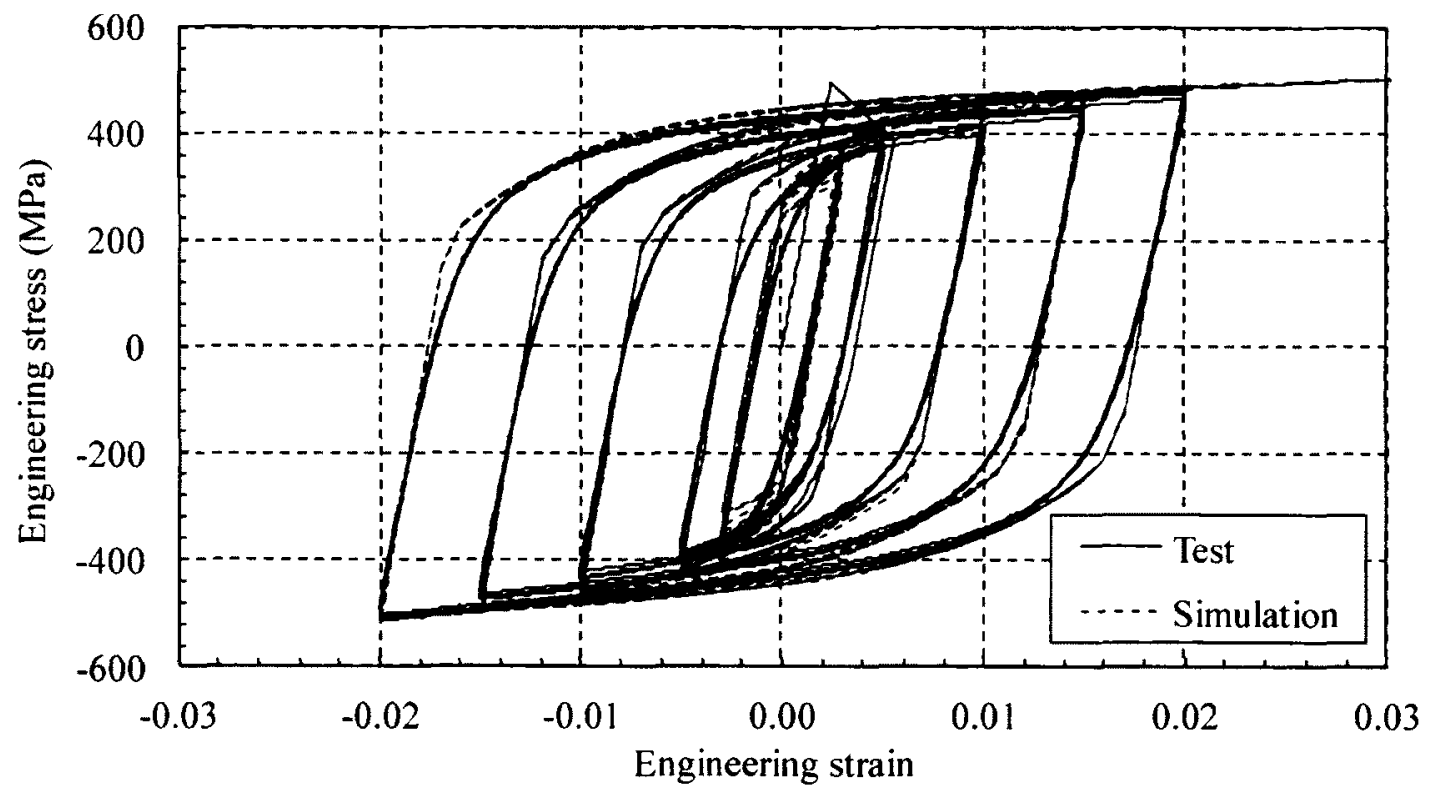

Figure 5.36 Test and predicted engineering stress versus engineering strain curves for the cyclic portion of ATE2I 


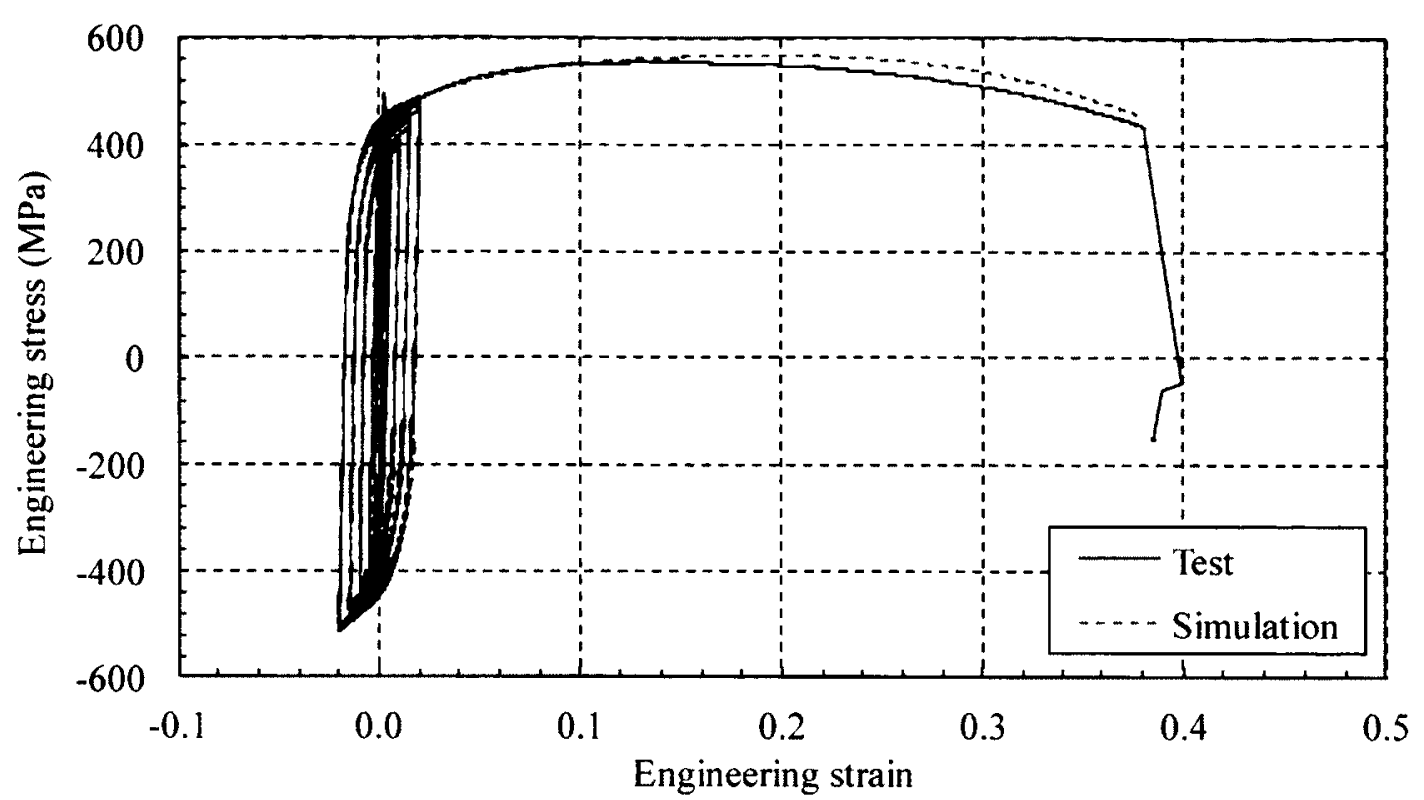

Figure 5.37 Test and predicted engineering stress versus engineering strain curves of ATE3I

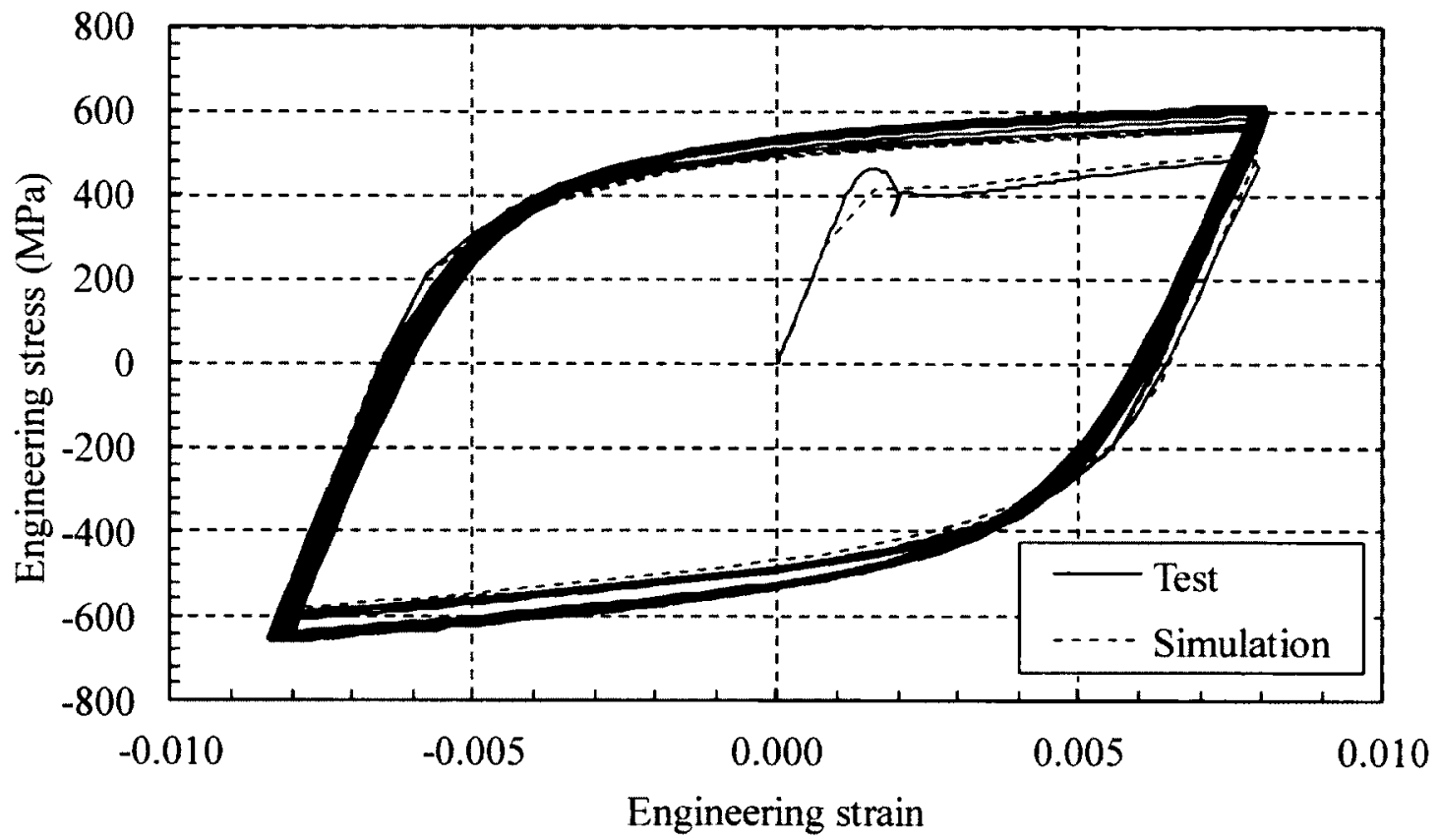

Figure 5.38 Test and predicted engineering stress versus engineering strain curves for 50 cycles of ANE4R4 


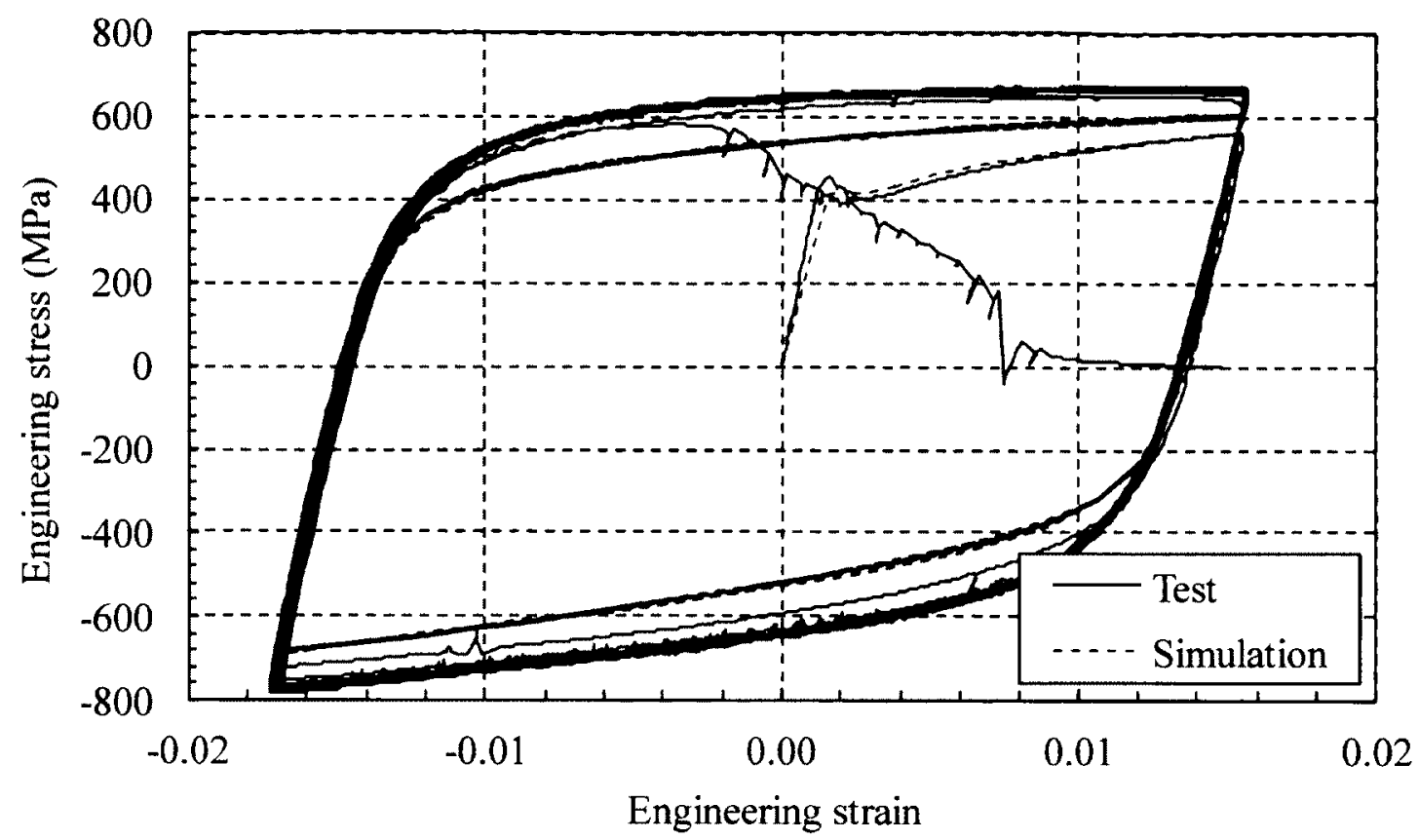

Figure 5.39 Test and predicted engineering stress versus engineering strain curves for 50 cycles of ANE4R8

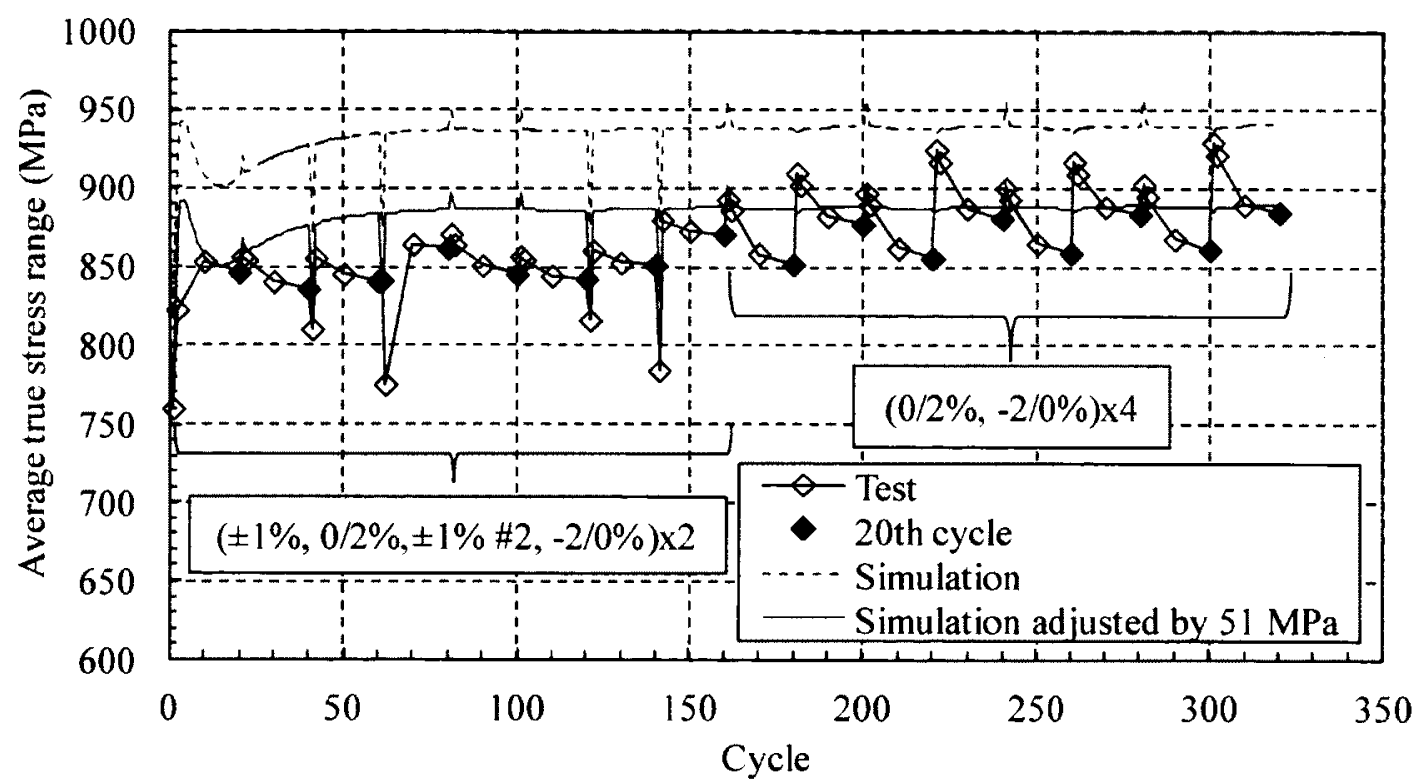

Figure 5.40 Test and predicted average true stress range versus cycle number for ATE2E 


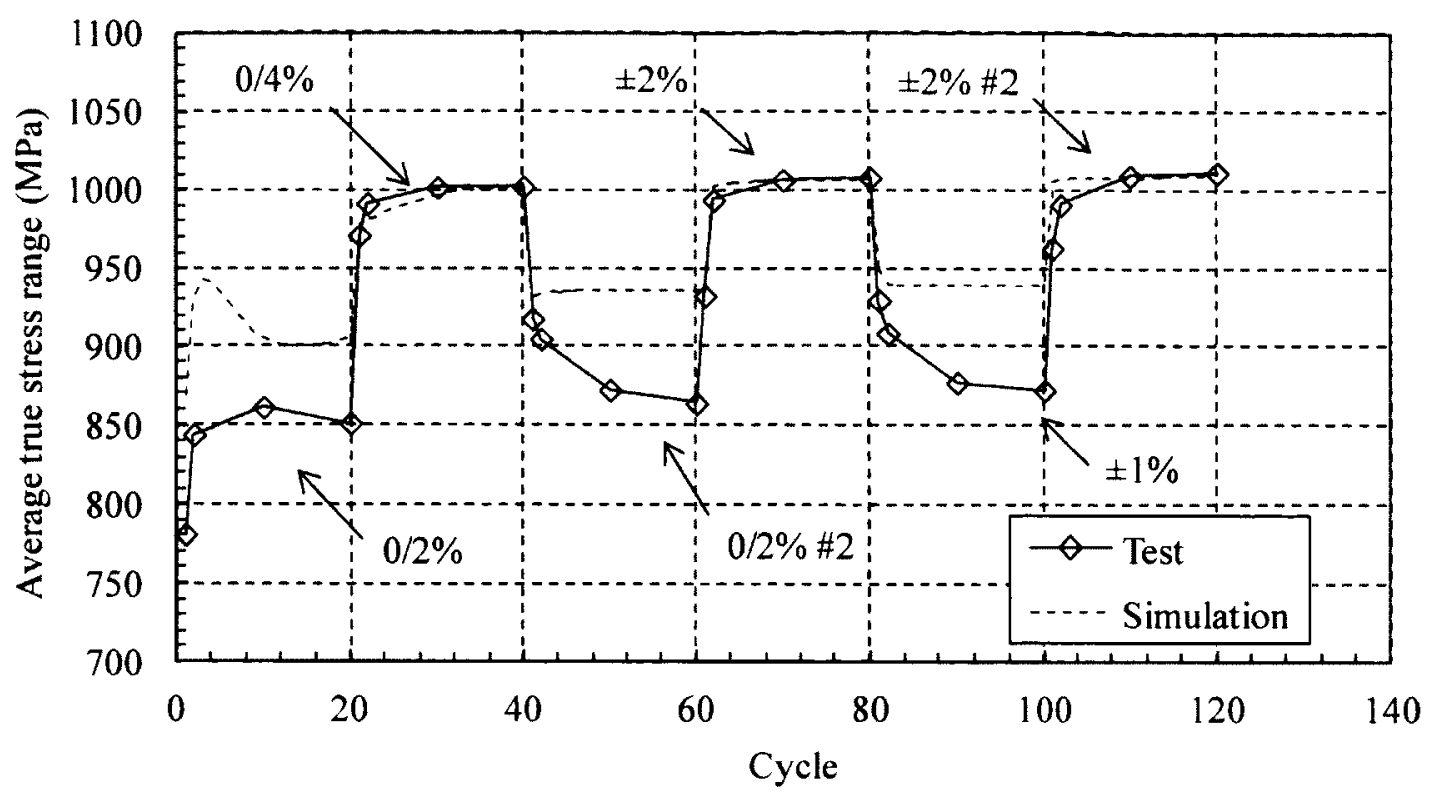

Figure 5.41 Test and predicted average true stress range versus cycle number for ATE2C-20 


\section{Chapter 6 Conclusions and Recommendations}

\subsection{Conclusions}

The following conclusions can be drawn from this study.

1) A positive rate sensitivity of stress within the first few cycles of loading can be seen for the strain rate from $10^{-4} \mathrm{~s}^{-1}$ to $10^{-1} \mathrm{~s}^{-1}$ on the material tested. Further into the cyclic tests of both tapered and notched specimens, a negative strain rate sensitivity of stress has been found for the strain rate range of $10^{-3}$ to $10^{-1} \mathrm{~s}^{-1}$ at a certain range of loading cycles. Evidence suggests that this is a result of adiabatic heating. With continual loading, notched specimen tests of material $\mathrm{A}$ at the strain rate of $10^{-1} \mathrm{~s}^{-1}$ show an increase in peak tensile stress with each successive loading cycle. This suggests that material A may be susceptible to dynamic strain aging (DSA). Nevertheless, a positive rate sensitivity has been observed for both tapered and notched specimens for the strain rates of $10^{-4} \mathrm{~s}^{-1}$ and $10^{-3} \mathrm{~s}^{-1}$ throughout the tests.

2) A negative rate sensitivity of both average true and engineering rupture strains from tapered specimens of both material A and B that were pulled to fracture has been observed for the strain rate range of $10^{-3}$ to $10^{-1} \mathrm{~s}^{-1}$. A reduction in fracture strain with an increasing number of loading cycles before being pulled to fracture can be seen in the test results in accordance to the reduction in fatigue life given by the Coffin-Manson relationship in Eq. (4.8).

3) The effect of monotonically increasing strain range loading on the stabilized cyclic stress-strain curve has been found to be minimal. Very little difference in 
the stress-strain curves has been observed between the $20^{\text {th }}$ cycle of a tapered specimen loaded at a strain range of $\pm 2 \%$ compared to one that has been loaded at incremental strain ranges from $\pm 0.3 \%$ to $\pm 2 \%$ for 10 cycles per strain range. As well, the effect of decreasing strain range loading on the flow stress has been found to be small for the strain ranges considered in this study if a sufficient number of cycles have been performed at a strain range such that the stress-strain curve has stabilized.

4) The effect of mean strain on the flow stress has been found to be small for the mean strains considered in this study if a sufficient number of cycles have been performed at each strain range such that the stress-strain curve has stabilized.

5) Notch specimens of material A have been found to show a slight positive rate sensitivity on the fatigue life for the strain rate range of $10^{-4}$ to $10^{-1} \mathrm{~s}^{-1}$, but the rate sensitivity decreases with the increase in loading strain range. However, the fatigue life of material $B$ was found to be mostly rate insensitive over the strain rate ranges of $10^{-4}$ to $10^{-1} \mathrm{~s}^{-1}$. Nevertheless, findings on the effect of strain rate on the fatigue life are not conclusive.

6) A procedure has been developed to obtain rate dependent yield stress versus true plastic strain curves that can be used as input for rate dependent numerical simulations under monotonic tension and cyclic loading using the mixed-mode hardening plasticity model by Lemaitre and Chaboche (1990). This procedure has been validated against test results through numerical simulations over the strain rate ranges of $10^{-4}$ to $10^{-1} \mathrm{~s}^{-1}$ for cyclic loading and $5 \times 10^{-5}$ to $10^{0} \mathrm{~s}^{-1}$ for monotonic tension loading. Finite element analyses using the input data generated have been 
found to be able to model the load-deformation behaviour of the tests in the absence of significant adiabatic heating. However, the numerical simulations have been less accurate on tests performed at a strain range other than the one used in the calibration to generate the input data.

\subsection{Recommendations}

The following recommendations are suggested for future work based on findings of the current study.

1) The significant work hardening seen in the later part of the fatigue test performed at the strain rate of $10^{-1} \mathrm{~s}^{-1}$ indicates that material $\mathrm{A}$ is susceptible to dynamic strain aging (DSA). A study can be conducted to explore how DSA can be accounted for in finite element analyses and to verify the susceptibility of material A to DSA. Generally, DSA studies are conducted under different constant temperatures and strain rates such that a matrix of test results can be formed. If material A is susceptible to DSA, an increase in flow stress, and possibly serrations, should be observed over a certain range of temperature for a given strain rate. For a higher strain rate test, the band of temperatures at which an increase in work hardening occurs should shift to higher values.

2) The methods of temperature measurement used in this study were employed to obtain only approximate results. An improved method of temperature measurement should be implemented in order to generate more accurate temperature data during loading. Surface mounted temperature gauges or temperature sensitive paints may be used to measure the surface temperature. It 
may also be possible to obtain the internal temperature measurement by drilling a small hole part way into a specimen and placing a thermocouple inside. However, tampering with the specimen in such a way may alter the response of the specimen.

3) To improve the numerical simulation of tests performed at the strain rates of $10^{-2} \mathrm{~s}^{-1}$ and higher, the effects of adiabatic heating should be accounted for in the constitutive modelling. In ABAQUS (Simulia 2010), the built-in Johnson-Cook isotropic hardening plasticity model is capable of modelling the strain rate and temperature effects on a yield stress versus true plastic strain curve. It may be possible to account for adiabatic heating with the same form of temperature modification factor, similar to that for the Johnson-Cook model, on the yield stress versus true plastic strain curve for the mixed-mode hardening plasticity model used in this study.

4) In the current study, results of numerical simulations are less accurate when the actual loading involved a strain range other than that used in the calibration to generate the data input. A study can be carried out to explore if the numerical simulation can be improved in modelling the response at different loading strain ranges by using more backstress (kinematic hardening) terms and/or using test data from more strain ranges in the calibration of the kinematic hardening parameters. In addition, using another model or performing improvements to the constitutive model by Lemaitre and Chaboche (1990) may be considered.

5) In this study, it has been found that the power law equation by Symonds and Cowper (1957) can be used to represent the relationship between the saturated 
yield stress and strain rate. Subsequently, the series of true flow stress versus true plastic strain curves at different strain rates have been generated using the differences in saturated yield stress given by this equation. The yield stress versus true plastic strain curves used in the numerical simulation are then calculated from these true flow stress versus true plastic strain curves. It may be worthwhile to explore if the power law equation used in the calculation of the saturated yield stress can be used as a strain rate dependent factor applied to a single yield stress versus true plastic strain curve in order to generate the yield stress versus true plastic strain curve for different strain rates. This may also help eliminate the convergence problem in numerical simulation associated with the input for strain rate dependent yield stress versus true plastic strain curves. 


\section{References}

1. Albertini, C. and Montagnani, M. (1980). Dynamic Uniaxial and Biaxial Stress-Strain Relationships for Austenitic Stainless Steel, Nuclear Engineering and Design, 57(1): $107-123$.

2. Alves, M., and Jones, N. (1999). Influence of Hydrostatic Stress on Failure of Axisymmetric Notched Specimens, Journal of the Mechanics and Physics of Solids, 47: 643-667.

3. Armstrong, P.J. and Frederick, C.O. (1966). A Mathematical Representation of the Multiaxial Bauschinger Effect, CEGB Report, RD/B/N/731, Berkeley Nuclear Laboratories, R\&D Department, California, USA.

4. ASTM (2004). Standard Practice for Strain-Controlled Fatigue Testing, ASTM E60604e1, American Society for Testing Materials, West Conshohocken.

5. ASTM (2007). Standard Specification for High-Strength Low-Alloy ColumbiumVanadium Structural Steel, American Society for Testing Materials, Pennsylvania, USA.

6. Avalos, M., Alvarez-Armas, I. and Armas, A.F. (2009). Dynamic Strain Aging Effects on Low-Cycle Fatigue of AISI 430F, Materials Science and Engineering A, 513-514: 1-7.

7. Barton, D.C., Sturges, J.L., Mirza, M.S., and Church, P. (1991). Deformation and Fracture; Modelling Techniques to Take Account of Strain-Rate Behaviour and Stress State, Journal De Physique IV, 1: 931-936. 
8. Begum, S., Chen, D.L., Xu, S., and Luo, A.A. (2009). Effect of Strain Ratio and Strain Rate on Low Cycle Fatigue Behaviour of AZ31 Wrought Magnesium Alloy, Materials Science and Engineering A, 517(1-2): 334-343.

9. Benson, D.K., and Hancock, J.R. (1974). The Effect of Strain Rate on the Cyclic Response of Metals, Metallurgical Transactions, 5(8): 1711-1715.

10. Bhanu Sankara Rao, K., Valsan, M., Sandhya, R., Ray, S.K., Mannan, S.L. and Rodriquez, P. (1985). On the Failure Condition in Strain-Controlled Low Cycle Fatigue, International Journal of Fatigue, 7(3): 141-147.

11. Blazynski, T.Z. (1983). Applied Elasto-Plasticity of Solids, Macmillan Press Ltd, London and Basingstoke.

12. Bridgman, P.W. (1944). The Stress Distribution at the Neck of a Tension Specimen, Transaction of the American Society for Metals, 32: 553-574.

13. Brindley, B. J. and Barnaby, J. T. (1966). Dynamic Strain Aging in Mild Steel, Acta Metallurgica, 14(12): 1765-1780.

14. Broek, D. (1979). The Practical Use of Fracture Mechanics, Kluwer Academic Publishers, Dordrecht, The Netherlands.

15. Bui-Quoc, T. and Biron, A. (1977). Comparison of Low-Cycle Fatigue Results with Axial and Diametral Extensometers, Experimental Mechanics, 17(1): 127-133.

16. Chaboche, J.L., Dang Van, K. and Cordier, G., (1979). Modelization of the Strain Memory Effect on the Cycle Hardening of 316 Stainless Steel, Transaction of SMiRT 5, vol. L, Paper No. L11/3.

17. Chai, H.-F. and Laird, C. (1987). Mechanisms of Cyclic Softening and Cyclic Creep in Low Carbon Steel, Materials Science and Engineering, 93: 159-174. 
18. Chang, K.-C. and Lee, G.C. (1987). Strain Rate Effect on Structural Steel Under Cyclic Loading, Journal of Engineering Mechanics, 113(9): 1292-1301.

19. Chen, J. (2010). An Experimental Study of Strain Rate Effects on Mild Steel, Department of Civil and Environmental Engineering, Carleton University, Ottawa, Ontario, Canada.

20. Cheng, J. and Nemat-Nasser, S. (2000). A Model for Experimentally-Observed HighStrain-Rate Dynamic Strain Aging in Titanium, Acta Materialia, 48(12): 3131-3144.

21. Chinh, N.Q., Horvath, G., Horita, Z. and Langdon, T.G. (2004). A New Consitutive Relationship for the Homogeneous Deformation of Metals Over a Wide Range of Strain, Acta Materialia, 52(12): 3555-3563.

22. Chiou, Y.-C. and Yip, M.-C. (2003). Effect of Mean Strain Level on the Cyclic Stress-Strain Behaviour of AISI 316 Stainless Steel, Materials Science and Engineering: A, 354(1-2): 270-278.

23. Christ, H.-J., Hoffmann, G. and Ottinger, O. (1995). History Effects in Meals During Constant and Variable Amplitude Testing. I: Wavy Dislocation Glide Behaviour, Materials Science and Engineering, A201: 1-12.

24. Coffin, L.F. (1954). A Study of the Effects of Cyclic Thermal Stresses on a Ductile Metal, Transactions of the ASME, 76: 931-950.

25. Cowper, G. R. and Symonds, P. S. (1957). Yield and Flow of Low-Carbon Steel at Medium Strain Rates, Proceedings of the Conference on the Physical Basis of Yield and Fracture, Oxford University, Oxford: 77-87.

26. CSA (2004). CSA G40.20/21, General Requirements for Rolled or Welded Structural Quality Steel, Canadian Standards Association, Mississauga, Ontario, Canada. 
27. Cunningham, S. (1999). Effect of Substitutional Elements on Dynamic Strain Aging in Steel, Department of Mining and Metallurgical Engineering, McGill University, Montreal, Quebec, Canada.

28. Dieter, G.E. (1986). Mechanical Metallurgy, $3^{\text {rd }}$ edition, McGraw-Hill Inc, United States of America.

29. Dusicka, P., Itani, A.M., and Buckle, I.G. (2007). Cyclic Response of Plate Steels Under Large Inelastic Strains, Journal of Constructional Steel Research, 63(2): 156164.

30. Feltner, C.E. and Laird, C. (1967). Cyclic Stress-Strain Response of F.C.C. Metals and Alloys - I Phenomenological Experiments, Acta Metallurgica, 15(10): 16211632.

31. Hahner, P. (1996). On the Physics of the Portevin-Le Chatelier Effect Part 1: The Statistics of Dynamic Strain Aging, Materials Science and Engineering A207(2): 208215.

32. Harmathy, T.Z. and Stanzak, W.W. (1970) Elevated-Temperature Tensile and Creep Properties of Some Structural and Prestressing Steels, American Society for Testing Materials, STP 464: 186-208.

33. Hong, S.G. and Lee, S.B. (2004). The Tensile and Low-Cycle Fatigue Behaviour of Cold Worked 316L Stainless Steel: Influence of Dynamic Strain Aging, International Journal of Fatigue, 26(8): 899-910.

34. Hong, S.-G., Lee, K.-O., Lee, S.-B. (2005). Dynamic Strain Aging Effect on the Fatigue Resistance of Type 3116L Stainless Steel, International Journal of Fatigue 27: 1420-1424. 
35. Johnston, W.G. and Gilman, J.J. (1959). Dislocation Velocities, Dislocation Densities, and Plastic Flow in Lithium Fluoride Crystals, Journal of Applied Physics, 30(2): 129-144.

36. Keh, A.S., Nakada, Y. and Leslie, W.C. (1968). Dynamic Strain Aging in Iron and Steel, Dislocation Dynamics, McGraw-Hill. p. 381.

37. Kliman, V. and Bily, M. (1980). The Influence of Mode Control, Mean Value and Frequency of Loading on the Cyclic Stress-Strain Curve, Materials Science and Engineering, 44(1): 73-79.

38. Krempl, E. (1979). An Experimental Study of Room-Temperature Rate-Sensitvity, Creep and Relaxation of AISI Type 304 Stainless Steel, Journal of the Mechanics and Physics of Solids, 27(5-6): 363-375.

39. Lemaitre, J. and Chaboche, J.-L. (1990). Mechanics of Solid Materials, Cambridge University Press, Cambridge.

40. Liu, H. and Wang, X. (2001). Dislocation Velocity Exponent and the Strain Rate, Journal of Materials Science and Technology, 17(3): 363-366.

41. Lukas, P. and Klesnil, M. (1973). Cyclic Stress-Strain Response and Fatigue Life of Metals in Low Amplitude Region, Materials Science and Engineering, 11(6): 345356.

42. Manjoine, M.J. (1944). Influence of Rate of Strain on Temperature on Yield Stresses of Mild Steel, Transactions of the American Society of Mechanical Engineers, Journal of Applied Mechanics, 66: A211-A218.

43. Manson, S.S. (1953). Behaviour of Materials Under Conditions of Thermal Stress, National Advisory Committee for Aeronautics, Report 1170. 
44. Manson, S.S. (1966). Thermal Stress and Low-Cycle Fatigue, Mc-Graw Hill Inc.

45. Miller, K.J., and Rizk, M.N. (1968). Effect of Strain Rate on Low-Endurance Torsional Fatigue in Commercially Pure Aluminum, Journal of Strain Analysis, 3(4): 273-280.

46. Moscato, M.G., Avalos, M., Alvarez-Armas, I., Petersen, C. and Armas, A.F. (1997). Effect of Strain Rate on the Cyclic Hardening of Zircaloy-4 in the Dynamic Strain Aging Temperature Range, Materials Science and Engineering A, 234-236: 834-837.

47. Ngo, T., Mendis, P., Gupta, A. and Ramsay, J. (2007). Blast Loading and Blast Effects on Structures - An Overview, Electronic Journal of Structural Engineering Special Issue: Loading on Structures: 76-91.

48. Prager, W. (1956). A New Method of Analyzing Stresses and Strains in WorkHardening Plastic Solids, Journal of Applied Mechanics, 23: 493-496.

49. Prasad, K., Sarkar, R., Ghosal, P. and Varma, V.K. (2008). The Influence of Dynamic Strain Aging on the Low Cycle Fatigue Behaviour of Near Alpha Titanium Alloy IMI 834, Materials Science and Engineering A, 494: 227-231.

50. Restrepo-Posada, J.I., Dodd, L.L., Park, R., and Cooke, N. (1994). Variables Affecting Cyclic Behaviour of Reinforcing Steel, Journal of Structural Engineering, $120(11): 3178-3196$.

51. Rodriguez, P. (1984). Serrated Plastic Flow, Bulletin of Materials Science, 6(4): 653663.

52. Sachs, N.W. (2007). Practical Plant Failure Analysis: A Guide to Understanding Machinery Deterioration and Improving Reliability, CRC Press, Taylor Francis Group, Boca Raton, Florida. 
53. Saeki, E., Sugisawa, M., Yamaguchi, T., and Wada, A. (1998). Mechanical Properties of Low Yield Point Steels, Journal of Materials in Civil Engineering, 10(3): 143-152.

54. Schoeck, G. (1984). The Portevin-Le Chatelier Effect. A Kinetic Theory, Acta Metallurgica, 32(8): 1229-1234.

55. Simulia (2010). Abaqus 6.10, Dassault Systemes Simulia Corp., Providence, RI, USA.

56. Soroushian, P. and Choi, K.B. (1987). Steel Mechanical Properties at Different Strain Rates, Journal of Structural Engineering, 113(4): 663-672.

57. Srinivasan, V.S., Sandhya, K., Bhanu Sankara Rao, S.L., Mannan, S.L. and Raghavan, K.S. (1991). Effects of Temperature on the Low Cycle Fatigue Behaviour of Nitrogen Alloyed Type 316L Stainlesss Steel, International Journal of Fatigue, 13(6): $471-478$.

58. Sung, J.H., Kim, J.H and Wagoner, R.H. (2010). A Plastic Constitutive Equation Incorporating Strain, Strain-Rate, and Temperature, International Journal of Plasticity, 26(12): 1746-1771.

59. Teresa Correia, M. and Fortes, M.A. (1981). The Initial Stage of Deformation Leading to Periodically Serrated Tensile Curves, Scripta Metallurgica, 15: 369-372.

60. Trampczynski, W. (1988). The Experimental Verification of the Evolution of Kinematic and Isotropic Hardening in Cyclic Plasticity, Journal of the Mechanics and Physics of Solids, 36(4): 417-441.

61. Tsuzaki, K., Matsuzaki, Y., Maki, T. and Tamura, I. (1991). Fatigue Deformation Accompanying Dynamic Strain Aging in a Pearlitic Eutectoid Steel, Materials Science and Engineering, A142: 63-70. 
62. Wen, Z. (2012). Modeling of Ductile Fracture in Steel Structures for Monotonic and Cyclic Loading, Department of Civil and Environmental Engineering, Carleton University, Ottawa, Ontario, Canada.

63. Weisse, M., Wamukwamba, C.K., Christ, H.-J. and Mughrabi, H. (1993). The Cyclic Deformation and Fatigue Behaviour of the Low Carbon Steel SAE 1045 in the Temperature Regime of Dynamic Strain Ageing, Acta Metallurgica et Materialia, 41(7): 2227-2233.

64. Wood, W. A. (1963). Removal of Internal Stresses from a Metal by Cyclic Strain, Journal of the Institute of Metals, 91: 193-196.

65. Zeghib, N.E. and Klepaczko, J.R. (1996). Work Hardening of Mild Steel Within Dynamic Strain Ageing Temperatures, Journal of Materials Science, 31(22): 60856088.

66. Zhao, H. and Abdennadher, S. (2004). On the Strength Enhancement Under Impact Loading of Square Tubes Made From Rate Insensitive Metals, International Journal of Solids and Structures, 41(24-25): 6677-6697.

67. Ziegler, H. (1959). A Modification of Prager's Hardening Rule, Quarterly of Applied Mathematics, 17(1): 55-65. 


\section{Appendix A Determination of True Strain Ranges for Notched Specimens}

True strain ranges of $\pm 2 \%, \pm 4 \%$ and $\pm 8 \%$ are considered for the notched specimens in this study. However, the resulting deformation in the radial direction for these true strain ranges is too small to allow for a reliable control through a diametral extensometer. Therefore, the loading for notched specimen tests was controlled through the axial extensometer. There is no general equation available that relates the axial extension applied on the specimen to the resulting true strain. For this reason, a finite element analysis (FEA) had been used to estimate the axial extensometer displacement required to produce the true strain ranges.

The analysis was carried out using ABAQUS (Simulia 2010). Utilizing symmetry, only the segment of the specimen over half of the gauge length of the $50.8 \mathrm{~mm}$ ( 2 inch) axial extensometer has been modelled using CAX8R, a bi-quadratic axisymmetric element with reduced integration. The finite element model used in the analysis is shown in Fig G.4 of Appendix G. A mixed-mode plastic hardening model by Lemaitre and Chaboche (1990) with two backstress terms has been used to model the material behaviour for this analysis. The true flow stress versus true plastic strain data has been generated using Eq. (5.2), and the backstress versus true plastic strain data for monotonic uniaxial loading has been generated using Eq. (2.18). The yield stress versus true plastic strain data for the numerical simulation were calculated from these generated flow stress and backstress using Eq. (2.21). Parameters calibrated by Wen (2012) for the same ASTM A572 Gr. 50 steel used in the current testing program have been used in generating the material property inputs for this analysis with the elastic modulus taken as $205600 \mathrm{MPa}$ and Poisson's ratio as 0.3. These parameters are summarized in Table A.1 
The numerical simulation involved a static analysis under either monotonic tension or compression. The predicted relationship between the axial engineering strain and average true strain is shown in Fig. A.1, with the average true strain taken as $\ln \left(A_{0} / A\right)$, where $A_{0}$ is the undeformed cross-sectional area and $A$ is the actual crosssectional area. Table A.2 shows the predicted axial engineering strains required to achieve the target average true strain. These compressive and tensile engineering strains have been used as the loading strain limits for both material A and B. 
Table A.1 Parameters used in generating the flow stress and backstress, taken from Wen (2012)

\begin{tabular}{|c|c|c|c|c|c|c|c|c|}
\hline \multicolumn{4}{|c|}{ Flow stress parameters } & \multicolumn{4}{c|}{ Kinematic hardening parameters } \\
\hline$\sigma_{t}^{y}(\mathrm{MPa})$ & $\varepsilon_{t}^{o}$ & $\sigma_{1}(\mathrm{MPa})$ & $d$ & $\varepsilon_{c}$ & $C_{1}(\mathrm{MPa})$ & $\gamma_{1}$ & $C_{2}(\mathrm{MPa})$ & $\gamma_{2}$ \\
\hline 362 & 0.0053 & 417 & 4.53 & 0.77 & 3625 & 39.46 & 711 & 3.39 \\
\hline
\end{tabular}

Table A.2 Estimated engineering strains to produce the desired true strains in the simulation

\begin{tabular}{|c|c|c|}
\hline $\begin{array}{c}\text { True strain range } \\
(\mathrm{mm} / \mathrm{mm})\end{array}$ & $\begin{array}{c}\text { Tensile engineering strain } \\
(\mathrm{mm} / \mathrm{mm})\end{array}$ & $\begin{array}{c}\text { Compressive engineering strain } \\
(\mathrm{mm} / \mathrm{mm})\end{array}$ \\
\hline $\pm 2 \%$ & $0.426 \%$ & $0.433 \%$ \\
\hline $\pm 4 \%$ & $0.795 \%$ & $0.819 \%$ \\
\hline $\pm 8 \%$ & $1.550 \%$ & $1.690 \%$ \\
\hline
\end{tabular}

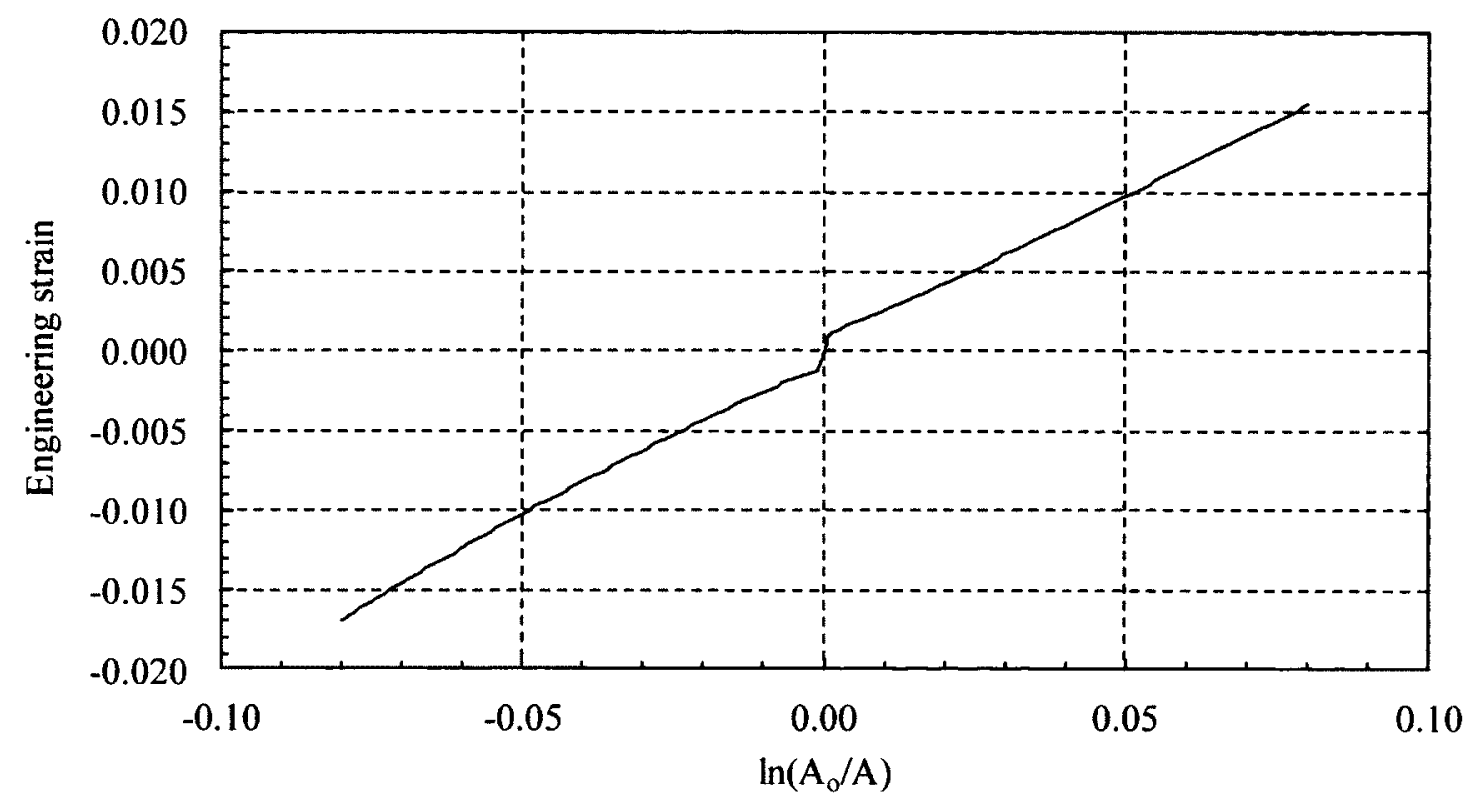

Figure A.1 Predicted engineering strain versus average true strain 


\section{Appendix B Additional Test Data}

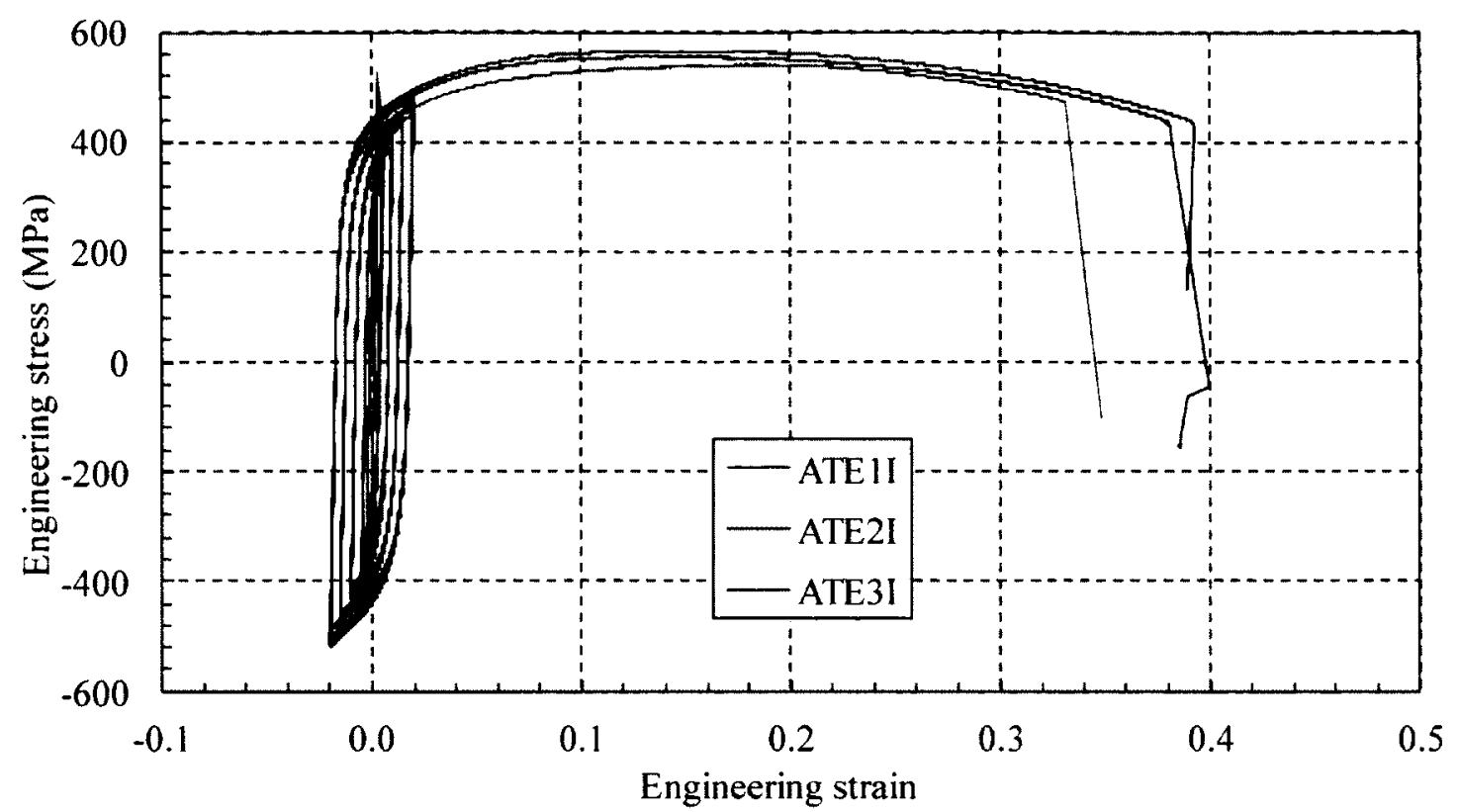

Figure B.1 Engineering stress versus engineering strain curve for ATE3I, ATE2I and ATE1I

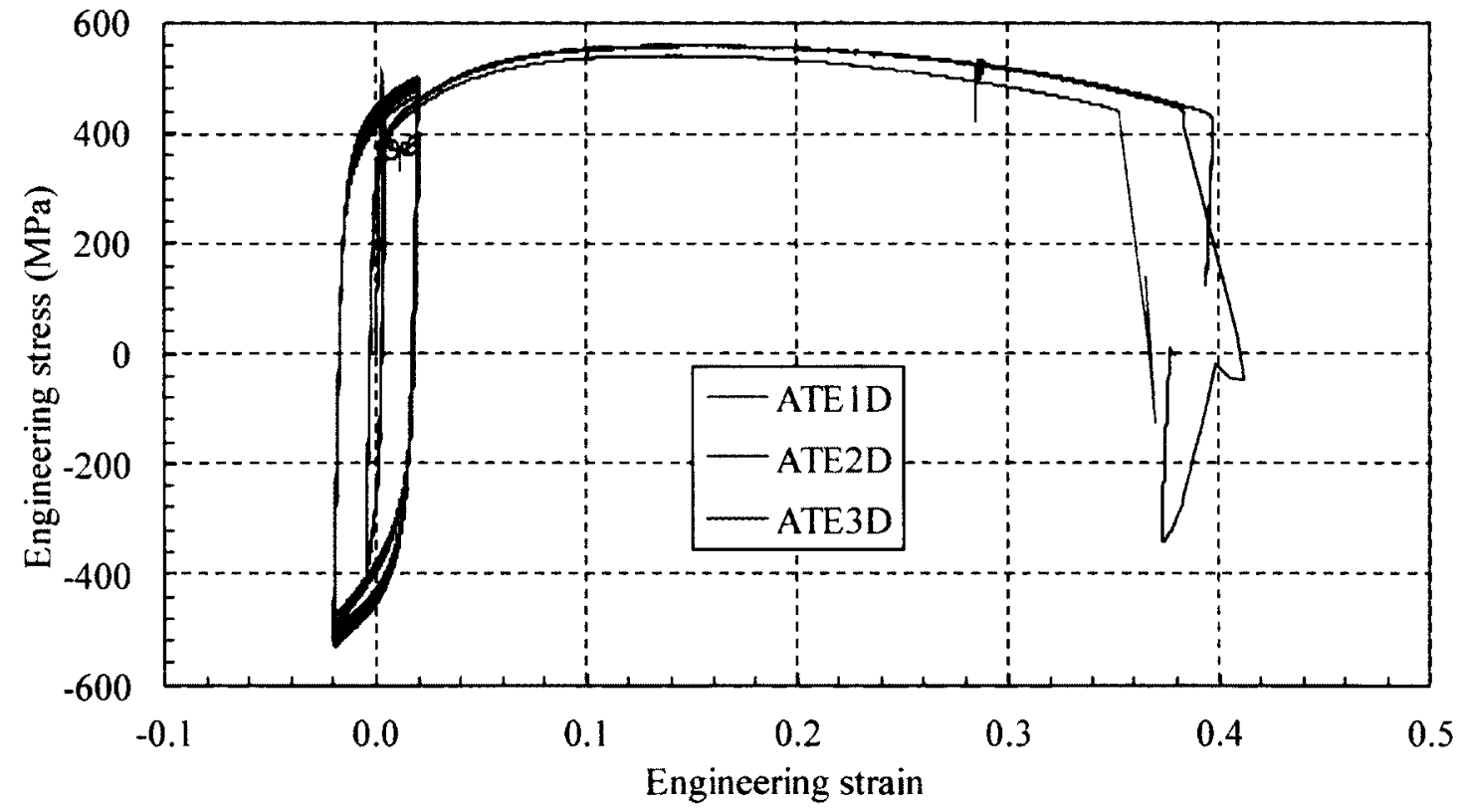

Figure B.2 Engineering stress versus engineering strain curve for ATE3D, ATE2D and ATE1D 


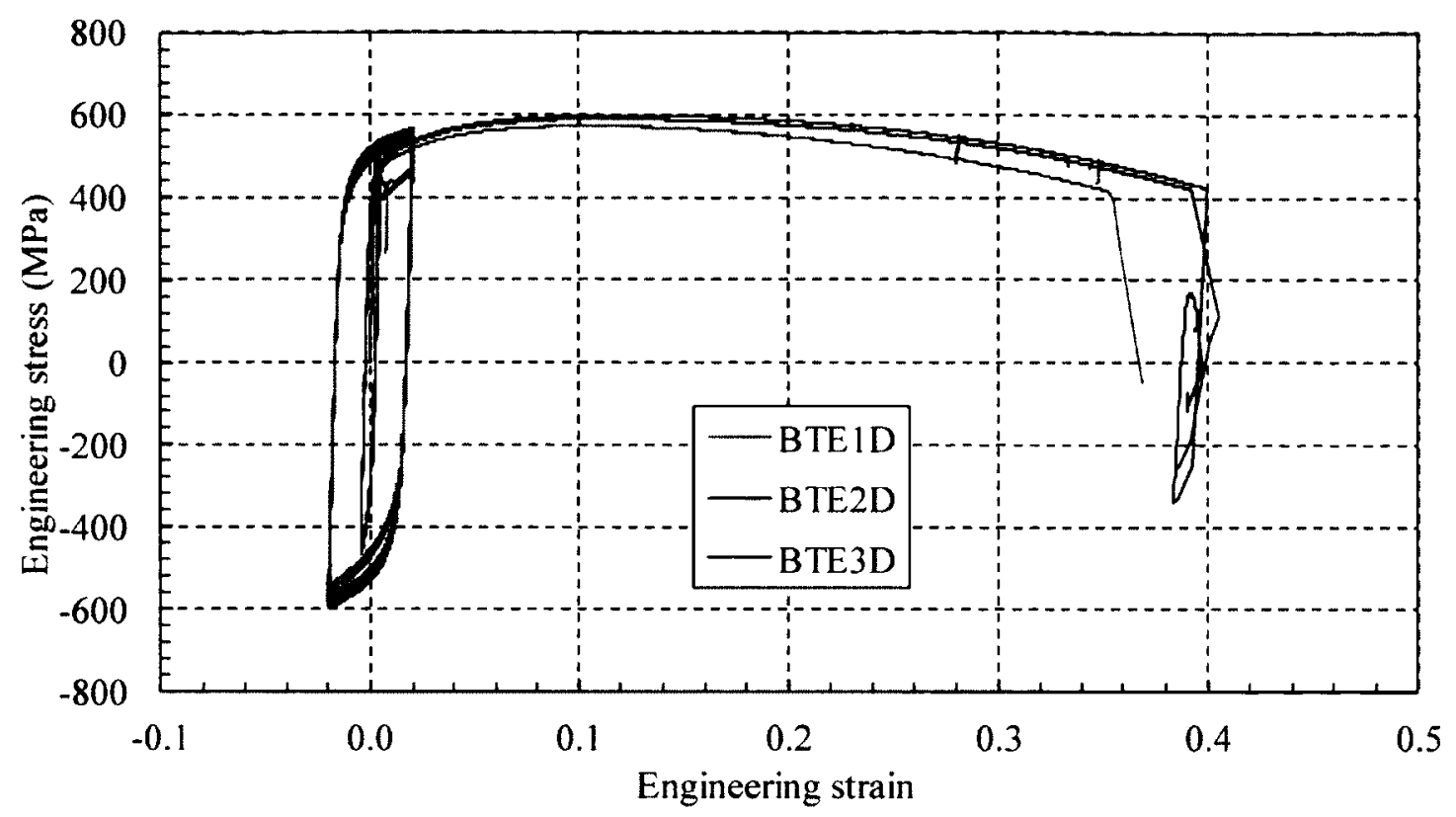

Figure B.3 Engineering stress versus engineering strain curve for BTE3D, BTE2D and BTE1D

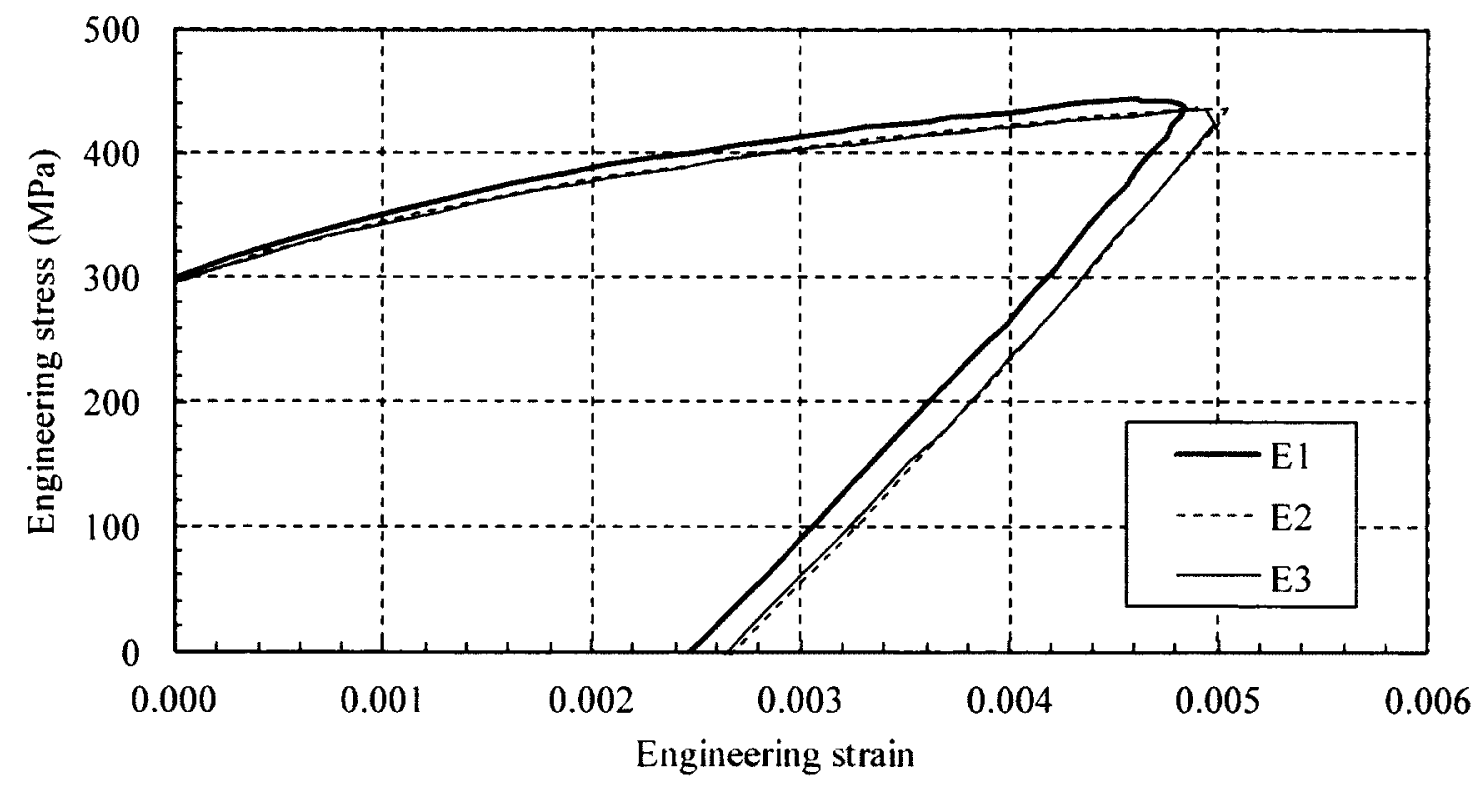

Figure B.4 Part of the $10^{\text {th }}$ cycle for BTE1I, BTE2I and BTE3I at the $\pm 0.5 \%$ strain range 


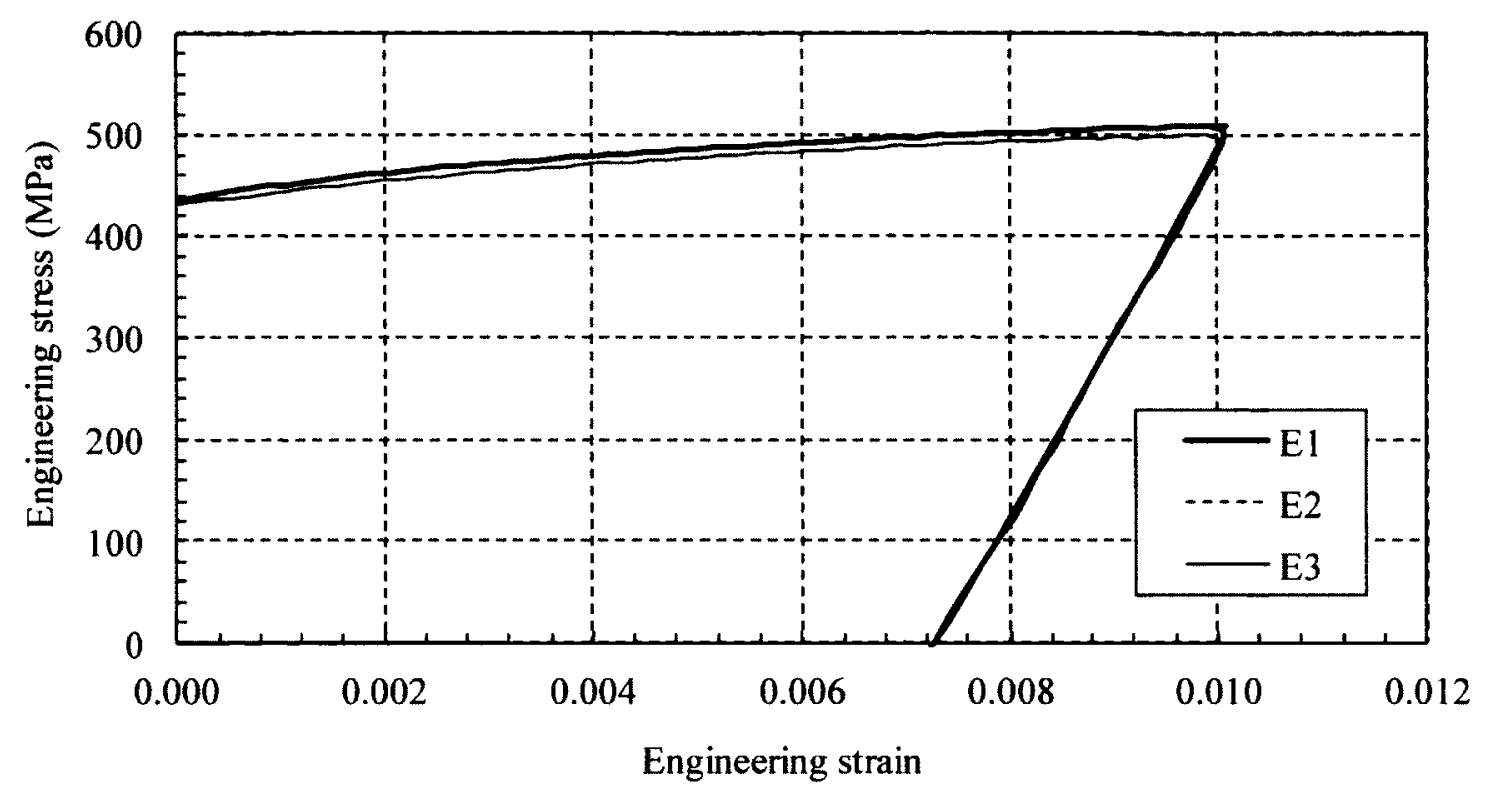

Figure B.5 Part of the $10^{\text {th }}$ cycle for BTE1I, BTE2I and BTE3I at the $\pm 1 \%$ strain range

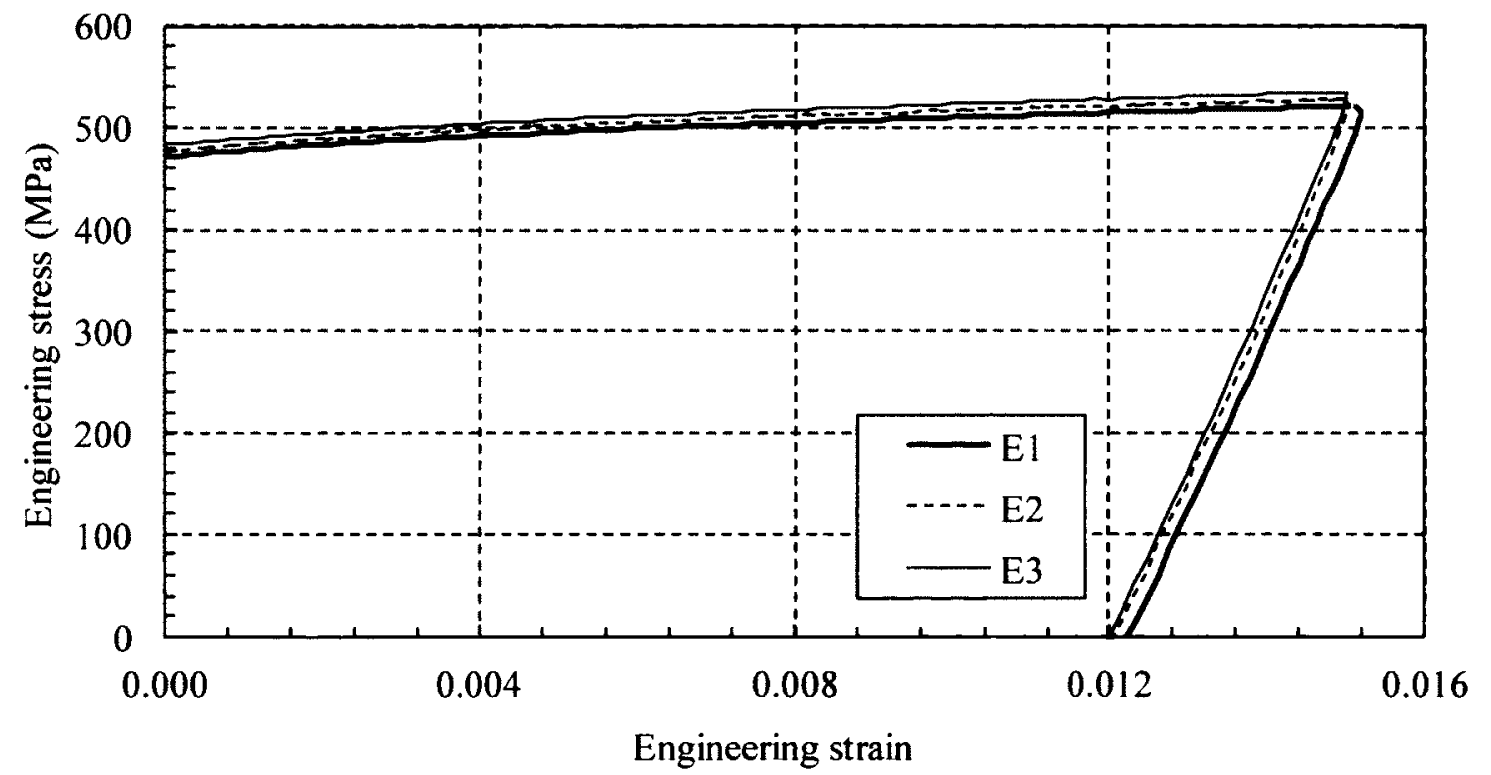

Figure B.6 Part of the $10^{\text {th }}$ cycle for BTE1I, BTE2I and BTE3I at the $\pm 1.5 \%$ strain range 


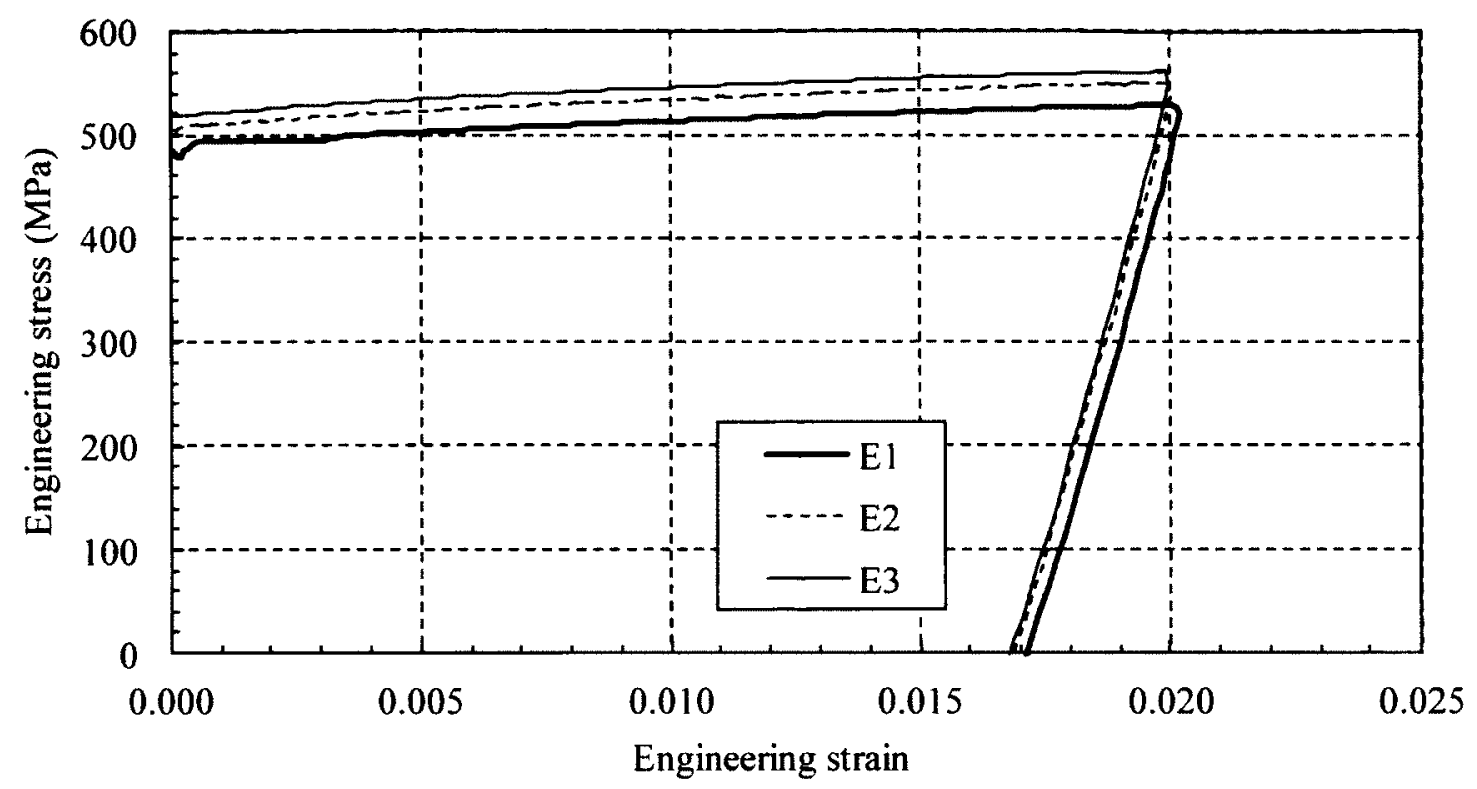

Figure B.7 Part of the $10^{\text {th }}$ cycle for BTE1I, BTE2I and BTE3I at the $\pm 2 \%$ strain range

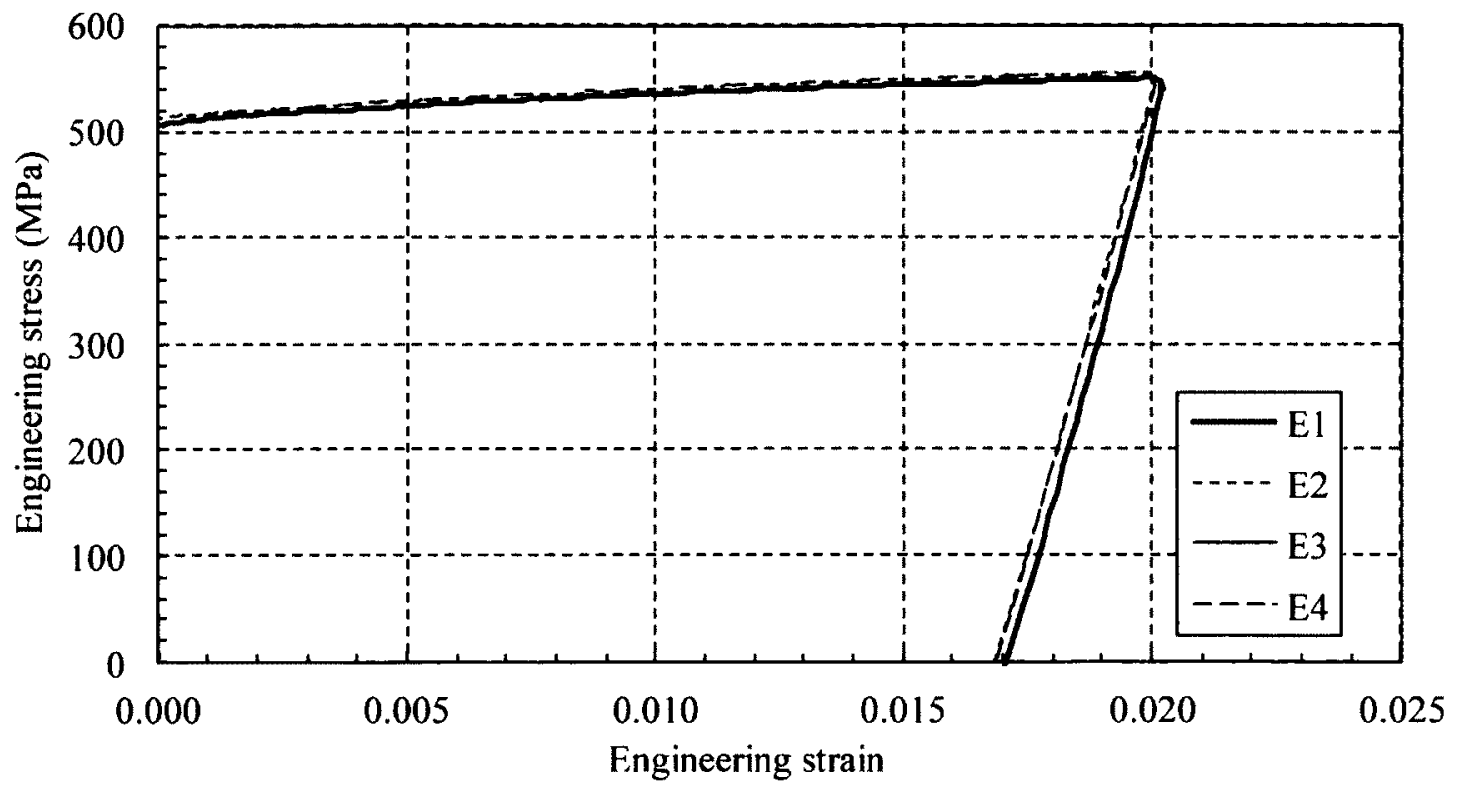

Figure B.8 Part of the $10^{\text {th }}$ cycle for BTE1D, BTE2D, BTE3D and BTE4D at the $\pm 2 \%$ strain range 


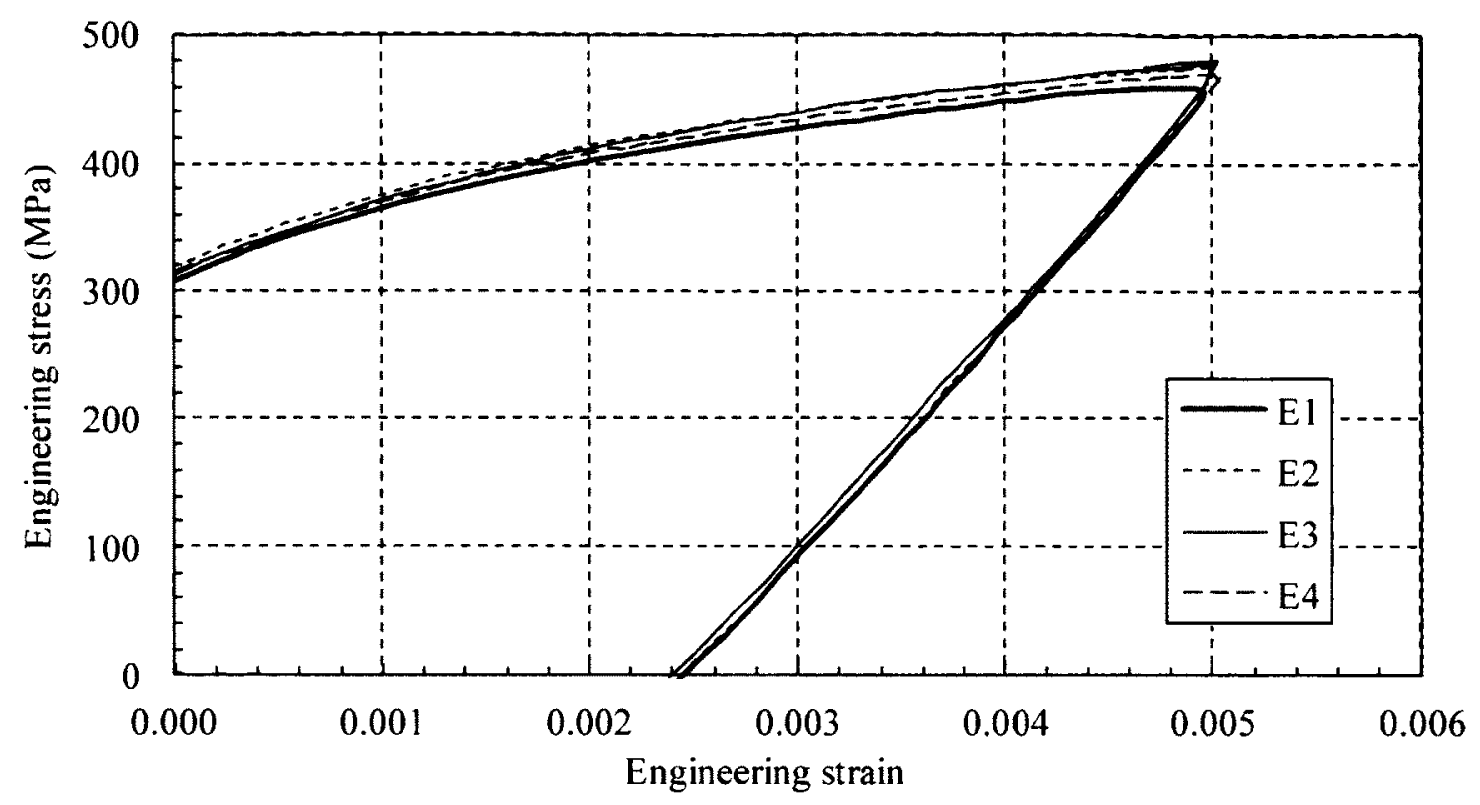

Figure B.9 Part of the $10^{\text {th }}$ cycle for BTE1D, BTE2D, BTE3D and BTE4D at the $\pm 0.5 \%$ strain range

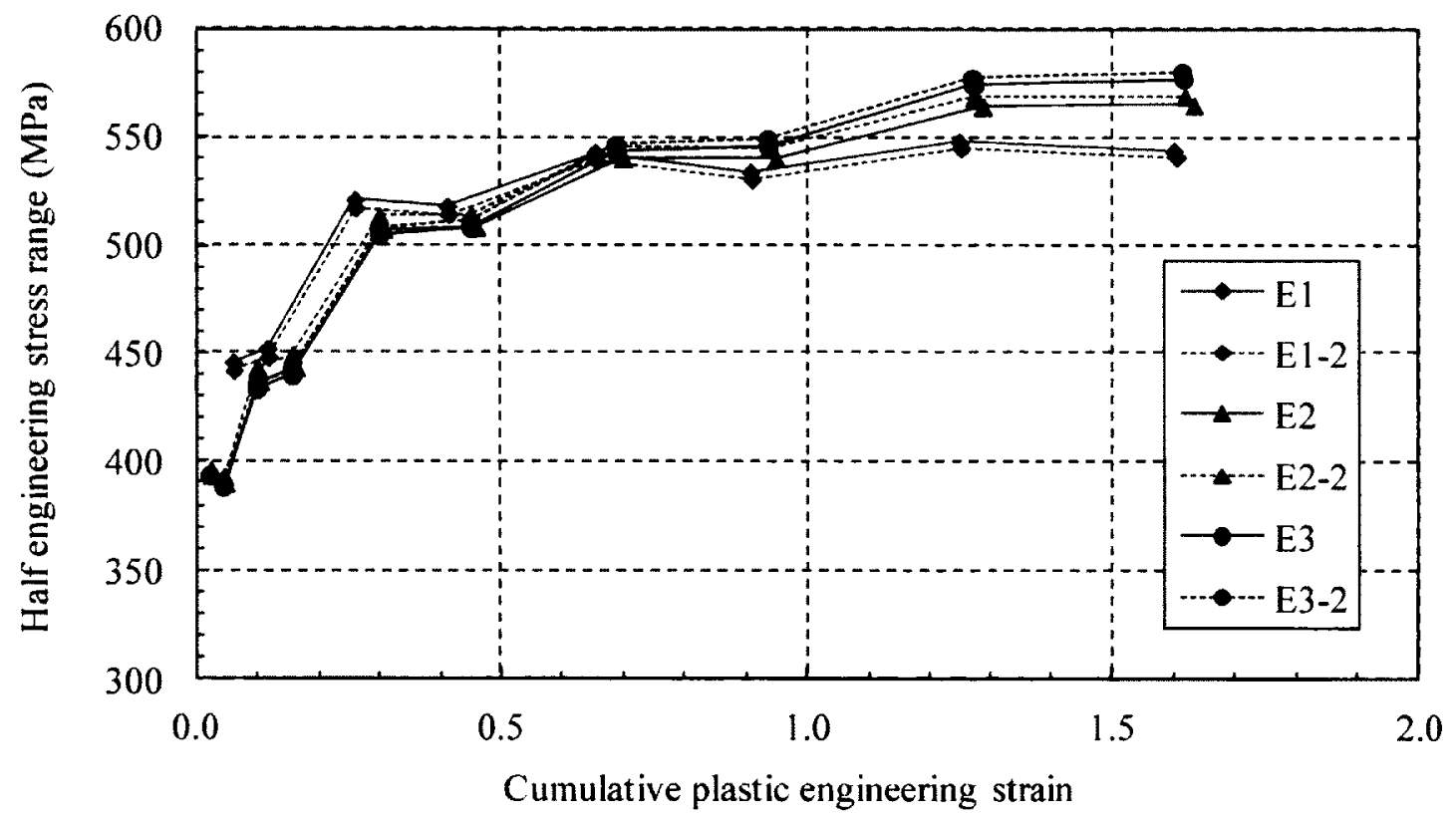

Figure B.10 Half engineering stress range versus cumulative plastic engineering strain at the $5^{\text {th }}$ and $10^{\text {th }}$ cycle of each strain range for BTEIs 


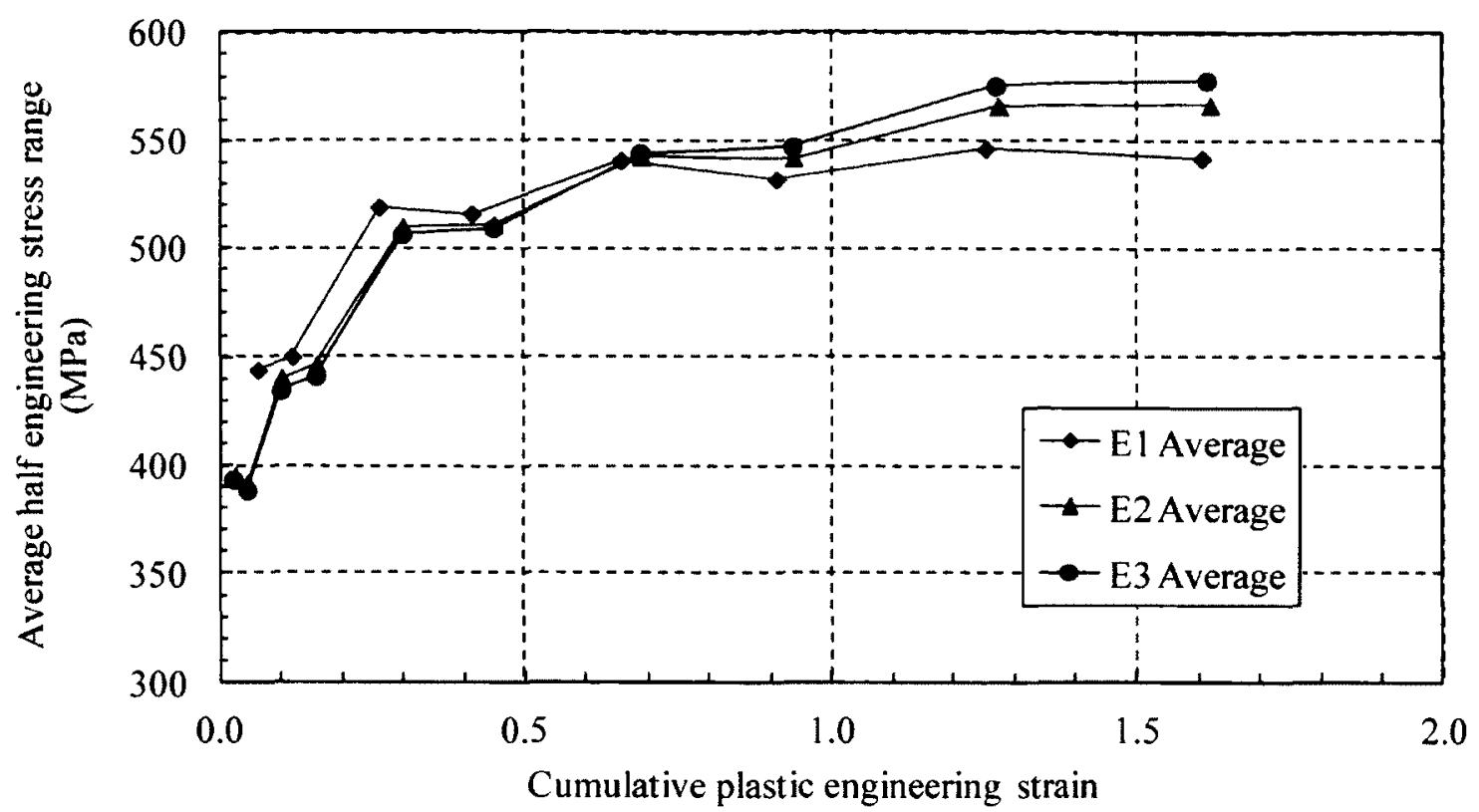

Figure B.11 Average of the half engineering stress range at each strain rate versus cumulative plastic engineering strain at the $5^{\text {th }}$ and $10^{\text {th }}$ cycle of each strain range for BTEIs

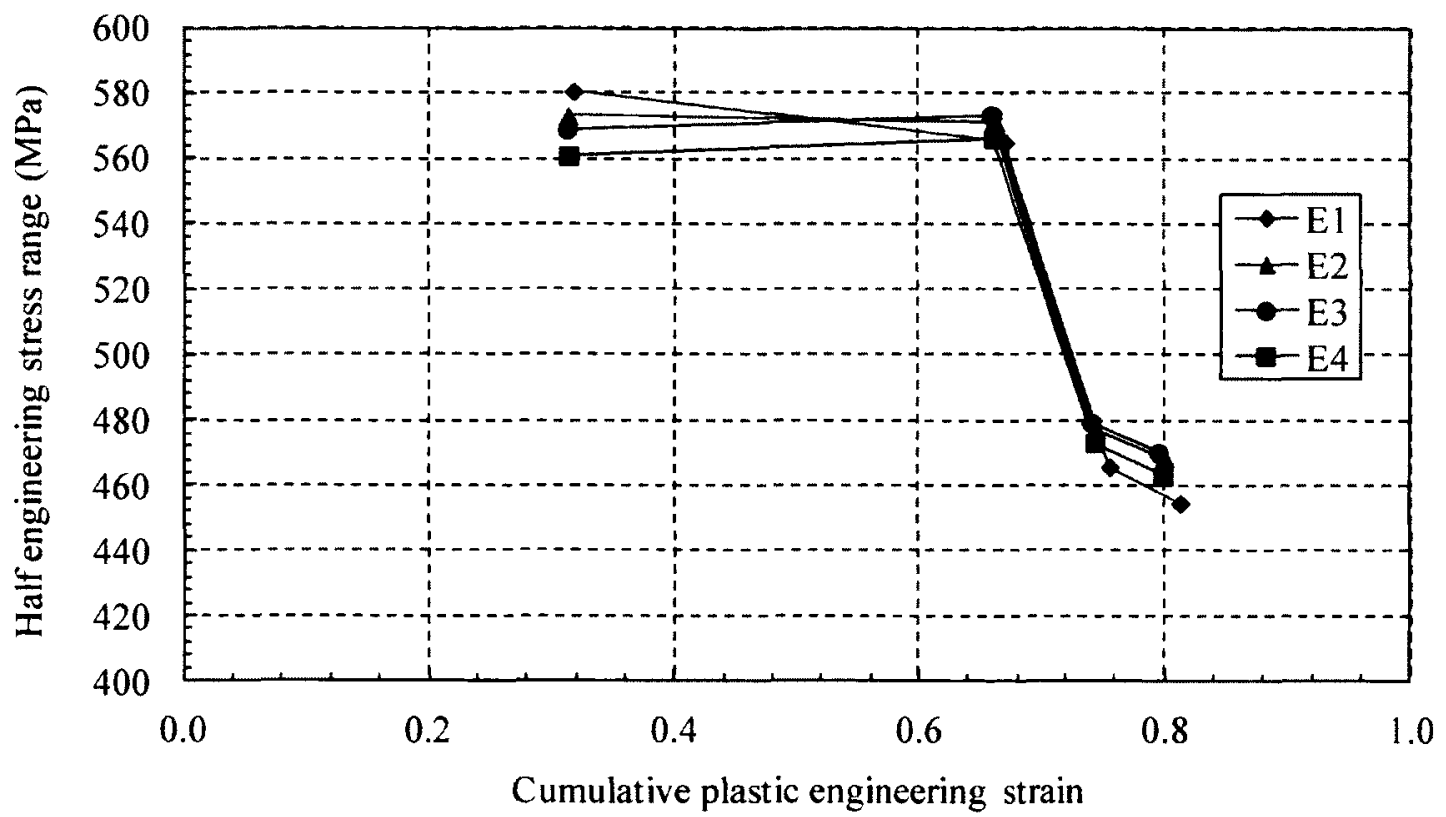

Figure B.12 Half engineering stress range versus cumulative plastic engineering strain at the $5^{\text {th }}$ and $10^{\text {th }}$ cycle of each strain range for BTEDs 


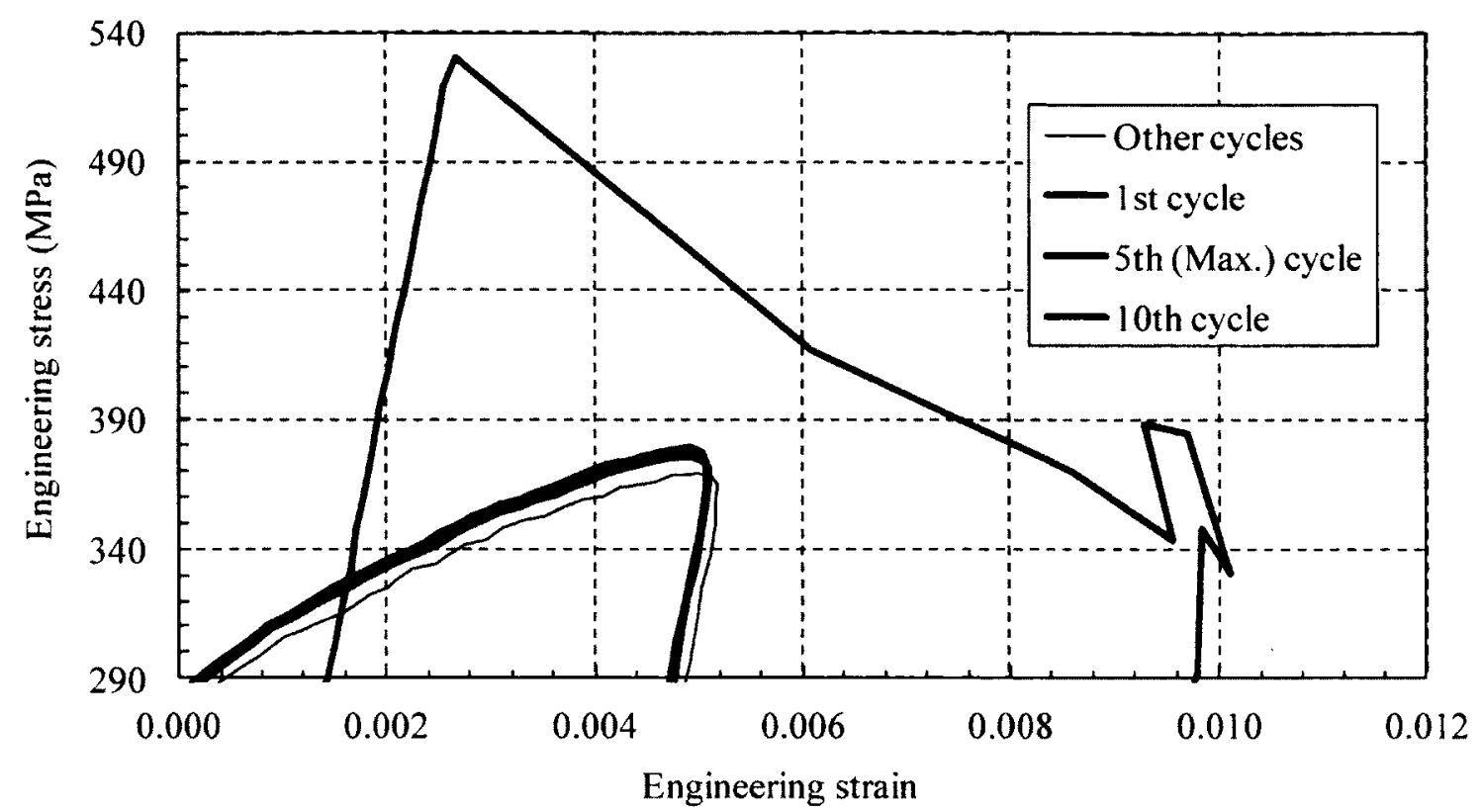

Figure B.13 Magnified view of the engineering stress versus engineering strain curves for ATE1I at the $\pm 0.5 \%$ strain range

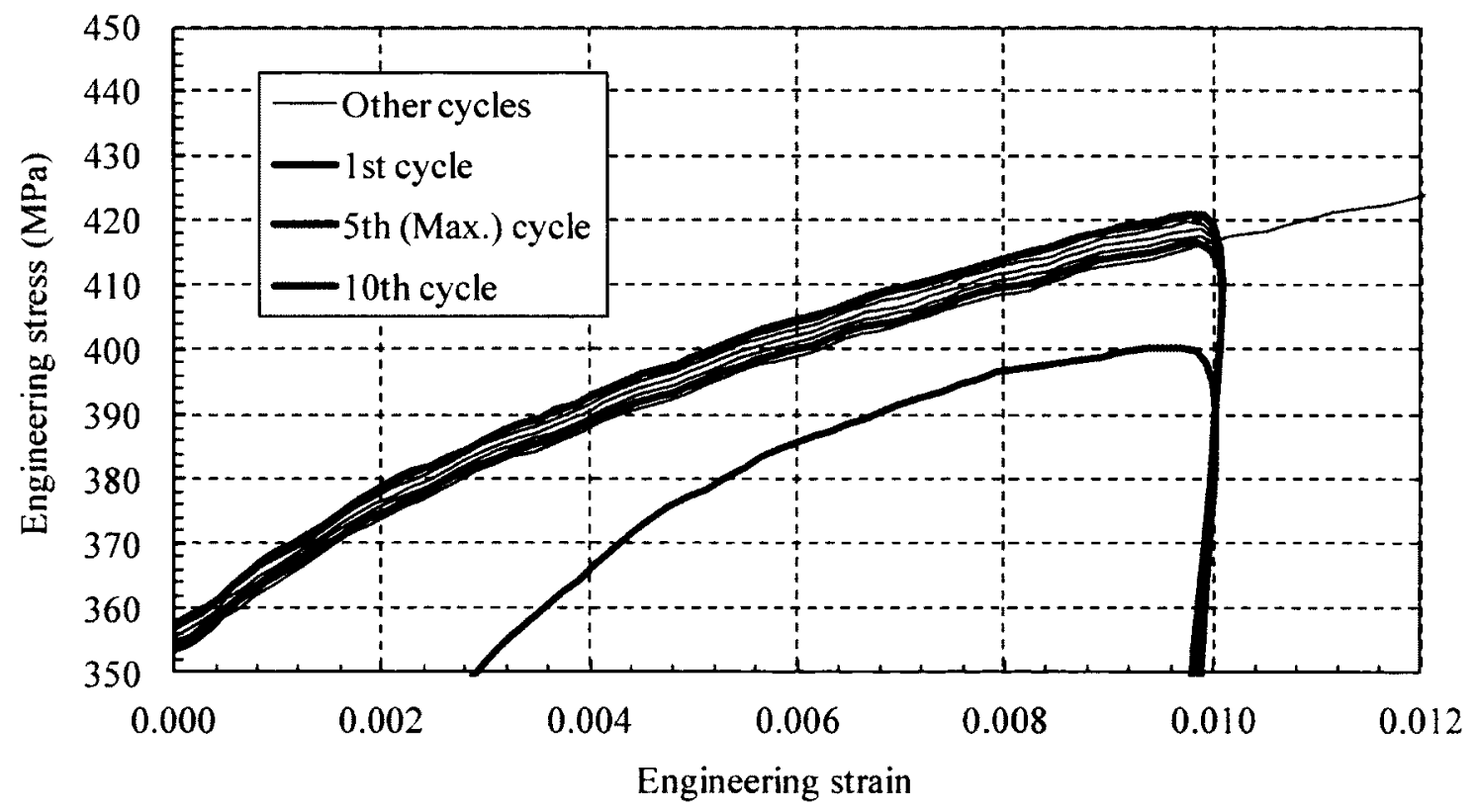

Figure B.14 Magnified view of the engineering stress versus engineering strain curves for ATE 1 at the $\pm 1 \%$ strain range 


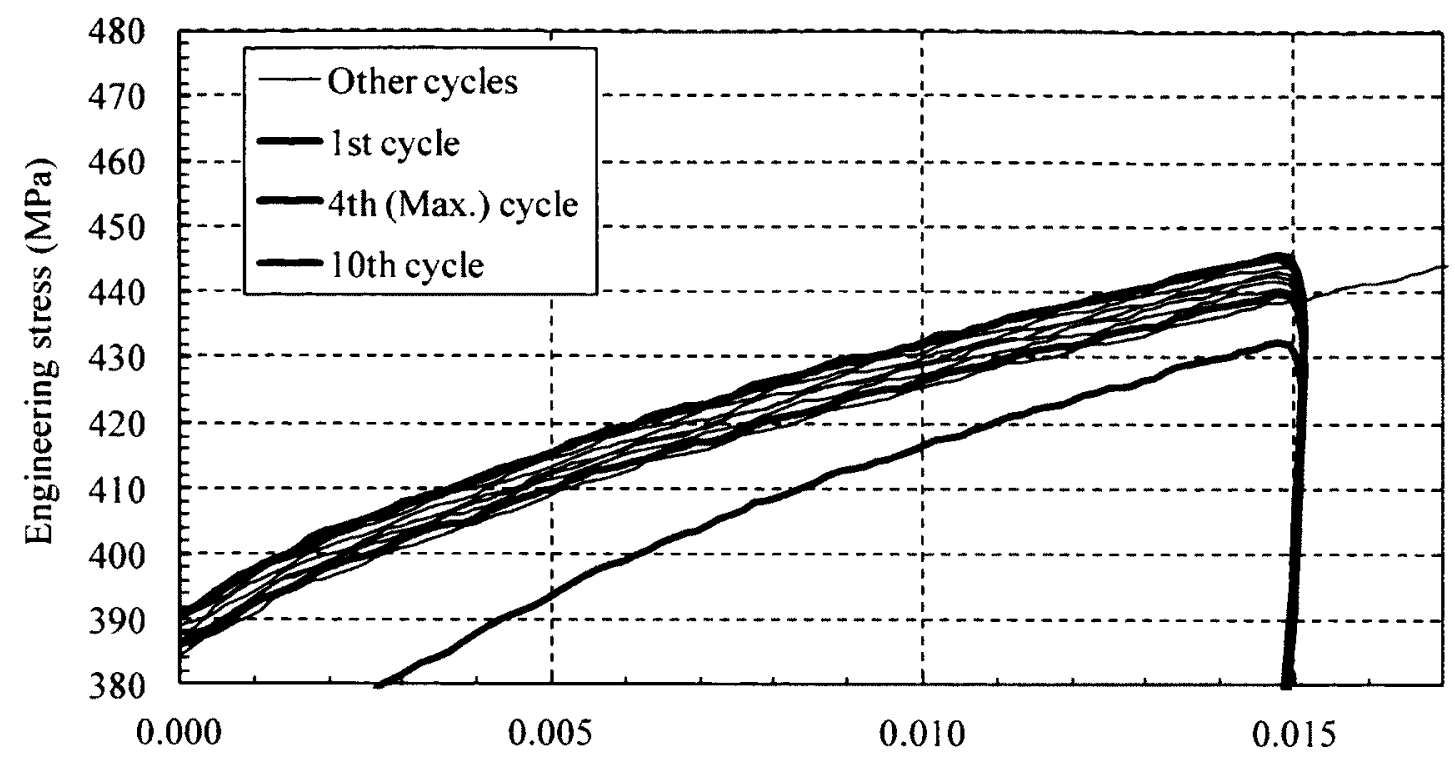

Engineering strain

Figure B.15 Magnified view of the engineering stress versus engineering strain curves for ATE1I at the $\pm 1.5 \%$ strain range

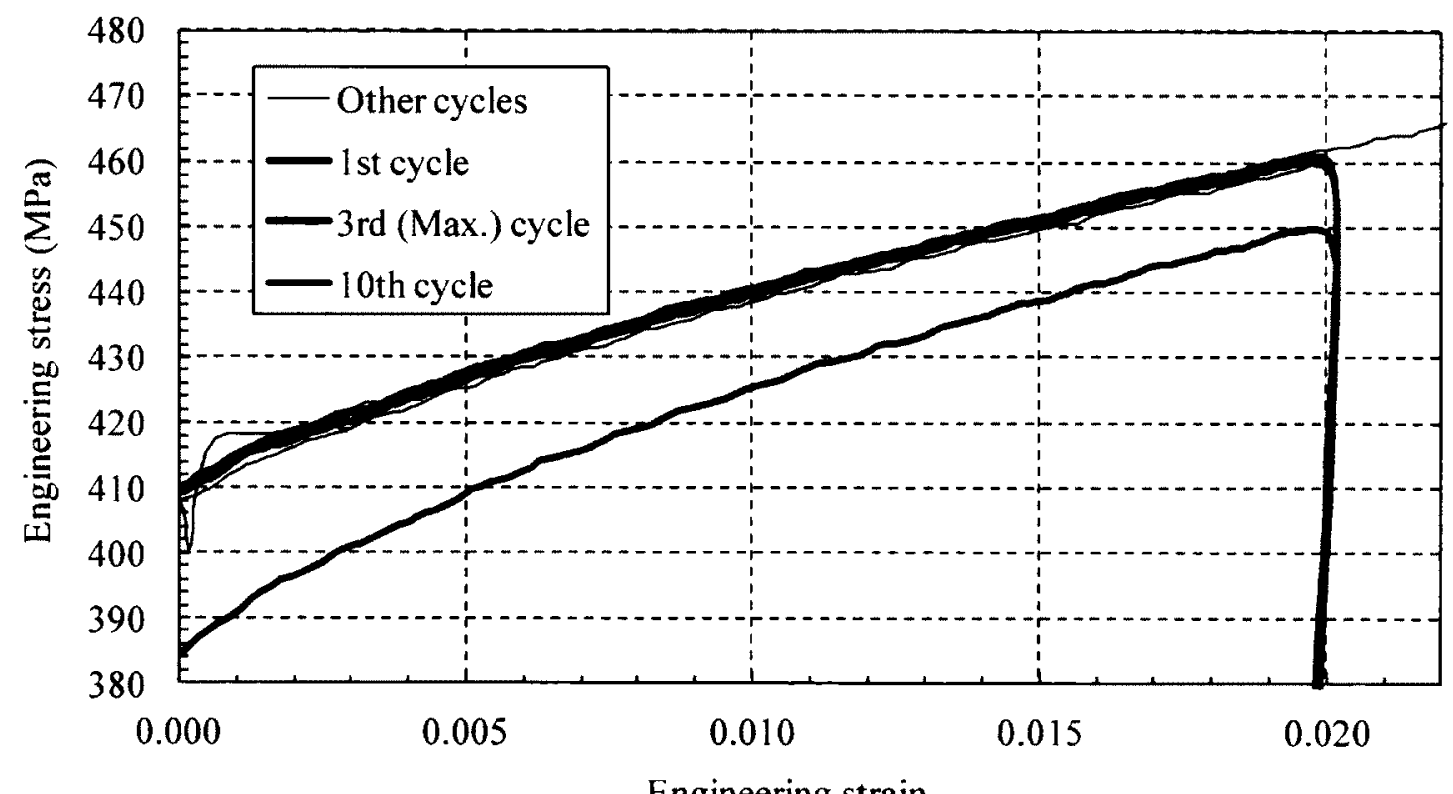

Figure B.16 Magnified view of the engineering stress versus engineering strain curves for ATE 1 at the $\pm 2 \%$ strain range 


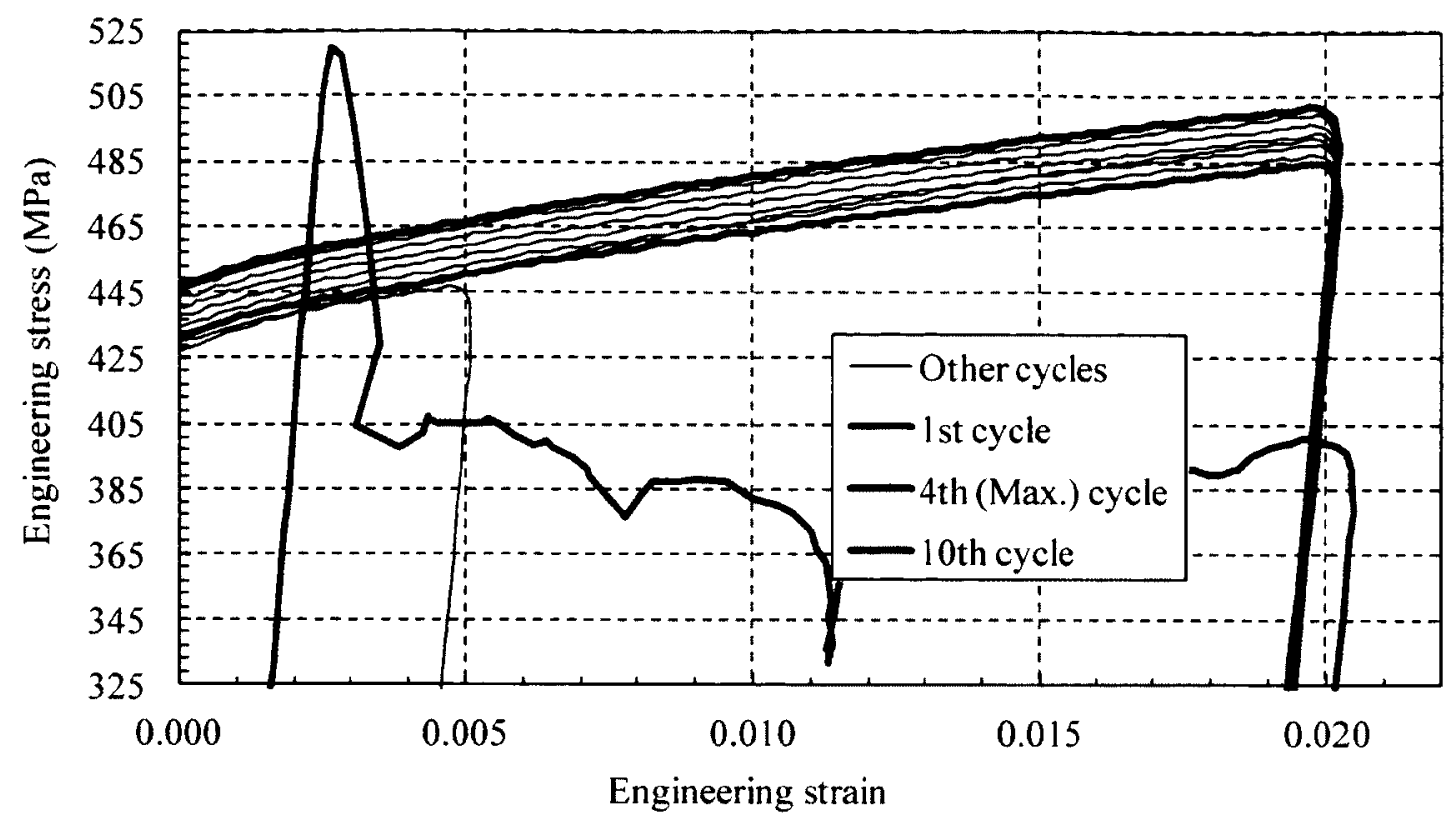

Figure B.17 Magnified view of the engineering stress versus engineering strain curves for ATE1D at the $\pm 2 \%$ strain range

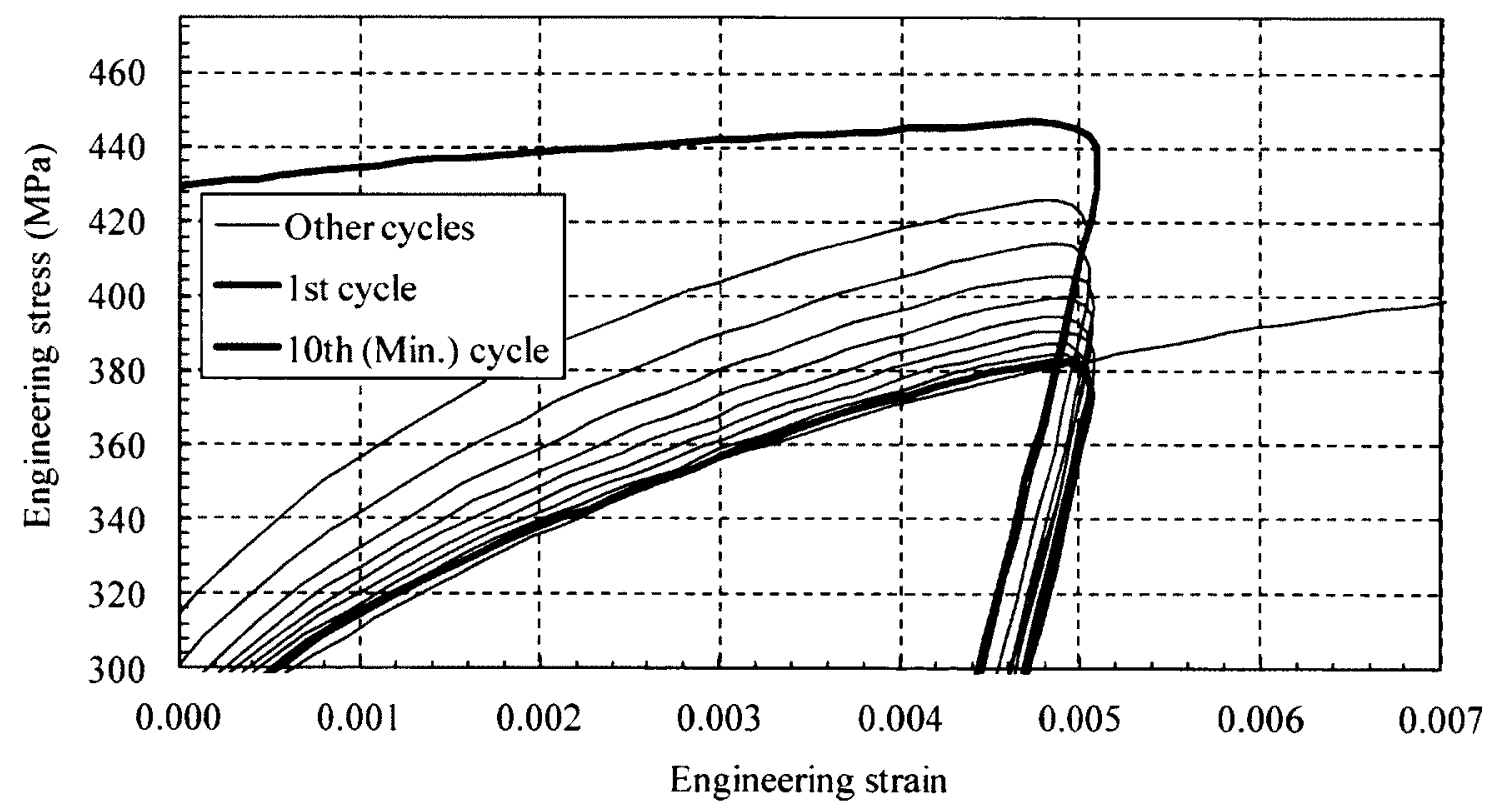

Figure B.18 Magnified view of the engineering stress versus engineering strain curves for ATE1D at the $\pm 0.5 \%$ strain range 


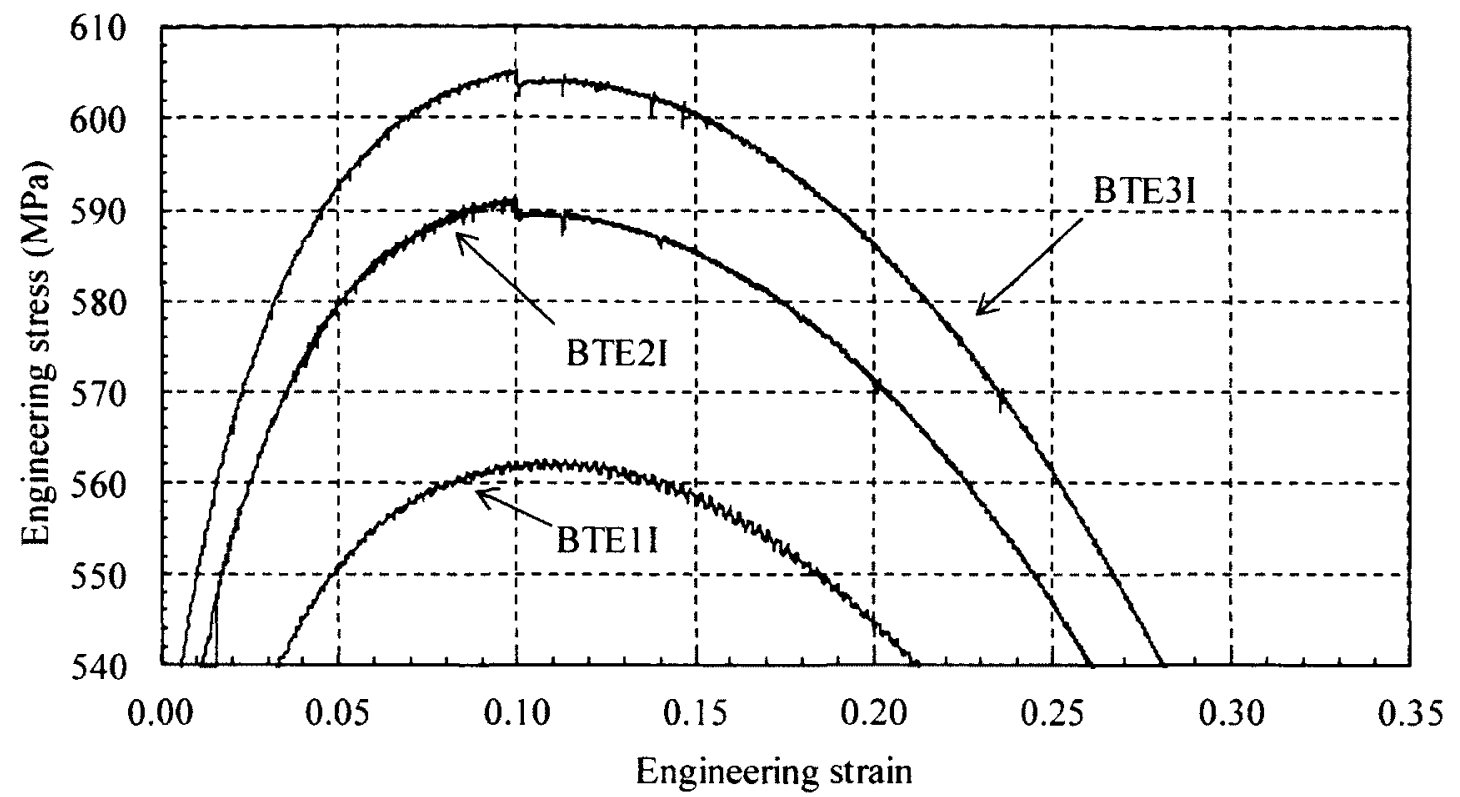

Figure B.19 Magnified view of the engineering stress versus engineering strain curves close to the peak stress for I series tests of material B when being pulled to fracture

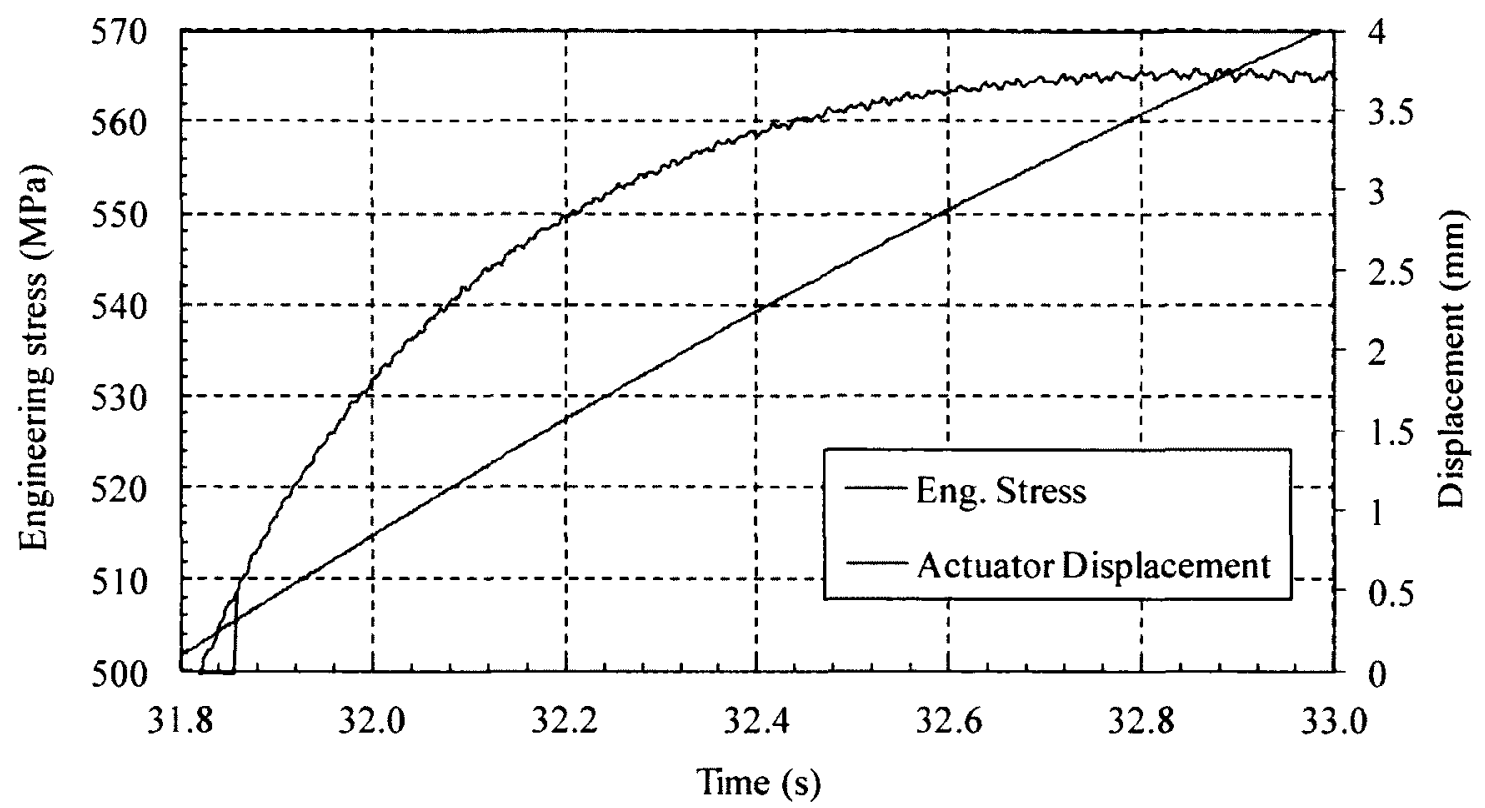

Figure B.20 Magnified view of the engineering stress and actuator displacement versus time between the approximate strain range of 0 and 0.1 during the pull to fracture for BTE1I 


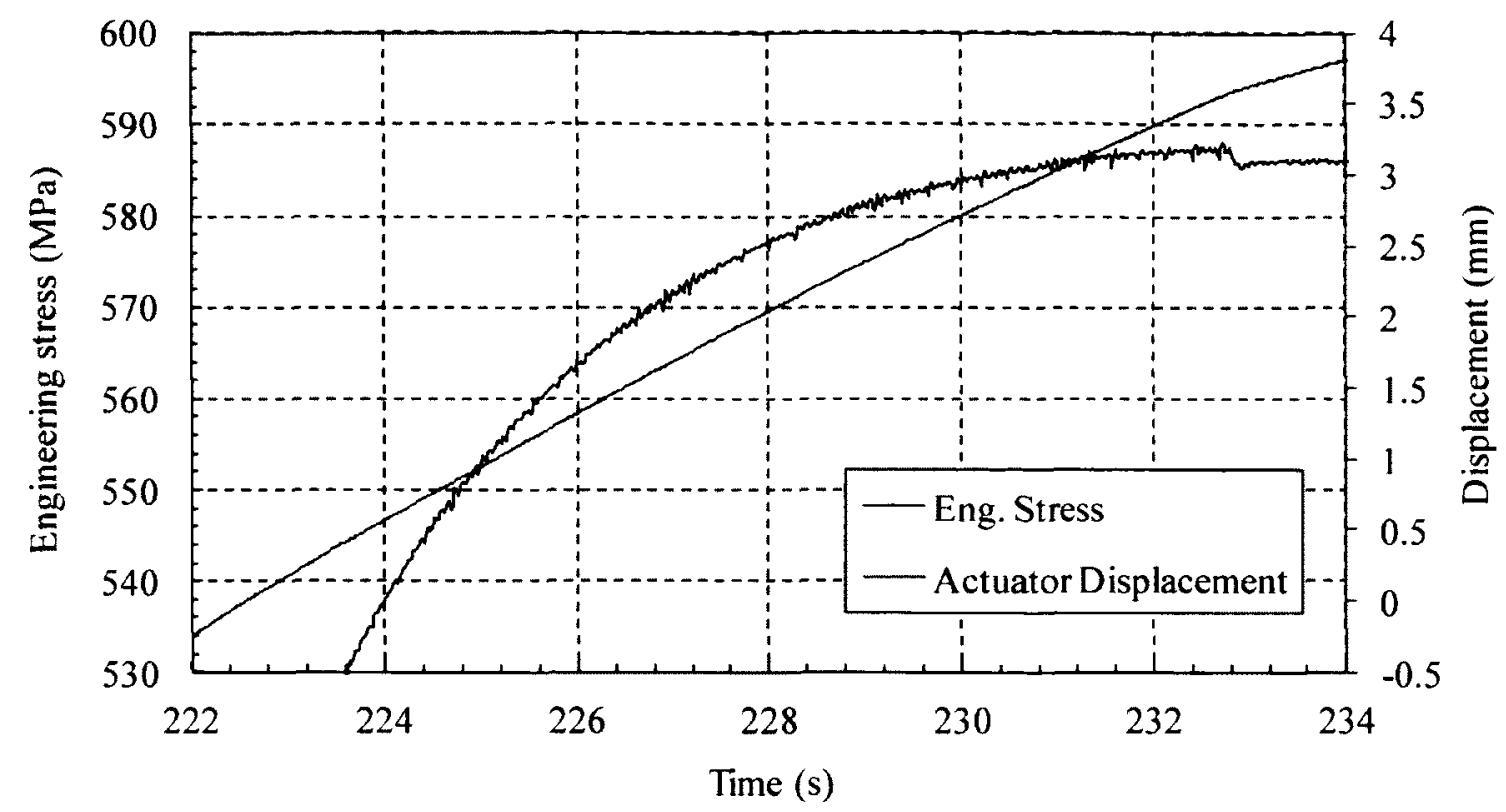

Figure B.21 Magnified view of the engineering stress and actuator displacement versus time between the approximate strain range of 0 and 0.1 during the pull to fracture for BTE2I

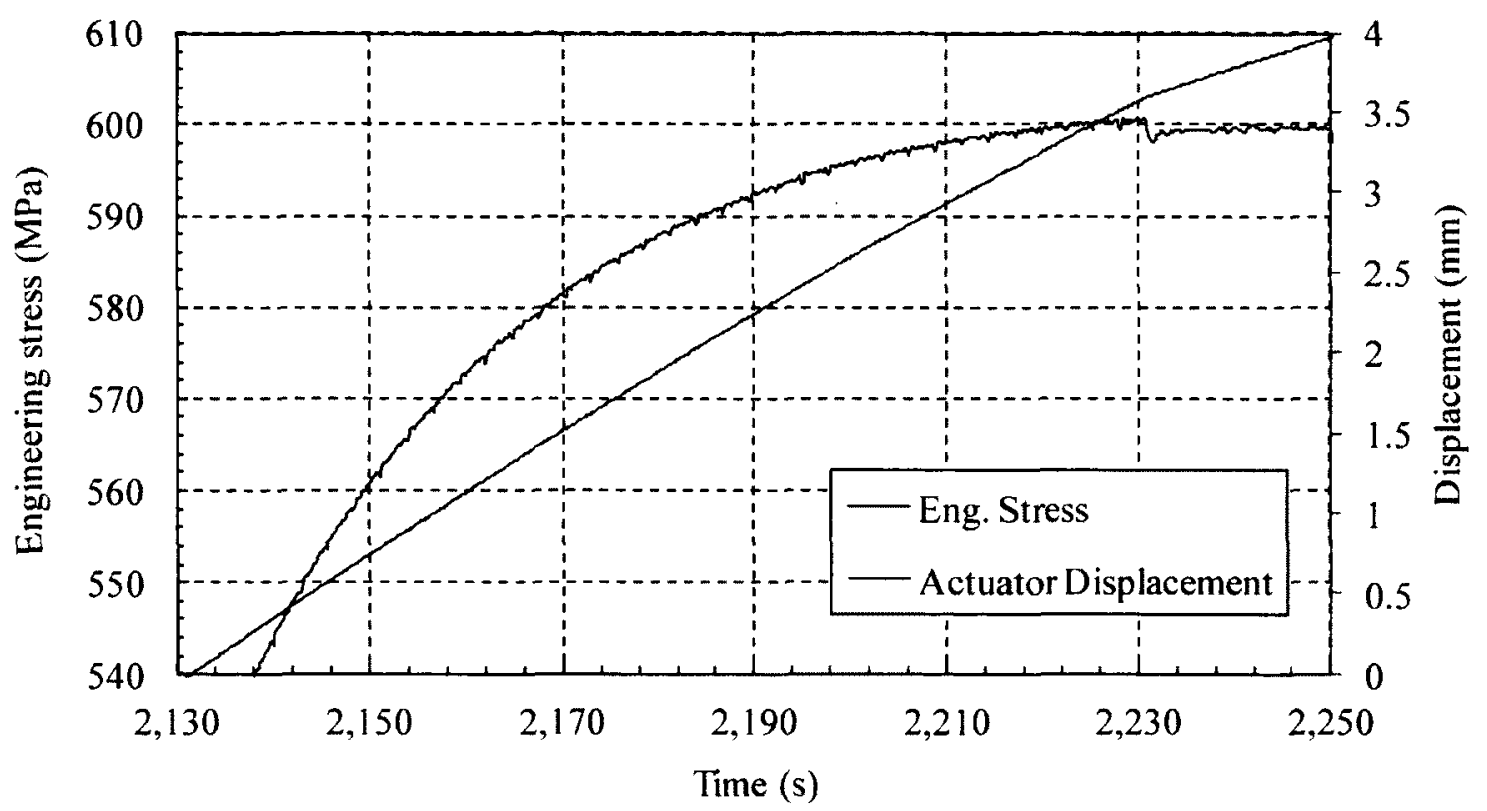

Figure B.22 Magnified view of the engineering stress and actuator displacement versus time between the approximate strain range of 0 and 0.1 during the pull to fracture for BTE3I 


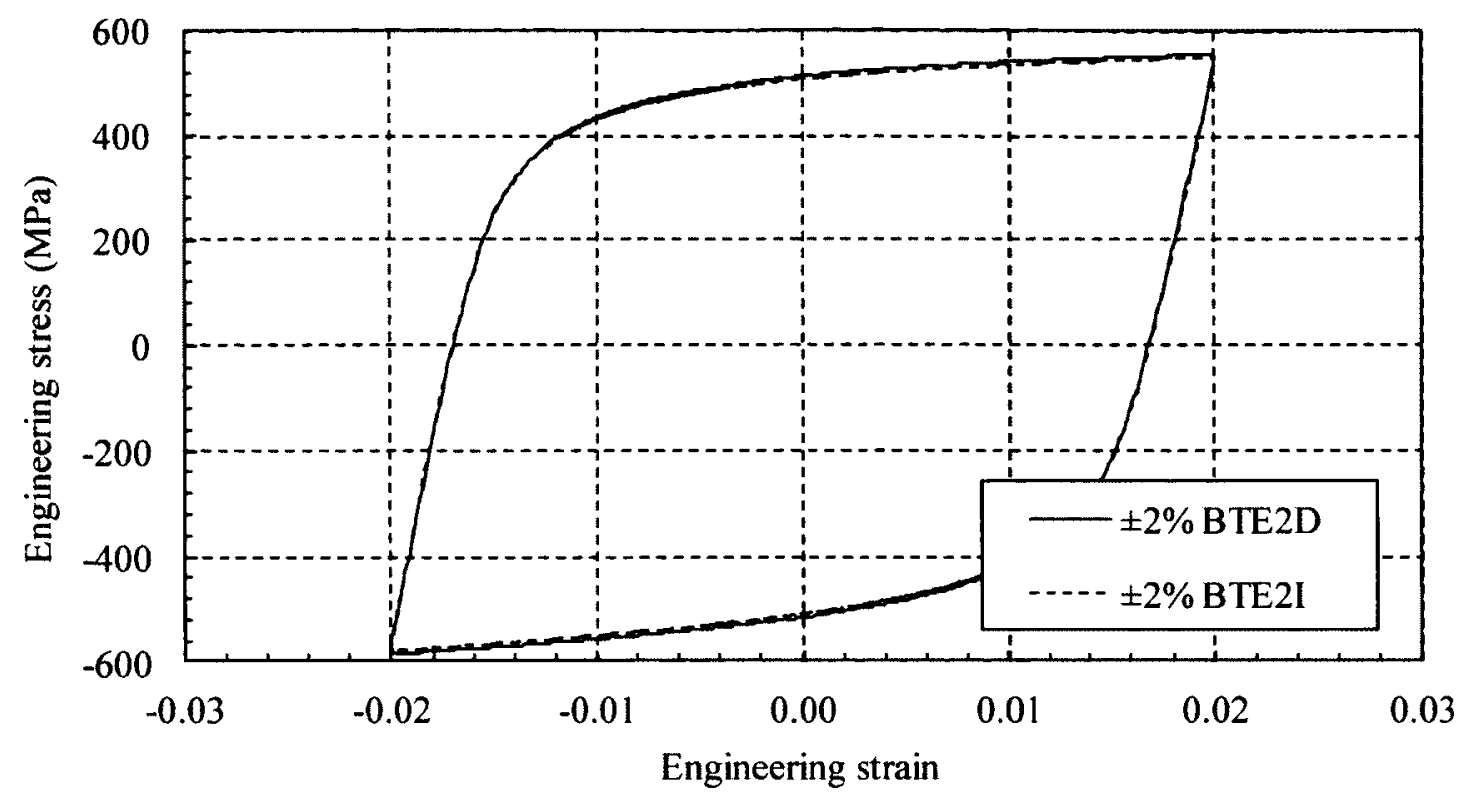

Figure B.23 The $10^{\text {th }}$ cycle of BTE2D and BTE2I at the $\pm 2 \%$ strain range

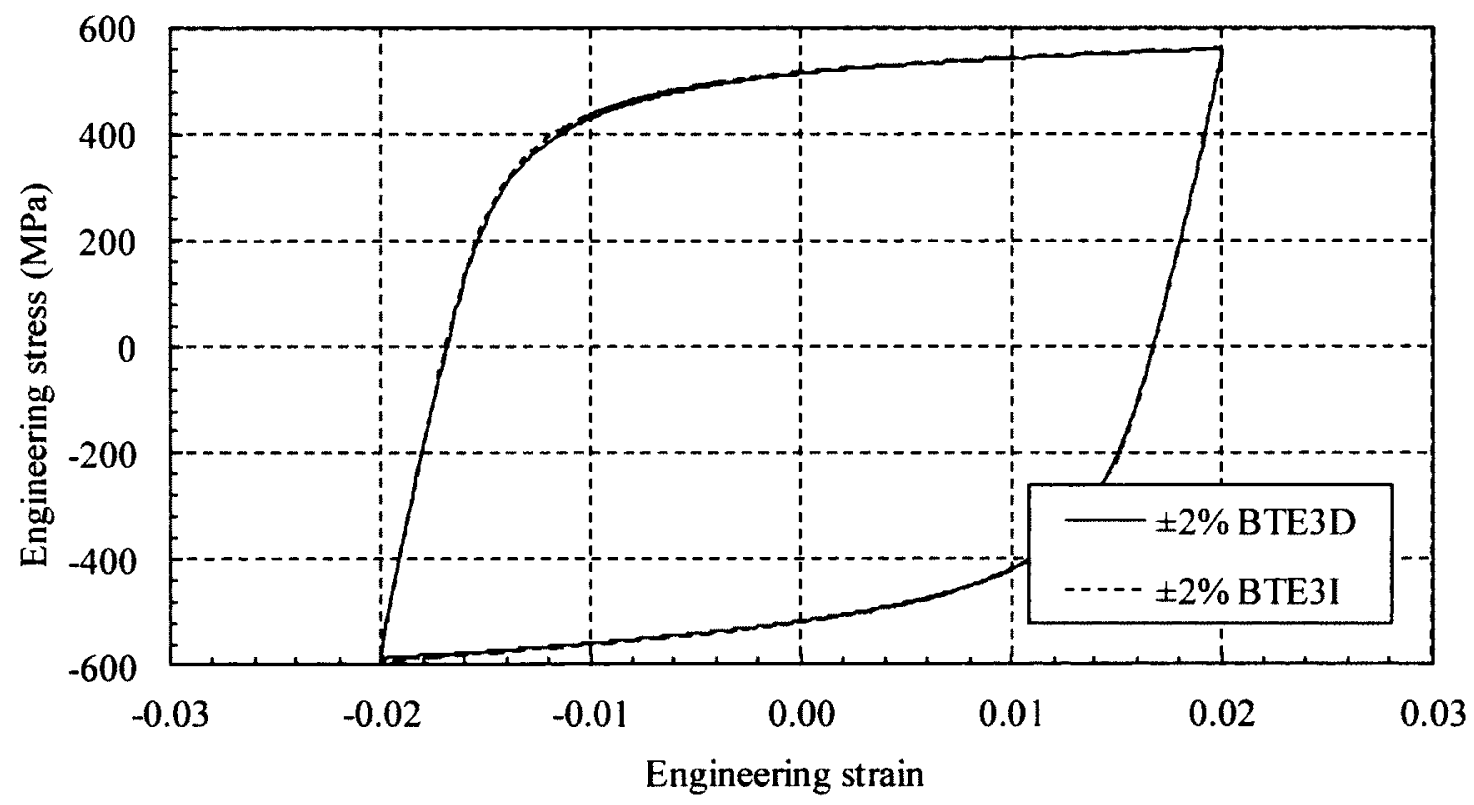

Figure B.24 The $10^{\text {th }}$ cycle of BTE3D and BTE3I at the $\pm 2 \%$ strain range 


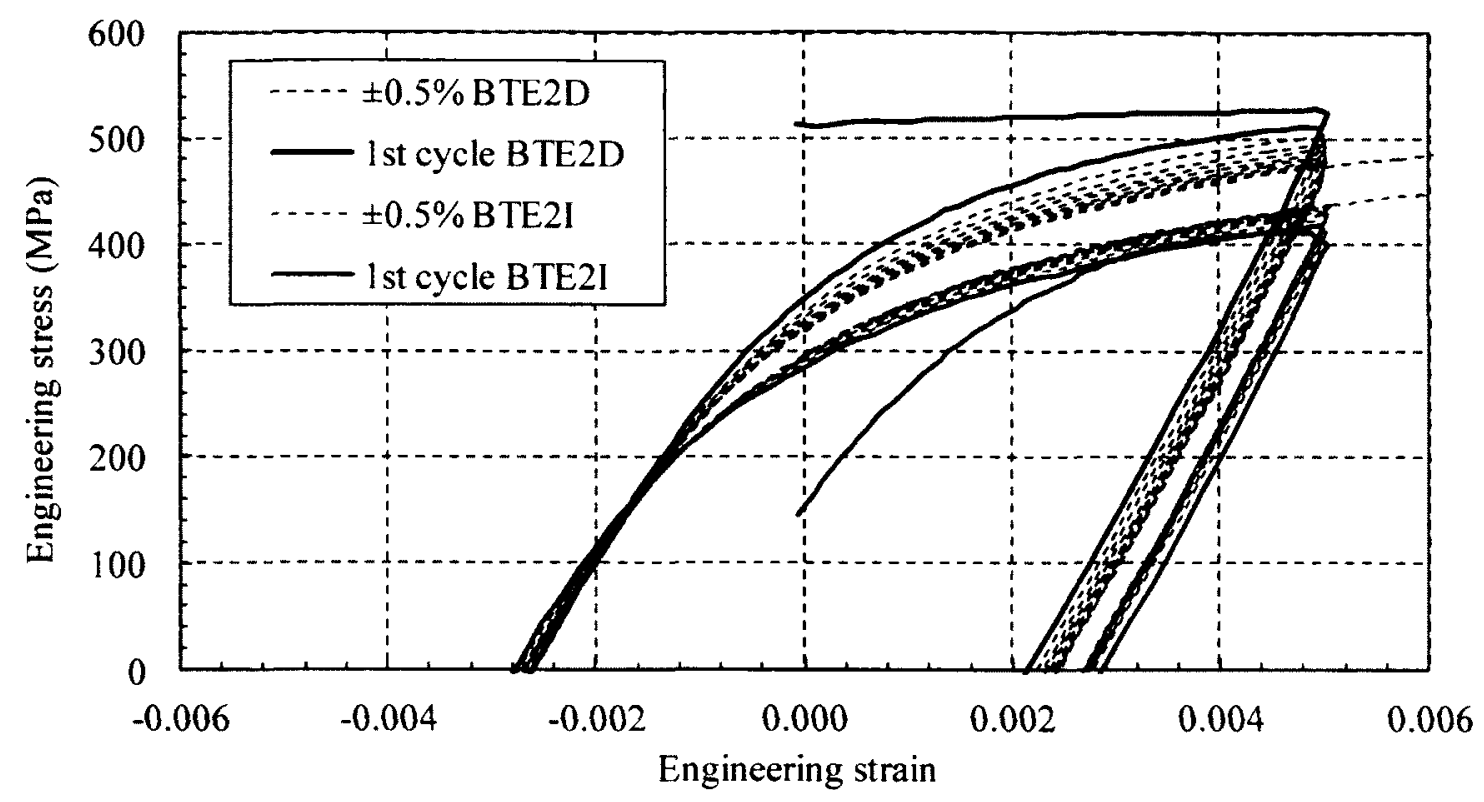

Figure B.25 Tensile behaviour of BTE2D and BTE2I at the strain range of $\pm 0.5 \%$

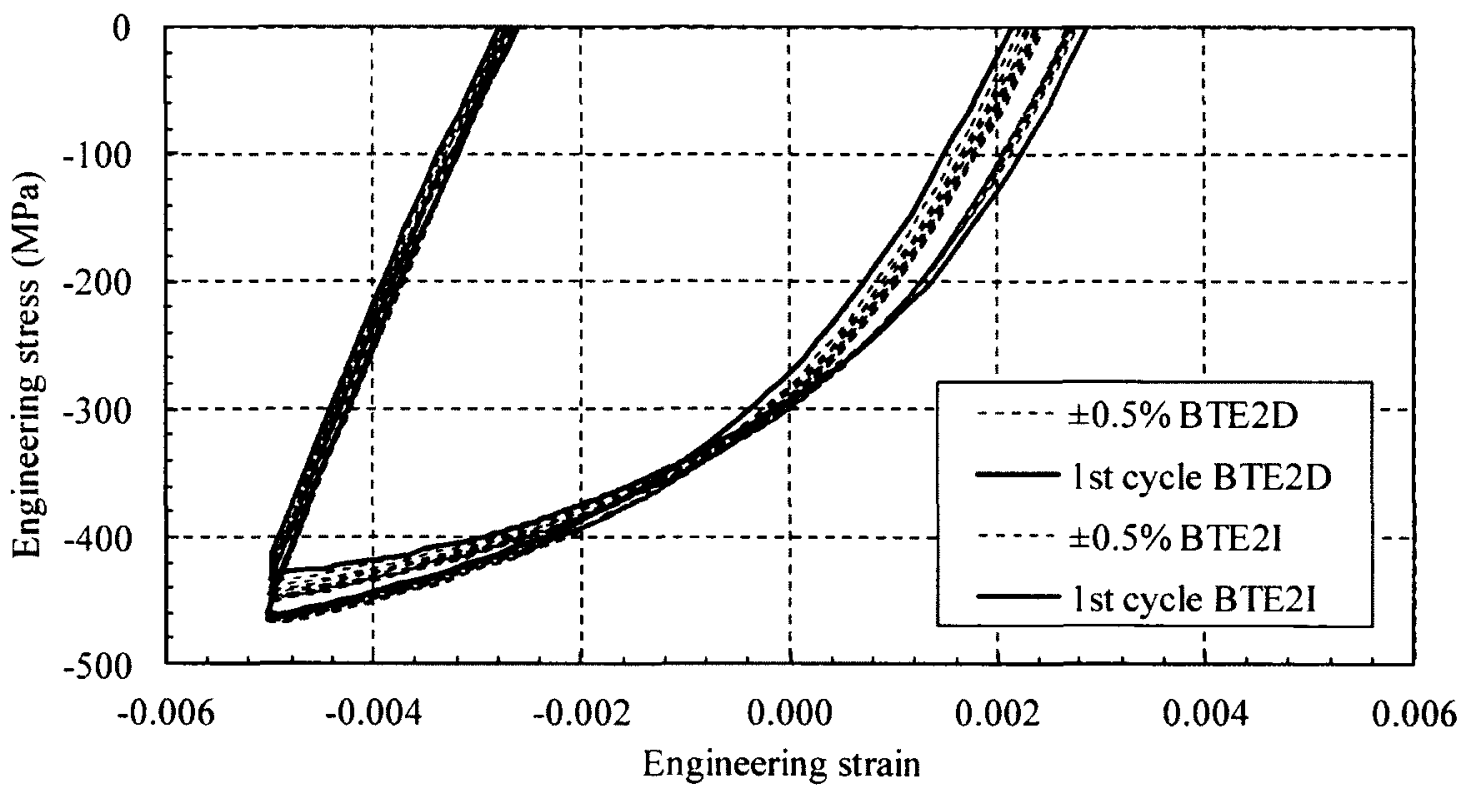

Figure B.26 Compressive behaviour of BTE2D and BTE2I at the strain range of $\pm 0.5 \%$ 


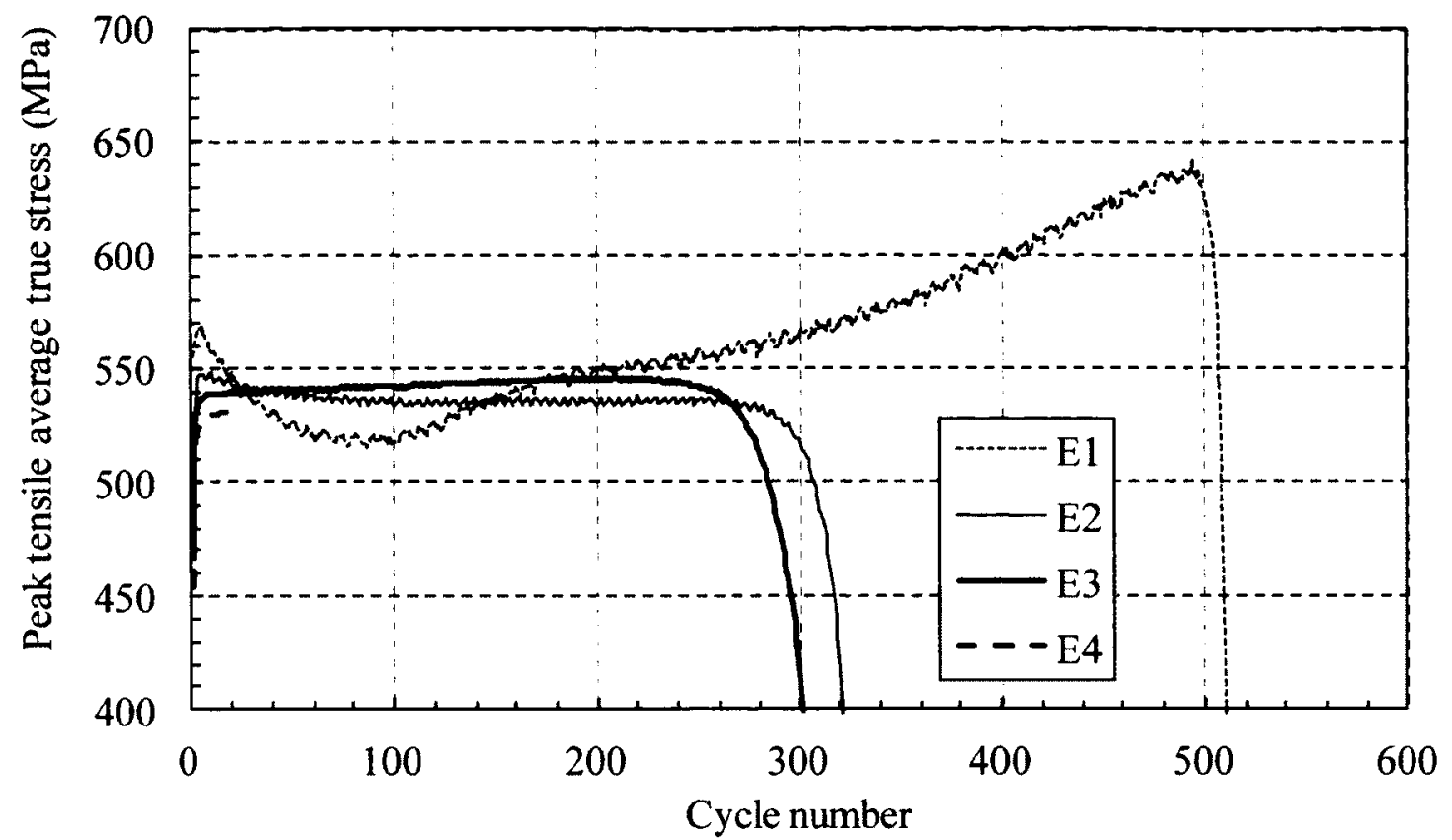

Figure B.27 Peak tensile average true stress versus cycle number for ANE1R2, ANE2R2, ANE3R2 and ANE4R2

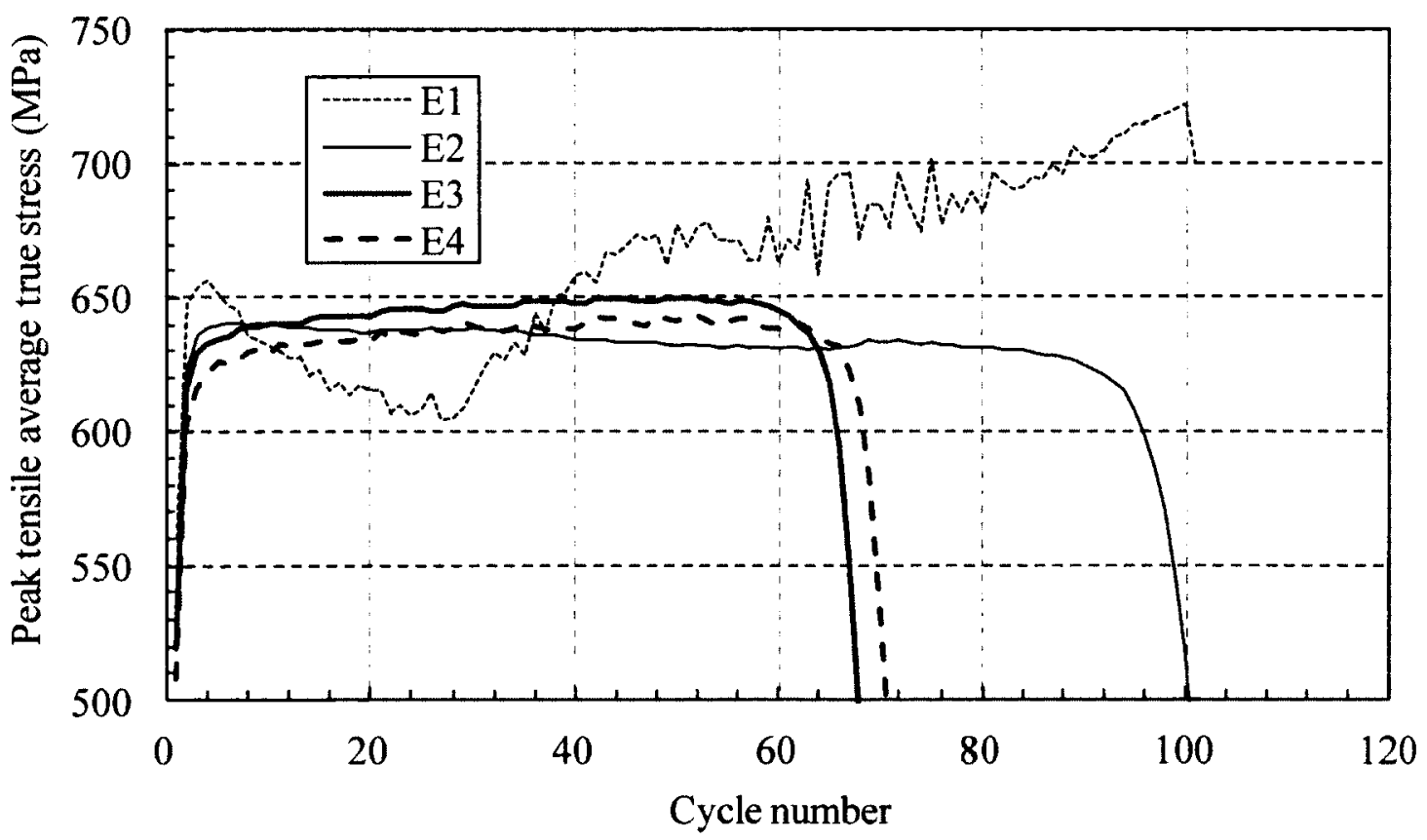

Figure B.28 Peak tensile average true stress versus cycle number for ANE1R4, ANE2R4, ANE3R4 and ANE4R4 


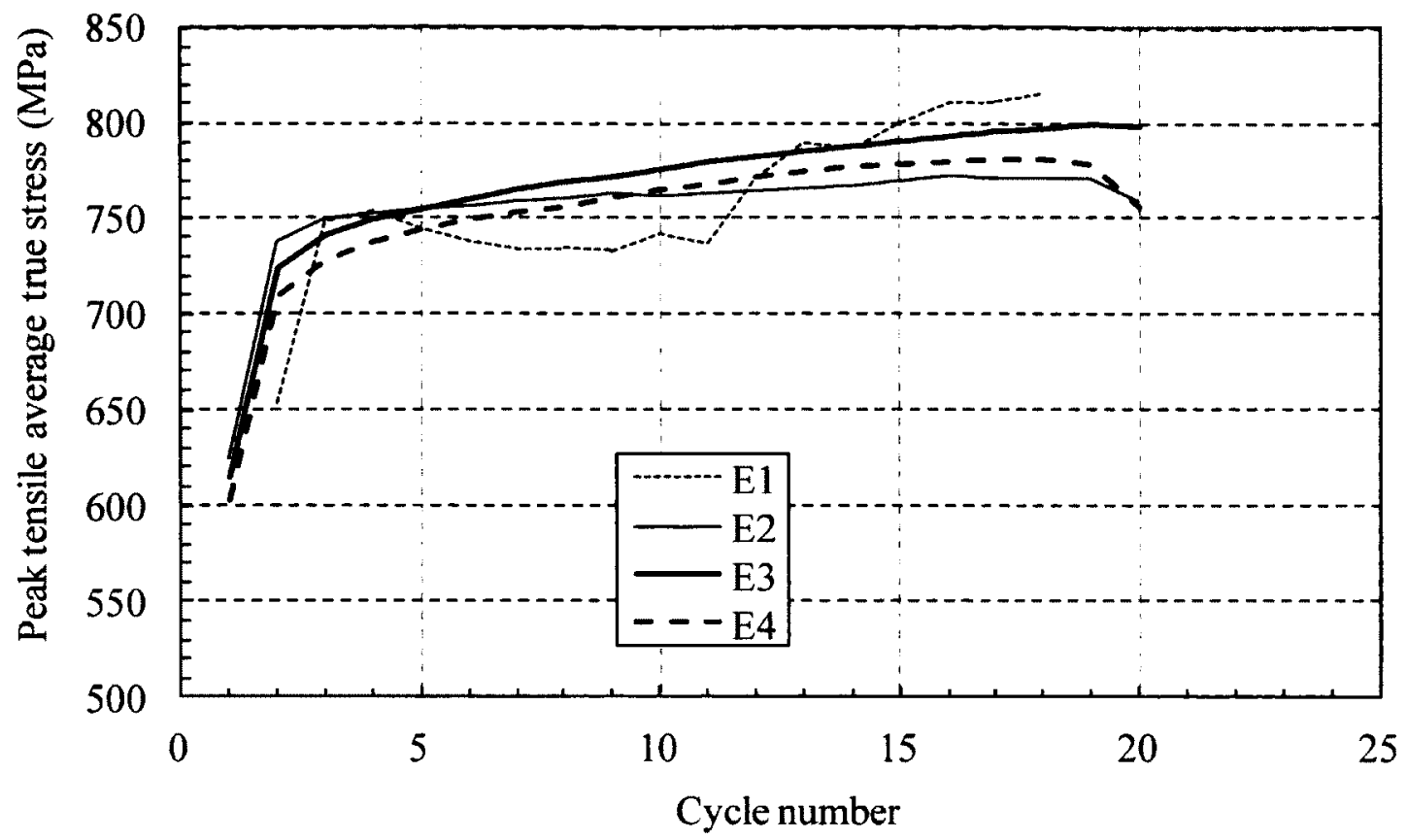

Figure B.29 Peak tensile average true stress versus cycle number for ANE1R8, ANE2R8, ANE3R8 and ANE4R8

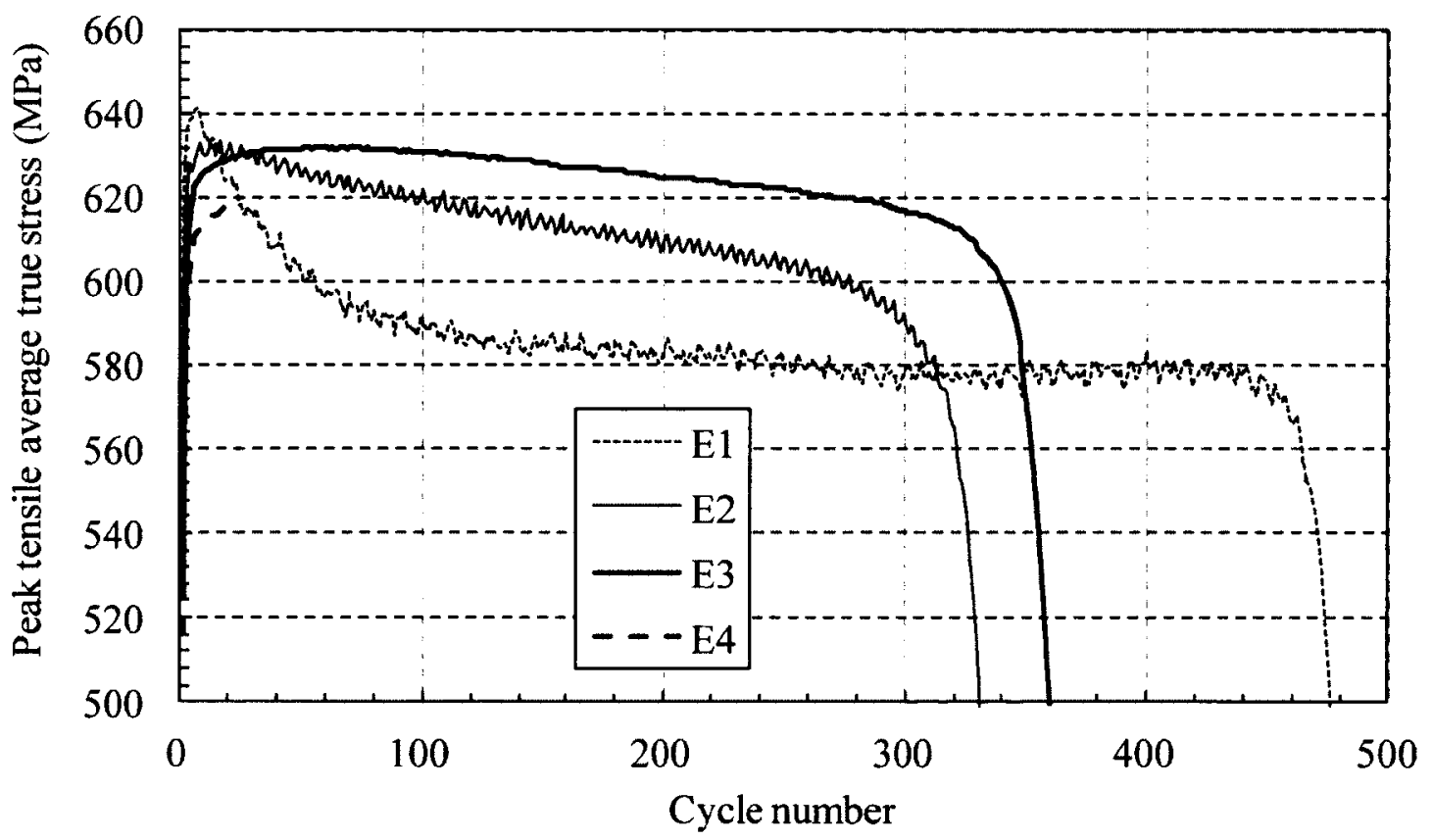

Figure B.30 Peak tensile average true stress versus cycle number for BNE1R2, BNE2R2, BNE3R2 and BNE4R2 


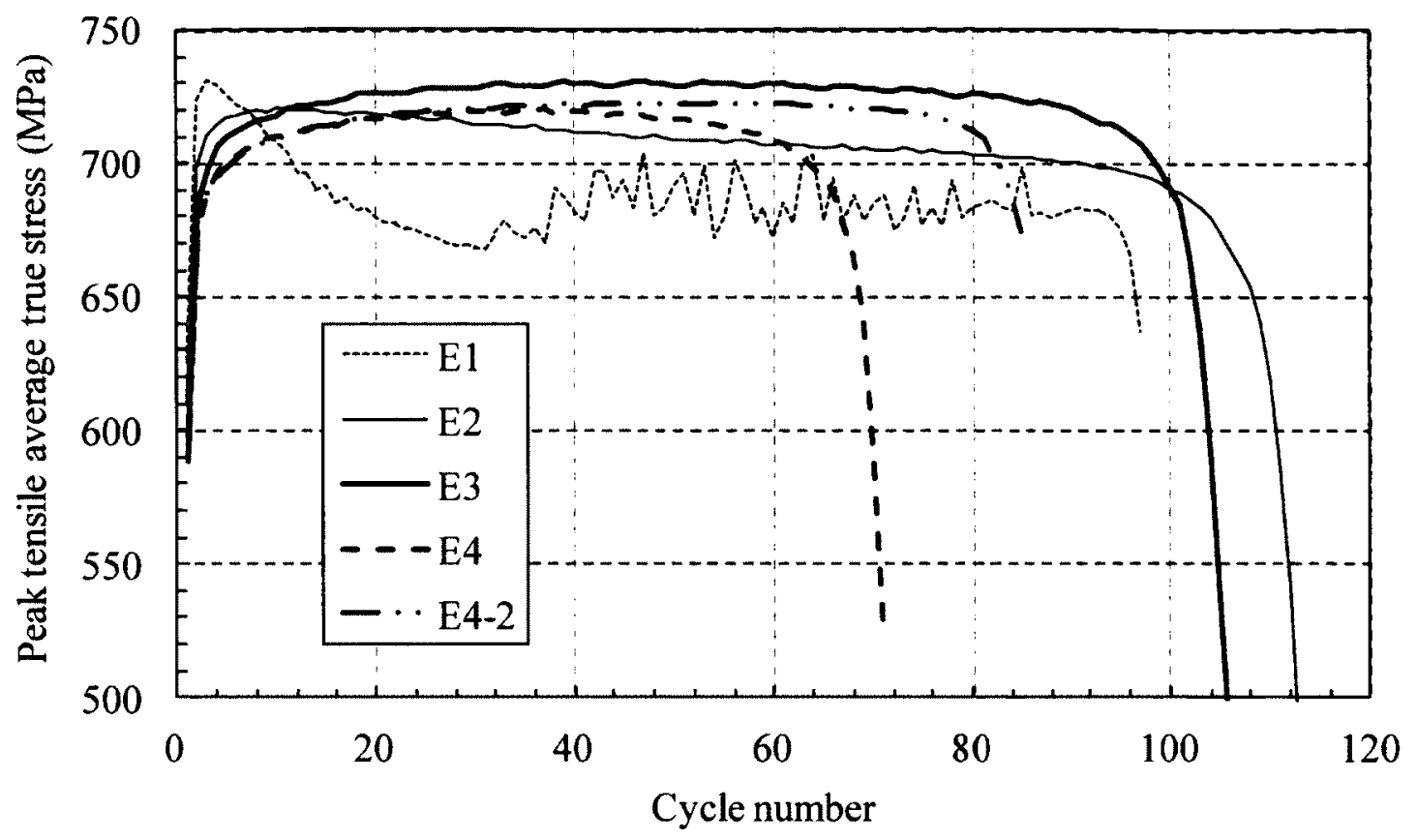

Figure B.31 Peak tensile average true stress versus cycle number for BNE1R4, BNE2R4, BNE3R4, BNE4R4 and BNE4R4-2

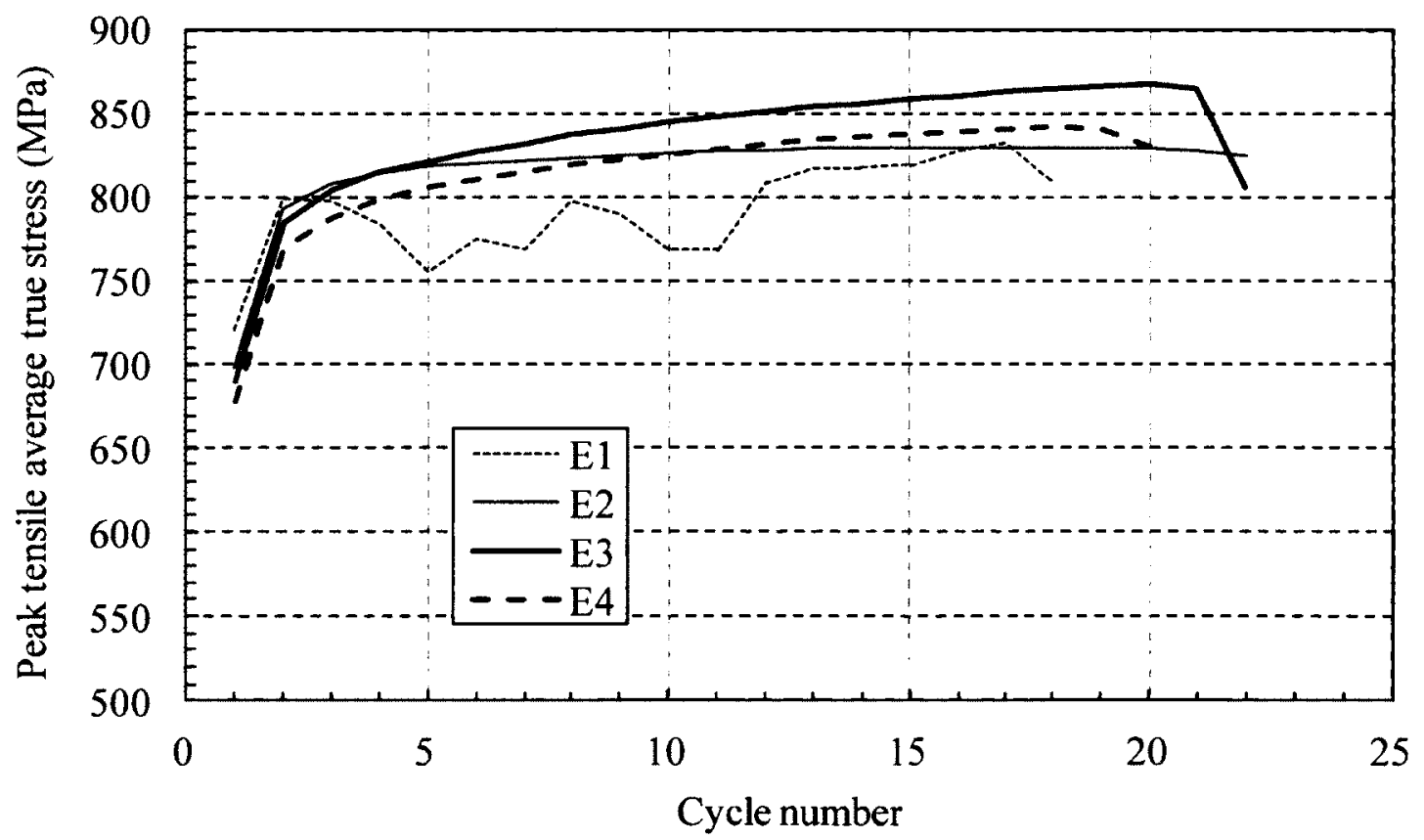

Figure B.32 Peak tensile average true stress versus cycle number for BNE1R8, BNE2R8, BNE3R8 and BNE4R8 


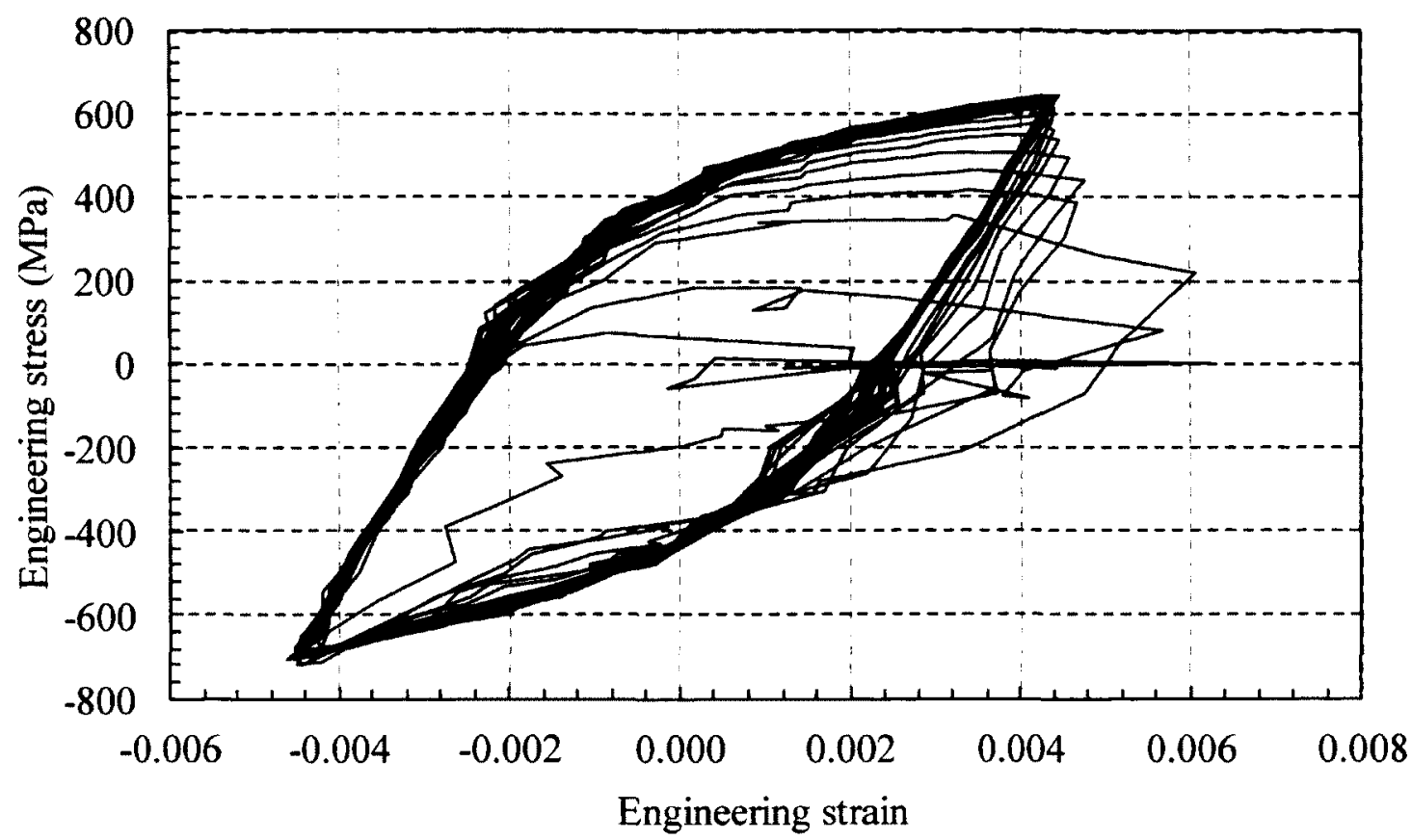

Figure B.33 Engineering stress versus engineering strain curve for the last few cycles of ANE1R2

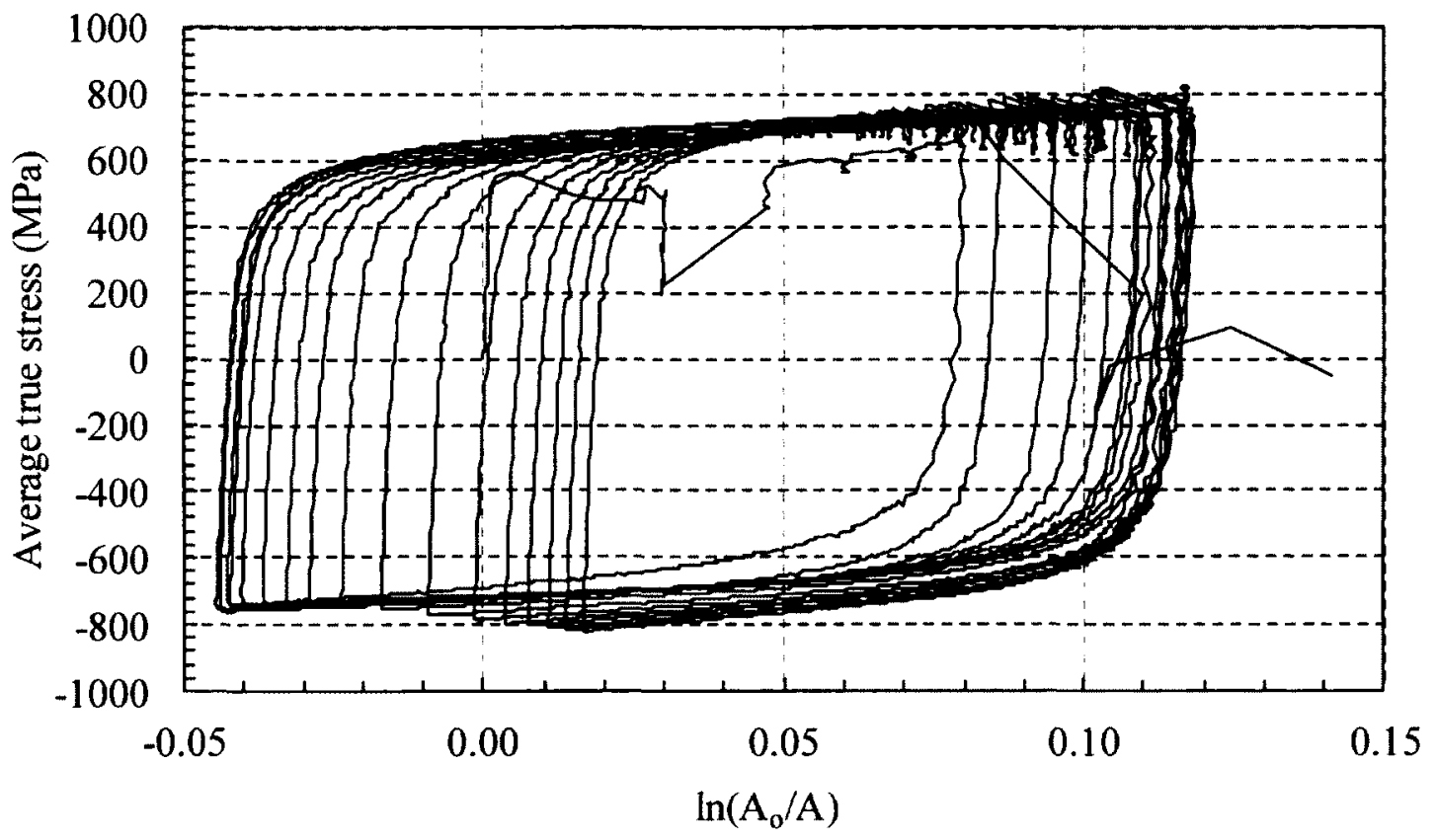

Figure B.34 Average true stress versus average true strain curve for ANE1R8 


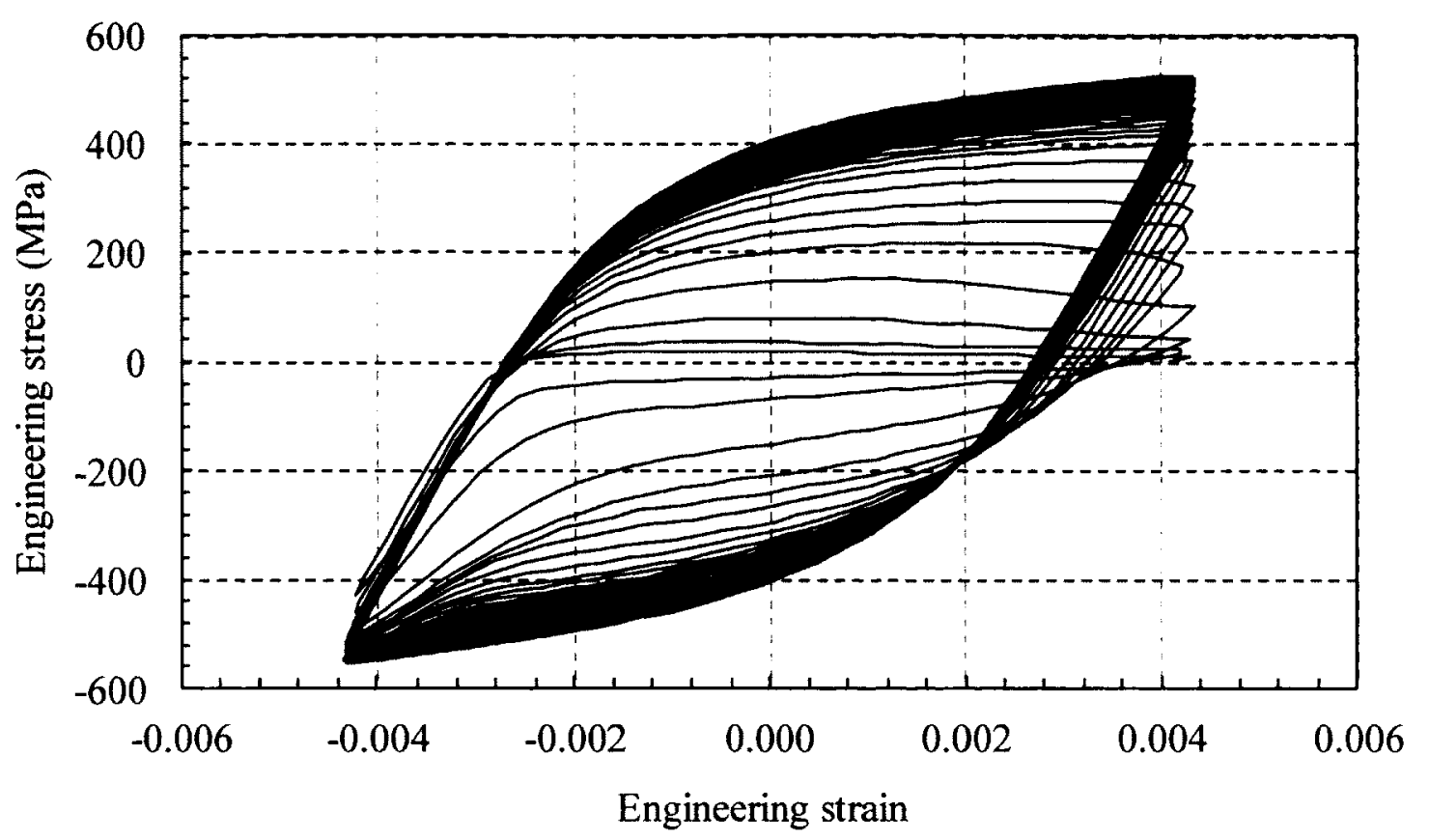

Figure B.35 Engineering stress versus engineering strain curve for the last few cycles of ANE2R2

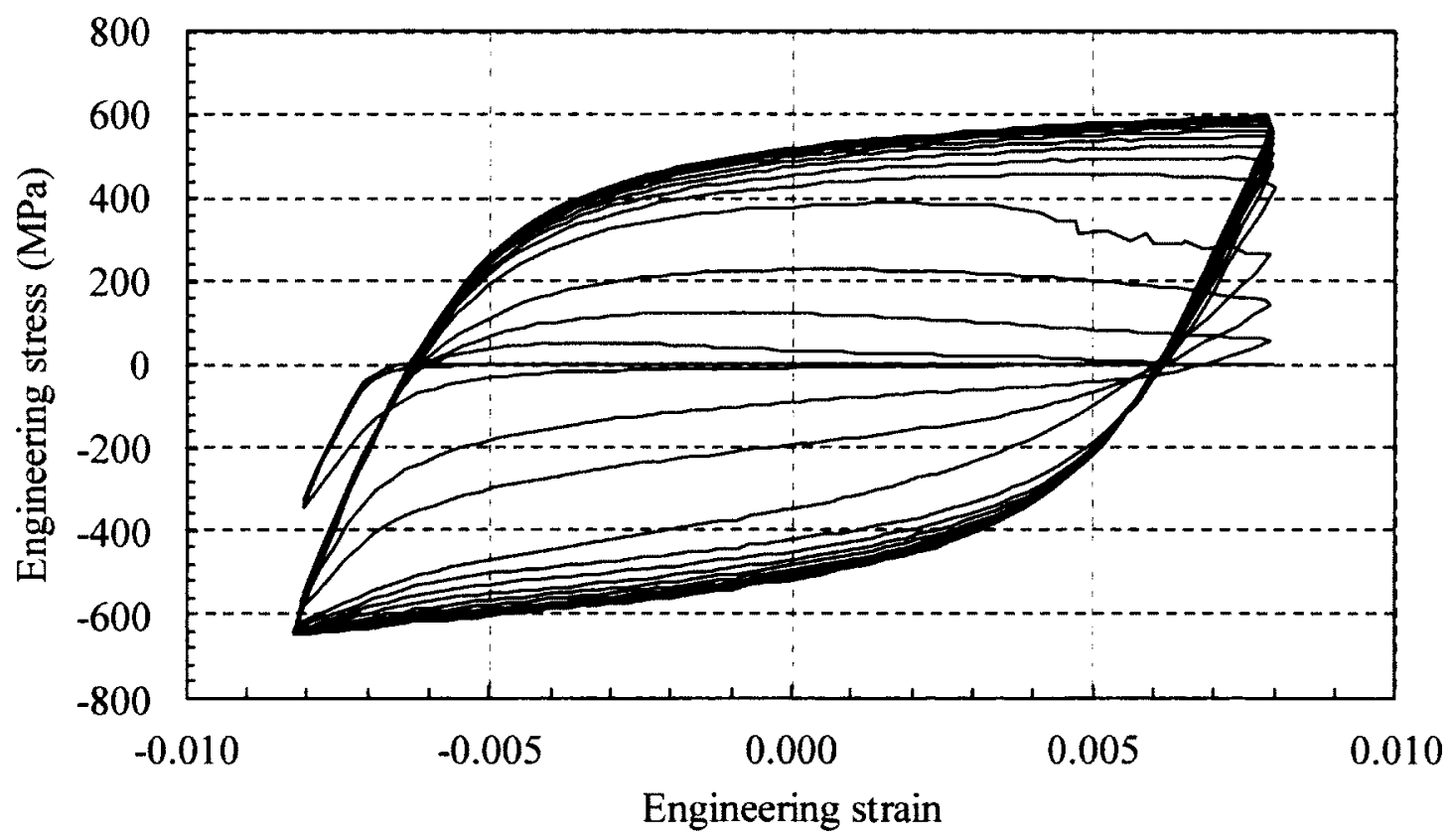

Figure B.36 Engineering stress versus engineering strain curve for the last few cycles of ANE2R4 


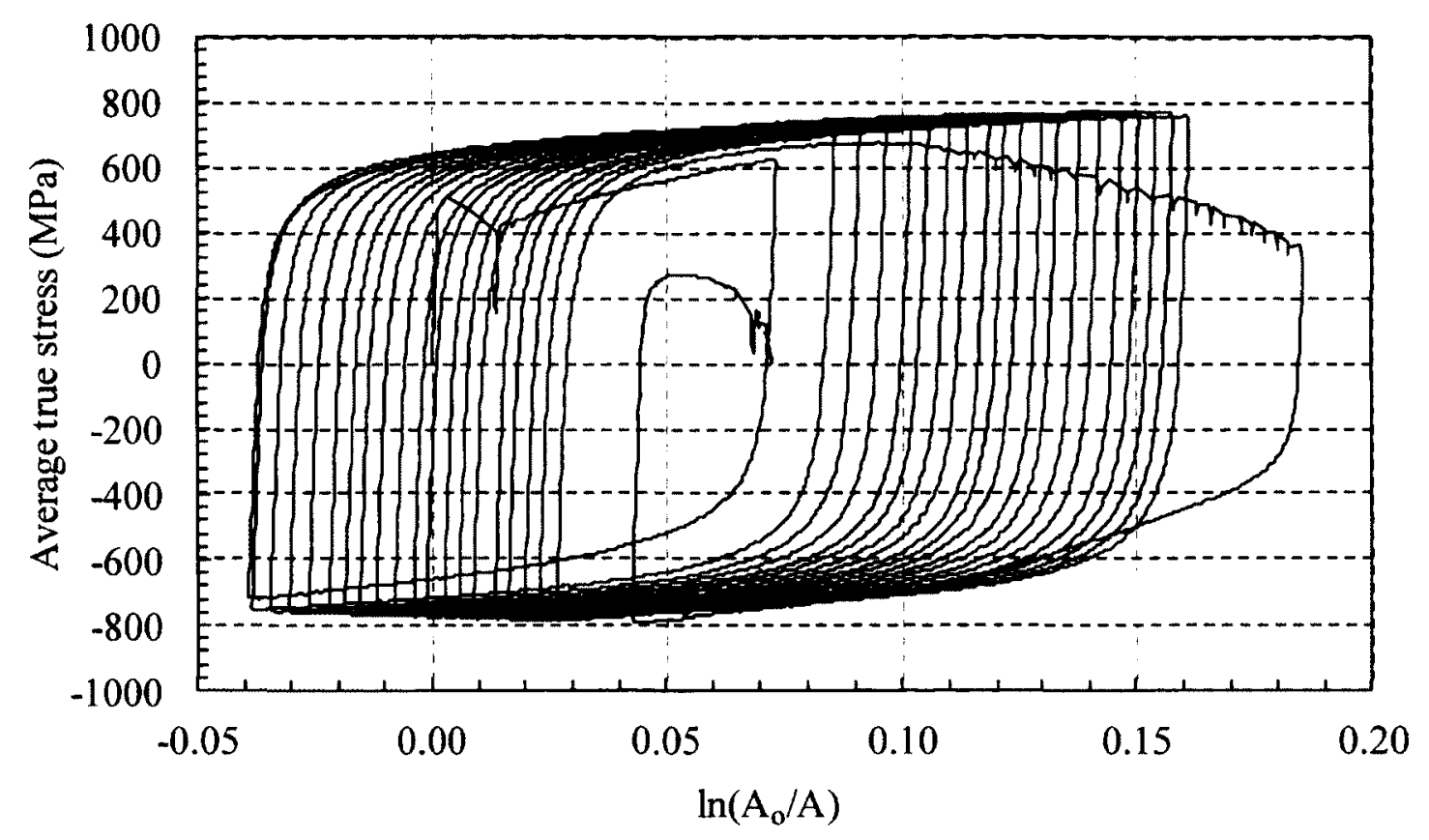

Figure B.37 Average true stress versus average true strain curve for ANE2R8

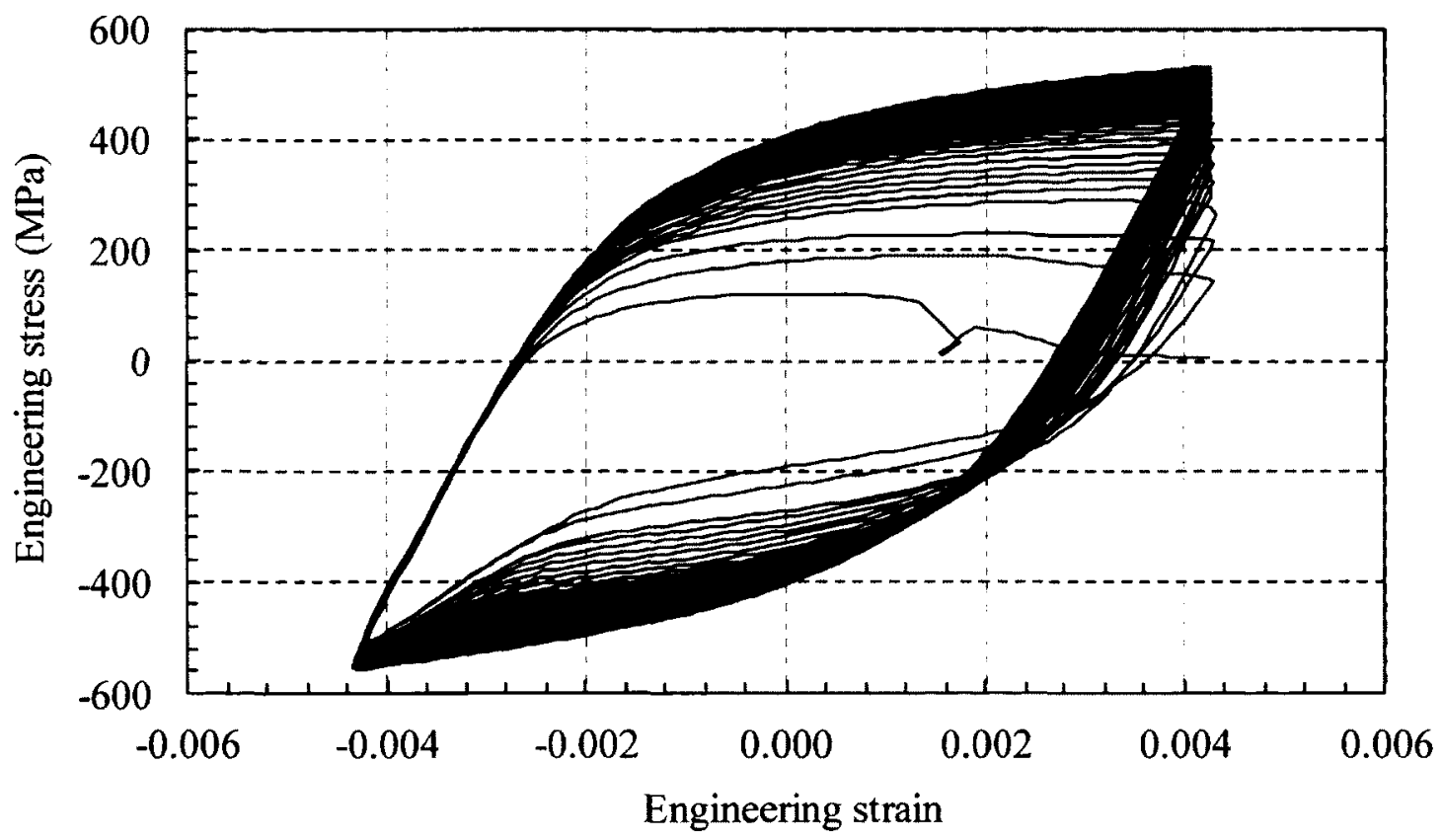

Figure B.38 Engineering stress versus engineering strain curve for the last few cycles of ANE3R2 


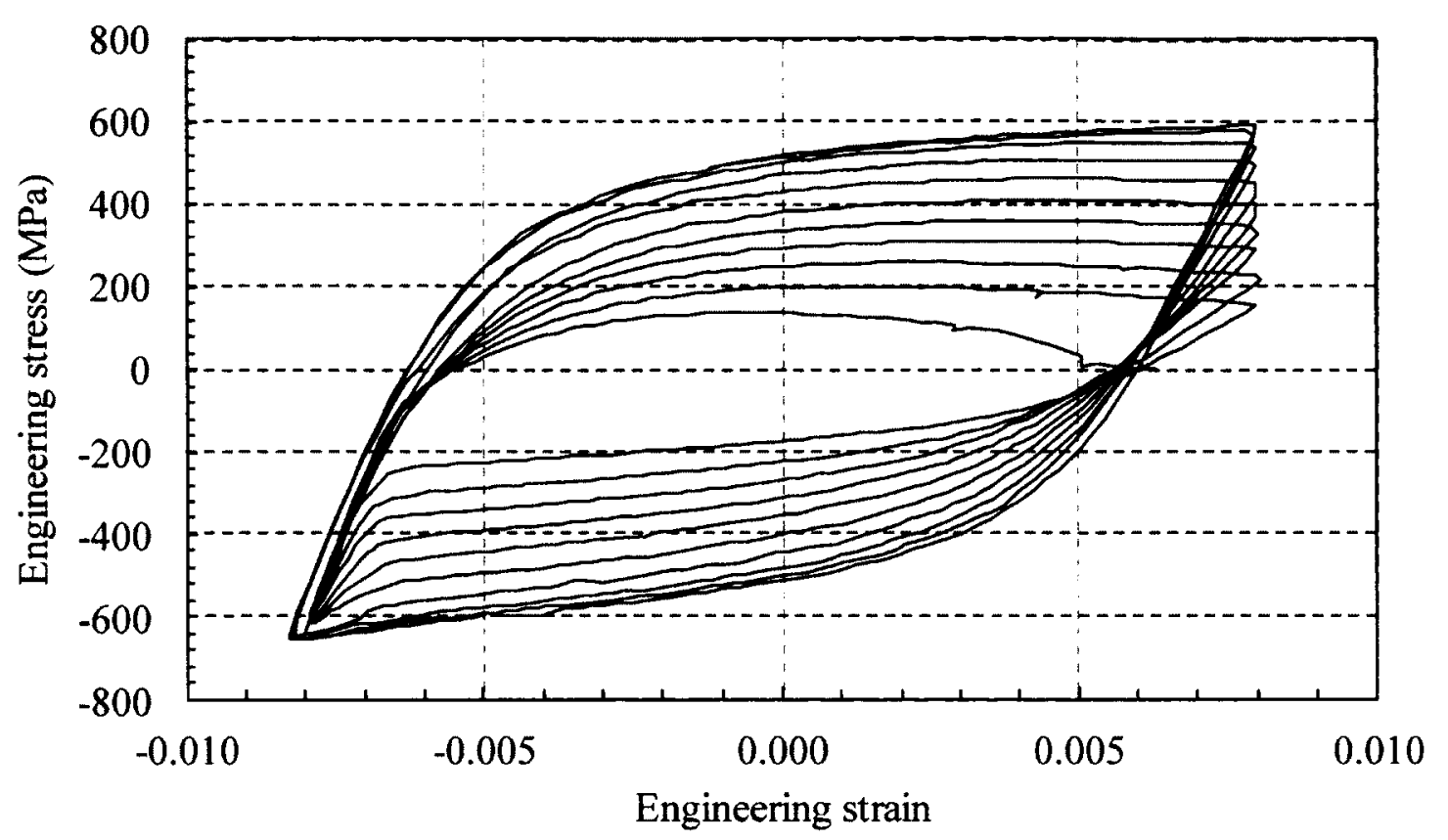

Figure B.39 Engineering stress versus engineering strain curve for the last few cycles of ANE4R4

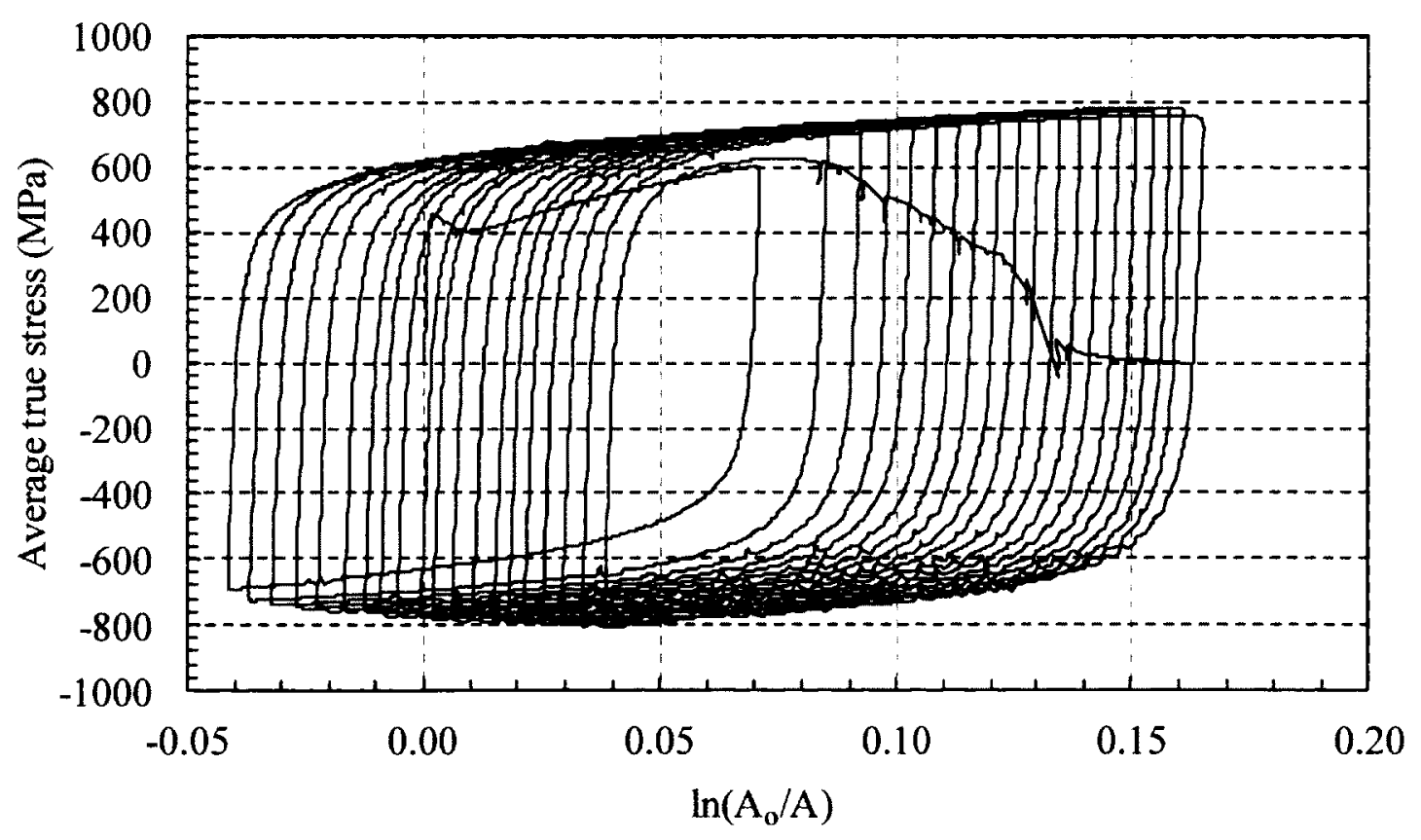

Figure B.40 Average true stress versus average true strain curve for ANE4R8 


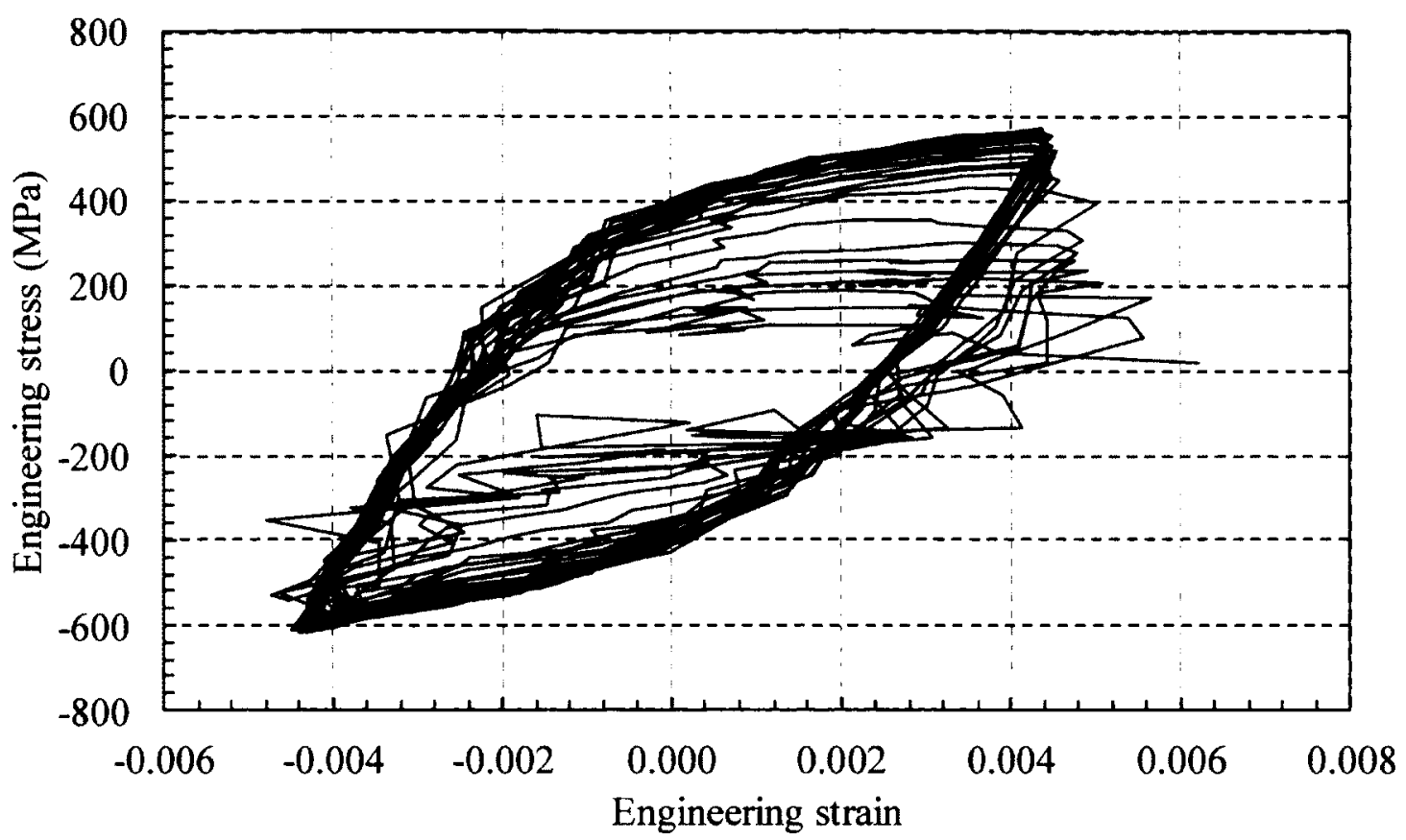

Figure B.41 Engineering stress versus engineering strain curve for the last few cycles of BNE1R2

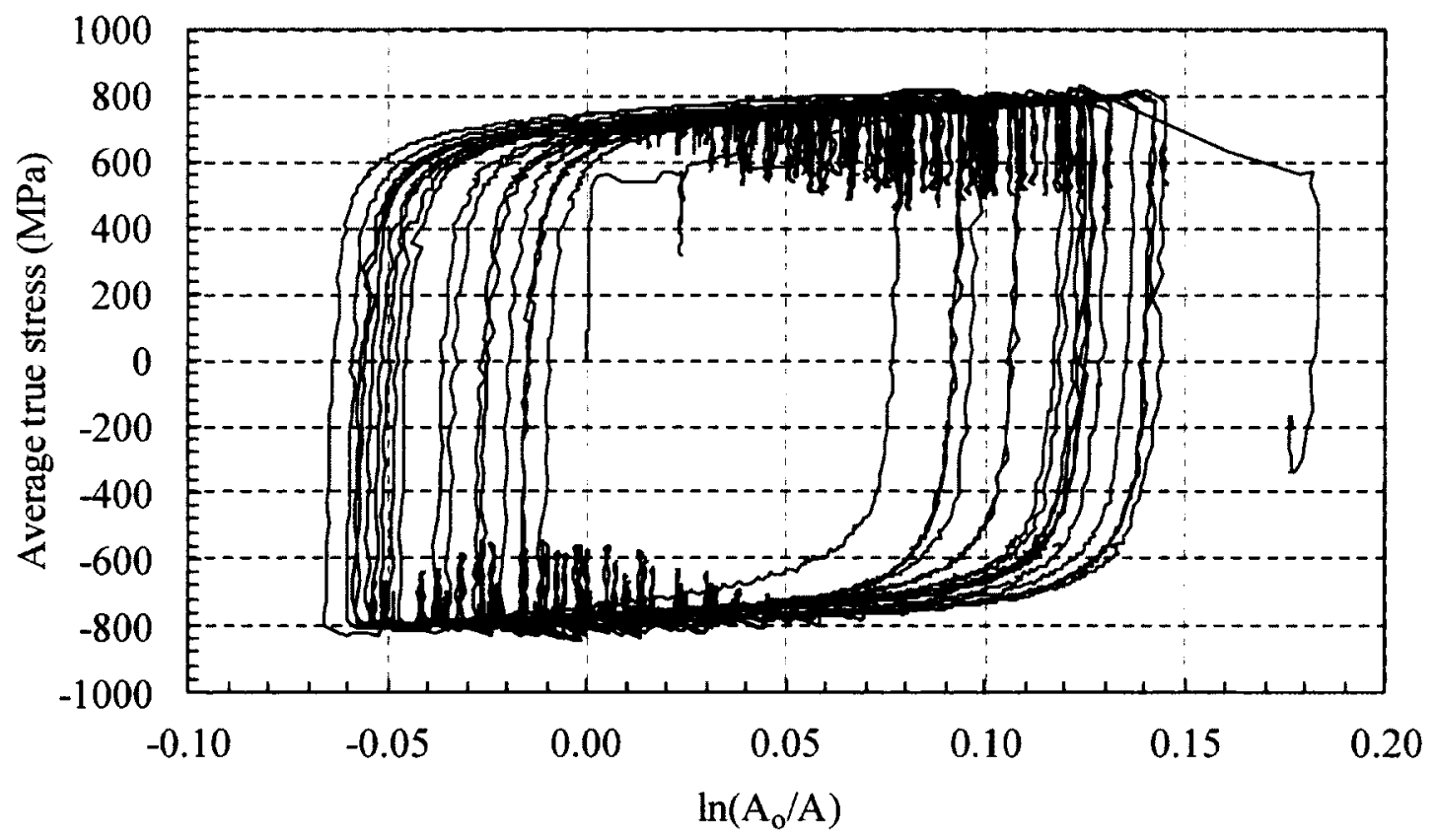

Figure B.42 Average true stress versus average true strain curve for BNE1R8 


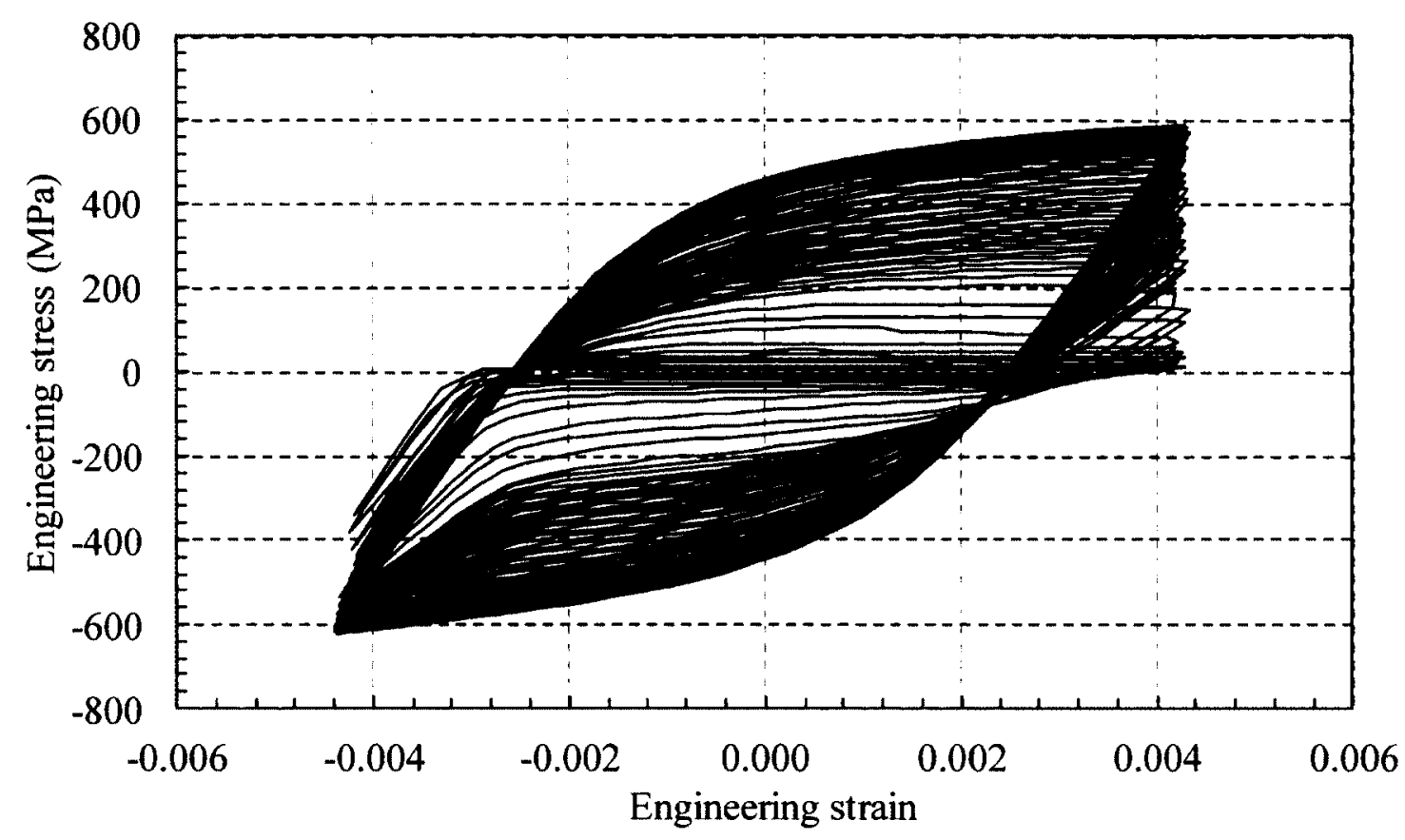

Figure B.43 Engineering stress versus engineering strain curve for the last few cycles of BNE2R2

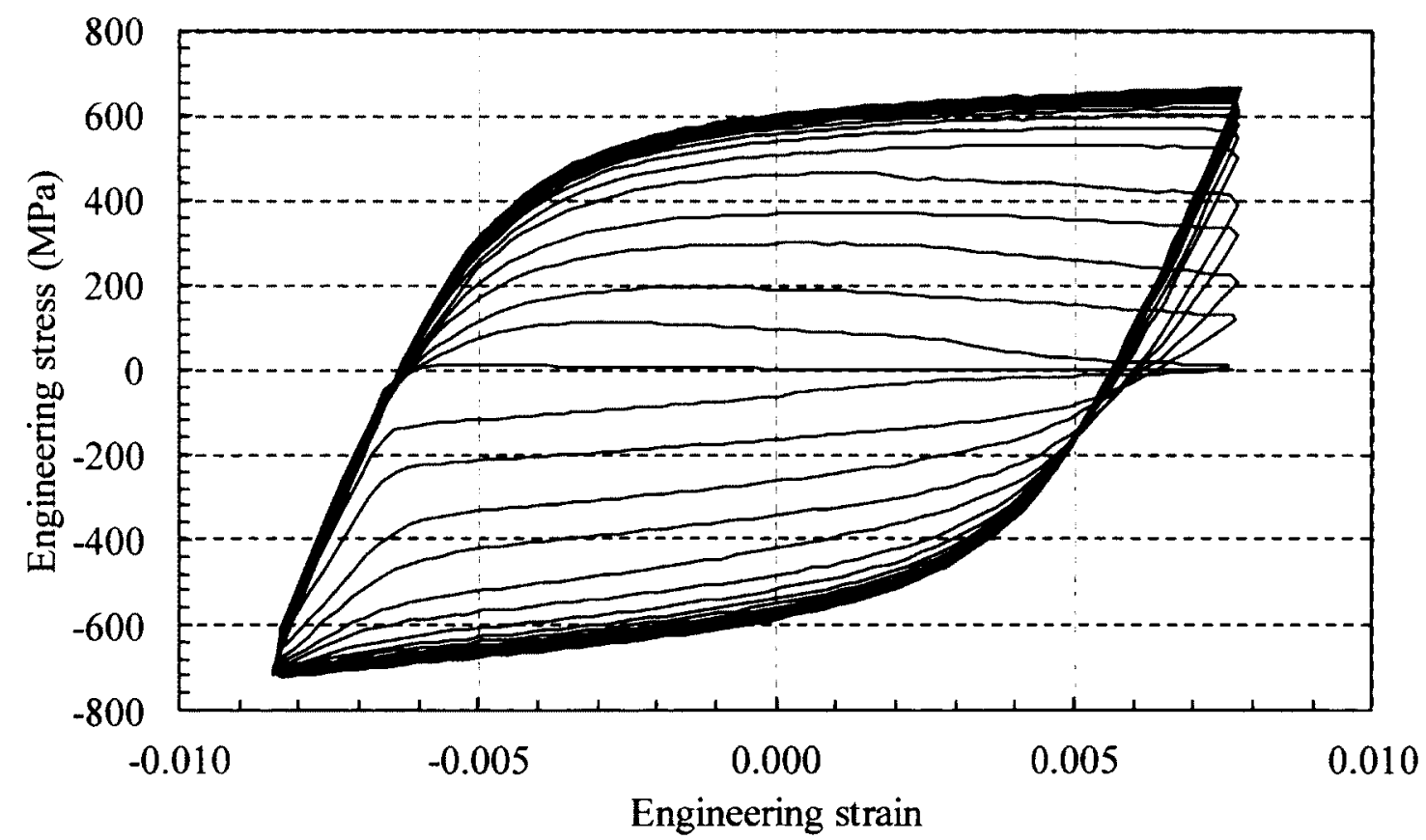

Figure B.44 Engineering stress versus engineering strain curve for the last few cycles of BNE2R4 


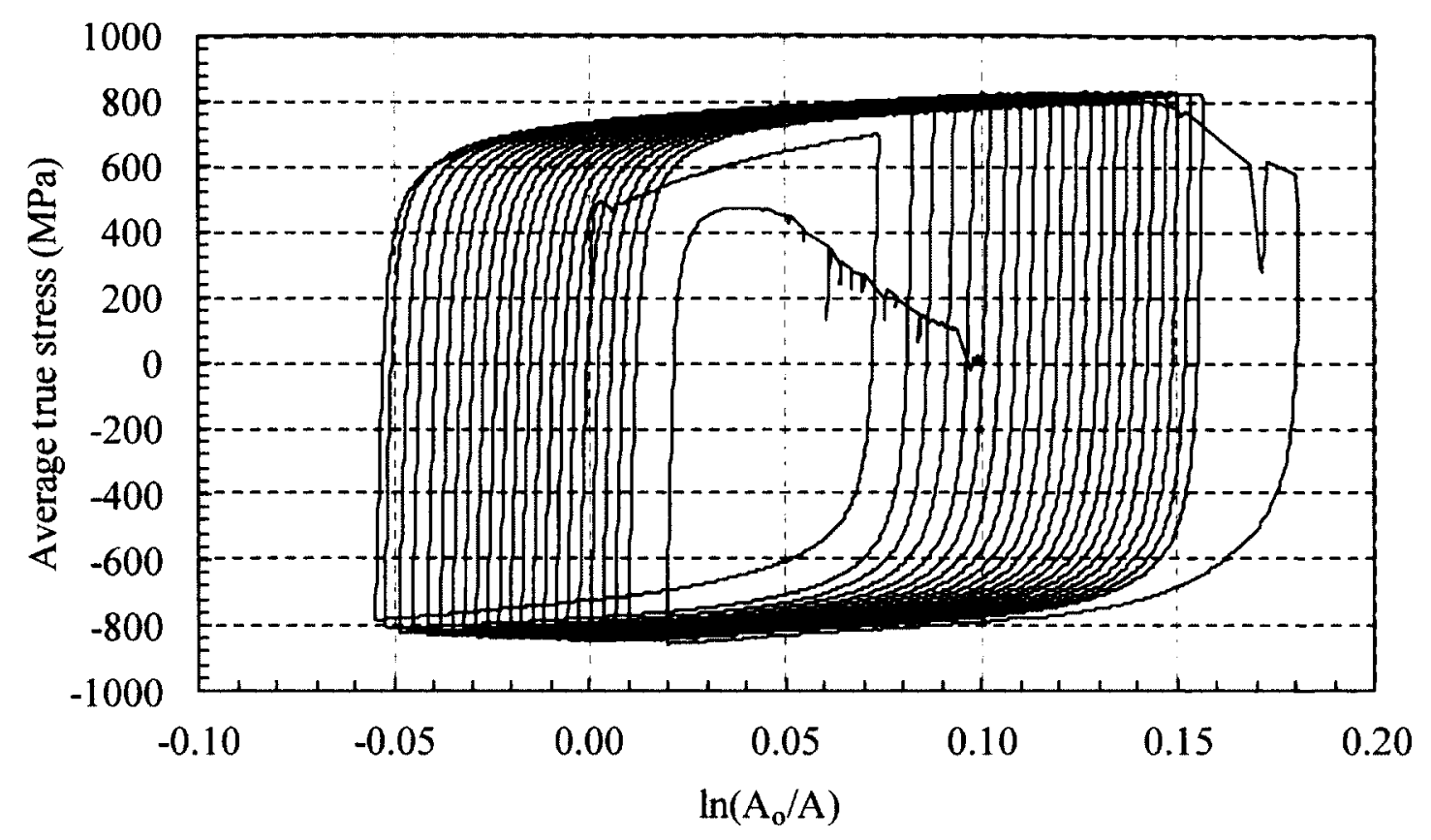

Figure B.45 Average true stress versus average true strain curve for BNE2R8

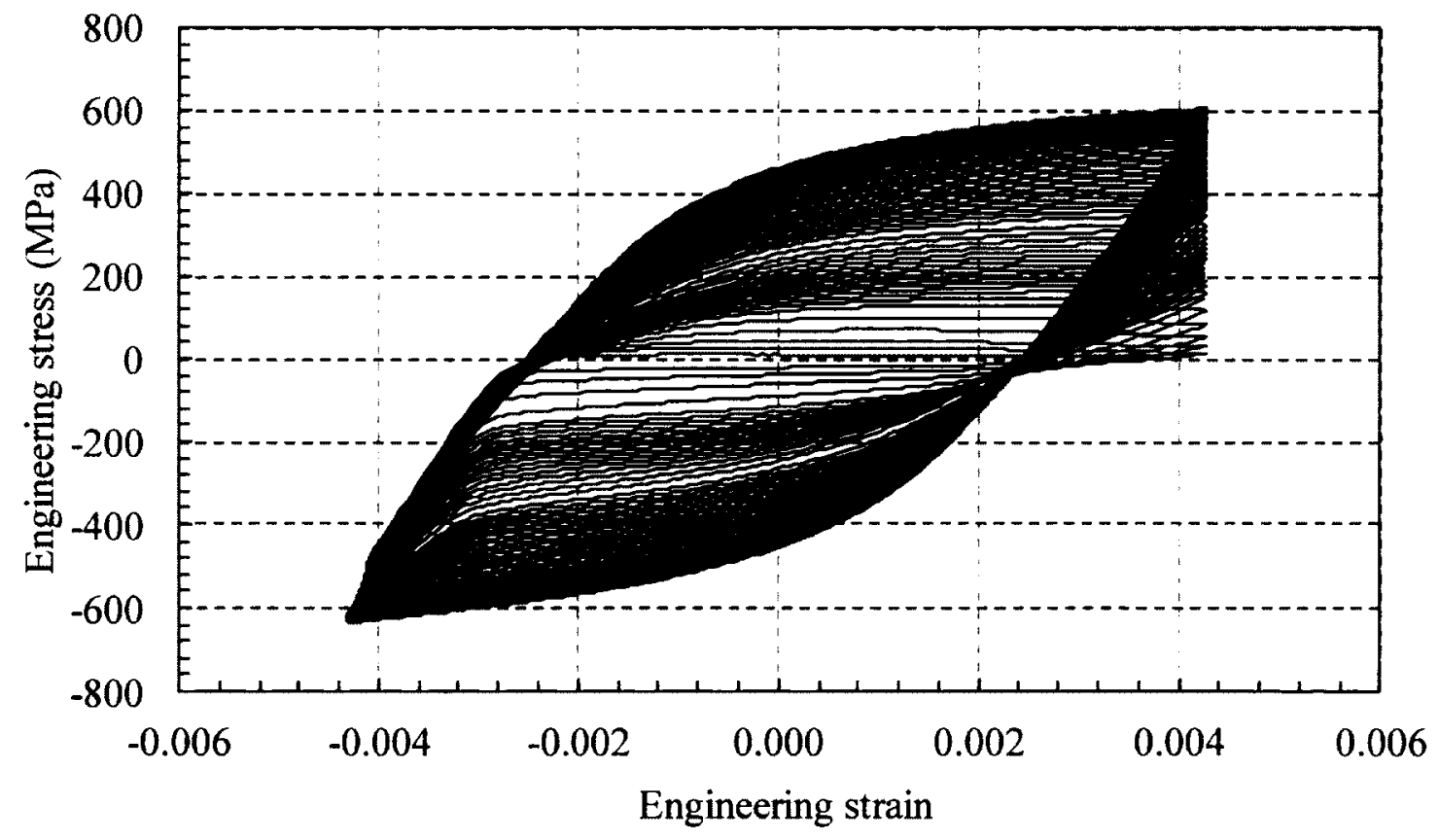

Figure B.46 Engineering stress versus engineering strain curve for the last few cycles of BNE3R2 


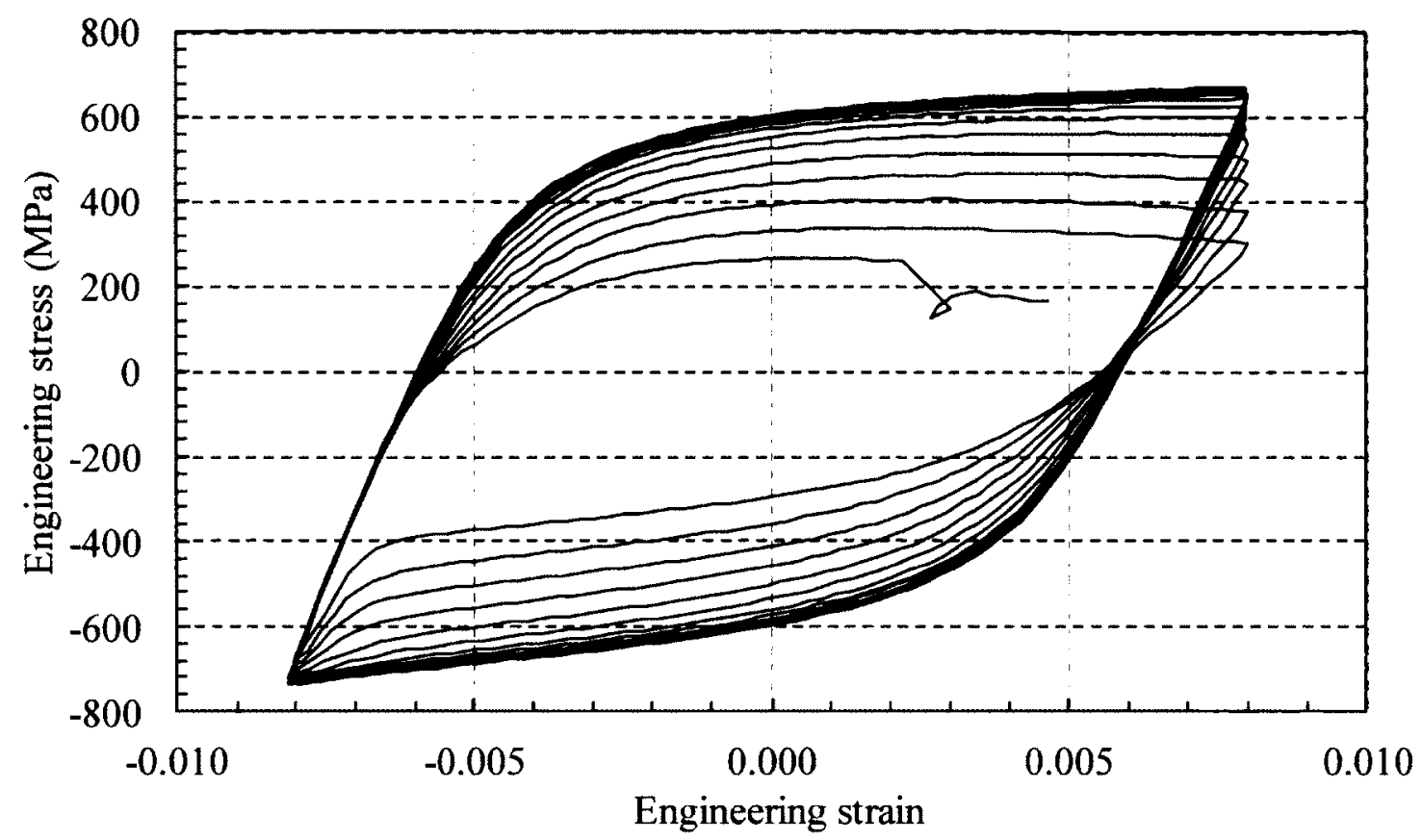

Figure B.47 Engineering stress versus engineering strain curve for the last few cycles of BNE3R4

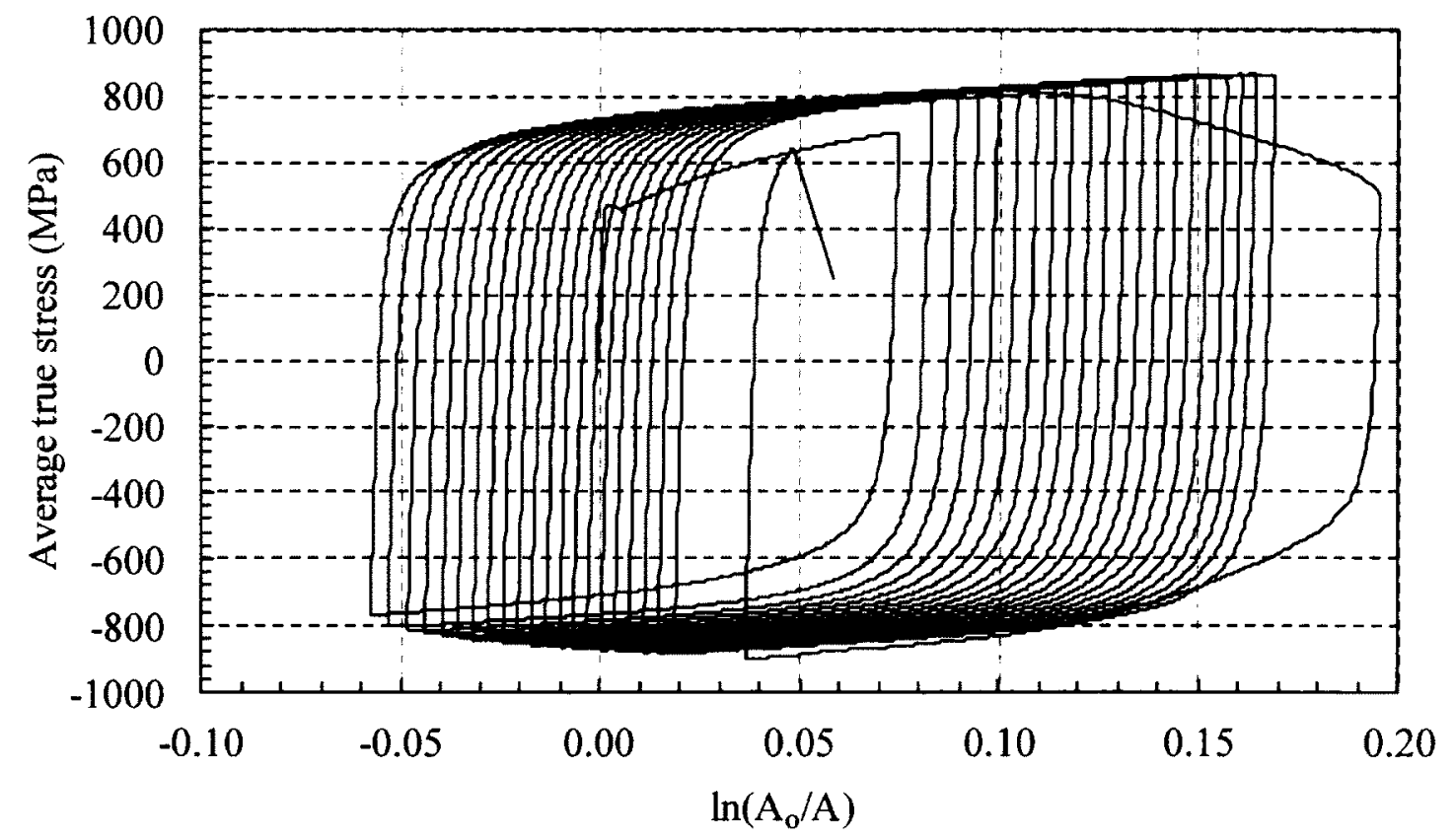

Figure B.48 Average true stress versus average true strain curve for BNE3R8 


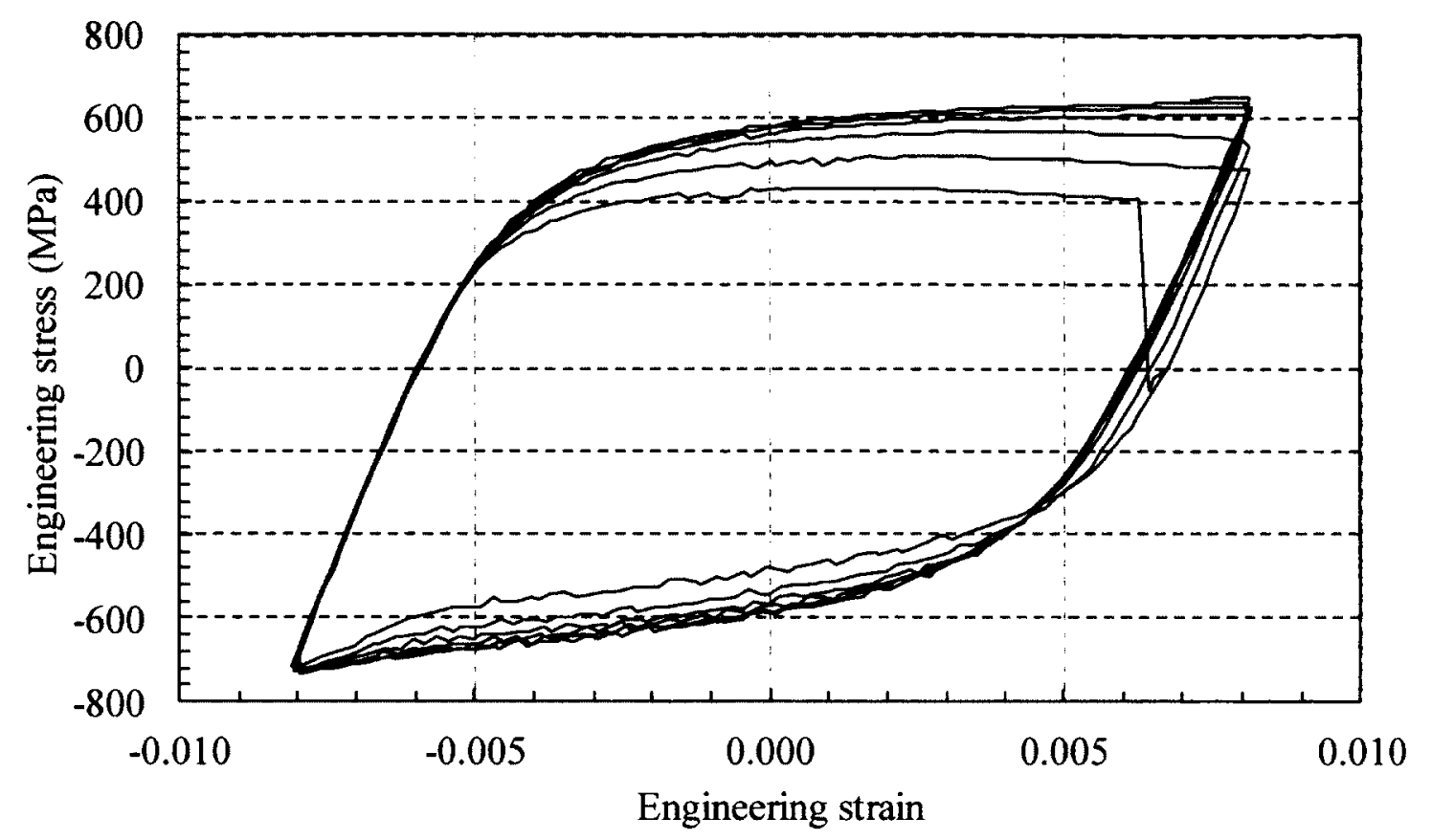

Figure B.49 Engineering stress versus engineering strain curve for the last few cycles of BNE4R4

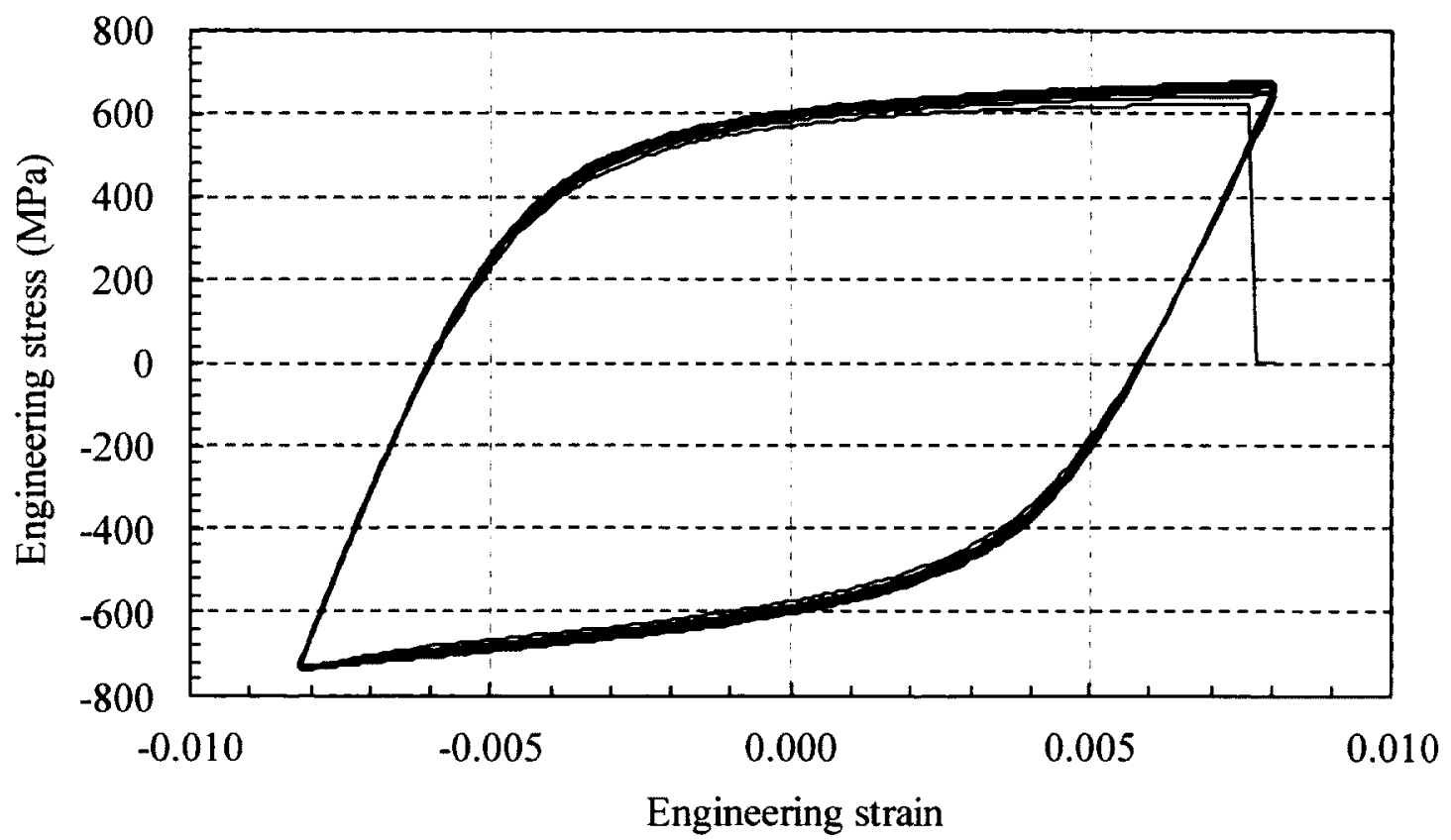

Figure B.50 Engineering stress versus engineering strain curve for the last few cycles of BNE4R4-2 


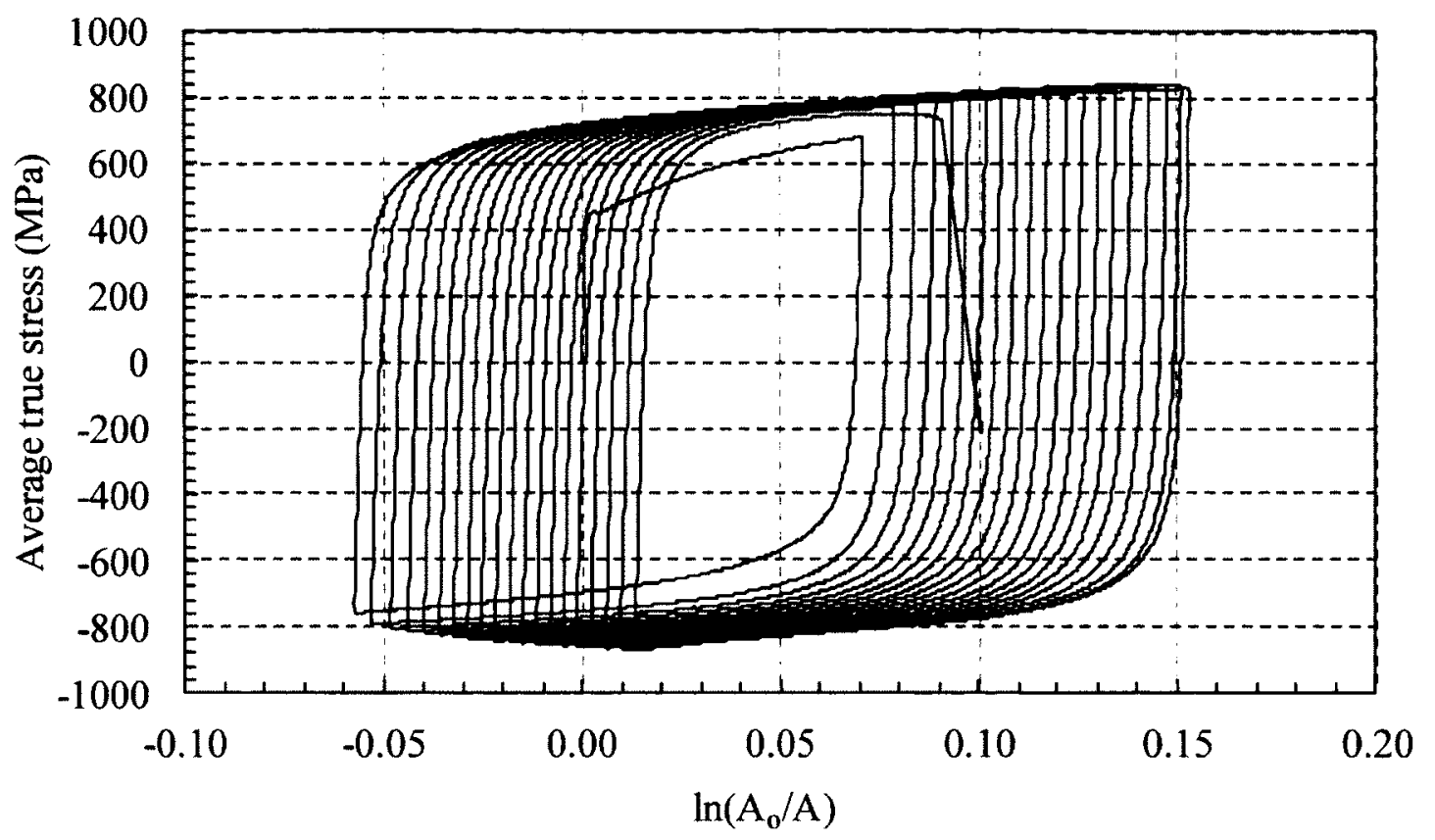

Figure B.51 Average true stress versus average true strain curve for BNE4R8 


\section{Appendix C Mesh Convergence Study}

A mesh convergence study has been carried out to improve the accuracy and efficiency of the finite element model. Necking occurs when a tapered specimen is being pulled to fracture. This produces a region with a highly non-uniform stress and strain distributions, similar that found in a notched specimen. A sufficiently fine finite element mesh is required to capture these complex stress and strain states in a numerical simulation accurately. However if a mesh is overly refined, computing effort increases without any significant improvement in accuracy.

Static analyses have been used in the numerical simulations of the tapered and notched specimens in the mesh convergence study. In the simulations, the tapered specimen is subjected to the loading of D test series while the notched specimen is subjected to the loading of ANE4R8 for 20 cycles. The material properties used in the numerical simulations are similar to those in Appendix A. Coarse and refined finite element meshes for the half-gauge length model of the tapered specimen are shown in Figs. C.1 and C.2, and notched specimen in Figs. C.3 and C.4. The element size of Scheme-1 in Fig. C.1 is double that of Scheme-2 in Fig. C.2. Similarly, the element size of Scheme-3 in Fig. C.3 is double that of Scheme-4 in Fig. C. 4 .

Comparisons of engineering stress versus engineering strain curves from the numerical simulations of the tapered specimens are shown in Figs. C.5 and C.6. It can be seen that the stress-strain curves with Scheme-1 and Scheme-2 are close to being identical. Similarly, comparisons of the average true stress versus average true strain curves and peak tensile average true stress versus cycle number curves for the notched 
specimen are shown in Fig. C.7 and C.8 respectively. Again, there is hardly any difference between results with Scheme-3 and Scheme-4.

Thus, the finite element mesh Scheme-1 has been chosen to be used to model tapered specimens and Scheme-3 to model notched specimens in the present study. 


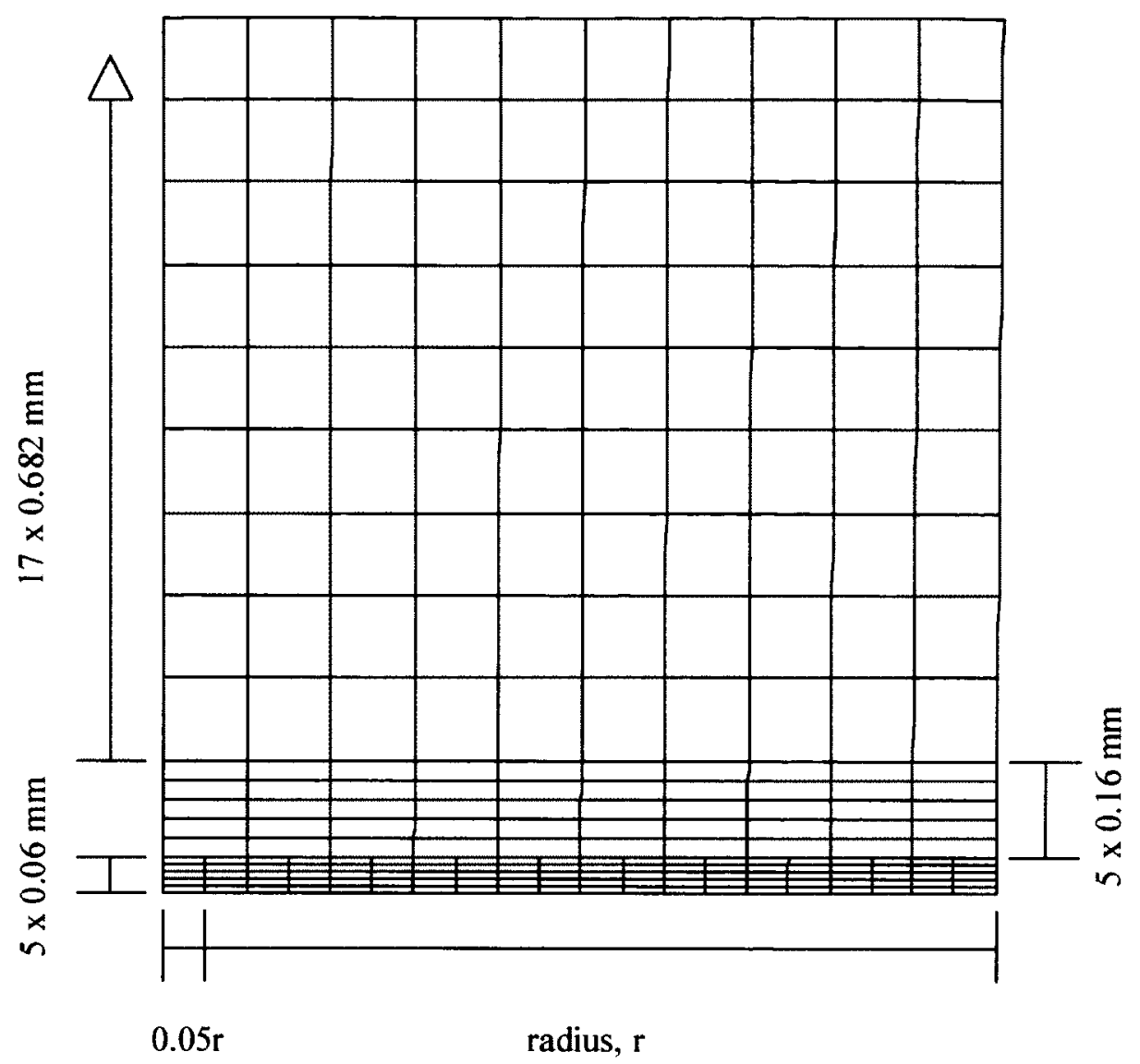

Figure C.1 Finite element mesh scheme-1 for the tapered specimen 


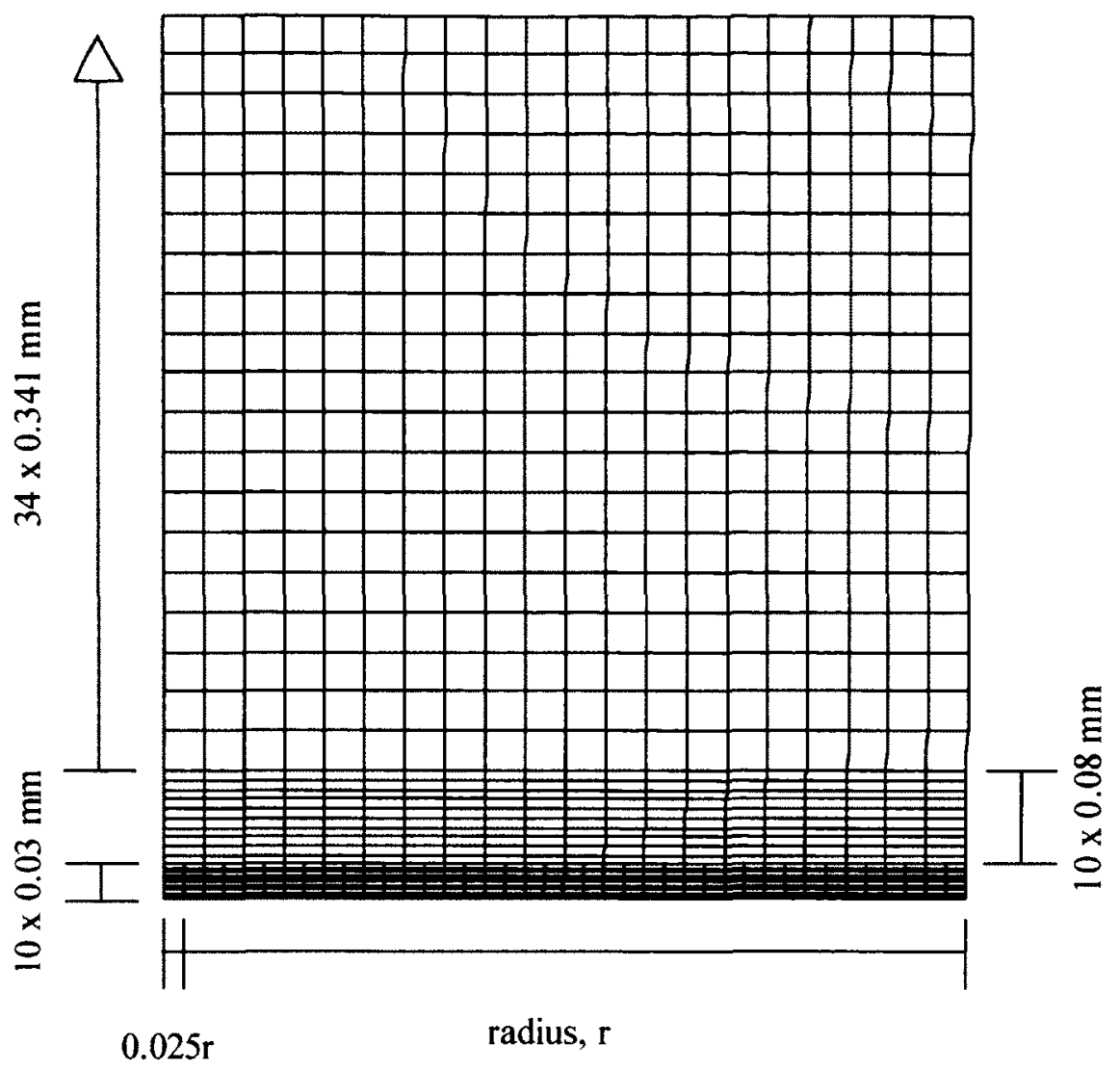

Figure C.2 Finite element mesh scheme-2 for the tapered specimen 


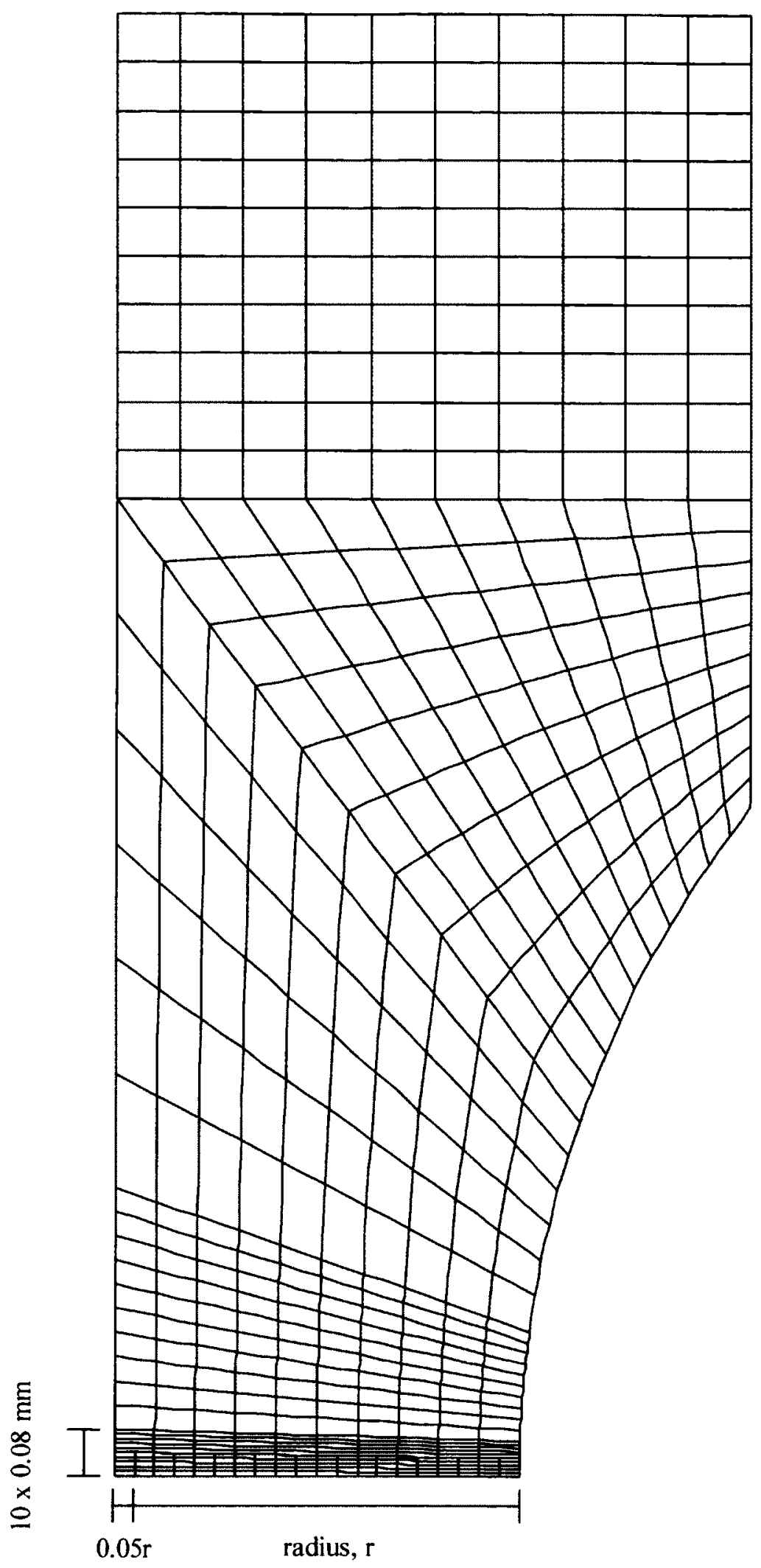

Figure C.3 Finite element mesh scheme-3 for the ANE4R8 specimen 


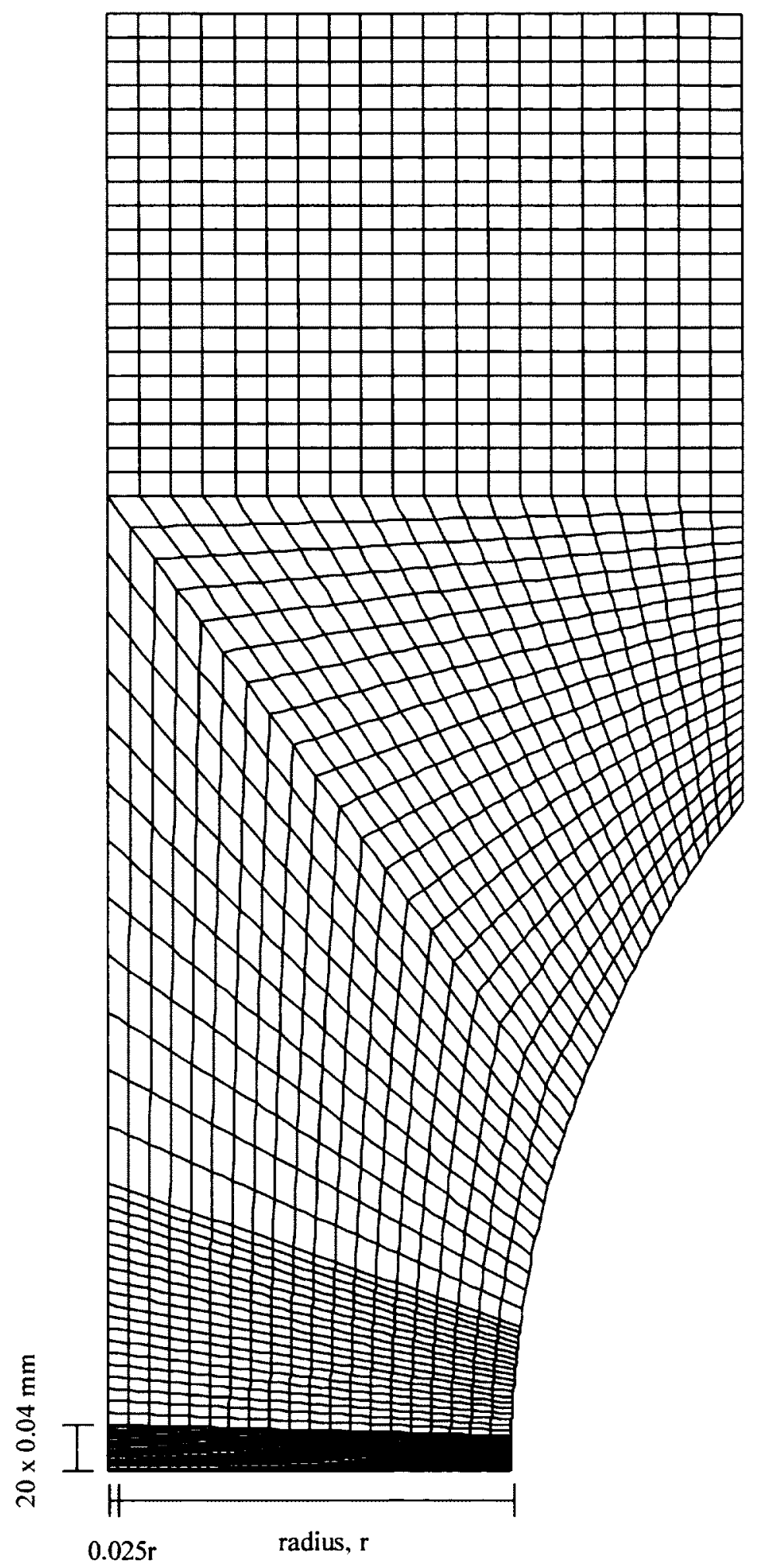

Figure C.4 Finite element mesh scheme-4 for the ANE4R8 specimen 


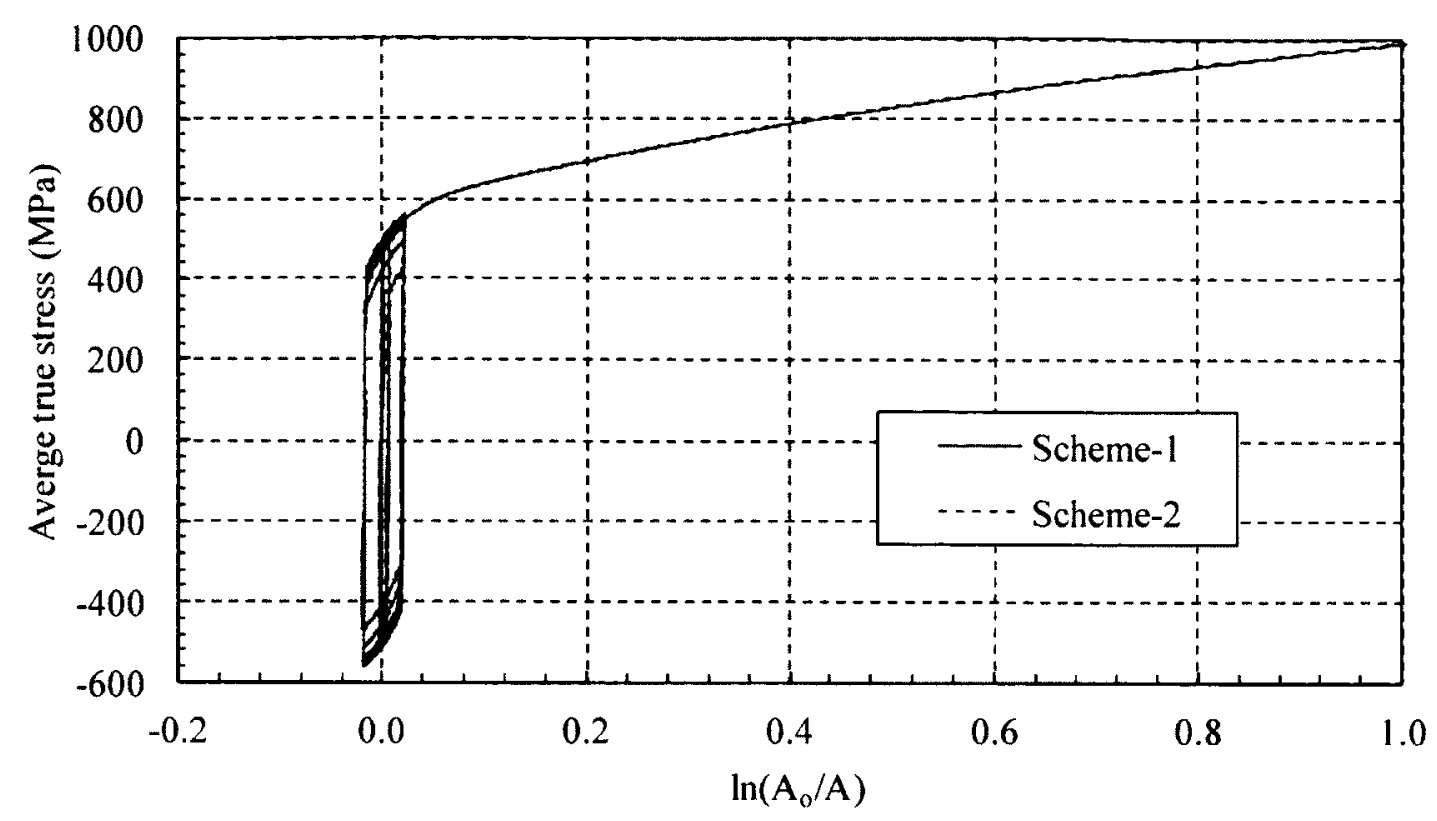

Figure C.5 Average true stress versus average true strain curves from FEA simulations of the tapered specimen for Scheme-1 and Scheme-2

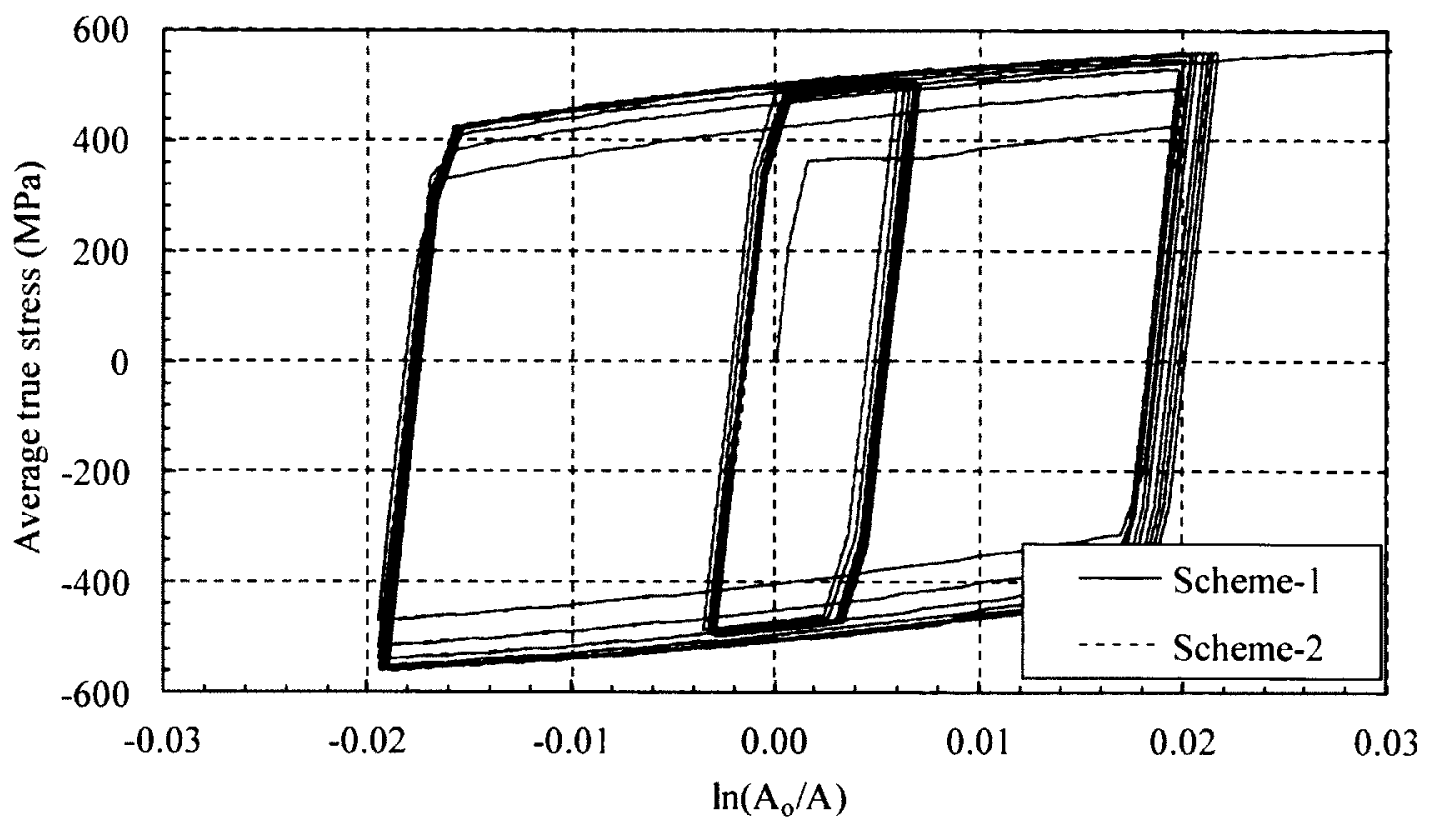

Figure C.6 Average true stress versus average true strain curves from FEA simulations of the tapered specimen for Scheme-1 and Scheme-2 during cyclic loading 


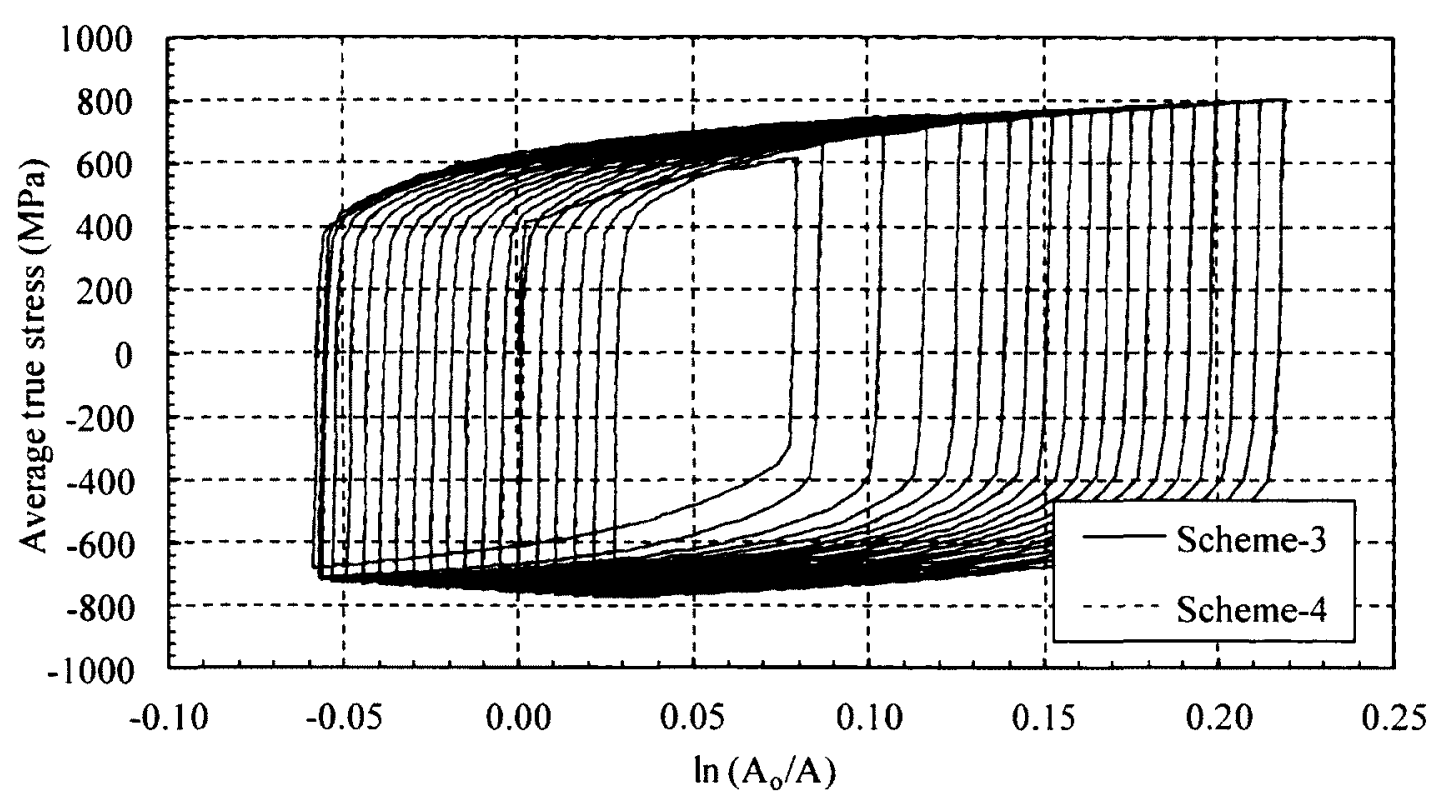

Figure C.7 Average true stress versus average true strain curves from FEA simulations of the notched specimen for Scheme-3 and Scheme-4

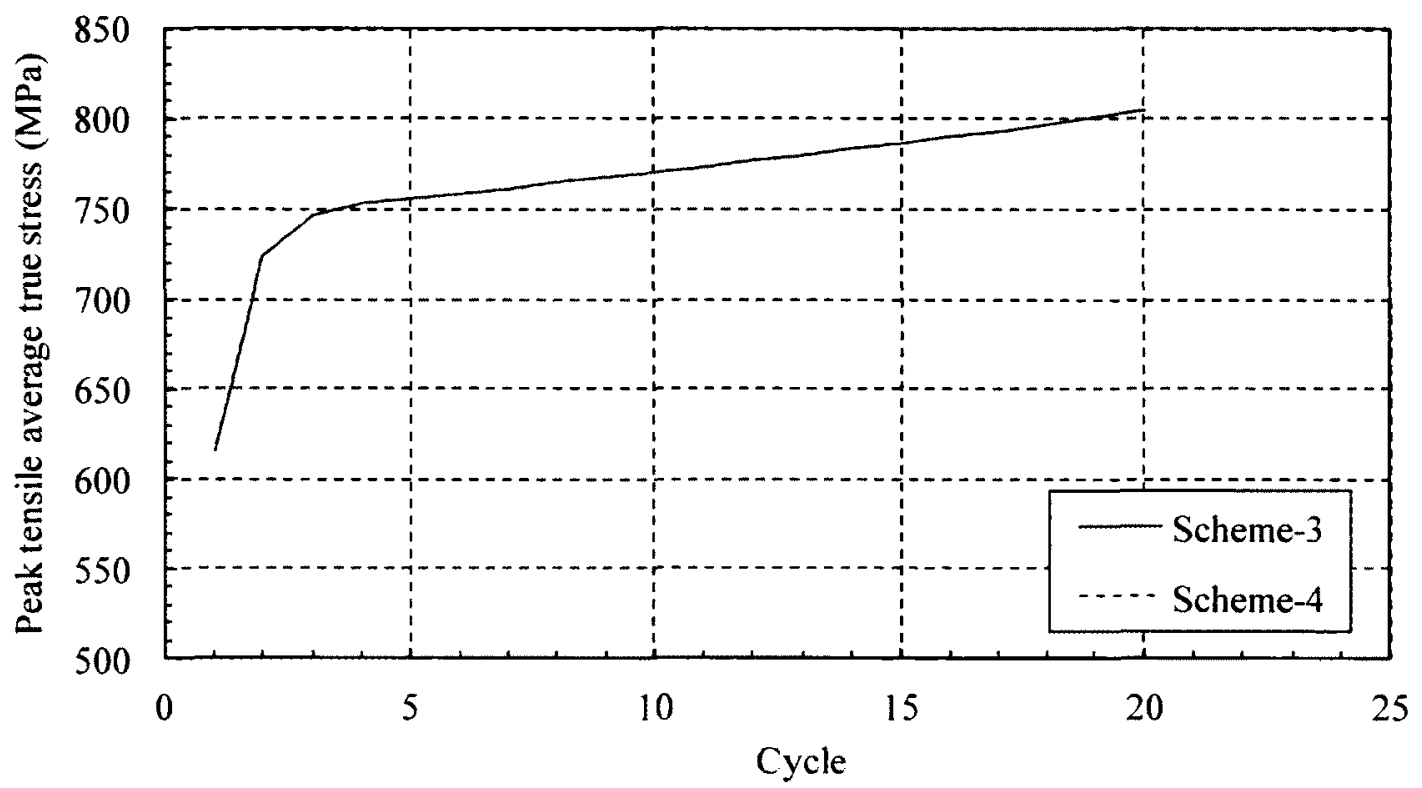

Figure C.8 Peak tensile average true stress versus cycle number curves from FEA simulations of the notched specimen for Scheme-3 and Scheme- 4 


\section{Appendix D Larger Diameter Specimen Taper Correction Factor for Monotonic and Cyclic Loading}

In Section 5.1.3, a factor has been calculated to convert the measured stress of a tapered specimen by Chen (2010) to that of an equivalent non-tapered (straight profile) specimen. It has been found through numerical simulation that a factor of $99 \%$ can be applied to the calculated average true stress for a $9 \mathrm{~mm}$ diameter tapered specimen to compensate for the effect of the increased stress triaxiality on the measured stress due to the tapered profile within the reduced section of the specimen. However in the current study, the reduced section of the tapered specimens has a diameter of $14 \mathrm{~mm}$ that tapers to a nominal minimum diameter of $13.72 \mathrm{~mm}$ at the middle of the reduced section (gauge length). Therefore, it is necessary to determine if the same conversion factor for the $9 \mathrm{~mm}$ diameter tapered specimens can be applied to a specimen of a larger cross-section. In addition, tapered specimens in the current study have been loaded cyclically before being pulled to fracture. Therefore, it is also necessary to determine if the conversion factor can be used under cyclic loading.

Using symmetry, half of the tapered and the non-tapered specimen have been modelled. The same number of elements are used to mesh the model for the $14 \mathrm{~mm}$ diameter specimen as that for the $9 \mathrm{~mm}$ diameter specimen shown in Fig. 5.3. As well, the element type, true stress versus true plastic strain curve, elastic modulus and Poisson's ratio are the same as those used in the numerical simulation to determine the conversion factor for the $9 \mathrm{~mm}$ diameter tapered specimens. Comparisons of the true flow stress versus true plastic strain curves calculated with Eqs. (4.3) and (4.6) and modified with Eq. (5.1) are shown in Fig. D.1 up to a true plastic strain of 1.0. Similarly, the engineering stress versus cross-sectional area ratio curves are shown in Fig. D.2. It can be 
seen that the engineering stress and true flow stress of the tapered specimen are about $1 \%$ higher than that for the non-tapered specimen. Therefore, the same conversion factor used for $9 \mathrm{~mm}$ diameter specimens can also be used for $14 \mathrm{~mm}$ diameter specimens.

The effect of the tapered profile on the measured stress of a tapered specimen compared to a non-tapered (straight profile) specimen under cyclic loading is being evaluated using the cyclic loading sequence of I test series. However, in order to simulate the applied loading of the I test series, the loading in the finite element analysis has to be applied at one end of the gauge length where the axial extensometer is attached to. Thus for cyclic loading, it is required to model only the segment of the specimen that is within the gauge length of the axial extensometer. So it is necessary to determine if the reduced length model can accurately simulate the behaviour of a full model. Using symmetry, numerical simulations have been carried out for the $14 \mathrm{~mm}$ diameter tapered specimens with ABAQUS (Simulia 2010) under monotonic tension loading using models of half the specimen and half of the reduced length (gauge length), and material properties used in the determination of the conversion factor for monotonic loading. The number of elements used in the models of the $14 \mathrm{~mm}$ diameter tapered specimen are the same as those used in the half full length and half gauge length models of the $9 \mathrm{~mm}$ diameter specimens shown Figs. 5.3 and 5.4. Figure D.3 shows the predicted engineering versus cross-sectional area ratio curves for the half full length and half gauge length models of the $14 \mathrm{~mm}$ diameter tapered specimen. It can be seen in Fig. D. 3 that there is no noticeable difference between results of numerical simulations from both models. Thus, the half gauge length model can be used in place of the half full length model in a numerical simulation. 
Numerical simulations have also been carried out with tapered and non-tapered half gauge length models for the cyclic loading sequence of the I test series with the same material property input data generated using the parameters in Table A.1 of Appendix A. The model of the non-tapered specimen has the same mesh configuration as that for the tapered specimen. Figures D.4 and D.5 show the respective predicted tensile and compressive average true stress versus average true strain for the non-tapered and tapered $14 \mathrm{~mm}$ diameter specimens with the average true stress of the tapered specimens reduced by $1 \%$. Overall, the maximum tensile and compressive average true stresses of the nontapered specimen at each cycle are slightly smaller than those for the tapered specimen with $1 \%$ correction, with increasing difference with the strain range. This difference is mainly due to the larger average true strain range experienced by the tapered specimens compared to the non-tapered specimens. The shifted curves may be used in the comparison in order to compensate for the difference in the average true strain range where maximum compressive average true stress points for both specimens have been shifted to the origin. Figure D.6 shows the shifted average true stress versus average true strain curves at the $10^{\text {th }}$ tension half-cycle of each strain range for both tapered and nontapered specimens. Ignoring the extension in average true strain for the tapered specimen due to a larger average true strain range, it can be seen in Fig. D.6 that the curve for nontapered specimens matches quite well with that for the tapered specimen, even at a large strain range. This shows that the conversion factor determined for monotonic loading can equally be applied to cyclic loading. 


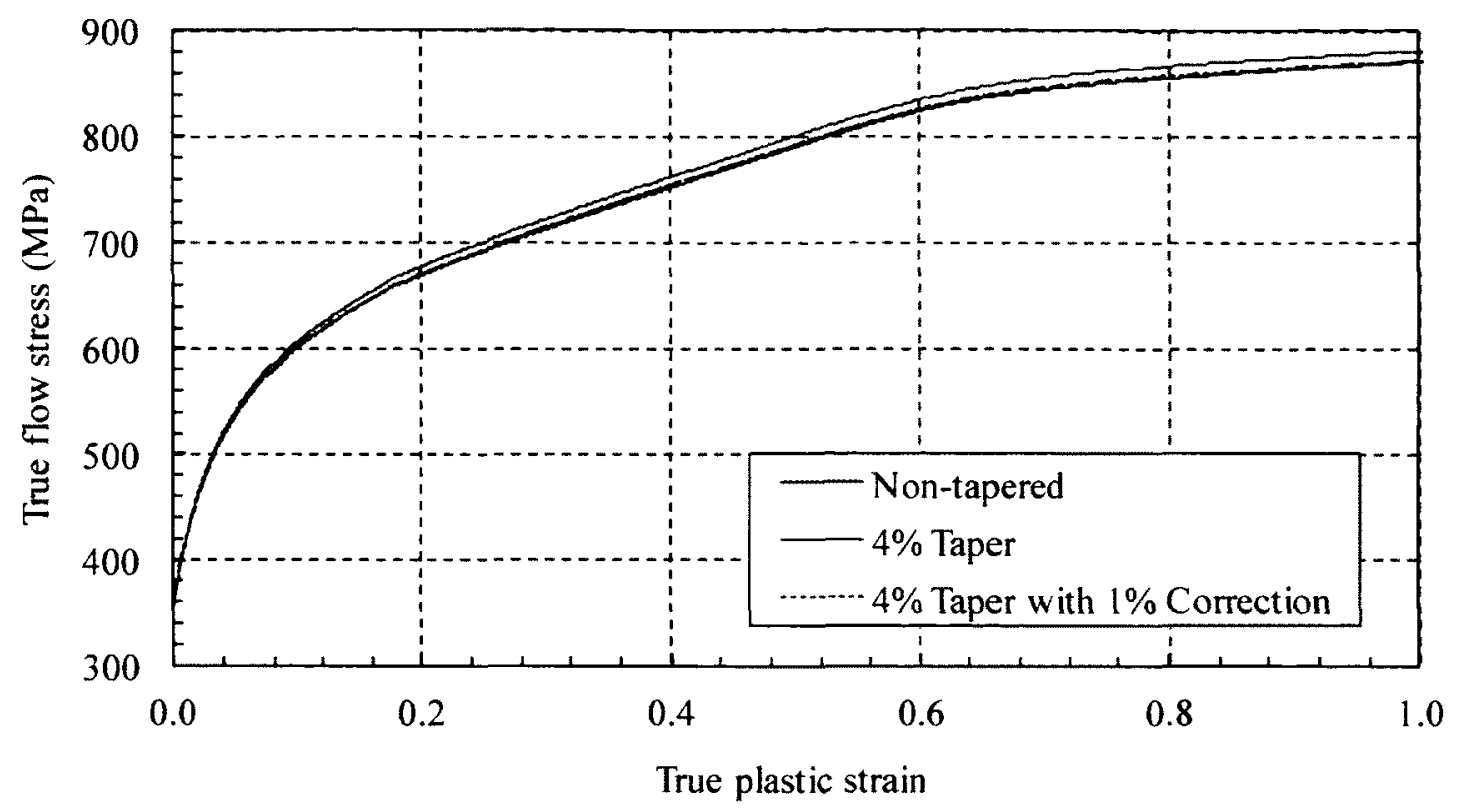

Figure D.1 True flow stress versus true plastic strain curves for tapered and non-tapered $14 \mathrm{~mm}$ diameter specimens

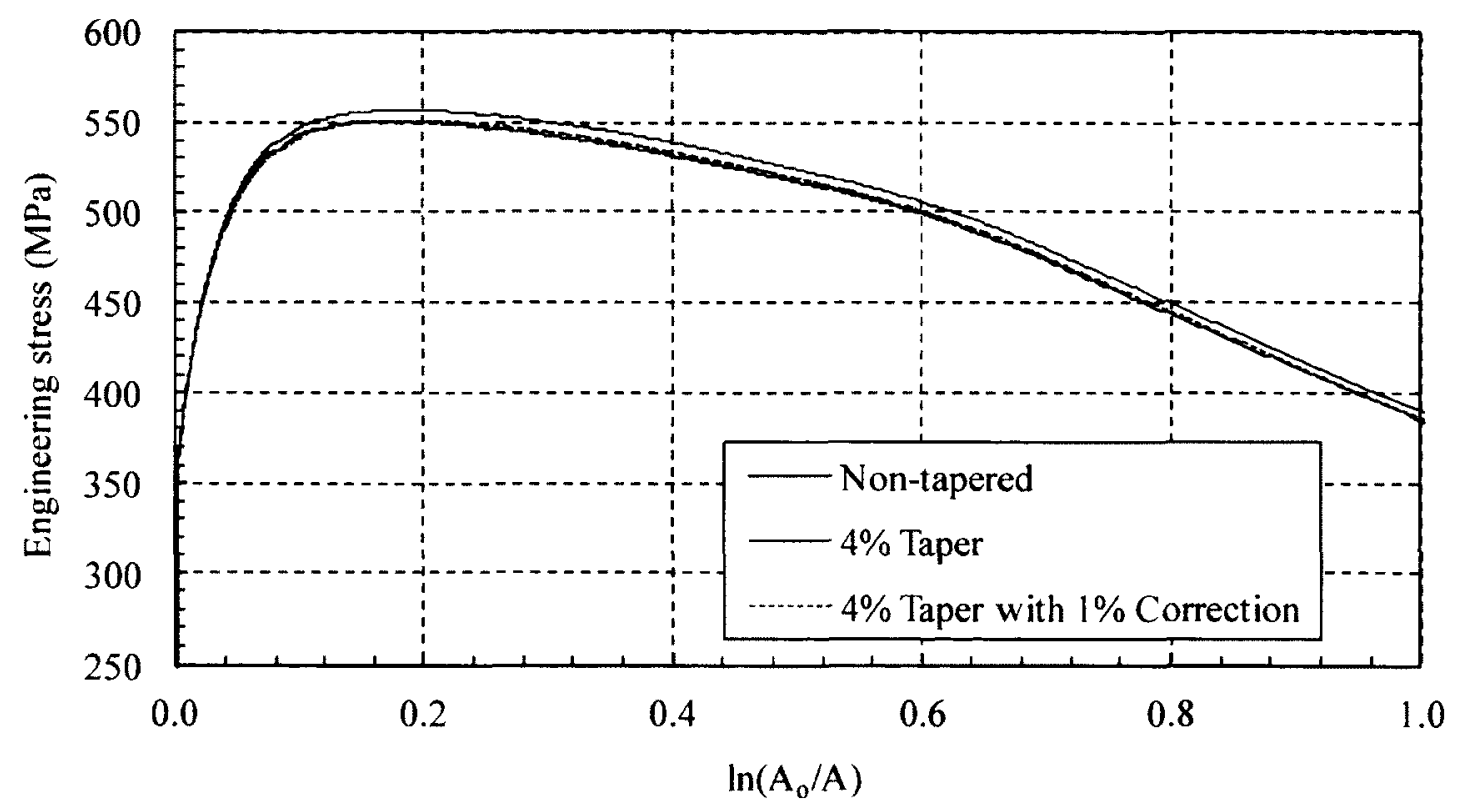

Figure D.2 Engineering stress versus cross-section area ratio curves for tapered and non-tapered $14 \mathrm{~mm}$ diameter specimens 


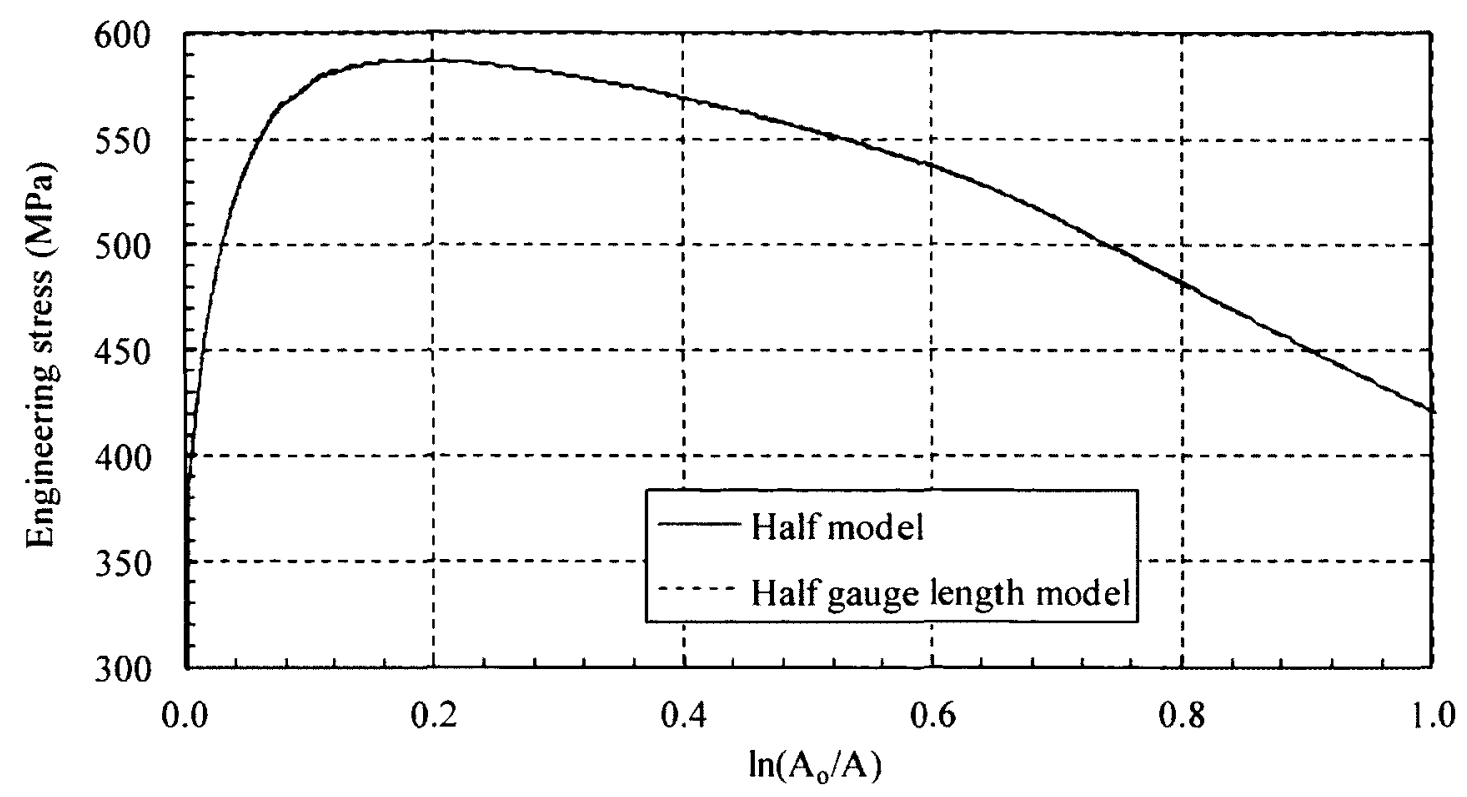

Figure D.3 Engineering stress versus cross-section area ratio curves for half gauge length and half models of $14 \mathrm{~mm}$ diameter tapered specimens

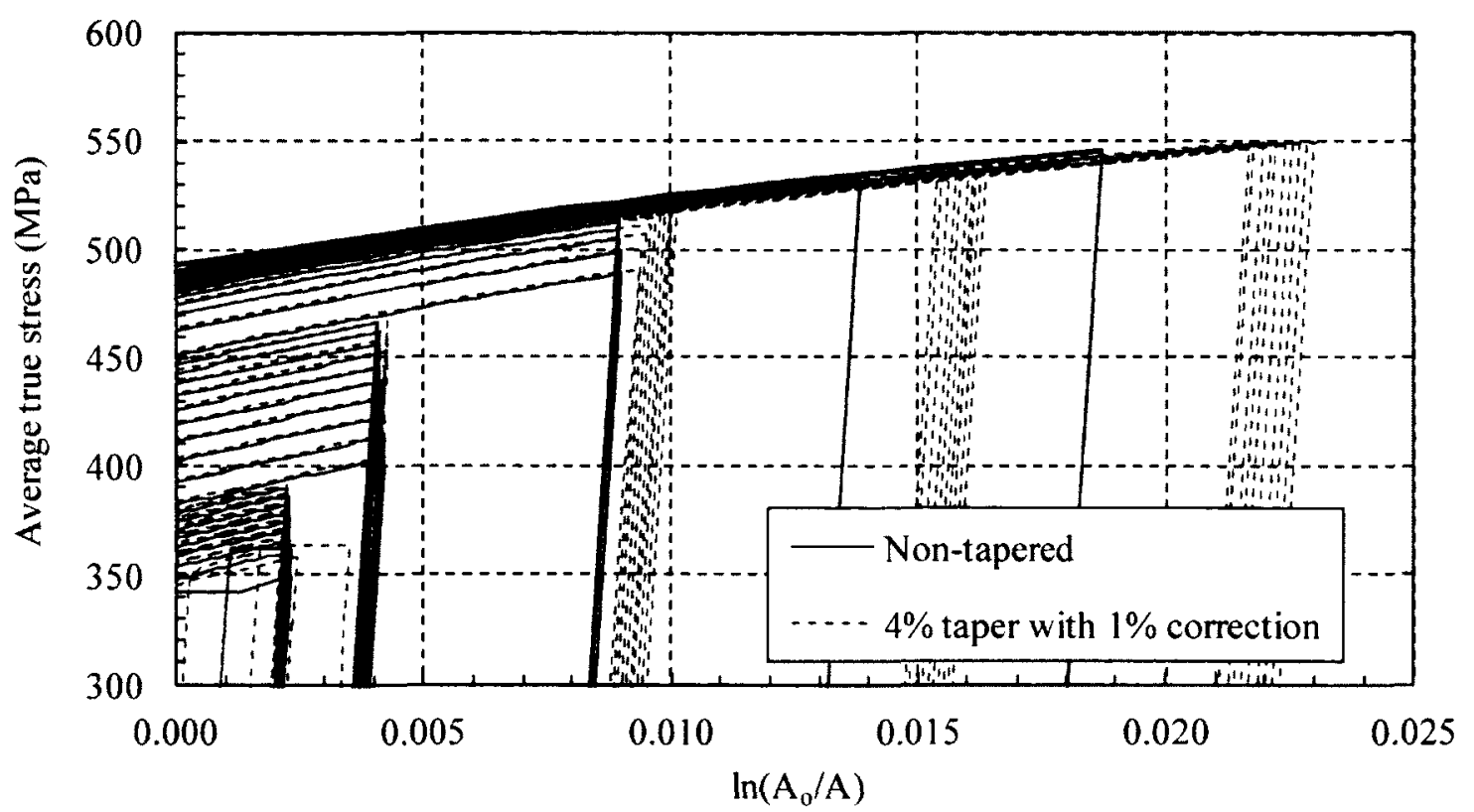

Figure D.4 Tensile average true stress versus average true strain curves for the nontapered and tapered $14 \mathrm{~mm}$ diameter specimens 


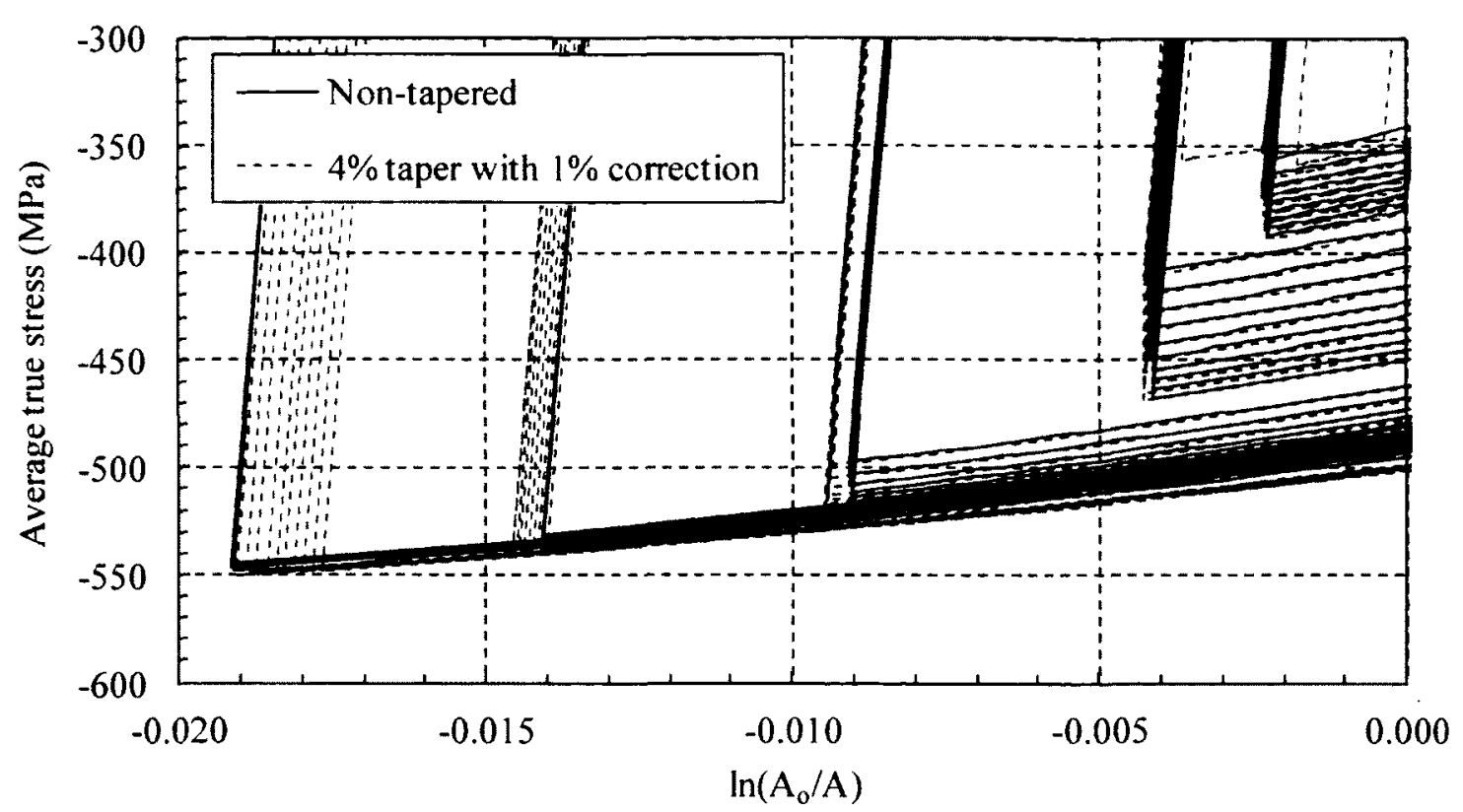

Figure D.5 Compressive average true stress versus average true strain curves for the non-tapered and tapered $14 \mathrm{~mm}$ diameter specimens

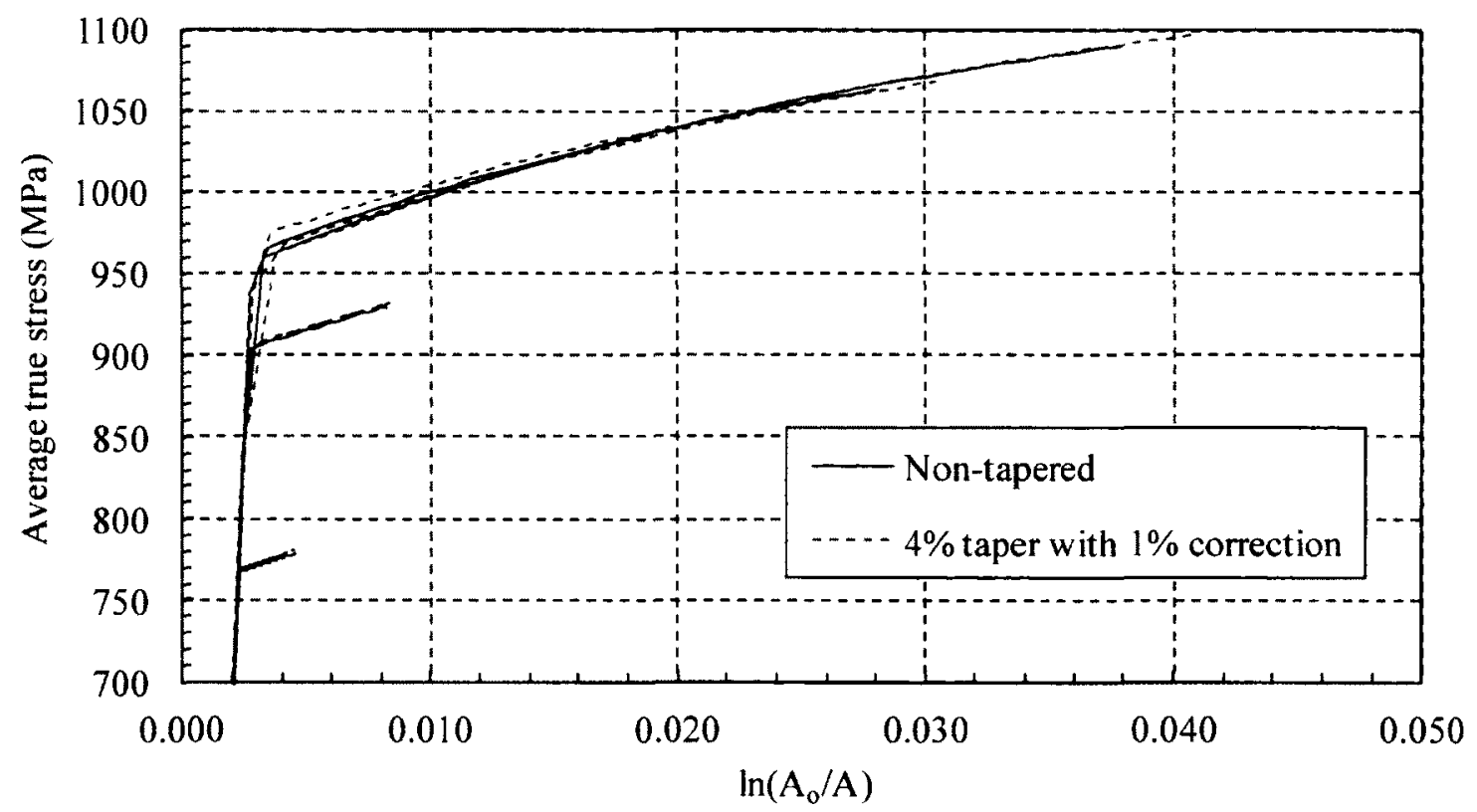

Figure D.6 Shifted average true stress versus average true strain curves at the $10^{\text {th }}$ tension half-cycle of each strain range 


\section{Appendix E Representing Strain Rate Effects on Flow Stress Using A Power Law Equation}

It has been shown by Cowper-Symonds (1957) that the effect of strain rate on the flow stress of metals can be represented by a power law equation in the form of Eq. (2.8). The power law equation gives a constant difference between values of stress at different strain rates since there is no strain term in Eq. (2.8). It can be seen in Figs. 5.1 and 5.2 that the difference between the true stress at various strain rates appears to be somewhat constant between the true plastic strains of 0.1 to 0.3 . At a true plastic strain higher than 0.3 , adiabatic heating and possible misplacement of the diametral extensometer may affect the flow stress measurement. However, at low strain rates in the absence of adiabatic heating, the difference between the peak tensile engineering stress at $10^{-4}$ and $10^{-3} \mathrm{~s}^{-1}$ strain rates for cyclic tests is maintained over a large number of loading cycles (range of cumulative plastic strain), as can be seen in Figs 4.51 and 4.52 . Therefore, it may be appropriate to try to represent the difference in the flow stress with a power law equation similar to Eq. (2.8). Test results have been used to explore the possibility of using Eq. (2.8) to represent the stress difference due to strain rate.

Values of true flow stress at a true plastic strain of 0.2 are taken from tests by Chen (2010), corrected for the tapered profile, at strain rates of $10^{-5}$ to $10^{0} \mathrm{~s}^{-1}$. Since kinematic hardening is assumed to be rate insensitive, the backstress $\left(\alpha_{\text {mono }}\right)$ can be assumed to be the same at the true plastic strain of 0.2 for all strain rates. Therefore, the flow stress difference between uniaxial monotonic tension tests of different strain rates at the same true plastic strain can be attributed to the yield stress. Assuming a trial backstress, the parameters for Eq. (2.8) can be determined by least-square error fitting through the yield stress calculated with the trial backstress. However, it has been found 
that there is very little difference in the results of the fitting over a large range of trial backstress. Therefore, a backstress $\left(\alpha_{\text {mono }}\right)$ of $200 \mathrm{MPa}$ has been taken to determine parameters of Eq. (2.8) to fit through the yield stress from monotonic tension tests for both materials $\mathrm{G}$ and $\mathrm{H}$. Parameters $\sigma_{s}, I$ and $J$ for Eq. (2.8) determined through leastsquare error fitting are listed in Table E.1. Test and predicted yield stresses are shown in Table E.2 and Fig. E.1. It can be seen in Fig. E.1 that there is an excellent agreement between the test and predicted yield stress for material $G$ and a reasonably good agreement for material $\mathrm{H}$.

Results of the cyclic tests show that values of peak tensile stress or half average stress range per cycle differ with the strain rate, even after many cycles. For material A, it can be seen in Fig E.2 that the half average true stress range difference between tests at strain rate of $10^{-3}, 10^{-2}$ and $10^{-1} \mathrm{~s}^{-1}$ of the I test series appears to have stabilized between the cumulative plastic engineering strain of 0.15 to 0.30 . For material $\mathrm{B}$, the same can be seen in Fig. E. 3 between the strain rates of $10^{-3}, 10^{-2}$ and $10^{-1} \mathrm{~s}^{-1}$ at the same cumulative plastic engineering strain range. For the $D$ test series, a near constant difference between the half average true stress range of the stabilized stress-strain curve (at the $5^{\text {th }}$ and $10^{\text {th }}$ cycle) of the $10^{-4}$ and $10^{-3} \mathrm{~s}^{-1}$ strain rate tests can be seen in Fig. E. 4 for material A and Fig. E.5 for material B. A similar trend can also be seen over a large cumulative plastic strain in the peak tensile engineering stress for the notched specimens between the $10^{-4}$ and $10^{-3} \mathrm{~s}^{-1}$ strain rate cyclic tests in Figs. 4.51 and 4.54 for materials $\mathrm{A}$ and $\mathrm{B}$ respectively up to approximately the $50^{\text {th }}$ to $60^{\text {th }}$ cycle. Therefore, it may be appropriate to attempt to represent the yield stress from the cyclic tests with Eq. (2.8), similar to that for the monotonic tests. 
The calibration of the parameters of Eq. (2.8) with the cyclic test data can be carried out using half of the average true stress range in a loading cycle, which is equivalent to the total of yield and backstress stresses at the maximum tensile applied strain. Values of half average true stress range, corrected for tapered profile, have been obtained from the I test series at a cumulative plastic engineering strain of 0.25 . For the D test series, values of corrected half average true stress range have been obtained at the $5^{\text {th }}$ cycle of the $\pm 2 \%$ loading strain range, which is at a cumulative plastic engineering strain of approximately 0.3 . These values of cumulative plastic engineering strain have been considered since they seem to represent a point in the test where the difference in the average true stress range appears to have stabilized and adiabatic heating has not significantly affected results at higher strain rates. Based on the mixed-mode hardening plasticity model by Lemaitre and Chaboche (1990) considered in this study, the saturated yield stress is assumed to be the same between monotonic and cyclic tests at the same strain rate. Thus, parameters for Eq. (2.8) are determined by fitting though the combined data of monotonic and cyclic tapered specimen tests.

For the I test series, values of (saturated) yield stress are calculated by subtracting an assumed backstress $\left(\alpha_{l}\right)$ of $100 \mathrm{MPa}$ from the values of half average true stress range for both material A and B. Similar to what has been found for the monotonic tests, changing the assumed backstress to a different reasonable value does not have a large effect on the outcome of the calibration. It can be expected that the values of backstress for the monotonic test $\left(\alpha_{\text {mono }}\right)$ and D test series $\left(\alpha_{D}\right)$ are different from that of the I test series due to differences in the loading. Therefore, $\alpha_{\text {mono }}$ and $\alpha_{D}$ have not be predefined in the calibration to have the same value as $\alpha_{I}$ but are instead allowed to vary with the 
parameters $\sigma_{s}, I$ and $J$ of Eq. (2.8) such that a minimum error could be obtained during least-square error fitting. Parameters $\sigma_{s}, I$ and $J$ for Eq. (2.8) along with $\alpha_{D}$ and $\alpha_{m o n o}$ determined through least-square error fitting of the cyclic and monotonic test data are listed in Table E.3. Test and predicted yield stresses are shown in Table E.4 and Fig. E.6. Again, it can be seen in Fig. E. 6 that there is a good agreement between the test and predicted yield stress for material A and a reasonably good agreement for material B. 
Table E.1 Parameters for Eq. (2.8) calibrated with monotonic test data and the assumed backstress

\begin{tabular}{|c|c|c|}
\hline & Material G & Material H \\
\hline $\mathrm{I}\left(\mathrm{s}^{-1}\right)$ & $6.032 \mathrm{E}+06$ & 4411 \\
\hline $\mathrm{J}$ & 9.78 & 4.64 \\
\hline$\sigma_{S}(\mathrm{MPa})$ & 423.0 & 379.3 \\
\hline$\alpha_{\text {mono }}(\mathrm{MPa})$ & 200.0 & 200.0 \\
\hline
\end{tabular}

Table E.2 Test and predicted yield stresses for monotonic tests of Chen (2010) calculated using Eq. (2.8) with calibrated parameters in Table E.1

\begin{tabular}{|c|c|c|c|c|c|c|}
\hline & \multicolumn{6}{|c|}{ Yield Stress (MPa) } \\
\cline { 2 - 7 } & \multicolumn{3}{|c|}{ Material A } & \multicolumn{3}{c|}{ Material B } \\
\hline $\begin{array}{c}\text { Strain rate } \\
\left(\mathrm{s}^{-1}\right)\end{array}$ & $\begin{array}{c}\text { Difference } \\
\text { with } \\
10^{-5} \mathrm{~s}^{-1} \\
\text { test data }\end{array}$ & Test & $\begin{array}{c}\text { Eq. } \\
(2.8)\end{array}$ & $\begin{array}{c}\text { Difference } \\
\text { with } \\
10^{-5} \mathrm{~s}^{-1} \\
\text { test data }\end{array}$ & Test & $\begin{array}{c}\text { Eq. } \\
(2.8)\end{array}$ \\
\hline 0.00001 & 0.0 & 450.2 & 449.5 & 0.0 & 488.5 & 484.5 \\
\hline 0.00005 & 3.3 & 453.4 & 454.2 & -6.9 & 481.5 & 486.6 \\
\hline 0.0001 & 6.4 & 456.6 & 456.5 & -3.8 & 484.7 & 487.8 \\
\hline 0.001 & 14.2 & 464.4 & 465.4 & 11.1 & 499.6 & 493.3 \\
\hline 0.01 & 27.5 & 477.7 & 476.6 & 12.1 & 500.5 & 502.3 \\
\hline 0.1 & 40.5 & 490.7 & 490.8 & 28.2 & 516.7 & 517.1 \\
\hline 1.0 & 58.5 & 508.7 & 508.8 & 53.2 & 541.6 & 541.4 \\
\hline
\end{tabular}

Table E.3 Parameters for Eq. (2.8) and backstresses calibrated with cyclic and monotonic test data with the assumed backstress $\left(\alpha_{l}\right)$

\begin{tabular}{|c|c|c|}
\hline & Material A & Material B \\
\hline $\mathrm{I}\left(\mathrm{s}^{-1}\right)$ & 45730 & 1585 \\
\hline $\mathrm{J}$ & 8.56 & 3.96 \\
\hline$\sigma_{S}(\mathrm{MPa})$ & 255.6 & 370.4 \\
\hline$\alpha_{D}(\mathrm{MPa})$ & 201.8 & 178.0 \\
\hline$\alpha_{\text {mono }}(\mathrm{MPa})$ & 370.6 & 305.6 \\
\hline$\alpha_{I}(\mathrm{MPa})$ & 100.0 & 100.0 \\
\hline
\end{tabular}


Table E.4 Test and predicted yield stresses from the monotonic (Chen 2010) and cyclic tests and calculated using Eq. (2.8) with the calibrated parameters and backstresses in Table E. 3

\begin{tabular}{|c|c|c|c|c|c|c|c|c|c|c|}
\hline \multirow{3}{*}{$\begin{array}{c}\text { Strain } \\
\text { rate } \\
\left(\mathrm{s}^{-1}\right)\end{array}$} & \multicolumn{10}{|c|}{ Yield Stress (MPa) } \\
\hline & \multicolumn{5}{|c|}{ Material A } & \multicolumn{5}{|c|}{ Material B } \\
\hline & $\begin{array}{c}\text { Difference } \\
\text { with } \\
5 \times 10^{-5} \mathrm{~s}^{-1} \\
\text { from Eq. }(2.8)\end{array}$ & Monotonic & $\begin{array}{l}\text { I test } \\
\text { series }\end{array}$ & $\begin{array}{l}D \text { test } \\
\text { series }\end{array}$ & $\begin{array}{l}\mathrm{Eq} . \\
(2.8)\end{array}$ & $\begin{array}{c}\text { Difference } \\
\text { with } \\
5 \times 10^{-5} \mathrm{~s}^{-1} \\
\text { from } \mathrm{Eq} .(2.8)\end{array}$ & Monotonic & $\begin{array}{l}\text { I test } \\
\text { series }\end{array}$ & $\begin{array}{l}D \text { test } \\
\text { series }\end{array}$ & $\begin{array}{l}\text { Eq. } \\
(2.8)\end{array}$ \\
\hline 0.00001 & -3.9 & 273.1 & - & - & 274.7 & -1.6 & 375.9 & - & - & 373.6 \\
\hline 0.00005 & 0.0 & 276.3 & - & - & 278.6 & 0.0 & 369.1 & - & - & 375.1 \\
\hline 0.0001 & 1.9 & 279.4 & - & 285.2 & 280.5 & 0.9 & 372.2 & - & 377.9 & 376.0 \\
\hline 0.001 & 9.6 & 287.2 & 292.0 & 286.4 & 288.2 & 5.4 & 386.9 & 379.7 & 385.1 & 380.5 \\
\hline 0.01 & 19.7 & 300.3 & 297.5 & 300.3 & 298.3 & 13.3 & 387.9 & 383.6 & 389.4 & 388.5 \\
\hline 0.1 & 32.8 & 313.1 & 308.4 & 306.5 & 311.4 & 27.5 & 403.9 & 408.4 & 395.3 & 402.7 \\
\hline 1.0 & 50.0 & 331.0 & - & - & 328.6 & 53.0 & 428.6 & - & - & 428.1 \\
\hline
\end{tabular}




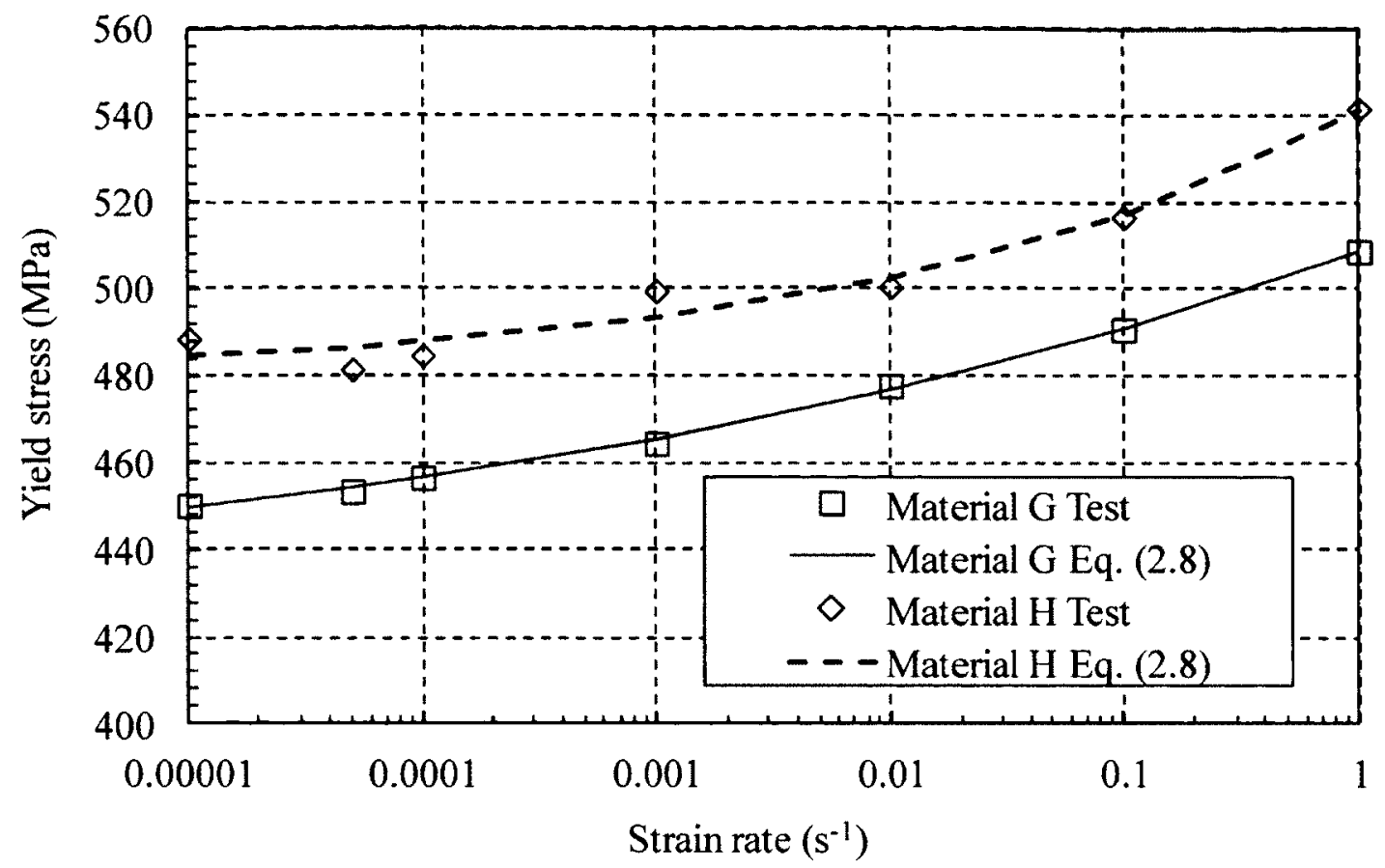

Figure E.1 Test and predicted yield stress versus strain rate for the monotonic tests by Chen (2010) at a true plastic strain of 0.2 with a backstress of $200 \mathrm{MPa}$

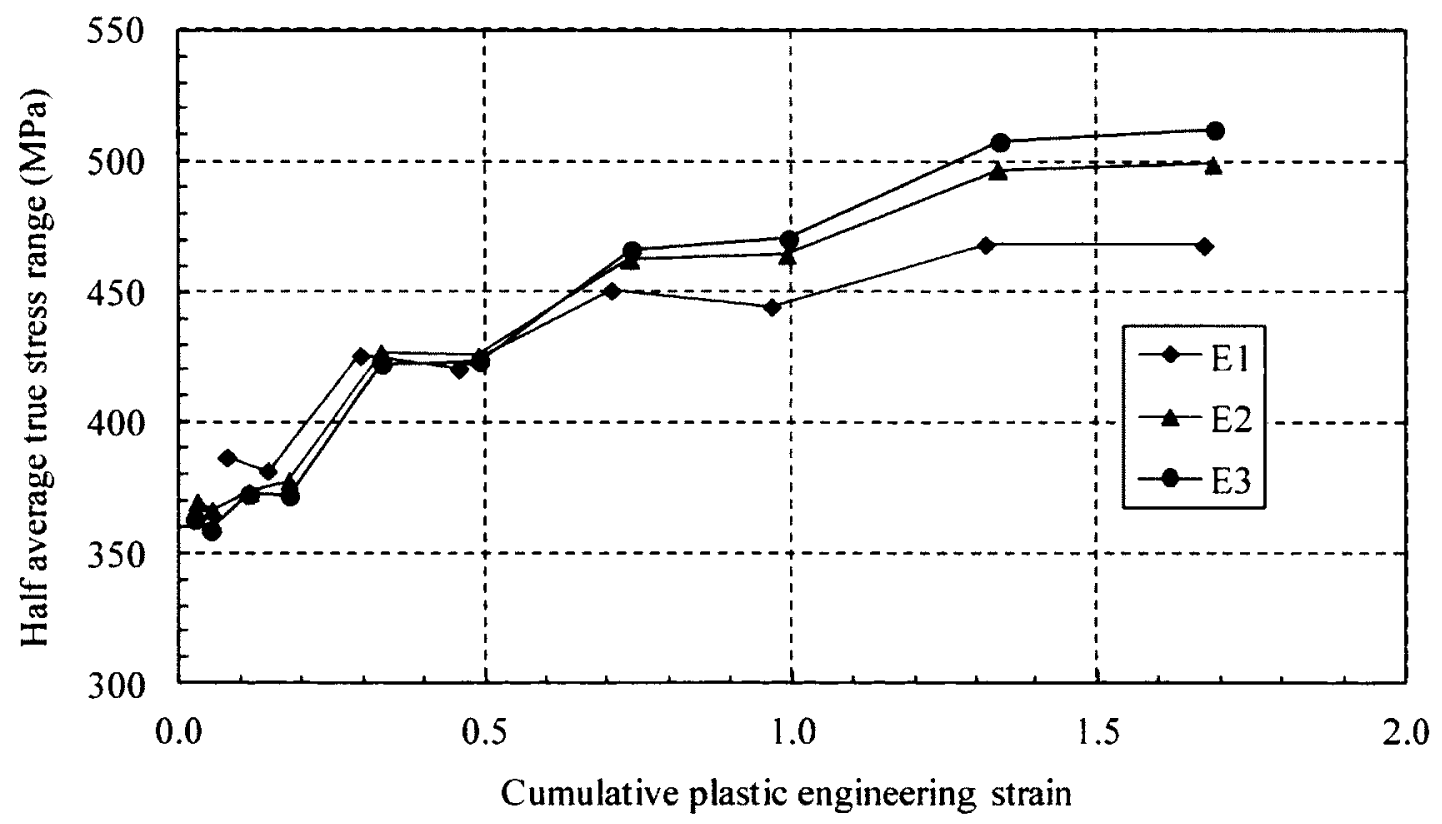

Figure E.2 Half average true stress range versus cumulative plastic engineering strain at the $5^{\text {th }}$ and $10^{\text {th }}$ cycle of each strain range for ATEIs 


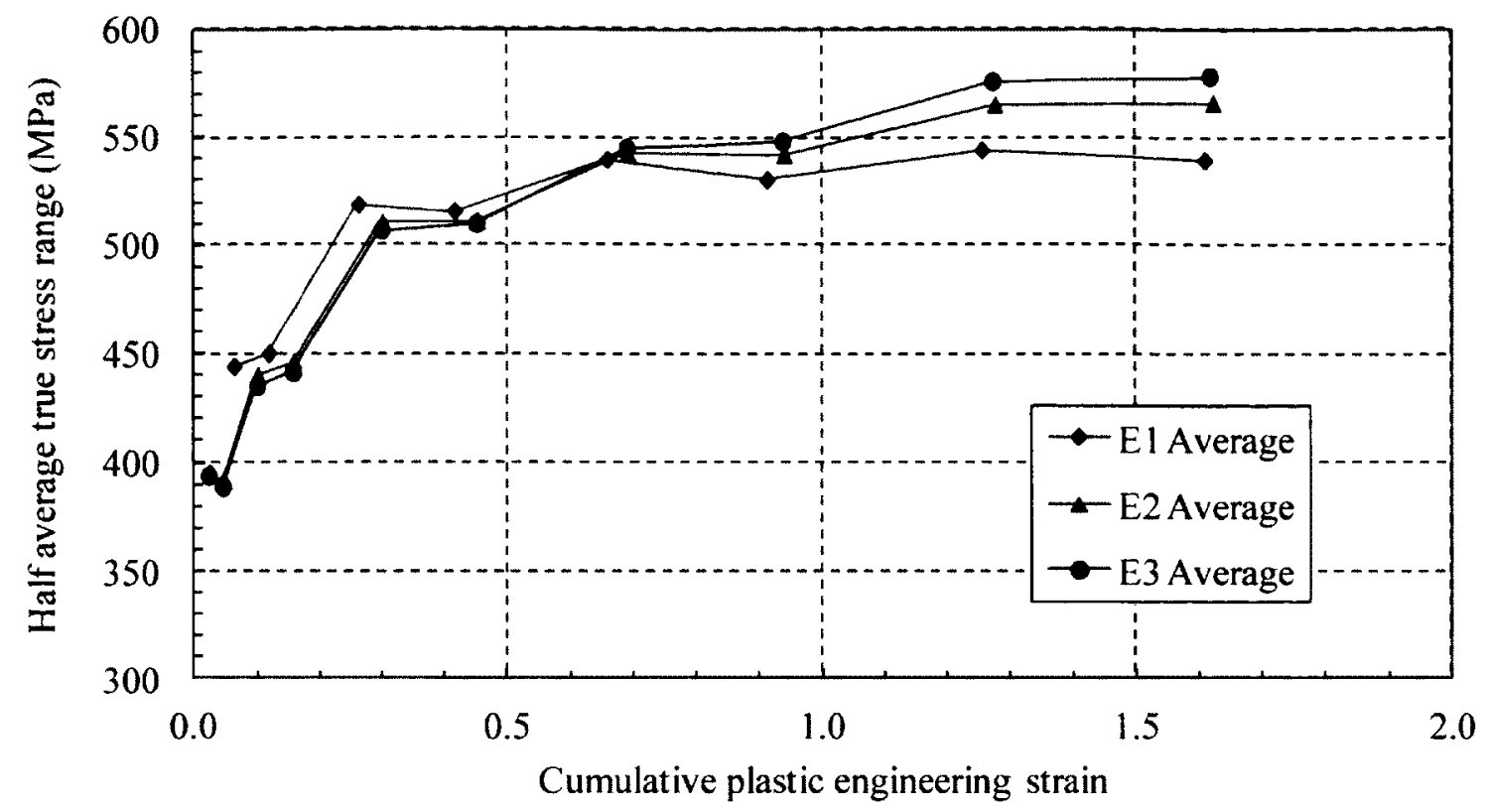

Figure E.3 Half average true stress range versus cumulative plastic engineering strain at the $5^{\text {th }}$ and $10^{\text {th }}$ cycle of each strain range for BTEIs

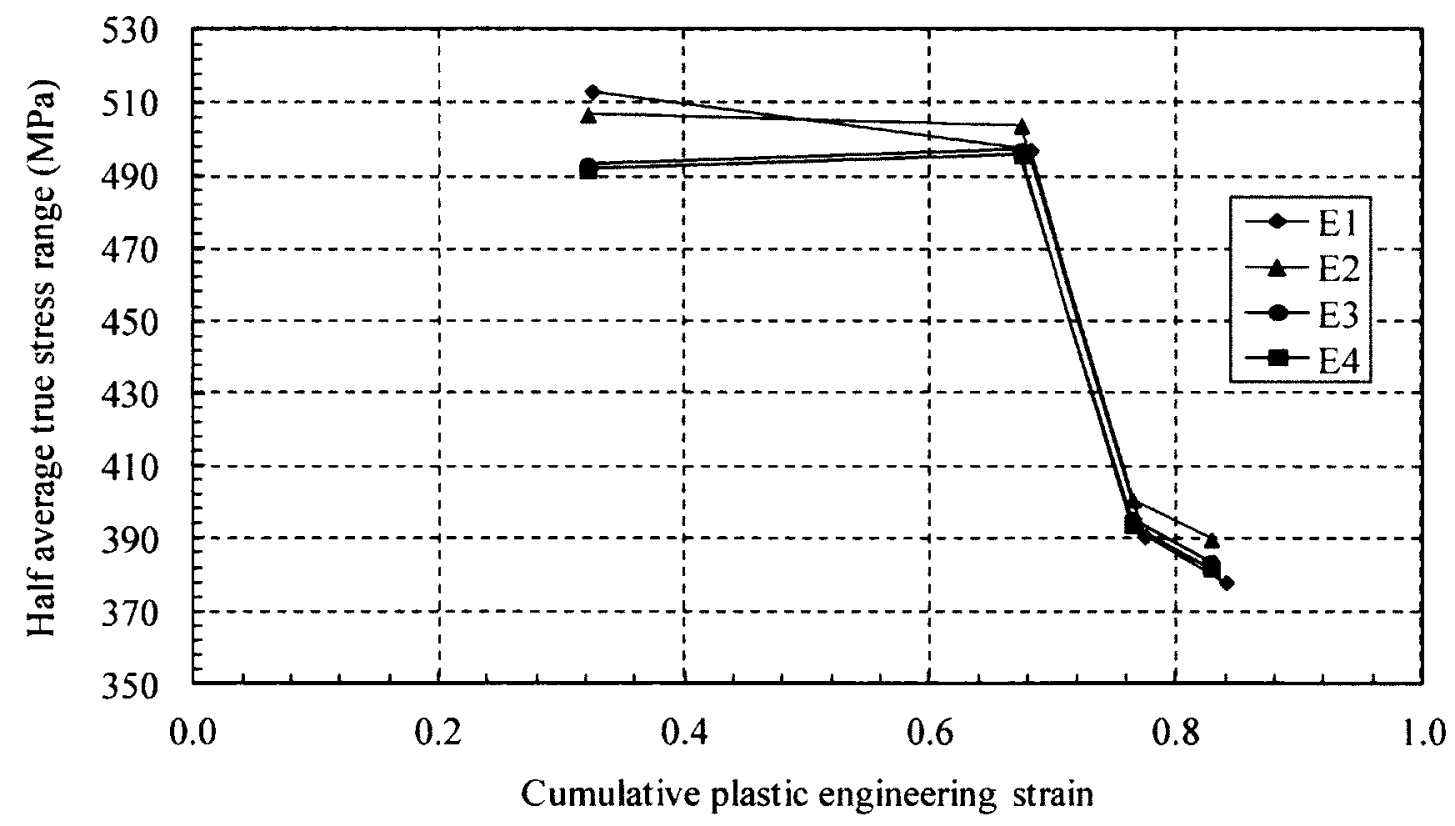

Figure E.4 Half average true stress range versus cumulative plastic engineering strain at the $5^{\text {th }}$ and $10^{\text {th }}$ cycle of each strain range for ATEDs 


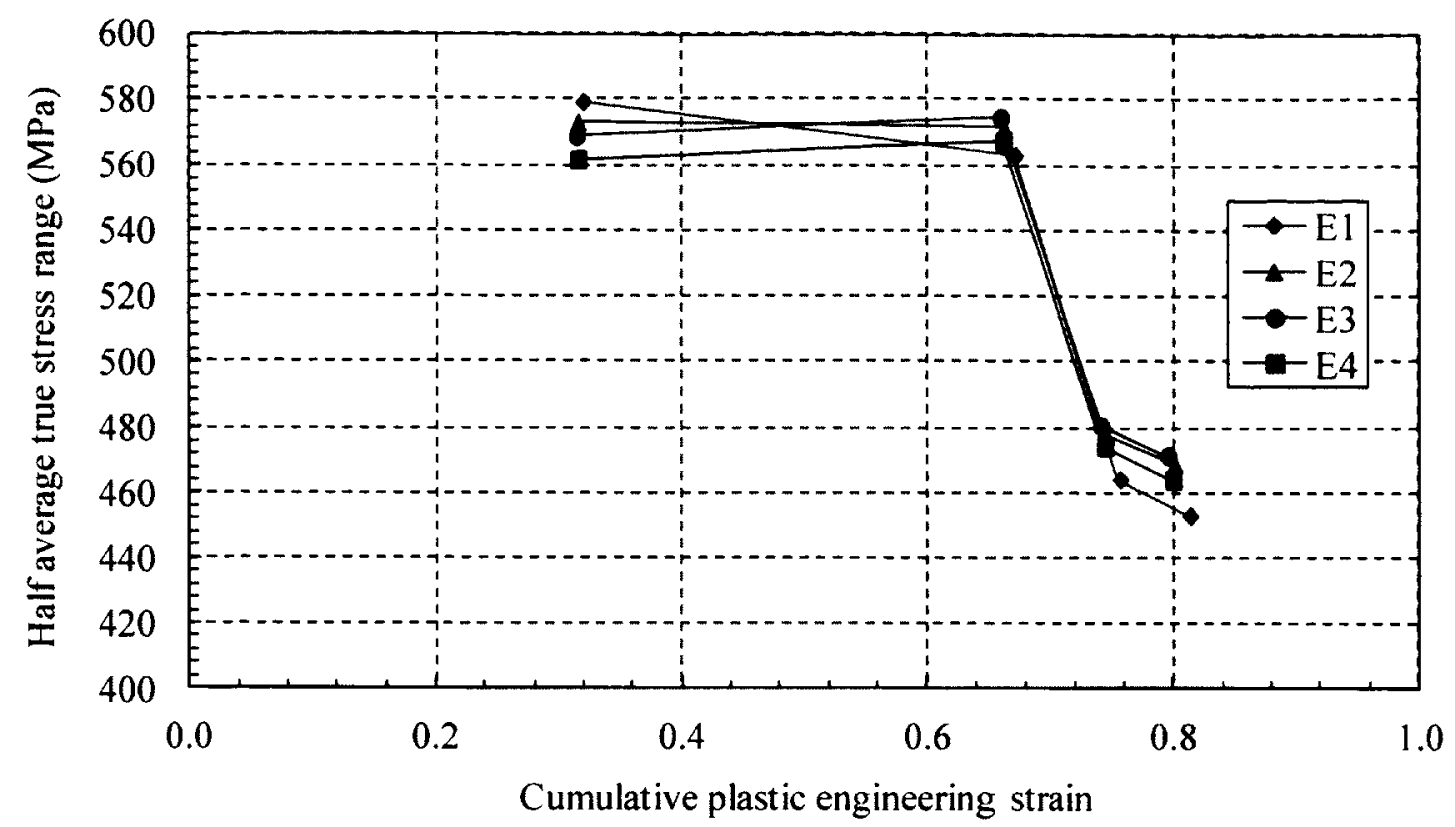

Figure E.5 Half average true stress range versus cumulative plastic engineering strain at the $5^{\text {th }}$ and $10^{\text {th }}$ cycle of each strain range for BTEDs

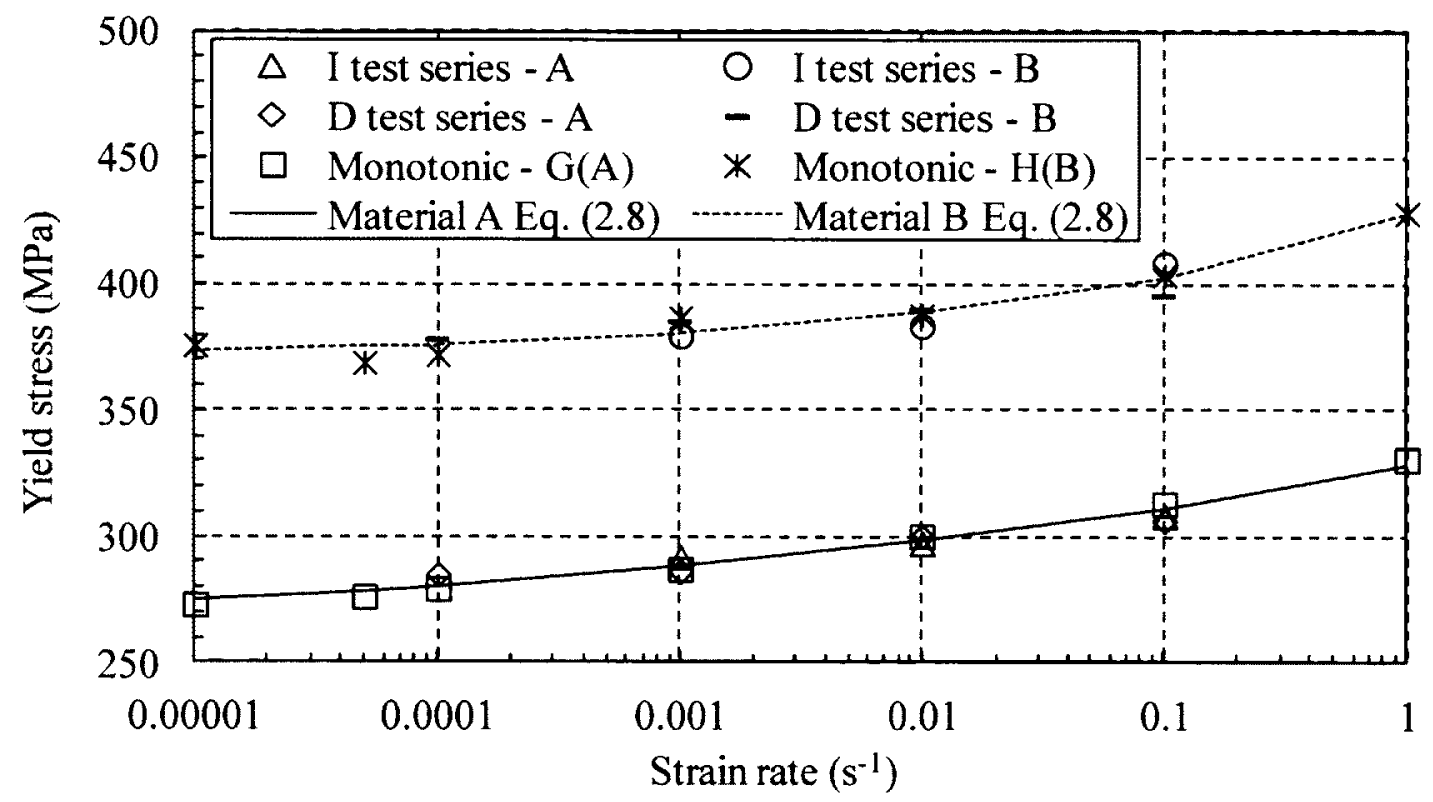

Figure E.6 Test and predicted yield stress versus strain rate for the combined monotonic and cyclic tests of tapered specimens with $\alpha_{l}=100 \mathrm{MPa}$ 


\section{Appendix F Additional Results of Numerical Simulations}

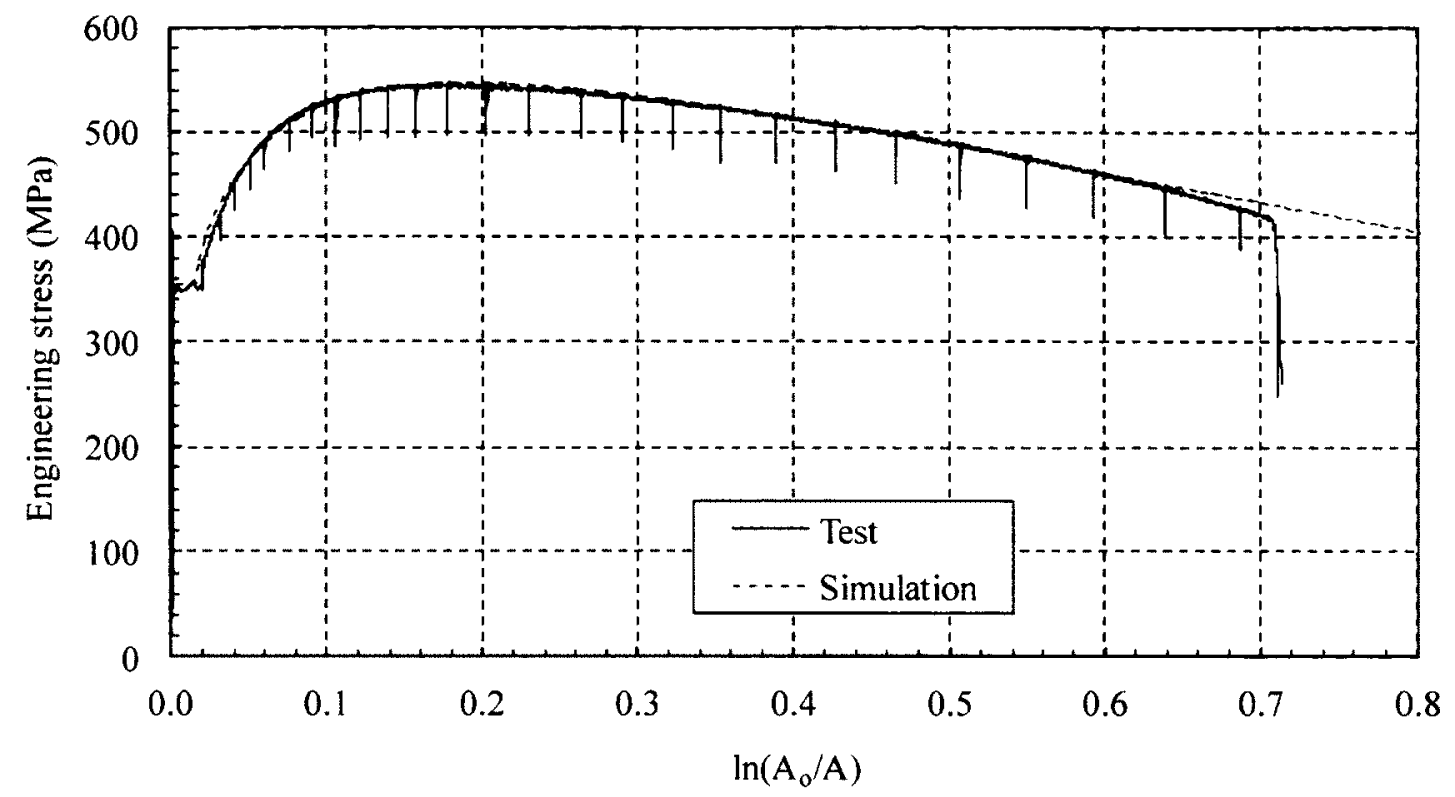

Figure F.1 Measured and predicted engineering stress versus cross section ratio curves for GTIE4ED

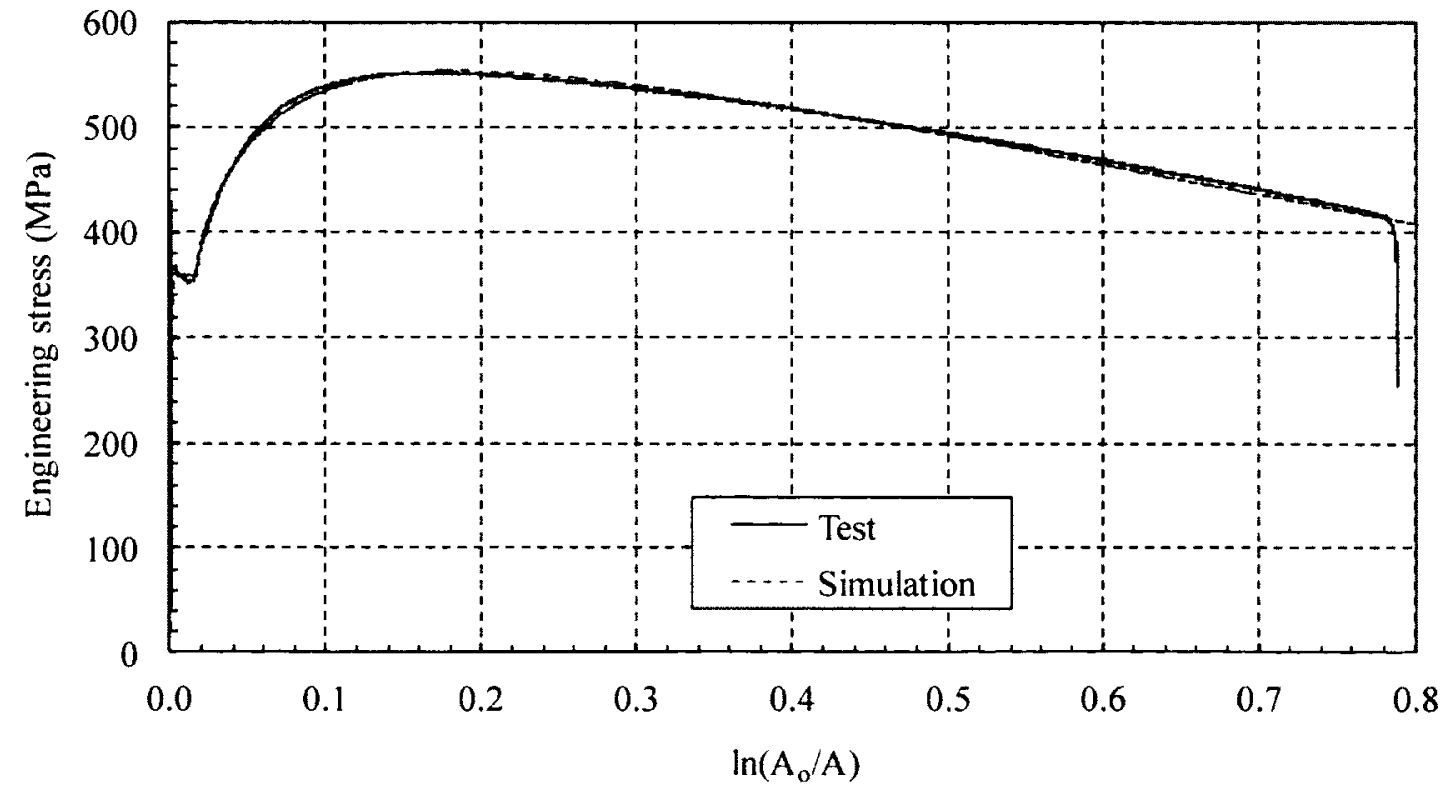

Figure F.2 Measured and predicted engineering stress versus cross section ratio curves for GT1E3ED 


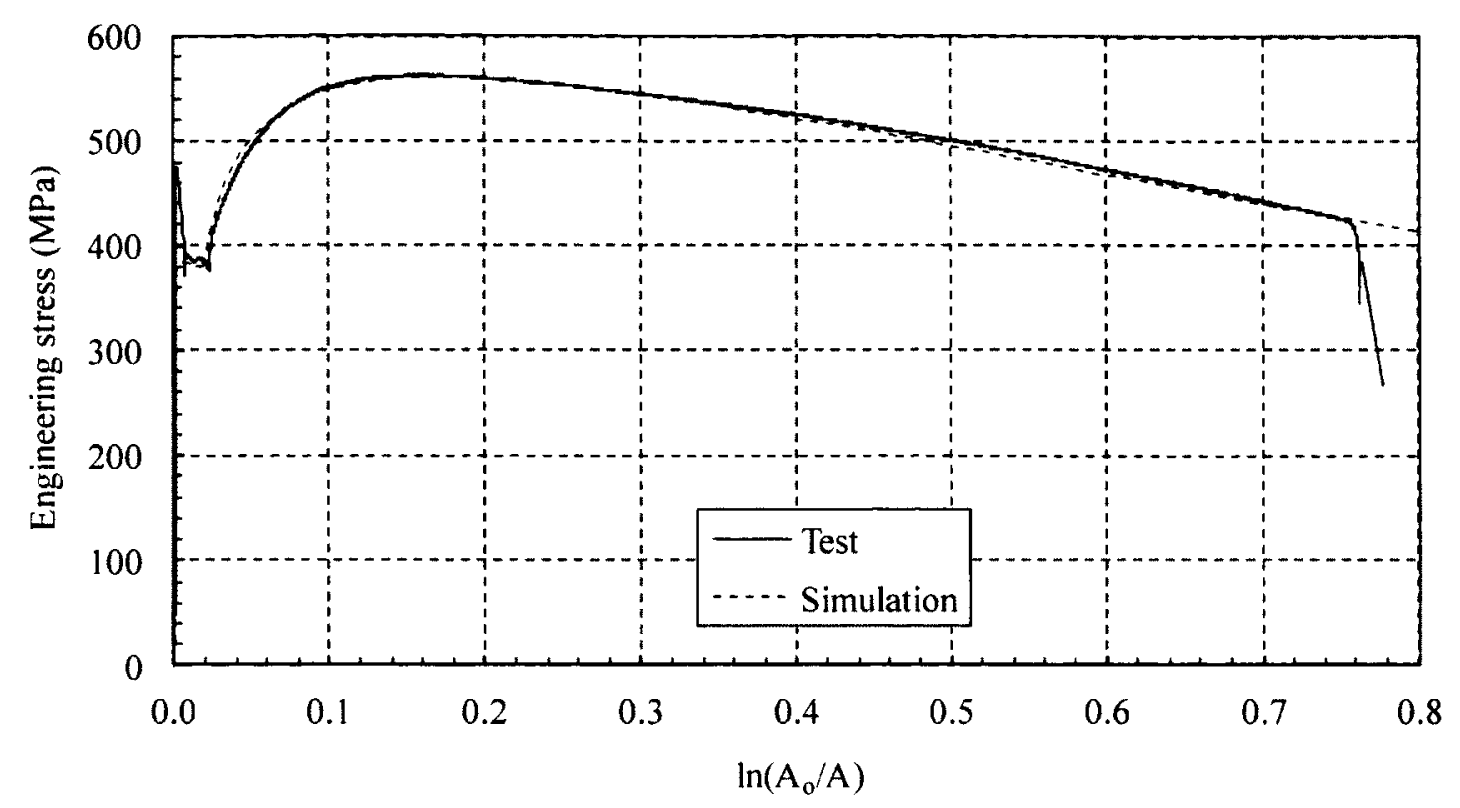

Figure F.3 Measured and predicted engineering stress versus cross section ratio curves for GT1E2ED

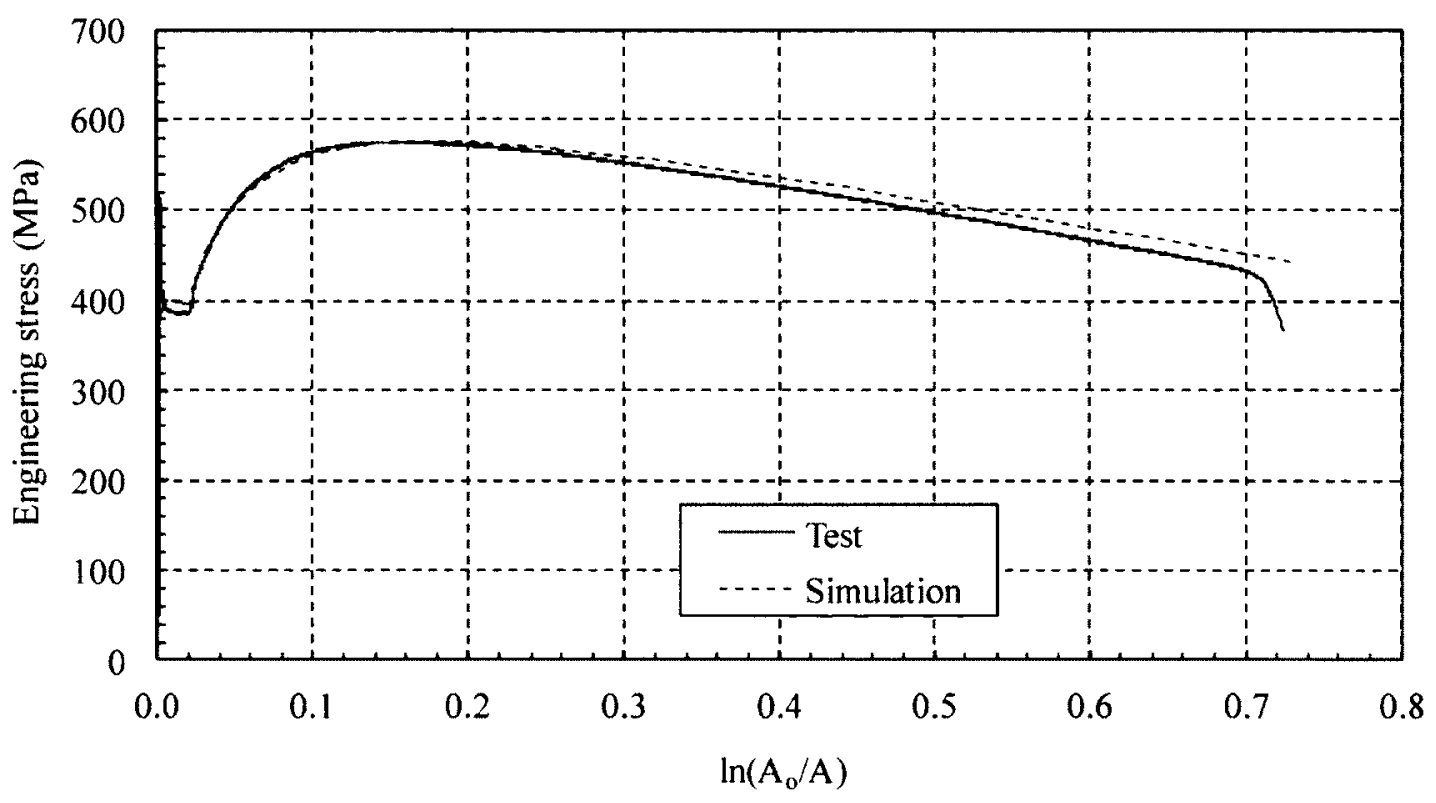

Figure F.4 Measured and predicted engineering stress versus cross section ratio curves for GT1E1E 


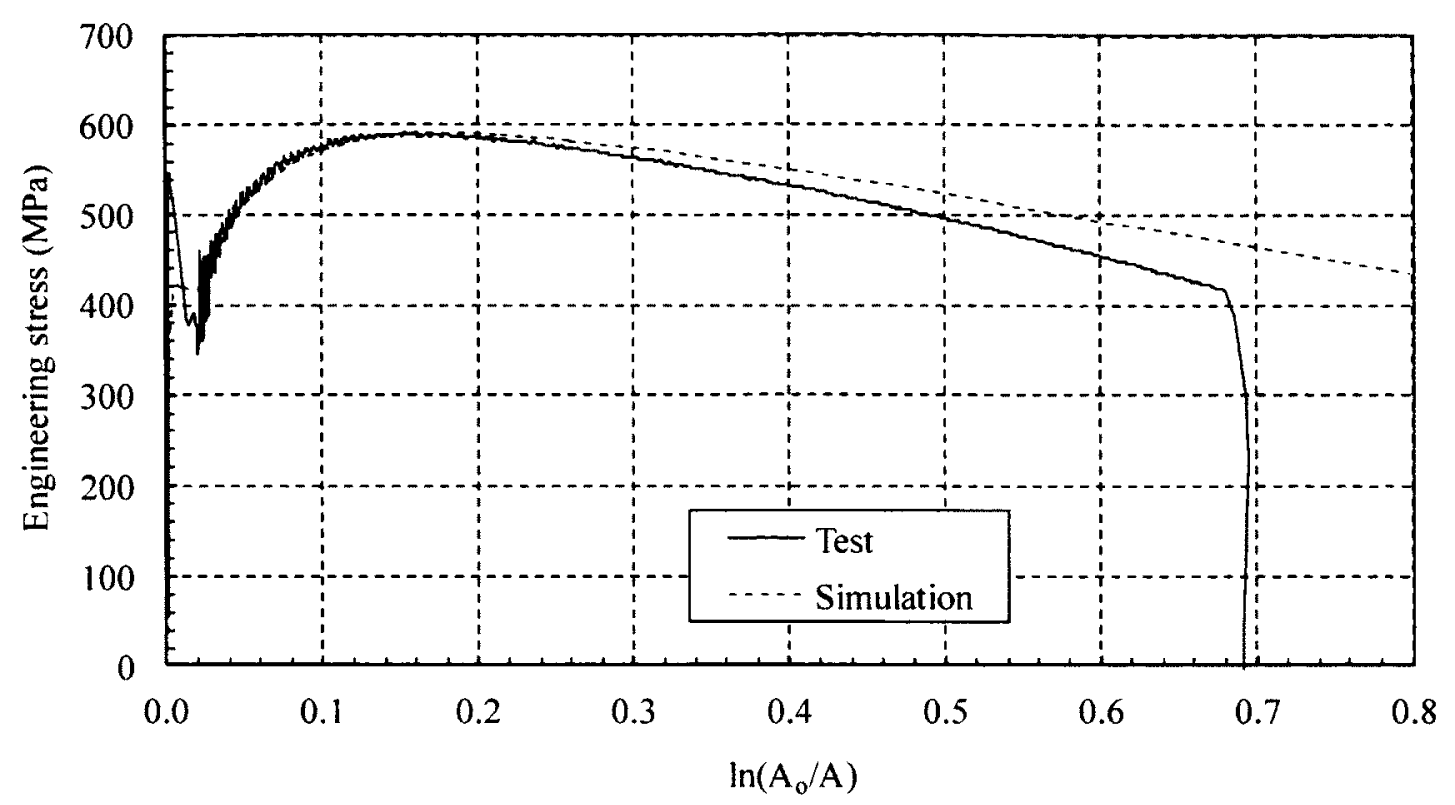

Figure F.5 Measured and predicted engineering stress versus cross section ratio curves for GT1E0E

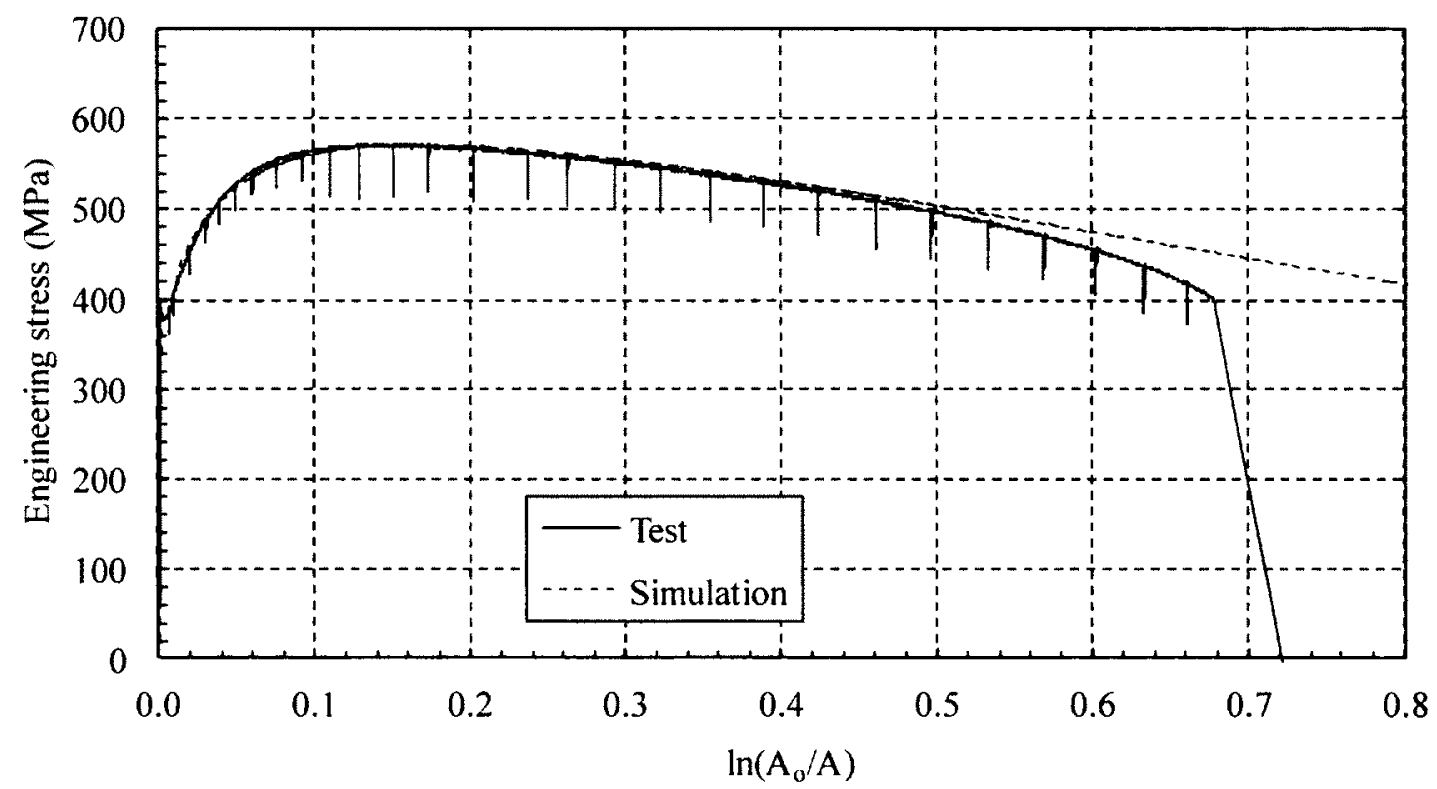

Figure F.6 Measured and predicted engineering stress versus cross section ratio curves for HT1E4ED 


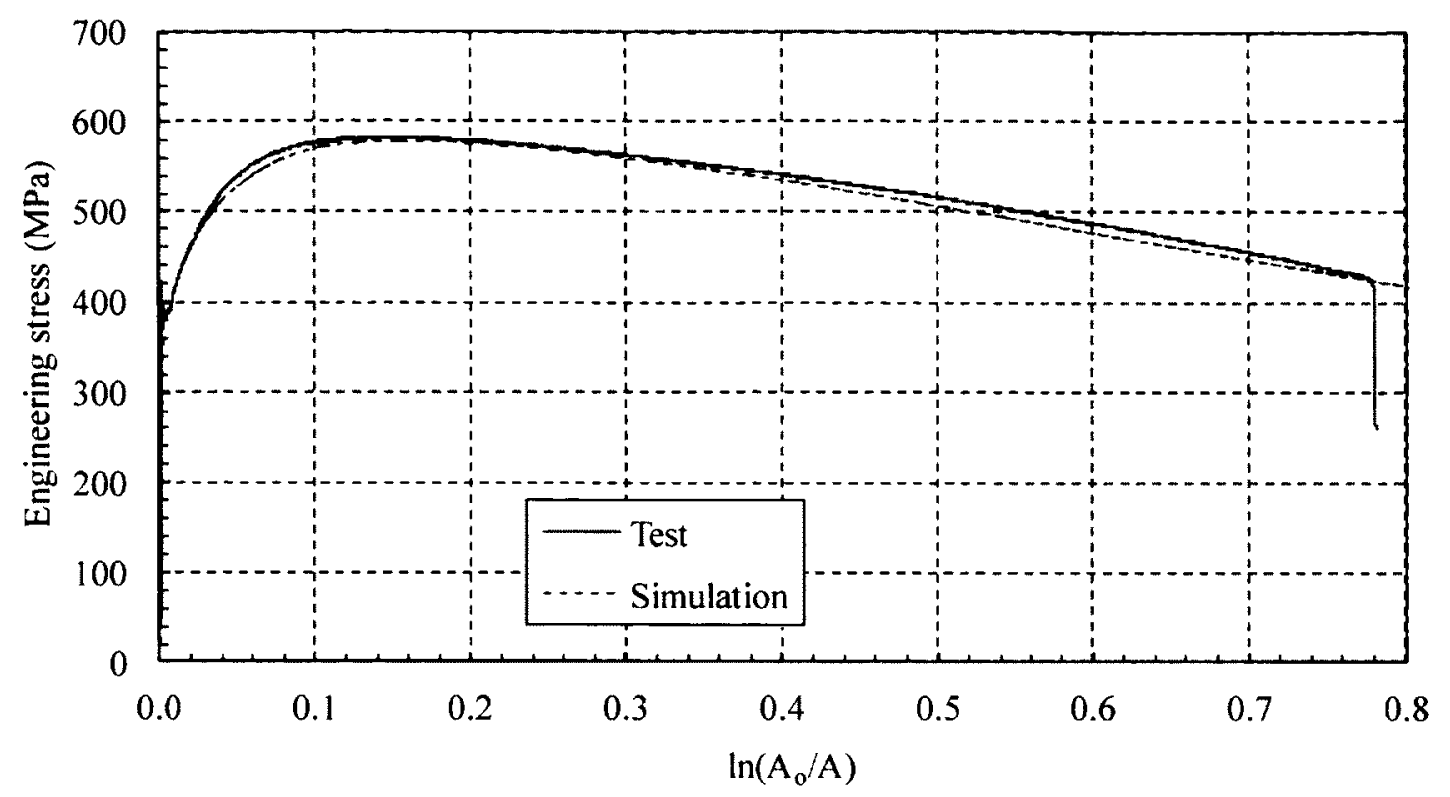

Figure F.7 Measured and predicted engineering stress versus cross section ratio curves for HT1E3ED

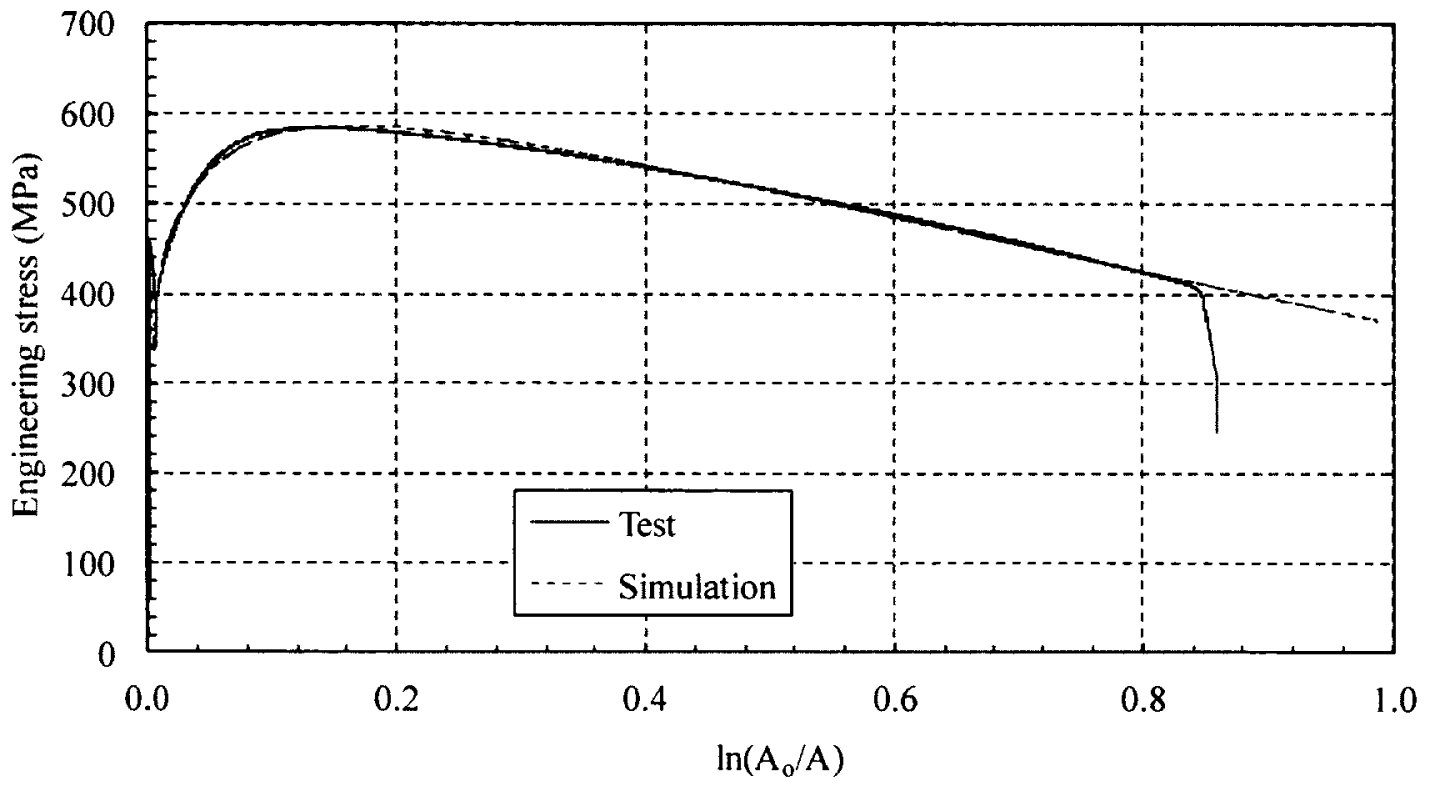

Figure F.8 Measured and predicted engineering stress versus cross section ratio curves for HT1E2ED 


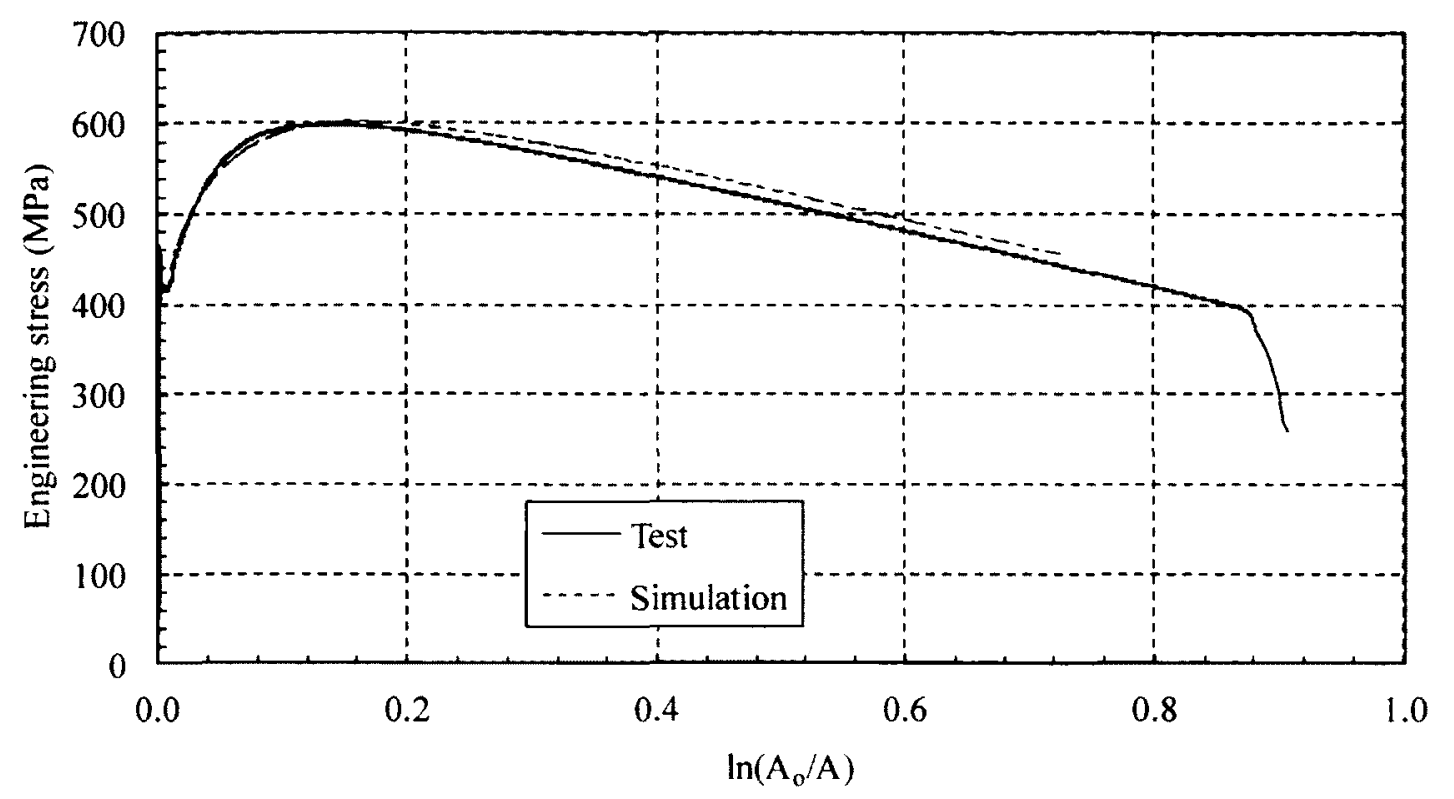

Figure F.9 Measured and predicted engineering stress versus cross section ratio curves for HTIE1E

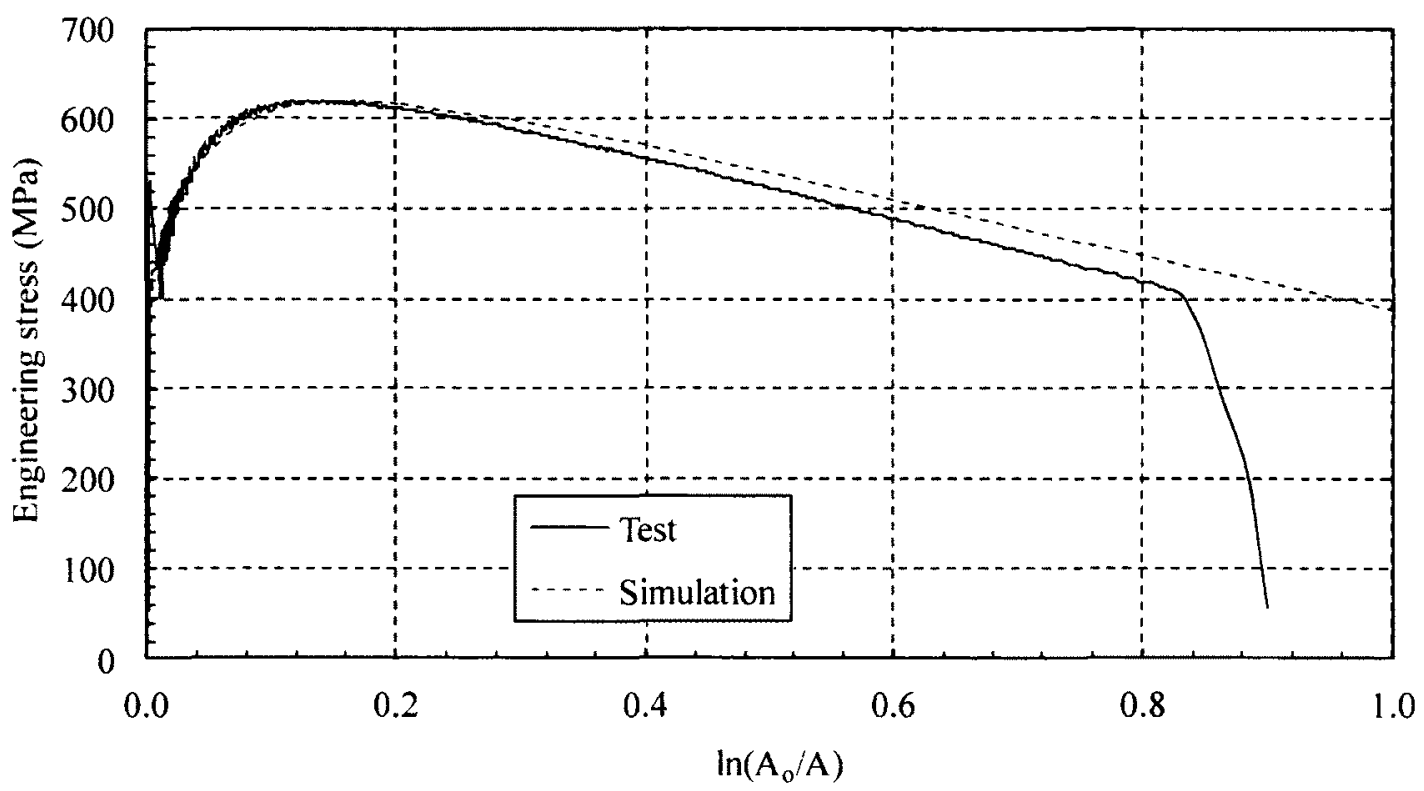

Figure F.10 Measured and predicted engineering stress versus cross section ratio curves for HT1E0E 


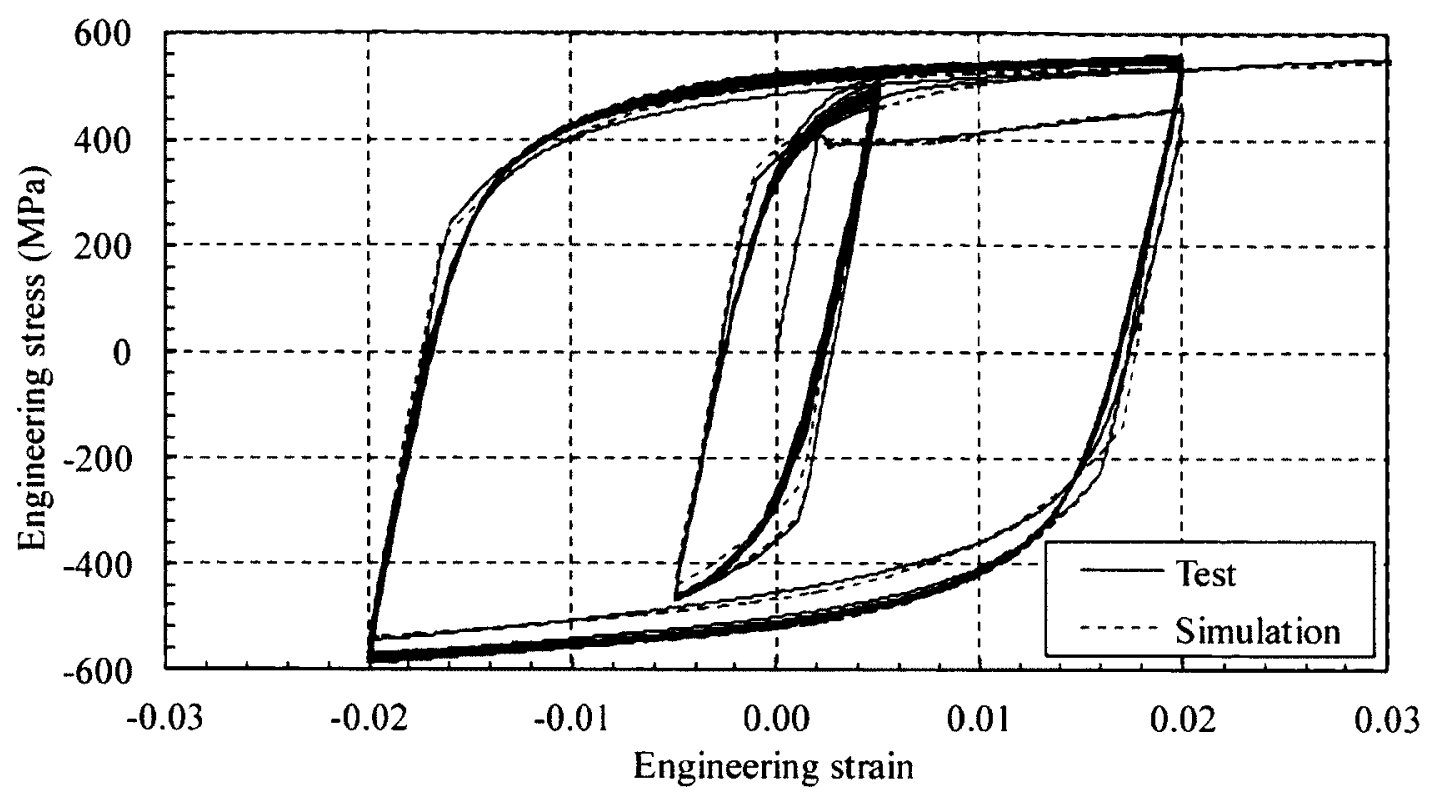

Figure F.11 Test and predicted engineering stress versus engineering strain curves for the cyclic portion of BTE3D

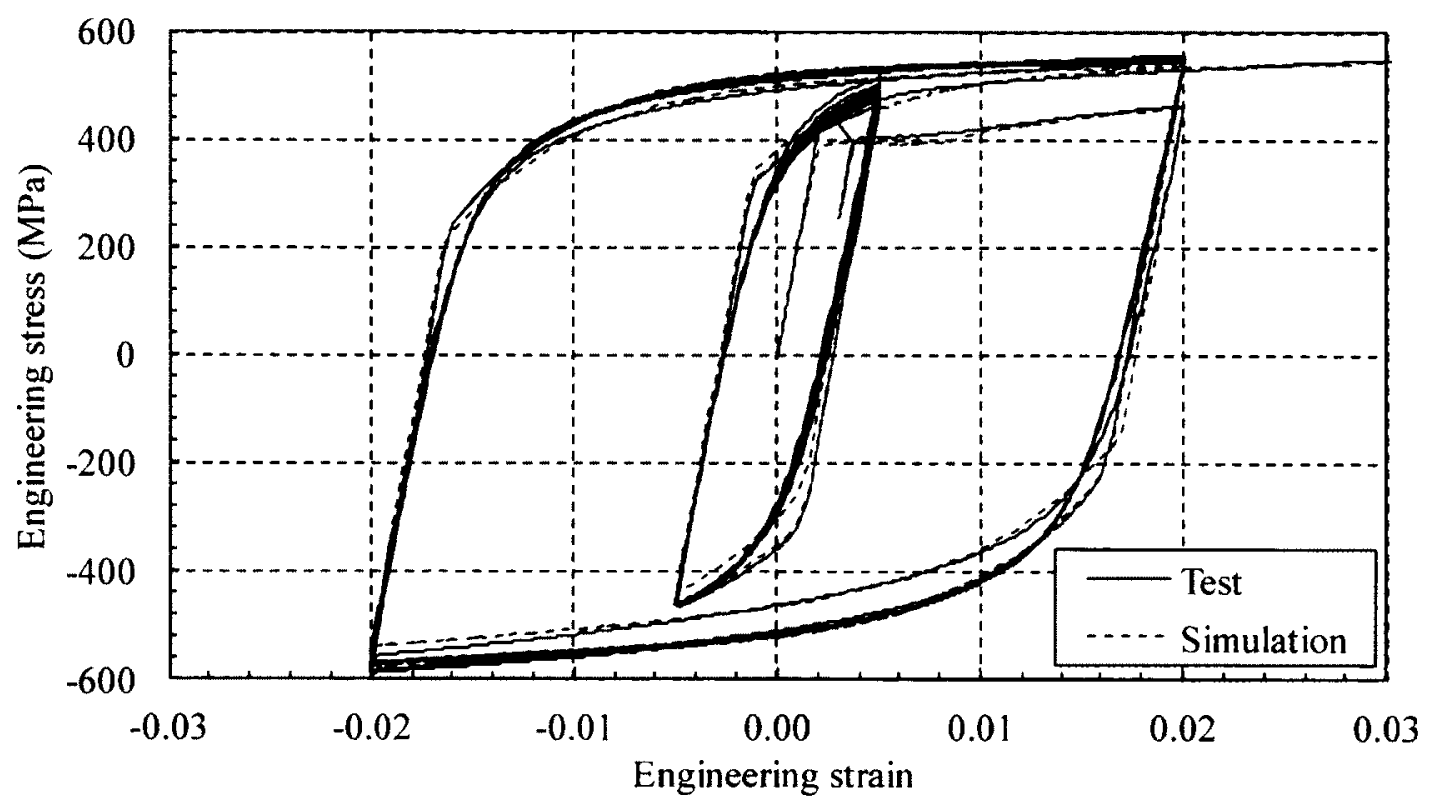

Figure F.12 Test and predicted engineering stress versus engineering strain curves for the cyclic portion of BTE2D 


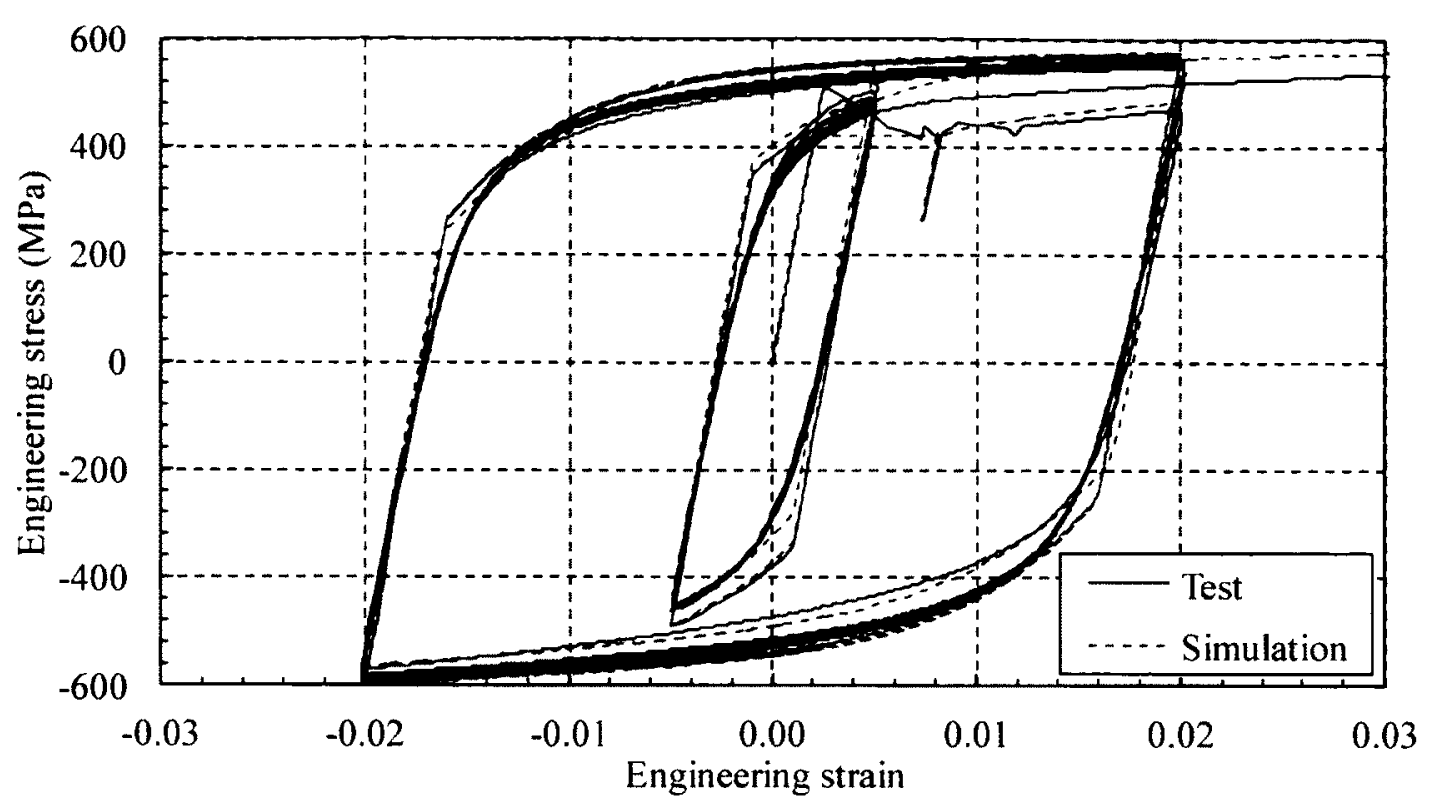

Figure F.13 Test and predicted engineering stress versus engineering strain curves for the cyclic portion of BTE1D

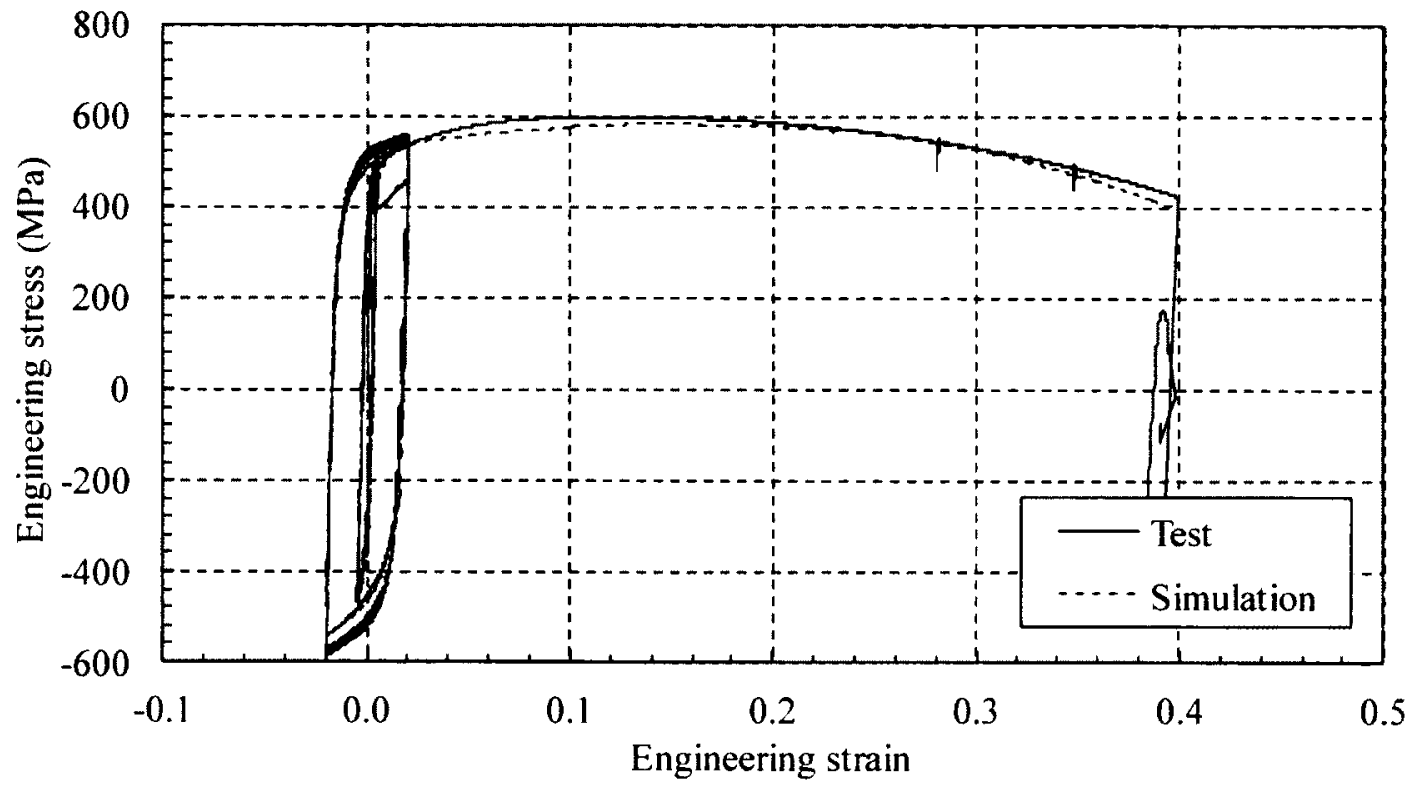

Figure F.14 Test and predicted engineering stress versus engineering strain curves of BTE3D 


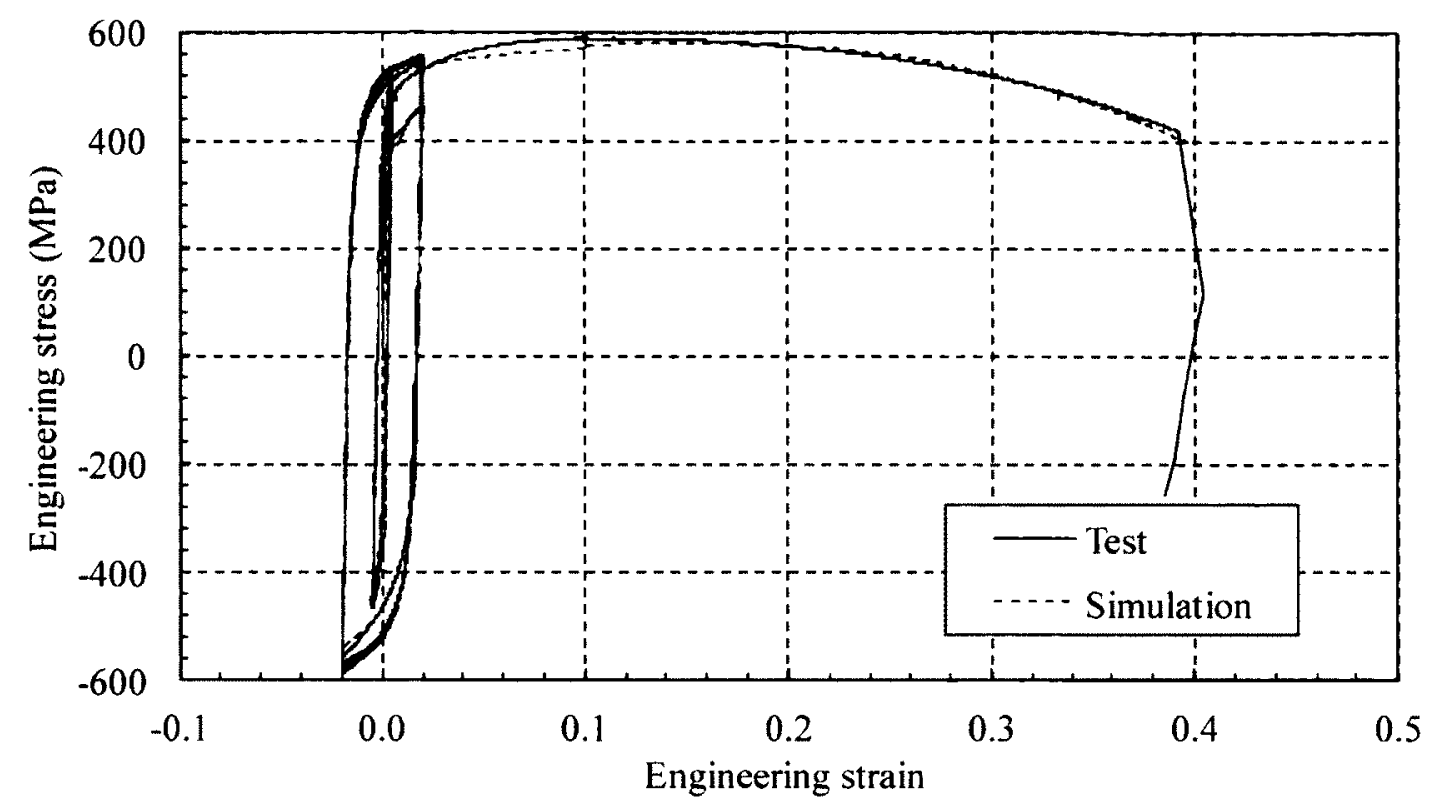

Figure F.15 Test and predicted engineering stress versus engineering strain curves of BTE2D

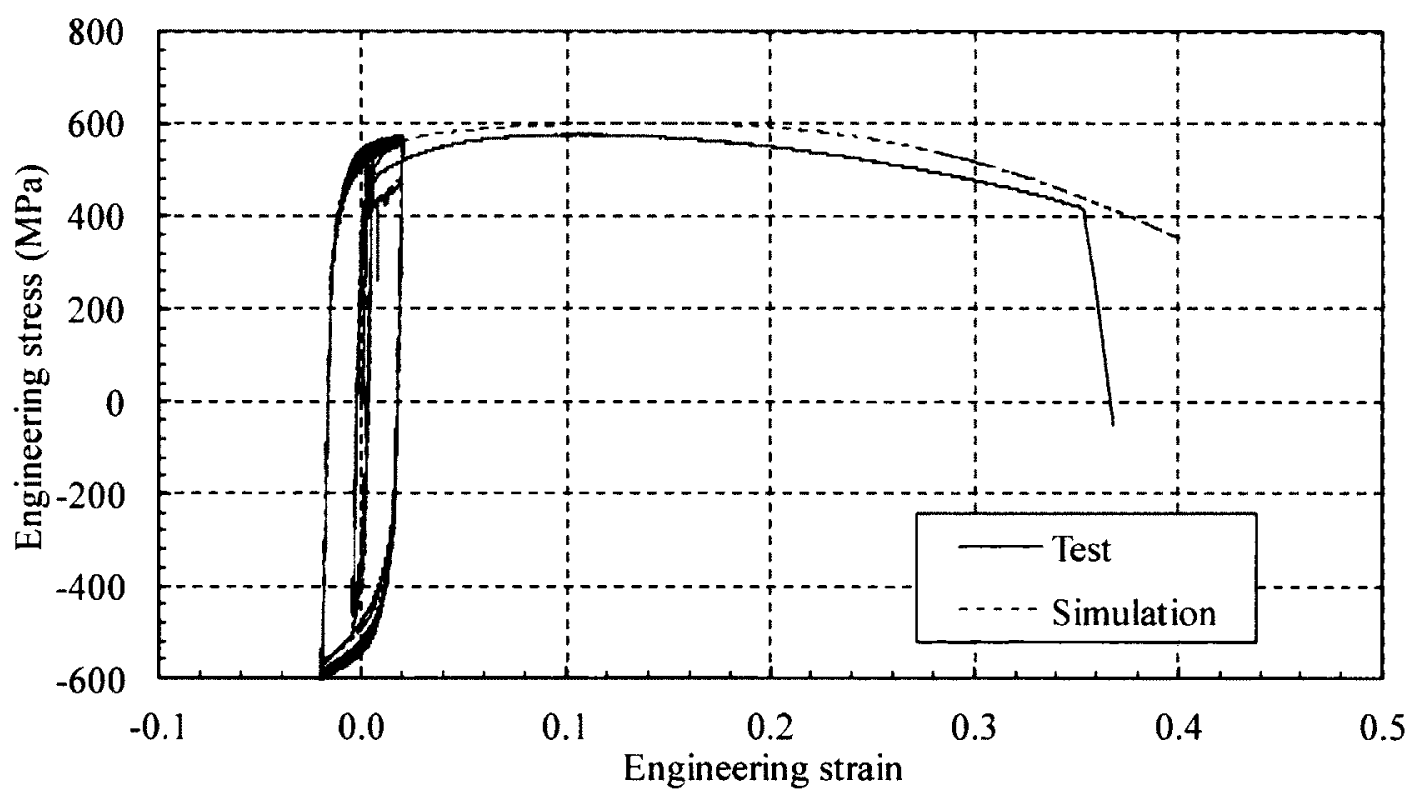

Figure F.16 Test and predicted engineering stress versus engineering strain curves of BTE1D 


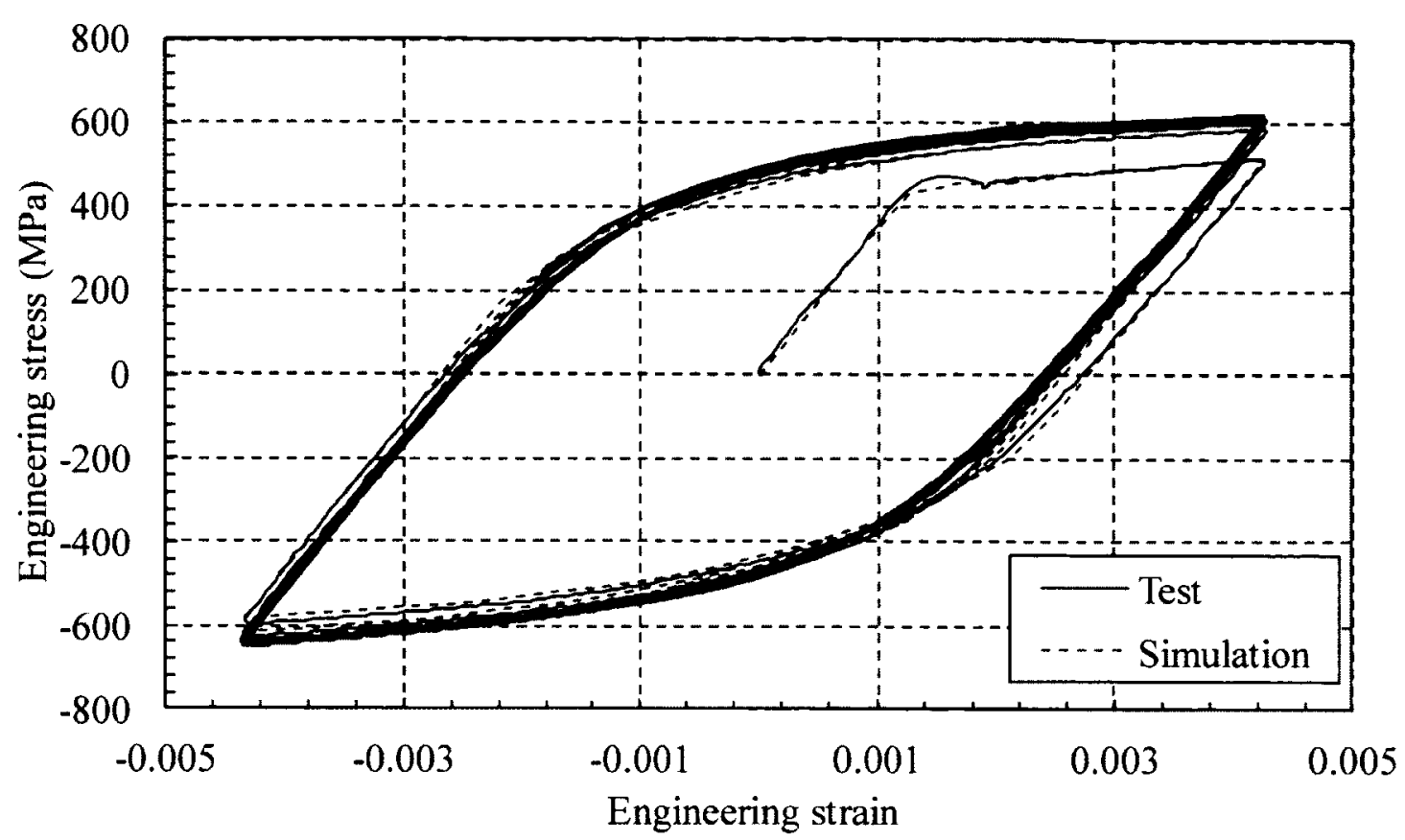

Figure F.17 Test and predicted engineering stress versus engineering strain curves for 50 cycles of BNE3R2

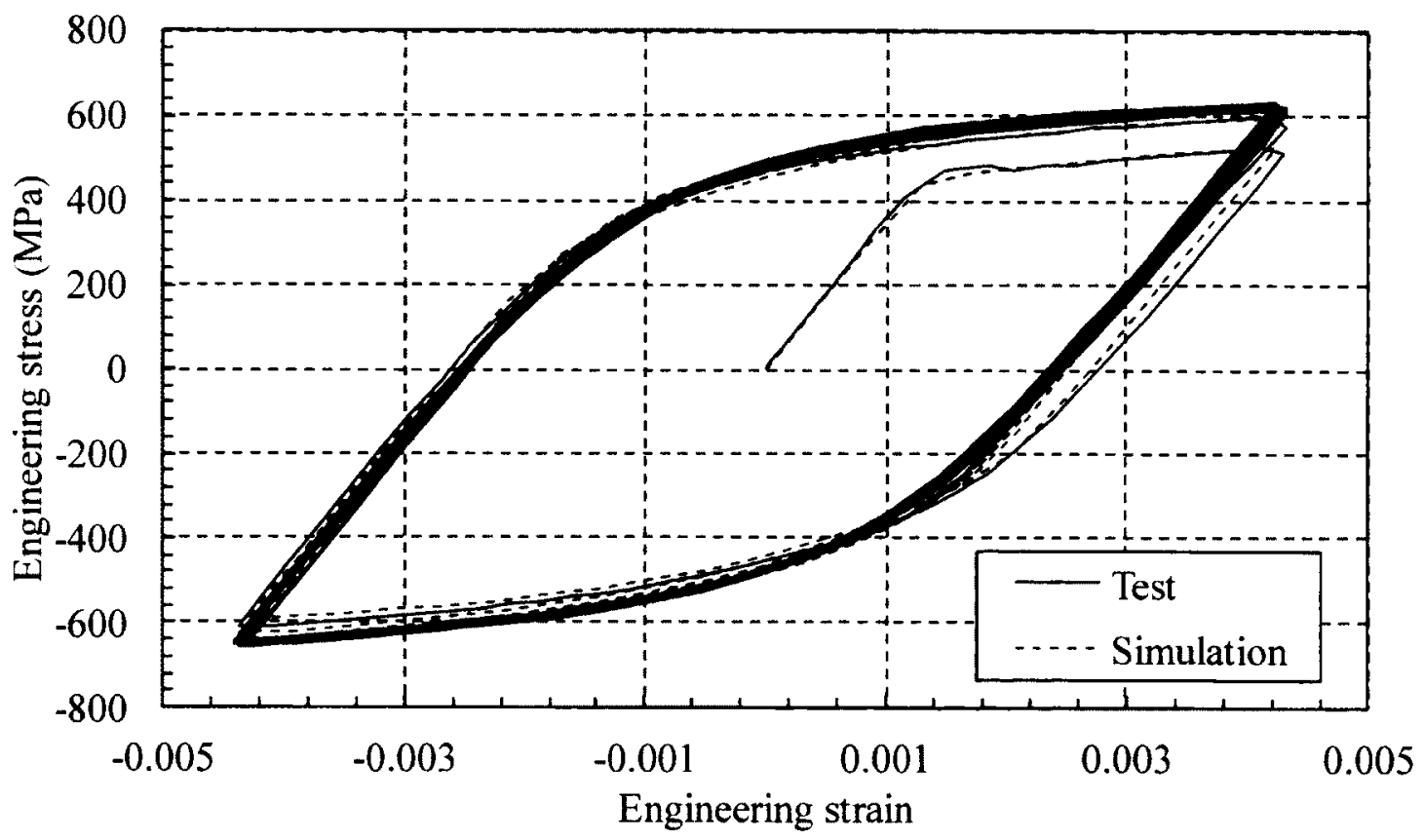

Figure F.18 Test and predicted engineering stress versus engineering strain curves for 50 cycles of BNE2R2 


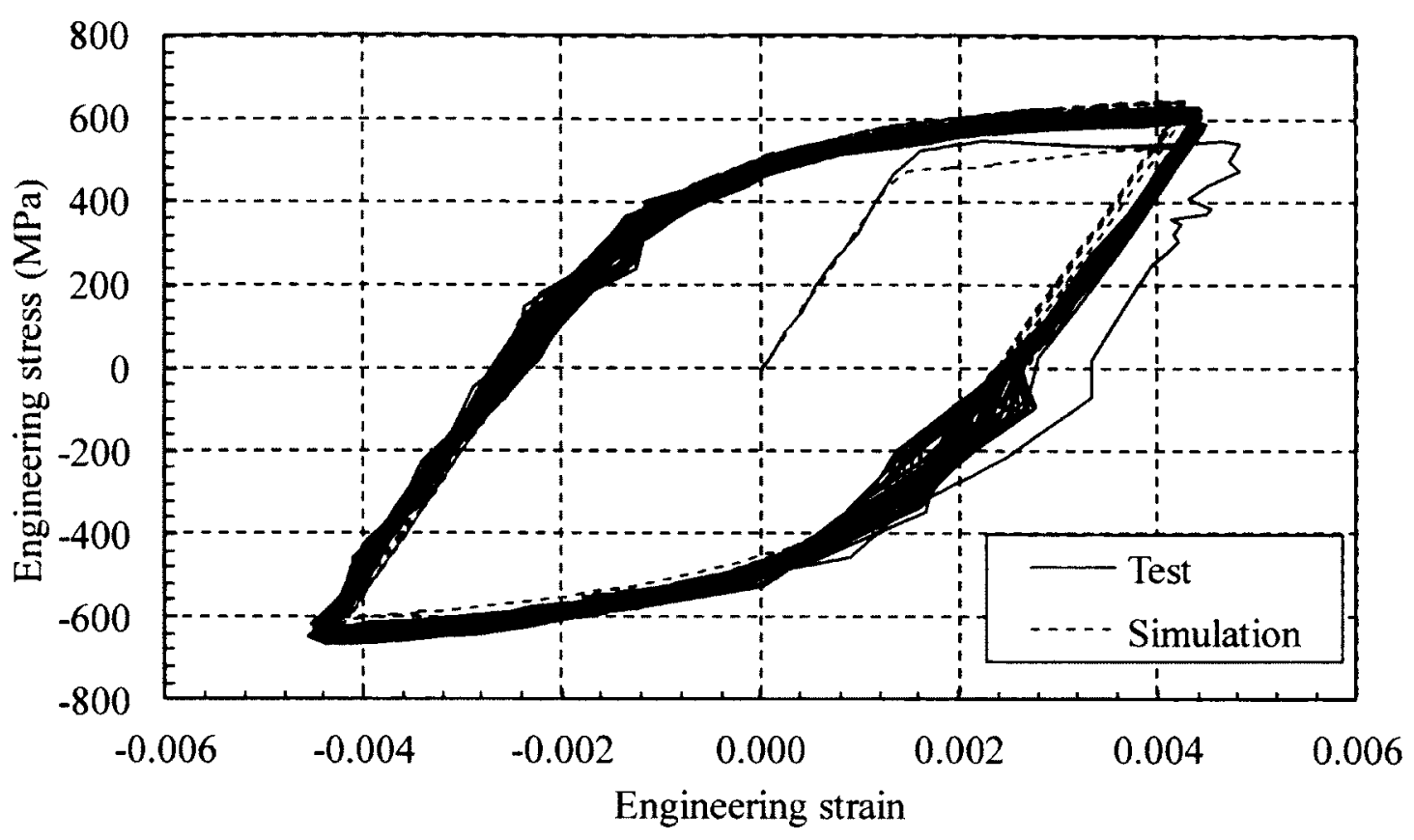

Figure F.19 Test and predicted engineering stress versus engineering strain curves for 50 cycles of BNE1R2

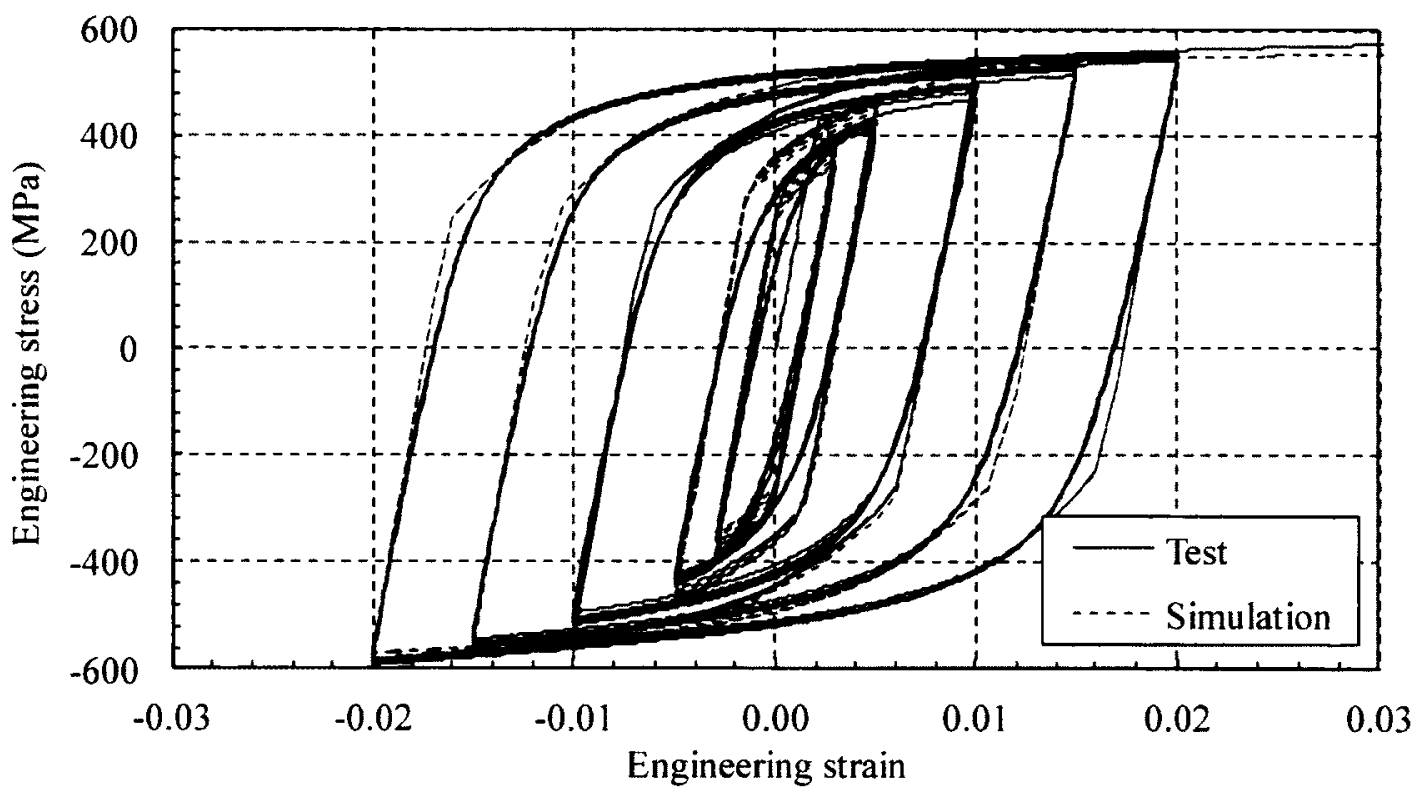

Figure F.20 Test and predicted engineering stress versus engineering strain curves for the cyclic portion of BTE3I 


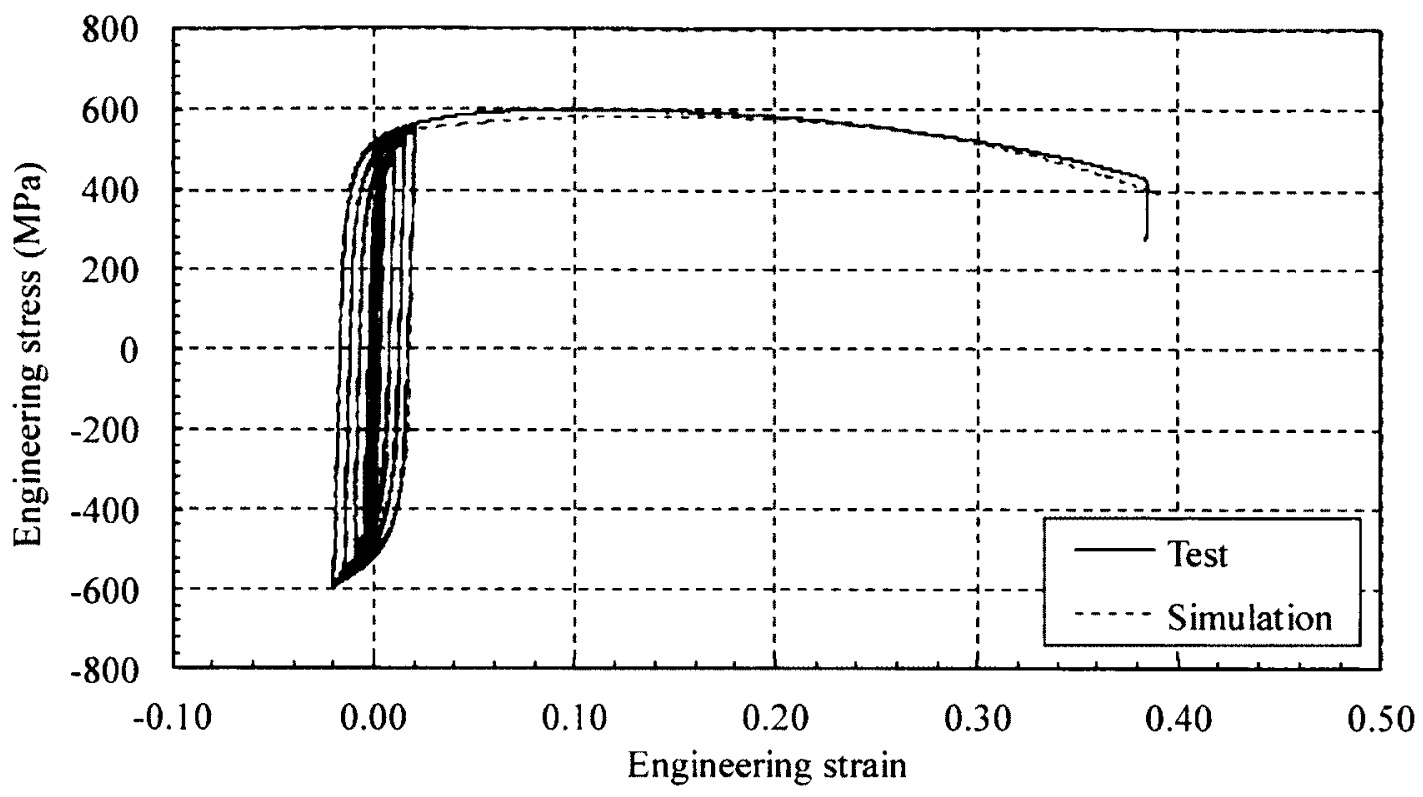

Figure F.21 Test and predicted engineering stress versus engineering strain curves for BTE3I 


\section{Appendix G Calibration and Optimization of the Kinematic Hardening Parameters}

In this appendix, the optimization and calibration of the kinematic hardening parameters used to carry out the numerical simulations of the tests in Chapter 5 are discussed. The procedures are employed in the calibration and optimization have been adopted from Wen (2012). These procedures have been discussed in greater details by Wen (2012).

\section{G.1 Calibration of Kinematic Hardening Parameters}

It has been discussed in Chapter 5 that effects of strain rate on the flow stress are accounted for through the change in the size of the yield surface. Thus, the kinematic hardening parameters $C_{n}$ and $\gamma_{n}$ can be calibrated using quasi-static test data at the strain rate of $10^{-4} \mathrm{~s}^{-1}$. Since, it is desirable to use the stabilized cyclic stress-strain curve over a large strain range in the calibration, the stress-strain curve of the $10^{\text {th }}$ cycle at the $\pm 2 \%$ loading strain range from ATE4D and BTE4D are selected to be used in the calibration of the kinematic hardening parameters of material A and B respectively. Similarly, the monotonic true flow stress versus true plastic strain curves for GT1E4ED and HT1E4ED up to a very large strain generated with the procedure outlined in Section 5.1.4 are used in the calibration. Kinematic hardening parameters for both material A and B are calibrated using the same procedures. However, only the calibration of the kinematic hardening parameters for material $\mathrm{A}$ is described in details below.

The estimated backstress versus the true plastic strain relationship at both small and large strains are used in the calibration. The relationship at small strain can be calculated from the stabilized true stress versus plastic strain curve of a uniaxial cyclic 
test. Therefore, the true flow stress of ATE4D, corrected for the tapered profile, from the tensile half-cycle at the $10^{\text {th }}$ cycle of the $\pm 2 \%$ loading strain range is used since the true stress versus true plastic strain curve appears to stabilize within the first few cycles of loading. The yield stress $\left(\sigma_{S}^{o}\right)$ can be assumed to be constant within one loading cycle in a stabilized cyclic stress-strain curve since the cumulative plastic strain at the $10^{\text {th }}$ cycle of the $\pm 2 \%$ loading strain range is close to 0.6 , the yield stress is close to being saturated as well. Thus, the yield stress can be subtracted from the flow stress of the tensile half-cycle of a stabilized stress-strain curve to obtain the backstress curve according to the equation

$$
\alpha=\sigma_{t}^{F}\left(\varepsilon^{p}\right)-\sigma^{o}
$$

It has been suggested by Wen (2012) that 15 evenly spaced backstress data points be used to calibrate the kinematic hardening parameters for small strain. For a stabilized stress-strain curve, the backstress can be generated according to Wen (2012) for each term of backstress as

$$
\alpha_{n}=-\frac{C_{n}}{\gamma_{n}}\left[\frac{1-e^{\left(-\gamma_{n} \Delta \varepsilon^{p}\right)}}{1+e^{\left(-\gamma_{n} \Delta \varepsilon^{p}\right)}}\right] e^{\left(-\gamma_{n} \varepsilon^{p}\right)}+\frac{C_{n}}{\gamma_{n}}\left[1-e^{\left(-\gamma_{n} \varepsilon^{p}\right)}\right]
$$

where $\varepsilon^{p}$ is the plastic strain taken as zero at the start of the half cycle, and $\Delta \varepsilon^{p}$ is the plastic strain range of the tensile half-cycle. The estimated backstress data and the true flow stress versus true plastic strain (shifted) curve used in the calculation are shown in Fig. G.1.

The estimated backstress versus true plastic strain at large strain can be calculated using the generated monotonic true flow stress versus true plastic strain curve for GT1E4ED up to a true strain of 10 using Eq. (5.2) with calibrated parameters in Table 5.2 and an assumed saturated yield stress. Results of the ATE4D test show that its cyclic stress-strain curve appears to have stabilized within a few cycles at the $\pm 2 \%$ strain range 
loading. Therefore, for the purpose of calibration, the yield stress is assumed to be saturated at a true plastic strain of 0.2 as suggested by Wen (2012). A saturated yield stress $\left(\sigma_{L}^{o}\right)$ different from that used for Fig. G.1 may be assumed in estimating the backstress at a large strain since the yield stress may not have been completely saturated at the $10^{\text {th }}$ loading cycle of the $\pm 2 \%$ loading strain range in the ATE4D test. The backstress can be calculated by subtracting the assumed saturated yield stress $\left(\sigma_{L}^{o}\right)$ from the flow stress according to Eq. (G.1). Wen (2012) has suggested that 30 evenly spaced backstress data from the plastic strain of 0.2 to 10.0 should be used together with the 15 backstress data points in Fig. G.1 for small strain to calibrate the kinematic hardening parameters $C_{n}$ and $\gamma_{n}$. A plastic strain upper limit of 10 is used in the calibration as the flow stress and generated backstress are expected to reach and maintain close to constant values after a true plastic strain of 5.0. Figure G.2 shows the estimated backstress data and the true flow stress versus true plastic strain curve used in the calculation. The backstress versus true plastic strain curve can be generated with Eq. (2.18) for monotonic uniaxial tension loading.

Using curve fitting, the kinematic hardening parameters are determined by minimizing the summation of squared error of fitting Eq. (G.2) through the backstress data points for small strain in Fig. G.1 and Eq. (2.18) through the backstress data points for large strain in Fig. G.2 together. Results of the curve fitting with the assumed yield stress $\left(\sigma_{S}^{o}\right)$ of $320 \mathrm{MPa}$ and saturated yield stress $\left(\sigma_{L}^{o}\right)$ of $330 \mathrm{MPa}$ are shown in Fig. G.1 and G.2. It can be seen that the generated backstress curve is able to fit the backstress data points quite well with two terms of backstress. Test and predicted engineering stress 
versus engineering strain curves are shown in Fig. G.3. It can be seen that there is a good agreement between the test and numerical simulation results.

\section{G.2 Optimization of the Kinematic Hardening Parameters}

Wen (2012) has shown that using the calibrated kinematic hardening $\left(C_{n}\right.$ and $\left.\gamma_{n}\right)$ parameters can provide an accurate modelling of the uniaxial cyclic loading test with which the test data have been used in the calibration. However, the numerical simulations using the same calibrated parameters are less accurate for tests of notched specimens. For this reason, Wen (2012) has proposed a procedure to obtain an optimum yield stresskinematic hardening parameters combination that allows for overall more accurate numerical simulations of both tapered and notched specimen tests incorporating notched specimen test data in the optimization process.

ANE4R2 and BNE4R2 notched specimen test data are used in the optimization, but other notched specimen tests can also be used. Using symmetry, the segment of notched specimen over half of the axial extensometer gauge length has been modelled with CAX8R, a bi-quadratic axisymmetric element with reduced integration. A mesh convergence study, discussed in Appendix C, has been performed to ensure the accuracy of the finite element mesh used in the numerical simulations. The resulting mesh selected for the notched specimen is shown in Fig. G.4. Due to the fact that the notched specimen is non-uniaxial, the average true strain rate experienced during a notched specimen test has been found to be 4 to 5 times higher than the applied nominal engineering strain rate. The calculated (measured) average true strain rate for each of the notched specimen test can be found in Table 4.6. Therefore, effects of strain rate on the flow stress have to be 
considered in order to carry out the numerical simulations properly. ABAQUS (Simulia 2010) allows for the consideration of strain rate effects on material properties with the mixed-mode hardening model in the form of a table of yield stress-true plastic strain data points for a series of strain rate. For this reason, the yield stress versus true plastic strain curves have been defined up to a strain rate of $10^{-3} \mathrm{~s}^{-1}$ using the monotonic uniaxial true flow stress versus true plastic strain curves generated in Section 5.2 for the numerical simulations of ANE4R2.

The procedure for optimization requires using different kinematic hardening parameters calculated with the procedure in Section G.1 by changing the assumed saturated yield stress $\left(\sigma_{L}^{o}\right)$ used in calculating the backstress for large strain while keeping the yield stress $\left(\sigma_{S}^{o}\right)$ used in calculating the backstress for small strain constant until the minimum error between the test and numerical results is obtained for that value of assumed $\sigma_{S}^{o}$. Comparisons are made between the test and numerical simulation results of ANE4R2 at zero (at each compressive and tensile half-cycle), maximum compressive and maximum tensile strains of every alternate cycle up to 20 cycles of loading on the engineering stress versus engineering strain curve. These locations are shown on the engineering stress versus engineering strain curve for the $20^{\text {th }}$ cycle of ANE4R2 in Fig. G.5. The summation of the square of the difference between the test and predicted engineering stress at these 40 points is minimized to obtain the optimum assumed saturated yield stress $\left(\sigma_{L}^{o}\right)$ for an assumed yield stress $\left(\sigma_{S}^{o}\right)$. Test and predicted engineering stress versus engineering strain and engineering stress versus cross-section ratio curves for ANE4R2 based on the kinematic hardening parameters calibrated with $\sigma_{S}^{o}=320 \mathrm{MPa}$ and $\sigma_{L}^{o}=330 \mathrm{MPa}$ are shown in Figs. G.6 and G.7 respectively. It can be 
seen that there is generally a good agreement between test and predicted engineering stress and engineering strain curves, and to a lesser extent the engineering stress versus cross-section change curves as well. Nevertheless, improvement can be made to the numerical simulation with different $\sigma_{S}^{o}$ and $\sigma_{L}^{o}$.

The next step in the optimization involves changing the value of $\sigma_{S}^{o}$ to determine the minimum error for the selected $\sigma_{S}^{o}$ using the method above. Repeating this procedure for different $\sigma_{S}^{o}$ values until the minimum overall error in terms of $\sigma_{S}^{o}$ is achieved gives the optimized values of $\sigma_{S}^{o}, \sigma_{L}^{o}, C_{n}$ and $\gamma_{n}$. Similar procedures have been used to calibrate the parameters for material B. The trial values of $\sigma_{S}^{o}, \sigma_{L}^{o}, C_{n}, \gamma_{n}$ and the associated error for each trial simulation, arranged sequentially in accordance to the optimization process, can be seen in Table G.1 and G.2 for material A and B respectively. The final optimized $\sigma_{S}^{o}, \sigma_{L}^{o}, C_{n}$ and $\gamma_{n}$ can be found in Table 5.4 for materials A and B. 
Table G.1 Material model parameters used in finding the optimum kinematic hardening parameters and their associated error for material A

\begin{tabular}{|c|c|c|c|c|c|c|}
\hline $\begin{array}{c}\sigma_{S}^{o} \\
(\mathrm{MPa})\end{array}$ & $\begin{array}{c}\sigma_{L}^{o} \\
(\mathrm{MPa})\end{array}$ & $\begin{array}{c}C_{1} \\
(\mathrm{MPa})\end{array}$ & $\gamma_{1}$ & $\begin{array}{c}C_{2} \\
(\mathrm{MPa})\end{array}$ & $\gamma_{2}$ & $\begin{array}{c}\text { Error } \\
\left(\mathrm{MPa}^{2}\right)\end{array}$ \\
\hline \multirow{4}{*}{320} & 310 & 25257 & 204.89 & 2129 & 6.40 & 3601 \\
\cline { 2 - 7 } & 320 & 24892 & 199.45 & 2000 & 6.22 & 2021 \\
\cline { 2 - 7 } & 330 & 24548 & 194.28 & 1872 & 6.04 & 6032 \\
\cline { 2 - 7 } & 318 & 24963 & 200.50 & 2025 & 6.26 & 1892 \\
\hline \multirow{5}{*}{340} & 320 & 18223 & 171.48 & 2165 & 6.37 & 6909 \\
\cline { 2 - 7 } & 330 & 17994 & 166.54 & 2033 & 6.20 & 2082 \\
\cline { 2 - 7 } & 340 & 17782 & 161.90 & 1904 & 6.02 & 3176 \\
\cline { 2 - 7 } & 333 & 17928 & 165.11 & 1994 & 6.14 & 1784 \\
\hline \multirow{4}{*}{330} & 340 & 12079 & 132.03 & 2072 & 6.19 & 4540 \\
\cline { 2 - 7 } & 350 & 11944 & 127.36 & 1935 & 6.00 & 2174 \\
\cline { 2 - 7 } & 360 & 11944 & 124.55 & 1807 & 5.82 & 6255 \\
\cline { 2 - 7 } & 349 & 11943 & 127.34 & 1935 & 6.00 & 2173 \\
\hline \multirow{4}{*}{320} & 20089 & 179.71 & 2115 & 6.32 & 3964 \\
\hline & 330 & 19828 & 174.71 & 1984 & 6.15 & 1837 \\
\cline { 2 - 7 } & 340 & 19589 & 170.04 & 1856 & 5.97 & 5532 \\
\cline { 2 - 7 } & 329 & 19852 & 175.20 & 1997 & 6.17 & 1784 \\
\hline
\end{tabular}


Table G.2 Material model parameters used in finding the optimum kinematic hardening parameters and their associated error for material B

\begin{tabular}{|c|c|c|c|c|c|c|}
\hline $\begin{array}{c}\sigma_{S}^{o} \\
(\mathrm{MPa})\end{array}$ & $\begin{array}{c}\sigma_{L}^{o} \\
(\mathrm{MPa})\end{array}$ & $\begin{array}{c}C_{1} \\
(\mathrm{MPa})\end{array}$ & $\gamma_{1}$ & $\begin{array}{c}C_{2} \\
(\mathrm{MPa})\end{array}$ & $\gamma_{2}$ & $\begin{array}{c}\text { Error } \\
\left(\mathrm{MPa}^{2}\right)\end{array}$ \\
\hline \multirow{4}{*}{380} & 370 & 31796 & 219.70 & 1484 & 5.57 & 4695 \\
\cline { 2 - 7 } & 380 & 31393 & 214.93 & 1365 & 5.35 & 3167 \\
\cline { 2 - 7 } & 390 & 31013 & 210.45 & 1251 & 5.13 & 6707 \\
\cline { 2 - 7 } & 378 & 31471 & 215.86 & 1389 & 5.39 & 3064 \\
\hline \multirow{4}{*}{400} & 380 & 24082 & 188.88 & 1525 & 5.57 & 7507 \\
\cline { 2 - 7 } & 390 & 23800 & 184.51 & 1406 & 5.36 & 3265 \\
\cline { 2 - 7 } & 400 & 23538 & 180.42 & 1291 & 5.14 & 4371 \\
\cline { 2 - 7 } & 393 & 23719 & 183.25 & 1371 & 5.29 & 3041 \\
\hline \multirow{4}{*}{420} & 400 & 17097 & 153.02 & 1450 & 5.38 & 6169 \\
\cline { 2 - 7 } & 410 & 16859 & 148.22 & 1323 & 5.13 & 4569 \\
\cline { 2 - 7 } & 420 & 16844 & 146.31 & 1219 & 4.95 & 8764 \\
\cline { 2 - 7 } & 408 & 17095 & 151.25 & 1357 & 5.21 & 4529 \\
\hline \multirow{4}{*}{390} & 380 & 27591 & 201.55 & 1442 & 5.45 & 3680 \\
\cline { 2 - 7 } & 390 & 27267 & 197.18 & 1326 & 5.24 & 3438 \\
\cline { 2 - 7 } & 400 & 26962 & 193.05 & 1212 & 5.01 & 8380 \\
\cline { 2 - 7 } & 385 & 27427 & 199.33 & 1383 & 5.35 & 2905 \\
\hline
\end{tabular}




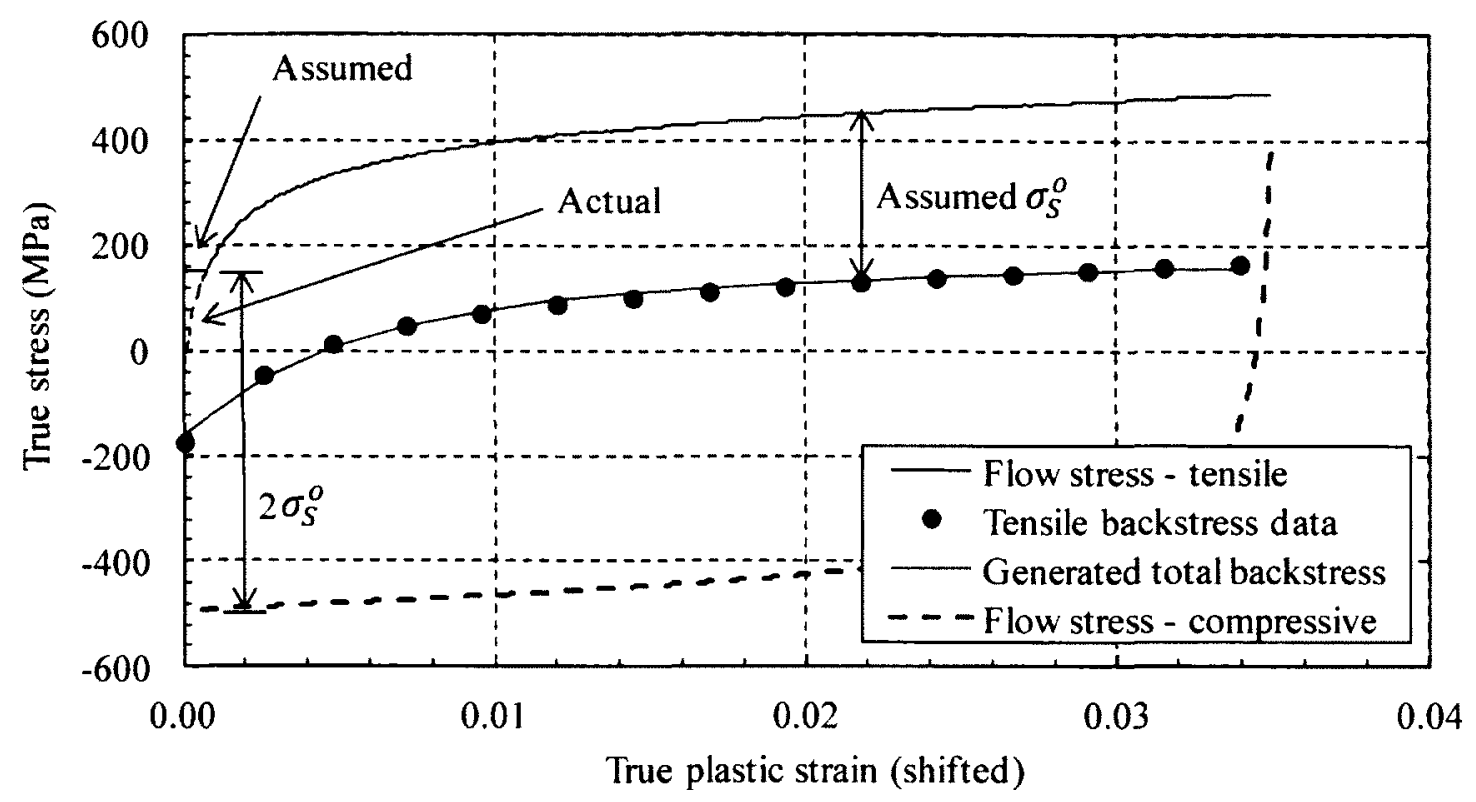

Figure G.1 Curve fitting of the backstress for small strain with an assumed yield stress $\left(\sigma_{S}^{o}\right)$ of $320 \mathrm{MPa}$ for ATE4D of material A

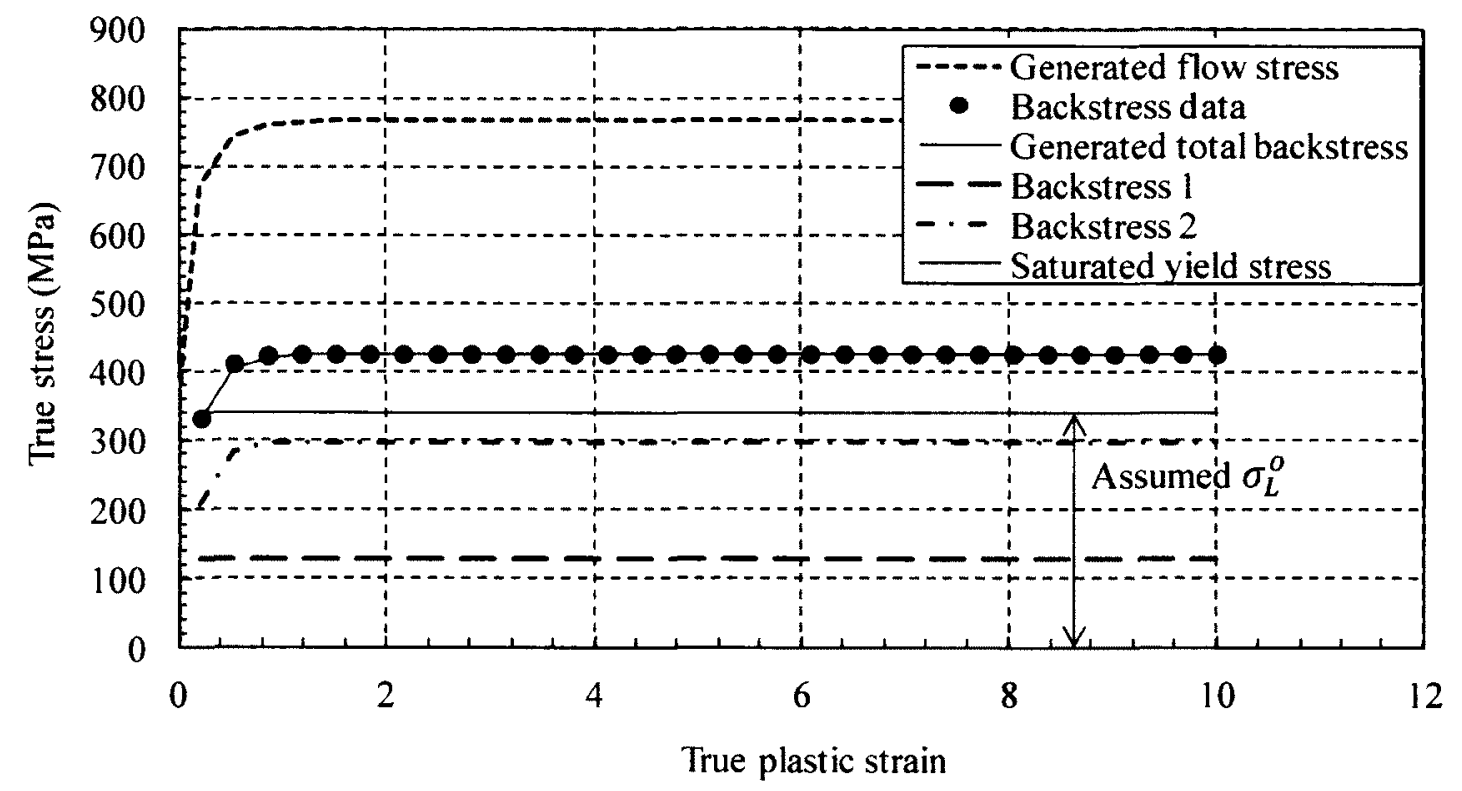

Figure G.2 Curve fitting of the backstress for large strain with an assumed saturated yield stress $\left(\sigma_{L}^{o}\right)$ of $330 \mathrm{MPa}$ for material A 


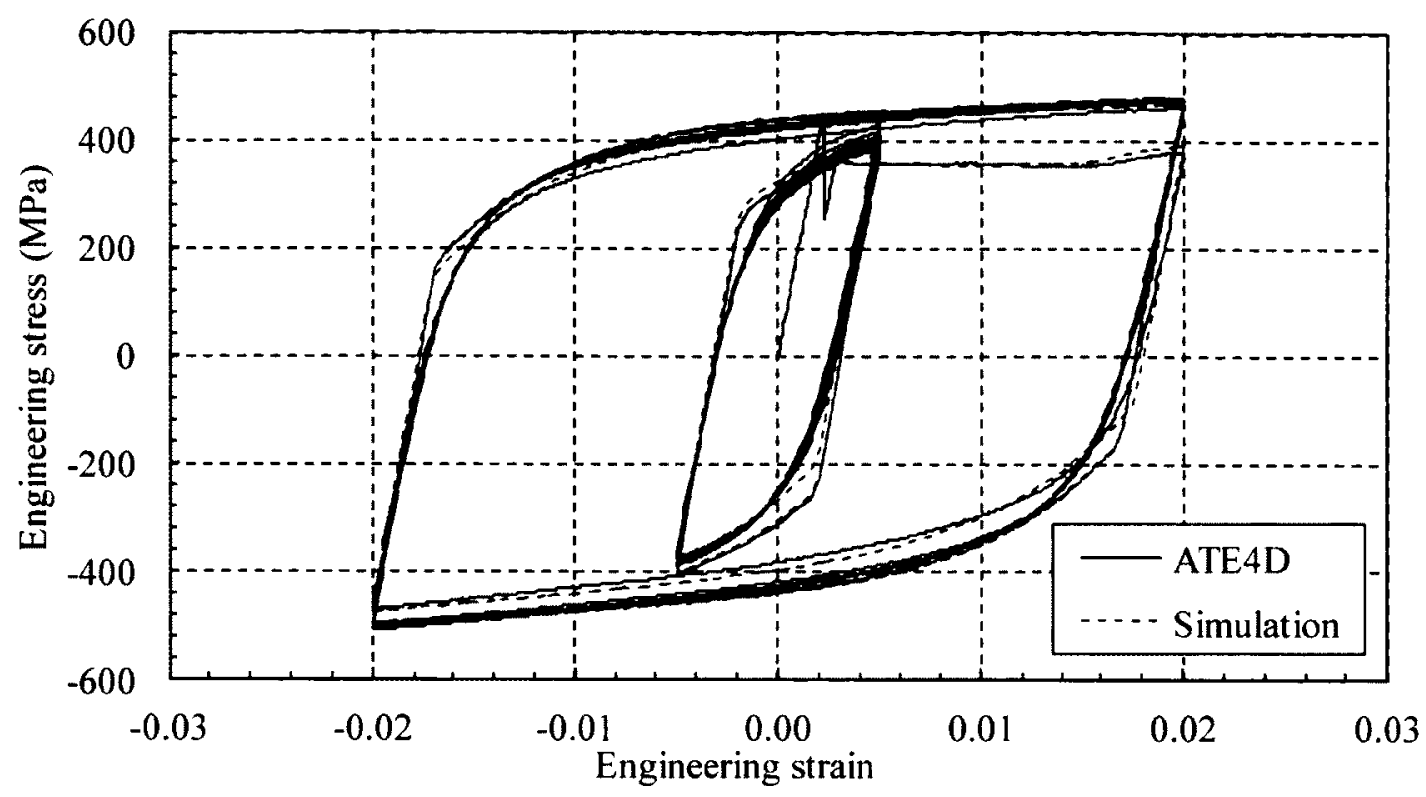

Figure G.3 Test and predicted engineering stress versus engineering strain for ATE4D using the kinematic hardening parameters calibrated with an assumed yield stress $\left(\sigma_{S}^{o}\right)$ of $320 \mathrm{MPa}$ and a saturated yield stress $\left(\sigma_{L}^{o}\right)$ of $330 \mathrm{MPa}$ 


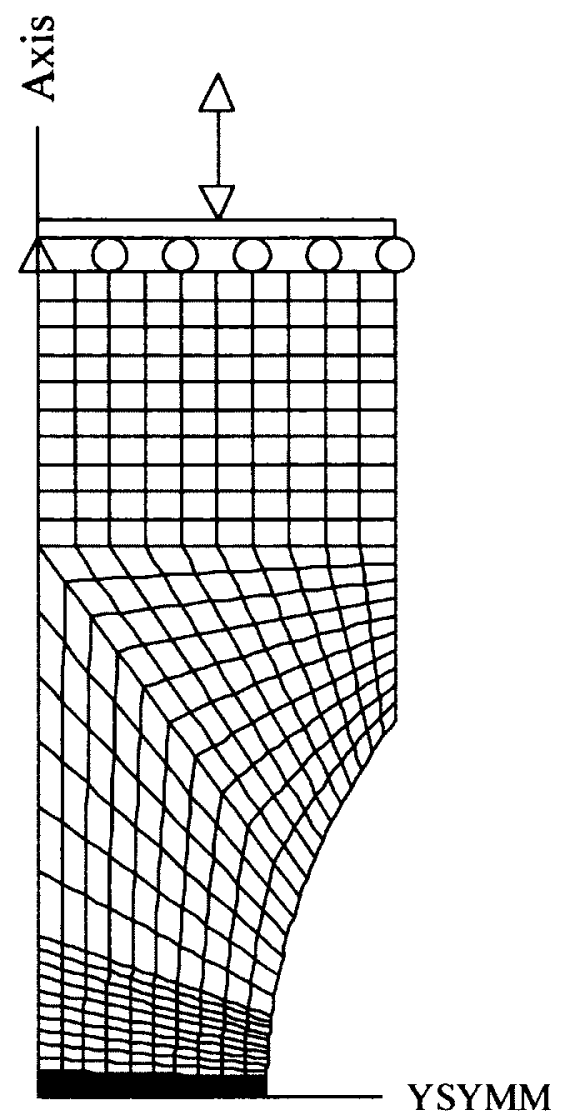

Figure G.4 The half gauge length model of the notched specimen 


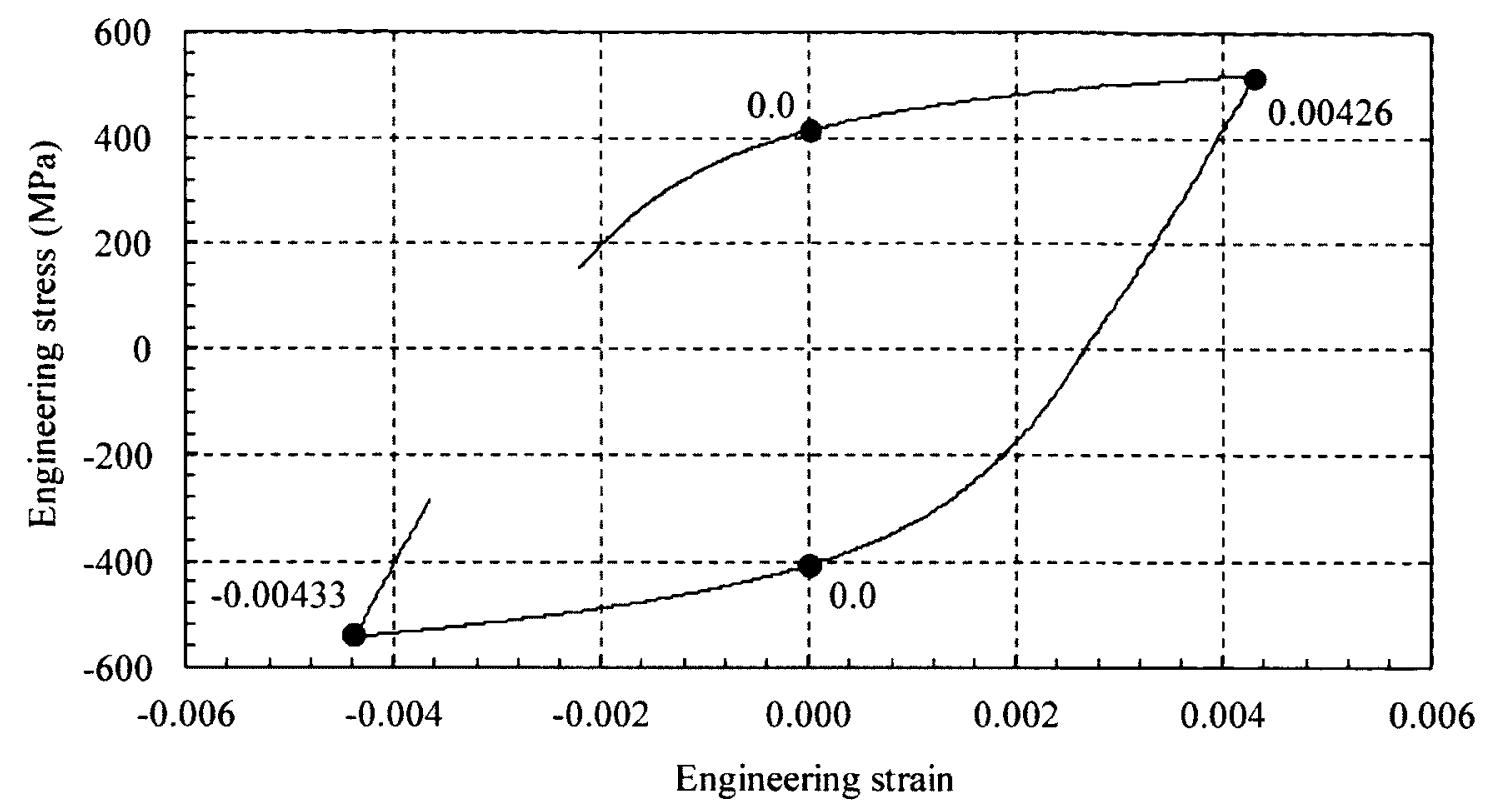

Figure G.5 Locations of engineering strains where errors are calculated for the $20^{\text {th }}$ cycle of ANE4R2

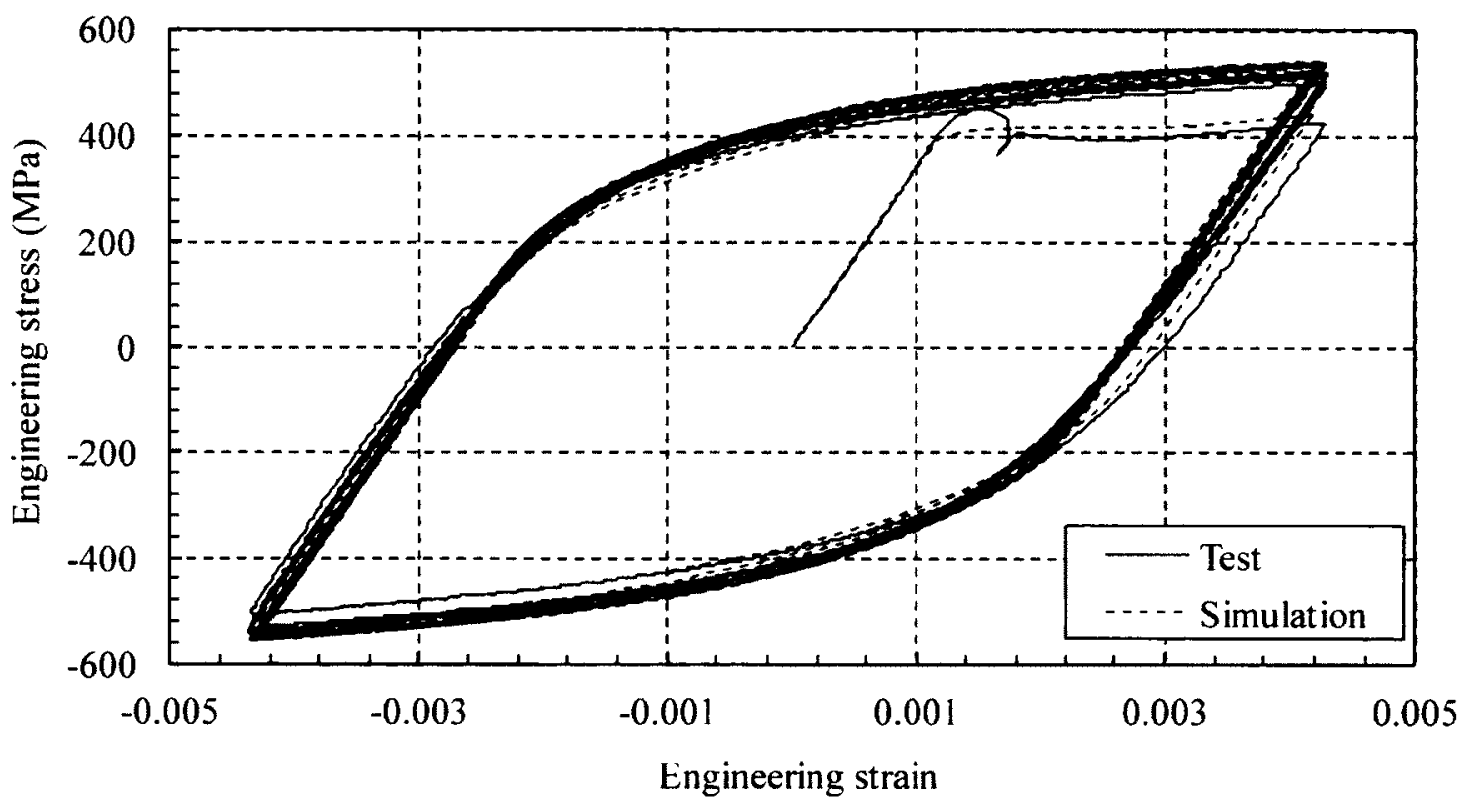

Figure G.6 Test and predicted engineering stress versus engineering strain for ANE4R2 using the kinematic hardening parameters calibrated with an assumed yield stress $\left(\sigma_{S}^{o}\right)$ of $320 \mathrm{MPa}$ and a saturated yield stress $\left(\sigma_{L}^{o}\right)$ of $330 \mathrm{MPa}$ 


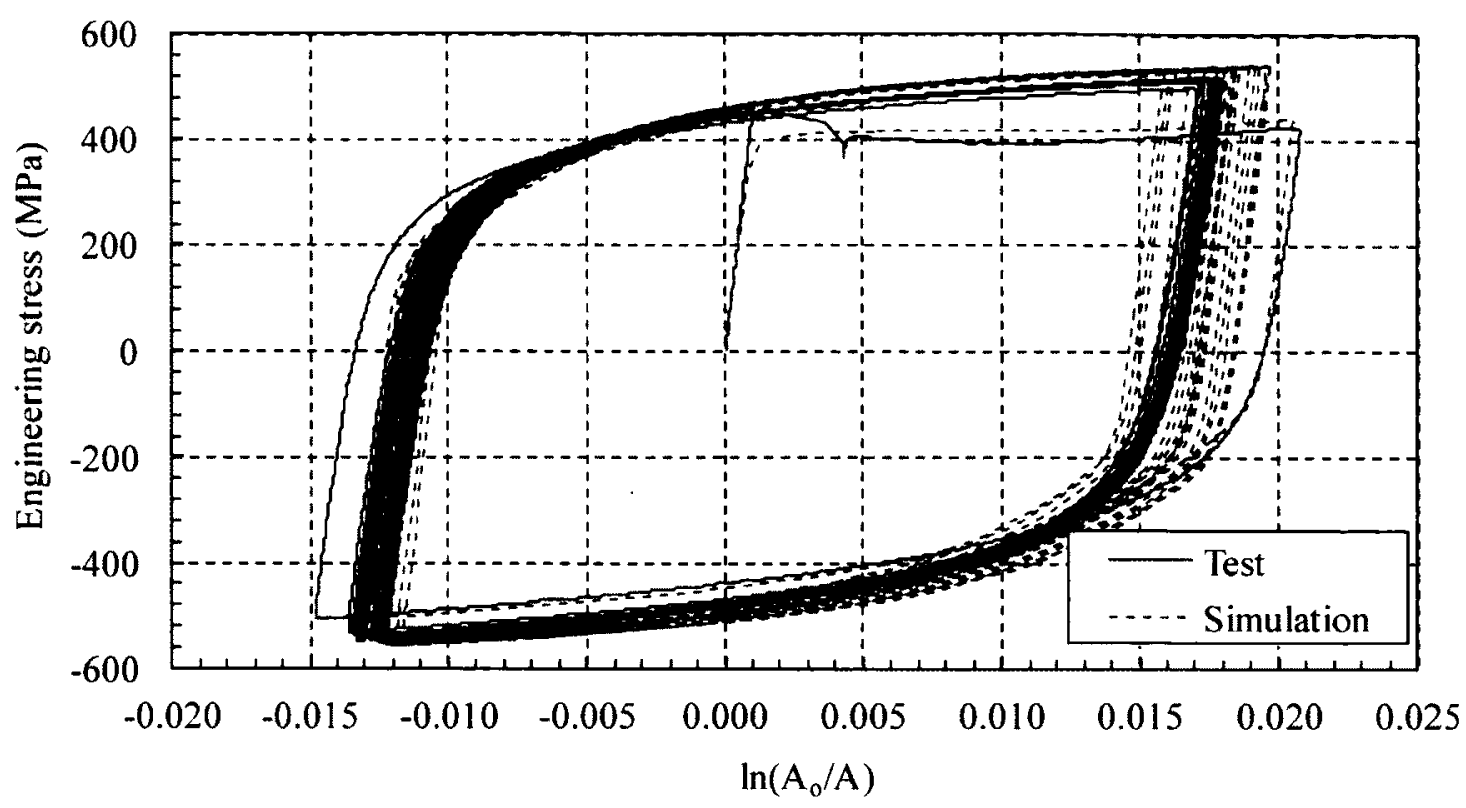

Figure G.7 Test and predicted engineering stress versus cross section change for ANE4R2 using the kinematic hardening parameters calibrated with an assumed yield stress $\left(\sigma_{S}^{o}\right)$ of $320 \mathrm{MPa}$ and a saturated yield stress $\left(\sigma_{L}^{o}\right)$ of $330 \mathrm{MPa}$ 